\title{
Biomedical Imaging and Sensing Conference
}

, "Biomedical Imaging and Sensing Conference," Proc. SPIE 11140, Biomedical Imaging and Sensing Conference, 1114001 (21 April 2019); doi: 10.1117/12.2535451

SPIE. Event: Optics and Photonics International Congress, 2019, Yokohama, Japan 


\section{PROCEEDINGS OF SPIE}

\section{Biomedical Imaging and Sensing Conference}

Toyohiko Yatagai

Editor

22-26 April 2019

Yokohama, Japan

Published by SPIE

Volume 11140 


\section{Contents}

$\begin{array}{ll}\text { vii } & \text { Author Index } \\ \text { xi } & \text { Conference Committee }\end{array}$

BISC-OMC JOINT SESSION

JS-3-02 Optical Tweezers in Biology

JS-3-03 Optical trap and laser interferometry in living cells

JS-3-04 Non-invasive NIR imaging of diseases in Living mice

JS-3-05 Isotropic Quantitative Differential Phase Contrast Microscopy with Vortex Asymmetric Illumination Patterns

JS-3-06 Monitoring mitochondrial dynamics within mitotic apparatus by lightsheet microscopy

\section{SUPER-RESOLUTION MICROSCOPY}

BISC-1-01 The molecular architecture of proteins at the base of primary cilia revealed by super-resolution microscopy

BISC-1-02 Subdiffraction-limit optical-pattern generation and its application to super-resolution imaging

BISC-1-03 Optically sectioned, super-resolution imaging by image scanning microscopy using optimized reconstruction algorithm

BISC-1-04 Applying terahertz solid immersion microscopy for sub-wavelength-resolution imaging of soft biological tissues

\section{OPTICAL COHERENCE TOMOGRAPHY}

BISC-2-01 Needle-Probe Optical Coherence Tomography for Intelligent Guidance of Epidural Anesthesia

BISC-2-02 Advances in Optical Coherence Elastography

BISC-2-03 Non-invasive 3D imaging for biology by using OCT technique

BISC-2-04 Swept-Source Optical Coherence Tomography Imaging of the Guinea Pig Cochlea 
BISC-2-05 A calibration method for central corneal thickness measurement from Scheimpflug imaging in a non-contact tonometer with pachymetry

\section{ADVANCED MICROSCOPY}

BISC-3-01 Optical microscopy with optical-frequency-comb

BISC-3-02 Blood glucose measurement based on mid-infrared absorption spectroscopy

BISC-3-03 Dynamic 3D SERS imaging of small molecules in live cells

BISC-3-04 Fluorescence imaging of microbeads by transport of intensity equation

DIGITAL HOLOGRAPHIC MICROSCOPY

BISC-4-01 Multimodal digital holographic microscopy

BISC-4-02 Planar lightwave circuit digital holographic microscope and biomedical imaging applications

BISC-4-03 Heterodyne terahertz holography of biological samples

STRUCTURED ILLUMINATION MICROSCOPY

BISC-5-01 Resolution enhancement methods of nonlinear microscopy based on structured illumination

BISC-5-03 Maximizing illumination contrast in arbitrary direction for Structured Illumination Microscopy

BISC-5-04 Dual-channel structured illumination microscopy setup based on Digital Micromirror Device

BISC-5-05 Simulating compressive three-dimensional structured illumination microscopy

BISC-5-06 Structured light imaging with selective spatial frequency domain filtering: A search for the right spatial frequencies to differentiate between benign and malignant tissue subtypes

BISC-IP JOINT SESSION

JS-4-01 Light-field acquisition and super-resolution with structured illumination

JS-4-02 Differential Phase Contrast Volume Holographic Incoherent Microscopy

JS-4-03 DOPPLER PHASE-SHIFTING OPTICAL LOW-COHERENCE TOMOGRAPHY 
JS-4-04 Multi-focal holographic differential confocal microscopy

JS-4-05 Multi-spectral digital holography with burst imaging method

JS-4-06 Imaging cytometry without image reconstruction (Ghost Cytometry)

JS-4-07 Simulation of Multi-Exposure Laser Speckle Contrast Blood Flow Imaging based on Multi-Tap Charge Modulator CMOS Image Sensor

JS-4-08 In vivo 3D image reconstruction of lamina cribrosa in glaucoma eyes

JS-4-09 Reversible transformation of DNA gels using light signals

\section{ADVANCED MICROSCOPY II}

BISC-6-01 Deep imaging techniques by spatio-temporal control of excitation pulses

BISC-6-02 Acousto-optic spatial and spectral filtering for incoherent phase imaging

BISC-6-03 High resolution, high sensitivity, X-ray phase contrast imaging obtained with a Hartmann mask based imaging system for mammography applications

BISC-6-04 Multi-focal holographic SAX microscopy

BISC-6-05 Real-time 3D interactive microscopy

ADVANCED MICROSCOPY AND SIGNAL PROCESSING

BISC-7-01 Label-free imaging of live cell morphology by path-length stabilized quantitative phase microscope

BISC-7-02 Holographic Light Sheet Microscopy for Live Caenorhabditis elegans

BISC-7-03 Simple intensity equalization methods in SLM generated multispots

BISC-7-04 A new signal processing in laser Doppler flowmetry

\section{POSTER SESSION}

BISC-P-01 Visualization of transcutaneous bilirubin, hemoglobin, and melanin based on hyperspectral diffuse reflectance imaging

BISC-P-02 High-Efficient Holographic Photopolymer Based on Fluorinated Epoxy Resin

BISC-P-03 Fast-physical optics modeling of two-photon microscopy with 3D-structured illumination 
BISC-P-04 Development of a Long-Wavelength Swept-Source Optical Coherence Tomography System for High-Content Ex Vivo Tissue Imaging

BISC-P-05 Label-fee dynamic imaging of mitochondria and related organelles inside live cells with simultaneous dual-wavelength photothermal microscopy

BISC-P-06 Lensless digital holographic imaging through diffusive glass with different diffusion angles

BISC-P-07 Lensless Phase Difference Microscopy with Designed Imaging Device

BISC-P-08 Suppression of speckle noise in digital holography using speckle correlation properties of outof-plane direction

BISC-P-09 Scan-less Full-field Fluorescence Microscopy by using 2D Spectral Disperser and Dual-comb Optical Beats

BISC-P-10 Femtosecond laser microsurgery of cells using confocal surface detection system

BISC-P-11 Enhanced sensitivity of non-enzymatic glucose sensor using $<$ strong $></$ strong $>$ different concentrations of Al-doped ZnO nanorod

BISC-P-12 Development of photoplethysmogram sensor with an LED and sampling rate assessment

BISC-P-13 Quantitative imaging of efflux pumps in planktonic and biofilm-associated bacteria through single-molecule localization microscopy

BISC-P-14 Implementation of Imaging Post Amplifier on Scan-less Confocal Dual-Comb Microscope

BISC-P-15 Dynamic phase imaging of Haematococcus pluvialis cells by transport of intensity equation

BISC-P-16 In vivo multimodal optical biopsy of skin cancer

BISC-P-17 Differentiation of the microvasculature with different blood flow speed based on variable interscan time analysis in OCT angiography skin imaging

BISC-P-18 Non-invasive measurement of blood glucose by attenuated total reflection spectroscopy with fixed-wavelength quantum cascade lasers

BISC-P-19 Phase-shifting digital holography with burst-imaging method

BISC-P-20 Total protein measurement features in venous and capillary blood using Raman spectroscopy method

BISC-P-21 Moisture measurement of oral mucosa based on near-infrared diffuse reflection spectroscopy by using optical fiber probe

BISC-P-22 Optical microscopy using annular full-color LED for quantitative phase and spectroscopic imaging of biological tissues

BISC-P-23 Non-invasive blood glucose measurement by using fast swept-wavelength quantum cascade laser 
BISC-P-24 New library of spectral reflectance and point spread function developed by Monte Carlo simulation with nine-layered skin model

BISC-P-25 Early diagnosis of teeth caries with non-invasive optical coherence tomography

BISC-P-26 Development of silicone-based skin tissue phantom and its spectral reflectance properties

BISC-P-27 Study on the option of the optimal time length of measurement in resting-state fNIRS brain imaging

BISC-P-28 Complex Measurements of Fluorescence and Speckle Contrast in Laboratory Mice during Pancreas Ischemia Modeling

BISC-P-29 Study on properties of near-infrared excitation upconversion nanoparticles for optogenetic applications

BISC-P-30 Tapered optical fiber temperature sensor coated with DNA based biopolymer

BISC-P-31 High spatial resolution ion imaging with potentiometric sensor using an electron beam

BISC-P-32 Single-shot recording of both front and rear surfaces of object by digital holography using a polarization-imaging camera

BISC-P-33 Imaging of sound by a digital holographic microscope

BISC-P-34 Glucose concentration measurement with enzymatic modified cuvette by heterodyne interferometer 


\section{Authors}

Numbers in the index correspond to the numbers in the table of contents.

Aizu, Yoshihisa, BISC-P-24, BISC-P-26, BISC-P-08 Akikusa, Naota, BISC-P-23

Ando, Jun, BISC-3-03

Arao, Kohei, BISC-P-32

Arimoto, Hidenobu, BISC-P-06, BISC-P-12

Artemyev, Dmitry, BISC-P-16, BISC-P-20

Awatsuji, Yasuhiro, BISC-3-04, BISC-4-01,

BISC-7-03, BISC-P-32, BISC-P-33

Bando, Kazuki, BISC-3-03

Banh, Tuan, JS-4-03

Bianchi, Silvio, BISC-6-05

Bratchenko, Ivan, BISC-P-16, BISC-P-20

Buranasiri, Prathan, JS-4-08

Capitanio, Marco, BISC-P-13

Cetin, Mujdat, BISC-5-05

Champagne, Justine, BISC-6-02

Chang, Teng-Chieh, BISC-P-04

Chang, Vincent, BISC-2-05

Chen, Bi-Chang, JS-3-06

Chen, Chin-Yi, JS-3-06

Chen, Hsin, BISC-2-04

Chen, Jung-Chih, BISC-P-27, BISC-P-29 Chen, SZU-Yu, BISC-5-01

Chen, Ting-Hao, BISC-P-04, BISC-P-17

Chen, Ting, BISC-2-04

Chen, Yu-Han, BISC-P-34

Chen, Zhongping, BISC-2-02

Chernomyrdin, Nikita, BISC-1-04

Chia, Chou-Min, JS-4-04

Chia-Yen Ke, BISC-P-25

Chia, Yu-Hsin, JS-4-02

Chien, Ju-Hsuan, BISC-7-02

Choi, Bernard, JS-4-07

Chuang, Ching-Cheng, BISC-P-27, BISC-P-29

Chuang, Yu-Hsuan, JS-3-05

Chueh, Chuan-Bor, BISC-P-04, BISC-P-17

Chueh, Chuan, BISC-2-04

Crouzet, Christian, JS-4-07

Dai, Ching-Liang, BISC-P-34

Davydkin, lgor, BISC-P-20

De La Rochefoucault, Ombeline, BISC-6-03

Deng, Dingnan, BISC-P-15

Dodo, Kosuke, BISC-3-03

Dolganova, Irina, BISC-1-04

Dong, Chunyang, BISC-4-03

Dong, Guan-Cheng, BISC-P-27

Dovillaire, Guillaume, BISC-6-03

Dremin, Viktor, BISC-7-04, BISC-P-28

Dunaev, Andrey, BISC-7-04, BISC-P-28 Favre-

Bulle, Itia, JS-3-02
Fujita, Katsumasa, BISC-3-03

Fukuda, Takahito, BISC-P-32

Funamizu, Hideki, BISC-P-08, BISC-P-26, BISC-P-24

Gardini, Lucia, BISC-P-13

Gryadunova, Anna, BISC-1-04

Hamada, Michito, JS-3-04

Haneishi, Hideaki, BISC-P-01

Harms, Fabrice, BISC-6-03

Hase, Eiji, BISC-P-09, BISC-P-14

Hasegawa, Satoshi, BISC-P-10

Hashisaka, Tomoki, BISC-P-24

Hayasaki, Yoshio, JS-4-03, JS-4-05, BISC-P-10,

BISC-P-19

Hellmann, Christian, BISC-P-03

Hirakawa, Tatsuya, BISC-P-32

Hisaka, Masaki, BISC-P-22

Hong, Seongjin, BISC-P-30

Horisaki, Ryoichi, JS-4-06

Hsiao, Haw, BISC-6-04

Hsieh, Ting-Yu, BISC-7-02

Hsu, Cheng-Chih, BISC-P-34

$\mathrm{Hu}, \mathrm{Xuemei,} \mathrm{BISC-P-07}$

Huang, BO, BISC-2-04

Huang, Kuang-Yuh, BISC-6-04

Huang, Tai-Ling, BISC-P-29

Huang, Yin-Peng, BISC-P-04, BISC-P-17

Huang, Zhijian, BISC-P-07

Hung, Jui-Ting, BISC-5-01

Hung, Yi-Ping, BISC-P-04, BISC-P-17

Inami, Wataru, BISC-P-31

Isobe, Keisuke, BISC-6-01

Itahashi, Yoko, JS-4-06

Iwanaga, Masashi, BISC-P-10

Jin, Guofan, BISC-5-03

Jung, Aeri, BISC-P-30

Kagawa, Keiichiro, JS-4-07

Kandurova, Ksenia, BISC-P-28

Karalkin, Pavel, BISC-1-04

Karasik, Valery, BISC-1-04

Kassirov, Dmitry, BISC-P-16

Kastelik, Jean-Claude, BISC-6-02

Kawahito, Shoji, JS-4-07

Kawata, Satoshi, BISC-3-03

Kawata, Yoshimasa, BISC-P-31

Khristoforova, Yulia, BISC-P-16

Kino, Saiko, BISC-3-02, BISC-P-18, BISC-P-23

Kobori, Yuta, BISC-P-24

Koike, Kota, BISC-3-03

Konovalov, Semen, BISC-P-16

Korn, Dietmar, BISC-6-03

Koshimizu, Yohei, BISC-2-03 
Koyama, Takuya, BISC-3-02, BISC-P-18

Kozlov, Igor, BISC-7-04

Kucheryavenko, Anna, BISC-1-04

Kumar, Manoj, BISC-3-04, BISC-4-01, BISC-7-03

Kuo, Wen-Chuan, BISC-2-0

Kuwahara, Teru, BISC-P-26

Kuzmina, Tatiana, BISC-P-20

Lai, Pei-Chen, BISC-P-34

Lee, Chia-Yen, BISC-P-27, BISC-P-29

Lee, Hsiang-Chieh, BISC-P-04, BISC-P-17,

BISC-P-25

Lee, Hsiang, BISC-2-04

Lee, Ya-Ju, BISC-P-25

Legrand, Jérôme, BISC-6-03

Levecq, Xavier, BISC-6-03

Li, He, BISC-4-03

Li, Wei-Che, BISC-P-29

Li, You-Xuan, BISC-P-34

Liao, Jung-Chi, BISC-1-01

Lin, Brian, BISC-2-05

Lin, Yu-Zi, JS-3-05

Lisauskas, Alvydas, BISC-4-03

Liu, Guoxuan, BISC-5-03, BISC-5-04

Liv, Xiaoli, BISC-P-15

Luo, Shi-Wei, BISC-P-11

Luo, Yuan, BISC-6-04, BISC-7-02, JS-3-05, JS-4-02, JS-4-04

Lykina, Anastasiya, BISC-P-16, BISC-P-20

Ma, Haowen, BISC-P-07

Maeda, Takaaki, BISC-P-26

Maloney, Benjamin, BISC-5-06

Mamoshin, Andrian, BISC-P-28

Manassakorn, Anita, JS-4-08

Mastropietro, Francesca, BISC-6-03

Matoba, Osamu, BISC-3-04, BISC-4-01, BISC-7-03,

BISC-P-32, BISC-P-33

Matsuura, Yuji, BISC-3-02, BISC-P-18, BISC-P-21, BISC-P-23

McClatchy, David, BISC-5-06

Meglinski, Igor, BISC-7-04

Michikawa, Takayuki, BISC-6-0

Midorikawa, Katsumi, BISC-6-01

Minakawa, Masashi, BISC-P-0 1

Minamikawa, Takeo, BISC-3-01, BISC-P-09,

BISC-P-14

Miura, Kenjiro, JS-4-01

Miura, Makoto, BISC-P-10

Miwa, Yoshihiro, JS-3-04

Miyawaki, Atsushi, BISC-6-01

Miyazaki, Jun, BISC-P-05

Mizuno, Daisuke, JS-3-03

Mizuno, Seiya, JS-3-04

Mizuno, Takahiko, BISC-P-09, BISC-P-14

Mochida, Shogo, BISC-P-32

Molchanov, Vladimir, BISC-6-02

Mori, Yuki, BISC-2-03

Moryatov, Alexander, BISC-P-16

Myakinin, Oleg, BISC-P-16

Nakano, Kazuya, BISC-P-01
Namiki, Kana, BISC-6-01

Nicolas, Lionel, BISC-6-03

Nii, Kiyohisa, BISC-P-31

Nishidate, Izumi, BISC-P-0 1

Nishimura, Takahiro, BISC-1-02, JS-4-09

Nishio, Kenzo, BISC-P-32, BISC-P-33

Nishizawa, Kenji, JS-3-03

Noji, Hiroyuki, JS-4-06

Ogura, Yusuke, BISC-1-02, JS-4-09

Oh, Kyunghwan, BISC-P-30

Ohya, Takehiro, BISC-P-24

Omatsu, Takashige, JS-3-01

Osakada, Fumitaka, BISC-6-01

Oshima, Kenta, JS-3-04

Ota, Sadao, JS-4-06

Ozgurun, Baturay, BISC-5-05

Panneer Selvam, Sivakumar, JS-4-07

Paulsen, Keith, BISC-5-06

Pavone, Francesco, BISC-P-13

Peng, Hsuan-Yuan, BISC-P-17

Peng, Xiang, BISC-P-15

Pham, Quang, JS-4-03

Piavchenko, Gennadii, BISC-P-28

Piponnier, Martin, BISC-6-03

Pogue, Brian, BISC-5-06

Quan, Xiangyu, BISC-3-04, BISC-4-01, BISC-7-03

Rajput, Sudheesh, BISC-3-04, BISC-P-33

Raupov, Dmitry, BISC-P-16

Rizzo, Elizabeth, BISC-5-06

Roskos, Hartmut, BISC-4-03

Rubinsztein-Dunlop, Halina, JS-3-02

Rungsawang, Rakchanok, BISC-6-03

Sakasai, Tomoki, JS-3-04

Sakazume, Yoshiki, BISC-P-08

Sato, Issei, JS-4-06

Seryogina, Evgeniya, BISC-P-28

Shamina, Lyudmila, BISC-P-16

Shi, Rui, BISC-P-03

Shibano, Satoru, BISC-P-31

Shibata, Naoto, BISC-P-23

Shimizu, Kazuki, BISC-P-33

Shimomura, Suguru, JS-4-09

Shinkawa, Daiki, BISC-1-02

Smith, Nicholas, BISC-3-03

Sodeoka, Mikiko, BISC-3-03

Song, Sanggwon, BISC-P-30

Stilgoe, Alexander, JS-3-02

Streeter, Samuel, BISC-5-06

Su, Yan-Kuin, BISC-P-11

Sugino, Yujiro, JS-3-03

Sugiyama, Atsushi, BISC-P-23

Sugiyama, Fumihiro, JS-3-04

Takahashi, Satoru, JS-3-04

Takase, Yuki, BISC-P-33

Tamada, Yosuke, BISC-1-02, BISC-4-01

Tan, Cheng-Zn, BISC-5-01

Tanaka, Junko, JS-3-04

Tang, Qijian, BISC-P-15

Tanida, Jun, BISC-1-02, JS-4-09

Tao, Ming, BISC-P-15 
Tao, Yue, BISC-P-07

Tomita, Hikaru, BISC-P-31

Toumon, Yasunobu, BISC-P-05

Tsai, Cheliang, BISC-2-05

Tsai, Meng-Tsan, BISC-P-17, BISC-P-25

Tsai, Meng, BISC-2-04

Tsai, Ting-Yen, BISC-P-04, BISC-P-17

Tsai, Ting, BISC-2-04

Tsai, Ying-Ju, JS-3-05

Tsai, You-Nan, BISC-P-04

Tsai, You, BISC-2-04

Tsuda, Takuya, BISC-P-14

Tuchin, Valery, BISC-1-04

Ujiie, Takumi, JS-4-05, BISC-P-19

Umeda, Katsuhiro, JS-3-03

Uozumi, Jun, BISC-P-08

Usuki, Shin, JS-4-01

Vignolini, Tiziano, BISC-P-13

Wake, Hiroaki, BISC-7-03

Wang, Chia-Cheng, BISC-P-27

Wang, Chih, BISC-2-04

Wang, Ching-Yu, BISC-P-04

Wang, I-Jong, BISC-2-05

Wang, Jen-Hsuan, BISC-P-29

Wang, Kuo-Jen, BISC-2-05

Wang, Wen-Cheng, JS-3-06

Wang, Yen-Li, BISC-P-25

Wang, Zi-Hao, BISC-P-1

Wares, Md. Abdul, BISC-P-01

Watanabe, Eriko, BISC-4-02

Watanabe, Wataru, BISC-P-06, BISC-P-12

Wells, Wendy, BISC-5-06

Wu, Chyan-Chyi, BISC-P-34

Wu, Hao-Hao, BISC-5-01

Wu, Jui-ching, BISC-7-02

Wu, Meng-Shan, BISC-P-17

Wu, Tso-Hua, JS-4-04, BISC-6-04

Wu, Yi-Chun, BISC-P-17

Wyrowski, Frank, BISC-P-03

Xia, Peng, BISC-P-33

Xiong, Wenzhen, BISC-5-04

Yamada, Hidenao, BISC-7-01

Yamamoto, Hirotsugu, BISC-P-09, BISC-P-14

Yamauchi, Toyohiko, BISC-7-01

Yan, Feng, BISC-P-07

Yang, Cheng, BISC-P-07

Yang, Chih-Chiang, BISC-P-11

Yang, Huaidong, BISC-1-03, BISC-5-03, BISC-5-04

Yasuhiko, Osamu, BISC-7-01

Yasui, Takeshi, BISC-P-09, BISC-P-14

Yasutomi, Keita, JS-4-07

Yatagai, Toyohiko, JS-3-01

Ye, Yan, BISC-P-02

Yeh, Chia-Hua, BISC-5-01

Yeh, J. Andrew, JS-3-05, JS-4-04

Yu, Hsin-Chieh, BISC-P-11

Yuan, Hui, BISC-4-03

Yuasa, Tomonori, BISC-P-24, BISC-P-26

Yushkov, Konstantin, BISC-6-02

Zakharov, Valery, BISC-P-20
Zayłsev, Kirill, BISC-1-04

Zeng, Hui, BISC-1-03

Zhang, Lu, BISC-P-15

Zhang, Sichun, BISC-5-03

Zhang, Site, BISC-P-03

Zhang, Xinrong, BISC-5-03

Zhao, YU, BISC-P-O2

Zhao, Yuxia, BISC-P-02

Zharkikh, Elena, BISC-7-04

Zhelnov, Vladislav, BISC-1-04

Zherebtsov, Evgeny, BISC-7-04

Zherebtsova, Angelina, BISC-7-04

Proc. of SPIE Vol. $111401114001-9$ 
Proc. of SPIE Vol. $111401114001-10$

Downloaded From: https://www.spiedigitallibrary.org/conference-proceedings-of-spie on 25 Apr 2023 Terms of Use: https://www.spiedigitallibrary.org/terms-of-use 


\title{
Conference Committee
}

\author{
Conference Chair
}

Toyohiko Yatagai, Utsunomiya University (Japan)

Conference Co-chairs

Yoshihisa Aizu, Muroran Institute of Technology (Japan)

Osamu Matoba, Kobe University (Japan)

Yasuhiro Awatsuji, Kyoto Institute of Technology (Japan)

Yuan Luo, National Taiwan University (Taiwan)

\section{Conference Program Committee}

Barry Cense, Utsunomiya University (Japan)

Wonshik Choi, Korea University (Korea, Republic of)

Shi-Wei Chu, National Taiwan University (Taiwan)

Katsumasa Fujita, Osaka University (Japan)

Yoshio Hayasaki, Utsunomiya University Center for Optical Research \& Education (Japan)

Masaki Hisaka, Osaka Electro-Communication University (Japan)

Wataru Inami, Shizuoka University (Japan)

Ichiro Ishimaru, Kagawa University (Japan)

Toshiaki Iwai, Tokyo University of Agriculture and Technology (Japan)

Hsiang-Chieh Lee, National Taiwan University (Taiwan)

Xingde Li, Johns Hopkins University (United States)

Takashi Kakue, Chiba University (Japan)

Myung K. Kim, University of South Florida (United States)

Robert Magnusson, The University of Texas at Arlington (United States)

Yuji Matsuura, Tohoku University (Japan)

Izumi Nishidate, Tokyo University of Agriculture and Technology

(Japan)

Goro Nishimura, Hokkaido University (Japan)

Yusuke Ogura, Osaka University (Japan)

Eiji Okada, Keio University (Japan)

Yukitoshi Otani, Utsunomiya University (Japan)

Yong-Keun Park, KAIST (Korea, Republic of)

Xiangyu Quan, Kobe University (Japan)

Manabu Sato, Yamagata University (Japan)

Shunichi Sato, National Defense Medical College (Japan)

Tatsuki Tahara, Kansai University (Japan)

Enrique Tajahuerce, University Jaume I (Spain)

Yosuke Tamada, National Institute for Basic Biology (Japan)

Eriko Watanabe, The University of Electro-Communications (Japan)

Peng Xia, AIST (Japan)

Yasui Takeshi, The University of Tokushima (Japan) 
Proc. of SPIE Vol. 11140 1114001-12

Downloaded From: https://www.spiedigitallibrary.org/conference-proceedings-of-spie on 25 Apr 2023 Terms of Use: https://www.spiedigitallibrary.org/terms-of-use 


\title{
Optical Tweezers in Biology
}

\author{
Alexander B. Stilgoe*a, Itia A. Favre-Bulle ${ }^{\mathrm{a}}$, and Halina Rubinsztein-Dunlop ${ }^{\mathrm{a}, \mathrm{b}}$

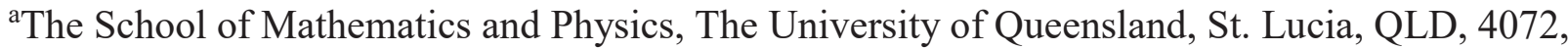 \\ Australia \\ ${ }^{\mathrm{b}}$ ARC Centre of Excellence for Engineered Quantum Systems, The University of Queensland, St. \\ Lucia, QLD, 4072, Australia
}

\begin{abstract}
Optical tweezers has enabled application and measurement of forces in a highly quantitative way and has been used to discover and study in depth a multitude of biological processes. In this tutorial presentation, we start with overview of the original work by Arthur Ashkins' and the first applications of optical tweezers in biological systems and we describe the principles of optical trapping showing how to measure optical forces and torques. We describe the broad use of optical tweezers with applications ranging from cell manipulation to molecular biology. We conclude the presentation with discussions of the current state of the art of this area of science and discuss further applications of optical tweezers and possible future directions.
\end{abstract}

Keywords: optical tweezers, forces, torques, cells, biophysics, molecular biology, beam shaping, light detection,

\section{Introduction}

Optical tweezers have been widely used in quantitative measurements of biological systems and processes since the first demonstration of the technique in 1986 by Arthur Ashkin ${ }^{1}$. They were utilized in biophysics and cell biology to shine light into the understanding of processes taking place in cells and molecules ${ }^{2,3}$. Here we describe optical tweezers, outlining the principal of operation and review their use in biological systems as well as in a number of other micro-systems.

\section{Body}

\section{Principal of operation and measurement}

Optical tweezers can trap, manipulate and measure forces acting on particles in a range from femto- to nanoNewtons (fN-nN). Optical forces occur due to the conservation of momentum when light scatters off an object. If a particle scatters light such that the average direction of the light is changed, then the particle must experience a force in the opposite direction. We outline various methods of modelling this process, including: Rayleigh scattering, geometrical optics, and an approach based on a full electromagnetic theory. We outline how the effect of the scattering can be measured using various detection systems, such as camera and position sensitive detector, and how these are incorporated into a system to measure forces acting in a broad variety of systems including biological and bio-medically relevant ones.

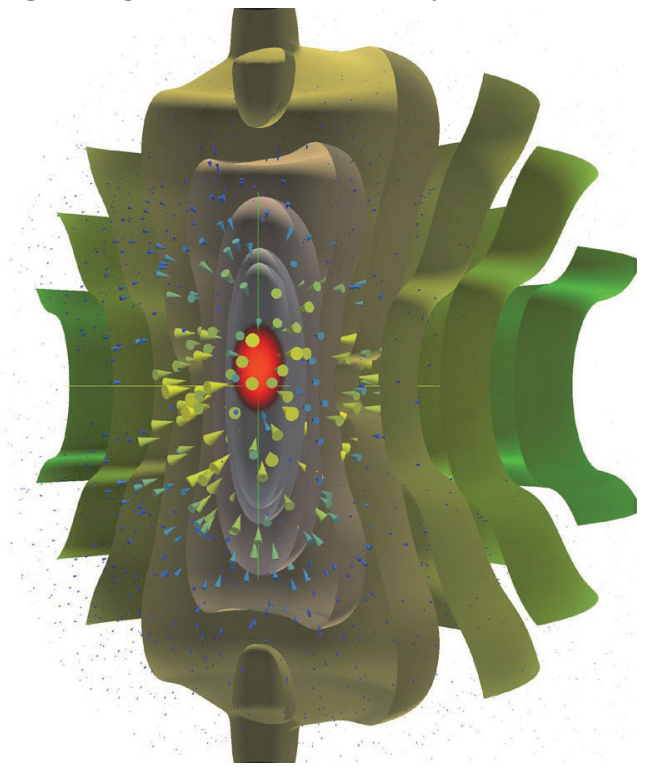

Figure 1: Model and simulation of an optically trapped particle in a highly focused laser beam. Cones depict relative magnitude of optical force. Ellipsoidal patch shows the distribution of particle location under Brownian motion. 3D contours represent the equipotential of intensity of a laser beam.

* stilgoe@physics.uq.edu.au 


\section{Applications to biology}

In the years since their first demonstration optical tweezers have been used to manipulate organelles ${ }^{4}$, and measure molecular motions, such as the compliance of flagella ${ }^{5}$ and kinesin stepping ${ }^{6}$. Optical tweezers have been particularly successful in the realm of biochemistry and have been used to precisely measure DNA, protein and enzyme function ${ }^{3}$. An optical tweezers ability to remotely, and without contact, manipulate has been essential to these experiments and applications. We cover the large range of methods used to illustrate the range of capabilities of the optical tweezers technique.
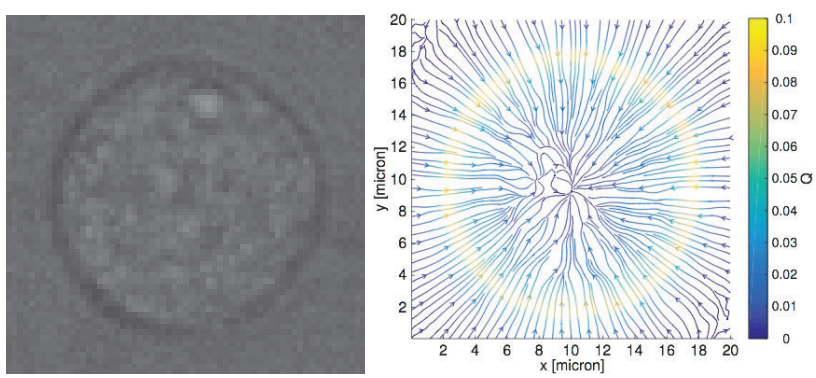

Figure 2: Digital micrograph of Chinese hamster ovary (CHO) cell and optical forces acting on it as a function of position. The overall spherical shape gives a net trapping force, relative changes of individual parts of the cell can be both manipulated and measured with optical tweezers.

\section{Current work, our work and future directions}

We have been using optical tweezers in a number of applications with particular emphases on measurement of optical torque and rotation. This enabled measurement of viscoelastic properties in biologically relevant systems. We also have been concerned with developing methods for using optical tweezers in in-vivo studies of model systems such as zebrafish and combining these with relevant imaging modalities to study in details neuronal systems.

The capabilities of optical tweezers are continually being extended, being now able to trap with greater degree of control over optical forces ${ }^{7,8}$, deeper into the system $^{9,10}$, with precision ${ }^{11}$, and with measurement beyond spherical particles to irregular and deformable particles $^{12,13}$.
Future application of optical tweezers will be considered for measurements inside cells, studies of biological swimmers and their characterization.

\section{References}

1. Ashkin A, Dziedzic JM, Bjorkholm JE, Chu S. Observation of a single-beam gradient force optical trap for dielectric particles. Opt Lett. 1986;11 (5):288-290.

2. Svoboda K, Block SM. Biological Application of Optical Forces. Annual Review of Biophysics and Biomolecular Structures. 1994;23:247-285.

3. Moffitt JR, Chemla YR, Smith SB, Bustamante C. Recent advances in optical tweezers. Annual Review of Biochemistry. 2008;77:205-228.

4. Ashkin A, Schütze K, Dziedzic JM, Euteneuer U, Schliwa M. Force generation of organelle transport measured in vivo by an infrared laser trap. Nature. 1990;348:346.

5. Block SM, Blair DF, Berg HC. Compliance of bacterial flagella measured with optical tweezers. Nature. 1989;338:514.

6. Svoboda K, Block SM. Force and velocity measured for single kinesin molecules. Cell. 1994;77(5):773-784.

7. Taylor MA, Waleed M, Stilgoe AB, Rubinsztein-Dunlop H, Bowen WP. Enhanced optical trapping via structured scattering. Nature Photonics. 2015;9(10):669-673.

8. Phillips DB, Padgett MJ, Hanna S, et al. Shapeinduced force fields in optical trapping. Nature Photonics. 2014;8:400.

9. Favre-Bulle IA, Stilgoe AB, Rubinsztein-Dunlop H, Scott EK. Optical trapping of otoliths drives vestibular behaviours in larval zebrafish. Nature Communications. 2017;8(1):630-630.

10. Zhong M-C, Wei X-B, Zhou J-H, Wang Z-Q, Li $\mathrm{Y}-\mathrm{M}$. Trapping red blood cells in living animals using optical tweezers. Nature Communications. 2013;4:1768-1768.

11. Huang R, Chavez I, Taute KM, et al. Direct observation of the full transition from ballistic to diffusive Brownian motion in a liquid. Nature Physics. 2011;7(7):576-580. 
12. Català F, Marsà F, Montes-Usategui M, Farré A, Martín-Badosa E. Extending calibration-free force measurements to optically-trapped rodshaped samples. Scientific Reports. 2017;7:42960.

13. Bui AAM, Kashchuk AV, Balanant MA, Nieminen TA, Rubinsztein-Dunlop H, Stilgoe AB. Calibration of force detection for arbitrarily shaped particles in optical tweezers. Scientific Reports. 2018;8(1):10798. 


\title{
Optical trap and laser interferometry in living cells Daisuke Mizuno, Umeda Katsuhiro, Sugino Yujiro, Kenji Nishizawa \\ Department of Physics, Kyushu University, Motooka 744 , Nishiku, Fukuoka 819-0395, Japan
}

\begin{abstract}
Mechanics of living cell interior are governed by cytoskeletons and cytosol. They are extraordinarily heterogeneous and their physical properties are strongly affected by the internally generated forces. In order to understand the out-ofequilibrium mechanics, we have developed a method of microrheology using laser interferometry and optical trapping technology. This method allowed us to probe mechanics and dynamics in living cells with a high spatio-temporal resolution. Microscopic probes in cells are stably trapped in the presence of vigorous cytoplasmic fluctuations, by employing smooth 3D feedback of a piezo-actuated sample stage. To interpret the data, we present a theory that adapts the fluctuation-dissipation theorem (FDT) to out-of-equilibrium systems. We discuss the interplay between material properties and non-thermal force fluctuations in the living cells that we quantify through the violations of the FDT.
\end{abstract}

Keywords: optical trap, laser interferometry, microrheology, cell mechanics

\section{Introduction}

Microrheology (MR) is a method to investigate the mechanical response of soft materials at microscopic $(\sim$ $\mu \mathrm{m})$ range. This technique enables us to measure the viscoelastic properties of the surrounding medium by tracking the motion of imbedded, micron-sized tracer beads [1]. One of the standard implementation of this method is active microrheology (AMR) where tracer beads are actively manipulated with an external force. Then we measure the displacement response of the beads, from which we obtain the viscoelasticity of surrounding media. The other method is passive microrheology (PMR) that passively tracks the imbedded bead's thermal fluctuations. PMR can provides material's rheological properties only at equilibrium since it relies on the fluctuation-dissipation theorem (FDT) to estimate the response function of the probe. Whereas FDT is satisfied only at equilibrium conditions, by comparing the result with PMR to AMR, we can estimate how strongly the system is driven out of equilibrium [2-5].

\section{MR with optical trap and laser interferometry}

Conventional active/passive microrheology has been typically performed using laser interferometry and optical trapping [1]. Laser interferometry (LI) is a technique to detect the position of the probe particle by illuminating it with a probe laser and measuring the interference pattern made between un-scattered and scattered lights [6]. Another laser (drive laser) is tightly focused on the same probe to trap it. Manipulating the focus positions, the arbitrarily controlled optical-trap (OT) force is applied to the probe. Both techniques (LI and OT) require keeping the probe well inside the sub- $\mu \mathrm{m}$-sized tightly-focused laser spot.

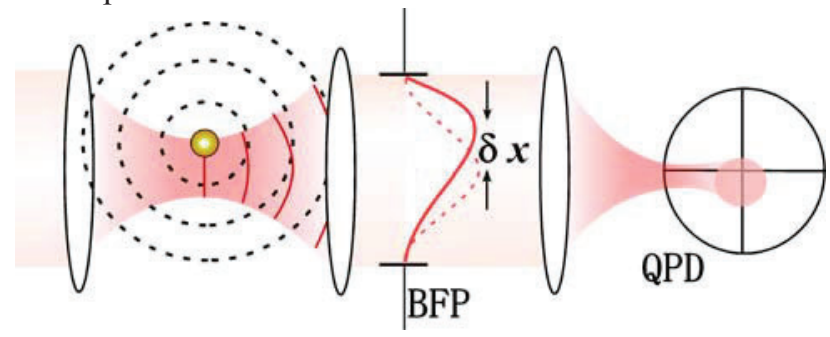

Fig. 1: Schematic illustration of laser interferometry. Image of the interference pattern at the back focal plane (BFP) was projected on to the quadrant photodiode (QPD).

In living cells, mechanoenzymes such as motor proteins create vigorous flows and fluctuations. The probe particle then moves out of the laser focus within the experimental time period. Although strong optical trapping force is able to retain the probe in the focus, a probe trapped in a strong optical potential does not display the mechanical properties of surrounding materials. 


\section{Feedback-tracking active microrheology}

To overcome these issues and perform MR in active environments (typically in living cells), we developed optical-trap-based MR implemented with threedimensional feedback-controlled sample stage [7]. The stage is manipulated by a piezo-mechanical sample stage to cancel large and slow movements of a probe particle so that high-bandwidth, large dynamic-range MR can be performed even in vigorous fluctuations e.g. HeLa cell.

The position of the sample stage $u_{\text {stage }}$ is controlled by the PID feedback as below. Probe position from the laser focus $u_{Q P D}$ measured by LI is used to control the piezo position as

$$
u_{\text {stage }}(t)=1 / \tau \int u_{Q P D} d t,
$$

where $\tau$ (set at $10 \sim 100 \mathrm{~ms}$ ) is the delay time for the feedback response of our experimental setup. Since the total displacement $u$ of the probe in the sample is given as $u=u_{Q P D}+u_{\text {stage }}$ (Fig. 2), eq. (1) yields frequency response relation:

$$
\hat{u}=(1-i \omega \tau) \hat{u}_{\text {stage }}=(1-1 / i \omega \tau) \hat{u}_{Q P D} .
$$

Hereafter, ${ }^{\wedge}$ denotes the amplitude of the sinusoidal signal.

Probe particles are manipulated by a sinusoidal lateral oscillation of the drive laser focus whereas the probe laser is stationary. The optical trapping force applied to the probe particle by drive and probe laser is $k_{d} L \exp (-i \omega t)-\left(k_{d}+k_{p}\right) u_{Q P D}$, where $k_{d}, k_{p}$ refer to the trap stiffness of the drive and probe laser respectively. $L$ is the amplitude of the drive laser oscillation. The Langevin equation for the probe particle under feedback control is then written as

$$
k_{p} u_{Q P D}(t)+\int_{-\infty}^{t} \gamma\left(t-t^{\prime}\right) \dot{u}\left(t^{\prime}\right) d t^{\prime}=k_{d}\left(L e^{-i o t}-u_{Q P D}(t)\right)+\zeta(t)+f(t),
$$

where $\dot{u}(t)$ denotes the velocity of the probe in the coordinate system traveling with the feedback-controlled piezo stage. $\zeta(t)$ and $f(t)$ are the thermal and nonthermal forces respectively, and $\gamma(t)$ is the friction function.. The Fourier transform of the ensemble or time average of eq. (3) yields the frequency-dependent response which we rewrite as

$\langle\hat{u}(\omega)\rangle^{F B}=\left\langle\hat{u}_{Q P D}(\omega)\right\rangle+\left\langle\hat{u}_{\text {stage }}(\omega)\right\rangle=\alpha(\omega)\left\{\hat{F}(\omega)-\left(k_{d}+k_{p}\right)\left\langle\hat{u}_{\text {OPD }}(\omega)\right\rangle\right\}$, where $\hat{F}(\omega) \equiv k_{d} L$. The superscript FB denotes the total displacement when the position of the piezo stage is feedback controlled.
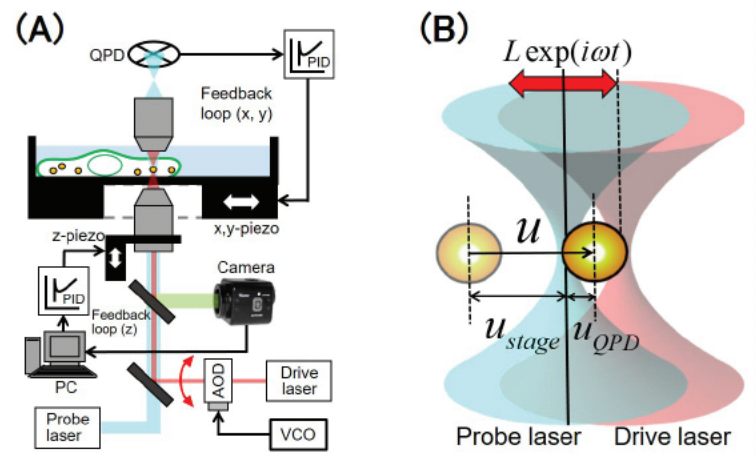

Fig. 2: (A) Schematic of the feedback-tracking microrheology setup. (B) Displacements of a probe and laser positions.

We define the "total" response of the probe movement to the wiggling force applied by the drive laser under the feedback control as $A_{F B} \equiv\langle\hat{u}(\omega)\rangle^{F B} / \hat{F}(\omega)$. By using this definition and substituting eq. (2) into eq. (4), we obtain the relationship between $A_{F B}(\omega)$ and intrinsic response function of a probe in the same media $\alpha(\omega)$ (i.e. response without trap) as

$$
A_{F B}(\omega)=\frac{\langle\hat{u}(\omega)\rangle^{F B}}{\hat{F}(\omega)}=\frac{\alpha(\omega)}{1+\beta \alpha(\omega)},
$$

where $\beta \equiv\left(k_{d}+k_{p}\right) /(1-i \omega \tau)$ is the parameter describing the correction under feedback. $\alpha(\omega)$ is then obtained from the total displacement as

$$
\alpha(\omega)=\frac{A_{F B}}{1-\beta A_{F B}(\omega)}=\frac{\langle\hat{u}(\omega)\rangle^{F B}}{\hat{F}(\omega)-\beta\langle\hat{u}(\omega)\rangle^{F B}} .
$$

The shear viscoelastic modulus $G(\omega)$ of the surrounding material is then obtained via the EinsteinStokes relation extended to frequency response, $G(\omega)=1 / 6 \pi \alpha(\omega) a$ where $a$ is the radius of the probe.

\section{Cell mechanics during cell-cycle progression}

As a typical application of the developed technique, we performed feedback-tracking active microrheology in HeLa cervical cancer cells. HeLa cells were seeded in the glass-bottom dish and incubated until epithelial-like sheets were formed. $2 a=1 \mu \mathrm{m}$ melamine particles (microParticles $\mathrm{GmbH})$ coated with polyethelene-glycol 
polymer brush were bombarded into cells with GeneGun (Biorad, 1652257J1).

In Fig. 3, we show shear viscoelasticity of the cytosols in HeLa cells as a function of frequency. We observed that the approximate power-law relation $G(\omega) \propto(i \omega)^{0.5}$ is satisfied in the wide range of frequencies. This response is in contrast to the cytoskeletal networks, for which elastic plateau response $\left[G(\omega) \propto(i \omega)^{0}\right]$ at low frequencies and $G(\omega) \propto(i \omega)^{3 / 4}$ at high frequencies have been typically observed for in vitro [2] and in vivo [7]. Recently, the anomalous viscoelastic response $\left[G(\omega) \propto(i \omega)^{0.5}\right]$ observed in living epithelium was attributed to the dynamics of cytosol rather than cytoskeletons $[7,8]$. In cells, various soft colloids, such as macromolecular assemblies, organelle, etc, are present at their highly condensed state. At the same time, they are forcedly fluidized because of the non-thermal mechanical energies originated from the active metabolism. In this sense, living cytosol is a typical representation of active colloidal glass, which is a current focus of study in the nonequilibrium statistical mechanics

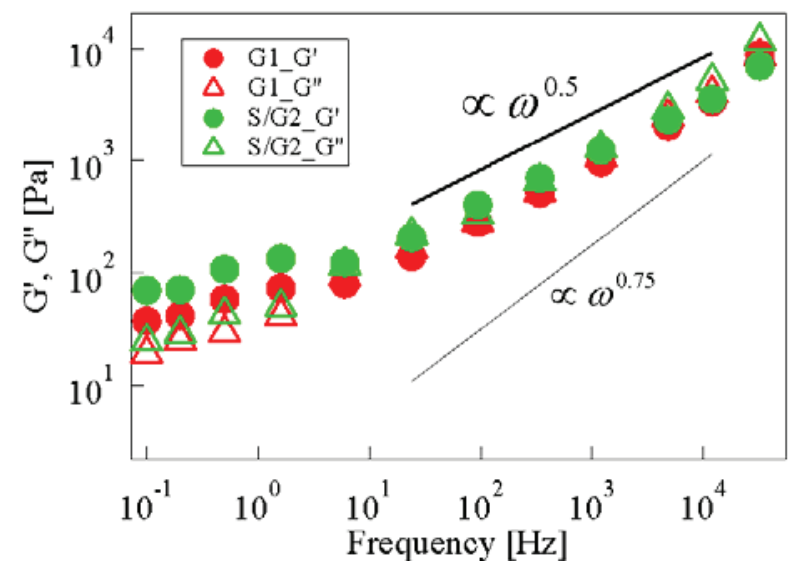

Fig.3: Shear viscoelastic spectrum of HeLa. Fucci2 cells in $\mathrm{G} 1$ and $\mathrm{S} / \mathrm{G} 2$ phases.

field.

The HeLa cells used in this study (HeLa. Fucci2) were labelled with Fucci system that enables to identify G1 and $\mathrm{S} / \mathrm{G} 2$ phases during the cell-cycle progression, by the fluorescence color in the cell nucleus [9]. We found little dependence of rheology during the cell-cycle progression. Although $G(\omega)$ in $\mathrm{S} / \mathrm{G} 2$ phase seems to be slightly increased, we believe this artifact. In this experiment, cells in G1 phase were subjected to microrheology experiments continually until they proceed to $\mathrm{S} / \mathrm{G} 2$ phases. The feedback-tracking microrheology technique allows us to perform experiments with less than $1 \mathrm{~mW}$ laser. For the sake of technical convenience, however, several $\mathrm{mW}$ laser was illuminated to the probe particle. We recently found that such prolonged exposure to several $\mathrm{mW}$ laser affects the probe response (data not shown).

In this study, cells in S, G2 and G1 phases were studied with microrheology. Cells in $M$ Phase could exhibit mechanical properties largely different from other phases. However, $M$ phase cells are rare to find and could be easily eliminated by observing the structure of nucleus under the microscope. Therefore, the finding in this study (little dependence of intracellular rheology on cell-cycle progression from $\mathrm{G} 1$ to $\mathrm{S} / \mathrm{G} 2$ ) allows us to investigate mechanics of cell interiors without strong concern on their cell-cycle dependence.

\section{References}

[1] D. Mizuno, D. A. Head, F. C. MacKintosh, and C. F. Schmidt, Macromolecules 41, 7194 (2008).

[2] D. Mizuno, C. Tardin, C. F. Schmidt, and F. C. MacKintosh, Science 315, 370 (2007).

[3] D. Mizuno, R. Bacabac, C. Tardin, D. Head, and C. F. Schmidt, Phys. Rev. Lett. 102, 168102 (2009).

[4] T. Harada, and S. Sasa, Phys. Rev. Lett. 95, 130602 (2005).

[5] T. Ariga, M. Tomishige, and D. Mizuno, Phys. Rev. Lett. 121 (2018).

[6] F. Gittes, and C. F. Schmidt, Opt Lett 23, 7 (1998).

[7] K. Nishizawa, M. Bremerich, H. Ayade, C. F. Schmidt, T. Ariga, and D. Mizuno, Sci Adv 3, e1700318 (2017).

[8] K. Nishizawa, K. Fujiwara, M. Ikenaga, N. Nakajo, M. Yanagisawa, and D. Mizuno, Sci Rep-Uk 7 (2017). [9] A. Sakaue-Sawano, H. Kurokawa, T. Morimura, A. Hanyu, H. Hama, H. Osawa, S. Kashiwagi, K. Fukami, T. Miyata, H. Miyoshi, T. Imamura, M. Ogawa, H. Masai, and A. Miyawaki, Cell 132, 487 (2008). 


\title{
Isotropic Quantitative Differential Phase Contrast Microscopy with Vortex Asymmetric Illumination Patterns
}

\author{
Yu-Hsuan Chuang, ${ }^{\mathrm{a}, \mathrm{b}^{*}}$, Ying-Ju Tsai ${ }^{\mathrm{b}, \mathrm{c}}$, Yu-Zi Lin ${ }^{\mathrm{b}}, \mathrm{J}$. Andrew Yeh ${ }^{\mathrm{a}}$ and Yuan Luo ${ }^{\mathrm{b}, \mathrm{d}^{*}}$ \\ ${ }^{a}$ Department of Power Mechanical Engineering, National Tsing Hua University, Hsinchu, 30013, Taiwan \\ ${ }^{\mathrm{b}}$ Institute of Medical Device and Imaging, National Taiwan University, Taipei 10051, Taiwan \\ ${ }^{c}$ Department of Electrical Engineering, National Taiwan University, Taipei, 10617, Taiwan \\ ${ }^{\mathrm{d}}$ Molecular Imaging Center, National Taiwan University, Taipei, 10672, Taiwan
}

\begin{abstract}
Differential phase contrast (DPC) microscopy is a well-known methodology to recover phase image of transparent specimen under multi-axis intensity measurements. We propose a novel illumination method to achieve isotropic differential phase contrast(iDPC) efficiently and meanwhile improve the accuracy and stability of phase recovery effectively. Our iDPC system is an add-on module setting on the commercial inverted microscope, using a programmable TFT shield to control the illumination pattern. We demonstrate our theoretical approach for iDPC and experimental results by using radial asymmetric pupils to show our accuracy on phase recovery. Besides, we also implemented the new illumination pattern with multiple wavelengths to achieve the same circularly symmetric phase transfer function result in high-speed operation.
\end{abstract}

Keywords: isotropic differential phase contrast; asymmetric pupil; phase recovery; multiple wavelengths

\section{Introduction}

Differential phase contrast(DPC) microscopy[1] based on asymmetric illumination has long been used to retrieve high-resolution qualitative image of a phase specimen. Typically, in DPC techniques, a half-circle pupil with 2axis measurements along vertical and horizontal directions is utilized to reconstruct quantitative images of weak phase samples (i.e. cells). However, some artifacts are produced in the process due to the missing frequencies of phase reconstruction since DPC transfer function is not circularly symmetric with only 2 -axis measurements. Therefore, DPC using 12-axis measurements along twenty-four different angles[2] has been reported recently to obtain missing frequency information such that DPC transfer function becomes circularly symmetric, and thus additional DPC measurements along other axes can significantly improve stability and accuracy for quantitative phase recovery. Here, we present a new approach by using radially asymmetric pupils $[3,4]$ in engineered illumination to acquire pair-wise images for phase recovery and efficiently achieve circularly symmetric transfer function under partially coherent condition.[5] In contrast to conventional DPC using 2 axis measurements, our isotropic DPC does not have missing frequency issue. Besides, compared to the half-circle based DPC method using 12-axis, our approach provides equivalently symmetric transfer function with only 3-axis measurements.

\section{Theory}

By using isotropic differential phase contrast method, we can utilize weak object transfer function to separate the phase from intensity and achieve quantitative phase measurement by pair-wise images[1,2]. Our system can be interpreted by Fourier optics. We use the TFT shield to control our radially asymmetric pupils, so under incoherent illumination condition, the resultant intensity captured on the camera can be written as[2]

$I(r)=\iint|\mathcal{F}\{\mathcal{F}[\mathrm{B}(\mathrm{u}, \mathrm{r})] \cdot[\mathrm{P}(\mathrm{u})]\}|^{2} d^{2} u$,

where $\mathrm{F}$ donates the Fourier transfer function and $\mathrm{u}$ and $\mathrm{r}$ denote spatial coordinates $(\mathrm{x}, \mathrm{y})$. I is the intensity on the camera and $\mathrm{P}$ is the pupil function in the objective lens. The transmission function of specimen can be presented as $O(r)=\exp (-\alpha(r)+i \phi(r))$, where $(r)$ denotes the spatial coordinates $(x, y), \alpha(r)$ is attenuation factor, and $\phi(r)$ is the optical phase. Under a weak object approximation, the 
intensity of a weakly scattering specimen in the Fourier space can be written as [6]

$I(u)=\widetilde{H}_{p h a s e} \cdot \widetilde{\phi}(u)+\widetilde{H}_{a m p} \cdot \tilde{\alpha}(u)+\widetilde{H}_{b} \cdot \tilde{\delta}(u)$,

where $\mathrm{u}, \tilde{\phi}(u)$, and $\tilde{\alpha}(u)$ represent as spatial frequency coordinates, phase and absorption of the specimen respectively. Therefore, the expression for $\widetilde{H}_{\text {phase }}$ can be written as [7]

$$
\widetilde{H}_{\text {phase }}=j\left[\begin{array}{l}
\iint S\left(\mathrm{u}^{\prime}\right) P\left(\mathrm{u}^{\prime}+\mathrm{u}\right) P^{*}\left(\mathrm{u}^{\prime}\right) d^{2} u^{\prime} \\
-\iint S\left(\mathrm{u}^{\prime}\right) P\left(u^{\prime}\right) P^{*}\left(u^{\prime}-u\right) d^{2} u^{\prime}
\end{array}\right],
$$

\section{Experiment Setup and Results}

In Figure 1, we compare a conventional half-circle pupil with a radially asymmetrical pupil in illumination to see their numerical results of different intensity computed by transfer function $\left(\sum_{\mathrm{i}}\left|\widetilde{\mathrm{H}}_{\text {phase }, x}\right|^{2}\right)$ in multiple axes $(\mathrm{x}=1,2,3)$. Besides, in Figure 1(g,h), we can see that by using our radially asymmetric pupils, we can achieve circularly symmetric intensity of transfer function with only 3-axis measurements, which can be viewed as direct evidence of avoiding artifacts of missing frequencies for phase reconstruction.

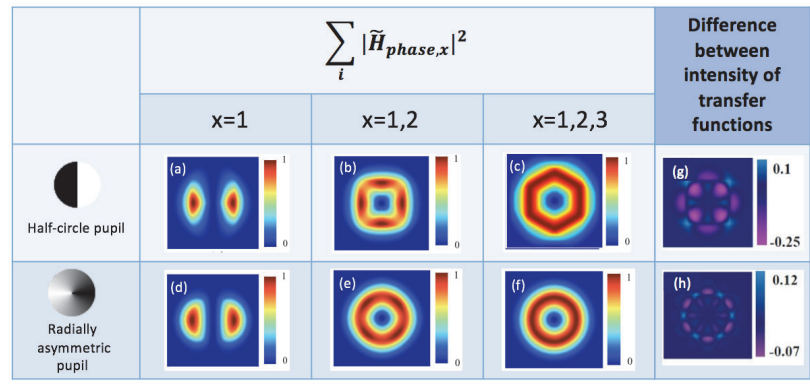

Figure 1. Simulation results of intensity of transfer

function $\left(\sum_{\mathrm{i}}\left|\widetilde{\mathrm{H}}_{\text {phase }, x}\right|^{2}\right)$ along different axes.

Figure 2 is a schematic diagram of the proposed iDPC system setup with the thin-film transistor (TFT) shield located on the Fourier plane of the condenser lens. The TFT shield is controlled by Arduino to show pair-wise radially asymmetric patterns along three different axes. Each pair of images generates a differential phase contrast image, which is utilized to reconstruct quantitative phase information and largely reduce phase reconstruction artifacts of missing frequencies.

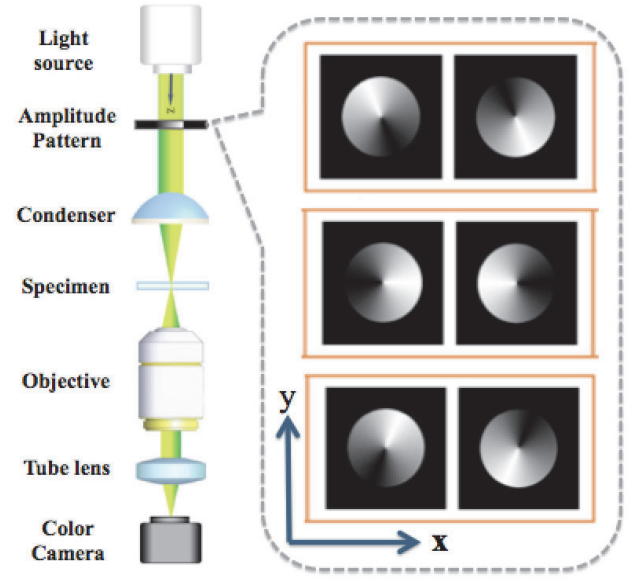

Figure 2. Schematic of our iDPC system setup, using a TFT shield as a programmable aperture.

Figure 3 shows quantitative phase images of live 3T3 mouse fibroblasts cells generated by computationally reconstruct three pairs of DPC images. The color bar in gray scales represents quantitative phase recovery of the cells, and the detailed information of live cells can be clearly observed from the image.
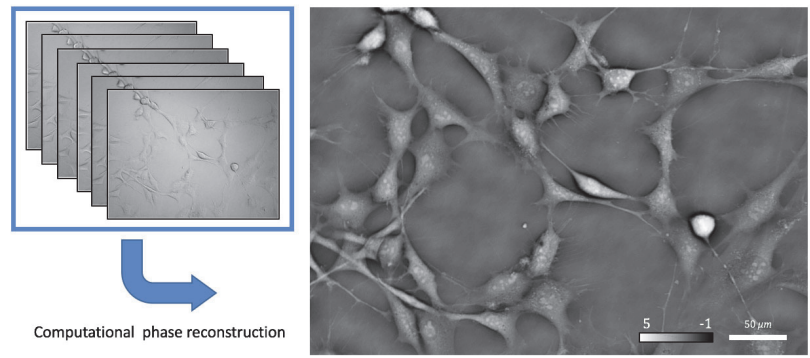

Figure 3. Quantitative phase images of live 3T3 mouse fibroblasts cells obtained using our iDPC system.

\section{Future Work}

Figure 4 We combine our radially asymmetric pattern with multiple wavelengths to shorten image acquisition time. However, different color spectrums may overlap on camera sensor, which is known as "color leakage" problem. This phenomenon will let the information of different color channels mix together and reduce the accuracy of reconstructed images significantly. In order to lower down the influence of color leakage problem, we 
choose the red and blue color in our pattern. The spectrum of red and blue is more separated than the combination with green, so that the visibility of specimen in the unprocessed image can be expected to be higher.

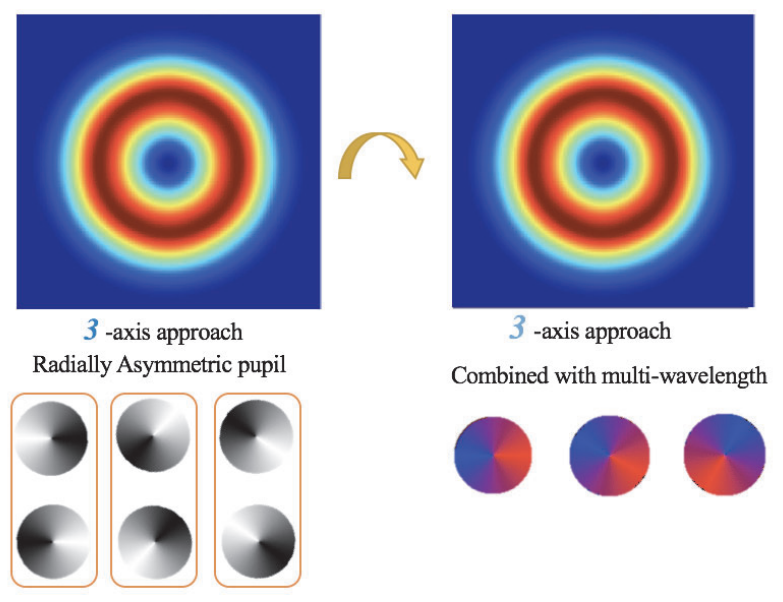

Figure 4. Combine our radially asymmetric pupil with different wavelengths based on the same isotropic circularly symmetric transfer function result.

Figure 5 (a)The quantitative phase image of $10 \mu \mathrm{m}$ microspheres using the multi-wavelength radially asymmetric pupil with 3-axis measurement after color leakage correction. (b)Measured phase distribution of the cross-section along the dashed lines of a zoomed-in microsphere at the solid box region of (a) The scalar bar is $50 \mu \mathrm{m}$.
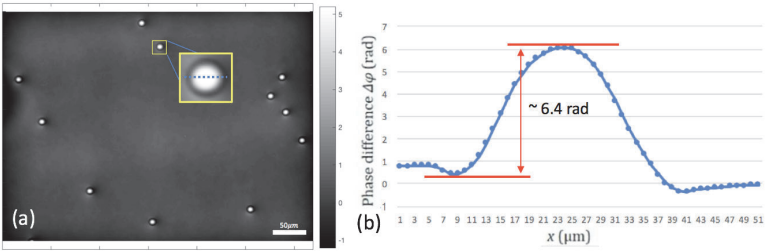

Figure 5. The quantitative phase image of $10 \mu \mathrm{m}$ microspheres and the cross-section phase profile

\section{Conclusions}

We have demonstrated the capability of iDPC to acquire quantitative phase images of label-free cell sample by using radially asymmetric pupils. The proposed iDPC shows isotropic intensity of transfer function, using three-axis measurements with compact system configuration as well as programmable TFT shield. The improvement of accuracy of phase recovery and stability using iDPC has potential to contribute to future study of biophotonics. Furthermore, radially asymmetric illumination can be implemented using multiple wavelengths to obtain quantitative phase images to achieve circularly symmetric phase transfer function, in high-speed operation as well.

\section{References}

[1] S. B. Mehta and C. J. R. Sheppard, "Quantitative phase-gradient imaging at high resolution with asymmetric illumination-based differential phase contrast," Opt. Lett. 34, 1924-1926 (2009).

[2] Tian, L. and Waller, L., "Quantitative differential phase contrast imaging in an LED array microscope." Optics Express, 2015. 23(9): p. 11394. [3] Y.-Z. Lin, K.-Y. Huang and Y. Luo, "Quantitative differential phase contrast imaging at high resolution with radially asymmetric illumination," Opt. Lett. 43, 2973-2976 (2018)

[4] Y.-H. Chuang, Y.-J. Tsai, Y.-Z. Lin and Y. Luo, "Isotropic Quantitative Differential Phase Contrast Microscopy with Radially Asymmetric Illumination," Optics \& Photonics Taiwan, the International Conference (OPTIC 2018)

[5] H.-H. Chen and Y. Luo, "Two-axis isotropic differential phase contrast transfer function using gradient amplitude modulation for phase imaging," in Imaging and Applied Optics 2017 (3D, AIO, COSI, IS, MATH, pcAOP), OSA Technical Digest (online) (Optical Society of America, 2017), CTh4B.5.

[6] Streibl, N., "3-Dimensional Imaging by a Microscope," Journal of the Optical Society of America a-Optics Image Science and Vision, 1985. 2(2): p. 121.

[7] Y.-Z. Lin, H.-H. Chen, K.-Y. Huang, and Y. Luo, "Isotropic Quantitative Differential Phase Contrast Microscopy." Optics \& Photonics Taiwan, the International Conference (OPTIC 2017) 


\title{
Monitoring mitochondrial dynamics within mitotic apparatus by lightsheet microscopy
}

\author{
Wen-Cheng Wang*, Chin-Yi Chen and Bi-Chang Chen \\ Research Center for Applied Sciences, Academia Sinica, Taipei 11529, Taiwan
}

\begin{abstract}
Mitochondria are major energy resources in cell. These highly dynamic organelles are constant fusion and fission inside of cell, especially while cells are at mitotic status. Fusion and fission of mitochondria allowed the inheritances (mitochondrial DNA; mtDNA) and mitochondrial proteins could be exchanged between mitochondria. Sharing the inheritances and mitochondrial proteins is necessary for mitochondria to maintain mitochondrial function. Here, we investigated dynamic of mitochondrial proteins during mitochondrial fusion and fission. We genetically expressed photoswitchable proteins (Dendra2) ${ }^{1}$ into mitochondria and optically monitored the inter-mitochondrial distribution of Dendra2 by lightsheet microscopy ${ }^{2}$. The advantages of lightsheet microscopy, low phototoxicity and fast image speed, make it possible to detect the fusion/fission events of mitochondria during mitosis. We observed that the small portion of photoswitched Dendra2 was transmitted to the whole cell within 15 minutes.
\end{abstract}

Keywords: lightsheet microscopy, mitochondrial protein, mitosis, Dendra2, photoswitch

\section{Introduction}

Frequently mitochondrial fission and fusion (mitochondria dynamics) is one of mechanisms for mitochondria to control the functional quality of mitochondria, ${ }^{3,4}$. The protein synthesis at cytoplasm would be imported into mitochondria via a recognition sequence. Targeting signals are usually removed once the protein has reached its correct inter-mitochondrial location. Furthermore, mitochondria also have their own mitochondrial DNA (mtDNA) and could synthesize peptides inside of mitochondria. For both types of proteins, they could be inter-mitochondrially redistributed via fusion and fission.

Mitochondrial matrix, lumen inside of inner mitochondrial membrane, is where metabolic process occurs. There are hundreds of enzyme and proteins required for tricarboxylic acid cycle, urea cycle, and apoptosis cascade and so on. Fusion and fission of mitochondria can result in mitochondrial matrix proteins transferred within mitochondria ${ }^{5,6}$. In addition, damaged proteins and mutated/damaged mtDNA would be asymmetrically segregated into two daughter cells during cell division ${ }^{7-9}$. Therefore, asymmetry division of mitotic or meiosis cell would generate a mixture of mtDNA ${ }^{10,11}$ within the daughter cells. Mechanisms of asymmetric division and excluding aging/damaged mitochondria regulate stemness preservation of stem cell and multipotency of neural progenitors ${ }^{12-15}$.

Since asymmetry division and fusion/fission play crucial roles in controlling cell function, we are wondering how mitochondria transfer their matrix proteins to mitochondria through fusion and fission. Also, mitotic cells turn into sphere, which become a hindrance for live imaging. To solve this problem, we overcame the obstacles of live imaging requirements by lightsheet microscopy. Increasing spatiotemporal resolutions and reducing phototoxicity to specimen allow us to observe several dynamic subcellular processes without sacrifice of substantial spatiotemporal resolution. By combining with photoswitchable fluorescent proteins, Dendra $2^{1}$, we could monitor the inter-mitochondrial dynamics of mitochondrial proteins during the cell division process of mitosis.

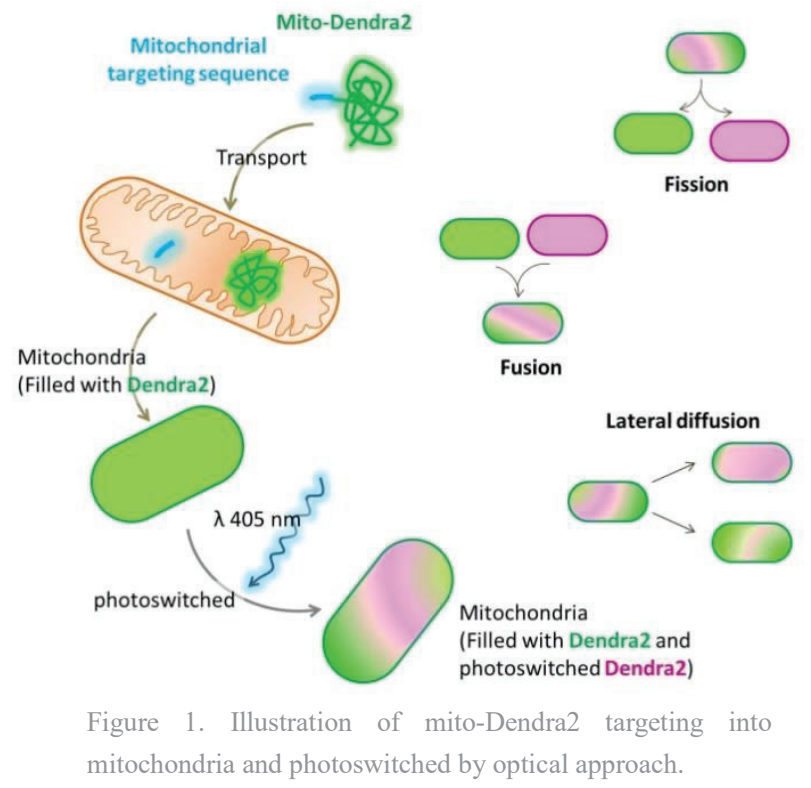




\section{Body}

In order to monitor how the mitochondrial matrix proteins shuttle between mitochondria, we generated a stable cell line with constant expression of Dendra2 in the mitochondrial matrix (Figure 1). The mitochondrial targeting of Dendra2 are achieved by tagging the mitochondrial matrix targeting sequence on Dendra2 (mito-Dendra2) ${ }^{16}$. The mitochondrial targeting sequence would lead the mito-Dendra2 shuttling between mitochondria and imported into mitochondrial matrix (Figure 1). After the correct transport of mito-Dendra2, the targeting sequence would be removed by the mitochondrial enzyme. The mitochondria filled with mito-Dendra2 could be observed by green fluorescent.

To optically mark a portion of mito-Dendra2, the laser $405 \mathrm{~nm}$ were applied on the localized area of cell (Figure 2A). The exposure to $405 \mathrm{~nm}$ laser would switch the mito-Dendra2 from green to red fluorescent. This results in a mixture population of mito-Dendra 2 within mitochondria (Figure 1 and 2). In the figure 2, a redistribution of optically labeled mitochondria with red mito-Dendra2 was observed before the cell entry into metaphase/anaphase. These indicated a rapid exchange and diffusion of mitochondrial material before the cell division. Asymmetry division was observed after the cytokinesis (unpublished data). Taken all together, our data indicated that we could combine the lightsheet microscopy and the optically labelling approach to mark a small population of mitochondria and monitor the dynamics of mitochondrial materials within mitochondria during mitosis.

To further quantify the dynamic of photoswitched mito-Dendra2, we generated a 3D mask to cover all mitochondria (Figure 3A). The 3D mask was used to filter the mito-Dendra2 signals; the photobleaching rate of live image was calculated from the masked green and red signals (Figure 3A). The total intensity of green mito-Dendra2 was gradually decreased (Figure 3B); the photoswitched mito-Dendra2 show slight decay during imaging. We adjusted the intensity decrease by photobleaching correction. The corrected intensity were used for calculating the 560/488 ratio (Figure 3C).

The ratio of 560/488 signals were shown in gray scale (Figure 3D); the ratio signal was gradually diffused from photoswitched area to distal area. To visualize the spatio-temporal change of 560/488 ratio, a color-coded ratio were demonstrated (Figure $3 \mathrm{E}$ ). In addition, we extract a small region of phtoswitched area to analyze the change of ratio (Figure $3 \mathrm{~F}$ ). We could observe a decreased ratio while the photoswitched mito-Dendra2 diffused and redistributed into distal area (Figure 3C).

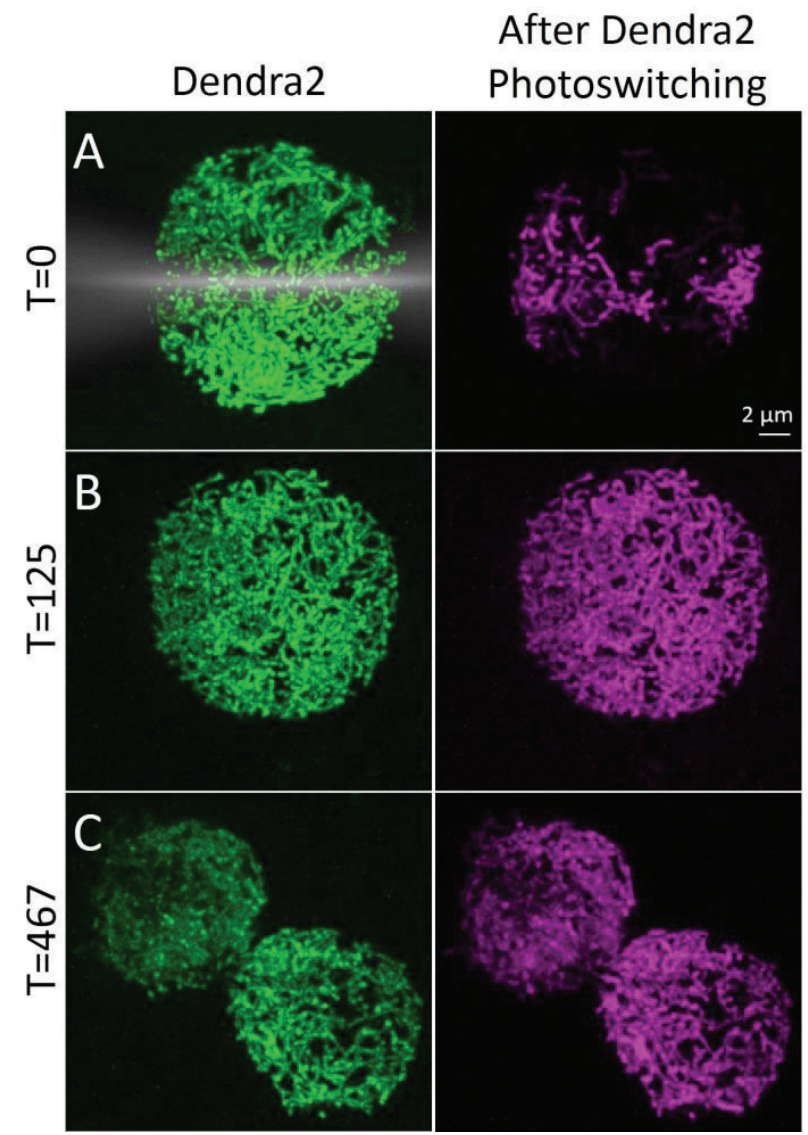

Figure 2. The 3D live images of mitotic cell labelling with green fluorescent mito-Dendra2. A portion of mito-Dendra2 were photoswitched into red fluorescent (Magenta) by 405 $\mathrm{nm}$ laser, as the horizontal lighsheet be illuminated at the central of cell. The time point of 125 shows the mito-Dendra2 which are redistributed and spread into whole cell. The end of time point $(\mathrm{T}=467)$ shows the segregation of photoswitched labeled mitochondria is asymmetry. Maxima intensity projection of 3D z-stack is shown; images are deconvoluted. Time $(\mathrm{T})$ interval $=4.4 \mathrm{sec}$.

As for the region without exposure to photoswitching (Figure 3G), the redistribution of mito-Dendra 2 results in an increase of ratio. According to these results, we could analyze, track and quantify the subcellular dynamics and redistribution of mitochondrial proteins during cell division by lightsheet and optical labelling.

\section{Results and Discussion}

A detailed dynamic of mito-Dendra 2 within single mitochondria would be quantified. These would improve our understanding about mito-Dendra2 diffusion and exchange by mitochondrial fusion/fission. Specific mitochondrial proteins with Dendra2 tag would be 

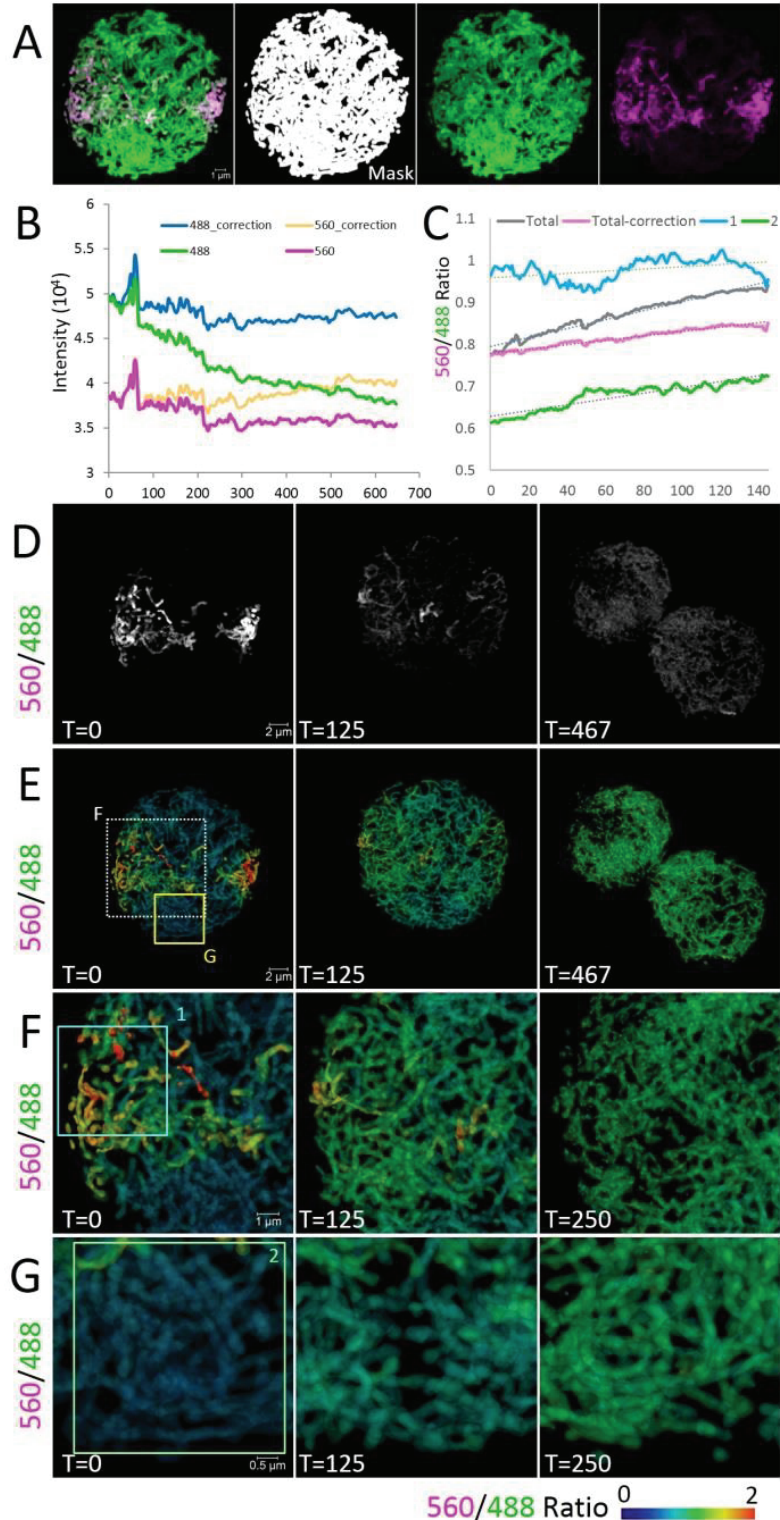

Figure 3. The quantification of mito-Dendra2 dynamic and redistribution. (A) The sum of green and red mito-Dendra2 was used for generated the 3D mask. The outside signals of both colors were removed by the mask. (B) The total fluorescence intensities were quantified and applied for bleaching correction. (C) The ratio was calculated from the masked signals of each color after bleaching correction. The $560 / 488$ ratio demonstrated in gray scale (D) and color-code (E-G) illustrate the relative population of mito-Dendra2. (F) A selected area form E (white dashed line box) shows the diffusion and decay of photoswitched mito-Dendra2. (G) A selected area from E (yellow box) shows the gradually increase of photoswitched mito-Dendra2. Quantification of F and $\mathrm{G}$ was shown in $\mathrm{C}$. studied. These investigations would reveal the correlation of mitochondrial proteins/inheritances and cell division. This research might expectantly increase the understanding of mitochondrial function and pathology of mitochondrial diseases in the future.

\section{References}

1 Chudakov, D. M., Lukyanov, S. \& Lukyanov, K. A. Tracking intracellular protein movements using photoswitchable fluorescent proteins PS-CFP2 and Dendra2. Nat Protoc 2, 2024-2032, doi:10.1038/nprot.2007.291 (2007).

2 Chen, B. C. et al. Lattice light-sheet microscopy: imaging molecules to embryos at high spatiotemporal resolution. Science 346, 1257998 , doi:10.1126/science.1257998 (2014)

3 Benard, G. et al. Mitochondrial bioenergetics and structural network organization. J Cell Sci 120, 838-848, doi:10.1242/jcs.03381 (2007).

4 Devine, M. J. \& Kittler, J. T. Mitochondria at the neuronal presynapse in health and disease. Nat Rev Neurosci 19, 63-80, doi:10.1038/nrn.2017.170 (2018).

5 Bockler, S. et al. Fusion, fission, and transport control asymmetric inheritance of mitochondria and protein aggregates. J Cell Biol 216, 2481-2498, doi:10.1083/jcb.201611197 (2017).

6 Zhou, C. et al. Organelle-based aggregation and retention of damaged proteins in asymmetrically dividing cells. Cell 159, 530-542, doi:10.1016/j.cell.2014.09.026 (2014).

7 Aguilaniu, H., Gustafsson, L., Rigoulet, M. \& Nystrom, T. Asymmetric inheritance of oxidatively damaged proteins during cytokinesis. Science 299, 1751-1753, doi:10.1126/science.1080418 (2003).

8 Youle, R. J. \& van der Bliek, A. M. Mitochondrial fission, fusion, and stress. Science 337, 1062-1065, doi:10.1126/science.1219855 (2012).

9 Lopez-Domenech, G. et al. Miro proteins coordinate microtubule- and actin-dependent mitochondrial transport and distribution. EMBO J 37, 321-336, doi:10.15252/embj.201696380 (2018).

10 Mishra, P. \& Chan, D. C. Mitochondrial dynamics and inheritance during cell division, development and disease. Nat Rev Mol Cell Biol 15, 634-646, doi:10.1038/nrm3877 (2014).

11 Dalton, C. M. \& Carroll, J. Biased inheritance of mitochondria during asymmetric cell division in the mouse oocyte. J Cell Sci 126, 2955-2964, doi:10.1242/jcs.128744 (2013).

12 Katajisto, P. et al. Stem cells. Asymmetric apportioning of aged mitochondria between daughter cells is required for stemness. Science $\mathbf{3 4 8}$, 340-343, doi:10.1126/science.1260384 (2015).

13 Teitell, M. A. Adult stem-like cells exclude "older" mitochondria. Cell Metab 21, 658-659, doi:10.1016/j.cmet.2015.04.023 (2015).

14 Suomalainen, A. Stem cells: Asymmetric rejuvenation. Nature 521, 296-298, doi:10.1038/521296a (2015).

$15 \mathrm{Lu}$, B., Jan, L. \& Jan, Y. N. Control of cell divisions in the nervous system: symmetry and asymmetry. Annu Rev Neurosci 23, 531-556, doi:10.1146/annurev.neuro.23.1.531 (2000).

16 Schatz, G. The protein import system of mitochondria. J Biol Chem 271, 31763-31766 (1996). 


\title{
The molecular architecture of proteins at the base of primary cilia revealed by super-resolution microscopy
}

\author{
Tzu-Yuan Chiu ${ }^{\mathrm{a}}$, Jung-Chi Liao*a \\ ${ }^{a}$ Institute of Atomic and Molecular Sciences, Academia Sinica, Taipei, 10617, Taiwan
}

\begin{abstract}
Primary cilia are an important organelle responsible for cell sensing and signaling. At the base of primary cilia, there are important proteins whose malfunctions result in serious diseases, i.e. ciliopathies. Using two super-resolution microscopy techniques, i.e. stimulated emission depletion (STED) microscopy and direct stochastic optical reconstruction microscopy (dSTORM), we map the molecular architecture of two essential regions, i.e. the transition zone and the distal appendages. Specifically, we find the relative localization of ciliopathy-associated proteins potentially suggesting their affinity to either the ciliary membrane or the axoneme. We also find a new structural part of the distal appendages, the distal appendage matrix, in between adjacent distal appendage blades. The matrix and blades are responsible for different functions for cilia.
\end{abstract}

Keywords: super-resolution, dSTORM, sptPALM, cilia, centriole, localization microscopy

\section{Introduction}

Primary cilia exist in many cell types in our body [14]. In each cell, a primary cilium functions like an antenna with a single rod-shaped protrusion from the plasma membrane or within the cell body. This rod-like organelle is about $250 \mathrm{~nm}$ in diameter and 3-5 $\mu \mathrm{m}$ in length. It contains a large number of signaling receptors which respond to the environmental changes by activating different signaling pathways to alter cell behaviors. For example, during development, the concentration gradient of sonic hedgehog determines the number of our finger digits, the location of our heart, and the morphology of our brain through the signaling of primary cilia [8]. In adult mice, ciliary mutation results in failed injury recovery of kidney [9]. Knowing the molecular architecture of the primary cilia would greatly enhance our understanding of functions of ciliary proteins.

Among all high-resolution techniques, electron microscopy (EM) has been regularly used for primary cilia imaging with its superior spatial resolution, but one can only obtain a static snapshot under vacuum preparation. Therefore, it is important to develop new methods for acquiring high-resolution images. Light microscopy, which utilizes different fluorescent dyes to amplify the target protein signals, requires procedures less complicated than those of EM and is able to acquire multi-color images once.

Several super-resolution techniques have recently evolved to break the diffraction limit of light, including photoactivated localization microscopy (PALM), stochastic optical reconstruction microscopy (STORM), structured illumination microscopy (SIM), and stimulated emission depletion microscopy (STED). PALM and STORM have great spatial resolution $(\sim 20 \mathrm{~nm})$. However, they require stacking thousands of stochastically activated images into one frame. The sampling rate is thus relatively too slow ( $\sim 1$ frame/min) to track dynamic intraflagellar transport (IFT) particles. SIM has $\sim 1$ frame/sec sampling rate but a spatial resolution of $120 \mathrm{~nm}$, slightly too large for transition zone (TZ) imaging. STED is a scanning based approach which requires one laser to excite a fluorophore and the other red-shifted laser to deplete the boundary of a diffractionlimited spot, yet it requires high power laser, increasing the possibility of photo-bleaching.

In this review, we show the super-resolution imaging by STED and direct STORM (dSTORM) to map the architecture of the ciliary proteins, and provide new ideas for primary cilia research.

\section{Results and Discussion}

Transition zone: Gating protein in and out from the cilia 
The $\mathrm{TZ}$ is a specific region at the ciliary base between the basal body (BB) and the axoneme proper. At the TZ, axonemes are cross-linked to ciliary membrane through the Y-shaped linkers (Y-links), and several organized protein complexes are involved. For instance, MKS/B9, TCTN1/2 and CEP290 complexes were hypothesized to compose parts of the Y-links. By using STED superresolution microscopy, we have mapped the proteins at the TZ of primary cilia of human retinal pigment epithelial (RPE-1) cells to elucidate their anchoring and tethering functions [1]. We found that CEP290 showed a rectangular-like shape, and RPGRIP1L occupied a similar width as CEP290 and the axoneme, whereas MKS1, TCTN2, and TMEM67 displayed two separate peaks, indicating that they were localized toward the outer periphery of the TZ. Setting centrin2-eGFP as a BB marker, we displayed that CEP290 is localized at a distinct axial level situated between $\mathrm{BB}$ and other $\mathrm{TZ}$ proteins.

The work described above provides an architectural map to find out the trafficking of the IFT proteins. Using dual-color STED imaging, we demonstrate that IFT88 is distributed across the $\mathrm{TZ}$ with a tilted angle from distal appendages (DAPs) to TZ, suggesting defined traveling paths of IFT particles at the ciliary base.

How do $\mathrm{TZ}$ proteins regulate IFT complex transportation? We found that IFT88 extended toward the BB due to TCTN2 depletion, and the ciliary length was clearly shortened. By performed dual-color dSTORM super-resolution imaging, we confirmed an extension of IFT88/IFT144 localization toward the BB in both TCTN2 and RPGRIP1L-deficient cells [2]. Furthermore, using imaging to quantify the full width at half-maximum, the diameter of IFT88 in the BB was smaller in TCTN2impaired cells compared to the wild-type, displaying the lumen occupancy of IFT88.

Distal appendages: Controlling ciliary membrane protein trafficking

To initiate primary cilium growth, membrane docking of centriole by its distal end is necessary. DAPs are defined as nine-bladed, pinwheel-like structures and are thought to be involved in precedent steps of ciliogenesis. For example, CEP83, CEP89, SCLT1, CEP164, and FBF1 are core DAP components recruited to distal ends of the centriole before ciliogenesis. Upon membrane docking, TTBK2 recruited intraflagellar transport proteins and release $\mathrm{CP} 110$ and CEP97, two negative regulators of ciliogenesis. By using dSTORM superresolution microscopy, we displayed the radial localization of DAP proteins in RPE-1 cells [3]. Most of the core DAP components showed nine-fold symmetric ring-like patterns. In contrast, FBF1 localized in different angular positions from those of CEP164. We monitored the axial and lateral views of the DAP proteins with series of two-color dSTORM images, and reconstructed the three-dimensional spatial map of DAPs. We found that instead of the 9 pinwheels, the DAPs form a cone shape containing 9 DAP blades and proteins between blades that we termed distal appendage matrix (DAM). ARL13B, a ciliary membrane protein, were decorated at FBF1 signals during cilium initiation, but excluded from proximal $\mathrm{TZ}$ after cilia maturation.

How FBF1 regulates these ciliary proteins trafficking? Smo and SSTR3, two transmembrane proteins, were no enriched in primary cilia in FBF1-deficient cells. This result is not attributed to $\mathrm{TZ}$ proteins since they are unaffected by FBF1 knockout. These data suggested an essential role in ciliary membrane protein gating by DAP proteins.

\section{References}

[1] Yang TT, Su J, Wang WJ, Craige B, Witman GB, Tsou MF, Liao JC." Super-resolution Pattern Recognition Reveals the Architectural Map of the Ciliary Transition Zone," Sci Rep. 5:14096. (2015)

[2] Weng RR, Yang TT, Huang CE, Chang CW, Wang WJ, Liao JC. "Super-Resolution Imaging Reveals TCTN2 Depletion-Induced IFT88 Lumen Leakage and Ciliary Weakening," Biophys J. 115(2):263-275. (2018)

[3] Yang TT, Chong WM, Wang WJ, Mazo G, Tanos B, Chen Z, Tran TMN, Chen YD, Weng RR, Huang CE, Jane WN, Tsou MB, Liao JC, "Super-resolution architecture of mammalian centriole distal appendages reveals distinct blade and matrix functional components," Nat Commun. 9(1):2023 (2018) 


\title{
Subdiffraction-limit optical-pattern generation and its application to super-resolution imaging
}

\author{
Yusuke Ogura*a $^{\text {a }}$ Daiki Shinkawa ${ }^{\mathrm{a}}$, Takahiro Nishimura ${ }^{\mathrm{b}}$, Yosuke Tamada ${ }^{\mathrm{c}}$, Jun Tanida ${ }^{\mathrm{a}}$ \\ ${ }^{a}$ Graduate School of Information Science and Technology, Osaka University, 1-5 Yamadaoka, \\ Suita, Osaka 565-0871, Japan \\ ${ }^{\mathrm{b}}$ Graduate School of Engineering, Osaka University, 2-1 Yamadaoka, Suita, Osaka 565-0871, Japan \\ ${ }^{\mathrm{c}}$ Division of Evolutionary Biology, National Institute for Basic Biology, 38 Nishigonaka, Myodaiji, \\ Okazaki, Aichi 444-8585, Japan
}

\begin{abstract}
Illumination is an important factor for many types of super-resolution imaging, including structured illumination microscopy and stimulated emission depletion microscopy. In this paper, we present generation of subdiffraction-limit optical patterns that are finer than the diffraction-limit spot, using a computer generated hologram (CGH). Such optical patterns can be utilized in super-resolution imaging as illumination to increase its performance. An algorithm has been constructed to design CGHs generating subdiffraction-limit optical patterns. We demonstrated the generation of subdiffraction-limit optical spots and a subdiffraction-limit light sheet. Furthermore, an array of subdiffraction-limit spots is applied to enhance the resolution in laser scanning microscopy. Simulation and experimental results show that the validity of using subdiffraction-limit optical spots in super-resolution imaging.
\end{abstract}

Keywords: subdiffraction limit, spot, light sheet, computer generated hologram, super-resolution

\section{Introduction}

Diffraction limit restricts the performance of optical technologies, not only imaging including microscopy but also generating optical patterns. Several types of superresolution microscopy utilize adequate modification of illumination [1]. Structured illumination microscopy, which uses stripe-shaped illumination, is a typical example. Stimulated emission depletion (STED) microscopy is based on miniaturization of an effective excitation area by combining illumination light for STED and excitation. These facts suggest that optical patterns as illumination are important for enhancing imaging performance.

We are studying about a method for generating subdiffraction-limit optical patterns that are finer than the diffraction limit spot in a local space, using a computer generated hologram (CGH) [2]. Because these optical patterns are generated using propagating light, they provide many benefits including spatial parallelism, noncontact accessibility to objects, and multiplexing capability. This paper presents recent results in generation of subdiffraction-limit optical spots and light sheets. We also discuss laser scanning microscopy using subdiffraction-limit spots as illumination.

\section{Subdiffraction-limit optical pattern generation}

The achievable optical patterns by using CGHs are usually constrained by the diffraction limit of light, and therefore fine optical-pattern generation requires careful treatment in design. Our design method is based on the Gerchberg-Saxton algorithm, but the constraint on the output plane is modified to consider the phase of optical patterns [2]. Let us consider that three optical spots are positioned in a line. When the phases of the sides' spots are different from the phase of the central spot by $\pi$, zerointensity points are created between adjacent spots, then the spots are separated and their size is reduced. The constraint in our algorithm is constructed on this basis.

Figure 1 shows an array of $3 \times 3$ subdiffraction-limit spots generated by a designed CGH. The optical pattern was captured on the focal plane of an objective lens with the numerical aperture (NA) of 0.46 . The size of the spot was reduced from $1.31 \mu \mathrm{m}$ to $0.93 \mu \mathrm{m}(71 \%)$. Note that the number and positions of spots can be determined flexibly.

Subdiffraction-limit optical patterns generated by our method is not limited to spot arrays. As an option, we investigated a subdiffraction-limit light sheet with micrometer-order thickness. For example, in light sheet 
(a)

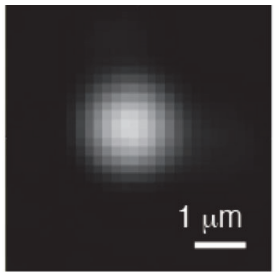

(b)

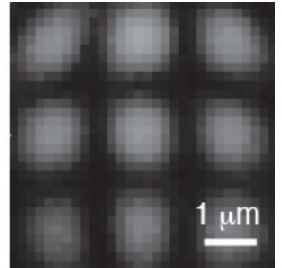

Fig. 1 (a) Diffraction-limit and (b) subdiffraction-limit spots captured in an experiment. (a)

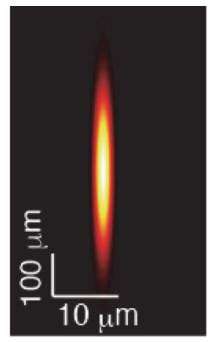

(b)

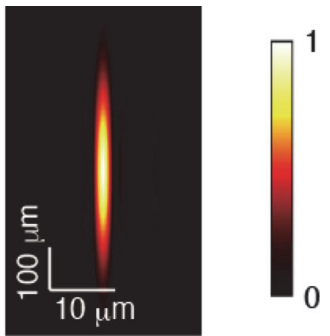

Fig. 2 Cross-sections of (a) a diffraction-limit light sheet and (b) a subdiffraction-limit light sheet.

fluorescence microscopy [3], use of a thinner light sheet as illumination is effective for enhancing the axial resolution, reducing the damage to a sample, and suppressing the photobleaching. Figure 2 shows calculated cross-sections of light sheets generated using a cylindrical lens (focal length: $100 \mathrm{~mm}$ ) and an objective lens (NA: 0.25). The thickness of the light sheet is $1.6 \mu \mathrm{m}$ for subdiffraction limit, whereas it is $2.0 \mu \mathrm{m}$ for diffraction limit.

\section{Super-resolution imaging using a subdiffraction-limit spot array}

Subdiffraction-limit optical patterns are generated using propagating light, thus they can be easily applied to illumination in microscopy. We consider super-resolution imaging by scanning an array of subdiffraction-limit spots. Laser scanning microscopy is an option for superresolution imaging, and its resolution depends on the size of an illuminating spot. Hence use of subdiffraction-limit spots is a promising strategy to enhance the resolution. In addition, parallel scan within the field of view is achievable by use of multiple subdiffraction-limit spots. This offers reduction in time for image acquisition. Fluorescence images are captured at the individual scan positions, and then a high-resolution image is reconstructed through digital processing. To confirm effectiveness of using a

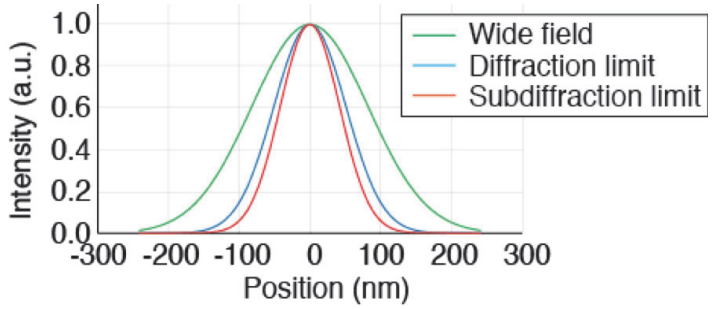

Fig. 3 Comparison of PSFs.

subdiffraction-limit spot, we calculated the point spread function (PSF) of an imaging system using an objective lens with the NA of 1.40 . Figure 3 shows comparison of PSFs obtained by uniform illumination, diffraction-limit spot scanning, and subdiffraction-limit spot scanning. The full width at half maximums (FWHMs) are $194 \mathrm{~nm}, 120$ $\mathrm{nm}$, and $99 \mathrm{~nm}$, respectively. The PSF is reduced by using the subdiffraction-limit spot instead of the diffraction-limit spot. We also experimentally observed fluorescent proteinlabeled tubulin and demonstrated the validity of the method.

\section{Conclusions}

CGHs are capable of generating subdiffraction-limit optical patterns, including spot arrays and light sheets. As an application, the resolution of laser scanning microscopy can be enhanced by using subdiffraction-limit spots as illumination. The features of the method should be useful in bio-imaging.

This research was supported by the grant of Joint Research by the National Institutes of Natural Sciences (NINS program No. 01111702), Research Foundation for Opto-Science and Technology, and JSPS KAKENHI Grant Number $17 \mathrm{H} 02799$.

\section{References}

[1] Huang, B., Bates, M., and Zhuang, X., "SuperResolution Fluorescence Microscopy," Annu. Rev. Biochem. 78, 993-1016 (2009).

[2] Ogura, Y., Aino, M. and Tanida, J., "Design and demonstration of fan-out elements generating an array of subdiffraction spots," Opt. Express 22(21), 2519625207 (2014).

[3] Power, R. M. and Huisken,J., "A guide to light-sheet fluorescence microscopy for multiscale imaging," Nat. Methods 14(4), 360-373 (2017). 


\title{
Optically sectioned, super-resolution imaging by image scanning microscopy using optimized reconstruction algorithm
}

\author{
HUI ZENG ${ }^{\text {** }}$, HUAIDONG YANG $^{\text {a }}$

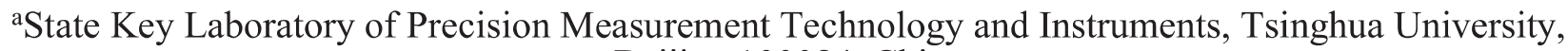 \\ Beijing 100084, China
}

\begin{abstract}
The datasets of image scanning microscopy contain sufficient information which can be used for simultaneous enhancement of lateral spatial resolution and axial sectioning ability. However, an iterative reconstruction method is rather time-consuming, and we report here a novel approach for utilizing this information in a straightforward manner. The approach, termed Optically sectioned super-resolution virtual structured microscopy (OS-SR vSIM), combing virtual modulation method and modified optical transfer function attenuation method, is able to improve the lateral resolution by a factor of 2, reduce the out of focus background and improve the optical sectioning ability. We demonstrated the performance of the reported approach by simulating imaging of designed three-layer sample.
\end{abstract}

Keywords: fluorescence microscopy, super resolution, image reconstruction algorithm

*zengh16@mails.tsinghua.edu.cn

\section{Introduction}

Fluorescence microscopy is an essential tool in life sciences study. However, the sophisticated sub-cellular structures are indistinguishable using conventional fluorescence microscopy because the resolution is limited by diffraction effect. The super resolution technique, which designed for breaking this diffraction barrier has played a significant role in enabling advanced biological research. In the past decade, various super resolution techniques have been developed [1-5]. One class of these techniques use illumination patterns to modulate the high-frequency information into the low-frequency passband. The structure illumination microscopy (SIM) technique $[6,7]$ and Imaging scanning microscopy (ISM)[8-10] are good examples to this end, and both of them are able to achieve a resolution doubling that of wide-field microscopy. SIM is a relatively mature technique. The use of sinusoidal pattern illumination in fluorescence microscopy allows the suppression of out of focus light and an increase in effective spatial resolution. The Optical Transfer Function (OTF) attenuation algorithm of SIM combines these two attributes, to allow optically sectioned, super resolution imaging[11, 12]. ISM is an ever-developing method and shows great potentials as a super-resolution technique. It can be considered as a confocal microscope with a detector array. Using pixel reassignment algorithm[9], ISM doubles the cutoff spatial frequency of 3D OTF in all three directions compared to conventional microscopy, but the 'missing cone' region is still present. The out of focus information is contained primarily with the 'missing cone' region close the center of 3D OTF, so the sectioning ability of ISM is weak. Although optical sectioning can be regained by limiting the size of the detector array, that give a compromise in resolution improvement and detection efficiency. We present an image reconstruction scheme of ISM for achieving simultaneous enhancement of spatial resolution and optical sectioning named Optically sectioned, super-resolution virtual structured illumination microscopy (OS-SR vSIM). The virtual modulation method[13] was utilized to convert the ISM data into SIM data and solve the super resolution frequency components. Then, the modified OTF attenuation algorithm of SIM was applied to perfectly fill the 'missing cone' region and remove the out of focus information.

\section{Method}

\section{Virtual modulation algorithm}

For a general ISM system, the imaging process can be expressed as follows:

$$
\begin{aligned}
& \mathrm{D}\left(\boldsymbol{r}_{\boldsymbol{d}}, \boldsymbol{r}_{\boldsymbol{s}}\right)=\iint h_{\text {ill }}\left(\boldsymbol{r}^{\prime}-\boldsymbol{r}_{s}\right) S\left(\boldsymbol{r}^{\prime}\right) h_{\text {det }}\left(\boldsymbol{r}_{\boldsymbol{d}}-\boldsymbol{r}^{\prime}\right) d^{2} \boldsymbol{r}^{\prime} \\
& \mathrm{D}\left(\boldsymbol{r}_{\boldsymbol{d}}, \boldsymbol{r}_{\boldsymbol{s}}\right) \text { is the signal recorded at a point } \boldsymbol{r}_{\boldsymbol{d}}=\left(x_{d}, y_{d}\right)
\end{aligned}
$$
in the plane of the detector when a two-dimensional fluorescent sample of strength $S\left(\boldsymbol{r}^{\prime}\right)$ is illuminated by a 
light spot $h_{i l l}$ at scan position $\boldsymbol{r}_{\boldsymbol{s}}=\left(x_{s}, y_{s}\right)$. and $h_{\text {det }}$ is the PSF of our detecting system. The virtual modulation algorithm is to modulate the signals of ISM via digital implementation in spatial domain. The image after the modulation can be expressed as

$$
\begin{aligned}
\mathrm{D}\left(\boldsymbol{r}_{\boldsymbol{d}}\right) & =\iiint \int h_{i l l}\left(\boldsymbol{r}^{\prime}-\boldsymbol{r}_{\boldsymbol{s}}\right) S\left(\boldsymbol{r}^{\prime}\right) h_{d e t}\left(\boldsymbol{r}_{\boldsymbol{d}}-\boldsymbol{r}^{\prime}\right) M\left(\boldsymbol{r}_{\boldsymbol{s}}\right) \\
& d^{2} \boldsymbol{r}_{\boldsymbol{s}} d^{2} \boldsymbol{r}^{\prime} \\
& =\left[M \otimes h_{i l l} \cdot \mathrm{S}\right] \otimes h_{d e t}
\end{aligned}
$$

Where $\mathrm{D}\left(\boldsymbol{r}_{\boldsymbol{d}}\right)$ denotes the virtual modulated image; $\otimes$ represents the convolution operation and $M$ denotes the virtual modulation, which is a sinusoidal pattern written as

$$
M\left(\boldsymbol{r}_{s}\right)=\cos \left(\varphi+2 \pi \boldsymbol{f} \cdot \boldsymbol{r}_{\boldsymbol{s}}\right)
$$

Where $\boldsymbol{f}=\left(f_{x}, f_{y}\right)$ denotes spatial modulation frequency and $\varphi$ represents the phase of sinusoidal pattern. The convolution between $M$ and $h_{i l l}$ can be simplified as

$$
M \otimes h_{i l l}\left(\boldsymbol{r}^{\prime}\right)=m M\left(\boldsymbol{r}^{\prime}\right)
$$

Where $m$ denotes the modulation factor introduced by $h_{i l l}$. By using Eq.(4) and convolution theorem, Eq.(2) can be illustrated at frequency domain as

$$
\begin{aligned}
D(\boldsymbol{k}) & =m S(\boldsymbol{k}) \otimes M(\boldsymbol{k}) \cdot H_{\text {det }}(\boldsymbol{k}) \\
= & \frac{m}{2}\left[\mathrm{~S}(\boldsymbol{k}-\boldsymbol{f}) e^{-i \varphi}+\mathrm{S}(\boldsymbol{k}+\boldsymbol{f}) e^{i \varphi}\right] \cdot H_{\text {det }}(\boldsymbol{k})
\end{aligned}
$$

where $H_{\text {det }}(\boldsymbol{k})$ is system optical transfer function (OTF). The ISM data is perfectly converted into SIM data and there is no direct current (DC) component in $D(\boldsymbol{k})$. Therefore, the number of required SIM raw images are reduced. It is worth noting that the modulation is implemented in a digital manner, the parameters of the illumination patterns like orientations, period and phases could be freely controlled.

2.Optically sectioned, superresolution reconstruction

In order to simultaneously provides a two-fold increase in spatial resolution and enhancement of optical sectioning, the SIM raw dataset with three pattern orientations spaced $60^{\circ}$ apart $(d=1,2,3)$ and $N$ modulation frequency $(N \geq 2)$ are required. The super resolution components $\mathrm{S}(\boldsymbol{k}) H_{\text {det }}\left(\boldsymbol{k}-\boldsymbol{f}_{n d}\right)(n=1,2, \ldots, N)$ can be calculated using conventional SIM algorithms. $\left|\boldsymbol{f}_{n d}\right|=\frac{n}{N} f_{\text {cut }}, f_{c u t}$ denotes the cutoff frequency.

Because the fluorescence signals are detected the same as wide-field format, out of focus fluorescence blur is inevitable, especially imaging thick or densely labeled samples. Therefore, reconstruction images of these conditions are easy to produce artifacts[14-16]. Exploiting the fact that defocus information is mainly contained in the area near the center and edge of each spatial frequency component $\mathrm{S}(\boldsymbol{k}) H_{\text {det }}\left(\boldsymbol{k}-\boldsymbol{f}_{n d}\right)$, Each component was multiplied by an attenuation function $a_{n d}(\boldsymbol{k})$ respectively to reduce the overall amount out of focus light in the reconstructed image $\mathrm{I}_{\text {rec }}$.

$$
\begin{gathered}
\mathrm{I}_{r e c}=\sum_{d=1}^{3} \sum_{n=-N}^{N} a_{n d}\left(\boldsymbol{k}-\boldsymbol{f}_{n d}\right) \mathrm{S}(\boldsymbol{k}) H_{\text {det }}\left(\boldsymbol{k}-\boldsymbol{f}_{n d}\right) \\
a_{n d}(\boldsymbol{k})= \begin{cases}1-e^{-\frac{|k|^{2}}{k_{\sigma}^{2}}}-e^{-\frac{\left(|k|-f_{c u t}\right)^{2}}{k_{\sigma}^{2}}} & n \neq N \\
1-e^{-\frac{|k|^{2}}{k_{\sigma}^{2}}} & n=N\end{cases}
\end{gathered}
$$

Where $k_{\sigma}$ is the standard deviation of Gaussian attenuation function. Due to the removed areas have high absolute signals, the performance of this optically sectioned algorithm can be degraded by noise. Thus, there exist a tradeoff between signal-to-noise ratio and gains in sectioning, so the parameters $k_{\sigma}$ should be adjusted according to the experiment situation. The $\mathrm{N}$ order frequency components, which extend to limit frequency, their peripheral zone should be preserved. Through this optimization algorithm, the ISM data from one focal plane can be used to reconstruct an optically sectioned superresolution image.

\section{Results and Discussion}

We designed a three-layer sample shown as Figure 1 to prove our approach's ability to simultaneously reject outof-focus background noises and enhance the lateral resolution. We simulated imaging using wide-field microscopy, conventional SIM, Pixel Reassignment ISM and our approach. We found that our approach achieved the best background suppression performance while kept the lateral super resolution.
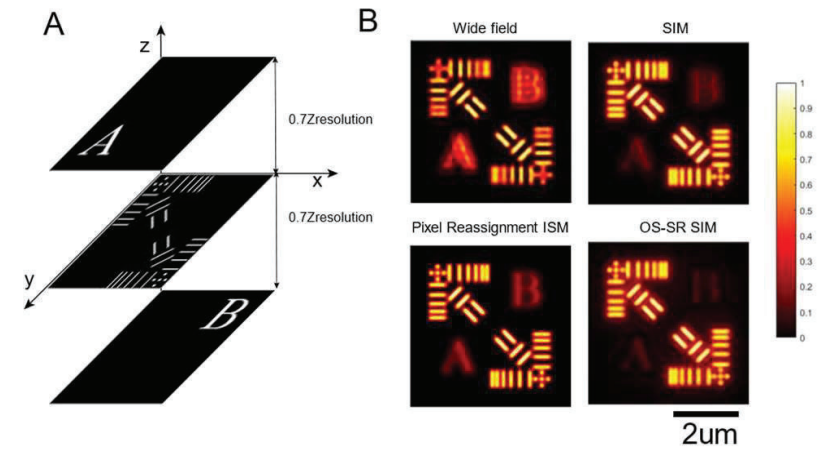
Figure 1. (A) The designed sample of simulation. The in-focus layer is a resolution test sample, and the defocus layers are two words ' $A$ ' and ' $B$ '. The distance from the defocus layers to in-focus layer is 0.7 times of system axial resolution. (B) Imaging results of wide field deconvolution, conventional SIM, Pixel Reassignment ISM, and OS-SR vSIM.

In summary, we put forward a new super resolution imaging approach for fluorescent samples by integrating the virtual modulation method and OTF attenuation algorithm. The approach provides a straightforward method to improve the lateral resolution by a factor of 2 and reduce the out of focus background, facilitating observation of structures in thick, densely labeled samples. This approach can be applied to process the data acquired under the multi-spot illumination system using microlens array. It has the potential to achieve high speed live-cell OS-SR imaging.

\section{References}

1. M. J. Rust, M. Bates, and X. Zhuang, "Subdiffraction-limit imaging by stochastic optical reconstruction microscopy (STORM)," Nat. Methods 3, 793-795 (2006).

2. S. W. Hell and J. Wichmann, "Breaking the diffraction resolution limit by stimulated emission: stimulated-emission-depletion fluorescence microscopy," Optics Letters 19, 780-782 (1994).

3. E. Betzig, G. H. Patterson, R. Sougrat, O. W. Lindwasser, S. Olenych, J. S. Bonifacino, M. W. Davidson, J. Lippincott-Schwartz, and H. F. Hess, "Imaging intracellular fluorescent proteins at nanometer resolution," Science 313, 1642-1645 (2006).

4. T. Dertinger, R. Colyer, G. Iyer, S. Weiss, and J. Enderlein, "Fast, background-free, 3D super-resolution optical fluctuation imaging (SOFI)," Proceedings of the National Academy of Sciences of the United States of America 106, 22287-22292 (2009).

5. G. Zhao, C. Zheng, C. Kuang, R. Zhou, M. M. Kabir, K. C. Toussaint, W. Wang, L. Xu, H. Li, P. Xiu, and X. Liu, "Nonlinear Focal Modulation Microscopy," Phys Rev Lett 120, 193901 (2018).

6. M. G. L. Gustafsson, "Surpassing the lateral resolution limit by a factor of two using structured illumination microscopy," Journal of Microscopy-Oxford 198, 82-87 (2000).

7. M. G. L. Gustafsson, L. Shao, P. M. Carlton, C. J. R. Wang, I. N. Golubovskaya, W. Z. Cande, D. A. Agard, and J. W. Sedat, "Three-dimensional resolution doubling in wide-field fluorescence microscopy by structured illumination," Biophysical Journal 94, 4957-4970 (2008). 8. C. B. Muller and J. Enderlein, "Image scanning microscopy," Phys Rev Lett 104, 198101 (2010).

9. C. J. R. Sheppard, S. B. Mehta, and R. Heintzmann, "Superresolution by image scanning microscopy using pixel reassignment," Optics Letters 38, 2889-2892 (2013). 10. M. Guo, P. Chandris, J. P. Giannini, A. J. Trexler, R. Fischer, J. Chen, H. D. Vishwasrao, I. Rey-Suarez, Y. Wu, X. Wu, C. M. Waterman, G. H. Patterson, A. Upadhyaya, J. W. Taraska, and H. Shroff, "Single-shot super-resolution total internal reflection fluorescence microscopy," Nat. Methods (2018).

11. K. O'Holleran and M. Shaw, "Optimized approaches for optical sectioning and resolution enhancement in 2D structured illumination microscopy," Biomedical Optics Express 5, 2580-2590 (2014).

12. M. Shaw, L. Zajiczek, and K. O'Holleran, "High speed structured illumination microscopy in optically thick samples," Methods 88, 11-19 (2015).

13. R. Cao, C. Kuang, Y. Liu, and X. Liu, "Superresolution via saturated virtual modulation microscopy," Optics Express 25, 32364 (2017).

14. D. Li, L. Shao, B.-C. Chen, X. Zhang, M. Zhang, B Moses, D. E. Milkie, J. R. Beach, J. A. Hammer, III, M. Pasham, T. Kirchhausen, M. A. Baird, M. W. Davidson, P. Xu, and E. Betzig, "Extended-resolution structured illumination imaging of endocytic and cytoskeletal dynamics," Science 349(2015).

15. S. J. Sahl, F. Balzarotti, J. Keller-Findeisen, M. Leutenegger, V. Westphal, A. Egner, F. Lavoie-Cardinal, A. Chmyrov, T. Grotjohann, and S. Jakobs, "Comment on "Extended-resolution structured illumination imaging of endocytic and cytoskeletal dynamics"," Science 352(2016).

16. D. Li and E. Betzig, "Response to Comment on "Extended-resolution structured illumination imaging of endocytic and cytoskeletal dynamics"," Science 352(2016). 


\title{
Applying terahertz solid immersion microscopy for sub-wavelength-resolution imaging of soft biological tissues
}

\author{
Anna S. Kucheryavenko, ${ }^{a, b}$, Nikita V. Chernomyrdin ${ }^{a, b}$, Irina N. Dolganova ${ }^{b, c}$, \\ Vladislav A. Zhelnov ${ }^{\mathrm{b}}$, Pavel A. Karalkin ${ }^{\mathrm{d}, \mathrm{e}}$, Anna A. Gryadunova ${ }^{\mathrm{d}, \mathrm{f}}$, Valery E. Karasik ${ }^{\mathrm{b}}$, \\ Valery V. Tuchin ${ }^{\mathrm{g}}$, and Kirill I. Zaytseve ${ }^{\mathrm{a}, \mathrm{b}}$ \\ aprokhorov General Physics Institute of the Russian Academy of Sciences, \\ Moscow 119991, Russia \\ ${ }^{b}$ Bauman Moscow State Technical University, Moscow 105005, Russia \\ ${ }^{\mathrm{c}}$ Institute of Solid State Physics of the Russian Academy of Sciences, \\ Chernogolovka 142432, Russia \\ d3D Bioprinting Solutions, Moscow 115409, Russia \\ eNational Medical Research Center of Radiology, Moscow 125284, Russia

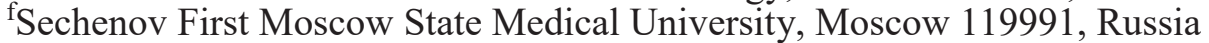 \\ ${ }^{\mathrm{g}}$ Saratov State University, Saratov 410012, Russia
}

\begin{abstract}
Terahertz (THz) imaging and spectroscopy have found numerous applications in biology and medicine. However, THz optical systems suffer from low spatial resolution due to relatively large $\mathrm{THz}$ wavelengths. Thus, conventional modalities of $\mathrm{THz}$ imaging are not suitable for studying low-scale inhomogeneity of biological tissues, such as microfibrils, separate cells and cell organelles. In order to mitigate the problem of improving the resolution of $\mathrm{THz}$ imaging of biological tissues, in our work, we developed a method of THz solid immersion microscopy, which allows for overcoming the Abbe diffraction limit and provides $0.15 \lambda$-resolution, where $\lambda$ is an electromagnetic wavelength. The proposed approach relies on the solid immersion phenomenon - i.e. on reduction in the dimensions of electromagnetic beam caustic, when the beam is focused in a free space, at a small distance $(<\lambda)$ behind a medium featuring high refractive index. In order to assemble the $\mathrm{THz}$ solid immersion microscope, we used a backward wave oscillator, as a continuous-wave emitter operating at $\lambda=500 \mu \mathrm{m}$, and a Golay cell, as a broadband detector of THz beam intensity.

Keywords: terahertz technology, terahertz imaging, sub-wavelength spatial resolution, Abbe diffraction limit, solid immersion phenomenon, biological tissue.

\section{Introduction}

Recently a remarkable progress in terahertz $(\mathrm{THz})$ spectroscopy and imaging was observed [1]. Among the applications of $\mathrm{THz}$ technology, medical diagnosis is of particular interest $[2,3]$. It was demonstrated that methods of $\mathrm{THz}$ imaging and spectroscopy yields label-free differentiation between healthy tissues and malignant tumors of various localization and nosology [4-6], as well as to analyze glycation of tissues [7-9]. However, some challenging problems are inherent to modern $\mathrm{THz}$ systems, among them we can note

- low performance (operation rate) or signal-tonoise ratio in $\mathrm{THz}$ images due to the limited efficiency of conventional $\mathrm{THz}$ wave emitters and detectors $[10,11]$;

- absence of efficient flexible fibers, hard waveguides and endoscopic systems for $\mathrm{THz}$ wave delivery [12];

- finally, low spatial resolution of $\mathrm{THz}$ spectroscopy and imaging [13].

In this work, we would focus on the last problem. Conventional $\mathrm{THz}$ imaging systems are based on spherical lenses and focusing mirror, which are characterized by diffraction-limited spatial resolution [14]. Such approaches as lens-less $\mathrm{THz}$ digital holography, synthetic aperture technique require complicated computations for resolving the inverse illposed problems [15-17]. Methods of $\mathrm{THz}$ near-field scanning-probe microscopy can provide spatial resolution up to $10^{-3} \lambda$ but require applying high-power sources, sensitive detectors and imply small distance between the probe and the object of interest [18,19]. Thereby, development of novel sub-wavelength-resolution modalities of $\mathrm{THz}$ imaging for studying soft biological tissues remains a challenging problem of $\mathrm{THz}$ science and technology.
\end{abstract}

Anna S. Kucheryavenko, E-mail: ans.kucher@mail.ru Nikita V. Chernomyrdin, E-mail: chernik-a@yandex.ru Kirill I. Zaytsev, E-mail: kirzay@gmail.com 
In our work, we developed $\mathrm{THz}$ imaging setup for studying biological objects and tissues with subwavelength spatial resolution. The core element of this system is a solid immersion lens, which provides high energy efficiency and $0.15 \lambda$-resolution. Such an advanced spatial resolution (it is beyond the $0.5 \lambda$ Abbe limit) is achieved thanks to the solid immersion effect. The results of experimental studies of various types of biological tissues using the proposed $\mathrm{THz}$ imaging modality reveal the sub-wavelength structural features of tissues; thus, highlighting a potential of the $\mathrm{THz}$ solid immersion microscopy in biophotonics.

\section{Results}

We assembled $\mathrm{THz}$ imaging setup based on backward wave oscillator, i.e. a continuous-wave source operating at $\lambda=500 \mu \mathrm{m}$, and a Golay cell, i.e. an opto-acoustic detector of electromagnetic beam intensity. The main element of the developed system is a solid immersion lens, which is comprised of three parts:

- an aspherical singlet, which is made of highdensity polyethylene (HDPE) and forms a converging beam with spherical wavefront and good aberrational correction [14];

- a hypo-hemispherical lens (a truncated sphere), which is made of high-resistivity float-zone silicon (HRFZ-Si) and mounted in front of the focal plane; its spherical surface is concentric to the convergent spherical wavefront of electromagnetic wave, while its flat surface is parallel to the image plane [13];

- a flat window, which is made of HRFZ-Si $\left(n_{\mathrm{Si}}=3.41\right)$ and being kept in touch with the flat surface of the HRFZ-Si hemisphere; this flat window serves simultaneously as a movable sample holder, on the top of which the sample is placed, and a part of the unitary optical element (HRFZ-Si hemisphere and flat window form a unitary HRFZ-Si resolution enhancer) [20].

In this solid immersion lens, the $\mathrm{THz}$ beam is focused in a free space, at the distance of about $l \simeq \lambda / 2$ behind the flat surface of the HRFZ-Si resolution enhancer. The caustic of electromagnetic beam is reduced $\sim n_{\mathrm{Si}}$-times, as compared to the electromagnetic wave focusing in a free space (without the HRFZ-Si resolution enhancer), thanks to the solid immersion effect $[13,20]$.

We applied the experimental setup for $\mathrm{THz}$ imaging of various test objects and biological tissues of different kind. During measurements, a sample is mounted onto the flat surface of the HRFZ-Si resolution enhancer and imaged in reflection-mode by raster scanning of its surface with the $\mathrm{THz}$ beam caustic. This scanning is performed by shifting the flat window (with the sample on the top of it) toward the hemisphere. By combining numerical simulations (using the finite-difference time- domain technique for solving the Maxwell's equations) and experimental studies (involving imaging of metal test objects featuring abrupt changes of reflectivity), we demonstrated that the abovementioned configuration of the $\mathrm{THz}$ solid immersion microscopy provides the impressive spatial resolution of $0.15 \lambda[13,20]$.

We applied developed setup for THz SIL microscopy of several types of biological tissues, among them:

- plant leaf blades [20,21];

- tissue spheroids made of chondrocytes and featuring the diameter of about $30 \mu \mathrm{m}[20,22]$; these spheroids are used in $3 \mathrm{D}$ bioprinting of tissues;

- soft biological tissues of the human body ex vivo $[20,23]$.

In our $\mathrm{THz}$ images, we observed sub-wavelength scale inhomogeneities of tissues, such as:

- veins in the leaf blades;

- separate cell spheroids;

- single large fat cells and their agglomerates in connective tissues of the breast ex vivo;

- channels in tissues of the breast ex vivo;

- muscle fibers in tissues of the tongue ex vivo.

$\mathrm{THz}$ images correlated with results of hematoxylin and eosin stained microscopy in the visible range. Abovementioned highlights the importance of taking into account the scattering properties of biological tissues during modeling of $\mathrm{THz}$ wave - tissues interactions.

\section{Acknowledgments}

This work is supported by the Russian Science Foundation, Project \#17-79-20346.

\section{References}

[1] Hu, B. B. and Nuss, M. C., "Imaging with terahertz waves," Opt. Lett. 20(16), 1716-1718 (1995).

[2] Yang, X., Zhao, X., Yang, K., Liu, Y., Liu, Y., $\mathrm{Fu}, \mathrm{W}$. and Luo, Y., "Biomedical Applications of Terahertz Spectroscopy and Imaging," Trends Biotechnol. 34(10), 810-824 (2016).

[3] Smolyanskaya, O. A., Chernomyrdin, N. V., Konovko, A. A., Zaytsev, K. I., Ozheredov, I. A., Cherkasova, O. P., Nazarov, M. M., Guillet, J.-P., Kozlov, S. A., Kistenev, Y. V., Coutaz, J.-L., Mounaix, P., Vaks, V. L., Son, J.-H., Cheon, H., Wallace, V., Shkurinov, A. P. and Tuchin, V. V., "Terahertz biophotonics as a tool for studies of dielectric and spectral properties of tissues and bioliquids related to water content," Prog. Quantum Electron. 62, 1-77 (2018).

[4] Woodward, R. M., Wallace, V. P., Pye, R. J., Cole, B. E., Arnone, D. D., Linfield, E. H. and Pepper, M., "Terahertz Pulse Imaging of ex vivo Basal Cell Carcinoma,” J. Invest. Dermatol. 120(1), 72-78 (2003).

[5] Joseph, C. S., Patel, R., Neel, V. A., Giles, R. H. and Yaroslavsky, A. N., "Imaging of ex vivo 
nonmelanoma skin cancers in the optical and terahertz spectral regions optical and terahertz skin cancers imaging," J. Biophotonics 7(5), 295-303 (2014).

[6] Zaitsev, K. I., Chernomyrdin, N. V., Kudrin, K. G., Reshetov, I. V. and Yurchenko, S. O., "Terahertz spectroscopy of pigmentary skin nevi in vivo," Opt. Spectrosc. 119(3), 404-410 (2015).

[7] Smolyanskaya, O. A., Lazareva, E. N., Nalegaev, S. S., Petrov, N. V., Zaytsev, K. I., Timoshina, P. A., Tuchina, D. K., Toropova, Y. G., Kornyushin, O. V., Babenko, A. Y., Guillet, J. P. and Tuchin, V. V., "Multimodal optical diagnostics of glycated biological tissues," Biochem. 84(Suppl. 1), S124-S143 (2019).

[8] Hernandez-Cardoso, G. G., Rojas-Landeros, S. C., Alfaro-Gomez, M., Hernandez-Serrano, A. I., SalasGutierrez, I., Lemus-Bedolla, E., Castillo-Guzman, A. R., Lopez-Lemus, H. L. and Castro-Camus, E., "Terahertz imaging for early screening of diabetic foot syndrome: A proof of concept," Sci. Rep. 7(1), 42124 (2017).

[9] Cherkasova, O., Nazarov, M. and Shkurinov, A., "Noninvasive blood glucose monitoring in the terahertz frequency range," Opt. Quantum Electron. 48(3), 217 (2016).

[10] Lavrukhin, D. V., Yachmenev, A. E., Glinskiy, I. A., Khabibullin, R. A., Goncharov, Y. G., Ryzhii, M., Otsuji, T., Spector, I. E., Shur, M., Skorobogatiy, M., Zaytsev, K. I. and Ponomarev, D. S., "Terahertz photoconductive emitter with dielectric-embedded highaspect-ratio plasmonic grating for operation with lowpower optical pumps," AIP Adv. 9(1), 015112 (2019).

[11] Lavrukhin, D. V., Yachmenev, A. E., Pavlov, A., Khabibullin, R. A., Goncharov, Y. G., Spektor, I. E., Komandin, G., Yurchenko, S. O., Chernomyrdin, N. V., Zaytsev, K. I. and Ponomarev, D. S., "Shaping the spectrum of terahertz photoconductive antenna by frequency-dependent impedance modulation," Semicond. Sci. Technol. (2019; accepted; in press).

[12] Katyba, G. M., Zaytsev, K. I., Chernomyrdin, N. V, Shikunova, I. A., Komandin, G. A., Anzin, V. B., Lebedev, S. P., Spektor, I. E., Karasik, V. E., Yurchenko, S. O., Reshetov, I. V, Kurlov, V. N. and Skorobogatiy, M., "Sapphire Photonic Crystal Waveguides for Terahertz Sensing in Aggressive Environments," Adv. Opt. Mater. 6, 1800573 (2018).

[13] Chernomyrdin, N. V., Schadko, A. O., Lebedev, S. P., Tolstoguzov, V. L., Kurlov, V. N., Reshetov, I. V., Spektor, I. E., Skorobogatiy, M., Yurchenko, S. O. and Zaytsev, K. I., "Solid immersion terahertz imaging with sub-wavelength resolution," Appl. Phys. Lett. 110(22), 221109 (2017).

[14] Chernomyrdin, N. V., Frolov, M. E., Lebedev, S. P., Reshetov, I. V., Spektor, I. E., Tolstoguzov, V. L., Karasik, V. E., Khorokhorov, A. M., Koshelev, K. I., Schadko, A. O., Yurchenko, S. O. and Zaytsev, K. I.,
"Wide-aperture aspherical lens for high-resolution terahertz imaging," Rev. Sci. Instrum. 88(1), 014703 (2017).

[15] Zhang, Y., Zhou, W., Wang, X., Cui, Y. and Sun, W., "Terahertz Digital Holography," Strain 44(5), 380-385 (2008).

[16] Krozer, V., Löffler, T., Dall, J., Kusk, A., Eichhorn, F., Olsson, R. K., Buron, J. D., Jepsen, P. U., Zhurbenko, V. and Jensen, T., "Terahertz imaging systems with aperture synthesis techniques," IEEE Trans. Microw. Theory Tech. 58(7 PART 2), 2027-2039 (2010).

[17] Guerboukha, H., Nallappan, K. and Skorobogatiy, M., "Exploiting k-space/frequency duality toward real-time terahertz imaging," Optica 5(2), 109 (2018).

[18] Chen, H.-T., Kersting, R. and Cho, G. C., "Terahertz imaging with nanometer resolution," Appl. Phys. Lett. 83(15), 3009-3011 (2003).

[19] Minin, I. V., Minin, O. V., Katyba, G. M., Chernomyrdin, N. V., Kurlov, V. N., Zaytsev, K. I., Yue, L., Wang, Z. and Christodoulides, D. N., "Experimental observation of a photonic hook," Appl. Phys. Lett. 114(3), 031105 (2019).

[20] Chernomyrdin, N. V., Kucheryavenko, A. S., Kolontaeva, G. S., Katyba, G. M., Dolganova, I. N., Karalkin, P. A., Ponomarev, D. S., Kurlov, V. N., Reshetov, I. V., Skorobogatiy, M., Tuchin, V. V. and Zaytsev, K. I., "Reflection-mode continuous-wave $0.15 \lambda$ -resolution terahertz solid immersion microscopy of soft biological tissues," Appl. Phys. Lett. 113(11), 111102 (2018).

[21] Chernomyrdin, N. V., Kucheryavenko, A., Malakhov, K., Schadko, A., Komandin, G., Lebedev, S., Dolganova, I., Kurlov, V., Lavrukhin, D., Ponomarev, D., Yurchenko, S., Tuchin, V. V. and Zaytsev, K., "Terahertz solid immersion microscopy for sub-wavelengthresolution imaging of biological objects and tissues," Proc. SPIE 10716, 1071606 (2018).

[22] Chernomyrdin, N. V., Kucheryavenko, A. S., Zaytsev, K. I., Kolontaeva, G. S., Katyba, G. M., Karalkin, P. A., Smolyanskaya, O. A., Karasik, V. E., Minin, O. V., Parfenov, V. A., Gryadunova, A. A., Norkin, N. E. and Minin, I. V., "A potential of terahertz solid immersion microscopy for visualizing subwavelength-scale tissue spheroids," Proc. SPIE 10677, C. Fournier, M. P. Georges, and G. Popescu, Eds., 106771Y, SPIE (2018).

[23] Chernomyrdin, N. V., Kucheryavenko, A. S., Rimskaya, E. N., Dolganova, I. N., Zhelnov, V. A., Karalkin, P. A., Gryadunova, A. A., Reshetov, I. V., Lavrukhin, D. V., Ponomarev, D. S., Karasik, V. E. and Zaytsev, K. I., "Terahertz microscope based on solid immersion effect for imaging of biological tissues," Opt. Spectrosc. (2019; accepted; in press) 


\title{
Needle-Probe Optical Coherence Tomography for Intelligent Guidance of
}

\section{Epidural Anesthesia}

\author{
Wen-Chuan kuo \\ Institute of Biophotonics, National Yang-Ming University, Taipei 112, Taiwan
}

\begin{abstract}
In this presentation, I will focus on our recent works where we developed an innovative image-guided technology using a needle probe optical coherence tomography (OCT) system with an automatic identification algorithm to reduce complications during epidural needle insertion. 3D monitoring of the dura puncture and blood patch process were also discussed. Finally, I will also introduce the multi-contrast OCT for the assessment of pathological processes, such as in the retina degeneration and tumor detection.
\end{abstract}

Keywords: optical coherence tomography, needle probe, epidural anesthesia, multi-contrast

\section{Epidural blockade and its key problems}

An epidural blockade is a useful technique to reduce the pain induced in surgery, labor, and delivery. By placing anesthetics within a local region of the spinal epidural space (ES), sensory, motor and sympathetic nerves within and near the spinal level will be blocked. For example, couples of the birth mothers have received painless labor (epidural analgesia) in Taiwan. For thoracic or other surgery, continuous drug infusion epidural catheters could be placed in order to provide intraoperative and postoperative analgesia. Besides acute pain management, epidural analgesia could also be used in the management of severe chronic pain in cancer patients with painful metastasis. Thus, epidural anesthesia could not only affect a spectrum of healthy people but also seriously ill patients, is an intervention with high societal impact. However, epidural needle insertion is traditionally a blind technique whose success relies mainly on tactile sensation, which depends on the experience of the operator. Complications could arise from uncertainty in the position of the needle tip relative to the dura and bone, or the catheter tip relative to blood vessels, and pockets of closed space. The most common complication of epidural anesthesia is inadequate pain control because of incorrect placement. These incorrect placements can also lead to complications including dural puncture and neural injury. The technique of the loss of resistance (LOR) to air or hanging drops is the most common method used to identify the ES. However, using the LOR technique can still cause an epidural failure rate of $3-10 \%$ (i.e., it is highly dependent on the technique of the operator) owing to incorrect catheter placement.

\section{ES detection methods}

\section{The comparison between several modalities}

Recently, many technologically assisted epidural needle systems have been proposed, including those employing mechanical force or pressure monitoring [1,2], electrical stimulation [3,4], and optical methods [5-8]. However, neither provides a visual image of the epidural tissue. Although ultrasound [9-16] can distinguish tissue types and therefore build a visual image of the axial anatomy, the technique does not provide adequate resolution to distinguish the tissue lasers where the needle travels through. Besides, the epidural and spinal spaces are surrounded by a complex bony structure, which limits the use of ultrasound to identify the ligamentum flavum (LF), ES, and dura well in adults, whose bones are fully calcified. Thus, ultrasound can only assist in landmark identification and reducing the contact with the bony structure. The epidural failure using sonographic-assisted neuraxial placement is still reported. An ideal method for locating the ES using direct sight has not yet been discovered. Clinicians are still searching for practical neuraxial techniques that allow "real-time" observation of the epidural catheter introduction and drug deposition. 
The comparison between several modalities is summarized in Table 1.

Table 1

\begin{tabular}{|c|c|c|}
\hline \multicolumn{2}{|c|}{ Method and Features } & Disadvantages \\
\hline \multicolumn{2}{|c|}{ Force or Pressure monitoring ${ }^{1,2}$} & Single point measurement \\
\hline \multicolumn{2}{|c|}{ Electrical stimulation ${ }^{3,4}$} & Neither provides a visual image of \\
\hline $\begin{array}{l}\text { Optical } \\
\text { method }^{5-8}\end{array}$ & $\begin{array}{l}\text { optical spectrum or dual wavelength } \\
\text { reflectance }\end{array}$ & the epidural tissue \\
\hline Ultrasound $d^{9-16}$ & $\begin{array}{l}\text { build a visual image of the axial anatomy, } \\
\text { can only assist in landmark identification } \\
\text { and reducing the contact with the bony } \\
\text { structure. }\end{array}$ & $\begin{array}{l}\text { 1.does not provide adequate } \\
\text { resolution } \\
\text { 2.epidural and spinal spaces are } \\
\text { surrounded by a complex bony } \\
\text { structure }\end{array}$ \\
\hline
\end{tabular}

\section{Optical coherence tomography (OCT)}

Optical coherence tomography (OCT) is a relatively new technology and fundamentally novel optical imaging modality invented in 1991. The resolution of OCT images is up to 10 times better than that of the conventional ultrasound and has a deeper imaging depth than confocal or multi-photon microscopy. The OCT technique was originally meant for imaging human retinas. Now, this method has become a critical diagnostic technology in the areas of ocular diseases. The advances of fast and highresolution OCT instruments during the past 10 years have opened new fields of applications and perspectives for further development of OCT imaging. Moreover, the use of fiber-optics and the ability to combine with endoscope and catheters allows OCT to access the small parts of the body. From the mid-1990s, the ability of intravascular OCT to provide high-resolution cross-sectional images of both in vitro human aorta and coronary arteries was demonstrated.

\section{OCT evaluation in epidural anesthesia}

A needle-probe time domain (TD) OCT system was proposed by Li et al. for guiding epidural anesthesia [17]. Their report presented an OCT system that monitors the axial information in front of the needle by providing Amode signals. However, the forward-looking probe is limited by the shallow OCT imaging penetration depth of several millimeters; it is not very useful for guidance purposes.

Recently, we have completed a prototype which guides the epidural block by employing a rotated side-looking fiber probe with a swept source (SS) OCT system, which provides a visual image underlying structures and thus mediates the needle-probe technique by identifying different biological tissues surrounding the needle (Fig. 1a). Our method [18] presents the first such demonstration of "live" two-dimensional (2D) images, which assists the navigation toward the ES, similar to the intravascular ultrasound (IVUS) currently used for cardiac imaging. As the tip of the epidural needle reaches the ES, whole circular images within the epidural channel can also be obtained (Fig. 1b). Through image feature analysis, we have designed an automatic tissue classification and integrated it into SSOCT to build an objective and intelligent decision-support system for epidural needle and catheter placement. (Fig. 1c) Moreover, the extension to $3 \mathrm{D}$ visualization (Fig. 1d) will benefit intraoperative imaging, not only for the clinical anesthesia but also for the blood patching for post dural puncture headache, lysis of adhesions for Post Lumbar Surgery Syndrome, and so on.

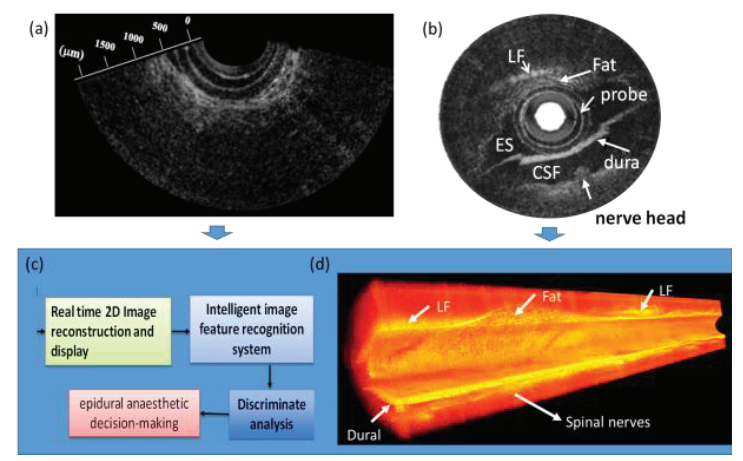

Figure 1. (a) In vivo radial images of epidural space (ES) in the lumbar region acquired using the sideimaging OCT needle probe. (b) A whole circular image within the epidural channel. (c) A flow chart of intelligent image recognition method design for automatic and objective discrimination of the ES from outside tissues. (d) A 3D OCT scanning.

\section{Contrast enhancement by functional OCT}

OCT technique relies on fluctuations in the refractive index to generate image contrast, therefore is still limited to nonspecific material properties as the scattering crosssection does not vary widely between molecular species. Functional extension of OCT combines the advantages of OCT with additional image contrast obtained by using the Doppler flow, spectroscopy, or polarization as a contrast source. Recently, we have successfully developed the multi-contrast OCT for the imaging of the retinal 
degeneration (Fig. 2a) and tumor angiogenesis (Fig. 2b). More results will be presented in this talk.

In summary, even though there have been many approaches to assist the anesthesiologists in performing neuroaxial anesthesia, none of the prior arts may be said as an unrestricted technique. The lack of a design that is with sufficient accuracy to the targets of interest and the automatic indication of needle placement makes it difficult to all-around implementation of field usage of objectiveness. We anticipate that the combined needle probe and the multi-contrast OCT imaging will allow us to increase the accuracy of epidural needle placement, and is helpful for intraoperative imaging.
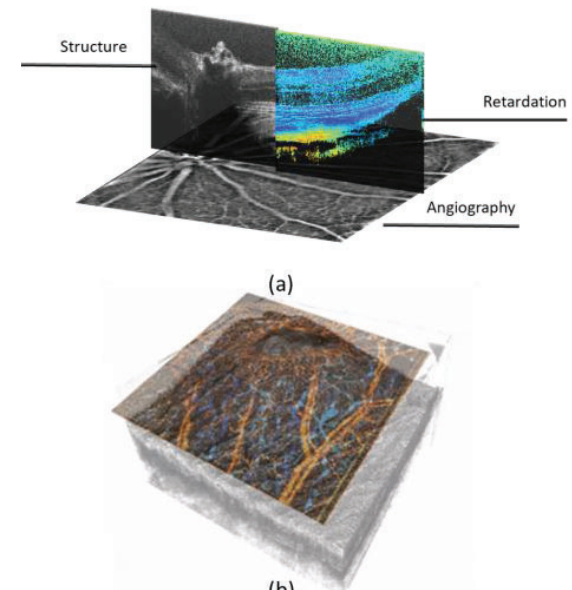

(b)

Figure 2. Schematic plot of multi-contrast OCT in (a) retina and (b) tumor.

\section{References}

1. Hiemenz L., Stredney D., Schmalbrock P., "Development of the forcefeedback model for an epidural needle insertion simulator," Stud Health Technol Inform 50, 272-277 (1998).

2. Magill J., Anderson B., Anderson G., Hess P., Pratt S., "Multi-axis mechanical simulator for epidural needle insertion," Medical Simulation 3078, 267-276 (2004).

3. Goobie S. M., Montgomery C. J., Basu R., McFadzean J., O'Connor G. J., Poskitt K., Tsui B. C., "Confirmation of direct epidural catheter placement using nerve stimulation in pediatric anesthesia," Anesth Analg 97, 984-988 (2003).

4. Tsui B. C., Emery D., Uwiera R. R, Finucane B., "The use of electrical stimulation to monitor epidural needle advancement in a porcine model," Anesth Analg 100, 1611-1613 (2005).

5. Ting C. K., Tsou M. Y., Chen P. T., Chang K. Y., Mandell M. S., Chan K. H., Chang Y., "A new technique to assist epidural needle placement: Fiberoptic-guided insertion using two wavelengths," Anesthesiology 112, 1128-1135 (2010).

6. Ting C. K., Chang Y., "Technique of fiber optics used to localize epidural space in piglets," Optics Express 18, 11138-11147 (2010).

7. Desjardins A. E., Hendriks B. H., van der Voort M., Nachabe R., Bierhoff W., Braun G., Babic D., Rathmell J. P., Holmin S., Soderman M., Holmstrom B., "Epidural needle with embedded optical fibers for spectroscopic differentiation of tissue: Ex vivo feasibility study," Biomed Opt Express 2, 1452-1461 (2011).

8. Rathmell J. P., Desjardins A. E., van der Voort M., Hendriks B. H., Nachabe R., Roggeveen S., Babic D., Soderman M., Brynolf M., Holmstrom B., "Identification of the epidural space with optical spectroscopy," An in vivo swine study. Anesthesiology 113, 14061418 (2010).

9. Arzola C., Davies S., Rofaeel A., Carvalho J. C., "Ultrasound using the transverse approach to the lumbar spine provides reliable landmarks for labor epidurals. Anesth Analg 104, 1188-1192 (2007).

10. Grau T., Bartusseck E., Conradi R., Martin E., Motsch J., "Ultrasound imaging improves learning curves in obstetric epidural anesthesia: A preliminary study," Can J Anaesth 50, 1047-1050 (2003).

11. Karmakar M. K., Li X., Ho A. M., Kwok W. H., Chui P. T., "Realtime ultrasound-guided paramedian epidural access: Evaluation of a novel in-plane technique," Br J Anaesth 102, 845-854 (2009).

12. Kerby B., Rohling R., Nair V., Abolmaesumi P., "Automatic identification of lumbar level with ultrasound," Conf Proc IEEE Eng Med Biol Soc 2008, 2980-2983 (2008).

13. Pan P. H., Sintay B. J., "Eyes to the needle: To assist identification of the epidural space," Anesthesiology 112, 1073-1075 (2010).

14. Rapp H. J., Folger A., Grau T, "Ultrasound-guided epidural catheter insertion in children," Anesth Analg 101, 333-339 (2005).

15. Chiang H. K., Zhou Q., Mandell M. S., Tsou M. Y, Lin S. P., Shung K. K., Ting C. K. "Eyes in the needle: novel epidural needle with embedded high-frequency ultrasound transducer--epidural access in porcine model. Anesthesiology 114, 1320-1324 (2011).

16. Tsen L. C., "The all-seeing eye? Ultrasound technologies for neuraxial techniques. Anesthesiology 114, 1274-1276 (2011).

17. Tingyu L., Kouichi N., Osamu M., Yoshimura T., "Range Technique in Scattering Medium Using a Needle-Fiber Optical Coherence Tomography System," Optical Review 4, 201-206 (2006).

18. Kuo W. C., Kao M. C., Chang K. Y., Teng W. N., Tsou M. Y., Chang Y., Ting C. K., "Fiber-needle Swept-source Optical Coherence Tomography System for the Identification of the Epidural Space in Piglets," Anesthesiology 122, 585-594 (2015). 


\title{
Advances in Optical Coherence Elastography
}

\author{
Zhongping Chen
}

Department of Biomedical Engineering, University of California, Irvine, Irvine, CA 92617, USA

\begin{abstract}
Abstracts Tissue mechanical properties provide valuable medical information in disease diagnosis and prognosis. We report a optical coherence elastography (OCE) system based on acoustic radiation force (ARF) excitation. We have demonstrated the first in vivo OCE imaging of retina in animal models. The ARF-OCE technology will have a broad range of clinical applications, including imaging and characterizing cardiovascular atherosclerotic lesions, imaging and diagnosing of early stage cancer, imaging and evaluating ophthalmic diseases such as keratoconus and age-related macular degeneration, and imaging and assessing blood coagulation.
\end{abstract}

Keywords: Optical coherence tomography, optical coherence elastography, acoustic radiation force, retinal diseases

\section{Introduction}

Age-related macular degeneration (AMD) develops in 200,000 people each year in the US and is the principle cause of blindness in those 60 years of age or older in North America and Europe [1, 2]. Early AMD is characterized by a spectrum of changes in the ageing eye before overt loss of central vision. It is known that tissue elastic change may be a precursor to neovascularization [3, 4]. Furthermore, it has been suggested that abnormalities in collagen or elastin in the Bruch's membrane, the outer retina or the choroid, may predispose towards GA [5]. Microvascular changes, drusen deposits and alterations in elastin may all contribute to elastic changes in the retina/choroid. While a variety of methods now exist for in vivo elastography, these are inapplicable to the retina/choroid either because of insufficient resolution or anatomic inaccessibility to direct compression. We report on the development of an ARF-OCE system that enables in-vivo imaging of the mechanical properties of the retina $[6,7]$. We demonstrate the first in-vivo elasticity mapping of the retina in rabbit animal model $[6,7]$.

\section{Methods}

Figure 1 shows the experimental setup of ARF-OCE, which includes an OCT unit for elastic wave detection and an ultrasound unit for tissue vibration/excitation. A 50 $\mathrm{kHz}$ spectral domain optical coherence tomography (SD-OCT) system with a central wavelength of $890 \mathrm{~nm}$ and bandwidth of $144 \mathrm{~nm}$ is used for the detection of tissue structure and response to stimulation. A $4.5 \mathrm{MHz}$ ring ultrasound transducer was used for pulsed tissue excitation. The excitation duration was limited to $1-2 \mathrm{~ms}$ while the optical detection speed was $50 \mathrm{kHz}$.

All rabbit experiments were performed according to the University of California, Irvine (UCI) Institutional Animal Care and Use Committee (IACUC) protocol. The rabbit was used for an AMD study, where it was exposed to a high fat diet, blue light, and nicotine for 8 weeks. Since the disease was localized, most of the central retina was still relatively healthy. The healthy region was used for this study. The rabbit was given $35 \mathrm{mg} / \mathrm{kg}$ of ketamine and 5 $\mathrm{mg} / \mathrm{kg}$ of xylazine subcutaneously for initial anesthesia. Two drops of proparacaine $\mathrm{HCl}$ and atropine solution were applied topically for further anesthesia and dilation of the eye for imaging, respectively. The unconscious rabbit was propped onto the imaging stage and the eye was proptosed. Additional anesthesia was given via subcutaneous injection of ketamine $(17.5 \mathrm{mg} / \mathrm{kg})$ if the heart rate or oxygen levels indicate distress. After imaging was completed, the rabbit was euthanized with an intravenous injection of euthasol. When death was confirmed, the rabbit eye was enucleated and fixed in $10 \%$ buffered formalin for histological analysis. 


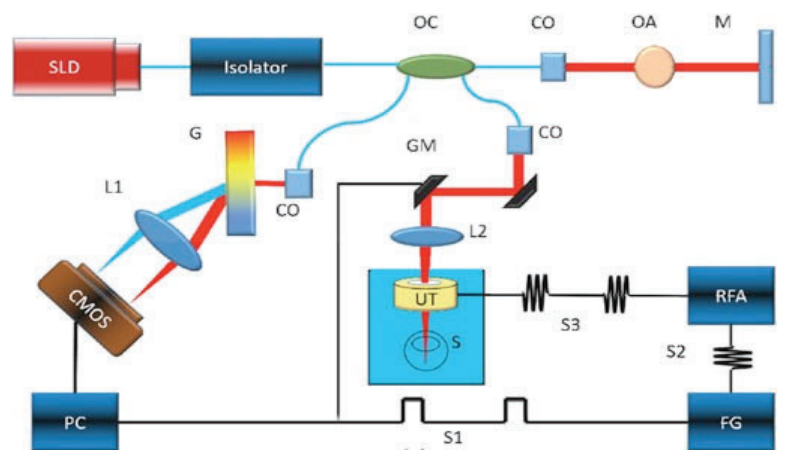

Fig. 1 Experimental setup ARF-OCE, which includes an OCT unit for elastic wave detection and an ultrasound unit for tissue vibration/excitation. SLD: superluminescent diode, OC: optical coupler, CO: collimator, OA: optical attenuator, M: mirror, GM: galvanometer mirrors, L1/L2: lens, UT: ul- trasound transducer, $\mathrm{S}$ : sample, RFA: radiofrequency amplifier, FG: function generator, G: grating, S1: baseband signal, S2: modulated signal, S3: amplified modulated signal (fom ref [6])

\section{Results and Discussion}

Propagation of shear wave in retina is shown in Figure 2. Within the 400 A-lines in M-mode at each location, the entire shear wave propagation through that point can be captured. In Fig. 2(a)-(c), a sample raw data for the porcine retina is shown where it is apparent that different locations on the retina correspond to different propagation speeds. The transducer focal area is on the left side of the images, and propagation is to the right. For example, the bottom most layer of the retina propagates the fastest and corresponds to a stiffer tissue component. It is also important to note that the ultrasound wave intensity decreases at regions of stiffer tissue, and the intensity of the displacement is not considered in the velocity calculations. (a)

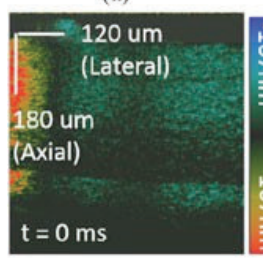

(b)

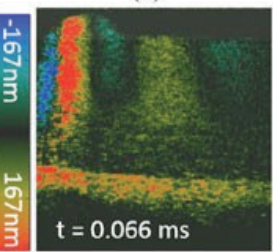

(c)

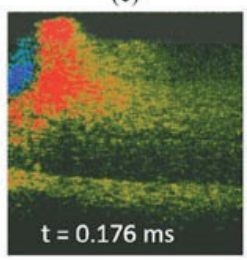

Fig. 3. (a-c) Cross-sectional raw data showing wave propagation of retinal layers at different time points for an ex-vivo pig retina. Shear wave induced axial displacement is shown by different colors corresponding to the color bar (fom ref [6])
ARF-OCE images of in-vivo rabbit model is shown in Figure 3. Imaging was performed on the central retina approximately $1.5 \mathrm{~mm}$ from the optic disc on the temporal side. The OCT intensity image is shown in Fig. 3(a), where segmentation was performed to isolate 5 different layers in the posterior globe. The shear velocity map is demonstrated in Fig. 3(b), and a speed of up to $5.8 \mathrm{~m} / \mathrm{s}$ can be visualized. The velocity was converted to the Young's modulus in Fig. 3(c). The elasticity of the first three layers from the ganglion side to the photoreceptor side are: $12.6 \pm$ $1.5,35.7 \pm 18.9,101.1 \pm 5.1 \mathrm{kPa}$. The bottom two layers of the eye could not be differentiated due to the fast propagation speed where the Young's modulus is over 100 $\mathrm{kPa}$. The shear wave is attenuated within $200 \mathrm{um}$ in the lateral direction and the current system setup is not fast enough to capture the wave propagation within such a distance for the bottom three layers. This issue can be resolved by increasing the imaging speed or extending the traveling distance of the shear wave with a higher ultrasound excitation power. According to the histology, the bottom two layers are close to the sclera and are expected to be stiffer than the retina and have a higher propagation velocity.

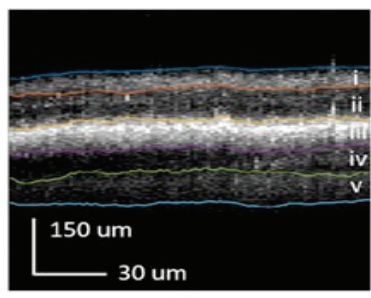

(a)

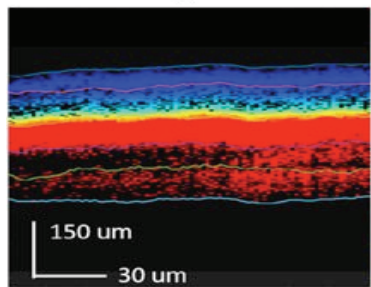

(c)

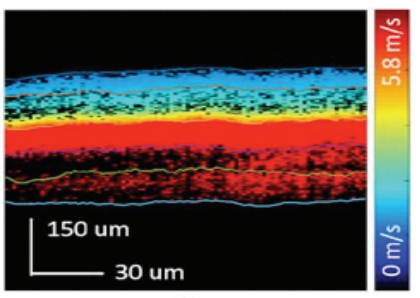

(b)

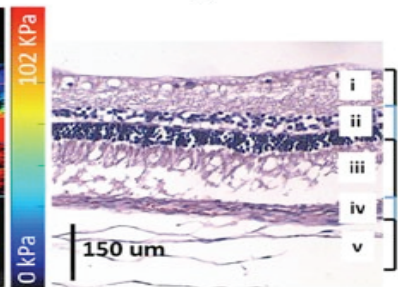

(d)
Fig. 5. In vivo rabbit elastography results. (a) OCT of rabbit central retina. (b) Shear wave velocity map. (c) Elastogram of corresponding region. (d) H\&E histology showing some retinal detachment. i: Nerve fiber, ganglion cell, \& inner plexiform; ii: inner nuclear, outer plexiform, \& outer nuclear; iii: RPE; iv: choroid; v: sclera (fom ref [6]). 


\section{Summary}

In summary, we have developed a novel ARF-OCE system to image and quantify the mechanical elasticity of the retinal layers in-vivo. The capability of imaging elastic properties of retina will provide new insights into the pathobiology of AMD and offer a new diagnostic technique for the detection of changes associated with disease development, progression and response to therapy.

\section{References}

[1] T. Wong, U. Chakravarthy, R. Klein, P. Mitchell, G. Zlateva, R. Buggage, et al., "The natural history and prognosis of neovascular age-related macular degeneration: a systematic review of the literature and meta-analysis," Ophthalmology, vol. 115, pp. 116-126. e1, 2008.

[2] T. Ayoub and N. Patel, "Age-related macular degeneration," Journal of the Royal Society of Medicine, vol. 102, pp. 56-61, 2009.

[3] L. Krishnan, J. B. Hoying, H. Nguyen, H. Song, and J. A. Weiss, "Interaction of angiogenic microvessels with the extracellular matrix," American Journal of Physiology-Heart and Circulatory Physiology, vol. 293, pp. H3650H3658, 2007.

[4] A. Mammoto, K. M. Connor, T. Mammoto, C. W. Yung, D. Huh, C. M. Aderman, et al., "A mechanosensitive transcriptional mechanism that controls angiogenesis," Nature, vol. 457, p. 1103, 2009.

[5] A. G. Marneros, H. She, H. Zambarakji, H. Hashizume, E. J. Connolly, I. Kim, et al., "Endogenous endostatin inhibits choroidal neovascularization," The FASEB Journal, vol. 21, pp. 3809-3818, 2007.

[6] Y. He, Y. Qu, J. Zhu, Y. Zhang, A. Saidi, T. Ma, et al., "Confocal Shear Wave Acoustic Radiation Force Optical Coherence Elastography for Imaging and Quantification of theIn VivoPosterior Eye," IEEE Journal of Selected Topics in Quantum Electronics, vol. 25, pp. 1-7, 2019.

[7] Y. Qu, Y. He, A. Saidi, Y. Xin, Y. Zhou, J. Zhu, et al., "In Vivo Elasticity Mapping of Posterior
Ocular Layers Using Acoustic Radiation Force Optical Coherence Elastography," Invest Ophthalmol Vis Sci, vol. 59, pp. 455-461, Jan 1 2018. 


\title{
Non-invasive3D imaging for biology by using OCT technique
}

\author{
Yohei Koshimizu, Yuki Mori \\ Bioscience \& Pharmaceuticals, SCREEN Holdings Co., Ltd., \\ 322 Furukawa-cho, Hazukashi, Fushimi-ku,Kyoto, 612-8486 Japan
}

\begin{abstract}
Non-invasive observation of internal structure like cavity and gap witch cannot be seen by microscope is possible by OCT. We introduce real three-dimensional imaging of organoids, spheroids and fertilized embryos, with some advanced technical information.
\end{abstract}

Keywords: OCT, SD-OCT, non-invasive, live cell imaging, live sample imaging.

\section{Introduction}

We have observed and analyzed true images of biological samples such as three-dimensional cultured cells, tissue sections of animals, and fertilized eggs by using OCT technology. Generally, OCT observation requires a dedicated observation conditions in order to prevent occurrence of ghosts and artificial signals from labware for cultures. Therefore, it was difficult to observe cultured cells during cultures. We have aimed to realize the dynamism of live culture, we have developed an OCT three-dimensional imager equipped with our advanced optical technology. In this report, we showed some case studies of true three-dimensional images and analyzed results with our novel OCT technology.

\section{Specifications}

\section{Product description}

System: SD-OCT (Spectrum Domain OCT Spectrometer). Resolution: High Resolution : 3 um (X, Y, Z directions) and Low Resolution: $10 \mathrm{um} \mathrm{(X,Y,} \mathrm{Z}$ directions) . Max. Fov: High Resolution: $1 \times 1 \mathrm{~mm}$ and Low Resolution: $10 \times 10 \mathrm{~mm}$. Max. detection depth: High Resolution : $1 \mathrm{~mm}$ and Low Resolution : $1 \mathrm{~mm}$ (according to sample). Wavelength: Center: 890nm and Band: $800 \mathrm{~nm}^{-}$970nm.

\section{Technical feature}

Our equipment was developed with the concept that it can observe from the bottom of the culture container such as plastic labware directly while biological samples are keeping in it. In order to realize the concept, we have developed an optical diaphragm technology that reduces the influence of the reflection from the culture container bottom. Together, we have developed a focus connection technology that reduces the reflection from the container when changing the focus.

In many cases, observed samples exists near the bottom of the culture container. Since this bottom surface is normally flat, it acts as a pseudo reference plane which causes a ghost image (Figure. 1). As a result, the condition makes an artificial signal and affects observation. An additional of a light shielding diaphragm in the optical pathway shields a regular reflection from the bottom surface, and then it prevents ghost occurrence. On the other hand, since half of a scattered light from the sample is transmitted through the light shielding diaphragm, an interference image can be obtained. Although this method has a loss of light quantity, it is useful for observation of a low contrast object such as a cell (Figure. 2).

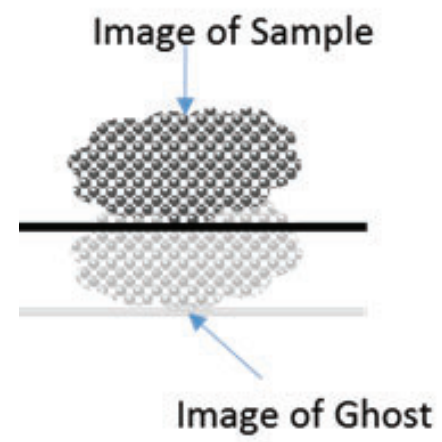

Figure 1. Relationship between sample image and ghost image. 


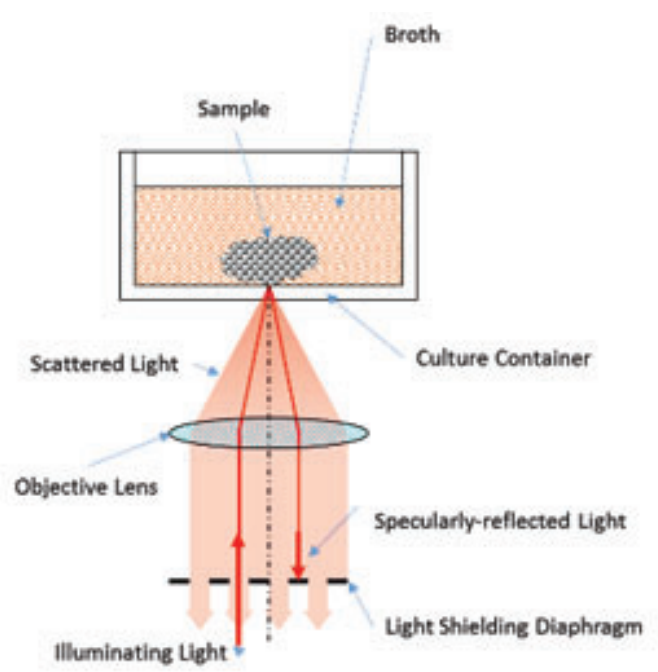

Figure 2. Optical system including a light shielding diaphragm

\section{Results and Discussion}

We captured true three-dimensional images of biosamples by using our OCT equipment. Cavity in spheroid with drug was captured. Our system cleared an inside structure of three dimensional culture, even if it is not slice section sample. (Figure 3)

A morphology of angiogenic sprouting were captured by OCT. It is not able to understand the accuracy morphology of angiogenic sprouting with a microscopy. The direction, length and so on were obtained from our three-dimensional images. (Figure 4 )*1
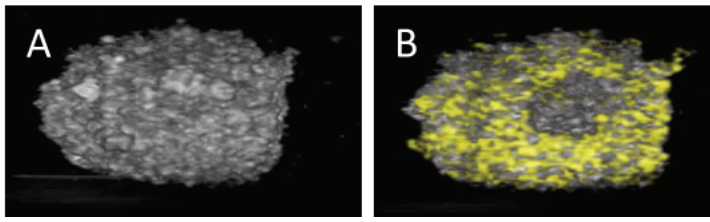

Figure 3. A is whole morphology of spheroid. The arrow shows the cavity in spheroids. Yellow surface is cross section with digital treatment (B).
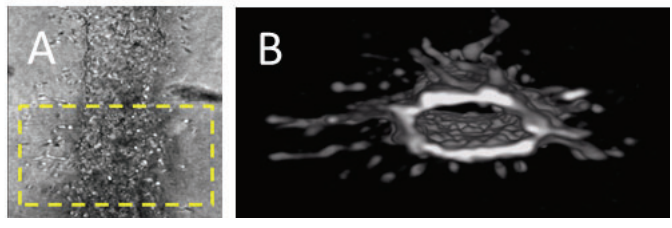

Figure 4. A is microscopy image. B is OCT captured image.

OCT captured a whole morphology of cell sheet like structure. A whole area thickness of the cell sheet were calculated without preparing a continuous sectional images. (Figure 5)

A dynamism of fertilized eggs were observed by OCT. The internal structures of blastocyst was segmented during growth with non-invasive. (Figure 6)
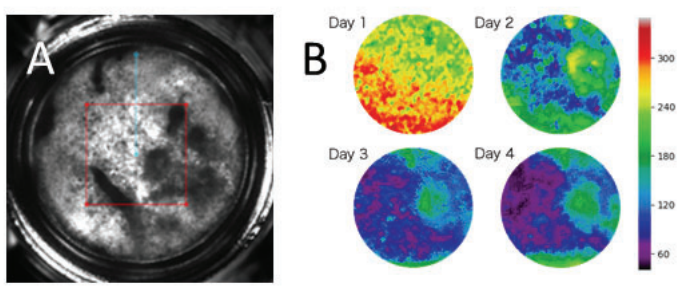

Figure 5. Whole well plate image captured by microscopy (A). Heat maps show the thickness of cell sheet during day in vitro (B).
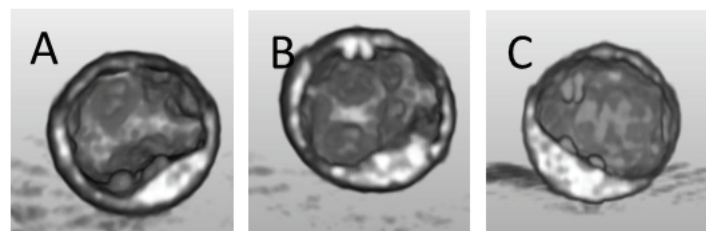

Figure 6. Live blastocyst images during growth. The viewing from Top-Left (A), Bottom-Left (B), and Right (C).

The novel developed optical systems in our OCT equipment achieved non-invasive observation of biological samples such as cultured cells in plastic labware. In addition, our system was able to obtain the accuracy morphology and internal structure of threedimensional biosamples without using sectional observation or fluorescent three-dimensional imaging 
(e.g., confocal microscope). By the existing methods, damage of the sample due to the invasive procedure occurred such as fluorescent staining. Therefor it was difficult to understand the dynamism of cultured cells or live samples during the culture process. Our system was shown to be a useful tool to clarify the dynamism of living cultured cells in regenerative medicine and drug discovery research.

\section{References}

*1 Haruko Takahashi, Keisuke Kato, Kenji Ueyama, Masayoshi Kobayashi, Gunwoong Baik, Yasuhiro Yukawa, Jun-ichi Suehiro \& Yukiko T. Matsunaga. Visualizing dynamics of angiogenic sprouting from a three-dimensional microvasculature model using stagetop optical coherence tomography. Scientific Reports volume 7, Article number: 42426 (2017) 


\title{
Swept-Source Optical Coherence Tomography Imaging of the Guinea Pig Cochlea
}

\author{
Ting-Hao Chen ${ }^{\mathrm{a}}$, Ting-Yen Tsai ${ }^{\mathrm{a}}$, Hsin-Chien Chen ${ }^{\mathrm{b}}$, Chuan-Bor Chueh ${ }^{\mathrm{a}}$, Bo-Huei Huang ${ }^{\mathrm{c}}$ \\ You-Nan Tsai ${ }^{\mathrm{a}}$, Meng-Tsan Tsai ${ }^{\mathrm{c}, \mathrm{d}}$, Chih-Hung Wang, ${ }^{\mathrm{b}, \mathrm{e},}$, Hsiang-Chieh Lee ${ }^{\mathrm{a}, \mathrm{f}, *}$ \\ ${ }^{a}$ Graduate Institute of Photonics and Optoelectronics, National Taiwan University, Taipei 10617, \\ Taiwan \\ ${ }^{b}$ Department of Otolaryngology-Head and Neck Surgery, Tri-Service General Hospital, \\ National Defense Medical Center, Taipei 11490, Taiwan. \\ ${ }^{\mathrm{c}}$ Department of Electrical Engineering, Chang Gung University, Taoyuan 33302, Taiwan \\ dDepartment of Dermatology, Chang Gung Memorial Hospital, Linkou and Taipei, Taiwan \\ 'Taichung Armed Forces General Hospital, Taichung 41168, Taiwan. \\ fMolecular Imaging Center, National Taiwan University, Taipei 10617, Taiwan \\ "Email: chw@ms3.hinet.net; "Email: hclee2@ntu.edu.tw
}

Abstract

OCT is an imaging modality that can be illustrated as the optical analog to ultrasound, where focused light illuminates a sample and backscattered light is collected under use of interferometry. On the other hand, the inner ear is a small and sophisticated organ, mainly comprising the vestibular system and cochlea. In this study, we leverage the technology of swept-source optical coherence tomography to obtain the ex vivo volumetric image of the guinea pig cochlea allowing a better understanding of the cochlear architectures, serving an important step for the subsequent in vivo applications. Moreover, two different center wavelength light sources, i.e. $1.06 \mu \mathrm{m}$ and $1.3 \mu \mathrm{m}$, were used to develop two separate OCT systems, enabling a more detailed investigation on the tissue architectures of the guinea pig cochlea at different regimes as well as different imaging resolutions.

Keywords: optical coherence tomography, cochlea, inner ear, sensorineural hearing loss, noise-induced hearing loss

\section{Introduction}

Hearing loss and deafness may result from congenital causes and acquired causes, such as birth asphyxia, chronic ear infections, the use of particular drugs, exposure to excessive noise [1]. Particularly, exposure to intense noise may result in the damage to the inner ear hair cells, causing noise-induced hearing loss (NIHL) or sensorineural hearing loss (SNHL) [2, 3]. Due to the lack of self-regenerative capacity of hair cells, the SNHL is not completely recoverable such that the patients having SNHL may be provided with the hearing aids, and the patients have the only choice of cochlear implants in case of severe to profound hearing loss [4]. On the other hand, optical coherence tomography (OCT) featuring a broadband light source and the interferometry technique, enabling volumetric imaging of the tissue architecture with an imaging resolution comparable to standard histopathology image scales. Recently, some literature has reported the mechanisms causing the blast-induced sensorineural hearing loss with the OCT technique $[5,6]$. In this work, we have developed two separate wavelength swept OCT systems with the central wavelength of 1060 $\mathrm{nm}$ and $1300 \mathrm{~nm}$, respectively to acquire the 3D anatomy of the guinea pig cochlea ex vivo. With the assistance of the software Matlab R2017b and ImageJ, the en-face and cross-sectional OCT image of the guinea pig cochlea can be analyzed facilitating the following comparisons.

\section{The experimental setup}

The SS-OCT system illustrated in figure 1 with a central wavelength $1060 \mathrm{~nm}$ swept source laser (Axsun Technologies), sweep bandwidth of $110 \mathrm{~nm}$, the repetition rate of $100 \mathrm{kHz}$ and average output power of $18 \mathrm{~mW}$ was configured such that the raw image data could be acquired. The interferometer schematic as shown in figure 1 consisted of four 50/50 fiber couplers, and a collimator lens as well as an achromatic doublet lens in the reference arm and sample arm, respectively. The reference arm also included a polarization controller to match the 
polarization of light from sample arm in which a pair of galvo mirrors (Thorlabs, Inc.) were configured to achieve the 2D and 3D OCT imaging. Moreover, the interference light signal is gathered and transferred into an electrical signal by a dual-balanced photodiode (DBPD; Thorlabs, Inc., PDB-480C-AC). An in-house developed interface based on $\mathrm{C}++$ programing language was used to control the beam scanning of the imaging specimen as well as the data acquisition where a high-speed 12-bit digitizer was used (Alazar Technologies Inc.). The configuration of another SS-OCT with a central wavelength $1300 \mathrm{~nm}$ swept source laser is analogous to figure 1. However, two $50 / 50$ fiber couplers before the collimator lens are replaced with two circulators, respectively.

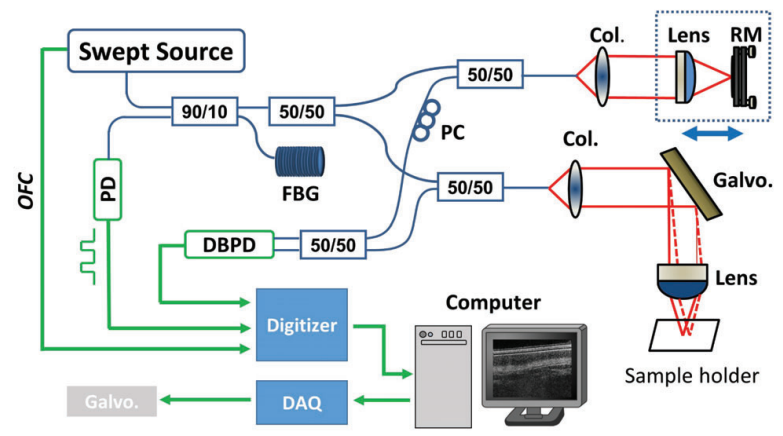

Figure 1 Schematic diagram of the swept-source optical coherence tomography (SS-OCT) system setup. (SS Laser: swept source laser, FC: fiberoptic coupler, lens: achromatic doublet, PC: polarization controller, M: mirror, DBPB: dual-balanced photodiode; Galvo: galvanometer scanner; Col: collimator).

\section{Results and discussion}

After the collection procedure, ex vivo guinea pig cochlea pre-treated with ethylenediaminetetraacetic (EDTA) acid to enhance the imaging depth was preserved in the Phosphate-buffered saline (PBS) solution before the OCT imaging session. In order to facilitate the analysis of various characteristic architectural features of the guinea pig cochlea, volumetric OCT imaging dataset over a twodimensional surface area of 4 x 4 mm (2000 x 1000 Ascans, X x Y-axis) was performed over each guinea pig cochlea sample. Each volumetric OCT dataset can be acquired in $\sim 20$ seconds using the $\mathrm{C}++$ acquisition interface aforementioned. As shown in figure 2(a), the presented cross-sectional OCT image clearly shows the inner structures as similarly illustrated in figure 2(f), a low magnification light micrograph of the guinea pig [7]. Also, the transverse scanning direction of figure 2(a) is described in figure $2(\mathrm{~g})$, where the cochlea is nearly vertical to the sample holder.
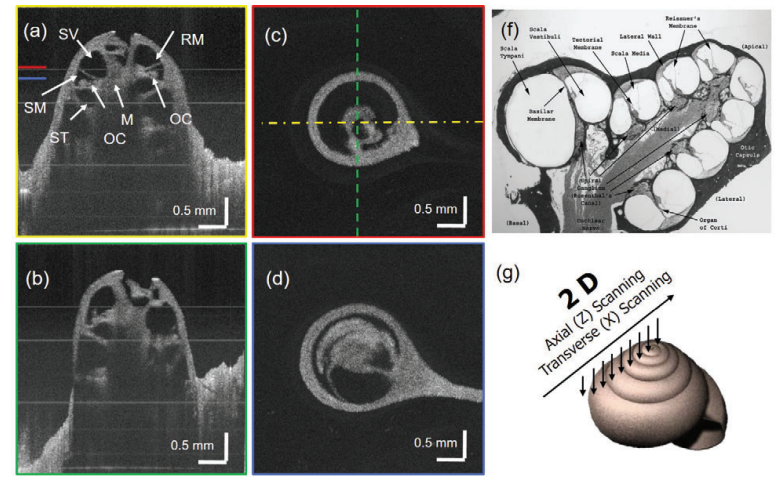

Fig. 2. (a) Cross-sectional OCT image of the guinea pig cochlea showed the features with lower OCT scattering intensity reminiscent of the structure in the guinea pig cochlea as shown in (f), the light micrograph of the guinea pig, and inner red line and blue line indicate the position of the en face images (c) and (d), respectively. Yellow and green lines in (c) indicate the position of the cross-sectional images (a) and (b), respectively. Note (f) is reprinted from a previous publication [7]. (M: modiolus; RM: Reissner's membrane, ST: scala tympani; SM: scala media; OC: organ of Corti; SV: scala vestibuli)

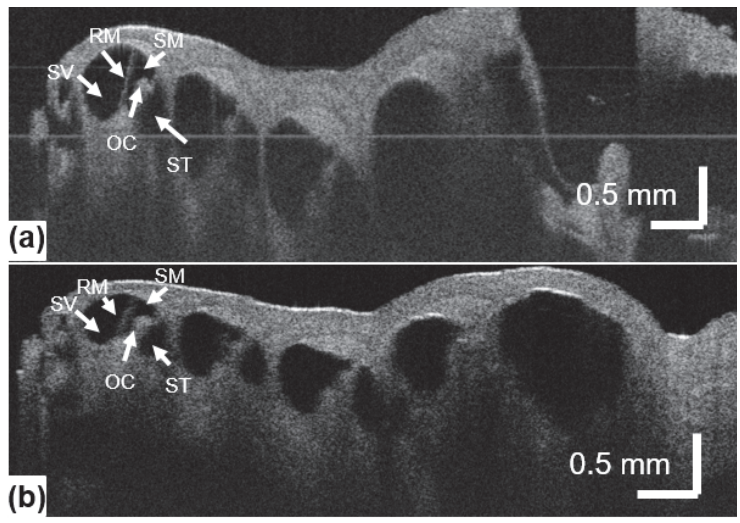

Fig. 3. (a) Cross-sectional OCT image of the guinea pig cochlea for $1060 \mathrm{~nm}$ SS-OCT system in terms of another transverse scanning direction. (b) for $1030 \mathrm{~nm}$ SS-OCT system. 
Due to slightly higher transverse and axial resolution for $1060 \mathrm{~nm}$ SS-OCT system over the $1300 \mathrm{~nm}$ SS-OCT system, the image for $1060 \mathrm{~nm}$ SS-OCT system provides better visualization of the fine anatomic features of the cochlea than the $1300 \mathrm{~nm}$ OCT system. On the other hand, $1300 \mathrm{~nm}$ OCT system enables OCT imaging of the guinea pig cochlear with an increased imaging depth than the $1060 \mathrm{~nm}$ OCT system. Although previous OCT studies have reported the use of OCT for imaging of the guinea pig cochlea, this study represents an important first study for the subsequent in vivo studies of either animal model or even human ear related disease. In sum, the developed swept-source optical coherence tomography system has preliminarily reconstructed the image of the guinea pig cochlea with two different center wavelength light source.

\section{Acknowledgment}

The authors acknowledge the support from the Young Scholar Fellowship Program by the Ministry of Science and Technology (MOST) of the Republic of China (ROC), Taiwan (MOST-107-2636-E-002-003).

\section{References}

[1] "http://www.who.int/en/news-room/factsheets/detail/deafness-and-hearing-loss, Deafness and hearing loss," World Health Organization, March 152018.

[2] T. N. Le, L. V. Straatman, J. Lea, and B. Westerberg, "Current insights in noise-induced hearing loss: a literature review of the underlying mechanism, pathophysiology, asymmetry, and management options," J Otolaryngol Head Neck Surg, vol. 46, no. 1, p. 41, May 232017.

[3] M. Waqas, S. Gao, S. Iram Us, M. K. Ali, Y. Ma, and W. Li, "Inner Ear Hair Cell Protection in Mammals against the Noise-Induced Cochlear Damage," Neural Plast, vol. 2018, p. 3170801 , 2018.

[4] G. M. Sprinzl and H. Riechelmann, "Current trends in treating hearing loss in elderly people: a review of the technology and treatment options a mini-review," Gerontology, vol. 56, no. 3, pp. 351-8, 2010.

[5] S. S. Gao et al., "Quantitative imaging of cochlear soft tissues in wild-type and hearing- impaired transgenic mice by spectral domain optical coherence tomography," Optics Express, vol. 19, no. 16, pp. 15415-15428, 2011/08/01 2011.

[6] J. Kim, A. Xia, N. Grillet, B. E. Applegate, and J. S. Oghalai, "Osmotic stabilization prevents cochlear synaptopathy after blast trauma," Proc Natl Acad Sci US A, vol. 115, no. 21, pp. E4853-E4860, May 222018.

[7] Y. Raphael and R. A. Altschuler, "Structure and innervation of the cochlea," Brain Res Bull, vol. 60, no. 5-6, pp. 397-422, Jun 152003. 


\title{
A calibration method for central corneal thickness measurement from Scheimpflug imaging in a non-contact tonometer with pachymetry
}

\author{
Wai W Wang ${ }^{\mathrm{a}}$, Kuo-Jen Wang ${ }^{\mathrm{a}}$, Brian Lin ${ }^{\mathrm{a}}$, Che-Liang Tsai*a, Vincent Chang ${ }^{\mathrm{a}}$, and I-Jong Wang \\ ${ }^{a}$ Crystalvue Medical Corporation, No.116, Ln. 956, Zhongshan Rd., Taoyuan Dist., Taoyuan City \\ 33072, Taiwan \\ ${ }^{\mathrm{b}}$ Department of Ophthalmology, National Taiwan University Hospital \\ No. 7, Chung-Shan South Rd., Taipei, Taiwan
}

\begin{abstract}
We propose a method to calibrate the pachymetry for central corneal thickness (CCT) measurement from a Scheimpflug imaging of a non-contact tonometer. Our calibration method takes into account not only the Scheimpflug imaging geometric distortion but also the slit width variation of the Scheimpflug illumination path. Based our method, CCT measurement with accuracy better than \pm 12 um is achieved.
\end{abstract}

Keywords: Scheimpflug image, pachymetry, tonometer

\section{Introduction}

Accurate determination of central corneal thickness (CCT) is crucial for the planning of refractive surgery, diagnosis of glaucoma, and monitoring of corneal edema ${ }^{1}$. In addition to ultrasonic measurement, Scheimpflug imaging is also a powerful tool and widely used for imaging the anterior segment and CCT measurement without contacting the cornea $^{2}$. To obtain cornea Scheimpflug image with accurate CCT readings, it is mandatory to correct the images from geometrical distortion caused by tilt of the object plane with respect to the optical axis of the instrument, further to correct the optical distortion caused by refraction from the different ocular surfaces ${ }^{3}$. Furthermore, the accuracy is also critically dependent upon the size of the slit width in the illumination path of a Scheimpflug system, because the beam width of a Scheimpflug system is proportional to the slit width. Consequently, the broader the beam size in the illumination path of a Scheimpflug system, the thicker the cornea image would be obtained. Therefore, to obtain accurate CCT measurement through Scheimpflug imaging system, the correction of CCT measurement must include not only the geometrical distortion but also the distortion induced by the variation of slit width in Scheimpflug system. In this report, we propose a method to calibrate the pachymetry from a Scheimpflug image which incorporated in an air-puff tonometer.

\section{Method}

The equipment used for this Scheimpflug imaging and CCT measurement study is a tonometer made by Crystalvue Medical Corporation, Taoyuan, Taiwan. The setup of the Scheimpflug imaging configuration is shown in Figure 1a and it is mainly composed of a LED light source with wavelength of $456 \mathrm{~nm}$ which is collimated through a slit with aperture width $30 \mathrm{um}$. The thickness of corneal image projected on the image sensor is closely related to the width of the slit, as shown Figure 1b.

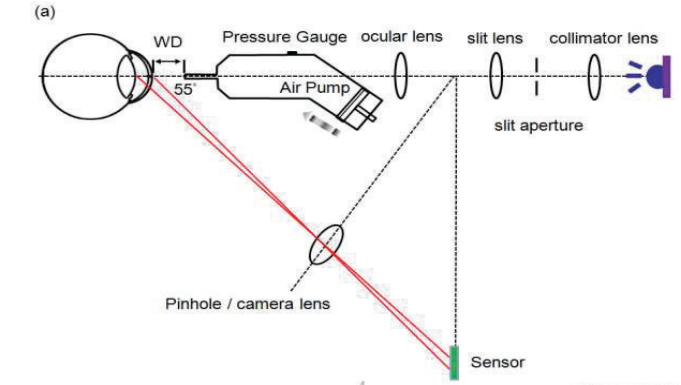

Fig. 1(a) The Scheimpflug imaging system for CCT measurement. 


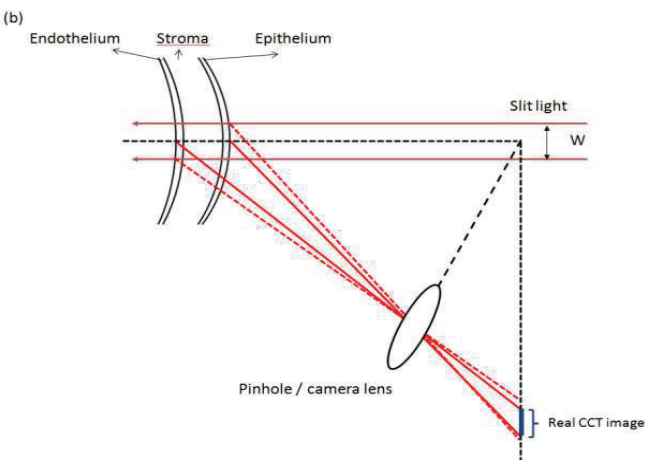

Fig. 1(b) The image of a CCT in Scheimpflug imaging system with slit width $\mathrm{W}$ in the illumination path.

\section{Modeling}

The simulation model of the Scheimpflug cornea image projected on the image sensor for CCT measurement is indicated in Figure 2. A collimated light beam passing through a narrow slit is incident on the cornea and guided to the cornea center by the tonometer automatic eye focusing/tracking system. The incident beam has a finite beam width $\mathrm{W}$ instead of an ideal narrow paraxial beam. The corneal scatters the incident light at its front as well as the rear surfaces, and the scattered light is captured to form an image projected on the image sensor, as shown in Figure 3. Besides the variations of curvatures of corneal front/rear surfaces and index of refraction of eye tissues, the variation of beam width of $\mathrm{W}$ induces the significant thickness variation of the cornea image, thus induces measured CCT variation.

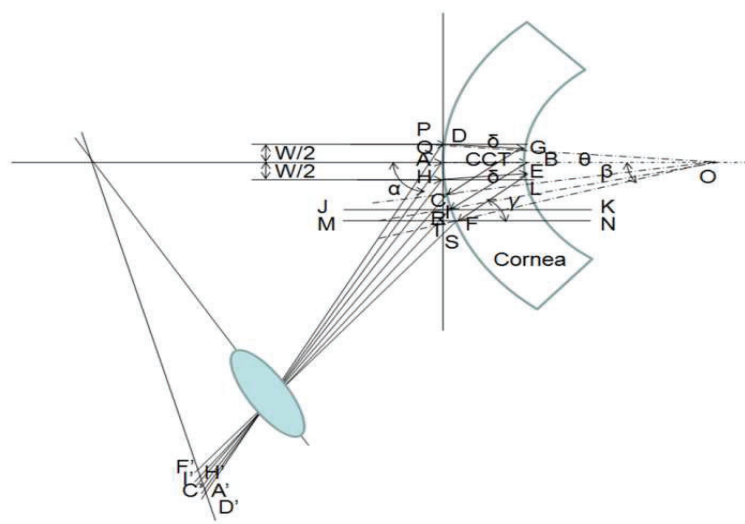

Fig. 2 Optical model of Scheimpflug imaging optics for CCT characterization.

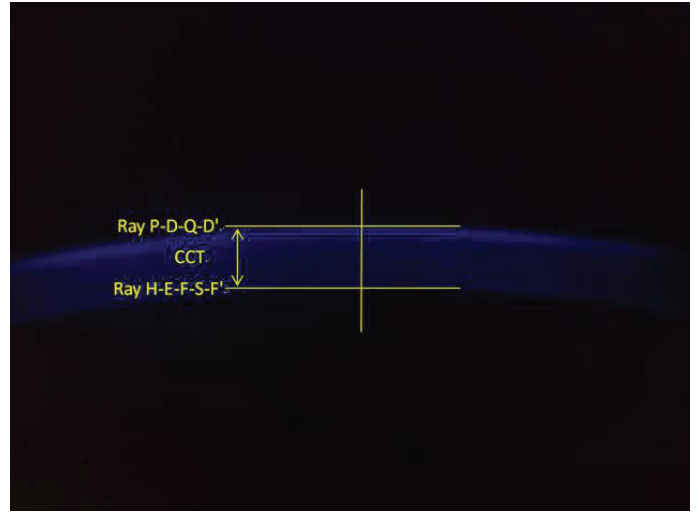

Fig. 3 Scheimpflug image of a human eye cornea

\section{Result}

Figure 4 shows the simulated and measured CCT values in pixels obtained through Scheimpflug imaging for both human and model eyes, where the y-axis is the pixel number of image sensor and the width of 1 pixel is $1.4 \mu \mathrm{m}$. The green line represents the simulated CCT and the circle dots represent the measured CCT from Scheimpflug image of human eyes, where the CCT were also validated by OCT measurement. The red line represents the simulated CCT and the cross marks represent the measured CCT from Scheimpflug image of model eyes, where the thickness of CCT were also validated by thickness gauge. The unique parameter difference used in human eye and model eye in the simulation model is that the index of refraction $n$, where the $n$ value of human eye is 1.3765 and 1.6060 for model eye used in our modeling.

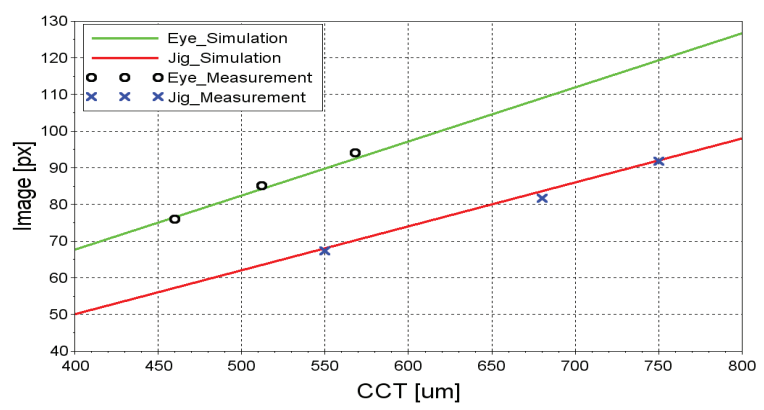

Fig. 4 Scheimpflug image simulation results of a human eye cornea and a model eye. 
The simulated CCT through Scheimpflug image is in good agreement with the measured central cornea thickness for both human eyes and model eyes with accuracy better than $99 \%$.

\section{Conclusion}

A calibration method for CCT measurement of Scheimpflug imaging in a non-contact tonometer with pachymetry is proposed. The calibration method takes into account not only the Scheimpflug imaging geometric distortion but also the CCT variation induced by the slit width of illumination path. Through this calibration, CCT measurement by Scheimpflug imaging system in a noncontact tonometer with accuracy \pm 12 um is achieved.

\section{References}

[1] Huang J, Pesudovs K, Wen D, Chen S, Wright T, Wang X, Li Y, and Wang Q, "Comparison of anterior segment measurements with rotating Scheimpflug photography and partial coherence reflectometry," J Cataract RefractSurg, 37(2), 341-348 (2011).

[2] Savini G, Barboni P, Carbonelli M, and Hoffer KJ,"Repeatability of automatic measurements by a new Scheimpflug camera combined with Placido topography," J Cataract RefractSurg, 37(10),18091816 (2011).

[3] Li T, Tian L, Wang L, Hon Y, Lam AK, Huang Y, Wang Y, and Zheng Y, "Correction on the distortion of Scheimpflug imaging for dynamic central corneal thickness," J Biomed Opt, 20(5), 56006 (2015). 


\title{
Optical microscopy with optical-frequency-comb
}

\author{
Takeo Minamikawa ${ }^{a, b, c}$ \\ ${ }^{\mathrm{a}}$ Graduate School of Technology, Industrial and Social Sciences, Tokushima University, 2-1 Minami- \\ Josanjima-cho, Tokushima, Tokushima, 770-8506, Japan \\ ${ }^{b}$ ERATO Intelligent Optical Synthesizer Project, Japan Science and Technology Agency (JST), 2-1 \\ Minami-Josanjima-cho, Tokushima, Tokushima 770-8506, Japan \\ bPRESTO, Japan Science and Technology Agency (JST), 2-1 Minami-Josanjima-cho, Tokushima, \\ Tokushima 770-8506, Japan
}

\begin{abstract}
We propose a novel optical microscopy employing optical-frequency-comb (OFC). OFC microscopy enables fast and unique spectroscopic imaging retrieving amplitude and phase information of light based on mechanical scanless Fourier transform spectroscopy. We provide a proof-of-principle demonstration of OFC microscopy in the variety of applications, such as laser scanning OFC microscopy, scanless confocal OFC microscopy, mechanically scanless spectroscopic ellipsometry, and so on. Our approach would be a useful and powerful tool for microscopic observation, and the concept of the OFC microscopy will be a new aspect of OFC technology.
\end{abstract}

Keywords: optical-frequency-comb, optical microscopy, amplitude imaging, quantitative phase imaging

\section{Introduction}

Optical microcopy is now widely used for the observation of microstructures and for the analysis of molecular and structural functions of samples, which has been applied in the field of industry, biology, medicine, and so on. General optical microscopy observes optical intensity for the analysis of samples via reflectance, scattering, absorbance, fluorescence and so on. Another contrasting method is using optical phase, which can enhance the image contrast of such high transparent materials and/or thin-structured films. However, the conventional microscopy utilized the limited capability of optical information for imaging.

In the present study, we propose a novel optical microscopy employing optical-frequency-comb (OFC). OFC is a broadband pulsed laser with highly stabilized repetition rate and career envelop offset, and is now proven technique in optical frequency metrology and spectroscopy. We applied OFC to optical microscopy, which enables fast and unique spectroscopic imaging retrieving both amplitude and phase information of light. We provided a proof-of-principle demonstrations of the OFC microscopy with some applications, such as laser scanning OFC microscopy[1], scanless confocal OFC microscopy[2], OFC-based single-pixel imaging[3], mechanically scanless spectroscopic ellipsometry[4], and so on. We discuss the benefit of the OFC microscopy for the various applications.

\section{Laser scanning OFC microscopy}

For the application of OFC to microscopy, we firstly developed an optical microscope employing laser scanning system as shown in Fig. 1. In the OFC microscopy, we utilized two OFCs with slightly different repetition rates, allowing the acquisition of a complete interferogram with an ultra-wide time span without mechanical scanning for the deduction of a highly resolved optical spectrum[5]. Furthermore, since dualcomb spectroscopy is based on Fourier transform spectroscopy, the full amplitude and phase spectra can be obtained through directly decoding from the interferograms of two OFC lasers.

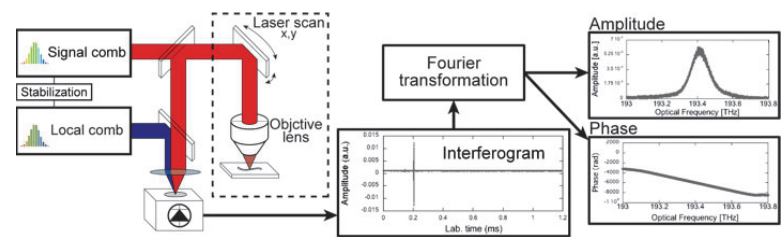

Figure 1. Laser scanning OFC microscopy.

We applied the laser scanning OFC microscopy to nanometer step-structured sample as shown in Fig. 2. The amplitude and phase images are obtained simultaneously. 
While the step structure $(50-300 \mathrm{~nm})$ was too small to be visualized by amplitude imaging, the phase imaging visualized the step structures of the sample.

(a)

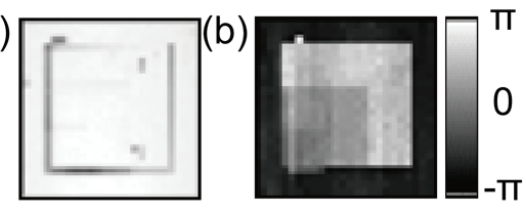

Figure 2. (a) Amplitude and (b) phase imaging of a nanometer step-structured sample by laser scanning OFC microscopy.

Importantly, this configuration enables simultaneous observation of amplitude and phase of light that reflected by a sample. Therefore, by using a polarization beam splitter and two detectors at the detection part, we can realize polarization measurement. We applied this method to spectroscopic ellipsometry as shown in Fig. 3. We obtained $\mathrm{SiO}_{2}$ film on $\mathrm{Si}$ substrate in terms of spectroscopic ellipsometry by using OFC. We realized an accuracy of $38.4 \mathrm{~nm}$ and a precision of $3.3 \mathrm{~nm}$ in the measurement of thin-film samples with a spectral resolution of up to $1.2 \times 10-5 \mathrm{~nm}$ throughout the spectral range of 1514-1595 $\mathrm{nm}$ and.

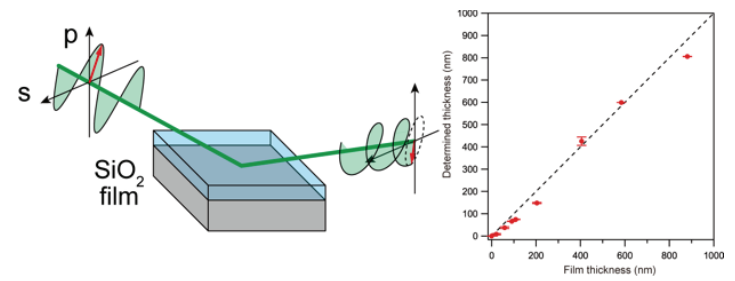

Figure 3. Spectroscopic ellipsometry by using OFC.

\section{Scanless OFC microscopy}

We also proposed a novel confocal imaging method by using OFC, namely, scanless confocal OFC microscopy. The concept of the proposed method is shown in Fig. 4a. This approach encoded the confocal image of a sample on OFC spectrum by using a wavelength disperser (Grating and VIPA). Since the reflected light from the sample can go through a confocal pinhole owing to the descan by the wavelength disperser, confocal amplitude and phase imaging can be realized without any mechanical scanning system via spectral information. We confirmed the imaging capability by using nanometer-step sample as shown in Fig. 1b.

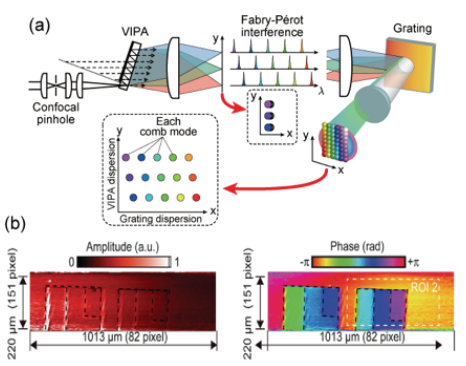

Figure 4. Scanless confocal imaging by using OFC.

\section{Conclusion}

We propose a novel optical microscopy employing optical-frequency-comb (OFC), which enables fast and unique spectroscopic imaging retrieving amplitude and phase information of light based on mechanical scanless Fourier transform spectroscopy. Our novel method will serve as an effective tool for the analysis of microscopic structures and functions of materials in biology and industry.

This work was supported by ERATO MINOSHIMA Intelligent Optical Synthesizer Project (JPMJER1304), from Japan Science and Technology Agency (JST), Japan, PRESTO (JPMJPR17PC) from JST, Japan, and JSPS KAKENHI (JP18H01901) from the Japan Society for the Promotion of Science (JSPS), Japan.

\section{References}

[1] Minamikawa, T., Nakano, S., Hase, E. et al., "Fourier transform spectroscopic optical microscopy using dual-comb spectroscopic technique," OSA Technical Digest. W4F.3 (2018).

[2] Hase, E., Minamikawa, T., Mizuno, T. et al., "Scanless confocal phase imaging based on dual-comb microscopy," Optica, 5(5), 634-643 (2018).

[3] Shibuya, K., Minamikawa, T., Mizutani, Y. et al., "Scan-less hyperspectral dual-comb single-pixelimaging in both amplitude and phase," Optics Express, 25(18), 21947-21957 (2017).

[4] Minamikawa, T., Hsieh, Y.-D., Shibuya, K. et al., "Dual-comb spectroscopic ellipsometry," Nature Communications, 8(1), 610 (2017).

[5] Coddington, I., Newbury, N., and Swann, W., "Dualcomb spectroscopy," Optica, 3(4), 414-426 (2016). 


\title{
Blood glucose measurement based on mid-infrared absorption spectroscopy
}

\author{
Yuji Matsuura*, Saiko Kino, Takuya Koyama \\ Graduate School of Biomedical Engineering, Tohoku University \\ 6-6-05 Aoba, Sendai 980-8579, Japan
}

\begin{abstract}
A blood glucose measurement system based on mid-infrared, attenuated-total-reflection (ATR) spectroscopy was developed. The system is composed of a Fourier-transform infrared spectrometer (FT-IR), hollow optical fibers, and a multi-reflection prism and by using this system, clear signatures of glucose are observed in the absorption spectra of human lips. It was shown that the intensity of an absorption peak of glucose coincided with the blood-glucose level well and that measurement errors were less than $20 \%$.
\end{abstract}

Keywords: blood glucose, infrared spectroscopy, hollow optical fibers

\section{Introduction}

Blood glucose measurement methods based on midinfrared absorption spectroscopy have advantages of higher absorption than near infrared spectroscopy because they detect the fundamental vibrations of glucose that are much stronger, sharper, and more isolated than the overtone peaks in the near infrared [1-3]. Those systems usually use an attenuated total reflection (ATR) prism to deal with the small penetration depth of midinfrared light. In most ATR measurement systems, the prism is in a rather bulky housing, so the area that can be measured is usually limited to skin surfaces such as the fingertip, which has a thick stratum corneum. In contrast, oral mucosa such as that of the inner lips has no keratinized layer and has interested many researchers as a target tissue for mid-infrared ATR spectroscopy.

In this paper, we introduce an FT-IR-based system comprising hollow optical fibers and multi-reflection ATR prism.

\section{Experiments and Results}

Our experimental setup for blood glucose measurement is schematically shown in Fig. 1. Infrared light from an FT-IR spectrometer was delivered to a trapezoidal ATR prism made of zinc sulfide by a hollow optical fiber. The prism whose dimensions are shown in the inset of Fig. 1 provides nine reflections. In the measurement, a subject holds the prism with the upper and lower lips and the measurement takes around 30 seconds.

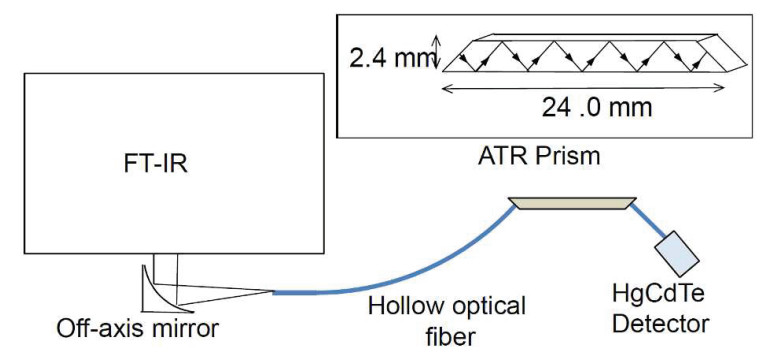

Figure 1. Schematic of measurement system

Figure 2 shows difference spectra measured for human lips. The spectra were obtained by subtracting from each measured spectrum taken at the higher blood glucose levels the spectrum measured in the fasting state. These difference spectra should correspond to absorption of only glucose and therefore can reduce errors caused by other components in human tissues that do not change with the blood glucose level. In this figure, a spectrum measured in the fasting state with a glucose level of $72 \mathrm{mg} / \mathrm{dl}$ was used as the subtrahend. Figure 2 also shows glucose solution spectra that were fitted to the measured spectra. 
The difference spectra clearly show the absorption peaks that fit those of the glucose solution.

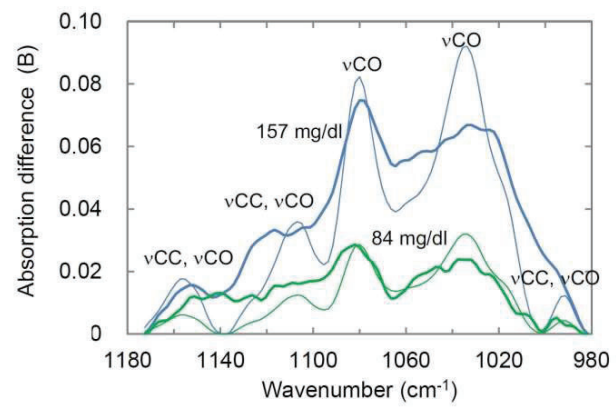

Figure 2. Difference spectra between the fasting state and state with higher blood glucose levels. Also shown (the thinner lines) are solution spectra created from least-squares fitting

We performed oral glucose tolerance tests for adult volunteers and first tried to obtain a calibration plot for blood glucose level by using the height and area of absorption peaks at 1080 and $1035 \mathrm{~cm}^{-1}$ because these peaks are larger in the height than others. However, the coefficient of determination $\mathrm{R}^{2}$ of the plot was smaller than 0.5 . This was mainly because there were random changes in the amplitude of the absorption peaks. These peaks originate from $\mathrm{C}-\mathrm{O}$ bonds of compounds other than glucose, such as phospholipids in oral mucosa that reflects compounds in ingested foods. We hence focused on the peak arising from the $\mathrm{C}-\mathrm{C}$ and $\mathrm{C}-\mathrm{O}$ combination at $1155 \mathrm{~cm}^{-1}$ because it originates from the pyranose ring structure of glucose.

Figure 3 shows a calibration plot drawn on a Clarke error grid. The reference values are blood glucose levels measured by blood sampling, and the measured values are calculated from the differential glucose absorption around $1155 \mathrm{~cm}^{-1}$ explained above. The figure shows that the certainty is improved and that the coefficient of determination $\mathrm{R}^{2}$ is 0.75 . The standard error in the glucose level is $12 \mathrm{mg} / \mathrm{dl}$, and all the measured values are in Region A, where the measured values are within $20 \%$ of the reference values.

\section{Conlusion}

We developed a mid-infrared ATR spectroscopy system that consists of hollow optical fibers for infrared light delivery, a trapezoidal multi-reflection ATR prism, and a conventional FT-IR spectrometer. In the analysis of in-vivo measurement data, difference spectra between the fasting state and states of higher glucose levels were calculated and clear signatures of glucose absorption were evident in them. We found that the amplitude of the peak at $1155 \mathrm{~cm}^{-1}$ followed changes in blood glucose level. We obtained a calibration plot with measurement errors less than $20 \%$ using the peak intensity.

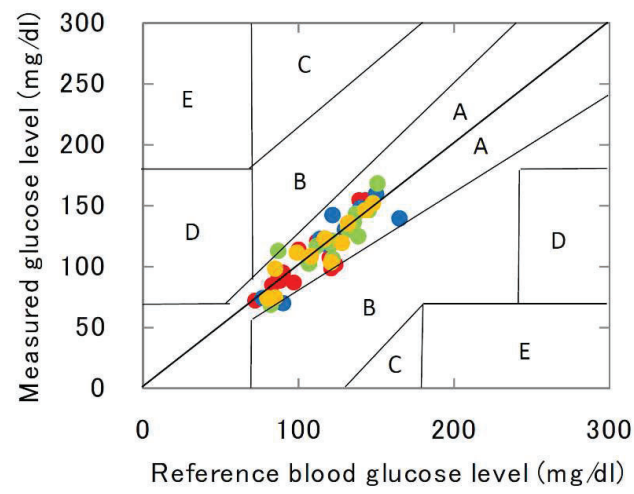

Figure 3. Calibration plot, drawn on Clarke error grid, of glucose levels measured at lip mucosa compared with reference blood glucose level.

\section{References}

[1] Y. C. Shen, et al., "The use of Fournier-transform infrared spectroscopy for the quantitative determination of glucose concentration in whole blood," Phys. Med. Biol. 48, 2023-2032 (2003).

[2] K. Kajiwara,et al., "Non-invasive measurement of blood glucose concentrations by analysing Fourier transform infra-red absorbance spectra through oral mucosa," Med. Biol. Eng. Comput. 31, S17-S22 (1993).

[3] H. M. Heise and R. Marbach, "Human oral mucosa studies with varying blood glucose concentration by non-invasive ATR-FT-IR-Spectroscopy," Cell. Mol. Biol. 44(6), 899-912 (1998) 


\title{
Dynamic 3D SERS imaging of small molecules in live cells
}

\author{
Kota Koike*a, b, Kazuki Bando ${ }^{\text {a, c }, \text { Jun Ando }}$, Nicholas I. Smith ${ }^{\text {d, Kosuke Dodo }}$, Satoshi Kawata ${ }^{\text {a c c }}$ \\ Mikiko Sodeoka ${ }^{\mathrm{e}}$, and Katsumasa Fujita ${ }^{\mathrm{a}, \mathrm{b}, \mathrm{f}}$ \\ aDepartment of Applied Physics, Osaka University, 2-1 Yamadaoka, Suita, Osaka, 565-0871, Japan \\ ${ }^{\mathrm{b}}$ Advanced Photonics and Biosensing Open Innovation Laboratory, AIST-Osaka Unverisity, 2-1 \\ Yamadaoka, Suita, Osaka, 565-0871, Japan \\ 'Serendip Research Ltd., 2-1 Yamadaoka, Suita, Osaka, 565-0871, Japan \\ dimmunology Frontier Research Center, Osaka University, 2-1 Yamadaoka, Suita, Osaka, 565-0871, \\ Japan \\ eSynthetic Organic Chemistry Laboratory, RIKEN, 2-1 Hirosawa, Wako, Saitama 351-0198, Japan \\ ${ }^{f}$ Transdimensional Life Imaging Division, Institute for Open and Transdisciplinary Research \\ Initiatives, Osaka University, 2-1 Yamadaoka, Suita, Osaka 565-0871, Japan
}

\begin{abstract}
Observing small molecules such as fatty acids, amino acids, nucleic acids, and low molecular weight drugs in live cells is important to understand living systems of the cells. Such molecules are not readily detected at low concentrations by spontaneous Raman scattering because of their small scattering cross-sections. We utilized surface-enhanced Raman scattering (SERS) to observe small molecules in live cells with high sensitivity. Gold nanoparticles introduced into living cells via endocytosis were used as SERS probes, and alkyne tag was used to detect target molecules separately from endogenous molecules. A slit-scanning 3D SERS microscope was developed to detect the target molecules in a cellular volume. Time-lapse 3D imaging successfully visualized the drug entry into living macrophages by detecting alkyne signature by SERS.
\end{abstract}

Keywords: Raman microscopy, surface-enhanced Raman scattering, small molecule, drug delivery, alkyne-tag

\section{Introduction}

Observing small molecules such as fatty acids, amino acids, nucleic acids, and low molecular weight drugs in live cells is important for studying and understanding living systems of the cells. Fluorescence microscopy has been a powerful tool to observe biological tissues and cellular organelles with a high spatial resolution by means of labeling with fluorescent proteins or organic dyes [Huang, B. 2009]. However, when the small molecules are labeled with the fluorescent probes which are relatively bulkier than the target small molecules, the original nature of the small molecules can be inhibited by the labeling. In addition, because of the photobleaching of the fluorescent tags, time-lapse observation of the dynamics of target molecules inside cells would be hard.

Raman microscopy directly observes the molecular distribution of live cells by detecting Raman spectrum from the molecules without labeling [Hamada, K. 2008]. Moreover, since alkyne shows a Raman peak where no
Raman peak from intracellular molecules present, the use of alkyne as a Raman tag allows observing the distribution of alkyne-tagged small molecules via Raman scattering of alkyne [Yamakoshi, H. 2011.] [Yamakoshi, H. 2012.]. However, because the detection sensitivity of Raman microscopy has been limited by the small scattering cross section, observing the temporal dynamics of the small molecules in live cells has not been realized.

Here, we propose a technique to detect alkyne-tagged small molecules in live cells using surface-enhanced Raman scattering (SERS). High sensitivity in the detection of small molecules is realized by the enhancement of Raman scattering from the alkyne at the surface of gold nanoparticles via localized plasmon resonance. The SERS signals from alkyne were detected when the molecules were at the vicinity of nanoparticles after penetrating the cellular membranes. Time-lapse SERS imaging enabled us the observation of uptake of the small molecules into cells and their dynamics of them 
inside the cells, which can be useful to understand the property of the small molecules in live cells through the interaction with intracellular molecules.

\section{Results and Discussion}

Slit-scanning SERS microscopy equipped with a 660 $\mathrm{nm}$ laser was developed to detect SERS spectra from gold nanoparticles in living cells. Slit-scanning Raman imaging allows the parallel detection of several hundreds of Raman spectra, resulting in the rapid Raman imaging [Hamada, K. 2008]. A Piezoelectric stage was used for scanning a water immersion objective lens of $60 \mathrm{x}$ NA1.27 for z-direction to perform 3D SERS imaging of the cells. As a target small molecule, alkyne-tagged acyloxymethyl ketone type inhibitor (Alt-AOMK) was chosen [Ando, J. 2016.]. Macrophages (J774A.1) were incubated for 20 hours with gold nanoparticles with a diameter of $50 \mathrm{~nm}$ at $37^{\circ} \mathrm{C}, 5 \% \mathrm{CO}_{2}$ in order to deliver gold nanoparticles into the cells via endocytosis. Time-lapse 3D SERS imaging of the cells was carried out with a temporal resolution of $22 \mathrm{sec} /$ volume for $53 \mathrm{~min}$. The alkyne-tagged small molecules were administrated into the sample with the final concentration of $5 \mu \mathrm{M}$ in the middle of the timelapse measurement. SERS signals of alkyne were detected in the cells at around 10 min after the administration, then the number of the SERS signal gradually increased by time. These results indicate the small molecules were taken up into the cells, and SERS signals from the terminal alkyne occurred when the small molecules were in the vicinity of gold nanoparticles. We also quantitatively monitored the entry of the small molecules into the cells by extracting SERS spectra of the small molecules and evaluating the number of SERS in each cell at each 3D SERS image.

The proposed technique can be useful to investigate the uptake efficiency of small molecules into cells and observe the dynamics of the target small molecules inside cells.

\section{References}

Ando, J. 2016. Alkyne-Tag SERS Screening and Identification of Small-Molecule-Binding Sites in Protein. Journal of the American Chemical Society 138(42), 13901-13910.
Hamada, K. 2008. Raman microscopy for dynamic molecular imaging of living cells. Journal of Biomedical Optics 13(4), 044027.

Huang, B. 2009. Super-Resolution Fluorescence Microscopy. Annual Review of Biochemistry 78, 993 1016.

Yamakoshi, H. 2011. Imaging of EdU, an Alkyne-Tagged Cell Proliferation Probe, by Raman Microscopy. Journal of the American Chemical Society 133(16), 6102-6105.

Yamakoshi, H. 2012. Alkyne-Tag Raman Imaging for Visualization of Mobile Small Molecules in Live Cells. Journal of the American Chemical Society 134(51), 20681-20689. 


\title{
Fluorescence imaging of microbeads by transport of intensity equation
}

\author{
Sudheesh K Rajput ${ }^{* 1}$, Manoj Kumar ${ }^{1}$, Xiangyu Quan ${ }^{1}$, Osamu Matoba ${ }^{1}$, Yasuhiro Awatsuji ${ }^{2}$ \\ ${ }^{1}$ Graduate School of System Informatics, Kobe University, Rokkodai 1-1, Nada, Kobe 657-8501, \\ Japan \\ ${ }^{2}$ Faculty of Electrical Engineering and Electronics, Kyoto Institute of Technology, Matsugasaki, \\ Sakyo-ku, Kyoto 606-8585, Japan
}

\begin{abstract}
We present a fluorescence imaging method based on transport of intensity equation (TIE) in which focus fluorescence image is retrieved from defocused intensity images. The focus image can be estimated from the phase image retrieved by TIE and the use of inverse Fresnel propagation under the partially coherence of microbeads. We present results for the focus measurement from experimentally recorded defocus fluorescence images.
\end{abstract}

Keywords: Fluorescence imaging, phase imaging, Fresnel propagation, phase retrieval, transport of intensity equation.

\section{Introduction}

Fluorescence imaging is the visualization of fluorescent biological samples as labels for the molecular structure. It is done by using epifluorescence where excitation light illuminates the sample through the same objective that is used to detect the emission from the sample [1]. A fluorescence filter separates the light by wavelength so that the emitted light can be imaged without interference from the excitation light [1-4]. The interferometric techniques such as digital holography imaging and noninterferometric imaging techniques such as transport of intensity equation (TIE) have also been proposed for quantitative phase imaging [3-9]. Among them, the TIE has an additional advantage of robustness against the disturbance under the partially coherent illumination $[5,6]$. Here, we explore the use of TIE for the focus image estimation in fluorescence imaging. Focus images can be obtained from the recorded three defocus images $[7,8]$.

\section{Imaging method}

The schematic of the proposed imaging system is shown in Fig. 1. In this system, a laser light source has been used to excite fluorescent beads and three defocus images are recorded by moving the stage along the vertical axis. After solving TIE by using these images, phase distribution can be retrieved [5-9]. Now the

*srskrajput@gmail.com complex amplitude is calculated from intensity image and retrieved phase, and this complex amplitude is inverse propagated. From back propagated distribution, intensity images can be estimated at different distances $[7,8]$.

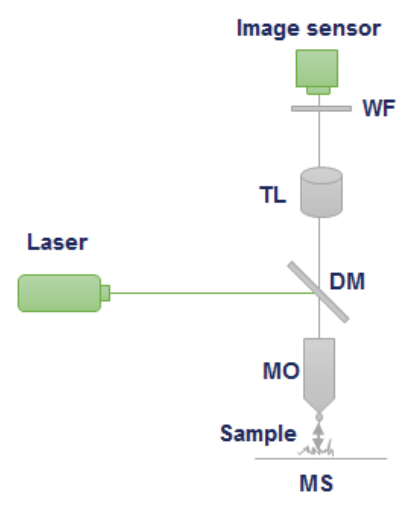

Fig. 1. Fluorescence imaging setup. TL: Tube Lens, MO: Microscope objective, MS: Movable stage, DM:

Dichroic mirror, and WF: Wavelength filter.

\section{Results and Discussion}

We have performed an experiment on fluorescence imaging using the optical setup as shown in Fig. 1. In this experiment, a blue laser of $473 \mathrm{~nm}$ wavelength and the microbeads of size $10.4 \mu \mathrm{m}$ have been used. The size of used image sensor is $1024 \times 1024$ with a pixel pitch of 13 $\mu \mathrm{m}$. We have recorded three defocus intensity images and 


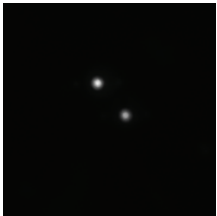

(a)

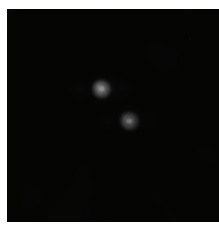

(c)

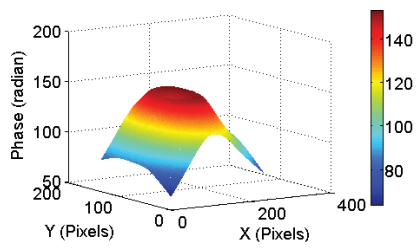

(e)

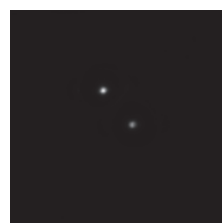

(f)

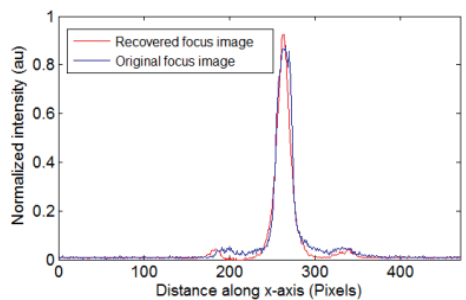

(h)

(g)

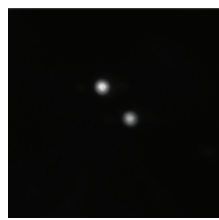

(b)

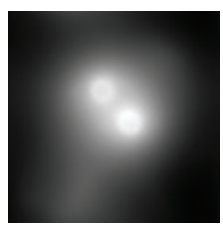

(d)

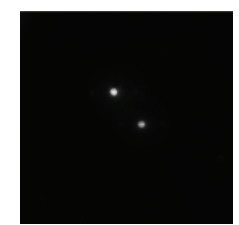

Fig. 2. Experimental results. (a) First defocus intensity
image, (b) second defocus intensity image, (c) third defocus intensity image, (d) the reconstructed phase image using TIE-based phase retrieval, (e) 3D plot of upper bead, (f) recovered focus image, (g) original focus image recorded in experiment, and (h) line plot for recovered and original focus image.

one focus intensity image. The defocus distances of three images from the focus image are $20 \mu \mathrm{m}, 30 \mu \mathrm{m}$ and 40 $\mu \mathrm{m}$, respectively. The fluorescent beads emit the light of wavelength ranging from $550 \mathrm{~nm}$ to $600 \mathrm{~nm}$. A band-pass filter centered at $575 \mathrm{~nm}$ was used in front of the image sensor.

The experimental results are shown in Fig. 2. Figures 2(a), (b), and (c) show the first, second and the third defocus intensity images, respectively. Figure 2(d) shows the reconstructed phase image using TIE-based phase retrieval algorithm when the image shown in Fig. 2(b) is considered as focused image and images shown in Figs. 2(a) and 2(c) are considered as defocus images. Figure 2(e) shows the 3D plot of the upper bead. Figure 2(f) shows the recovered focus image obtained by the proposed method, which is corresponds to the original focus image recorded in the experiment and shown in Fig $2(\mathrm{~g})$. Figure 2(h) shows the line plot across the upper bead of the recovered and original focus images. From these results, it can be seen that the focus image can be obtained successfully from the defocus images.

\section{Conclusions}

We have presented a focus estimation scheme for fluorescence imaging using TIE. The phase image retrieved from three defocus images using TIE and the inverse Fresnel propagation can estimate the focus image.

Acknowledgment- A part of this work has been supported by Japan Society for the Promotion of Science (JSPS) KAKENHI (17F17369, 18H03888, 17F17370) and JST CREST Grant Number JPMJCR1755.

\section{References}

[1] Kubitscheck, U., Fluorescence Microscopy: From Principles to Biological Applications, 2nd ed. (Wiley-VCH, 2017).

[2] Wang, Z., Cai, Y., Liang, Y., Zhou, X., Yan, S., Dan, D., Bianco, P. R., Lei, M., and Yao, B., "Single shot, threedimensional fluorescence microscopy with a spatially rotating point spread function,” Biomed. Opt. Express, 8(12), 5493-5506 (2017).

[3] Rosen, J., and Brooker, G., "Non-scanning motionless fluorescence three-dimensional holographic micoroscopy," Nature Photonics, 2, 190-195 (2008).

[4] Quan, X., Matoba, O., Xia, P., and Awatsuji, Y., "Image recovery from defocused $2 \mathrm{D}$ fluorescent images in multimodal digital holographic microscopy," Opt. Lett. 42(9), 1796-1799 (2017).

[5] Paganin, D., and Nugent, K.A., "Non interferometric phase 
imaging with partially coherent light," Phy. Rev. Lett. 80(12), 2586-2589 (1998).

[6] Yu, W., Tian, X., He, X., Song, X., Xue, L., Liu, C., and Wang, S., "Real time quantitative phase microscopy based on single-shot transport of intensity equation (ssTIE) method," Appl. Phy. Lett. 109, 071112, (2016).

[7] Tian, X., Meng, X., Yu, W., Song, X., Xue, L., Liu, C., and Wang, S., "In-focus quantitative intensity and phase imaging with the numerical focusing transport of intensity equation method," J. Opt. 18(10), 105302 (2016).

[8] Komura, K., and Nomura, T., "Object plane detection and phase amplitude imaging based on transport of intensity equation," Opt. Rev. 24(5), 626-633 (2017).

[9] Xia, P., Awatsuji, Y., Ura, S., Nishio, K, Matoba, O., “Oneshot complex amplitude measurement using transport of intensity equation," Technical Diest of Digital Holography and 3D Imaging, DTh5A.3 (2013).

Proc. of SPIE Vol. $111401114001-58$ 


\title{
Multimodal digital holographic microscopy
}

\author{
Manoj Kumar ${ }^{\mathrm{a}}$, Xiangyu Quan ${ }^{\mathrm{a}}$, Yasuhiro Awatsuji ${ }^{\mathrm{b}}$, Yosuke Tamada ${ }^{\mathrm{c}}$, and Osamu Matoba ${ }^{\mathrm{a}}$ \\ ${ }^{a}$ Graduate School of System Informatics, Kobe University, Rokkodai 1-1, Nada-ku, Kobe 657-8501, \\ Japan \\ ${ }^{b}$ Faculty of Electrical Engineering and Electronics, Kyoto Institute of Technology, Matsugasaki, \\ Sakyo-ku, Kyoto 606-8585, Japan \\ ${ }^{\mathrm{c} N a t i o n a l ~ I n s t i t u t e ~ f o r ~ B a s i c ~ B i o l o g y, ~ O k a z a k i, ~ J a p a n ~}$
}

\begin{abstract}
In this paper, we present a new multimodal approach for biological applications which comprises of a common-path off-axis incoherent digital holographic microscope to obtain 3D fluorescence imaging and another off-axis coherent digital holographic microscope to retrieve 3D phase imaging. The concept of the multimodal system to achieve both the 3D fluorescence and phase images is demonstrated on fluorescence beads and biological specimen. The experimentally obtained results authenticate the usefulness of the multimodal imaging system. The proposed system could found a beneficial role for numerous biomedical investigations.
\end{abstract}

Keywords: 3D fluorescence imaging, phase imaging, digital holographic microscopy, multimodal imaging system

\section{Introduction}

Multimodal imaging systems have found rapid progress in the last decade and demonstrated in various studies. By incorporating holographic fluorescence and phase imaging systems in a common hybrid imaging platform, it is possible to precisely visualize and characterize the structural and functional information. Here we present two holographic microscopes separately, one is efficient of recording and retrieving $3 \mathrm{D}$ fluorescence imaging while another 3D phase imaging of an object.

The phase imaging technique by digital holography has established a robust technique for numerous investigations, particularly eminent, presently in biological investigations [1-2]. The technique exploits the principle of optical interferometry and measures the optical phase delay induced by the variation of refractive index between a sample and a medium. The measured phase delay further can be utilized for analysis of various chemical and morphological properties of the biological specimen.

1manojklakra@gmail.com, Phone: +81-078-803-6240
Holographic fluorescence microscopy, on the other hand, plays an important role in investigations of functional information of a biological sample. The unprecedented molecular specificity of a biological specimen can be obtained by labeling of the target molecules with the fluorescence probes. The multimodal imaging system realized by combining the holographic fluorescence microscopy and phase imaging on a common platform could be an efficient and powerful tool to retrieve multi-functional information at a single observation. Various multimodal imaging configurations have been developed and the research in this direction is continuously progressing [3-6]. We are planning to develop a new configuration of the multimodal imaging system which comprises of an off-axis incoherent digital holographic microscope for 3D fluorescence imaging and an off-axis coherent digital holographic microscope for the 3D phase imaging, demonstrated separately in this paper on the same object, with their 3D imaging capabilities.

\section{Methodology}

The holographic fluorescence imaging is accomplished by utilizing a common-path off-axis incoherent digital 
holography in which a focusing lens, of focal length 800 $\mathrm{mm}$, with a diffraction grating, grating period 300 micrometer, is displayed onto a phase-mode spatial light modulator (SLM), (Holoeye pluto, 1920 $\times 1080$ pixels, 8 $\mu \mathrm{m}$ pixel pitch, phase-only modulation) [7,8]. This pattern (i.e. the combination of the lens function and the grating period) splits the incident light from the fluorescent sample into two light waves which are propagating slightly different propagation directions, depending on the period of the diffraction grating, and therefore, an off-axis interference is occurred on the image sensor, EMCCD sensor (Model-iXon 888, sensor format:1024×1024 pixels, pixel size of $13 \mu \mathrm{m}$, sensor diagonal of $18.8 \mathrm{~mm}$ ), between these two wavefronts. This interference pattern called a hologram, is recorded in the computer and the 3D object information can be obtained by using an appropriate digital holographic reconstruction algorithm, say the Fresnel propagation algorithm.

To obtained the phase information of the object, an offaxis coherent digital holographic system working in transmission configuration is exploited, where a He-Ne laser $(\lambda=632.8 \mathrm{~nm})$ is used as an illumination source. The expanded and collimated laser beam is divided into the object and the reference beams. The object beam is allowed to pass through the sample and diffracted beam from the sample is combined with the reference beam at the image sensor plane, CMOS camera (Sony Pregius IMX 249, sensor format-1920 $\times 1200$ pixels, pixel size$5.86 \mu \mathrm{m})$. The off-axis configuration is achieved by introducing a small angle between the object and the reference plane waves. In this case, two digital holograms are recorded: one in the presence of the sample and another without the sample. These two holograms were processed to obtain the phase information of the sample.

\section{Experimental Results}

Various experiments on fluorescence beads and biological samples were performed to verify the capabilities of the individual digital holographic microscopic systems. First, holographic fluorescence imaging is carried out on the fluorescent beads and then the holographic phase imaging is accomplished on the same sample. The results obtained from both the holographic microscopes are demonstrated in the following section.

\section{3D Fluorescence Imaging}

The fluorescent beads used in the experiment are of the size 10-14 $\mu \mathrm{m}$. In the holographic fluorescence microscopy, the $\mathrm{Nd}: \mathrm{YVO}_{4}$ laser (wavelength, $\lambda=532$ $\mathrm{nm}$ ) was used as a light source to excite the fluorescent beads, which emits the fluorescent light of a wavelength ranging from 550-600 nm. Fig. 1(a) shows the focused image of the fluorescent beads whereas Fig. 1(b) shows the recorded hologram, obtained by moving the translational stage in the $z$-direction by an amount of 80 $\mu \mathrm{m}$ and then displaying a lens function of focal length $800 \mathrm{~mm}$, with a diffraction grating, grating period 300 $\mu \mathrm{m}$ on to the SLM. Fig. 1(c) shows the retrieved reconstructed image of the fluorescent beads obtained by Fresnel reconstruction algorithm.
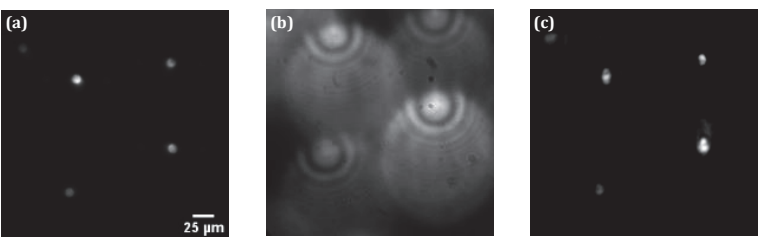

Fig. 1: Fluorescence imaging results: (a) the focused image, (b) incoherent hologram, and (c) reconstructed fluorescent image.

\section{3D Phase Imaging}

The 3D phase imaging of the same beads is accomplished by utilizing an off-axis digital holographic microscopy. The phase imaging results of the same beads are shown in Figs. 2(a) and 2(b). the obtained wrapped phase of the beads is shown in Fig. 2(a) and corresponding unwrapped phase map of the beads is depicted in Fig. 2(b).
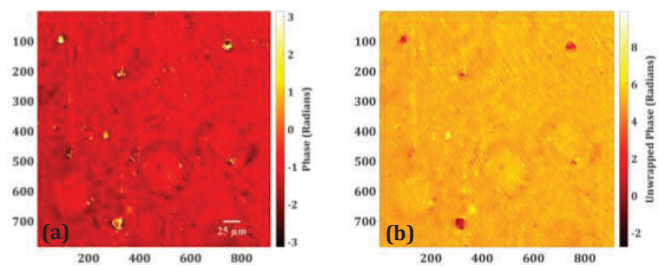

Fig. 2: Phase imaging results: (a) phase image, and (b) corresponding unwrapped phase map. 


\section{Conclusion}

The experimentally obtained results of both the holographic systems acknowledge their capabilities for the investigations of $3 \mathrm{D}$ fluorescence and phase information. In future, both the holographic systems will be integrated on a single platform in order to obtain a simultaneous recording of 3D fluorescence and phase imaging of the biological specimen. The proposed system could be utilized in analysis and investigations of various biophysical processes occurring in a biological specimen, since fluorescence imaging provides the function information of the fluorescentlabeled molecules/nucleus, whereas the phase imaging provides the structural information of the biological specimen.

Acknowledgments - Japan Society for the Promotion of Science (JSPS) KAKENHI (18H03888, 17F17370), and JST CREST Grant Number JPMJCR1755

\section{References}

[1] Popescu, G., [Quantitative Phase Imaging of Cells and Tissues], McGraw-Hill, New York, (2011).

[2] Kim, M. K., "Principles and techniques of digital holographic microscopy," SPIE Reviews 1(1), 018005 (2010).

[3] Park, Y., Popescu, G., Badizadegan, K., Dasari, R. R., Feld, M. S., "Diffraction phase and fluorescence microscopy," Opt. Express 14, 8263-8268 (2006).
[4] Pavillon, N., Benke, A., Boss, D., Moratal, C., Kühn, J., Jourdain, P., Depeursinge, C., Magistretti, P. J., Marquet, P., "Cell morphology and intracellular ionic homeostasis explored with a multimodal approach combining epifluorescence and digital holographic microscopy," J Biophoton 3, 432-436 (2010)

[5] Quan, X., Nitta, K., Matoba, O., Xia, P., Awatsuji, Y., "Phase and fluorescence imaging by combination of digital holographic microscopy and fluorescence microscopy," Opt. Rev. 22, 349-353 (2015).

[6] Matoba, O., Quan, X., Xia, P., Awatsuji, Y., Nomura, T., "Multimodal imaging based on digital holography," Proc. IEEE 105, 906-923 (2017).

[7] Kumar, M., Quan, X., Awatsuji, Y., Matoba, O., “Threedimensional fluorescence imaging of beads and cancer cells by off-axis incoherent digital holography," In Digital Holography and Three-Dimensional Imaging, pp. DTh1C3. Optical Society of America, 2018.

[8] Quan, X., Kumar, M., Matoba, O., Awatsuji, Y., Hayasaki, Y., Hasegawa, S., Wake, H., "Three-dimensional stimulation and imaging-based functional optical microscopy of biological cells," Opt. Lett. 43, 5447-5450 (2018). 


\title{
Planar lightwave circuit digital holographic microscope for imaging of biological cells
}

\author{
Eriko Watanabe $^{\mathrm{a}}$, Shutaro Kodama ${ }^{\mathrm{a}}$, Katsutoshi Inomoto ${ }^{\mathrm{a}}$, Kanami Ikeda ${ }^{\mathrm{b}}$ and Katsunari Okamoto ${ }^{\mathrm{c}}$ \\ ${ }^{a}$ The University of Electro-Communications, 1-5-1, Chofugaoka, Chofu, Tokyo, 182-28585, Japan \\ ${ }^{b}$ Osaka Prefecture University, 1-1, Gakuen-chou, Naka-ku, Sakai, Osaka, 599-8531, Japan \\ ${ }^{\circ}$ Okamoto laboratory
}

\begin{abstract}
We describe our developed planar lightwave circuit digital holographic microscope (PLC-DHM) with no moving parts and lenses. The PLC-DHM can accurately measure the microscopic intensity and phase distribution, has a spatial resolution of $1.6 \mu \mathrm{m}$, and provides highly accurate quantitative phase imaging. We also demonstrate three-dimensional and quantitative phase imaging of several biosamples using our PLC-DHM.
\end{abstract}

Keywords: Digital holographic microscopy, Planar lightwave circuit

*phase@fourier.ghrdp.uec.ac.jp; phone +81 42 443-5960

\section{Introduction}

A digital holographic microscope (DHM) can measure quantitative phase information, such as the thickness and refractive index of microscopic objects by a non-contact method and is therefore used in the medical field to identify breast cancer cells and normal cells based on quantitative phase information ${ }^{1)}$. We developed a DHM, which can be miniaturized by not using an objective lens for magnifying objects ${ }^{2}$. We have also developed a planar lightwave circuit digital holographic microscope (PLCDHM), with no moving parts and lenses that can measure microscopic intensity and phase distribution accurately by using an optimized design of the PLC and optical systems ${ }^{3)}$.

In this paper, we describe the PLC-DHM system and demonstrate three-dimensional and quantitative phase imaging of several biological cells using our PLC-DHM.

\section{Digital Holographic Microscope based on Planar Lightwave Circuit}

The experimental setup of PLC-DHM is shown in Fig. 1. The PLC-DHM is composed of a laser, optical fiber, PLC, beam splitter, and camera.

As a light source, a He-Ne laser with a wavelength of $632.8 \mathrm{~nm}$ is used. The light passes through the polarization-maintaining optical fibers while maintaining the fiber planes of polarization, then enters the PLC, and is divided into two beams emitted from the end of the
PLC as point light sources. One of the light beams is used as the reference light, called reference beam, and the other light beam is incident on the sample, denoted as the object beam. The reference and object beams are caused to interfere with each other via the beam splitter. The interference fringes on the imaging element surface are recorded and digitally reconstructed using a computer to obtain an intensity image and a phase image.

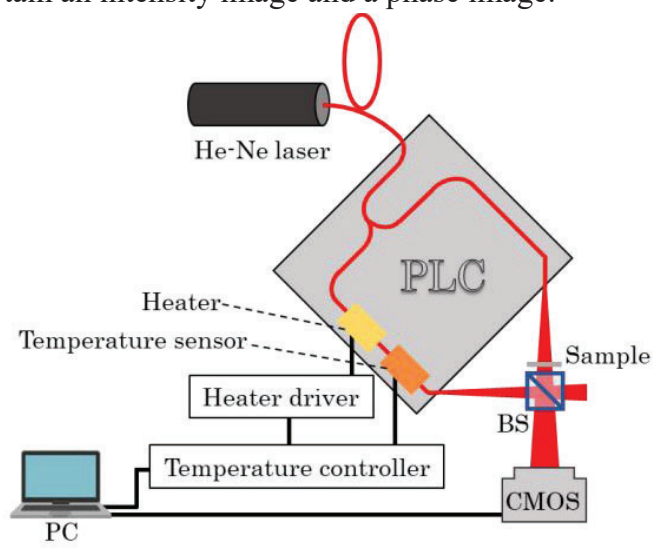

Fig. 1 PLC-DHM setup

We used the phase shifting method, based on the thermooptic effect ${ }^{4}$ ), for removing the $0^{\text {th }}$-order light in this system because the $0^{\text {th }}$ order and -1 order light are overlapped. The thermo-optic effect is a phenomenon in which heat is applied to a medium through which light propagates and a refractive index gradient occurs owing to a change in temperature. The refractive index changes, 
and as a result, the optical path length (phase) also changes. The thermo-optic effect can be generated by applying an external direct current, an alternating current, or pulsed heat ${ }^{5)}$. The optical path length variation ${ }^{6)} \Delta \mathrm{L}$ is expressed as

$$
\Delta L=n \alpha \Delta T+l \Delta L \frac{d n}{d T},(1)
$$

where, $n$ is the refractive index, $l$ is the temperature range, $\alpha$ is the coefficient of linear expansion, $d n / d T$ is the temperature coefficient, and $\Delta T$ is the increase in temperature.

\section{Experiment results}

As shown in Fig. 2 (a), three-dimensional imaging was performed for samples with different depths from different biological cells. As biological cells, zygnematales, cosmarium, and micrasterias thomasiana were used. The difference in depth of each biological cell was $1.27 \mathrm{~mm}$; the cells were separated by a slide glass with a refractive index of 1.524. Cosmarium has a constriction in the center and is $100 \mu \mathrm{m}$ or lesser in size. Micrasterias has multiple tips, which is divided into two branches, and is approximately $160 \mu \mathrm{m}$ to $100 \mu \mathrm{m}$ in size.

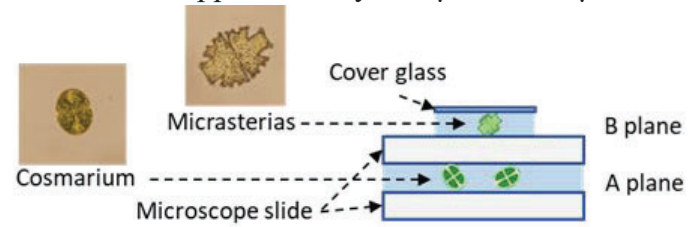

(a)

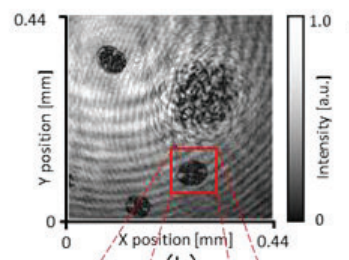

(b)

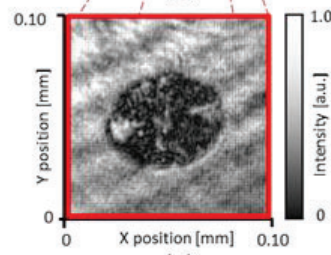

(d)

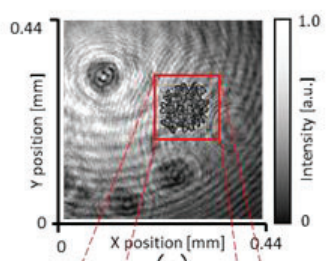

(c)

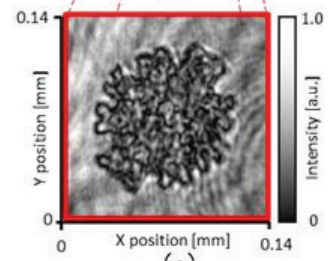

(e)
Fig. 2 3D imaging of biological samples.

(a) Sample set up; (b) Focusing A plane; (c) Focusing B plane; (d) Reconstructed image of cosmarium; reconstructed image; (e) Reconstructed image of micrasterias.

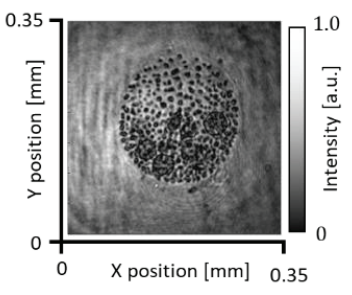

(a)

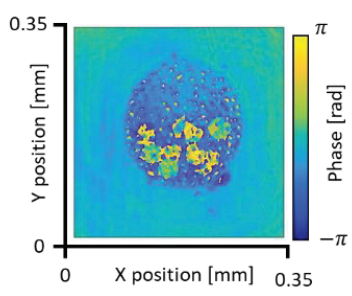

(b)
Fig. 3 (a) Intensity imaging and (b) quantitative phase imaging of volvox

By adjusting the focus to the A plane, the reconstructed intensity image of cosmarium was obtained as shown in Fig. 2(d). The distinctive contours of micrasterias in Fig. 2. (a) can be confirmed from the reconstructed image in Fig. 2 (e). As an example of quantitative phase imaging, a quantitative phase image of volvox is shown in Fig. 3 (b). The internal structure was obtained clearly in the quantitative phase imaging of volvox.

\section{Conclusions}

Three-dimensional imaging and quantitative phase imaging of biological cells were performed using the PLC-DHM. In quantitative phase imaging, the characteristics of the biological cells were reconstructed, which are difficult to observe in the intensity image.

\section{Acknowledgment}

This research partly supported by the JKA Foundation.

\section{References}

[1] P. Girshovitz and N. T. Shaked, "Generalized cell morphological parameters based on interferometric phase microscopy and their application to cell life cycle," Biomed Opt Express 3, 1757(2012).

[2] E. Watanabe, K. Hoshino, and S. Takeuchi, "Portable digital holographic microscope using spherical reference beam," Opt. Rev. 22, 342C (2015).

[3] K. Inomoto, H. Satake, K. Ikeda, S. Kodama, K. Okamoto, and E. Watanabe, ISOM'18 Mo-F-03(2018).

[4] T. Goh, S. Suzuki, and A. Sugita, "Estimation of waveguide phase error in silica-based waveguides," J. Lightwave Technol. 15,2107(1997).

[5] K. Okamoto, H. Aoyagi, and K. Takada, "Fabrication of Fourier-transform, integrated-optic spatial heterodyne spectrometer on silica-based planar waveguide," Opt. Lett. 35, 2103(2010).

[6] I. Yamaguchi, J. Kato, S. Ohta, and J. Mizuno. "Image formation in phase-shifting digital holography and applications to microscopy," Appl. Opt. 40(34), 6177(2001). 


\title{
Heterodyne terahertz holography of biological samples*
}

\author{
Hui Yuan ${ }^{\mathrm{a}}$, Alvydas Lisauskas ${ }^{\mathrm{a}}$, Hartmut G. Roskos*a ${ }^{*}$ Chunyang Dong ${ }^{\mathrm{b}}, \mathrm{He} \mathrm{Li}^{\mathrm{b}}$ \\ aPhysikalisches Institut, Johann Wolfgang Goethe-Universität, Frankfurt am Main, Germany \\ ${ }^{b}$ Tieling Central Hospital, Tieling, China
}

\begin{abstract}
Abstracts The field of $\mathrm{THz}$ radiation detection has been greatly improved and comprehensively expanded in recent years, resulting in imaging with $\mathrm{THz}$ radiation has gained great interest in multiple applications. Among these, holography as an effective coherent imaging method to employee both $\mathrm{THz}$ intensity and phase imaging has a great potential in diagnostic, quality monitoring and nondestructive detection etc. fields. In this work, we describe a novel continuous wave terahertz holography imaging system for bone structure monitoring. In the system, two 300-GHz electrical multiplier-chain sources combined with a narrow-band TeraFET detector are utilized to build up a heterodyne detection mechanism. Thanks for the heterodyne detection art, not only the intensity but also the phase of the hologram are recorded. With a digital recovery algorithm, the internal structure of a chicken leg bone is reconstructed. The dynamic range of the imaging result is $60 \mathrm{~dB}$ and a $1 \mathrm{~mm}$ resolution is achieved with a $60 \times 60 \mathrm{~mm}^{2}$ detection area, which shows this method a potential in medical diagnosis.
\end{abstract}

Keywords: terahertz, holography, diagnosis, high dynamic, biological

\section{Introduction}

The unique properties of Terahertz $(\mathrm{THz})$ radiation, such as non-ionizing but penetrates non-polar materials, absorbed by water and many organic substances and high chemical sensitivity, result in various applications of $\mathrm{THz}$ radiation in areas of stand-off explosive detection [1], medical imaging [2], non-destructive real-time imaging [3], biological applications [4], etc. Since $\mathrm{THz}$ radiation can penetrate many materials which are opaque for optical radiation, imaging biological samples in nondestructive configuration with $\mathrm{THz}$ radiation has gained great interest. The shorter wavelength (around 100um) THz in-line holography with focal arrays have been intensively investigated for biological structures imaging [5], while the possibility of holography imaging at long wavelength which has higher transmission rate with heterodyne detection art has not been considered in further extend. In this study, we demonstrate a heterodyne holography imaging setup at $300 \mathrm{GHz}$ for biological medical imaging uses. With the complex hologram recorded by the antenna-coupled field-effect transistor (TeraFET) which is known to be not only highly sensitive power detectors but also capable heterodyne receivers $[6,7]$, one can numerically retrieve the internal bone structure from amplitude and phase information of the hologram ultilizing the angular spectrum algorithm.

\section{Setup and Results}

The schematic of the $300 \mathrm{GHz}$ terahertz holography system is shown in fig.1. The experiment setup mainly consists of two synchronized electronic multiplier chain sources driven by the synthesizers and one narrow band CMOS TeraFET detector which is especially sensitive at $300 \mathrm{GHz}$. To record both the amplitude and the phase information of the hologram, heterodyne detection was employed in this set-up. The outputs from both sources mix on the detector to generate the beat signal, while the intermediate frequency from the un-multiplied signal of both synthesizers is fed to a lock-in amplifier which then amplifies the so-called beat signal from the detector. To generate the reference and the beat signal, a slight frequency offset between two synthesizers is needed. The detector and the local oscillate source fixed with it works in the raster-scanning mode to record the complex hologram propagating from the sample. With digital reconstruction algorithm, the complex information of the sample can be reconstructed. 


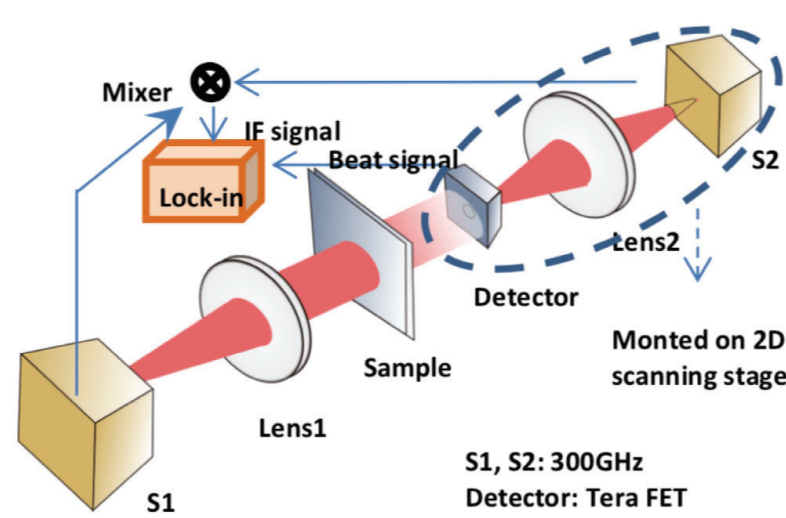

Fig. 1. Schematic Fourier imaging setup

To demonstrate the imaging ability for biological structures based on the system, a chicken leg bone was illuminated by the collimated $\mathrm{THz}$ radiation which was projected onto the single pixel detector (see Fig. 1). In principle, a detector array could be placed in the focus plane to quickly acquire amplitude and phase information in the Fourier plane. Here, we used one single pixel detector in a scanning configuration. The scanning area was $60 \times 60 \mathrm{~mm}^{2}$ with a sampling pitch of $0.5 \mathrm{~mm}^{2}$ resulting in $120 \times 120$ pixels, interpolating $50 \mathrm{~ms}$ for each point endowing the system with the potential of real-time imaging for detector array detection.

We used the mathematics derived in Ref. [8] to implement an angular spectrum algorithm using the inverse two dimensional FFT of MATLAB which calculates the light field at any given distance in front of the objects. One has to keep in mind that a wider scanning area in the hologram plane translates to a higher resolution of the object and a higher resolution in the Fourier plane translates to a bigger field of view in the object plane. For the previous stated parameter and a $5 \mathrm{~cm}$ imaging distance, the inverse transformation to the image plane yields a resolution and field of view of $0.83 \mathrm{~mm}$ and $10 \mathrm{~cm}$, respectively. By padding zeros around the taken raw data, the calculated image resolution is increased through interpolation and, therefore, gains recognisability. In our experiment, the large field of view is not of greater use since the actual illuminated area is very limited to a diameter of approximately $6 \mathrm{~cm}$. Fig. 2 shows the retrieved bone structures with a padding of 100 zeros around the raw date resulting in a resolution of around $1 \mathrm{~mm}$. One can clearly see the periodic structured holes,

where the intensity has been normalized to the maximum value. As expected, the calculation also reveals an underlying Gaussian intensity profile, so the normalization of the results by a previously acquired reference beam is adopted here.

Fig.2 is the optical image of the chicken bone sample and the imaging results. Since the bone is not a thin sample for the wavelength we select, we select three different recover distances $4.5 \mathrm{~cm}, 5 \mathrm{~cm}$ and $5.5 \mathrm{~cm}$ for the digital calculation section. From the results we can see that the intensity results nearly have nothing important to us as the first recover results, while we can obviously recognize the cavity and the marrow of the bone by different phase change at each position. This shows that the holography based on heterodyne detection is feasible in the $\mathrm{THz}$ regime and could provide the assistant images for bone structure diagnosis.

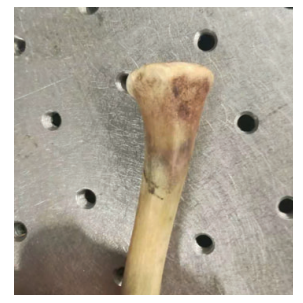

(a)

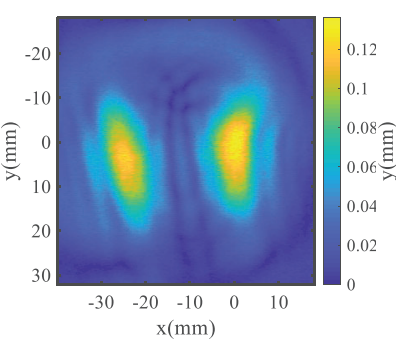

(b)

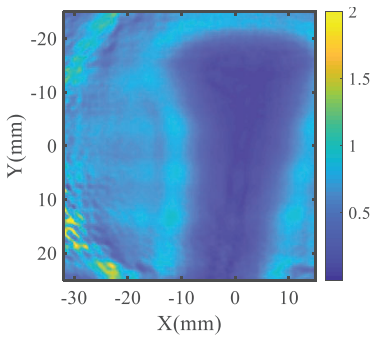

(d)

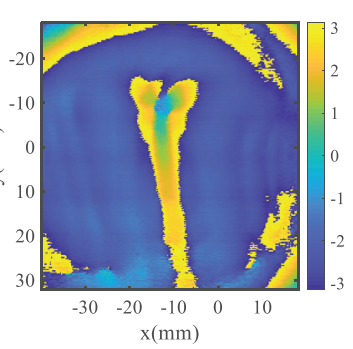

(c)

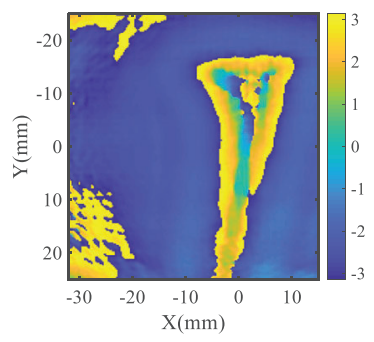

(e) 


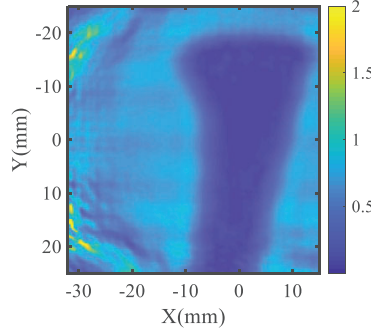

(f)

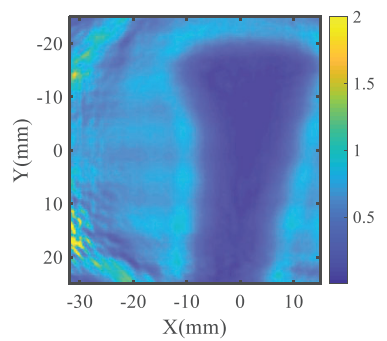

(h)

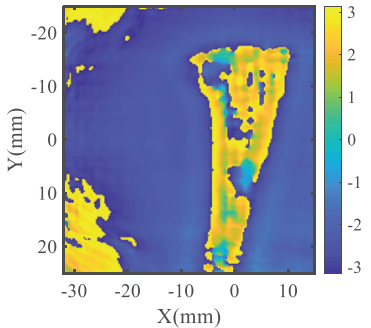

(g)

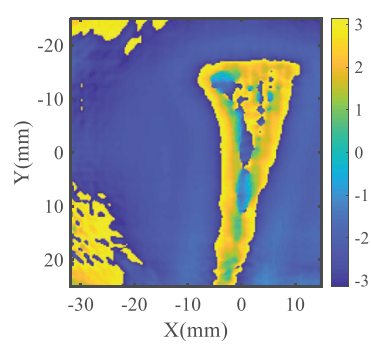

(i)
Fig. 2. Optical picture, the hologram and imaging results of the bone sample. (a) Optical picture of the bone. (b) Intensity hologram. (c) Phase hologram. (d) Intensity recover result for $4.5 \mathrm{~cm}$ recover distance. (e) Phase recover result for $4.5 \mathrm{~cm}$ recover distance. (f), (g), (h) and (i) Intensity and Phase recover results for $5 \mathrm{~cm}$ and $5.5 \mathrm{~cm}$ respectively.

\section{Discussion and Conclusion}

From the imaging results, one can see the internal structures of the chicken bone can be imaged. The images from different positions can show different structures of the positions, for the bone was located at the position of roughly $5 \mathrm{~cm}$ we can see the size of the result recovered from $5 \mathrm{~cm}$ distance is consistent with the size of the imaging sample, while off the center, for example $4.5 \mathrm{~cm}$ and $5.5 \mathrm{~cm}$ the recover results are a little more narrow than the size of the real sample because of the cylinder structure of the bone. This point of view also reviews the $3 \mathrm{D}$ imaging possibility of the system.

To conclude, a $300 \mathrm{GHz}$ terahertz holography system was presented in this paper. With a hologram recording area of $60 \times 60 \mathrm{~mm}^{2}$ and $5 \mathrm{~cm}$ imaging distance (from the center of the bone) from the imaged sample to the recording plane, the images including the internal information of the bone was achieved. Thanks to the complex information in the recording plane, not only the structure of the bone at $5 \mathrm{~cm}$ can be recovered, the structured of the bone off the center plane can also be recovered, indicating the $3 \mathrm{D}$ imaging capability of the imaging system and the possibility of a more robust imaging diagnosis tool.

\section{References}

[1] H. Zhong, A. Redo, Y. Chen, X.C. Zhang, "Standoff distance detection of explosive materials with $\mathrm{THz}$ waves", in: Jt. 30th Int. Conf. Infrared Millim. Waves, 42-43 (2005).

[2] Z.D. Taylor, R.S. Singh, D.B. Bennett, P. Tewari, C.P. Kealey, N. Bajwa, et al., "THz Medical Imaging: in vivo hydration sensing", IEEE Trans. Terahertz Sci. Technol. 1, 201-219 (2011).

[3] B. Hils, M. D. Thomson, T. Löffler, W. von Spiegel, C. am Weg, H. G. Roskos, P. de Maagt, D. Doyle, R. D. Geckeler, "Terahertz profilometry at $600 \mathrm{GHz}$ with $0.5 \mu \mathrm{m}$ depth resolution", Optics Express 16, 1128911293 (2008).

[4] H.B. Liu, G. Plopper, S. Earley, Y. Chen, B. Ferguson, X.C. Zhang, "Sensing minute changes in biological cell monolayers with $\mathrm{THz}$ differential time-domain spectroscopy", Biosens. Bioelectron. 22, 1075-1080 (2007).

[5] L. Rong etc., "Terahertz in-line digital holography of human hepatocellular carcinoma tissue", Sientific Repoert, 5, 8445 (2015).

[6] J. Grzyb, U. Pfeiffer, THz Direct Detector and Heterodyne Receiver Arrays in Silicon Nanoscale Technologies, JIMT, 36, 998 - 1032 (2015).

[7] M. Bauer, A. Rämer, S. Boppel, S. Chevtchenko, A. Lisauskas, W. Heinrich, V. Krozer, H.G. Roskos, "High-Sensitivity Wideband THz Detectors Based on GaN HEMTs with Integrated Bow-Tie Antennas", Proc. 10th European Microwave Integrated Circuits, Paris, France, Sep. 7-8, pp. 1-4 (2015).

[8] J. W. Goodman, "Introduction to Fourier optics", The McGraw-Hill companies, San Francisco (1968). 


\title{
Resolution enhancement methods of nonlinear microscopy based on
}

\section{structured illumination}

\author{
Szu-Yu Chen*, Chia-Hua Yeh, Cheng-Zn Tan, Hao-Hao Wu, and Jui-Ting Hung \\ Department of Optics and Photonics, National Central University, Taoyuan, 32001, Taiwan R.O.C.
}

\begin{abstract}
Second harmonic generation microscopy (SHGM) plays a significant role in revealing the noncentrosymmetric structures in biomedical researches. Without real-state transition during SHG generation, fluorescence-based superresolution methods cannot be applied to SHGM. To improve the spatial resolution of SHGM, fringe-scanning SHGM (FS-SHGM) system, which combines SHGM with the concept of structured illumination based on a pointscanning geometry, is introduced in this paper. The scanning path of focusing beam is periodically modulated to generate effective illumination pattern on the specimen. Due to the coherence nature of SHG signals, the mathematical model of image formation and reconstruction was established. To demonstrate the system performance, both simulation and experiments were carried out. In experiment, the improvement factor of FS-SHGM can achieve 1.4 in lateral and 1.56 in axial.
\end{abstract}

Keywords: nonlinear microscopy, second harmonic generation, superresolution, structured illumination

\section{Introduction}

In biomedical research and clinical applications, there is always the necessity to reveal the fine structures of biotissues. Two-photon microscopies, including twophoton fluorescence (TPF) and second harmonic generation (SHG) microscopy, have inherent optical sectioning power and high contrast in axial due to the nonlinearity of multi-photon excitation [1]. Among various kinds of nonlinear microscopy, SHGM makes use of the 2nd-order nonlinearity of the materials and can provide contrast in noncentrosymmetric structures like collagen fibers and tendons without staining [2]. Because of the coherence of SHG, the diffraction limit of SHGM is related to the amplitude transfer function (ATF) of the imaging system. The frequency components beyond the cut-off frequency of the ATF are eliminated. To achieve superresolution of SHGM, subtractive SHGM [3] and second harmonic superresolution microscopy (SHaSM) [4] were introduced using the concepts of stimulated emission depletion (STED) and stochastic optical reconstruction microscopy (STORM), respectively. Since SHG involves no real-level transitions, the fluorescebased modulation methods cannot be employed. Instead, nanosized photoswitchable probe which can be switched on/off by controlling the excitation polarization [4] was used in SHaSM, while subtracting two images acquired with different excitation polarizations was applied in the subtractive SHGM [3]. To achieve the resolution improvement, we introduce the structured illumination SHGM (SI-SHGM) which combines the concept of structured illumination [5] and SHGM, based on a pointscanning geometry required by two-photon excitation [6]. Due to the coherence of SHG, the mathematic models of the image formation and reconstruction are built instead of that based on TPF [6]. Both the simulation and experiments on chicken tendons are carried out to show the ability of SI-SHGM to get resolution improved.

\section{Principles}

SHG exhibits a square dependence on the excitation intensity [7] and thus, ultrafast laser pulses and tightly focusing are required to excite SHG. Therefore, a pointscanning geometry is commonly used in SHGM. Figure 1 shows the concept of illumination pattern generation based on the point-scanning geometry and quasi-comb scanning. During the scanning period of a frame (SPF), the excitation spot is scanned zia-zag throughout a rectangular area. The SHG arising from every excitation spot will be integrated and recorded by the camera. For each SPF, an SHG can be obtained. To get one 
conventional SHGM (C-SHGM) image with uniform illumination, two adjacent fast scan lines come very close to each other. Simply increasing the separation between two fast scan lines can produce fringe-scanning-SHGM (FS-SHGM) images with structured illumination pattern. In contrast to the temporally intensity modulating used in the previous study of laser scanning two-photon structured illumination microscope (LSTP-SIM) [8], the quasi-comb scanning method needs no additional intensity modulation element so that the system complexity can be reduced. The period and duty cycle of the illumination patterns can be easily adjusted by assigning a new scanning path. This makes it more convenient to integrate SIM with a conventional SHGM. Moreover, since the quasi-comb scanning can provide 0to-1 dynamic range of intensity modulation, the contrast of the illumination pattern can be increased as well.

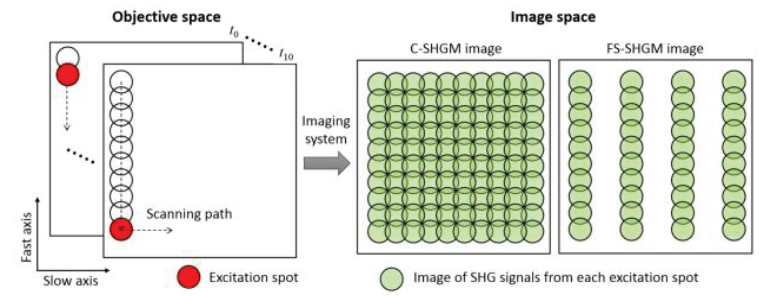

Figure 1. The concept of illumination pattern generation based on the point-scanning geometry and quasi-comb scanning.

\section{Experiment setup}

Fig. 2 shows FS-SHGM's system configuration. A 1064-nm femto-second laser (FPL-03UFF0 with Calmar pulse compressor) was utilized for SHG excitation. A 2D galvo-mirror (GVSM002/M, Thorlabs) was programmed to create the scanning pattern. Within a frame time, the fast-axis galvo mirror is actuated by a triangle wave to scan the laser beam back and forth. The laser beam after the galvo-mirror was expended to fill the back aperture of the objective (UPLSAPO 60XW, NA 1.2, Olympus) and was then tightly focused onto samples. The excited SHG signals were backward collected by the same objective and imaged onto a sCMOS camera (ORCA Flash 4.0 LT, Hamamatsu) point by point. The image magnification of this system is $116 \mathrm{X}$ and one pixel of the sCMOS camera corresponds to $55.7 \times 55.7 \mathrm{~nm}^{2}$ on the sample. A motorized-stage with step resolution of $0.2 \mu \mathrm{m}$ moves the sample along $\mathrm{z}$-axis so that $3 \mathrm{D}$ image can be obtained.

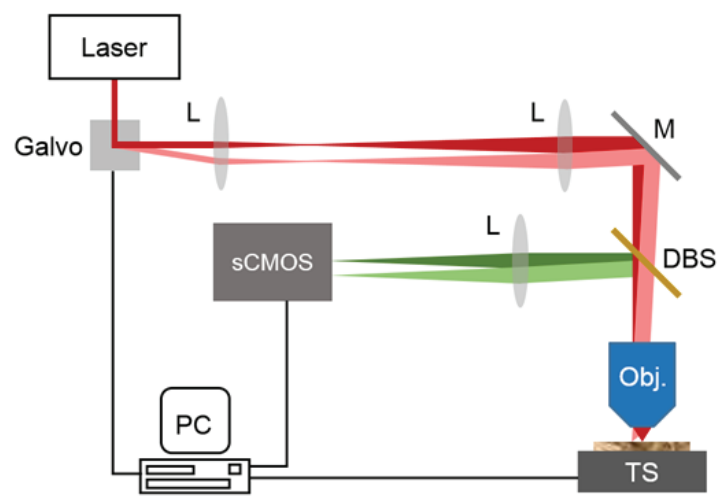

Figure 2. The experiment setup of the FS-SHGM.

\section{Results and Discussions}

To demonstrate the resolution improvement, unsectioned chicken tendon without staining was utilized as sample. After excised from chicken wings, the chicken tendon was immersed in the phosphate-buffered saline (PBS). The excitation power at the sample is $30 \mathrm{~mW}$. Fig. 3(a) and 3(b) are the C-SHGM images of the chicken tendon aligned along $\mathrm{x}$ - and $\mathrm{y}$-direction, respectively. Fig $3(c)$ and $3(d)$ are the FS-SHGM images corresponding to Fig. 3(a) and 3(b). For each FS-SHGM image, 6 patterned images ( 3 with $\mathrm{x}$-direction patterns and 3 with y-direction patterns) were acquired. The period of the scanning pattern is $1030 \mathrm{~nm}$ and the duty cycle is 0.3 . To get a patterned image with SNR higher than 3, the exposure time for one patterned image is 1.9 s. Fig. 3(e) shows the normalized intensity profiles along the yellow lines in the enlarged boxed regions in Fig. 3(a) and 3(c), while Fig. 3(f) shows the normalized intensity profiles in Fig. 3(b) and 3(d). After applying SIM, the contrast of the FSSHGM images are apparently improved in contrast to the C-SHGM images. By fitting these curves with Gaussian functions, the lateral resolution of C-SHGM and FSSHGM can be determined. The full width at half maximum (FWHM) of the fitting curves in Fig. 3(e) were measured as $345 \mathrm{~nm}$ for C-SHGM and $249 \mathrm{~nm}$ for FSSHGM. The FWHM of the fitting curves in Fig. 3(f) were measured as $329 \mathrm{~nm}$ for C-SHGM and $231 \mathrm{~nm}$ for FSSHGM. The resolution improving factors in Fig. 3(e) and 3(f) are 1.39 and 1.42, respectively. However, a lower 
improving factor of 1.21 was expected theoretically. The difference may be resulted from the sample thickness. In simulation, the sample was assumed to be thinner than the axial resolution, while in experiment, the sample was much thicker. Using the sCMOS camera as detector, SHG signals scattered by the surrounding tissues will result in background noises in the C-SHGM image. Which will reduce the effective lateral resolution of the C-SHGM image. During the SIM reconstruction process, the background noises in the C-SHGM can be removed along with the lateral resolution improvement. With the aid of the increased contrast, the efficient resolution improving factor was thus measured higher than the theoretical value.

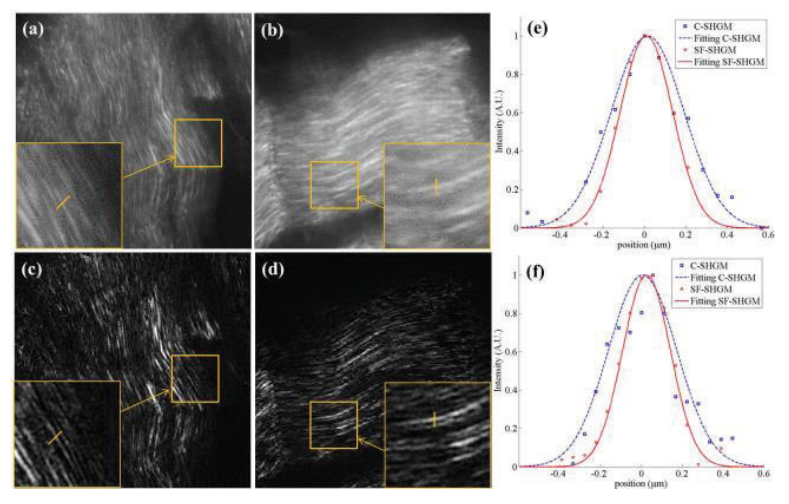

Figure 3. (a) and (b) are the C-SHGM images of the chicken tendon aligned along $\mathrm{x}$ - and $\mathrm{y}$-direction, respectively; (c) and (d) are the FS-SHGM images corresponding to (a) and (b); (e) the normalized intensity profiles along the yellow lines in (a) and (c); (f) the normalized intensity profiles in (b) and (d).

\section{References}

[1]. P. J. Campagnola, M. Wei, A. Lewis, and L. M. Loew," High-resolution nonlinear optical imaging of live cells by second-harmonic generation.” Biophys J, vol. 6, pp. 3341-3340, (1999).

[2].R. Gauderon, P. B. Lukins, and C. J. R. Sheppard, "Three-dimensional second-harmonic generation imaging with femtosecond laser pulses." Opt. Lett., vol. 23, pp. 1209-1211, (1998).

[3].N. Tian, L. Fu and M. Gu, "Resolution and contrast enhancement of subtractive second harmonic generation microscopy with a circularly polarized vortex beam," Sci. Rep., vol. 5, pp. 13580, (2015).
[4].J. Liu, I. H. Cho, Y. Cui and J. Irudayraj, "Second harmonic super-resolution microscopy for quantification of mRNA at single copy sensitivity," ACS Nano., vol. 8, pp. 12418-12427, (2014).

[5]. M. G. L. Gustafsson, "Surpassing the lateral resolution limit by a factor of two using structured illumination microscopy," J. Micro., vol. 198, pp. 8287, (2003).

[6]. C. H. Yeh, and S, Y. Chen, "Resolution enhancement of two-photon microscopy via intensity-modulated laser scanning structured illumination” Appl. Opt., vol. 54, pp. 2309-2317, (2015).

[7].Y. R. Shen, "Surface properties probed by secondharmonic and sum-frequency generation," Nature 337, 519-525 (1989).

[8]. C. H. Yeh, and S, Y. Chen, "Resolution enhancement of two-photon microscopy via intensity-modulated laser scanning structured illumination" Appl. Opt. 54, 2309-2317 (2015). 


\title{
Maximizing illumination contrast in arbitrary direction for Structured Illumination Microscopy
}

\author{
Guoxuan Liu ${ }^{\mathrm{a}}$, Huaidong Yang*a, Sichun Zhang ${ }^{\mathrm{b}}$, Xinrong Zhang ${ }^{\mathrm{b}}$, Guofan Jin ${ }^{\mathrm{a}}$ \\ ${ }^{a}$ State Key Laboratory of Precision Measurement Technology and Instruments, Tsinghua University, \\ Haidian District, Beijing, 100084, China \\ ${ }^{b}$ Department of Chemistry, Beijing Key Laboratory for Microanalytical Methods and \\ Instrumentation, Tsinghua University, Haidian District, Beijing, 100084, China
}

\begin{abstract}
In structured illumination microscopy (SIM), polarization direction is crucial to obtain high contrast of illumination pattern, guaranteeing the performance of SIM in noisy situation. We propose a polarization-state controlling method using vortex half-wave plate (VHWP), a special half-wave plate of which the fast axis rotates continuously. With proper linearpolarized incident light, VWHP produces azimuthal polarized emergent light, maximizing the contrast of SIM illumination patterns. Our proposed method works in arbitrary illumination direction, eliminating the constrain of fixed illumination directions of former method. Thus, multiple SIM working modes, such as linear and non-linear SIM, can be performed on the same setup, increasing the versatility of SIM.
\end{abstract}

Keywords: superresolution, structured illumination microscopy

\section{Introduction}

Structured illumination microscopy (SIM) is a highly concerned super-resolution microscopies owning to its fast imaging specialty and potential in live cell imaging[1-4]. SIM requires periodic pattern illumination, mostly sinusoidal stripes, to mixed normally inaccessible information into raw data and recover them to obtain a super-resolution image. In practical implementation, the contrast of sinusoidal stripes should be as high as possible to prevent the modulated super-resolution covered by the noise. Thus, the polarization direction of illumination light should be controlled.

At the beginning, a polarizer is used to determine a linear polarization direction in which SIM illumination pattern in three directions share nearly equal, but not maximized, contrast[5]. Then, a polarization rotator consist of liquid crystal retarder and quarter-wave plate is placed at the spectrum plane of SIM illuminator to rotate the linear polarization direction[1-4]. However, such rotator complicates the SIM setup and the controlling process. After that, a segmented polarizer was applied for simplification[4,6]. Although the segmented polarizer rotates the polarization direction passively, greatly simplifying the SIM setup and the controlling process, it requires fixed configuration of illumination directions, only in several fixed directions. Flexibly adjustment of illumination direction is seriously constrained.

Here we propose a polarization-state controlling method in SIM using vortex half-wave plate (VHWP). The VHWP is a special half-wave plate of which the fast axis rotates continuously over the optical surface. With proper linear-polarized incident light, VWHP produces azimuthal polarized emergent light. Such azimuthal polarized light maximizes the contrast of SIM illumination patterns in arbitrary direction[5]. With the help of our proposed method, it is possible to perform multiple imaging modes that require different configurations of illumination directions, such as linear[7] and non-linear SIM[8], on the same setup. The versatility of SIM is greatly increased.

\section{Method}

\section{Optical path configuration}

In SIM setup, the illumination light is generally modulated by the spatial light modulator (SLM) and then filtered by a spatial filter at the spectrum plane. The VHWP is placed at the spectrum plane, right close to the spatial filter, to turn the polarization-state of the illumination light into azimuthal polarization, maximizing the contrast of the SIM illumination patterns formed by 2beams interference in arbitrary direction. 


\section{Polarization-state controlling}

The detail process of polarization-state controlling at the spectrum plane is shown in Figure 2.

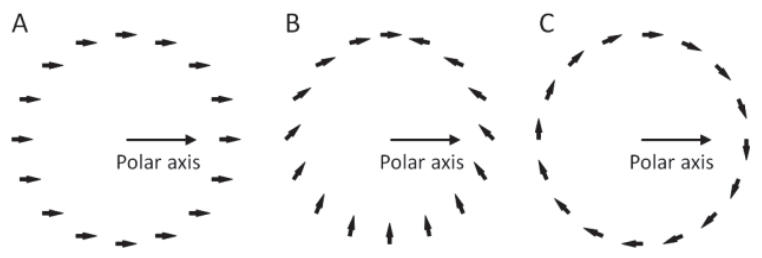

Figure 1. Detail process of polarization-state controlling at spectrum plane using VHWP. (A)

Polarization direction of the induced light. (B) Fast axis directions of VHWP. (C) Polarization direction after controlled by VHWP.

After being spatial filtered, only \pm 1 order of the SLM pattern spectrum are left, focused on spectrum plane and distributed symmetrically on a circle. Assume the filtered light is polarized along the polar axis (see Figure 1A). The VHWP is mounted where direction of fast axis is (see Figure 1B):

$\phi=\frac{1}{2}\left(\theta-\frac{\pi}{2}\right)$

where $\theta$ is the azimuthal angle. The polarization directions of incident and emergent light of VHWP are symmetrical about the fast axis. Thus, we can obtain the polarization distribution of emergent light (see Figure 1C):

$\varphi=2 \phi=\theta-\frac{\pi}{2}$

which is exactly azimuthally polarized.

\section{Results and Discussion}

We performed a Matlab simulation of SIM imaging process (Figure. 2), with different contrasts of illumination patterns and signal-to-noise ratios of raw images, correspondingly. The simulation result indicated that the low contrast of the illumination patterns might result in serious artifact and decreased resolution during noisy SIM imaging (Figure. 2C). Thus, it is necessary to keep high contrast of SIM illumination patterns.
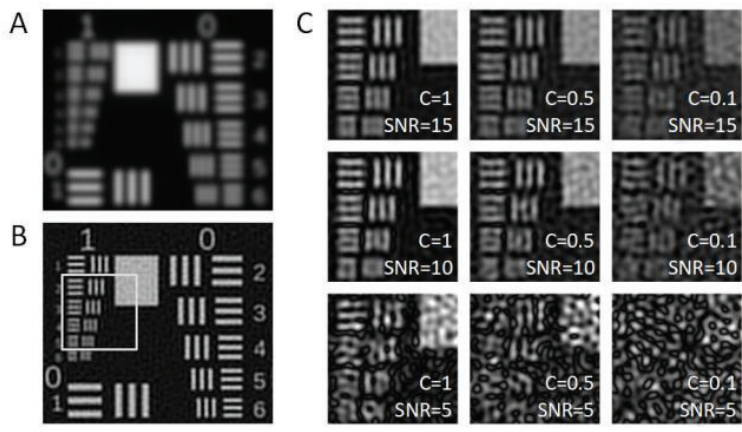

Figure 2. Simulation results of SIM. (A) Wide-field image. (B) Super-resolution SIM image. (C) Enlarged ROI of SIM images, with different contrast, C, of illumination patterns and signal-to-noise ratios, SNR, of raw images.

Applying VHWP for polarization-state controlling, the contrast of illumination patterns is maximized in arbitrary direction. A theoretical contrast curve of SIM pattern was demonstrated in Figure 3A. In comparison, the SIM pattern contrasts of both methods using VHWP and segmented polarizer (equivalently segmented into six sectors) for polarization-state controlling were studied.

The VHWP-controlled contrast curve remained 1, being maximized, in all directions while the segmentedpolarizer-controlled contrast curve periodically decayed, down to nearly 0.5 at the edge of the sectors. One thing to be mentioned is that the application of both VHWP and segmented polarizer are wavelength depended, they only work in single wavelength situation.
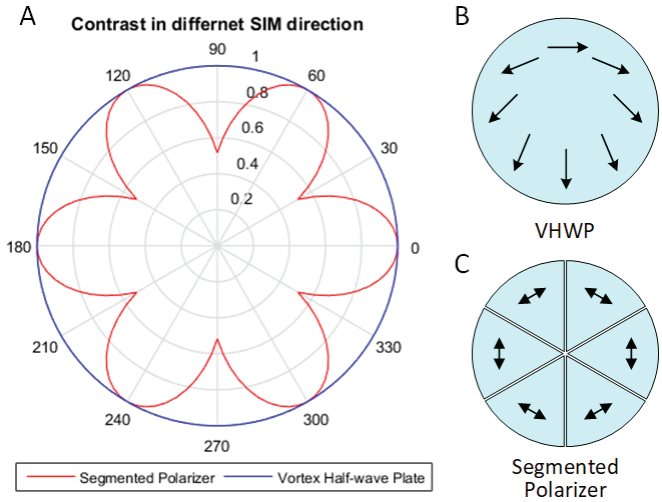

Figure 3. (A) Theoretical contrast curve of SIM pattern in all illumination directions. (B) Vortex halfwave plate (VHWP). The arrows indicate the 
directions of fast axis. (C) Segmented Polarizer. The arrows indicate the polarization directions.

In summary, our proposed method is capable of maximizing the illumination contrast of SIM in arbitrary direction, eliminating the constrain of the fixed configuration of illumination directions in former method. Such progress make it possible to perform multiple SIM working modes that require different configurations of illumination directions, such as linear (three illumination directions[7]) and non-linear SIM (five illumination directions[3,8]), utilizing the same SIM setup, increasing the versatility of the instrument.

\section{References}

[1] P. Kner, B. B. Chhun, E. R. Griffis, L. Winoto, and M. G. Gustafsson, "Super-resolution video microscopy of live cells by structured illumination," Nat Methods 6, 339-342 (2009).

[2] L. Shao, P. Kner, E. H. Rego, and M. G. Gustafsson, "Super-resolution 3D microscopy of live whole cells using structured illumination," Nat Methods 8, 10441046 (2011).James, D. 1980. United States fruit and vegetable harvest projections _ 1990. USDA-1007. Washington, D.C.: GPO.

[3] D. Li, L. Shao, B. C. Chen, X. Zhang, M. Zhang, B. Moses, D. E. Milkie, J. R. Beach, J. A. Hammer, 3rd, M. Pasham, T. Kirchhausen, M. A. Baird, M. W. Davidson, P. Xu, and E. Betzig, "ADVANCED IMAGING. Extended-resolution structured illumination imaging of endocytic and cytoskeletal dynamics," Science 349, aab3500 (2015).U.S. House Committee on Conservation Needs and Opportunities. 1986. Soil conservation: Assessing the national resource inventory, vol. 1. Washington, D.C.: National Academy Press.

[4] X. Huang, J. Fan, L. Li, H. Liu, R. Wu, Y. Wu, L. Wei, H. Mao, A. Lal, P. Xi, L. Tang, Y. Zhang, Y. Liu, S. Tan, and L. Chen, "Fast, long-term, super-resolution imaging with Hessian structured illumination microscopy," Nature Biotechnology (2018).

[5] K. O’Holleran, and M. Shaw, "Polarization effects on contrast in structured illumination microscopy," Optics Letters 37, 4603-4605 (2012).

[6] H. W. Luwalther, M. Kielhorn, R. Förster, A. Jost, K. Wicker, and R. Heintzmann, "fastSIM: a practical implementation of fast structured illumination microscopy," Methods \& Applications in Fluorescence 3, 014001 (2015).

[7] M. G. L. Gustafsson, "Surpassing the lateral resolution limit by a factor of two using structured illumination microscopy," J. Microsc.-Oxf. 198, 82-87 (2000).

[8] M. G. Gustafsson, "Nonlinear structured-illumination microscopy: wide-field fluorescence imaging with theoretically unlimited resolution," Proc Natl Acad Sci U S A 102, 13081-13086 (2005). 


\title{
Dual-channel structured illumination microscopy setup based on Digital Micromirror Device
}

\author{
Wenzhen Xiong, Guoxuan Liu, Huaidong Yang \\ State Key Laboratory of Precision Measurement Technology and Instruments, Tsinghua University, \\ Beijing, 10084, China
}

\begin{abstract}
Structured illumination microscopy (SIM) possesses higher imaging speed among SR imaging technologies, beneficial to living cell research. With the increase of application requirement, SIM has met challenges in multi-color imaging. The current multi-color SIM imaging is generally time-lapse imaging, making it unsuitable for living cell observation. Thus, we proposed a dual-channel SIM setup based on Digital Micromirror Device (DMD). DMD enables the same modulation of excitation lights simultaneously, and opto-splitter achieves dual-channel images collection simultaneously. We verified our dual-color SIM setup in experiment and its imaging speed is no longer limited by the wavelength. Compared to other dual-color SIM imaging methods, our method doubles the multi-color imaging speed, making it prospective to fast, multicolor SR imaging in the future study of living cells.
\end{abstract}

Keywords: SIM, multi-color imaging, dual channel

\section{Introduction}

SR imaging technology has become a significant method in the study of life science recently. SIM possesses higher imaging speed among SR imaging technologies, beneficial to living cell research. With the increase of application requirements, SIM has met opportunities and challenges in multi-color imaging. Compared with conventional monochromatic imaging, multi-color imaging not only has the ability to mark the morphology of different cell structures, but also provides the life process for the transportation and interaction of multiple substances inside cells, which is of great significance to life science research. Consequently, the multi-color SIM imaging is one of the important research fields of current SR imaging technologies.

The current multi-color SIM imaging is generally timelapse imaging whose multi-color imaging speed would reduce with the increase number of wavelengths, making it unsuitable for living cell observation. If we could get multi-color acquisition one time, the imaging speed of multi-color SIM would increase significantly. To achieve that, two issues have to be solved. The first is multi-color structured illumination excitation simultaneously. The current methods in the modulation of illumination generally are spatial light modulation (SLM). However, SLM is the wavelength dependent device, so the pattern corresponding to a specific wavelength to achieve its maximum diffraction efficiency always cannot attain the same diffraction efficiency of another wavelength, which would affect the modulation depth of structured illumination pattern. The second is multi-color image acquisition simultaneously. The original image of SIM is dense wide-field image, making it impossible to disperse the original image data by utilizing dispersion object, such as prism, directly like STORM and STED.

In order to solve the challenge that it is difficult to achieve multi-color excitation simultaneously and multicolor acquisition simultaneously, we proposed a dualchannel SIM setup based on DMD modulation. DMD enables the same modulation of two excitation lights simultaneously, and opto-splitter achieves dual-channel images collection simultaneously. We verified our dualcolor SIM setup in experiment and its imaging speed is no longer limited by the wavelength. Compared to other dual-color SIM imaging methods, our method doubles the multi-color imaging speed, making it prospective to fast, multi-color SR imaging in living cells study.

\section{Method}

We have built a dual-channel SIM setup based on DMD modulation, as depicted in Fig. 1. The excitation laser of $488 \mathrm{~nm}$ and $561 \mathrm{~nm}$ is used as light source in 
system. After passing through single mode fiber, beam collimator and expander separately, the two laser beams achieve mode cleaning and beam expansion, and finally have obliquely incidence on DMD surface respectively.

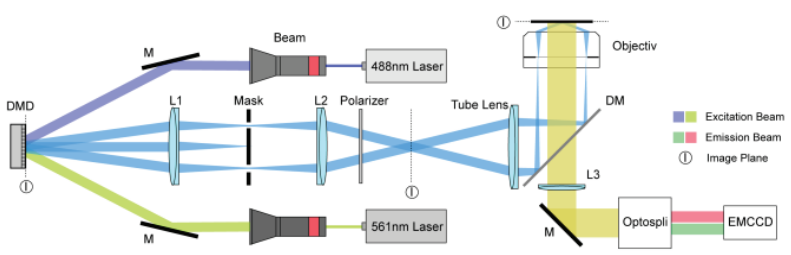

Fig. 1. Schematic diagram of the dual-channel SIM setup

DMD consists of several hundred thousand microscopic mirrors (pixel size: $10.8 \mathrm{um}$ ) arranged in a rectangular array corresponding to the pixels in the displayed image. The micromirror can be individually rotated $\pm 12^{\circ}$ to achieve light control. Setting the micromirror array a fixed periodicity enables DMD become an amplitude blazed grating, with constant line spacing $129.6 \mathrm{um}$ and blaze angle $12^{\circ}$. In multiwavelength modulation, SLM is the phase grating, thus a pattern corresponding to a wavelength's maximum diffraction efficiency always cannot achieve the same diffraction efficiency at another wavelength. DMD is the amplitude grating independent of wavelength, making it possible to achieve the same diffraction efficiency among different wavelengths. Since the pattern displayed on DMD is structured illumination pattern, the DMD needs to be placed in the image plane where its working plane is conjugated to the sample plane.

$$
d(\sin \alpha+\sin \beta)=m \lambda
$$

This is the grating equation, where $\mathrm{d}$ is the line spacing, $\alpha$ and $\beta$ is incidence angle and diffraction angle respectively, and $\mathrm{m}$ is diffraction order. To get diffraction direction vertical to DMD's working plane, that is $\beta$ equals to zero, the incidence angle of $488 \mathrm{~nm}$ and $561 \mathrm{~nm}$ is adjusted to $24^{\circ}$. Considering high diffraction order makes diffraction angle sensitive to changes in wavelength, the incident lights of $488 \mathrm{~nm}$ and $561 \mathrm{~nm}$ have to illuminate DMD surface separately from two sides, as shown in Fig. 1. At the vertical diffraction direction, it is not necessary to exist diffraction order for the diffraction intensity distribution vary from wavelengths to wavelengths. Thus, fine tune the incidence angle in both sides to satisfy that a diffraction order, which would be used for subsequent illumination, is exactly vertical to DMD's working plane.

Two excitation lights separately illuminate the preset periodical pattern loaded on DMD at its fine-tuning incidence angle and the pattern here is equivalent to two complementary gratings for these two incident lights illuminating from two sides. Diffracted by DMD, the illumination light produces several diffraction orders on the back focal plane (BFP) of L1 ( $\mathrm{f}=150 \mathrm{~mm}$, Thorlabs). In order to make the illumination light interfere at the sample plane, \pm 1 diffraction orders are kept, and other unwanted orders are blocked by a mask. It is a customized metal sheet with four holes at the positions of the desired first diffraction orders, placed on the BFP of L1. This mask filter is efficient for it saves efforts and time compared to the rotating slit.

After passing through L2 ( $\mathrm{f}=150 \mathrm{~mm}$, Thorlabs), the illumination light becomes two collimated lights with a certain angle. We referred to the scheme that place a polarizer behind the L2 to control the polarization of illumination light. By rotating the polarizer in the appropriate position, the modulation depth of interference fringe in different directions could almost be the same, attaining better SR image.

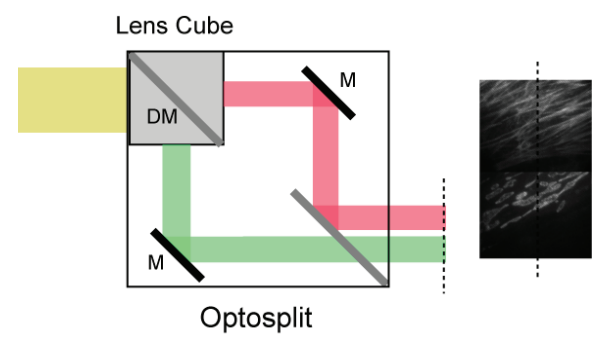

Fig. 2. Schematic diagram of opto-splitter

L2 and the illumination tube lens ( $\mathrm{f}=400 \mathrm{~mm}$, Thorlabs) image the spatial frequency plane into the BFP of the objective. The objective ( $\times 100 / 1.49$ oil, Zeiss $)$ generates two-beam interference, producing sinusoidal structured illumination pattern at the sample plane. In the experiment, the sample is multi-labeled fluorescent 
sample (FluoCell, Prepared Slide \#1, Thermo Fisher) and two fluorescent dyes are excited. One (Alexa Fluor 488) labeled the cell tubules and the other (Mito Tracker Red) labeled the mitochondria. The emitted fluorescent light collected by the same objective is imaged by the optosplitter (Cairn) onto the EMCCD (iXon Ultra 888, Andor). The opto-splitter mainly consists of a dichroic mirror (DM) (LP, 567nm, Thorlabs), a short pass filter (550nm, Thorlabs), and a band pass filter $(600 \mathrm{~nm} \pm 40 \mathrm{~nm}$, Thorlabs), Fig. 2. The emitted fluorescent light is split into two fluorescent signals by opto-splitter, attaining two images at different positions of EMCCD. Low background noise of EMCCD can improve the imaging quality. Fine adjustment of opto-splitter could adjust the size and distance of two images, achieving dual channel detection simultaneous.

\section{Image Registration}

Since two fluorescent signals is imaged at different positions of detection, the two images need to know their exact relative position before combined into one image. To get the exact relative position of the two images, image registration is necessary.

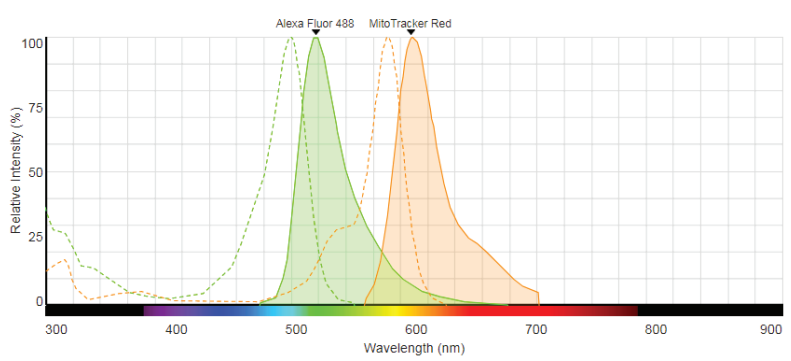

Fig. 3. The fluorescent spectrogram of Alexa Fluor 488 and Mito Tracker Red

Considering that the sample is prepared slide, we could not add additional fluorescent microsphere as reference signal. From the fluorescent spectrogram of sample in Fig. 3 from Thermo Fisher, the emitted fluorescent light, under the excitation light of $488 \mathrm{~nm}$ only, can pass through the $\mathrm{DM}$ and be divided into a strong optical signal and a relatively weak optical signal. Therefore, we took out the band pass filter in opto-splitter and kept the other experimental conditions unchanged. Under the excitation light of $488 \mathrm{~nm}$, the tubules of sample was imaged onto the detection plane of EMCCD with a strong signal channel and a weak signal channel. Attain two images from channels and utilize the autocorrelation algorithm to get the translation vector for image registration. Verify the translation vector in other imaging field of sample and finally the vector could be used in subsequent experimental.

\section{Result}

Under the excitation light of $488 \mathrm{~nm}$ and $561 \mathrm{~nm}$, we simultaneously imaged cell tubules and mitochondria and acquired with one camera simultaneously in the experiment. Six dual-channel original images with three different phases in each direction were attained. Due to the different fluorescence efficiency of two fluorescent dyes, we repeatedly adjusted the intensity of two illumination lights separately to ensure that the intensity of dual-channel images were close in the same exposure time. The excitation power in the experiment is generally lower than $12 \mathrm{w} / \mathrm{cm}^{2}$, greatly reducing the photodamage to the biological sample.

After the segmentation, cropping of the dual-channel original images, six component images of each channel were acquired. The wide-field deconvolution images and SR images of cell tubules and mitochondria were attained by utilizing the reconstruction algorithm. Fig. 4 and Fig. 5 illustrates the experiment results. 

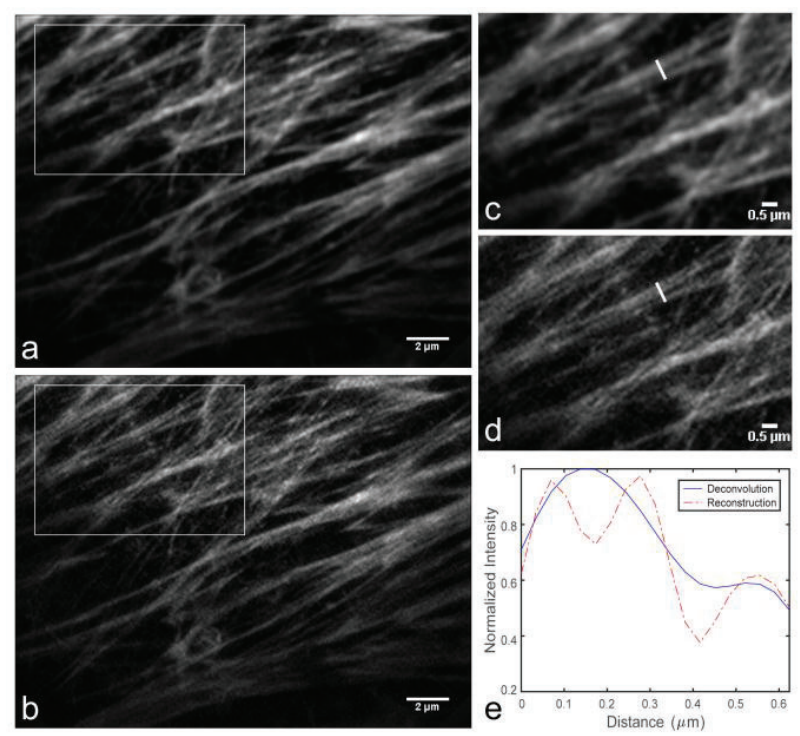

Fig. 4. The experiment result of tubules in single channel
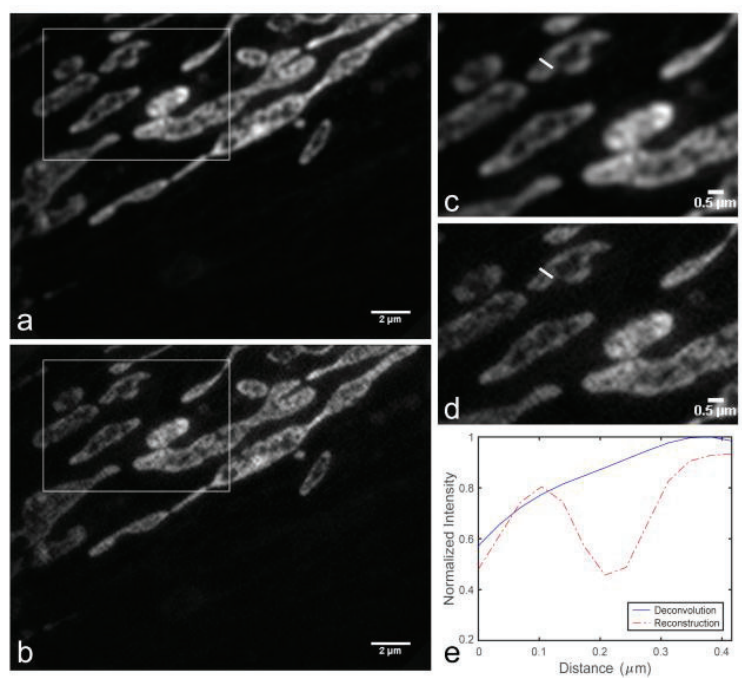

Fig. 5. The experiment result of mitochondria in single channel

Superimposing the two SR images of cell tubules and mitochondria by translation vector, we acquired dualchannel grayscale image and pseudo color image, correspondingly, its extended spatial frequency domain in reciprocal space, shown in Fig. 6.

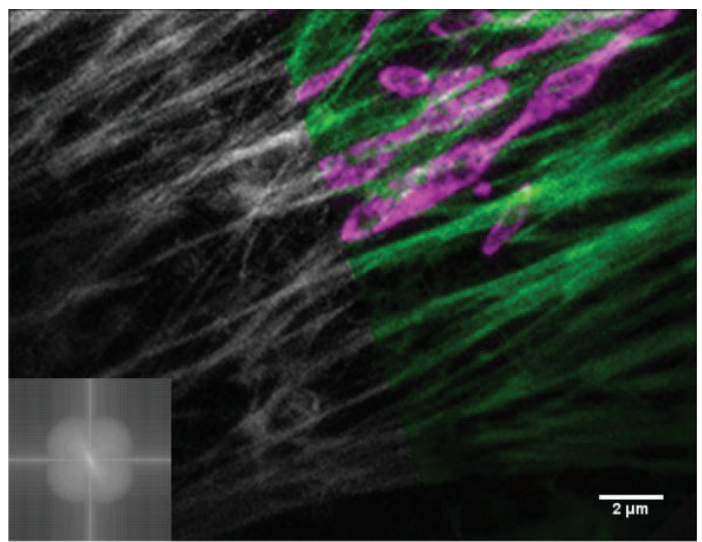

Fig. 6. The experiment result of dual channels

\section{Conclusion}

We have proposed a dual-channel SIM setup based on DMD modulation. DMD enables the same modulation of excitation lights simultaneously, and opto-splitter achieves dual-channel images collection simultaneously. We experimentally verified our dual-color SIM setup and attained dual-color SR image. Importantly, the imaging speed of our setup is no longer limited by the wavelength. Compared to conventional dual-color SIM imaging methods, our method doubles the multi-color imaging speed, making it prospective to fast, multi-color SR imaging in the future study of living cells.

\section{Reference}

Gustafsson, M. G. "Surpassing the lateral resolution limit by a factor of two using structured illumination microscopy". Journal of Microscopy, 198(2), 82-87 (2000).

Li, D., Shao, L., Chen, B., Zhang, X., Zhang, M., Moses, B., ... \& Betzig, E. "Extended-resolution structured illumination imaging of endocytic and cytoskeletal dynamics". Science, 349(6251) (2015).

Huang, X., Fan, J., Li, L., Liu, H., Wu, R., Wu, Y., ... \& Chen, L. "Fast, long-term, super-resolution imaging with Hessian structured illumination microscopy". Nature Biotechnology, 36(5), 451-459 (2018).

Brunstein, M., Wicker, K., Herault, K., Heintzmann, R., \& Oheim, M. "Full-field dual-color 100-nm super- 
resolution imaging reveals organization and dynamics of mitochondrial and ER networks". Optics Express, 21(22), 26162-26173 (2013).

Forster, R., Luwalther, H., Jost, A., Kielhorn, M., Wicker, K., \& Heintzmann, R. "Simple structured illumination microscope setup with high acquisition speed by using a spatial light modulator". Optics Express, 22(17), 20663-20677 (2014).

Oholleran, K., \& Shaw, M. "Polarization effects on contrast in structured illumination microscopy". Optics Letters, 37(22), 4603-4605 (2012).

Lal, A., Shan, C., Zhao, K., Liu, W., Huang, X., Zong, W., ... \& Xi, P. "A Frequency Domain SIM Reconstruction Algorithm Using Reduced Number of Images". IEEE Transactions on Image Processing, 27(9), 4555-4570 (2018).

Dan, D., Lei, M., Yao, B., Wang, W., Winterhalder, M., Zumbusch, A., ... \& Zhao, W. "DMD-based LEDillumination Super-resolution and optical sectioning microscopy". Scientific Reports, 3(1), 1116-1116 (2013). 


\title{
Simulating compressive three-dimensional structured illumination microscopy
}

\author{
Baturay Özgürün ${ }^{\mathrm{a}, \mathrm{b}}$, Müjdat Çetin ${ }^{\mathrm{b}, \mathrm{c}}$ \\ ${ }^{a}$ School of Engineering and Natural Sciences, Istanbul Medipol University, Istanbul, Turkey \\ ${ }^{b}$ Faculty of Engineering and Natural Sciences, Sabancı University, Istanbul, Turkey \\ ${ }^{c}$ Department of Electrical and Computer Engineering, University of Rochester, Rochester, NY, USA
}

\begin{abstract}
Three-dimensional structured illumination microscopy (3D-SIM) requires at least fifteen raw images to extend the spatial resolution to the half of the diffraction limit in both axial and lateral directions. This requirement can cause motion artifacts in a reconstructed super-resolution image. Therefore, reducing the acquisition duration of raw images is of interest. Here, we propose a novel method, which applies compressed sensing (CS) to 3D-SIM and enables faster raw image acquisitions. CS allows us to record a scene with a photomultiplier tube, which is much faster than a camera. Furthermore CS can recover the scene from a small number of measurements. The combination of these two facts leads to significant reduction of the overall acquisition time. In preliminary experiments, a computer-generated ground truth image is utilized to simulate the proposed method, and the spatial resolution of an image produced by the proposed method is compared with conventional optical microscopy and 3D-SIM.
\end{abstract}

Keywords: compressive sensing, super resolution, three-dimensional, structured illumination microscopy

\section{Introduction}

An optical microscope is widely used tool in biology laboratories. It enables scientists to examine biological specimens. However, diffraction limits the spatial resolution, and peak-to-peak distance of two points in the specimen can be optically resolved up to $200 \mathrm{~nm}$. This prevents the scientists from observing tiny objects such as most viruses and molecules. Super-resolution microscopy techniques surpass the diffraction limit, and hence finer structures of the specimen can be observed. Threedimensional structured illumination microscopy (3D-SIM) is one of the super-resolution microscopy techniques. It excites the sample with three-dimensional illumination pattern. This down-modulates the higher frequency information of the sample into the optical transfer function (OTF) of the microscope objective. Exciting the sample with several combinations of the illumination pattern and utilizing a dedicated reconstruction algorithm, the spatial resolution can be extended to the half of the diffraction limit in both axial and lateral directions ${ }^{1}$. Moreover, $3 \mathrm{D}$-SIM solves the out-of-focus light problem because three-dimensional illumination pattern modulates the OTF in such a way that the missing cone of the OTF is covered.

Although 3D-SIM extends the spatial resolution to half of the diffraction limit and it eliminates out-of-focus light problem, this technique requires at least fifteen raw images to acquire a single super-resolution image. This requirement can cause motion artifacts in the reconstructed image. Moreover, the specimen is exposed to excitation light over a long period of time, which can lead to photobleaching ${ }^{2}$. Therefore, it is desirable to reduce the raw image acquisition duration. In this study, we propose a novel method, which extends the compressed sensing (CS) framework to 3DSIM, to record raw images faster. CS can be good candidate to eliminate motion artifacts in the super-resolution image and photobleaching, since CS enables us to record a scene with a photomultiplier tube (PMT), which is much faster than a camera, and CS recovers the scene with several measurements. The cost of this method is that scene has to be recovered by a non-linear recovery algorithm, which is time consuming. In other words, the raw images cannot be reconstructed within a second. Parallel computing methods can overcome this computational cost but this is out of scope of this study. Here, we simulate the proposed method using a computer-generated ground truth image, and we compare the result with those of conventional optical microscopy and 3D-SIM.

\section{Application of CS to 3D-SIM}

To the best of our knowledge, there is only one study ${ }^{3}$ that applies the CS framework to SIM in the literature. In this study, under-sampling is performed by a camera in the 
output port of the microscope, and hence the sampling pattern does not suffer from diffraction. Moreover, that study involves only two-dimensional structured illumination microscopy. We propose that the sampling and downmodulation of an object scene are simultaneously performed with a single digital micromirror device (DMD), and PMT is utilized for recording the scene. In addition, the study proposed here is performed for 3D-SIM. DMD is useful device to display three-dimensional sinusoidal and random sampling patterns at the same time, but diffraction of the sampling pattern should be considered in the recovery algorithm. Our proposed approach is demonstrated with simulations but the preliminary results provide a proof-ofprinciple that encourages us to design an experiment to test our approach on real data.

All simulations are performed in MATLAB. In the simulation, a three-dimensional sparse scene is generated, and then the scene is simultaneously exposed to threedimensional sinusoidal illumination pattern and random sampling. An approach from previous work ${ }^{4}$ is utilized to generate the intensity distribution of the illumination pattern, and the zeros and ones are used to construct the sampling pattern. After interaction of the illumination and the sampling patterns with the scene, diffraction is performed using a three-dimensional point spread function (PSF). Eventually, the diffracted product is collected by a PMT or single photodiode. This provides a single measurement. To acquire the second measurement, a new sampling pattern, whose weights are randomly changed, and the same illumination pattern are utilized. Once the measurement vector is generated, NESTA recovery algorithm $^{5}$ is used to recover a raw SIM image. Changing the illumination pattern parameters and using the sampling pattern sequences lead to acquisition of another raw SIM image. After all raw images are collected, a 3D-SIM reconstruction algorithm such as fairSIM ${ }^{6}$ is utilized to acquire a super-resolution 3D-SIM image.

\section{Experimental Results}

Here, we utilize a computer-generated and sparse fluorescent microspheres as a ground truth image. The diameter of each microsphere is $50 \mathrm{~nm}$. In Figure 1a, the ground truth image on the focal plane is illustrated. This image is diffracted with a three-dimensional PSF, whose parameters are adjusted with 1.4 NA and $560-\mathrm{nm}$ emission light, to obtain a conventional optical microscope image (Figure 1b). In the optical microscope, the out-of-focus problem and low resolution are observed. The ground truth image is used to simulate the 3D-SIM technique (Figure 1c). It can be clearly observed that the 3D-SIM method substantially alleviates the out-of-focus light problem and enhance the spatial resolution. Eventually, the image produced by the proposed method, combines CS and 3DSIM, is presented in Figure 1d. This image is acquired with the sampling rate of 25 percent. In other words, the amount of data used the proposed method is four times lower than that used by the conventional method. Despite the data reduction, the spatial resolution of the image produced by the proposed method is in very good agreement with the ground truth image.

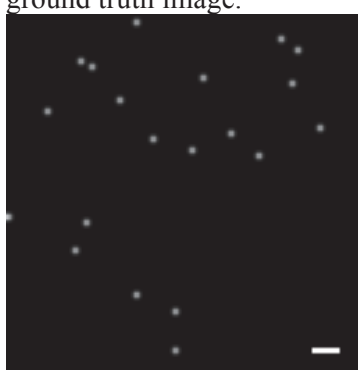

(a)

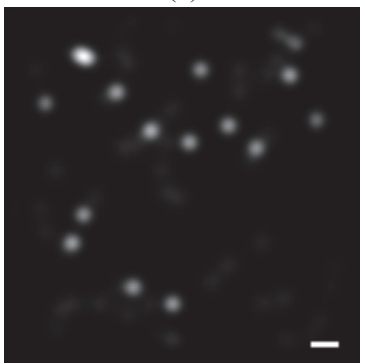

(c)

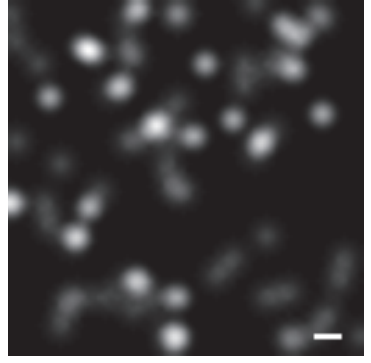

(b)

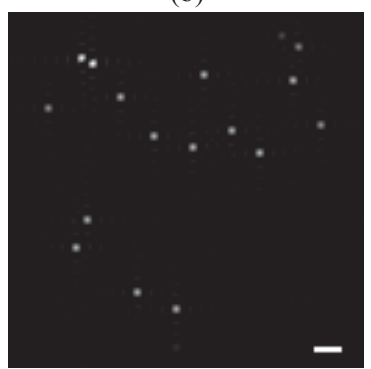

(d)
Figure 1. Experimental results on computergenerated, sparse fluorescent microspheres. (a) Focal plane ground truth image. (b) Conventional optical microscopy result. (c) Conventional 3DSIM result. (d) Result on the proposed CS-based 3D-SIM method. Scale bar is 500-nm.

\section{Conclusion}

Here, we proposed a novel approach, which combines compressed sensing and three-dimensional structured illumination microscopy. This study is motivated by the desire to eliminate motion artifacts in a three-dimensional structured illumination microscopy image. Results of the proposed approach have been demonstrated with simulations on computer-generated images. The simulation results provide a proof-of-principle that encourages us to design an experiment to test our approach on real data. 


\section{References}

[1] Gustafsson, M. G., Shao, L., Carlton, P. M., Wang, C. J., Golubovskaya, I. N., Cande, W. Z., Agard, D. A. and Sedat, J. W., "Three-dimensional resolution doubling in wide-field fluorescence microscopy by structured illumination," Biophys. J. 94(12), 4957-4970 (2008).

[2] Dobrucki, J. W., "Confocal microscopy: Quantitative analytical capabilities," Methods Cell Biol. 75, 41-72 (2004).

[3] Meiniel, W., Spinicelli, P., Angelini, E. D., Fragola, A., Loriette, V., Orieux, F., Sepulveda, E. and Olivo-Marin, J. C., "Reducing data acquisition for fast structured illumination microscopy using compressed sensing," IEEE Int. Symp. Biomedical Imaging, 32-35 (2017).

[4] Huang, H. C., Chang, B. J., Chou, L. J. and Chiang, S. Y., "Three-beam interference with circular polarization for structured illumination microscopy," Opt. Express 21(20), 23963-23977 (2013).

[5] Becker, S., Bobin, J. and Candes, E. J., "NESTA: A fast and accurate first-order method for sparse recovery," SIAM J. Imaging Sci. 4(1), 1-39 (2011).

[6] Müller, M., Mönkemöller, V., Hennig, S., Hübner, W. and Huser, T., "Open-source image reconstruction of superresolution structured illumination microscopy data in ImageJ," Nat. Comm. 21, (2016). 


\title{
Structured light imaging with selective spatial frequency filtering: A search for the optimal spatial frequencies to differentiate between benign and
}

\author{
malignant tissue subtypes
}

\author{
Samuel S. Streeter*a, Benjamin W. Maloney ${ }^{\mathrm{a}}$, David M. McClatchy ${ }^{\mathrm{a}}$, \\ Elizabeth J. Rizzo ${ }^{b}$, Wendy A. Wells ${ }^{\mathrm{b}}$, Keith D. Paulsen ${ }^{\mathrm{a}}$, Brian W. Pogue ${ }^{\mathrm{a}}$ \\ aThayer School of Engineering at Dartmouth, Dartmouth College, Hanover, USA, 03755 \\ ${ }^{b}$ Department of Pathology, Geisel School of Medicine at Dartmouth, Hanover, USA, 03755
}

\begin{abstract}
High spatial frequency (HSF) structured light imaging (SLI) is a wide field-of-view, non-destructive, rapid, costeffective optical imaging modality that provides enhanced sensitivity to surface tissue scatter and is being investigated as a tool for surface tissue characterization of breast tumors resected during breast conserving surgery (BCS). A recent clinical study involved imaging bread-loafed cut BCS tumor specimens using structured illumination at four wavelengths $(490,550,600,700 \mathrm{~nm})$ and four spatial frequencies $\left(0.00,0.15,0.61,1.37 \mathrm{~mm}^{-1}\right)$. Subsequent analysis focused on the highest spatial frequency $\left(1.37 \mathrm{~mm}^{-1}\right)$ and shortest wavelength $(490 \mathrm{~nm})$ illumination data, which exhibited the highest sensitivity to backscatter. Selective bandpass filtering in the spatial frequency domain revealed important textural features for differentiating pairs of tissue subtypes and indicated that the highest frequencies offer superior signal-to-noise ratios.
\end{abstract}

Keywords: structured light imaging, spatial frequency domain imaging, breast conserving surgery, texture analysis

\section{Introduction}

Breast conserving surgery (BCS) with radiation treatment is the current procedure of choice for early stage breast cancer. Unfortunately, $15-35 \%$ of BCS patients require a second surgery due to incomplete initial resection of cancerous tissue. ${ }^{1}$ Spatial frequency domain imaging, referred to here at structured light imaging (SLI), is an optical imaging modality that provides depth resolution within turbid media. ${ }^{2}$ High spatial frequency (HSF) SLI uses structured illumination that is finer than the length scale of diffuse photon propagation, leading to sub-diffusive photon propagation (i.e., spatial frequencies of at least $\sim 0.5$ $\mathrm{mm}^{-1}$ in human breast tissue). ${ }^{3,4}$ HSF SLI intrinsically confines photons to sub-millimeter depths into tissue, and the backscattered signal is dependent upon surface tissue morphology and ultrastructure. SLI is a wide field-of-view, non-destructive, and rapid imaging modality that requires relatively few cost-effective components (a light source, projector, and a camera).

Recent work by McClatchy et al. demonstrates that HSF SLI can characterize a range of benign and malignant surface tissues and suggests that HSF SLI could be useful for intraoperative BCS margin assessment. $^{3-5}$ The general workflow of SLI data

* Samuel.S.Streeter.TH@,Dartmouth.edu collection is illustrated in Figure 1.

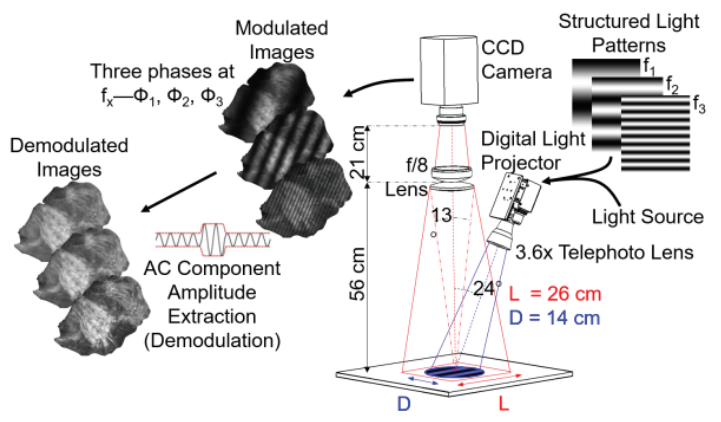

Figure 1. The general workflow of SLI data collection progresses from right to left. The schematic highlights the key SLI data collection components. ${ }^{5}$

SLI is commonly used to quantify optical properties, such as the reduced scattering coefficient, absorption coefficient, and phase function parameter. ${ }^{2-5}$ However, this study focused on the innate textural information visualized under HSF SLI that is undetectable by typical, planar illumination. Specifically, texture metrics quantified after selective bandpass filtering in the spatial frequency domain revealed the important spatial scales (and corresponding spatial frequencies) of features for differentiating pairs of tissue subtypes. Knowledge of these key diagnostic spatial scales (frequencies) may help guide the development of a next 
generation HSF SLI system for intraoperative BCS specimen margin assessment.

\section{Methods}

\section{Clinical Study Protocol and Dataset}

A recent clinical study at the Dartmouth Hitchcock Medical Center (DHMC) in Lebanon, New Hampshire, USA, involved collecting SLI data of freshly resected and bread-loafed specimens from BCS. The study was approved by the Committee for the Protection of Human Subjects, the Institutional Review Board at Dartmouth College and DHMC, and all procedures followed the approved protocol.

After standard-of-care histological processing and staining with hematoxylin and eosin, microscopic regions of interest (ROIs) were manually co-registered to SLI data. The ROIs analyzed here were conservatively outlined within the histopathologic ROIs. The specimens contained a total of 120 ROIs of confirmed and homogenous histological categories (69 benign and 51 malignant).

While the SLI dataset included many specimens and tissue subtypes, the work herein focused on a single, representative breast tissue specimen containing three distinct tissue subtypes (two benign, one malignant) to demonstrate the value of this type of bandpass filtering analysis.

\section{Imaging System and Data Collection Protocol}

The imaging system used in this study was previously developed and validated by McClatchy et $a l .{ }^{5}$ The system combines SLI and micro-computed tomography within the same light-tight, fully shielded Perkin Elmer In Vivo Imaging System (IVIS) SpectrumCT unit. SLI data were collected at four wavelengths $(490,550,600,700 \mathrm{~nm})$ and four spatial frequencies $\left(0.00,0.15,0.61,1.37 \mathrm{~mm}^{-1}\right)$.

The imaging routine for a single specimen across all wavelengths and spatial frequencies took approximately 5 minutes and is outlined in Figure 2. For the duration of the data collection phase of this study, the imaging system was housed in the DHMC pathology laboratory.

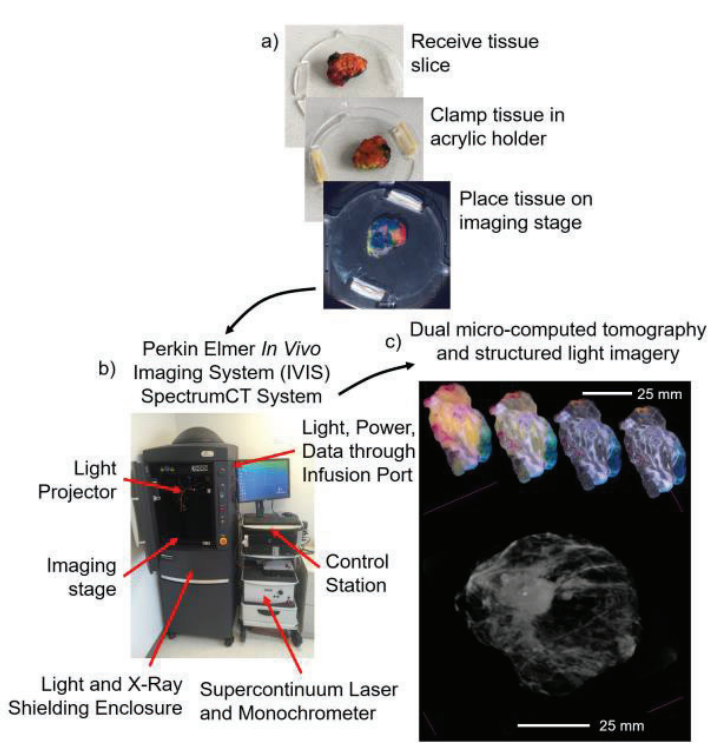

Figure 2. The overall workflow of data collection: a) a specimen is received in the DHMC pathology laboratory and placed in the imaging system; b) the key components of the previously developed and validated dual-modality imaging system; ${ }^{5}$ c) dual SLI (top images) and micro-computed tomography (bottom image) data were collected for every specimen.

\section{Texture Analysis}

The focus of this analysis was the highest spatial frequency $\left(1.37 \mathrm{~mm}^{-1}\right)$ and shortest wavelength (490 $\mathrm{nm}$ ) illumination imagery, because these settings exhibited the highest sensitivity to backscatter and provided the highest contrast to small-scale surface tissue textures. All images were converted to calibrated reflectance intensities using a previously outlined procedure $^{5}$ and were subsequently normalized to the range $[0,1]$ for comparing ROIs within and between specimens.

The $1.37 \mathrm{~mm}^{-1}, 490 \mathrm{~nm}$ images were selectively bandpass filtered in the spatial frequency domain across 12 feature sizes in the center of the passband $(0.25$ to $3.02 \mathrm{~mm}$, corresponding to spatial frequencies of 1.98 to $0.17 \mathrm{~mm}^{-1}$, respectively) and 12 bandwidths (corresponding to feature size ranges of \pm 0.13 to \pm 1.51 $\mathrm{mm}$ ), creating a total of 144 filtered versions of each ROI. Filtering was performed using the Bandpass Filter function in the Fiji distribution of ImageJ. ${ }^{6}$ MIJ, a Java package that facilitates image exchange between MATLAB and ImageJ, ${ }^{7}$ was used to integrate ImageJ's Bandpass Filter functionality with MATLAB-based 
image processing and scripting. ${ }^{8}$ After every bandpass filtering iteration, each ROI was masked before further processing. The selective bandpass filtering and ROI masking is illustrated in Figure 3, which shows a representative specimen containing adipose tissue, benign fibrocystic disease (FCD), and malignant tubular carcinoma. Figure 3 a) shows an RGB color space reconstruction of the specimen using planar $(0.00$ $\mathrm{mm}^{-1}$ ) illumination, and Figure $3 \mathrm{c}$ ) shows the specimen with HSF (1.37 $\left.\mathrm{mm}^{-1}\right)$, monochromatic (490 nm) illumination. The pair of images demonstrates that HSF, blue light illumination provides enhanced contrast to small-scale surface textures.

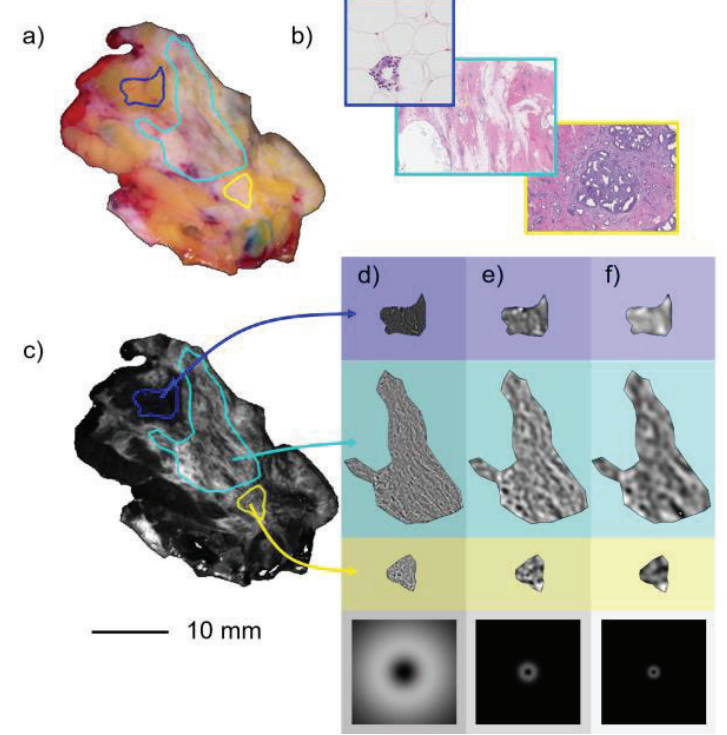

Figure 3. A representative breast tissue specimen: a) RGB color space, planar illumination $\left(0.00 \mathrm{~mm}^{-1}\right)$ image with three ROIs outlining adipose tissue (dark blue), benign FCD (cyan), and malignant tubular carcinoma (yellow); b) histology of each tissue; c) the same specimen under $1.37 \mathrm{~mm}^{-1}, 490$ $\mathrm{nm}$ illumination. Bandpass filtering at sequentially lower spatial frequencies is shown in columns d), e), and f); the bottom row in each column displays the corresponding frequency domain bandpass filter generated by ImageJ.

Metrics were quantified for all 144 (12x12) filtered versions of each ROI, including average normalized ROI intensity, ROI standard deviation, Euler number normalized by ROI area (based on binarized images using Otsu's Method $)^{8}$, fractal dimension, ${ }^{9,10}$ and grayscale level co-occurrence matrix (GLCM) statistics of contrast, correlation, and homogeneity. ${ }^{8}$ Each GLCM was based on a 10-level (matrix dimensions of 10x10) intensity normalization, a displacement distance of one pixel, and vector directionality symmetric about $0^{\circ}$, $45^{\circ}, 90^{\circ}$, and $135^{\circ}$. Reported values were averaged over the four angles, because texture features were assumed to be rotationally invariant. ${ }^{11,12}$ The signal-to-noise ratio (SNR) was computed for pairs of filtered ROIs containing different tissue subtypes. SNR variations with respect to spatial frequency domain bandpass filter center frequency and bandwidth revealed the most important spatial scales (and corresponding spatial frequencies) for differentiating pairs of tissue subtypes.

\section{Results and Discussion}

A subset of texture metrics for each ROI in the representative specimen (Figure 3) are shown in Figure 4. Each column in Figure 4 corresponds to a different tissue subtype, while each row is a different metric.
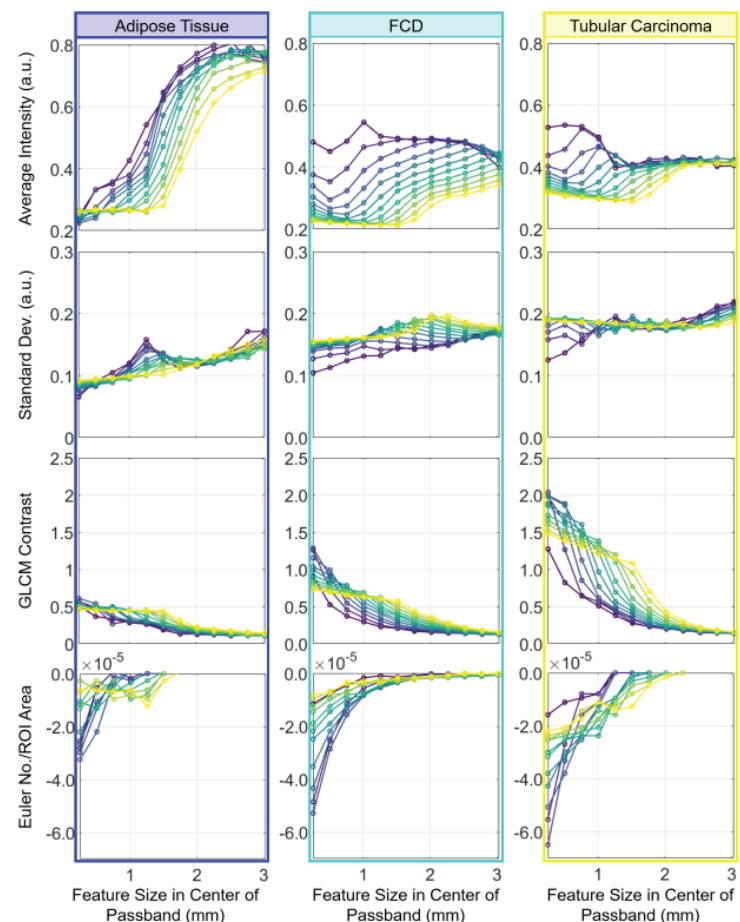

Passband (mm)

Passband (mm)

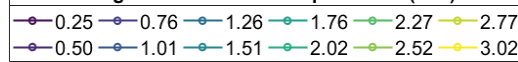

Figure 4. Texture metrics for the ROIs in the representative specimen (Figure 3). Subplot curves represent different filter bandwidths that correspond to constant spatial domain feature size ranges.

From the first to the last row, the subplots show the average normalized ROI intensity, the ROI standard deviation, GLCM contrast, and normalized Euler number. In cases when half of the passband feature size 
range drops below $0 \mathrm{~mm}$ for a given passband center feature size, all small-scale (high spatial frequency) content is retained.

The selection of texture metrics in Figure 4 show that selectively filtering in the spatial frequency domain draws out unique features within each tissue subtype relative to the other subtypes. For example, average normalized ROI intensity demonstrates that adipose tissue is dominated by relatively large-scale (low spatial frequency) features, while FCD and tubular carcinoma contain predominately small-scale (high spatial frequency) features. Additionally, GLCM contrast at passband center feature sizes less than $\sim 2 \mathrm{~mm}$ is significantly different between FCD and tubular carcinoma.

SNR, defined here as the signal intensity divided by the standard deviation of the background, can be used to identify optimal spatial frequency ranges for differentiating benign and malignant tissue subtype pairs. Figure 5 demonstrates this process by quantifying the SNR for the representative specimen (Figure 3) using the average ROI intensity and ROI standard deviation data for each benign-malignant tissue pair (Figure 4).
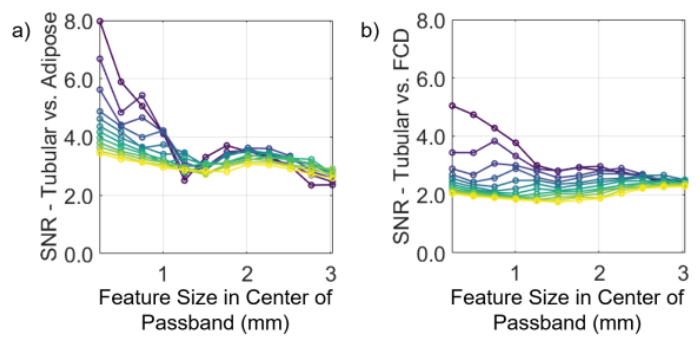

$$
\begin{aligned}
& \text { Range of feature sizes in passband ( } \mathrm{mm} \text { ) } \\
& \begin{array}{l}
\circ-0.25-0.76-0-1.26-1.76-2.27-2.77 \\
-0.50 \rightarrow-1.01-0-1.51-2.02-2.52-3.02 \\
\hline
\end{array}
\end{aligned}
$$

Figure 5. SNR plots for malignant tubular carcinoma (signal) versus a) adipose tissue (background) and b) FCD (background).

The results in Figure 5 indicate that isolating just the smallest spatial features (the highest bandpass filter center frequency) provides optimal SNR for differentiating tubular carcinoma from adipose tissue and tubular carcinoma from FCD. The maximum SNR in each subplot of Figure 5 occurs with the smallest filtered spatial scale, $0.25 \pm 0.13 \mathrm{~mm}(0.25 \mathrm{~mm}$ overall passband width), which corresponds to a filter center spatial frequency of $1.98 \mathrm{~mm}^{-1}$ and bandwidth of \pm 0.06 $\mathrm{mm}^{-1}$.

The selective bandpass filtering analysis presented here is limited to only one representative, heterogenous breast tissue specimen. To test the generalizability of the approach between specimens and the wide range of possible tissue subtypes, and to quantify the statistical significance of these filtered ROI metrics, the analysis will be expanded to include all specimens and ROIs in the clinical study dataset.

\section{Acknowledgements}

This work was made possible by a National Institutes of Health (NIH) National Cancer Institute (NCI) Academic-Industrial Partnership under grant number NIH/NCI R01 CA192803.

\section{References}

[1] Maloney, B. W., McClatchy, D. M., Pogue, B. W., Paulsen, K. D., Wells, W. A., and Barth, R. J., "Review of methods for intraoperative margin detection for breast conserving surgery," J Bio Opt, 23(10), 100901 (2018).

[2] Cuccia, D. J., Bevilacqua, F., Durkin, A. J., Ayers, F. R., and Tromberg, B. J., "Quantitation and mapping of tissue optical properties using modulated imaging," J Bio Opt, 14(2), 024012 (2009).

[3] McClatchy, D. M., Rizzo, E. J., Wells, W. A., Cheney, P. P., Hwang, J. C., Paulsen, K. D., Pogue, B. W., and Kanick S. C., "Wide-field quantitative imaging of tissue mircostructure using sub-diffuse spatial frequency domain imaging," Optica, 3(6), 613-621 (2016).

[4] Mcclatchy, D. M., Hoopes, P. J., Pogue, B. W., and Kanick, S. C., "Monochromatic subdiffusive spatial frequency domain imaging provides in-situ sensitivity to intratumoral morphological heterogeneity in a murine model," J Biophotonics, 10(2), 211-216 (2017).

[5] McClatchy, D. M., Rizzo, E. J., Meganck, J., Kempner, J., Vicory, J., Wells, W. A., Paulsen, K. D., and Pogue, B. W., "Calibration and analysis of a multimodela mico-CT and structured light imaging system for the evaluation of breast tissue," Phys Med Biol, 62, 8983-9000 (2017).

[6] Schindelin, J., Arganda-Carreras, I., Frise, E., Kaynig, V., Longair, M., Pietzsch, T., Preibisch, S., Rueden, C., Saalfeld, S., Schmid, B., Tinevez, J., White, D. J., Hartenstein, V., Eliceiri, K., Tomancak, P., Cardona, A., "Fiji: an open-source platform for biological-image analysis," Nature Methods, 9(7), 676-682 (2012).

[7] Sage, D., Prodanov, D., Tinevez, J., and Schindelin, J., "MIJ: Making Interoperability Between ImageJ and Matlab Possible," ImageJ User and Developer Conference, 24-26 (2012). 
[8] MathWorks, "MATLAB Image Processing Toolbox," 10.2, https://www.mathworks.com/products/image.html $\quad(1$ February 2019).

[9] Costa, A., "Hausdorff (Box-Counting) Fractal Dimension," MATLAB File Exchange, 18 December 2013, https://www.mathworks.com/matlabcentral/fileexchange/3 0329-hausdorff-box-counting-fractal-dimension February 2019).

[10] Costa, A. F., Mamani, G. H., and Traina, A., "An Efficient Algorithm for Fractal Analysis of Textures," Conference on Graphics, Patterns, and Images, 39-46 (2012).

[11] Haralick, R. M., Shanmugam, K., and Dinstein, I., "Textural Features for Image Classification," IEEE Trans Systems Man Cyber, 3(6), 610-621 (1973).

[12] Laughney, A. M., Krishnaswamy, V., Rizzo, E. J., Schwab, M. C., Barth, R. J., Pogue, B. W., Paulsen, K. D., and Wells, W. A., "Scatter Spectroscopic Imaging Distinguishes between Breast Pathologies in Tissues Relevant to Surgical Margin Assessment," Clin Can Res, 18(22), 6315-6325 (2012). 


\title{
Light-field acquisition and super-resolution with structured illumination
}

\author{
Shin Usuki*a ${ }^{*}$ Kenjiro T. Miura ${ }^{b}$

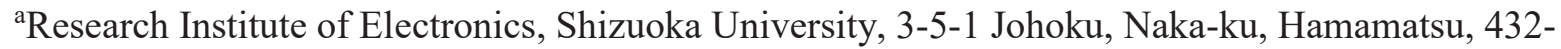 \\ 8561, Japan \\ ${ }^{\mathrm{b}}$ Graduate School of Science and Technoloty, Shizuoka University, 3-5-1 Johoku, Nakaku, \\ Hamamatsu, 432-8561, Japan
}

\begin{abstract}
The structured illumination microscopy is a well-known super-resolution technique and commercialized for wide-field bio-imaging applications. Since it is limited to a fluorescent imaging, not available in case of observations without fluorescent staining. Furthermore light-field can't be acquired because optical phase information of the structured illumination is lost in incoherent imaging. In this study, two-beam interference of coherent light was employed as the structured illumination for non-fluorescent imaging with super-resolution and light-filed acquisition. Experimental results showed that the super-resolution non-fluorescent imaging was possibly implemented. For further improvement on computations of plural images, reconstruction based on light-field deconvolution was proposed.
\end{abstract}

Keywords: structured illumination, non-fluorescent imaging, light field, super-resolution, deconvolution

\section{Introduction}

The structured illumination microscopy is a wellknown super-resolution technique and commercialized for wide-field bio-imaging applications. Since it is limited to a fluorescent imaging, not available in case of observations without fluorescent staining. Furthermore light-field can't be acquired because optical phase information of the structured illumination is lost in incoherent imaging. In this study, two-beam interference of coherent light was employed as the structured illumination for non-fluorescent imaging with superresolution and light-filed acquisition. The phase of the structured illumination was detected as a fringe signal with another interferometric measurement system in which a relatively low time coherence light source (e.g. a super luminescent diode SLD) was employed for both coherent imaging and low-speckle imaging. We constructed an optical system consisting of two set of Michelson interferometers, which allow the lowcoherence interference fringes to be manipulated on a sample and the phase to be detected on another image sensor. Feeding back the phase of structured illumination through computations of plural images allows robust imaging despite vibrations, drifting, and environmental changes. Some experimental results showed that the super-resolution non-fluorescent imaging was possibly performed. For further improvement on computations of plural images, reconstruction based on light-field deconvolution was proposed.

\section{Example of Experimental Results}

The experimental specifications of our system are listed in Table 1. Figure 1 shows comparison between the diffraction-limited image and high-resolution nonfluorescent image with the structured illumination. There are 2 results; one is super-resolution experiment for dots structure and the other is for line and space. Although intensity unevenness is apparent in the resulting images, the $0.4 \mu \mathrm{m}$ pitch, which is smaller than the diffraction limit, is surely resolved.

Table 1. Experimental specifications of the developed system.

\begin{tabular}{|l|l|}
\hline Items & Values \\
\hline SLD-as-light-source wavelength $\lambda$ & $0.67 \mu \mathrm{m}$ \\
\hline Structured illumination pitch $P$ & $0.47 \mu \mathrm{m}$ \\
\hline Observation magnification $M$ & 100 \\
\hline Numerical aperture of objective $N A$ & 0.55 \\
\hline Diffraction limit under the SLD $D L$ & $0.74 \mu \mathrm{m}$ \\
\hline
\end{tabular}




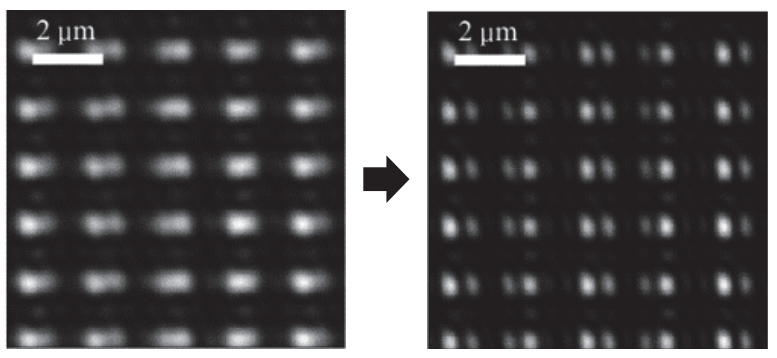

Super-resolution for dot structure
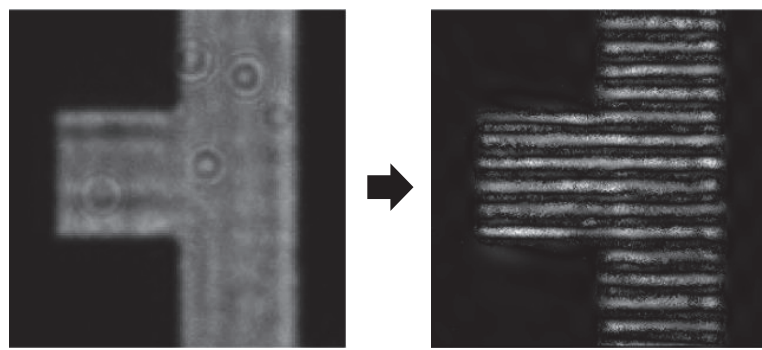

Super-resolution for line and space

Figure 1. Comparison between the diffraction-limited image and high-resolution non-fluorescent image with the structured illumination.

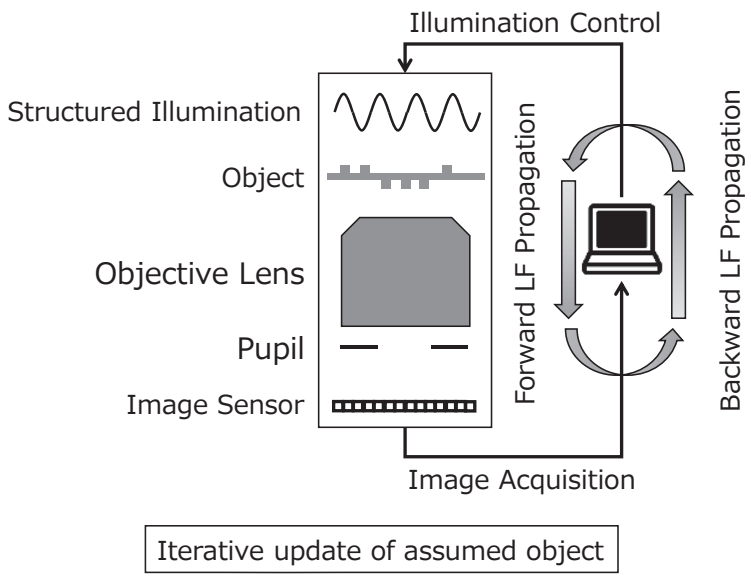

Figure 2. Conceptual diagram of computational lightfield deconvolution.

\section{Improvement of Computation}

A computational light-field deconvolution with structured illumination was proposed for light-field acquisition. A conceptual diagram of both experiments and computations is shown in Figure 2. In this concept, the structured illumination acts as light-field as it is generated by two beam interference of coherent light. Also initial object is assumed as light field. In the forward light-field propagation, the objective lens (including the imaging lens) perform a Fourier transform of light-field from the assumed object with structured illumination. The pupil of the objective lens performs low-pass filtering before imaging on the image sensor. The backward lightfield propagation is carried out by an inverse Fourier transform of light-field with constraint of the pupil function of the objective lens. Then difference between the forward light-filed of the object and the backward light-filed of the object is added to the initial assumed object with appropriate coefficient and weight. This object update is repeated with plural images acquired according to the structured illumination control until the solution converges. In this computation, both a phase recovery problem and a high spatial frequency extrapolation problem can be solved simultaneously for light-field acquisition and super-resolution respectively.

\section{References}

M.G.L. Gustafsson, Surpassing the lateral resolution limit by a factor of two using structured illumination microscopy, J. Microsc. 198 (2) (2000) 82-87.

Lin Shao, Berith Isaac, Satoru Uzawa, David A. Agard, John W. Sedat, Mats G.L. Gustafsson, I5S: Wide-field light microscopy with 100-nmscale resolution in three dimensions, Biophys. J. 94 (June) (2008) 4971-4983.

Diane S. Lidke, Keith A. Lidke, Advances in highresolution imagingtechniques for three-dimensional imaging of cellular structures, J. Cell Sci. 125 (2012) 2571-2580.

S. Usuki, H. Nishioka, S. Takahashi, K. Takamasu, Experimental verification of super-resolution optical inspection for semiconductor defect by using standing wave illumination shift, Int. J. Adv. Manuf. Technol. 46 (9) (2010) 863-875.

S. Usuki, T. Takada and K. T. Miura, Optical Microscopy with Improved Resolution Using Two-beam Interference of Low-coherence Light, Measurement, 78, (2016)373-380. 


\title{
Differential Phase Contrast Volume Holographic Incoherent Microscopy
}

\author{
Yu-Hsin Chia ${ }^{1}$, Yuan Luo ${ }^{1,2,3}$ \\ 1. Institute of Medical Device and Imaging, National Taiwan University, 10051, Taiwan, R. O. C. \\ 2.Molecular Imaging Center, National Taiwan University, Taipei, 10672, Taiwan, R. O. C. \\ 3. YongLin Institute of Health, National Taiwan University, Taipei, 10087, Taiwan, R. O. C. \\ Authore-mail address: r06458002@ntu.edu.tw
}

\begin{abstract}
We present an asymmetric illumination based differential phase contrast volume holographic microscopy (DPC-VHM) to enhance the contrast of the weak phase object. Under the incoherent illumination, the DPC-VHM can use single shot to obtain the one depth phase contrast image of biological samples, and it also has the ability to acquire multi-depth phase contrast images of specimens without axial scanning. Differential phase contrast images of basswood stem and USAF resolution chart are obtained and compared with conventional bright field VHM images.
\end{abstract}

Key Word - Phase contrast, Three-dimensional imaging, Incoherent, Holography, Microscopy.

\section{Introduction}

Several optical microscopy systems have been proposed to obtain three-dimensional (3D) images for biomedical request. However, most of these systems still require mechanical or electro-optic axial scanning mechanism to construct multi-depth images. Recently, the volume holographic microscopy (VHM) has been developed that can obtain multi depth images of biological specimen in single shot [1]. Using conventional VHM it is difficult to capture the images of weak phase object. Although the phase contrast VHM has been proposed to enhance phase information of all different depth images within transmittance specimen, the systems need to use the knife-edge component in the recording or imaging setup [2-3], so they still can be improved. Here, an asymmetric illumination based differential phase contrast volume holographic microscopy (DPC-VHM) is presented. For the single depth image, the proposed DPC-VHM inserts the multiplex wavelength coded volume holographic gratings (MWC-VHGs) that makes different wavelength probe beam can generate corresponding diffraction beam, and the CCD can simultaneously capture these diffraction beams to do the DPC imaging process. For the multi depth images, the system uses the multiplex volume holographic gratings (MVHGs) to function like spatial-spectral filters to obtain weak phase objects with multi-plane images without axial scanning.

\section{Fabrication and design of MWC-VHGs and MVHGs}

The proposed DPC-VHM uses the photosensitive materials PQ-PMMA to form high spectral and angular Bragg selective VHGs. An $\mathrm{Ar}^{+}$laser operated at wavelength $488 \mathrm{~nm}$ is used as light source to form the MVHGs. The input beam is divided into a reference and signal beams. Figure 1 (a) shows the K-sphere diagram for the MWC-VHGs that include two gratings $\mathrm{K}_{\mathrm{g} 1} \mathrm{~K}_{\mathrm{g} 2}$ designed for the wavelength $633 \mathrm{~nm}$ and $488 \mathrm{~nm}$ respectively. Here, we used collimated reference and signal beams. The signal beam maintains the same $45^{\circ}$ incident angles with respect to the surface normal of photopolymer substrate for both the gratings $\mathrm{K}_{\mathrm{g} 1} \mathrm{~K}_{\mathrm{g} 2}$. In order to record two gratings, we changed the angle of the reference beam by $\Delta \theta=21.5^{\circ}$ in two successive recordings of hologram. The advantage of MWC-VHGs is that different wavelength probe beams can use the same reconstruction angle to generate corresponding diffraction angles. Figure 1 (b) shows the K-sphere for MVHGs, the signal arm inserts a lens (L1) and adjust its position to produce different depth point sources for each grating. The reference arm need to use different incident angle for each exposure, so the MVHGs can function like multi-focal lens [4].

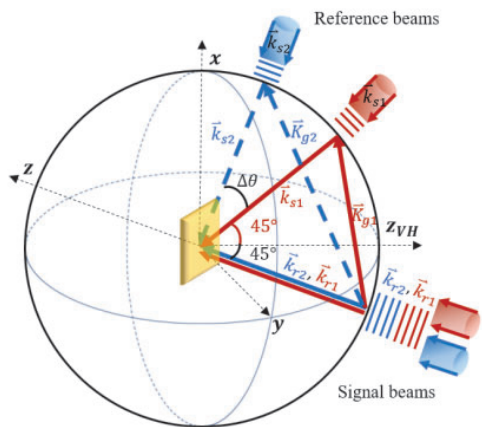

(a)

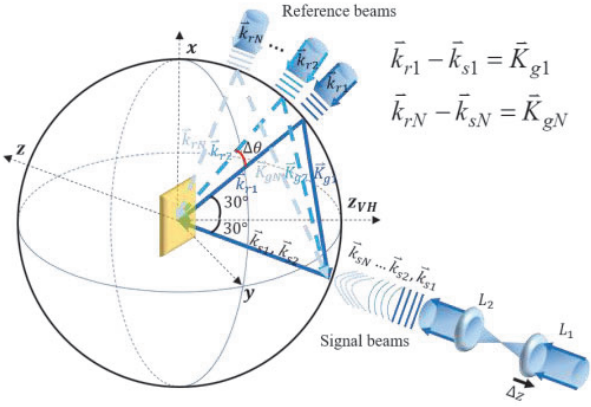

(b)

Fig.1 K-sphere diagram for recording of (a) MWC-VHGs (b) MVHGs.

\section{Experimental setups and results}


Figure 2 shows the proposed DPC-VHM setup. The TFT panel and condenser (LA1951-A, THORLABS) insert to the LED illumination part (LIUCWHA, THORLAB, $\lambda=425 \sim 700 \mathrm{~nm}$ ) of the VHM system. The TFT panel shows one redblue gradient circle pattern and make it at the front focal plane of the condenser as shown in the Figure 2 (a), so the light go through the pattern and condenser can simultaneously produce different direction asymmetric illumination beams with corresponding wavelength. An objective (ULWDMSPlan50X, OLYMPUS) and a tube lens (MPlanAPO20X, MITUTOYO) are used to assemble the 4- $f$ imaging system, with the MWC-VHGs located in the Fourier plane. When the MWC-VHGs satisfy Bragg match condition, it can make different wavelength beam to diffract to the corresponding direction that are captured by the CCD (GE1650, PROSILICA). The present system just need a single shot to acquire the one depth DPC image. For multi-depth images, the TFT panel need to sequentially show the left and right half circle patterns to generate two different direction asymmetric illumination images as shown in the Figure 2 (b), and the MVHGs also need to locate at Fourier plane to function like a multi focal lens. After taking two images, the proposed DPC-VHM can do the image processing to simultaneously obtain multi-depth DPC images without axial scanning. Figure 3 is the experimental images of basswood stem. Figure 3 (a) shows depth-resolved bright-field VHM images, while Figure 3 (b) shows DPC-VHM images. The DPC-VHM images obviously enhance the weak phase features for multi depth in the same time. Figure 4 is the experimental images of the Air Force Resolution Chart, the contrast of high frequency edge part in Figure 4 (b) is largely improved as comparison with bright field VHM images in Figure 4 (a).

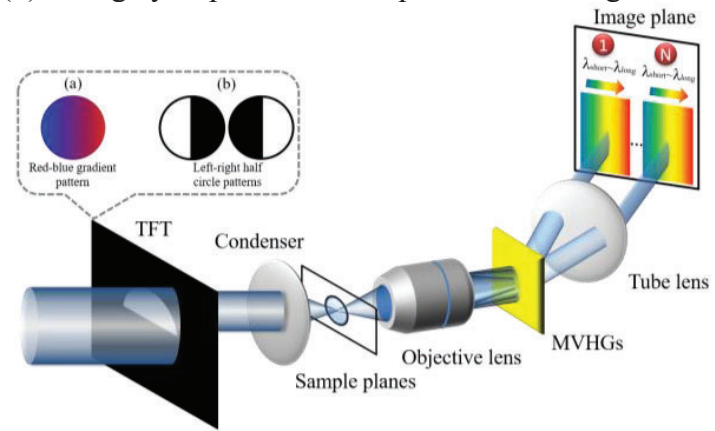

Fig.2 The proposed DPC-VHM setup.

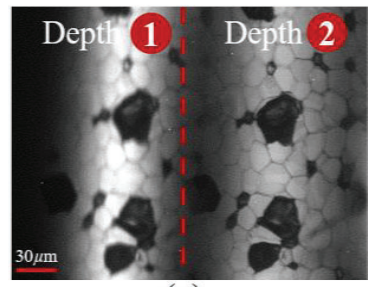

(a)

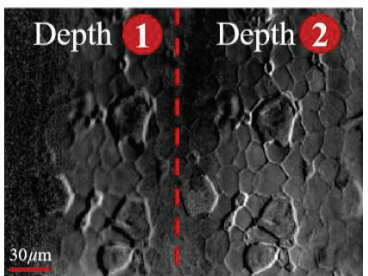

(b)

Fig.3 Two depth-resolved basswood stem (a) bright field, (b) DPC images acquired by the DPC-VHM.

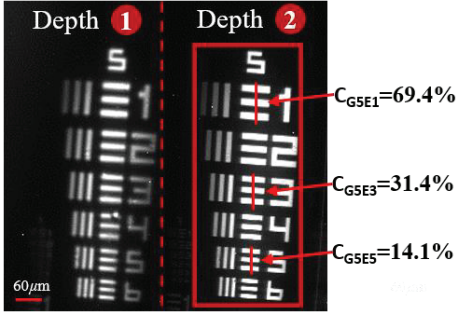

(a)

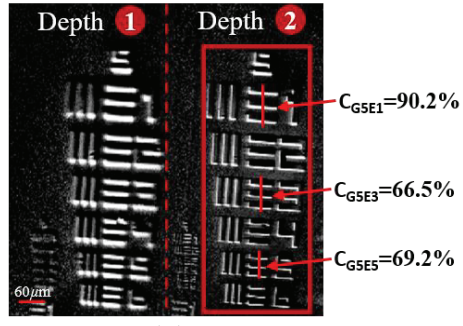

(b)

Fig.4 Two different depth resolution targets (a) bright field, (b) DPC images obtained by the DPC-VHM.

\section{Conclusion}

In summary, an asymmetric illumination based DPC-VHM has been proposed. By introducing the MWC-VHGs, the system can use one shot to obtain phase contrast image of biological samples. With the help of the MVHGs technique, the ability to simultaneously acquire multiple depth-resolved differential phase contrast images of specimens without axial scanning can be easily achieved. Our system can also combine the MWC-VHGs with quantitative differential phase system to obtain multi depth quantitative phase contrast information in the same time.

\section{References}

[1] Y. Luo, P. J. Gelsinger-Austin, J. M. Watson, G. Barbastathis, J. K. Barton, and R. K. Kostuk, "Laser-induced fluorescence imaging of subsurface tissue structures with a volume holographic spatial-spectral imaging system," Optics Letters Vol. 33, 2098-2100 (2008).

[2] Y. Luo, E. Leon, J. Castro, J. Lee, J. K. Barton, R. K. Kostuk, and G.Barbastathis, "Phase-contrast volume holographic imaging system," Optics Letters Vol. 36, 1290-1292 (2011).

[3] S. B. Oh, Z. Q. J. Lu, J. C. Tsai, H. H. Chen, G. Barbastathis and Y. Luo, "Phase-coded volume holographic gratings for spatial-spectral imaging filters," Optics Letters Vol. 38, 477-479 (2013).

[4] Yu-Hsin Chia, Hung-Chun Wang, Yuan Luo, "Incoherent holographic imaging of subsurface structures with volume holographic gratings", Proc. 


\title{
DOPPLER PHASE-SHIFTING OPTICAL LOW-COHERENCE TOMOGRAPHY
}

\author{
Quang Duc Pham¹, Quoc Tuan Banh ${ }^{1}$ and Yoshio Hayasaki² \\ ${ }^{1}$ Laboratory for optical fiber laser research development and application, NACENTECH, \\ No. 25, Le Thanh Tong, Phan Chu Trinh, Hoan Kiem, Hanoi, Vietnam. \\ ${ }^{2}$ Center for Optical Research and Education (CORE), Utsunomiya University 7-1-2 Yoto, Utsunomiya 321-8585, Japan \\ E-mail address: phamducquang1010@gmail.com
}

\begin{abstract}
A new as a hybrid method of TD-OCT and FD-OCT with an ultra-broad band light source and Doppler achromatic phase-shifter was proposed to achieve the depth image of the object with very high lateral and axial resolution.
\end{abstract}

\section{Introduction}

Optical Coherence Tomography (OCT) that is capable of non-invasive imaging tissue microstructure has become a power full tool in many medical applications. Optical coherence tomography is categorized by time domain optical coherence tomography (TD-OCT) and frequency domain optical coherence tomography (FD-OCT). [1-2] In TD-OCT, the reference mirror is scanned and a series of interference images between the optical wave reflected from the object and the reference wave is recorded to produce cross-correlation function, the peaks of the cross-correlation functions construct the structure of the tissue. The time consuming and the low resolution related to scanning speed of the reference mirror and the bandwidth of the light source is considered as the limitation of this technique. [1] In FD-OCT, the mechanical movement of reference mirror removed, the broadband light source is employed but the interference signal of the object wave and reference wave corresponding to each wavelength of the light source is separately acquired by a wavelength tuneable light source or a dispersive detector such as a grating or a linear detector array. Because of the Fourier relation between depth information of the object and the spectrum of the light source, a Fourier transform from acquired spectral intensity interference images enable to reconstruct the depth image of the object. [2] The wavelength scanning improves imaging speed dramatically, but the resolution is limited by the number of wavelength and scanning range.

In this research, we proposed a new method with an ultra-broad band light source and Doppler achromatic phaseshifter that is carried by shifting the reference mirror with constant velocity. [3] The achromatic phase-shifter gives difference frequencies shifting for respective spectral frequencies and the arbitrary selection of signals in the temporal frequency domain enables us to acquire the complex amplitude of the object for each corresponding wavelength. The Fourier transform of complex amplitudes allows us to achieve the depth image of the object with very high lateral and axial resolution. The series of interference images is recoded by scanning reference mirror like in TD-OCT and the depth image of the object is reconstructed by Fourier transform as in FD-OCT so the proposed method is considered as a hybrid method of TD-OCT and FD-OCT.

\section{Method}

The proposed method was described in Fig.1, and it is carried out by three steps. In the first step, the reference mirror was moved with a constant velocity, a series of interference image were recorded. In the second steps, the complex amplitude the object corresponding to each wavelength of the light source was reconstructed from interference images. Consiquently, in the third step, the spatial fourier transform of the complex amplitudes of the object is figured the intensity images in different layer of the object.

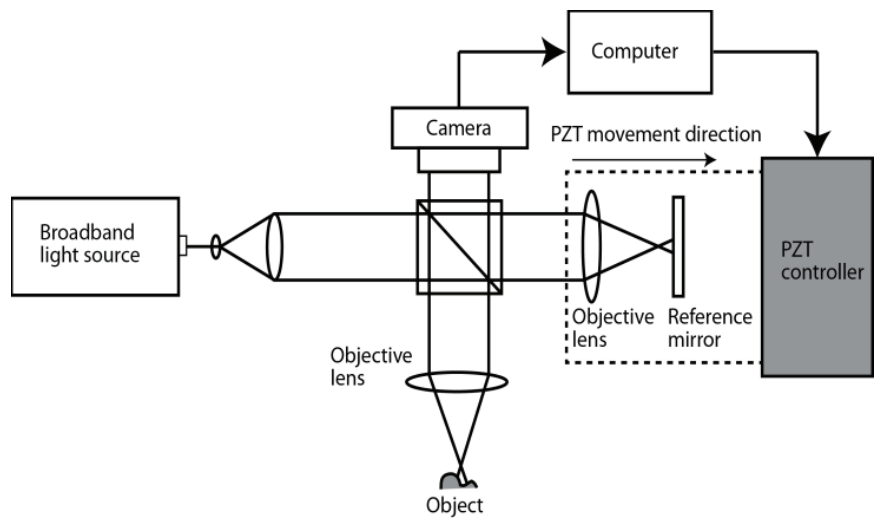

Fio 1 Princinle of the nronosed method 


\section{Experimental results}

The experiment was carried out to demonstrate the proposed method with a sample of onion cells. The velocity of the reference mirror was set at $20 \mu \mathrm{m} / \mathrm{s}$. The frame rate of the camera was set at $500 \mathrm{fps}, 1000$ of interference signal images were recorded by CMOS image sensor (Redlake; MotionScope M5) with a pixel size of $13.68 \mu$ m. The interference signal at a pixel of the camera was shown in Fig. 2(a) and the corresponding The spectrum of the light reflected from the object was overlapped on the pixel of the object
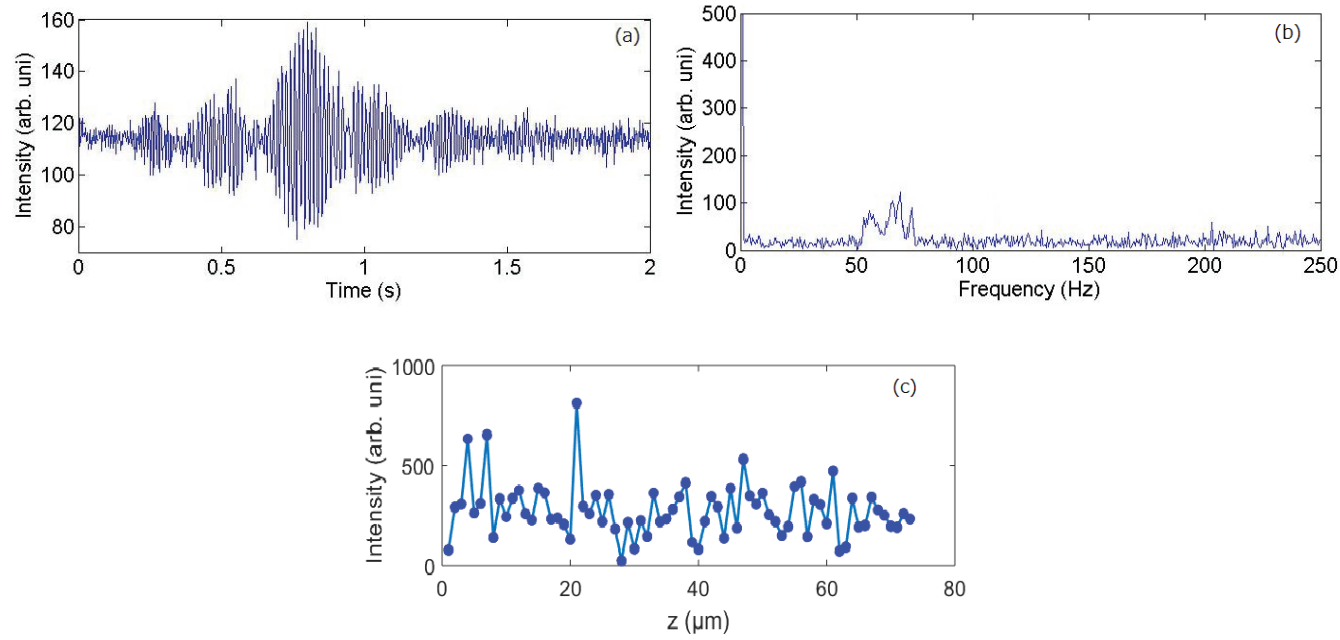

Fig 2. (a) The interference signal at a pixel recoded when the reference mirror was moved, (b) the Fourier transform related to the spectrum of the light reflected from the object and (c) the intensity image at different depth of the object was reconstructed by Fourier transform of the spectral function.
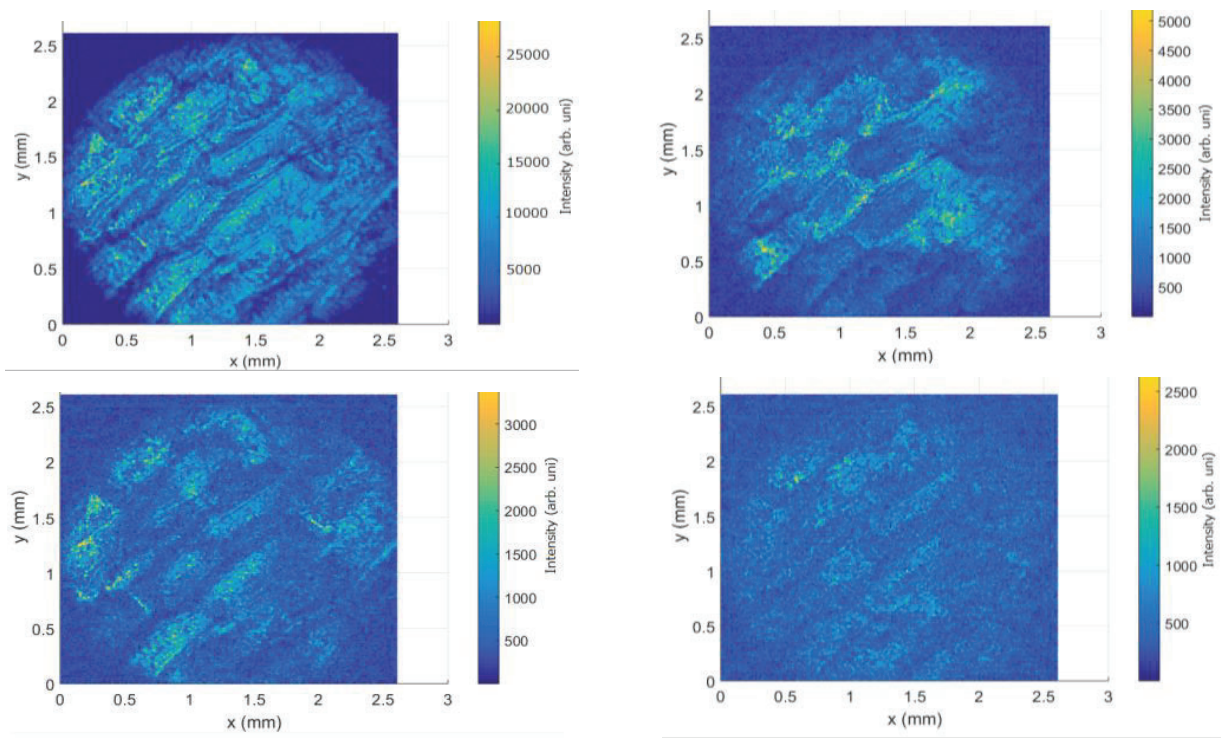

Fig 3. The intensity image of the object was reconstructed at different depth

\section{Conclusion}

The proposed method is new method to reconstruct the depth image of an object that is a combination the time domain and frequency domain tomographic method. The resolution is very high (e.g. $v_{R}=20 \mu \mathrm{m} / \mathrm{s}, f_{s}=500 \mathrm{fps}$, the resolution is $80 \mathrm{~nm})$. The measurement range is can be changed by using faster camera and larger velocity PZT $\left(z_{\operatorname{lmax}}=v_{R} N_{s} / f_{s}, v_{R}=\right.$ $20 \mu \mathrm{m} / \mathrm{s}, f_{s}=500 \mathrm{fps}, N_{s}=500 \mathrm{fr}$, the measurement range is $\left.40 \mu \mathrm{m}\right)$. The measurement time is only in $1 \mathrm{~s}$ or few seconds depending on the resolution requirement. The setup is simple and cheap with a lot of wavelengths can be employed. The detailed of the proposed method will be discussed in our presentation.

\section{References}

[1] K. Takada, I. Yokohama, K. Chida, J. Noda, Appl Opt. 26, 1987, pp. 1603-1606.

[2] M. Choma, M. Sarunic,C. Yang, J. Izatt, Opt Exp. 11, 2003, pp. 2183-2189.

[3 ]Q. D. Pham, S. Hasegawa, T. Kiire, D. Barada, T. Yatagai, and Y. Hayasaki, Opt. Express. 20, 2012, pp. 19744-19756. 


\title{
Multi-focal holographic differential confocal microscopy
}

\author{
Tso-Hua Wu ${ }^{1,2}$, Chou-Min Chia ${ }^{2}$, J. Andrew Yeh ${ }^{1}$, Yuan Luo ${ }^{2,3,4 *}$ \\ 1. Institute of NanoEngineering and MicroSystems, National Tsing Hua University, Hsinchu 30013, Taiwan, R.O.C. \\ 2. Institute of Medical Device and Imaging, National Taiwan University, Taipei 10051, Taiwan, R.O.C. \\ 3. Molecular Imaging Center, National Taiwan University, Taipei, 10672, Taiwan, R. O. C. \\ 4. YongLin Institute of Health, National Taiwan University, Taipei, 10087, Taiwan, R. O. C. \\ Author e-mail address: yuanluo@ntu.edu.tw
}

\begin{abstract}
Confocal microscopy has been widely applied to obtain optical sectioning image. However, scanning of conventional confocal microscopy is time-consuming to construct the three-dimensional images. Here, a non-axial-scanning differential confocal microscopy is presented. The proposed system combines multiplex volume holographic gratings (MVHGs) and differential confocal microscopy (DCM). The resolution is measured through resolution target and multi-depth information can be obtained by the proposed system.

Key Word-- Multiplex holography, Volume gratings, Differential confocal microscopy.
\end{abstract}

\section{Introduction}

Confocal microscopy can reconstruct high resolution three-dimensional (3D) images by adopting a pinhole to block out of focus background noise. Therefore, confocal microscopy has been widely utilized in medical imaging and life sciences [1] due to optical sectioning capability. However, conventional confocal microscopy scans point by point and layer by layer at lateral and axial direction. That is, scanning time is a dominate factor, such as in cell in-vivo research $[2,3]$.

To improve the efficiency, many methods were developed to reduce the scanning time at lateral and axial direction. Line scanning confocal is utilized to decrease the time of lateral direction dramatically [4-6]. Earlier a differential confocal microscopy was developed to speed up the scanning in axial direction [7-9]. Here, we describe a non-axialscanning multi-focal differential confocal microscopy, by combining multiplexed volume holographic gratings (MVHGs) [10] and differential confocal microscope. The proposed system can obtain multi-depth information simultaneously and reduces axial scanning time.

\section{System setup}

Fig 1 shows a schematic diagram of the proposed multi-focal holographic confocal microscope system. Here, we used MVHGs in the illumination part of the microscope. Two collimated light laser beams $(\lambda=488 \mathrm{~nm})$ incident to MVHGs and reconstruct two diffracted beams [10]. These two diffraction beams, reflected by a beam splitter, are focused at different height of a sample by an objective lens. At imaging arm, light from the sample surface pass through corresponding pinholes onto APDs. Out of focus background is eliminated by pinholes. Lastly, APD can obtain optical section images to continue subsequent differential algorithm.

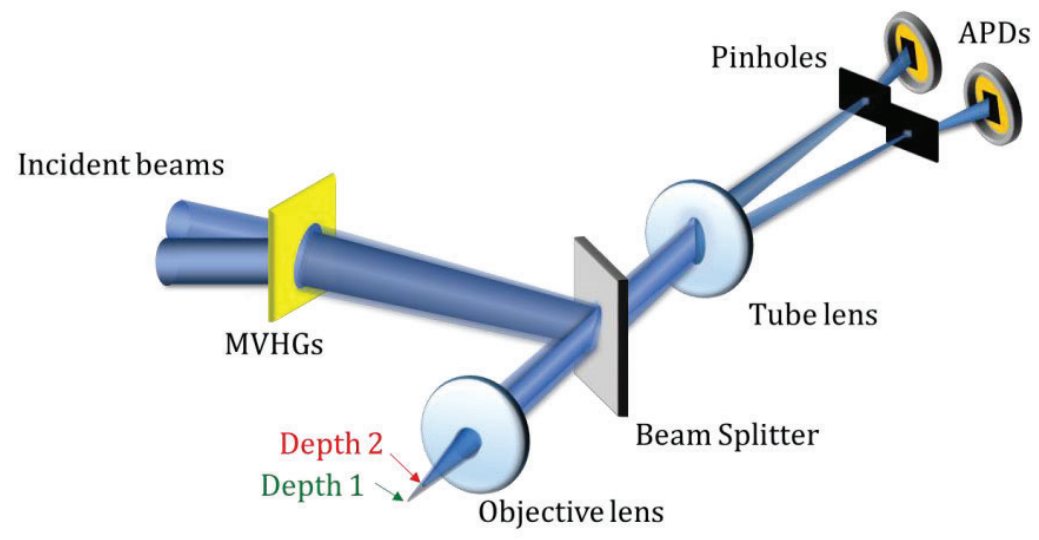

Fig. 1. Schematic diagram of multi-focal holographic differential confocal microscopy.

\section{Results and discussion}

First, we obtain the axial response curve by scanning the sample in axial direction. At the slope of response curve, 
be converted to height information of the sample. By moving the objective lens we can implement the focus offset, the differential subtraction of intensity responses is obtained and shown in Fig 2. Finally, the proposed system can reconstruct the topography of the sample.
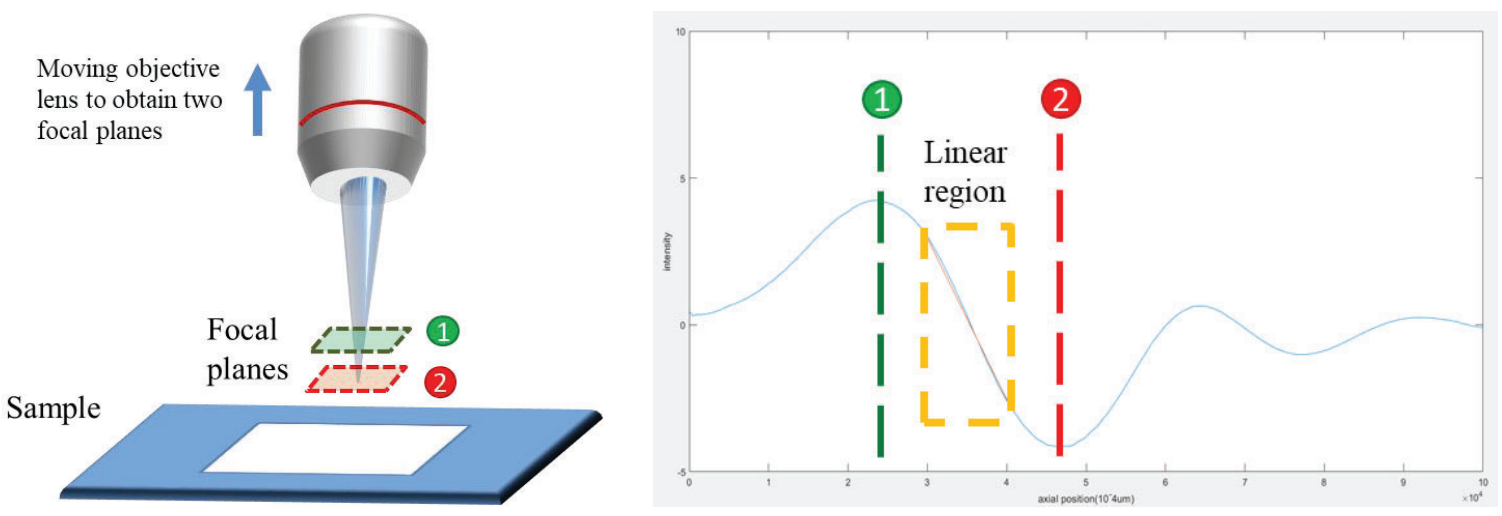

Fig. 2. The principle of the proposed system to obtain the differential result and the corresponding linear region.

The measurement of the lateral resolution was measured using an Air Force Resolution Chart (AFRC) 1951 standard. Fig 3 shows the lateral resolution of the proposed system is $0.488 \mu \mathrm{m}$. The axial resolution is determined by number of sampling points, instead of the width of the confocal response curve.

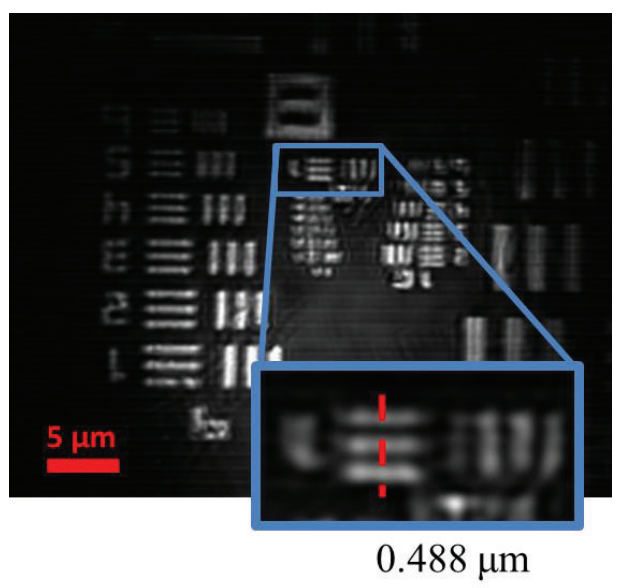

Fig. 3. Image intensity of resolution target by the proposed system.

\section{Conclusion}

The MVHG-base differential confocal microscopy is developed to obtain multi depth information simultaneously. The lateral resolution of the proposed system is $0.488 \mu \mathrm{m}$. For future work, we will use our system to reconstruct 3D images of samples.

\section{References}

[1] J. B. Pawley, Handbook of Biological Confocal Microscopy (Springer-Verlag, 2006).

[2] Yashvinder S. Sabharwal, Andrew R. Rouse, Latanya Donaldson, Mark F. Hopkins, And Arthur F. Gmitro, "Slit-Scanning Confocal Microendoscope for High-Resolution In Vivo Imaging," Appl. Opt.38, 7133-7144 (1999)

[3] Tavakoli M, Hossain P, Malik Ra. "Clinical Applications of Corneal Confocal Microscopy," Clinical Ophthalmology (Auckland, Nz). 2008;2(2):435-445.

[4] Kang-Bin Im, Sumin Han, Hwajoon Park, Dongsun Kim, And Beop-Min Kim, "Simple High-Speed Confocal Line-Scanning Microscope," Opt. Express 13,5151-5156 (2005).

[5] Chau-Chang Wang, "Laser Scanning, Theory and Applications," April 26, 2011.

[6] Charles J. Koester, "Scanning Mirror Microscope with Optical Sectioning Characteristics: Applications In Ophthalmology," Appl. Opt. 19, 17491757 (1980).

[7] Chau-Hwang Lee, Jyhpyng Wang, "Noninterferometric differential confocal microscopy with 2-nm depth resolution,” Optics Communications 135 233-237 (1997)

[8] W Zhao, Y Sun, Y Wang, L Qiu, R Shao, H Cui, “Three-dimensional super-resolution correlation differential confocal microscopy with nanometer axial focusing accuracy,” Optics Express, Vol. 26, Issue 12, pp. 15759-15768 (2018)

[9] Wei-ChihLiu, Meng-HaoChou, Kuang-YaoChang, Da-WeiLiu, Jim-WeiWu, Li-Chen Fu, "A Self-Designed Laser Scanning Differential Confocal Microscopy with a Novel Vertical Scan Algorithm for Fast Image Scanning,” IFAC-PapersOnLine, Volume 50, Issue 1, July (2017)

[10] Chou-Min Chia, Hung-Chun Wang, J. Andrew Yeh, Dipanjan Bhattacharya, Yuan Luo, " Multiplexed holographic non-axial-scanning slit confocal fluorescence microscopy ", Biomedical Imaging and Sensing Conference, Vol. 10711, 1071108 (2018) 


\title{
Multi-spectral digital holography with burst imaging method
}

\author{
Yu-Hsuan Huang, Takumi Ujiie, and Yoshio Hayasaki \\ Center for Optical Research and Education (CORE), Utsunomiya University \\ 7-1-2 Yoto, Utsunomiya 321-8585, Japan \\ hayasaki@cc.utsunomiya-u.ac.jp
}

\begin{abstract}
Application of a burst imaging method to a digital holography with multi-spectral illuminations was proposed. The burst imaging method is intermittently to capture several images with a high iteration while taking a rest time. When we assume that the wavelength switching and the image capturing in the multi-spectral digital holography should be faster than the changing speed of the object, it can be avoided one fatal problem in moving and deformation of an object while capturing multi-spectral imaging. In this research, the multi-spectral burst digital holography is demonstrated using fast wavelength switching implemented by a white-light continuum laser with acousto-optic modulator and a high-speed camera.
\end{abstract}

\section{Introduction}

Digital holography (DH) [1] is executed by three processes of recording, development and reconstruction. In the recording process, interference fringes formed by object light and reference light are acquired by an image sensor. In the development process, a hologram is generated from the interference fringes recorded on the computer. In the reconstruction process, the complex amplitude on a desired plane is obtained by light propagation calculation of the hologram. The DH is typically applied to a shape measurement of industrial products and a quantitative phase imaging of biological cells [2].

A recording of multiple images is effective for image quality improvement, acquisition of a stereoscopic image, super resolution, and polarization image measurement. The simplest method is to get for multiple interference fringes at the multiple times with a single image sensor, but it is not useful when an object has movements and changes. To solve this problem, the imaging methods of multiple image sensors [3] and the space division of one image sensor [4]. The former requires lateral and axial alignments of the image sensors and the latter requires a special image sensor.

In multispectral digital holography (MSDH), transmitted light and scattered light of an object are recorded with interference fringes at several wavelengths and reconstructed at respective wavelengths. To do this in practice, using a large number of image sensors is not practical from a cost, space and alignment point of view. Spatial division of image sensors involves the creation of special wavelength filter arrays and the reduction of spatial resolution due to the increase in the number of wavelengths. Therefore, it is possible to reconsider the method using one image sensor. Here we give one assumption. If it is possible to execute the multiple imaging that is sufficiently faster than the change of the object, it is equivalent to capture the images at the same time with multiple image sensors. In general, the image transfer rate from the image sensor in the camera to the memory is high, and the image transfer speed of the memory in the computer from the memory in the camera is low. Therefore, after shooting with the camera for the necessary number of times at short time intervals, the process of transferring these images to the computer over time is repeated to apply the continuous burst shooting. The MSDH is performed by a switching of the emission wavelength of the light source [5] and a switching of the transmission wavelength of the filter. Both methods are equivalent in principle.

In this research, we realize MSDH using a wavelength switch with an acousto-optic modulation for a white light continuum laser and a high-speed camera with a frame rate of $8.8 \mathrm{kHz}$, which has a minimum switching time of $100 \mu$ s. The performance of the MSDH is evaluated using a moving object.

\section{Burst-imaging method}

Figure 1 shows a time sequence that has a highly-iterative (bursty) image capturing period and an image transfer period. The image-capturing time sequence is called as a burst imaging method. If the changing amount of the object in the image capturing period is much smaller than a required resolution, it can be regarded as the object has no change. A camera with high-frame rate is used to achieve the above condition. Then two problems appear: one is an image processing time and another is a data transfer rate from the image sensor to a main computer. If the image processing can be performed within the frame interval, real time processing can be achieved. If real-time image processing is required, and no choice to choose a decrease of the frame rate is selected, the burst imaging method should be selected.

A data transfer rate from an image sensor to a buffer memory located near the photodetector in the image sensor is high, but the rate from the memory in a camera to the memory in a computer is relatively slow. Therefore, it is difficult to perform a continuous transfer of images captured by a high-speed camera images to a computer, and the number of images is limited by the capacity of the buffer memory. In this aspect, the concept of the burst imaging method is also useful. The images are bursty captured, and the image data stored in the buffer memory are transferred to the main memory after capturing, the data in the buffer memory is cleared to take the next bursty captured images. 


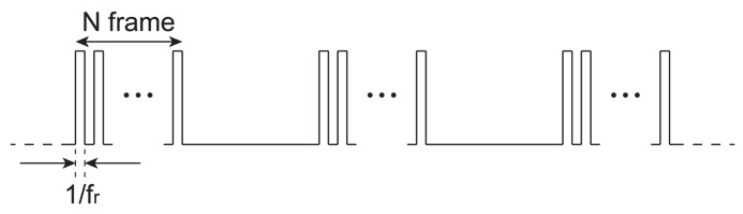

Fig. 1 Burst-imaging method.

\section{Experimental setup}

Figure 2 shows the experimental system. The light source is a white-light continuum laser (SuperK EXTREME, NKT Photonics) which generates light from $480 \mathrm{~nm}$ to $2300 \mathrm{~nm}$. A desired wavelength was selected from the broadband light by an acousto-optic tunable filter (AOTF) (SuperK SELECT, NKT Photonics) with an external radio frequency driver (SuperK External RF driver, NKT Photonics). The AOTF was controlled at a switching time of $250 \mu$ s. Up to eight wavelengths with a wavelength switching circuit (SuperK Command, NKT Photonics). The signal given to that wavelength switching circuit was given by a microcomputer (UNO R3, Arduino). In this study, it was changed every 20 $\mathrm{nm}$ from $520 \mathrm{~nm}$ to $660 \mathrm{~nm}$. The optical system was a Mach-Zehnder interferometer equipped with a microscope optical system with a magnification of 12.12 times constituted by an objective lens with a NA of 0.25 , a focal length of $16.5 \mathrm{~mm}$ and a lens with a focal length of $200 \mathrm{~mm}$ both on the object and on the reference optical paths. The interference fringes were captured by a high-speed CMOS image sensor (MotionPro Y4, IDT) with the maximum frame rate of $8800 \mathrm{fps}$ when acquiring $512 \times 512$ pixels. The sample was Closterium stained with green in the preparation.

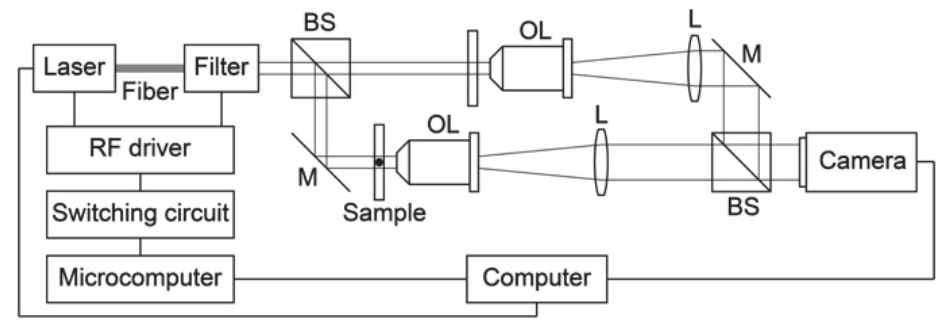

Fig. 2 Experimental setup.

\section{Experimental results}

Figure 3 shows the amplitude and the phase images at 520 to $660 \mathrm{~nm}$ every $20 \mathrm{~nm}$. Because the power of light source and sensitivity of the camera are different from the wavelength, we present the amplitude image with its transmittance. From the results in Fig. 3(c), the transmittance is about 100\% in the background (the part in slide glass without object), and the green band transmittance is higher in 5 points on Closterium.
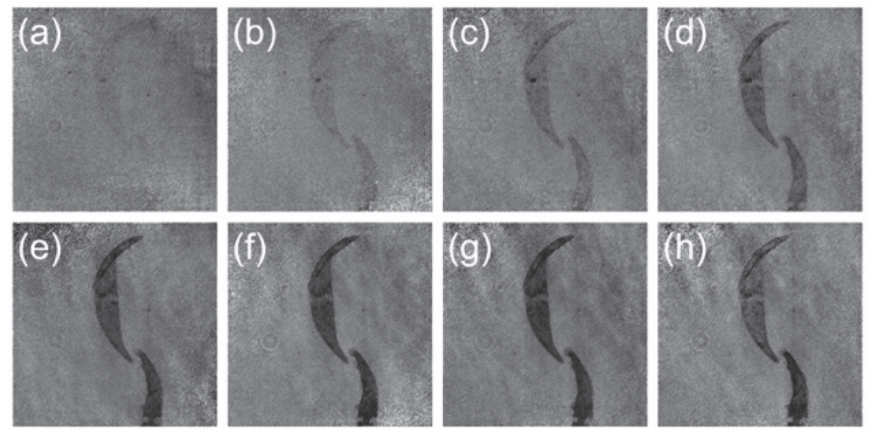

Fig. 3 Amplitude images at 520, 540, 560, 580, 600, 620, 640, and $660 \mathrm{~nm}$.

\section{Conclusion}

We proposed a new system of digital holography using high speed wavelength switching and high speed camera. We obtained the shape information and the spectral information of an object. When the normalized speed of image moves one pixel, the MAE was 34\%. And if the tolerance error is limited below one percent, the result will be the same as in stationary when the normalized speed is $1 / 34$ pixel.

\section{References}

[1] J. W. Goodman and R. W. Lawrence, "Digital image formation from electronically detected holograms," Appl. Phys. Lett. 11, 77-79 (1967).

[2] M. K. Kim, "Principles and techniques of digital holographic microscopy," SPIE Reviews 1, 018005 (2010).

[3] J. Zhang, Y. Xie, G. Li, Y. Ye, and B. E. Saleh, "Single-shot phase-shifting digital holography," Opt. Eng. 53, 1123316 (2014).

[4] Y. Awatsuji, M. Sasada, and T. Kubota, "Parallel quasi-phase-shifting digital holography," Appl. Phys. Lett. 85, 1069-1071 (2004).

I51 S. Chen. J. Rvu. K. Lee. and Y. Zhu. "Swent source digital hologranhic nhase microsconv." Ont. Lett. 41. 665-668 (2016). 


\author{
Imaging cytometry without image reconstruction (ghost cytometry) \\ Sadao Ota ${ }^{* a b}$, Ryoichi Horisaki ${ }^{\text {cd }}$, Yoko Kawamura ${ }^{b}$, Issei Sato ${ }^{\text {abe }}$, Hiroyuki Noji ad \\ a'University of Tokyo, 7-3-1 Hongo, Bunkyo-ku, Tokyo 113-8654, Japan \\ ${ }^{b}$ ThinkCyte Inc., 7-3-1 Hongo, Bunkyo-ku, Tokyo 113-8654, Japan \\ ${ }^{c}$ Department of Information and Physical Sciences, Graduate School of Information Science and \\ Technology, Osaka University, 1-5 Yamadaoka, Suita, Osaka 565-0871, Japan. \\ dJST, 4-1-8 Honcho, Kawaguchi-shi, Saitama 332-0012, Japan. \\ 'RIKEN AIP, 1-4-1 Nihonbashi, Chuo-ku, Tokyo, 103-0027, Japan.
}

\begin{abstract}
Imaging and analysis of many single cells holds great potential in our understanding of heterogeneous and complex life systems and in enabling biomedical applications. We here introduce a recently realized image-free "imaging" cytometry technology, which we call ghost cytometry. While a compressive ghost imaging technique utilizing object's motion relative to a projected static light pattern allows recovery of their images, a key of this ghost cytometry is to achieve ultrafast cell classification by directly applying machine learning methods to the compressive imaging signals in a temporal domain. We show the applicability of our method in analysis of flowing objects based on the reconstructed images as well as in that based on the imaging waveform without image production.
\end{abstract}

Keywords: imaging flow cytometry, machine learning, compressive sensing, optofluidics, single cell analysis

\section{Introduction}

Imaging and analyzing a static number of single cells is of importance in a wide range of biological and medicinal research fields ${ }^{1-3}$. Despite remarkable advances in the developments of ultrafast imaging methods ${ }^{4-7}$, speeding up imaging-activated cell sorting has been challenging mainly due to difficulties in compatibility of the acquisition of high dimensional image data and the image analysis including its reconstruction at the high speed in real-time.

We recently developed an image-free "imaging" cytometry approach, wherein we performed a direct analysis of compressive imaging waveforms with machine learning methods without image production, which we call ghost cytometry (GC) $)^{8}$. This GC skips the most computer-intensive process in the cell image analysis, which is reconstruction of two-dimensional images from raw signals, and thereby significantly relieved the computational bottleneck for the high-speed analysis and sorting based on morphological information ${ }^{8}$. Here we show the applicability of our method both in analysis of flowing objects based on the reconstructed images and in that based on the imaging waveform without image production. These results are compared with those obtained using a commercialized flow cytometer and an imaging flow cytometer.

\section{Body \\ 1. Ghost motion imaging (GMI)}

Compressive ghost imaging is a technique utilizing the compressive sensing methods to produce an image using a single pixel detector ${ }^{9-11}$. In our GMI, we adopted this ghost imaging technique to cells under motion: as cells are passing through a static, randomly patterned illumination area, fluorophores within the cells are continuously excited and the signals are detected by a single pixel detector. This measurement allows us to acquire temporally modulated waveforms of the fluorescence intensity from each cell experiencing multiple illumination spots at a time without switching the illumination pattern (Fig 1A). Lastly, combinatorial use of the acquired temporal waveform and a priori knowledge of the illumination pattern allows us to computationally reconstruct the cell image (Fig 1B).

\section{Ghost cytometry (GC)}

The more significance lies in our demonstration wherein the morphology-based cell classification was realized by directly applying machine learning-based 
analysis to the temporal waveforms without producing cell images (Fig 1C) $)^{8}$. GC starts with training of a machine learning-based classifier: many temporal waveforms of ghost imaging signals were acquired and labeled by cell types, fluorescence or other markers, to be used as a training data set. In testing, in turns, the trained model predicts the label from the imaging signals without using information of the labels. We used support vector machine (SVM) in this work.
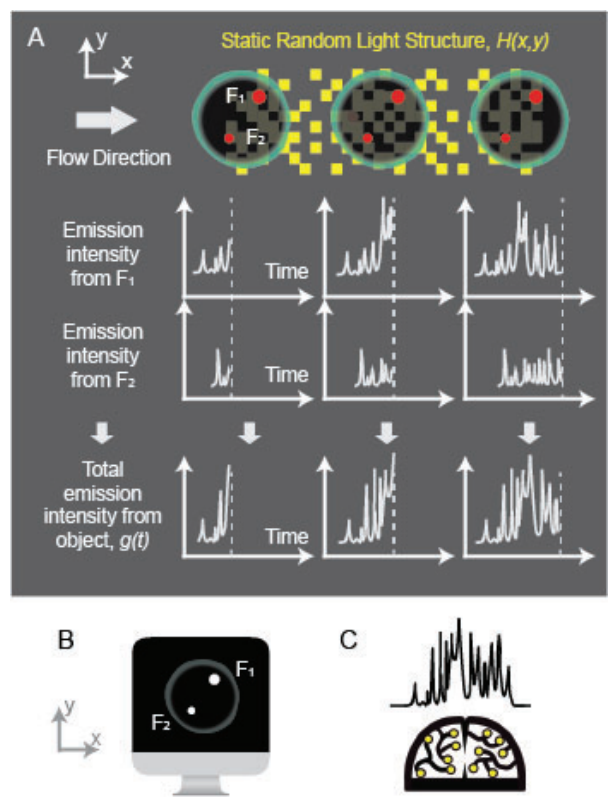

Figure 1. (A) Compressive ghost imaging process of objects under motion, wherein total fluorescence emission intensity is temporally modulated and recorded by a single pixel detector as the flowing cell experiences different excitation patterns according with time. While the image can be recovered (B), by training and testing a machine learning model, the imaging waveform signals can be directly analyzed and classified without image recovery $(\mathrm{C})$.

\section{Results and Discussion}

\section{GMI-based image recovery of flowing fluorescent} beads and its analysis

We show image-production capability of GMI by acquiring and analyzing images of flowing fluorescence beads having different size and intensity (Fig 2). We performed the GMI of 600 fluorescent beads, consisting of 300 each of two different sizes and intensities, under a hydrodynamic focus in flow. Using an experimentally measured illumination pattern, we computationally processed the waveforms to build a library of the GMI images (Fig 2A). From this library, we analyzed singlet bead images to determine bead diameter and total fluorescence intensity by Canny edge detection and integrating the cropped images, respectively. As shown in Fig 2B, obtained histograms of both diameter and fluorescence intensity produce a characteristic bimodal distribution with peaks at the expected diameters and intensities. Furthermore, when we compare these histograms with those of forward scatter and total fluorescence intensity measurements of the same bead samples using a standard flow cytometer (JSAN, Bay Bioscience), gated to include only singlets, the results show good consistency (Fig 2C).
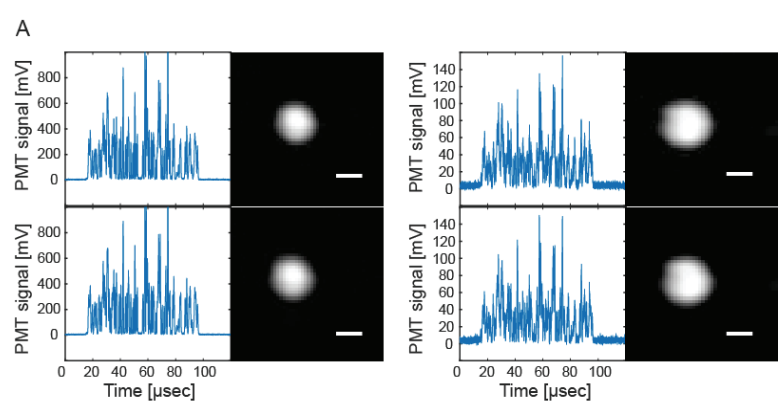

B
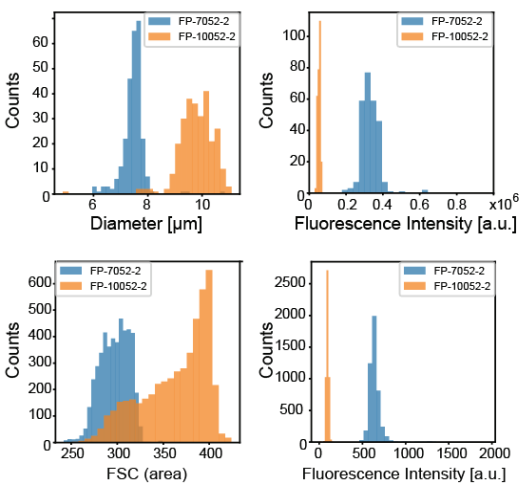

Figure 2. GMI image reconstruction and analysis in flow implementations. (A) Example waveforms from fluorescence beads in a high-speed flow and their reconstructed images (cropped). Left and right panels were from different kinds of beads (Fluorescent Yellow Particles, FP-7052-2 and FP-10052-2, Spherotech, with mean diameters of $7.4 \mu \mathrm{m}$ and 10.2 
$\mu \mathrm{m}$, respectively). (B) Left and right panels are histograms of diameters and total fluorescence intensity, respectively. We obtained them by using the single-bead images from a library of the reconstructed images. We cropped the reconstructed images into a size of 32 by 32 pixels and calculated their diameters and total intensities by Canny edge detection and integrating the cropped images, respectively. (C) Left and right panels are histogram of forward scattering and fluorescence intensity, respectively, measured by using a commercialized flow cytometer (JSAN). All scale bars $=5 \mu \mathrm{m}$.

Moreover, we performed PSNR measurements of fluorescence beads in the PDMS device used previously ${ }^{8}$. Using a reference bead image taken by a conventional microscope (Fig 3A), we obtained moderately good PSNRs of $28.8 \mathrm{~dB}$ and $29.0 \mathrm{~dB}$ for two example reconstructed bead images, respectively (Fig. 3B).
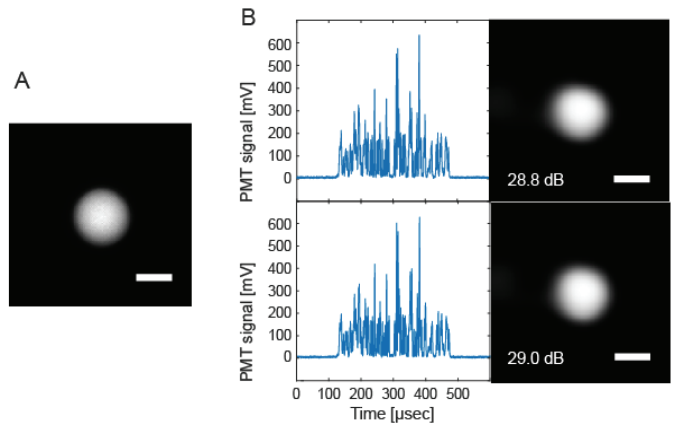

Figure 3. (A) Left panel is a fluorescence image of a fluorescent bead (FP-7052-2), taken by a conventional microscope (Olympus), used as a reference for calculating PSNR. This image was taken on a slide glass with a 20x objective (UPLSAPO 20x, Olympus) and an arrayed pixel camera (ZWO ASI1600MM). (B) Right panels show example compressive waveforms from the same kind of beads flowing in a PDMS fluidic device and their reconstructed and cropped images. The PSNRs were calculated with images adjusted to scale, cropped to 134 by 134 pixels, and normalized by intensity. Each bead image shown here is normalized with their max intensities for enhancing their visibilities. All scale bars $=5 \mu \mathrm{m}$.

While achieving moderately good PSNRs, it is also worth noting that, in general, image recovery in compressive sensing is computationally costly, and image qualities are affected by calibration errors rather than the theoretical limit. Our image-free imaging cytometry bypasses both of these issues by utilizing machine learning directly on the waveforms.

\section{GC-based image-free classification of cells}

Using the training and testing process of $\mathrm{GC}$, which is concisely described above, we classified MCF-7 and MIA PaCa-2 cells that are morphologically similar but different. In the experiment, both cells were fluorescently stained using green dyes (LIVE/DEAD ${ }^{\mathrm{TM}}$ Fixable Green Dead Cell Stain Kit, for $488 \mathrm{~nm}$ excitation), measured at short temporal width of $<100 \mu \mathrm{sec}$, and classified by applying the machine learning model on the fluorescence "imaging" waveforms. The classification result recorded the area under a receiver operating characteristic (ROC) curve (AUC) of 0.971 over about fifty thousands of cells ${ }^{8}$.

In addition, we separately confirmed the morphological characteristics of the MCF-7 and MIA PaCa-2 cells to further understand the high accuracy of our GC-based classification. It is well known that different samples of even the same cell lines can have different sizes $^{12-13}$, depending on various conditions including culture and fixing methods. In our case, the two cell lines are experimentally shown similar in average size (Fig 4A) and have different morphological characteristics (Fig 4B). In the experiment, the cells were fixed and stained using the green dyes of fixable green. By flowing the two cell types separately into a commercialized flow cytometer (JSAN), we first measured the peak intensity of the green fluorescence and the forward scattering signals from each cell. The resultant plots in Fig $\mathbf{4 A}$ shows that the two cell types share their fluorescence intensity and size. By flowing the two cell types separately into an image flow cytometer (ImageStreamX, 20x objective lens), we then generated a library of fluorescence images of the cell populations. We preprocessed the data set in this image library using IDEAS ${ }^{\circledR}$ software (Merck Millipore Inc.) to identify a population of focused single cells ${ }^{14}$ before cropping raw images around the center of mass into images of $28 \times 28$ pixels and randomly rotating them with a multiple of 90 degrees, wherein error images (pixel saturation or part of cells are cut off) were removed. 1,000 of single MCF-7 cells and 1,000 of single MIA PaCa-2 
cells were used for training, respectively, while 100 of single MCF-7 cells and 100 of single MIA PaCa-2 cells were used for testing, respectively. To the image dataset which was lastly normalized in a cell-type independent way, we performed an image-based SVM classification. The AUCs obtained was 0.967 , confirming that the cell types are morphologically distinguishable even at relatively low (20x) magnification, corresponding to an image pixel size of approximately $1 \mu \mathrm{m}$.

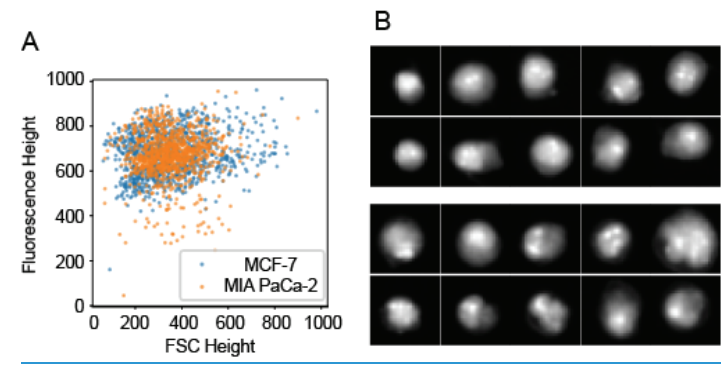

Figure 4. (A) Scattering plot of the intensity height of green fluorescence and the height of forward scattering intensity for MCF-7 and MIA PaCa-2 cells, obtained using a commercialized flow cytometer (JSAN), showing that the cell lines are similar in the fluorescence intensity and size. (B) Example images of the data set (top ten images are MCF-7 cells and bottom ten images are MIA PaCa-2 cells), obtained using a commercialized image flow cytometer (ImageStreamX, using 20x objective lens). This image data set was used for an image-based classification using the SVM-based model.

Combining the comprehensive discussions above, the two cell lines are similar in average size but have different morphological characteristics. In addition to this morphological difference, the classifier could detect other factors such as flowing velocities, which can be encoded in the waveforms as well. Such velocity difference could arise from morphological characteristics such as size and shape, as well as other physical characteristics such as deformability. The effect of such velocity difference also may remain in the reconstructed images in other reported flow-scanned imaging flow cytometry technologies unless the obtained imaging signals are appropriately normalized. Direct learning of waveforms without image production is not degraded by the velocity difference; the image-free approach utilizes such difference for a more robust classification. In addition to further exploring the potential of image-free approach for extracting essential cell features, we thus think that analysis of the whole waveform holds potential to utilize various types of characteristics of cells comprehensively.

\section{References}

[1] Thaunat, O., et al., "Asymmetric segregation of polarized antigen on B cell division shapes presentation capacity," Science, 335, 475-9 (2012).

[2] Samsel, L., McCoy, J. P., "Imaging flow cytometry for the study of erythroid cell biology and pathology," J. Immunol. Methods., 423, 52-59 (2015).

[3] Knoblich, J. A., “Asymmetric cell division: recent developments and their implications for tumour biology," Nat. Rev. Mol. Cell Biol., 11, 849-860 (2010).

[4] Goda, K., Tsia, K. K., Jalali, B., "Serial time-encoded amplified imaging for real-time observation of fast dynamic phenomena," Nature, 458, 1145-1149 (2009).

[5] Diebold, E. D., Buckley, B. W., Gossett, D. R., Jalali, B., "Digitally synthesized beat frequency multiplexing for sub-millisecond fluorescence microscopy," Nature Photonics, 7, 806-810 (2013).

[6] Han, Y., Lo, Y.-H., "Imaging Cells in Flow Cytometer Using Spatial-Temporal Transformation," Scientific Reports, 5, 13267 (2015).

[7] Han, Y., Gu, Y., Zhang, A. C., Lo, Y-H., "Review: imaging technologies for flow cytometry," Lab Chip, 16, 4639-4647, (2016).

[8] Ota, S., Horisaki, R. Kawamura, Y. Ugawa, M., Sato, I., Hashimoto, K., Kamesawa, R., Setoyama, K., Yamaguchi, S., Fujiu, K., Waki, K., Noji, H. "Ghost cytometry", Science, 360, 1246-1251 (2018).

[9] Bromberg, Y., Katz, O., Silberberg, Y., "Ghost imaging with a single detector," Phys. Rev. A., 79, 53840 (2009).

[10] Shapiro, J. H., "Computational ghost imaging," Phys. Rev. A, 78061802 (2008).

[11] Katz, O., Bromberg, Y., Silberberg, Y., “Compressive ghost imaging," Appl. Phys. Lett., 95, 131110 (2009).

[12] Nguyen, A. V. et al, "Stiffness of pancreatic cancer cells is associated with increased invasive potential", Integrative Biology, 8, 1232-1245 (2016).

[13] Sato, N., Mizumoto, K., Kusumoto, M., Nishino, S., Maehara, N., Urashima, T., Ogawa, T., Tanaka, M. "Up- 
regulation of telomerase activity in human pancreatic cancer cells after exposure to etoposide," Br. J. Cancer, 82, 1819-2816 (2000).

[14] Eulenberg, P., Köhler, N., Blasi, T., Filby, A., Carpenter, A.E., Rees, P., Theis, F.J., Wolf, F.A,

"Reconstructing cell cycle and disease progression using deep learning," Nature Communications, 8, 463 (2017).

Proc. of SPIE Vol. $111401114001-100$ 


\title{
Simulation of Multi-Exposure Laser Speckle Contrast Blood Flow Imaging based on Multi-Tap Charge Modulator CMOS Image Sensor
}

\author{
Panneer Selvam Sivakumar ${ }^{1}$, Keiichiro Kagawa², Christian Crouzet ${ }^{3}$, Bernard Choi ${ }^{3}$, \\ Keita Yasutomi ${ }^{2}$, Shoji Kawahito ${ }^{2}$ \\ ${ }^{I}$ Graduate School of Science and Technology, Shizuoka University, 3-5-1, Johoku, Hamamatsu 432-8011, Japan \\ TEL: +81-53-478-1342, FAX: +81-53-412-5481, \\ ${ }^{2}$ Research Institute of Electronics, Shizuoka University, 3-5-1, Johoku, Hamamatsu 432-8011, Japan, TEL: +81-53-478-1342, FAX: +81-53-412-5481 \\ ${ }^{3}$ Beckman Laser Institute, University of California, Irvine, 1002 Health Sciences Rd, Irvine, CA92612, USA \\ e-mail:kagawa@idl.rie.shizuoka.ac.jp, siva@idl.rie.shizuoka.ac.jp
}

\begin{abstract}
The laboratory-designed multi-tap charge modulator CMOS image sensor with multiple exposure patterns can realize blood flow imaging at about the video rate. The multi-tap image sensor can acquire different exposure time images simultaneously, therefore high frame rate camera is not necessary. Thus, low power is achieved and low processing capability is required. The captured images with a 4-tap image sensor were simulated with real measured images by a high-speed camera whose frame rate was $40 \mathrm{k}$ frames per second. Two different exposure patterns, where the ratio of the shortest to longest exposure time were 1:4 and 1:8, respectively, were compared.
\end{abstract}

\section{Introduction}

Multi-exposure laser speckle contrast imaging (MELSI) is a wide-field and non-contact optical method to image the blood flow velocity[1] and it has been applied widely to clinical studies. When coherent laser light illuminates a moving sample like red blood cells, a speckle pattern is generated and the pattern fluctuates over the range of exposure time, $T$. The fluctuation is characterized by the correlation time, $\tau_{\mathrm{c}}$. The relationship between the velocity, $v$, the wavelength, $\lambda$, and $\tau_{\mathrm{c}}$ is given by $v=\lambda /\left(2 \pi \tau_{\mathrm{c}}\right)$. The conventional MELSI systems can reproduce wide-field blood flow velocity maps by using high-speed cameras operating at a high frame rate of around 1000fps. In general, the rate of blood flow change caused by pulsation is about $1 \mathrm{~Hz}$. To observe the pulsation, the video rate is satisfactory. However, high frame rate is required due to fast changing of the speckle pattern. The aim of this study is to build the MELSI system to monitor the blood flow change with a moderate frame rate of around the video rate (typically $30 \mathrm{fps}$ for progressive scan), which contributes to low power consumption and requires less processing capability.

\section{MELSI with 4-tap charge modulator}

To bridge the gap between 30fps and 1000fps, a laboratory-designed CMOS image sensor based on a multi-tap charge modulator[2] shown in Fig. 1 is utilized in this work. This image sensor modulates the photo-generated charges at a high frequency to detect the speckle fluctuation with four pieces of charge storage and puts out the images simultaneously at around the video rate. In other words, the four images for sub-frame exposures, which are programmable, are read out. The generated photo-charges in the photodiode (PD) is quickly transferred to one of the storage diodes (FDs) and the drain. GD is a gate which drains unnecessary charges. This turns on while reading images. The charge transfer to the storage diode is controlled by G1-4 that correspond to FD1-4, respectively. While GX (X=1-4) is on, FDX is exposed. Thus, arbitrary exposure is implemented by programming the GX signal. Two examples of exposure pattern are discussed in this paper (Fig. 2). The unit exposure time is denoted by $T_{0}$. The effective exposure times are synthesized with the four images that are obtained with the exposure patterns G1-4. Because multiple images for the same effective exposure time are synthesized in some cases, the SNR is increased by summing them up. The exposure pattern in Fig. 2 (b) has longer exposure time, so that this can be suitable for observing low velocities.

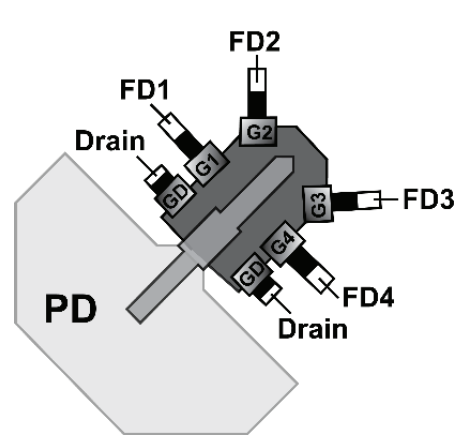

Fig. 1. Example pixel structure of 4-tap charge modulator

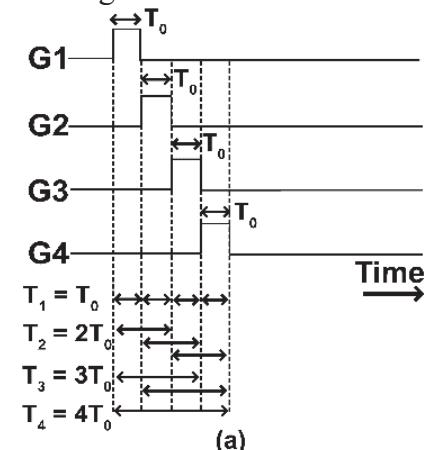

(a)

Fig. 2. Exposure patterns: (a) equal exposure and (b) exponential-like exposure

\section{Measurement Setup and Simulation}

A ground glass plate placed on a motorized stage was used as a sample to emulate the blood flow and was illuminated by expanded laser beam at the wavelength of $785 \mathrm{~nm}$. Speckle images $(160 \times 32$ pixels $)$ were captured with a high-speed 
imaging lens with the focal length of $25 \mathrm{~mm}$ and F/1.6 was used. The acquisition of speckle images was carried out for $1 \mathrm{~s}$ and nine data sets each of which is as long as $102.4 \mathrm{~ms}$ (composed of 4096 images) were prepared. As a reference, dense contrast curves that consists of 13 exposure times $(25 \mu \mathrm{s}-102.4 \mathrm{~ms})$ were calculated based on a binary tree method[3]. Multi-tap images $\left\{I^{i}(x, y)\right\}$ (i $\left.=1-4\right)$ were emulated as follows:

$$
I^{i}(x, y)=\sum_{n=0}^{4095} I\left(x, y ; n T_{\min }\right) G_{i}\left(n T_{\min }\right)
$$

where $(x, y)$ denotes the pixel position. $i$ is the index of tap. $I(x, y ; t)$ represents captured high-speed images. $G_{\mathrm{i}}(t)$ is an exposure pattern function for tap-i. $T_{\min }$ and $T_{0}$ were $25 \mu \mathrm{s}$ and $3.2 \mathrm{~ms}$, respectively, in simulation. Speckle contrast, $K^{2}$, was calculated in the region of interest (ROI) of $12 \times 12$ pixels.

\section{Results and Discussion}

The measured or simulated speckle contrast was fitted by MATLAB fminsearch function with the following equation[4].

$$
K^{2}=\left[\left\{\beta \rho^{2}\left(\frac{e^{-2 x}-1+2 x}{2 x^{2}}\right)+4 \beta \rho(1-\rho)\left(\frac{e^{-x}-1+x}{x^{2}}\right)+v_{n}\right\}\right]
$$

In fitting, we assumed that all photons were Doppler-shifted, which made $\rho$ equal unity. In the reference high-speed camera case, $\beta$, which describes the speckle averaging effect, $x=T / \tau_{\mathrm{c}}$ ( $T$ is an effective exposure time), and the noise factor, $v_{\mathrm{n}}$, were estimated. However, in the 4-tap cases, $\beta$ was determined in a different method and only two parameters were estimated because estimating three parameters from noisy four measured images was not easy. In simulation, $\beta$ of 0.0672 , that was given by the $K_{\max }^{2}$ for the shortest exposure time, $25 \mu \mathrm{s}$, in the static condition was used[3]. Fig. 3 shows simulated contrast values and fitted contrast curves for the two exposure patterns. Fig. 4 shows the relationship between the actual and estimated velocities. In the equal exposure case, the standard deviation for high velocities is smaller than that of the exponential case because the number of averaged contrast images is larger. However, the change in the estimated velocity is small for low velocities because the longest effective exposure was shorter than that of the exponential case.

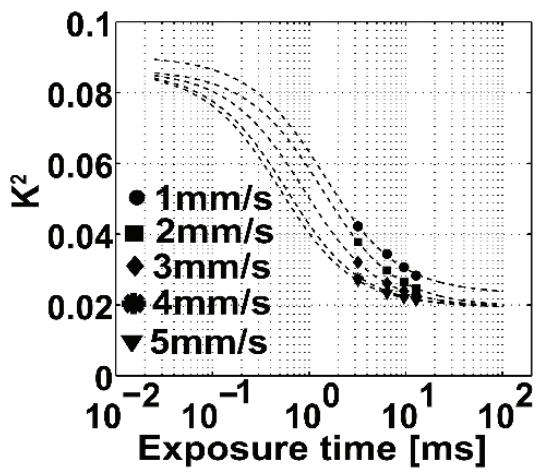

Fig. 3 Simulated contrast values and fitted speckle contrast curves for (a) equal and (b) exponential exposure patterns

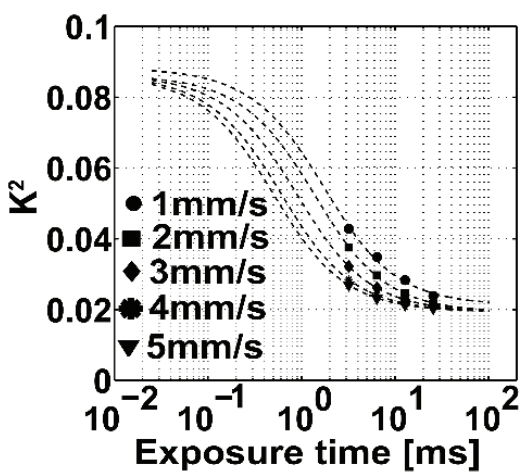

Exposure time [ms]

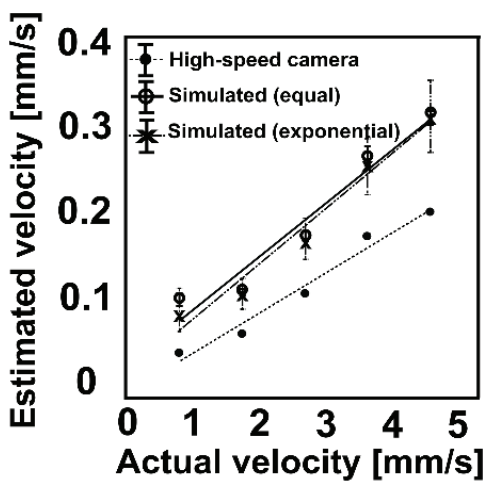

Fig. 4. Comparison of estimated velocity

\section{Conclusion}

The MELSI system with lower frame rate requirement was built with a laboratory-designed CMOS image sensor based on a multi-tap charge modulator. Two exposure patterns with different ratios of the shortest and longest exposure times were simulated with real high-speed camera images. Comparison of the estimated velocities showed that the equal exposure case was able to measure high velocities with small errors. From the simulation, it is confirmed that the multitap charge modulator CMOS image sensor can be applied to monitor wide-field blood flow change.

Acknowledgement: This work is partially supported by Grant-in-Aid for Scientific Research (B) Number $18 \mathrm{H} 01497$ and (S) Number $18 \mathrm{H} 05240$.

\section{References}

[1] Ashwin B. Parthasarathy et al., "Robust flow measurements with multi-exposure speckle imaging," Optics Express 16, 1975-1989 (2008).

[2] Keita Kondo et al., "A built-in drift-field PD based 4-tap lock-in pixel for time-of-flight CMOS range image sensors," Int'l Conf. on Solid State Devices and Materials, J-7-03 (2018).

[3] Martin Hultman et al., "A 15.6 frames per second 1-megapixel multiple exposure laser speckle contrast imaging setup," J. Biophotonics 11, e20170069 (2018) 


\title{
In vivo 3D image reconstruction of lamina cribrosa in glaucoma eyes
}

\author{
Jutamash Wongwai ${ }^{\mathrm{a}}$, Anita Manassakorn ${ }^{\mathrm{b}}$, and Prathan Buranasiria* \\ ${ }^{a}$ Department of Physics, Faculty of Science, King Mongkut's Institute of Technology Ladkrabang, Bangkok, Thailand; \\ ${ }^{b}$ Department of Ophthalmology, Chulalongkorn University, King Chulalongkorn Memorial Hospital, Bangkok, Thailand \\ "prathan.bu@kmitl.ac.th
}

\begin{abstract}
The purpose of the study was to reconstruction of lamina cribrosa (LC) in human eyes in vivo by using the image from optical coherence tomography (OCT). The study uses the image from a glaucoma subject where glaucoma subjects have an abnormally optic disc and visual field. The 3D image of $\mathrm{LC}$ is reconstructed by image processing and $3 \mathrm{D}$ reconstruction processing from the $2 \mathrm{D}$ image that acquired by OCT. The results showed that the structure of LC is meshwork.
\end{abstract}

\section{Introduction}

Glaucoma is a group of eye diseases that the optic nerves have been damaged. This damage is often caused by abnormally high pressure in the eye. Glaucoma is one of the causes of blindness. The target for glaucomatous damage is the LC, a meshwork of collagen fibers which allow nerve fibers of the optic nerve pass through the sclera [1-3]. The resulting glaucomatous damage is characterized by pattern loss of visual field. Currently, there are more than 70 million glaucoma patients around the world. About $10 \%$ of them finally have been losing their vision. In Thailand, there are about 1.7-2.4 million glaucoma patients. Many patients have vision loss due to not receive the appropriate treatment. There is no signal warning in all forms of glaucoma developing. Therefore, there is no treatment of patients who have beginning glaucoma disease. There are many risk factors: having high internal eye pressure, being over age 60, and having a family history of glaucoma. In the diagnosis, ophthalmologist checks the eye: visual acuity (VA) testing, eye pressure testing, visual field (VF) testing, and the optic disc and retina testing. In addition, OCT have recently been used to measure the thickness of the nerve fiber layer as well.

\section{OCT}

OCT is a non-invasive, non-contact, and depth-resolved imaging technique based on low-coherence interferometry. The principle of OCT is based on Michelson interferometer with a light source of the short coherence length. After passing through a beam splitter, the light is split into two beams, where one is sent to the reference mirror, and the other beam is sent into the sample. The backscattered signal from the sample and the reflected signal from reference mirror combine and generate an interference pattern. By changing the path length of the reference arm, the signal backscattering reflected from the layers at different depth of the sample as an A-scans. Conversely, changing the path length of the sample arm, the signal is acquired by the transverse section of the sample as a B-scan. The image is generated by interference of the signal. The early OCT system is known as time-domain OCT (TD-OCT). As a result, an A-scan image is produced. The later of OCT system is Fourier-domain OCT (FD-OCT) that includes two difference term of system setup: spectral-domain OCT (SD-OCT) and swept-source OCT (SS-OCT) [4].

\section{Image processing}

Image processing is the method to convert an image into digital form and perform some operations on it, in order to get an enhanced image, remove noise out of the image, and segment of the interested image for getting useful information from the signals.

\section{Image enhancement}

Image enhancement is the process of adjusting the image so that the results are more suitable than the original image for the specific application. For example, sharpening or brightening an image, and removing noise out. Image enhancement methods can be done on either spatial or frequency domain techniques.

\section{Thresholding}

Image thresholding is a simple method of image segmentation that converts the image into a binary format which the two levels are assigned to pixels that are below or above the specified threshold value. These methods can separate dark side and light side of the image. Image thresholding method can be separated into two steps as following:

1) determining a threshold value by maximizing the total variance between the object and background, 2) converting the original image into the threshold image by using a threshold value.

\section{D image reconstruction}

$3 \mathrm{D}$ image reconstruction is the method for creating of three-dimension image from a group of 2D-images as following: 
2) convert into grayscale,

3) invert grayscale image,

4) image enhancement processing,

5) image threshold processing,

6) convert $2 \mathrm{D}$ images into a $3 \mathrm{D}$ image.

\section{Result}

In our investigation, we used 10 slides of 2D image from OCT as our input as described in section 3 . The images were proceeded with image processing method and then were converted into a 3D image using method in section 3. Analysis revealed that the structure of LC in glaucoma eye as the meshwork as shown in Fig. 1. Then, the 3D image was cut cross section into 10 slides to show that the structure of LC was clearly meshwork as shown in Fig. 2.
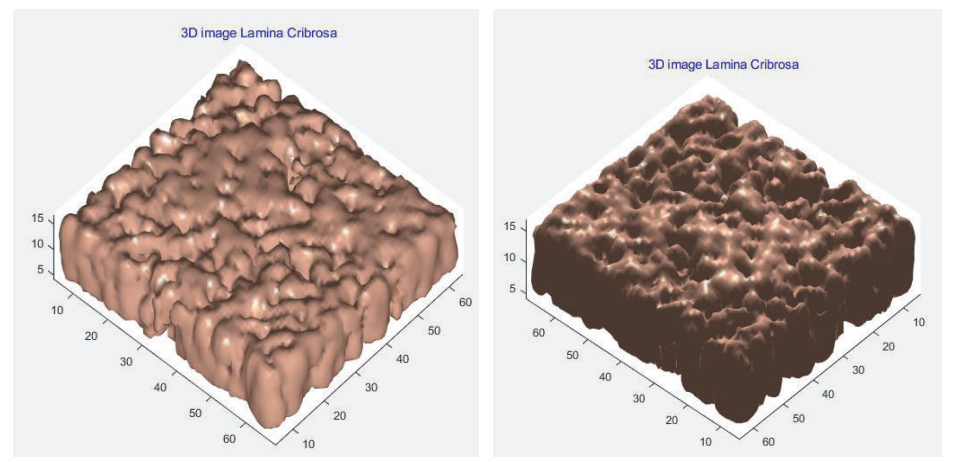

Fig. 1: Example of 3D reconstruction images of LC from glaucoma subject

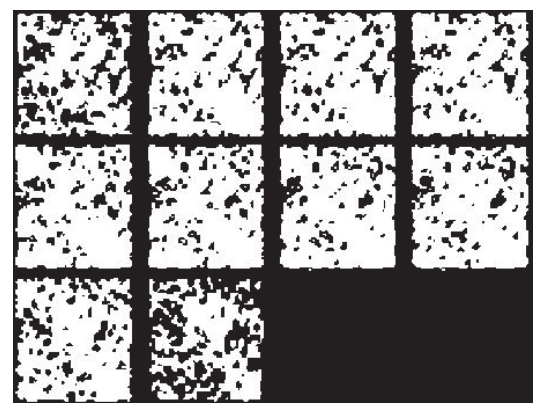

Fig. 2: The slide of 2D image from OCT with image processing

\section{Conclusion}

The study was designed to reconstruction the structure of 3D image LC in glaucoma eye from 2D images from OCT imaging. From our cross section images of our 3D reconstructed image using our process, the structure of LC in glaucoma is meshwork which is followed the real structure of LC.

\section{Reference}

[1] Zach Nadler, Bo Wang, Gadi Wollstein, Jessica E Nevins, Hiroshi Ishikawa, Richard Bilonick, Larry Kagemann, Ian A. Sigal, R Daniel Ferguson, Ankit Patel, Daniel X. Hammer, and Joel S. Schuman, "Repeatability of in vivo 3D lamina cribrosa microarchitecture using adaptive optics spectral domain optical coherence tomography," Biomedical Optics Express, Vol. 5, No. 4, 1114-1123 Apr 2014.

[2] Tae-Woo Kim, Larry Kagemann, Michel J. A. Girard, Nicholas G. Strouthids, Kyung-Rim Sung. Christopher K. Leung, Joel S. Schuman, and Gadi Wollstein, "Imageing of the Lamina Cribrosa in Glaucoma: perspectives of Pathogenesis and Clinical Applications," Curr Eye Res, 903-909 Sep 2013.

[3] Bo Wang, Jessica E. Nevins, Zach Nadler, Gadi Wollstein, Hiroshi Ishikawa, Richard A. Bilonick, Larry Kagemann, Ian A. Sigal, Ireneusz Grulkowski, Jonathan J. Liu, Martin Kraus, Chen D. Lu, Joachim Hornegger, James G. Fujimoto, and Joel S. Schumann, "In Vivo Lamina Cribrosa Micro-Architecture in Healthy and Glaucoma Eyes as Assessed by Optical Coherence Tomography,” Investigative Ophthalmology \& Visual Science, Vol. 54, No. 13, 8270-8274 December 2013.

「41 Chieh-Li Chen and Ruikang K. Wang. “Optical coherence tomography based angiography 「invitedl.” Biomedical Optic Express. Vol. 8. No. 2. 


\title{
Reversible transformation of DNA gels using light signals
}

\author{
Suguru Shimomura*a, Takahiro Nishimura ${ }^{\mathrm{b}}$, Yusuke Ogura ${ }^{\mathrm{a}}$ and Jun Tanida ${ }^{\mathrm{a}}$ \\ ${ }^{\text {a }}$ Graduate school of Information Science and Technology, Osaka University, 1-5 Yamadaoka, Suita, \\ Osaka 565-0871, Japan \\ ${ }^{\mathrm{b}}$ Graduate School of Engineering, Osaka University, 2-1 Yamadaoka, Suita, Osaka 565-0871, Japan
}

\begin{abstract}
In this study, we propose a method for reversible transformation of DNA gels using light signals. Use of light is effective to induce gel-sol transition with spatial and temporal control. By changing the viscosity of DNA gels in association with the gelation and solation according to optical signals, the mobility of micrometer-sized objects such as cells can be controlled. The reversible transitions of DNA gels were induced by the denaturation control of double-stranded DNA utilizing photothermal effect of quenchers. In the experiment, we demonstrated the spatial formation and decomposition of DNA gels according to light signals with two wavelengths.
\end{abstract}

Keywords: DNA gel, Optical transition, photothermal conversion.

\section{Introduction}

A DNA gel is a high-viscosity material constructed by self-assembly of many DNA motifs [1]. Its property changes according to the design of DNA sequences and their modifications with specific molecules. The DNA gels are biocompatible and thus they are useful in biomedical applications including protein production and molecular detection $[2,3]$. Moreover, the state change of DNA gels in response to epithelial cell adhesion molecules provides selective trapping of living cells because the DNA gels can regulate cell's mobility owing to the high viscosity [4]. Therefore, flexible state transition of DNA gels enables to control the motion of cells which leads to estimation of cell migrations.

In this research, we propose an optical method for reversible transformation of DNA gels. Use of optical control enables to change the shape of DNA gels spatially, temporally and remotely. By the reversible transformation according to light signals, the viscosity of DNA gels can be changed on demand, and therefore the mobility of micrometer-sized objects is dynamically controlled. So far, we demonstrated optical formation and decomposition of DNA gels as separated schemes using different light signals $[5,6]$. By combining them into a single scheme, reversible transformation of DNA gels can be achieved. To demonstrate reversible transformation of DNA gels, we irradiated a DNA solution containing designed DNA components on a slide glass with two wavelengths of light and observed it with a fluorescence microscope to show validity of the method.

\section{II optical transformation of DNA gels}

Figure 1 shows a schematic diagram of an optically transformable DNA gel. DNA solution for the reversible transformation of DNA gels consists of Y-motif DNAs (YDNAs), Linker DNAs (L-DNAs), and Cap-DNA (CDNAs). DNA gels are constructed by linking of Y-DNAs through L-DNAs. For reversible transformation of DNA gels, the sticky ends of the L-DNA and the both end of the C-DNA are modified with different kinds of quenchers. At initial state, C-DNAs bind with the sticky ends of Y-DNA and prevent Y-DNAs from binding L-DNAs. When excitation light of the quenchers which are attached to $\mathrm{C}$ DNAs irradiates the DNA solution, thermal energy is generated near C-DNAs. This energy induces the denaturation of double-stranded DNA, and C-DNAs are separated from Y-DNAs [7]. Then, the separated Y-DNAs can bind with L-DNAs. As a result, DNA gels can be formed in the irradiation area. For decomposition of the DNA gels, the quenchers which are attached to the sticky ends of L-DNA are optically excited. The bonds between Y-DNA and L-DNA are cleaved, and then DNA gels are decomposed. After that, the cleaved Y-DNAs can bind with C-DNAs again, and the DNA solution returns to the initial state. These reactions depend on the wavelengths of the irradiation light for excitation of each quencher. Thus, DNA gels can be transformed according to light signals.

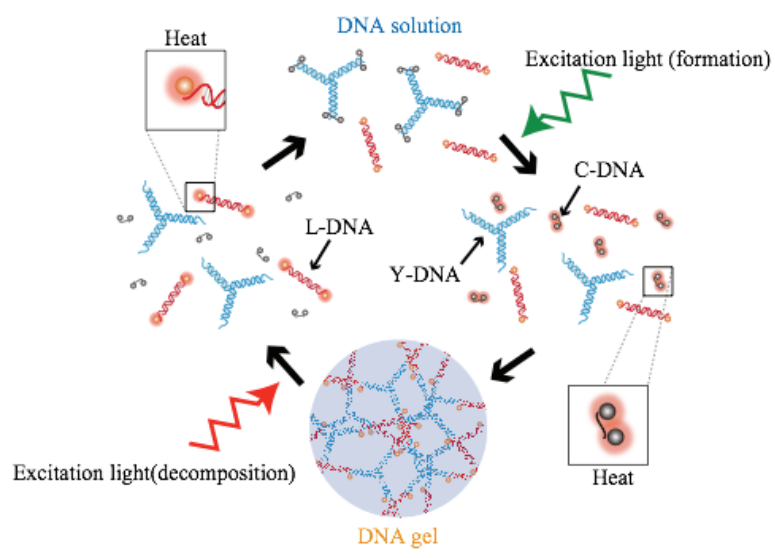

Figure 1. Schematic diagram of an optically transformed DNA gel 


\section{Transformation of DNA gels corresponding to light irradiation with two wavelengths}

To investigate that the designed DNA gels can be transformed according to light signals, we observed the DNA solution under the light irradiation by using a fluorescence microscope. Figure 2 shows an optical set up for the light irradiation and the observation of DNA gels. The quenchers which are attached to the C-DNA and the L-DNA were BHQ-1 (absorption wavelength: $534 \mathrm{~nm}$ ) and BBQ-650 (absorption wavelength: $650 \mathrm{~nm}$ ), respectively. The wavelengths of the excitation light to form and to decompose DNA gels were $532 \mathrm{~nm}$ (light intensity: 137.1 $\mathrm{W} / \mathrm{cm}^{2}$ ) and $660 \mathrm{~nm}$ (light intensity: $35.7 \mathrm{~W} / \mathrm{cm}^{2}$ ). Each excitation light was focused at the sample plane. For observation of DNA gels, the DNA solution was stained with fluorescence dye DAPI (excitation wavelength: 360 $\mathrm{nm}$, fluorescence wavelength: $460 \mathrm{~nm}$ ).

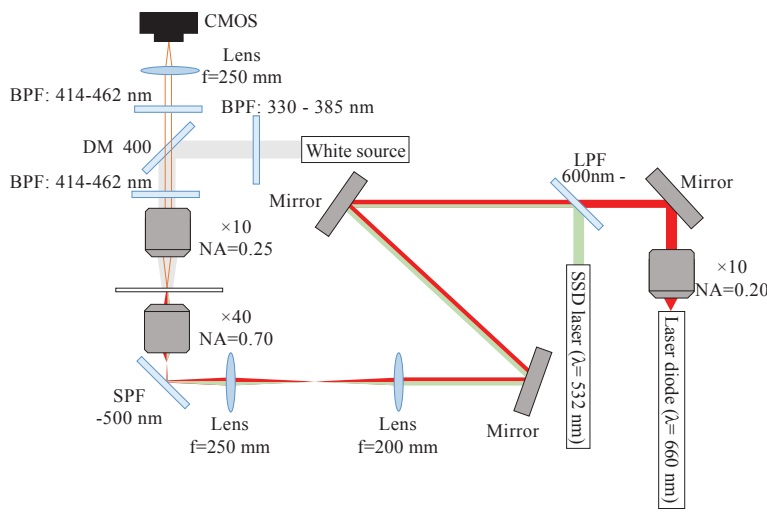

Figure 2. Optical setup. LPF: long-pass filter, BPF: band-pass filter, DM: dichroic mirror, f: focal length.

Figures $3(a, b)$ show the fluorescence images of the DNA solution before and after the light irradiation to form the DNA gels. The DNA solution was irradiated with the excitation light (wavelength: $532 \mathrm{~nm}$ ) for $30.0 \mathrm{sec}$. The fluorescence intensity profiles before and after the irradiation are shown in Fig 3 (c). Each intensity value is normalized so that the minimum and the maximum intensities in each line are 0 and 1 . The local fluorescence intensity after the irradiation became higher locally than that before the irradiation. These results indicate that DNA gels are formed by the excitation light.

Next, we irradiated the DNA gels with the excitation light (wavelength: $660 \mathrm{~nm}$ ) to decompose them. Figures $4(\mathrm{a}, \mathrm{b})$ show the fluorescence images before and after the irradiation for $450.0 \mathrm{sec}$, and Fig 4 (c) shows the profiles of fluorescence intensity along blue and red lines. In the irradiation area, the fluorescence intensity decreased. This result indicates that DNA gels were locally decomposed by excitation light for the decomposition. Based on these results, we experimentally demonstrated the reversible transformation of DNA gels according to the wavelengths of excitation light.
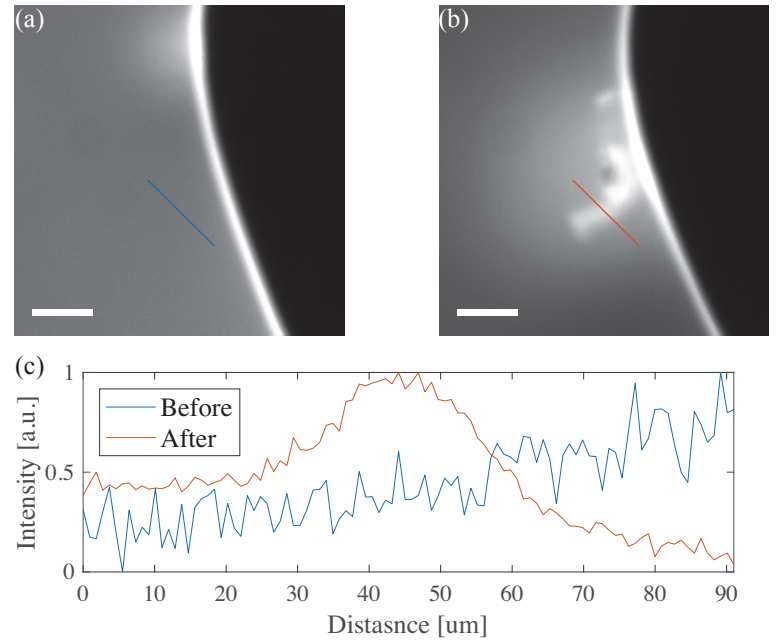

Figure 3. Fluorescence images (a) before irradiation and (b) after irradiation for $30.0 \mathrm{sec}$. Scale bars indicate $30.0 \mu \mathrm{m}$. (c) Intensity profiles along blue and red lines in Fig. $3(a, b)$.
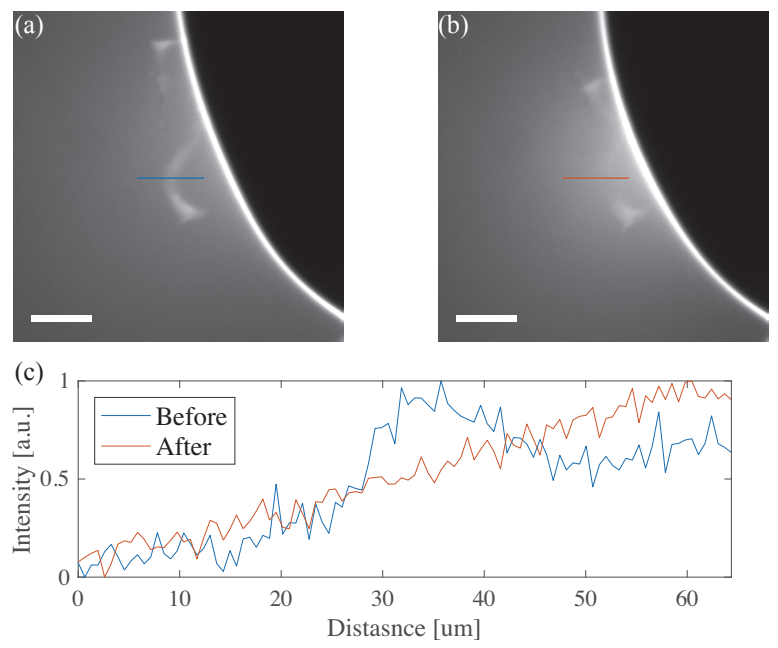

Figure 4. Fluorescence images (a) before irradiation and (b) after irradiation for $450.0 \mathrm{sec}$. Scale bars indicate $30.0 \mu \mathrm{m}$. (c) Intensity profiles along blue and red lines in Fig. 4 (a, b).

\section{Conclusion}

In this study, we demonstrated that DNA gels were formed and decomposed reversibly according to light signals. 
Future works include spatio-temporal generation of viscosity distribution by transformation of DNA gels according to light.

\section{Acknowledgement}

This research was supported by "Program for Leading Graduate Schools" of the Ministry of Education, Culture, Sports, Science and Technology, Japan, and by JSPS KAKENHI Grant Number 18K19922.

\section{References}

[1] Li, Y., Tseng, Y. D., Kwon, S. Y., d'Espaux, L., Bunch, J. S., McEuen, P. L., and Luo, D, "Controlled assembly of dendrimer-like DNA," Nature materials, 3(1), 38 (2004).

[2] Park, N., Um, S. H., Funabashi, H., Xu, J., and Luo, D, "A cell-free protein-producing gel," Nature materials, 8(5), 432 (2009).

[3] Zhang, L., Lei, J., Liu, L., Li, C., and Ju, H, "Selfassembled DNA hydrogel as switchable material for aptamer-based fluorescent detection of protein, " Analytical chemistry, 85(22), 11077-11082 (2013).

[4] Song, P., Ye, D., Zuo, X., Li, J., Wang, J., Liu, H., Wang, J., Liu, H., Hwang, M. T., Chao, J., Su, S., Wang, L., Shi, J., Wang, L, Huang, W., Lal, R., and Fan, C., "DNA hydrogel with aptamer-toehold-based recognition, cloaking, and decloaking of circulating tumor cells for live cell analysis, " Nano letters, 17(9), 5193-5198 (2017).

[5] Shimomura, S., Nishimura, T., Ogura, Y., and Tanida, J, "Photothermal fabrication of microscale patterned DNA hydrogels," Royal Society open science, 5(2), 171779 (2018)

[6] Shimomura, S., Nishimura, T., Ogura, Y., and Tanida, $\mathrm{J}$, "Patterning of DNA hydrogels by photodecomposition with visible light," Microfluidics, BioMEMS, and Medical Microsystems XVII , SPIE Photonics West 2019 BIOS, 10875-22 (3 February 2019).

[7] Ogura, Y., Onishi, A., Nishimura, T., \& Tanida, J. "Optically controlled release of DNA based on nonradiative relaxation process of quenchers, " Biomedical optics express, 7(6), 2142-2153 (2016). 


\title{
New methodology for tumor detection in mammograms image.
}

\author{
Luis Cadena ${ }^{* a}$. \\ ${ }^{a}$ Department of Electric and Electronic Engineering, Universidad de las Fuerzas Armadas ESPE, \\ Av. Gral Ruminahui s/n, Sangolqui, Ecuador
}

\begin{abstract}
Breast cancer is a serious and become common disease that affects thousands of women in the world each year. Early detection is essential and critical for effective treatment and patient recovery. This work gives an idea of extracting features from the mammogram image to find affected area, which is a crucial step in breast cancer detection and verification. We present the affected area identification through in which place the tumor cells are extracted directly from the grey scale mammogram image. To remove noise from the mammogram image this work presents a simple and efficient technique using fast average filter, to determine the pixel value in the noise less image. To contour detection used shearlet transform and classic filters as like Sobel, and others. To evaluate the quality of contour used SSIM measure. Our experimental results demonstrate that our approach can achieve the better performance in time duration of reduce noise and with shearlet transform select affected area with high efficiency.
\end{abstract}

Keywords: medical image, mammogram image, fast average filter, edge detection, shearlet transform, SSIM measure.

\section{Introduction}

Biomedical Breast cancer has been one of the major causes of death among women since the last decades and it has become an emergency for the healthcare systems of industrialized countries. This disease became a commonest cancer among women. If the cancer can be detected early, the options of treatment and the chances of total recovery will increase. American statistics classify this cancer as the second leading cause of death among women with an age between 40 and 55 years. Early detection is the key to improving breast cancer prognosis consequently many countries have established screening programs. These programs yield large volumes of mammograms. Mammogram image diagnosis is very big problem for the radiologist to get conclusion about cancer findings. The presence of cyst formation in the breast gives wrong assumption about the growth of cancer cells. Because, a cyst and a tumor can feel the same on a physical exam and they can also look the same on a mammogram. Simple cysts are benign (not cancer)

* ecuadorx@gmail.com; phone +593997221212 and don't need to be biopsied. If a mass is not fluid-filled, it is of more concern and might need to be biopsied to be sure it isn't cancer. To confirm that a mass is really a cyst, a breast ultrasound is often done. Another option is to remove (aspirate) the fluid from the cyst with a thin, hollow needle. If a mass is not a simple cyst (that is, if it's at least partly solid), more imaging tests may be needed. Some masses can be watched with regular mammograms or ultrasound, while others may need a biopsy. The size, shape, and margins (edges) of the mass may help the radiologist determine if cancer is likely to be present. The ability to improve diagnostic information from medical images can be further enhanced by designing computer processing algorithm, applications and software intelligently. Several preprocessing steps of algorithm gives initial step for cancer cell findings with in regulator mammogram from the node of image. [1-6]

The digital processing of images consists of algorithmic processes that transform an image into another in which certain information of interest is highlighted, and/or the information that is irrelevant to the application is attenuated or eliminated. Image processing techniques plays important role in medical 
image to diagnostic and detection the sicknesses and monitor the patient from this sicknesses.

Medical images are often contaminated by impulsive, additive or multiplicative noise due to a number of nonidealities in the imaging process. The noise usually corrupts medical images by replacing some of the pixels of the original image with new pixels having luminance values near or equal to the minimum or maximum of the allowable dynamic luminance range. The identification of type of noise in the medical image is carried out in two stages. In the first stage, a criterion is used to detect the presence of the impulsive noise. If the result of this criterion is negative, the image is then submitted to second stage of another criterion in order to identify either the additive or the multiplicative nature of the noise [7-9]

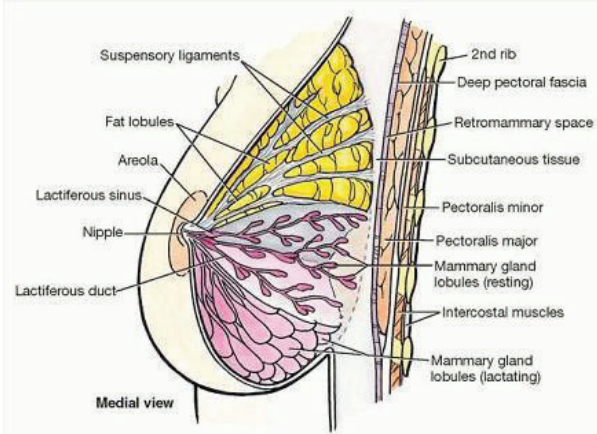

Figure 1 - Sagittal section of female breast (Moore, 2004).

This work presents a simple and efficient technique to remove noise from the medical images using fast average filter, to determine the pixel value in the noise less image. For contour detection used shearlet transform and classic filter: Sobel, Prewitt, Roberts, Canny and LoG, and to evaluate the quality egde used SSIM measure. Following we describe these methods.

\section{Image processing tools}

Average fast filter for enhancement. The arithmetic classic average filter is defined as the average of all pixels spectrum within a local region of an image. The fast average filter obtained by accumulation of the neighborhood of pixel $\mathrm{P}(\mathrm{y}, \mathrm{x})$, shares a lot of pixels in common with the accumulation for pixel $\mathrm{P}(\mathrm{y}, \mathrm{x}+1)$. This means that there is no need to compute the whole kernel for all pixels except only the first pixel in each row.
Successive pixel filter response values can be obtained with just an add and a subtract to the previous pixel filter response value $[8,12]$.

A medical image with different size was processed with the fast average filter.
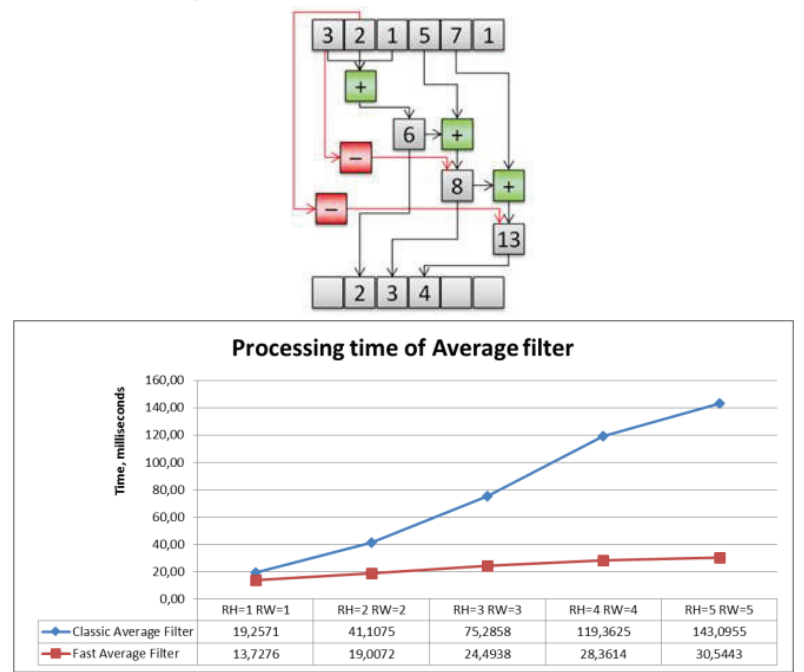

Figure 2. Comparison of classical and fast average filter. Timing of the proposed algorithm.

\section{Filters for edge detection.}

Used following filters to edge detection: Shearlet transform, Prewitt, Sobel, Roberts, LoG and Canny, and compared its by SSIM measure.

Edge by Shearlet transform. To detection edge of the image we used and modify algorithm FFST[10] and found that the edge of objects can be obtained as the sum of the coefficients shearlet transform a fixed value for the scale and the last of all possible values of the shift parameter as follow [10-11]:

$$
f_{\text {cont }}=\sum_{k=0}^{k_{\max }} \sum_{m=0}^{m_{\max }} s h_{\psi}(f(j *, k, m)),
$$

where $\operatorname{sh}_{\psi}$ assigns the coefficients of the function $f \mathrm{sh}_{\psi} f(\mathrm{j} *, \mathrm{k}, \mathrm{m})$, obtained for the last scale $\mathrm{j}^{*}$, orientation $\mathrm{k}$ and displacement $\mathrm{m}$, where $\mathrm{k}_{\max }$ - the maximum number of turns, $\mathrm{m}_{\max }$ - the maximum number of displacements.

SSIM metric. The structural similarity (SSIM) is used for measuring the similarity between two images. The SSIM index is a full reference metric; in other words, the measurement or prediction of image quality is based on an initial uncompressed or distortion free image as 
reference. SSIM is designed to improve on traditional methods such as peak signal to noise ratio (PSNR) and mean squared error (MSE), which have proven to be inconsistent with human visual perception.

The difference with respect to other techniques mentioned previously such as MSE or PSNR is that these approaches estimate absolute errors; on the other hand, SSIM is a perception based model that considers image degradation as perceived change in structural information, while also incorporating important perceptual phenomena, including both luminance masking and contrast masking terms. Structural information is the idea that the pixels have strong interdependencies especially when they are spatially close. These dependencies carry important information about the structure of the objects in the visual scene. Luminance masking is a phenomenon whereby image distortions (in this context) tend to be less visible in bright regions, while contrast masking is a phenomenon whereby distortions become less visible where there is significant activity or "texture" in the image. $[13,14]$

The mean structural similarity index is computed as follows:

Firstly, the original and distorted images are divided into blocks of size $8 \times 8$ and then the blocks are converted into vectors. Secondly, two means and two standard derivations and one covariance value are computed from the images. Thirdly, luminance, contrast, and structure comparisons based on statistical values are computed, the structural similarity index measure between images $\mathrm{x}$ and $\mathrm{y}$ is given by:

$$
\operatorname{SSIM}(x, y)=\frac{\left(2 \mu_{x} \mu_{y}+c_{1}\right)\left(2 \sigma_{x y}+c_{2}\right)}{\left(\mu_{x}^{2}+\mu_{y}^{2}+c_{1}\right)\left(\sigma_{x}^{2}+\sigma_{y}^{2}+c_{2}\right)}
$$

where $\mathrm{c}_{1}$ and $\mathrm{c}_{2}$ are constants [13]

First selected images were converted into gray images using the function in Matlab, then the metrics were implemented upon these images and last a comparison has been done between six objective evaluations.

\section{Results}

\section{Methodology}

This work propose follow algorithm to select tumor from mammograms image.

1.- Data acquisition.
2.- Image Preprocessing. Remove noise by fast average filter.

3.- Find contour using shearlet transform and classic filters as Sobel, Prewitt, Roberts, Canny, LoG.

4.- Contour evaluation with SSIM measure.

5.- Select tumor breast from shearlet transform image.

6.- Result can see in figures 3-22.

For the experiment used Notebook ASUS K75VJ core i7, 16Gb RAM, software Matlab 2015 (The MathWorks).

The figure 5 show the results obtained from the tumor search for the mammogram images.

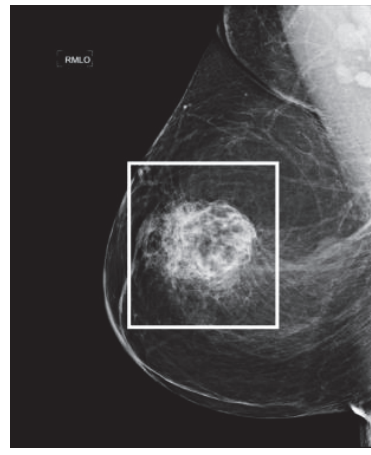

a)

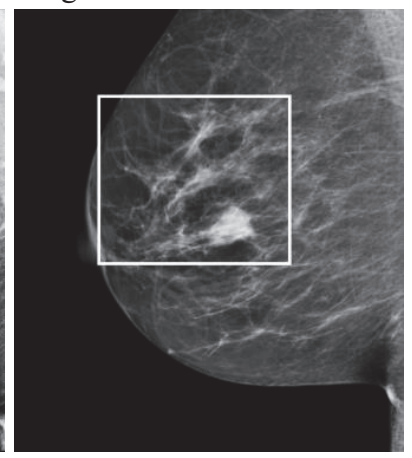

b)
Figure 3. Model images tumor breast for experiment.

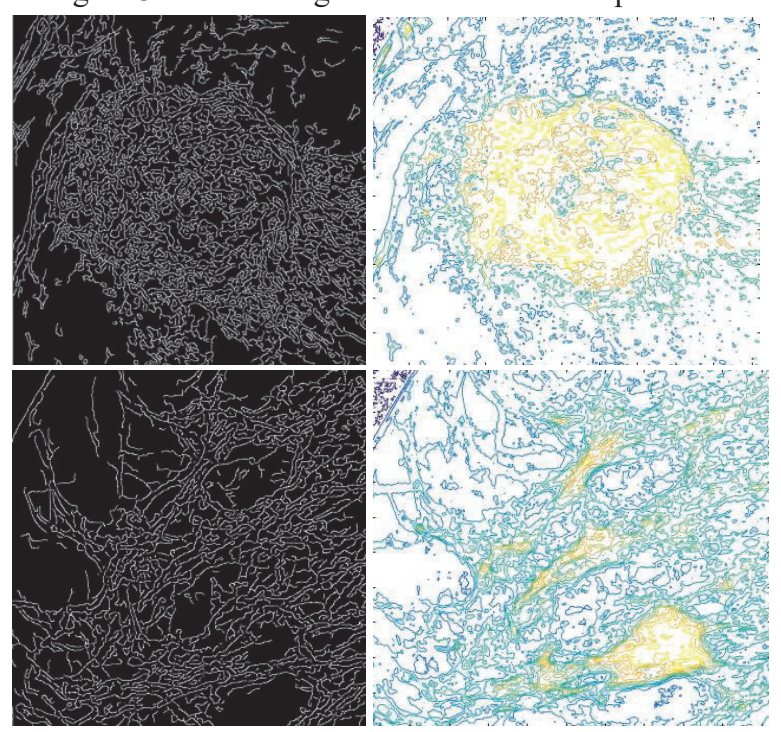

Figure 4. Contour processed by canny filter and shearlet transform for model images $a, b$.

Table 1. SSIM values for different filters for image a.

\begin{tabular}{|l|l|l|l|l|l|l|}
\hline & Shearlet & Sobel & Prewitt & Roberts & Canny & LoG \\
\hline Shearlet & 1.0000 & 0.6827 & 0.6827 & 0.6795 & 0.7135 & 0.7015 \\
\hline Sobel & 0.6827 & 1.0000 & 0.9999 & 0.9993 & 0.9939 & 0.9967 \\
\hline
\end{tabular}




\begin{tabular}{|l|l|l|l|l|l|l|}
\hline Prewitt & 0.6827 & 0.9999 & 1.0000 & 0.9993 & 0.9939 & 0.9967 \\
\hline Roberts & 0.6795 & 0.9993 & 0.9993 & 1.0000 & 0.9934 & 0.9964 \\
\hline Canny & 0.7135 & 0.9939 & 0.9939 & 0.9934 & 1.0000 & 0.9954 \\
\hline LoG & 0.7015 & 0.9967 & 0.9967 & 0.9964 & 0.9954 & 1.0000 \\
\hline
\end{tabular}

Table 2. SSIM values for different filters for image $b$.

\begin{tabular}{|c|c|c|c|c|c|c|}
\hline & Shearlet & Sobel & Prewitt & Roberts & Canny & LoG \\
\hline Shearlet & 1.0000 & 0.8968 & 0.8968 & 0.8953 & 0.9110 & 0.9044 \\
\hline Sobel & 0.8968 & 1.0000 & 0.9999 & 0.9994 & 0.9954 & 0.9978 \\
\hline Prewitt & 0.8968 & 0.9999 & 1.0000 & 0.9994 & 0.9954 & 0.9978 \\
\hline Roberts & 0.8953 & 0.9994 & 0.9994 & 1.0000 & 0.9952 & 0.9976 \\
\hline Canny & 0.9110 & 0.9954 & 0.9954 & 0.9952 & 1.0000 & 0.9962 \\
\hline LoG & 0.9044 & 0.9978 & 0.9978 & 0.9976 & 0.9962 & 1.0000 \\
\hline
\end{tabular}

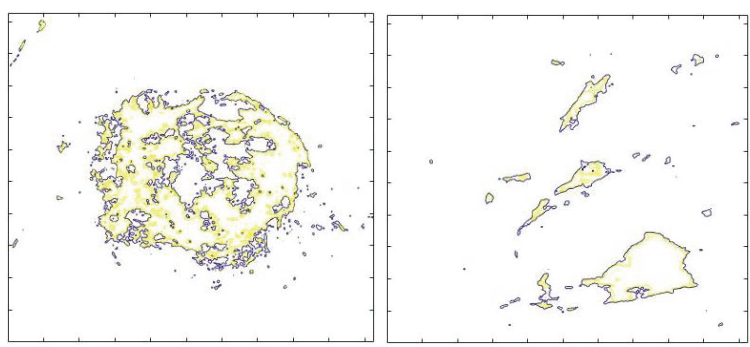

Figure 5. Select tumor breast for images a, b.

\section{Conclusion}

During experiments used fast average filter algorithm for noise suppression, which give us acceleration about 34 times for small kernel $(3 \times 3)$ and around 13 times for large kernel $(11 \times 11)$. Using shearlet transform and classics filters Sobel, Prewitt, Roberts, Canny and LoG we obtained contour of tumor of mammograms, and for evaluate quality contour we used SSIM measure with good results which show in Tables I, II. For select tumor of mammograms we used contour obtained with shearlet transform, with great precision. Our experimental results demonstrate that our approach can achieve the better performance in time duration of reduce noise and with shearlet transform select affected area with high efficiency.

\section{References}

[1] S. Saheb Basha, K. Satya Prasad. Automatic detection of breast cancer mass in mammograms using morphological operators and fuzzy c -means clustering. Journal of Theoretical and Applied Information Technology. (C) 2005 - 2009 JATIT. 704709.

[2] N. Angayarkanni, D. Kumar. Mammogram Breast
Cancer Detection Using Pre-processing Steps of Binarization and Thinning Technique. Pensee Journal. Vol 76, No. 5;May 2014. 60-65.

[3] Zhu H. Medical image processing Overview. University of Calgary. 2010. Preprint.

[4] Elizabeth A. Morris. Breast cancer imaging with MRI. Radiol Clin N Am 40 (2002) 443- 466.

[5] B. Alhadidi, M. Zubi, H. Suleiman. Mammogram breast cancer image detection using image processing functions. Information Technology Journal 6(2):217221, 2007. ISSN 1812-5638.

[6] Rita Teixeira. Computer analysis of mammography images to aid diagnosis. Monography. Universidade do Porto. 2012.

[7] Gonzalez RC, Woods RE. Digital Image Processing 3rd edition, Prentice-Hall, 2008. ISBN-13: 9780131687288, 2008.

[8] Kovalevsky Vladimir. Noise suppression. 2010. Available at http://www.kovalevsky.de/ImProc/ Noise_e.htm

[9] Abdulaziz Saleh Yeslem Bin-Habtoor et al. Removal Speckle Noise from Medical Image Using Image Processing Techniques. (IJCSIT) International Journal of Computer Science and Information Technologies, Vol. 7 (1) , 2016, 375-377.

[10] Hauser S., 2011"Fast finite shearlet transform: a tutorial," University of Kaisers-lautern. http://www.mathematik.uni-kl.de/fileadmin/image/ haeuser/software/FFST.zip

[11] Kutyniok G., Labate D. Introduction to shearlets. In Shearlets. Multiscale analysis for multivariate data. Boston, MA: Birkhauser, 2012. P. 1-38.

[12] Luis Cadena et al. "Noise Reduction Techniques for Processing of Medical Images," Lecture Notes in Engineering and Computer Science: Proceedings of The World Congress on Engineering 2017, 5-7 July, 2017, London, U.K., pp496-500.

[13] Guan-Hao Chen, et al. Edge-based structural similarity for image quality assessment. 142440469X/06/\$20.00 C2006 IEEE. ICASSP 2006.

[14] Wang Zhou, Alan C. Bovik, Hamid R. Sheikh, and Eero P. Simoncelli, "Image Quality Assessment: From Error Measurement to Structural Similarity", IEEE Transactions on Image Processing, Vol. 13, No.4, pp600-613, April 2004. 


\title{
Deep imaging techniques by spatio-temporal control of excitation pulses*
}

\author{
Keisuke Isobe ${ }^{* a}$, Kana Namiki ${ }^{b}$, Takayuki Michikawaa,b, Atsushi Miyawakia,b, Fumitaka Osakada, \\ Katsumi Midorikawa ${ }^{a}$ \\ ${ }^{a}$ RIKEN Center for Advanced Photonics, 2-1 Hirosawa, Wako, Saitama 351-0198, Japan \\ ${ }^{b}$ RIKEN Center for Brain Science, 2-1 Hirosawa, Wako, Saitama 351-0198, Japan \\ ${ }^{\mathrm{c}}$ Graduate School of Pharmaceutical Sciences, Nagoya University; Furo-cho, Chikusa-ku, Nagoya \\ 464-8601, Japan
}

\begin{abstract}
We demonstrate that a spatio-temporal modulation technique using a focal distribution modulator at $10 \mathrm{MHz}$ provides dual-color imaging with background rejection and enhanced spatial resolution. We also show the other spatio-temporal modulation technique for the wavefront measurement and its correction. In addition, we propose a dual-plane two-photon imaging system, which can image two independent focal planes at $15 \mathrm{~Hz}$.
\end{abstract}

Keywords: deep imaging, wavefront correction, dual-plane imaging, two-photon microscopy, spatio-temporal modulation

\section{Introduction}

Two-photon excited fluorescence (TPEF) microscopy has become a powerful tool for deep imaging of a biological tissue [1, though it is intrinsically difficult. Even if we use TPEF microscopy, the out-of-focus background fluorescence limits the fundamental imaging depth [2]. Wavefront distortion, which is caused by the inhomogeneous refractive index distribution of tissues, also makes deep imaging difficult because it results in resolution degradation and signal reduction [3]. In addition, there is a trade-off between the temporal resolution and imaging area. In this presentation, we present the spatio-temporal control of excitation pulses to solve these problems.

\section{Results and Discussion}

\section{The-out-of-focus background rejection}

In order to reject the out-of-focus background fluorescence, we employ nondegenerate TPEF signals excited by a combination of two-color pulses. As shown in Fig. 1(a), the spatial intensity distribution of the one excitation pulse is modulated only near the focal region by modulating the spatial phase at the pupil of an objective lens whereas that of the other excitation pulse is fixed. As a result, the spatial overlap between the two color pulses is modulated. Since the nondegenerate TPEF generation requires the spatial overlap between the two- color pulses, the TPEF intensity is modulated only near the focal region. Then, the frequency dependence of the TPEF intensity modulation varies from the center to the edge in the focal volume. Thus, by extracting the frequency component at the center, the spatial resolution can be enhanced and the out-of-focus background TPEF can be rejected. In addition, the degenerate TPEF signal excited by the modulated pulse can be used for the same purpose as the nondegenerate TPEF signal.

We applied spatial overlap modulation (SPOM) microscopy using a focal distribution modulator at 10 $\mathrm{MHz}$ to dual-color deep imaging of green and red fluorescent beads in the tissue-like phantom. Figure 1(b) shows the images observed by conventional TPEF and SPOM microscopies. We found that the two-color background TPEF was dramatically suppressed and the imaging depth could be extended by the SPOM technique.

\section{Wavefront correction}

Wavefront information can be retrieved from interference patterns created at the focal plane by selfreferencing interferometry with local phase modulation. In self-referencing interferometry, the phase of a focused beam is locally modulated at the pupil of a focusing lens by a spatial light modulator (SLM). The modulated field interferes with the unmodulated field at the focal plane. The interference patterns of the examined beam can be 


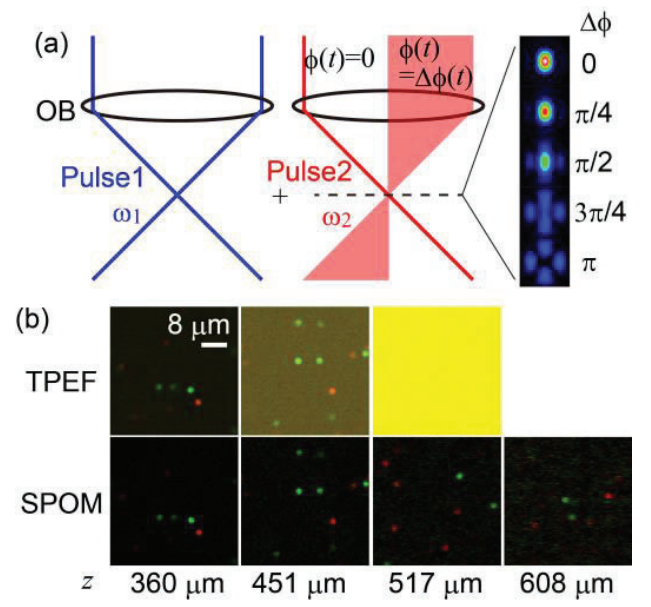

Figure 1. (a) Spatial overlap modulation (SPOM) by using a focal intensity modulator. (b) Fluorescence images of $2-\mu \mathrm{m}$ beads at various imaging depth

read through a nonlinear optical interaction of the examined beam with a probe in the same way as for TPEF microscopy. Thus, this technique has advantages for deep imaging with TPEF microscopy. Using four-wave mixing as a nonlinear optical process, the wavefront can be measured at all places in the sample without guide stars. The retrieved wavefront is fed back to the SLM and then the wavefront can be corrected.

We applied this technique to compensate for wavefront distortion in deep imaging of a mouse brain tissue. Figures 2(a) and (b) show the TPEF images before and after wavefront correction, respectively. The result clearly indicates the improvement of the signal intensity and the spatial resolution by wavefront correction.
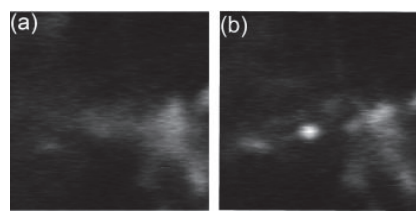

Figure 2. TPEF images of a mouse brain tissue (a) before and (b) after wavefront correction.

\section{Dual-plane imaging}

We propose a simple dual-plane imaging technique, in which two beams with different beam divergences are rapidly switched by an optical chopper. If the chopper frequency is set to a half of imaging frame rate, the irradiation of the two beams is switched at each frame. The beam divergences can be independently controlled by a SLM or a valifocal lens.

We performed in-vivo dual-plane imaging of layer $2 / 3$ neurons and layer 5 neurons in a transgenic mouse expressing GCaMP6, which is genetically encoded calcium sensors. Distance of each imaging plane was 200 $\mu \mathrm{m}$. As shown in Fig. 3, we obtained spontaneous calcium responses in layer $2 / 3$ neurons and layer 5 neurons from the dual-plane images at $15 \mathrm{~Hz}$. By using dual-plane imaging technique, the relationship between neurons in different layers could be analyzed.
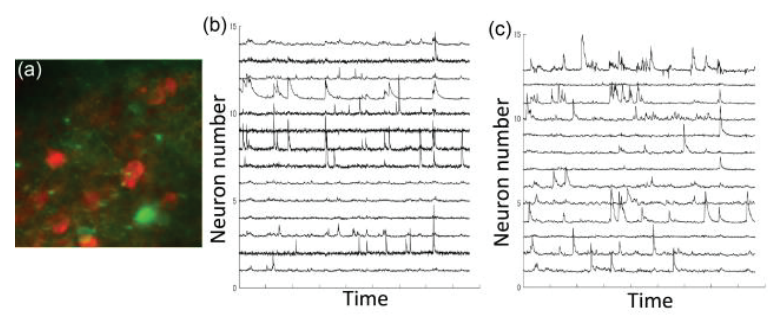

Figure 3. (a) Maximum intensity projections from TPEF images of layer $2 / 3$ neurons (green) and layer 5 neurons (red). (b,c) Spontaneous calcium responses in (b) layer 2/3 and (c) layer 5 neurons for $5 \mathrm{~min}$.

\section{Acknowledge}

This work was supported by JST PRESTO Grant Number JPMJPR14F2, JST CREST Grant Number JPMJCR1851, MEXT/JSPS KAKENHI Grant Number JP18H04750 "Resonance Bio", and the Nakatani Foundation for Advancement of Measuring Technologies in Biomedical Engineering grant program.

\section{References}

[1] Helmchen F. and Denk W. "Deep tissue two-photon microscopy," Nat. Methods 2, 932-940 (2005).

[2] Theer P. and Denk W. "On the fundamental imagingdepth limit in two-photon microscopy," J. Opt. Soc. Am. A 23, 3139-3149 (2006).

[3] Herz J. "Expanding two-photon intravital microscopy to the infrared by means of optical parametric oscillator,” Biophys. J. 98 715-723 (2010). 


\title{
A high resolution, high sensitivity, X-ray phase-contrast imaging system for applications in biology and medical imaging
}

\author{
Martin Piponnier*a, Ombeline de La Rochefoucauld ${ }^{\mathrm{a}}$, Guillaume Dovillaire ${ }^{\mathrm{a}}$, Fabrice Harms ${ }^{\mathrm{a}}$, \\ Dietmar Korn $^{\mathrm{a}}$, Jérôme Legrand ${ }^{\mathrm{a}}$, Xavier Levecq ${ }^{\mathrm{a}}$, Francesca Mastropietro ${ }^{\mathrm{b}}$, Lionel Nicolas ${ }^{\mathrm{a}}$, \\ Rakchanok Rungsawang ${ }^{\mathrm{a}}$ \\ ${ }^{a}$ Imagine Optic, 18 rue Charles de Gaulle, 91400 Orsay, France \\ bInstitut Bergonié, 229 cours de l'Argonne, 33000 Bordeaux, France
}

\begin{abstract}
Early cancer diagnosis is of paramount importance when targeting optimal treatment and prognosis, in particular for high occurrence cancer types as breast cancer. Regarding this type of cancer, it has been shown that knowing the chemical composition of breast microcalcifications helps to differentiate malign vs benign tumors. This composition is directly related to the refractive index of materials, which opens the door to non-invasive diagnosis through optical phase measurement, in-vivo, thus avoiding unnecessary biopsies. To this aim, we propose a design of an X-ray phase-contrast imaging system based on a Hartmann mask. We demonstrate high resolution $(20 \mu \mathrm{m})$ and high sensitivity $(1 \mu \mathrm{rad})$ phasecontrast imaging using a first prototype.
\end{abstract}

Keywords: X-ray, phase-contrast imaging, Hartmann, mammography, wavefront sensing

\section{Introduction}

$\mathrm{X}$-ray imaging is an essential tool for medical diagnosis. Many technical approaches and corresponding studies have been performed, targeting an increase of image-based cancer diagnosis. These studies aim at enriching the nature or increasing the quality of information recorded in X-ray images, in order to facilitate the conclusions of clinicians and avoid misdiagnosis.

Breast microcalcifications, which are recognized as a tumor marker, can be classified as malign or benign. Their chemical compositions differ and therefore their refraction indices too [1]. Absorption properties between the two are very similar, in particular for X-ray energies currently used in mammography, making it difficult to differentiate them at an early stage through X-ray absorption imaging. As the real part of their refraction indices differs, a precise measurement of the phase has the potential to provide a reliable classification of microcalcifications. X-ray phase-contrast imaging thus represents a good opportunity to achieve this aim.
Different imaging approaches have already been demonstrated [2-3]. We are developing a new approach for phase-contrast imaging, based on a Hartmann mask, targeting mammography applications. We present the principle of our phase-contrast imaging system, then report first X-ray images of nylon wires and metal parts obtained with a first prototype, in order to quantify the performance of this initial design.

\section{Phase-Contrast Imaging system}

\section{Description of the imaging system}

The system is composed of a Hartmann mask, a scintillator, a relay lens and a CCD camera (Fig. 1). The mask is made of a regular grid of square holes with a $20 \mu \mathrm{m}$ pitch, which diffracts the incident X-ray light up to the scintillator. The scintillator converts $\mathrm{X}$-rays to visible light, that is collected by a relay optical system up to the CCD camera. This design is optimized for an energy range between 5 to $25 \mathrm{keV}$. The relay system contains a lead glass to block X-rays and prevent damages to the camera.

*mpiponnier@imagine-optic.com; phone 0033164861560 


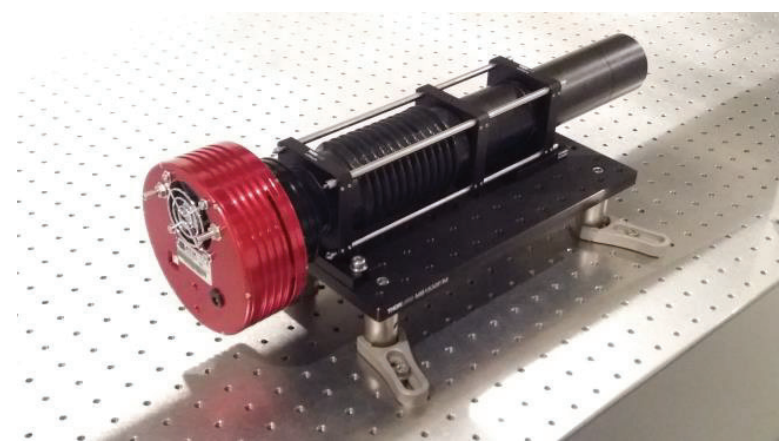

Figure 1. Picture of the imaging system $\left(50 \times 15 \times 15 \mathrm{~cm}^{3}\right)$.

\section{Phase-contrast imaging with a Hartmann mask}

The Hartmann mask is a device that is commonly used for wavefront sensing. Each hole in the mask generates a bright spot on the camera and the lateral position of the spot is proportional to the local derivative of the wavefront. Practically, a first calibration measurement is done using a well-defined wavefront (typically a plane wave or spherical wave) to get the reference position of the spots. Then an arbitrary wavefront creates a new set of spot positions. The difference between the new and the reference positions is directly linked to the deformation of the wavefront relatively to the reference beam.

In the X-ray domain, the Hartmann mask is particularly interesting, because it is easy to make (contrary to lenses) and the low level of diffraction minimizes interferences between spots.

\section{Results and Discussion}

To demonstrate the interest of our Hartmann approach for the phase-contrast imaging of breast microcalcifications, we firstly chose a nylon fiber with a $120 \mu \mathrm{m}$ diameter as a phase object and a hex key as an absorption object. Our system was placed $60 \mathrm{~cm}$ away from an Excillum Metaljet X-ray source. The sample was placed between the source and the system, at roughly $30 \mathrm{~cm}$ of the Hartmann mask. The raw image obtained with our system is presented in Figure 2.

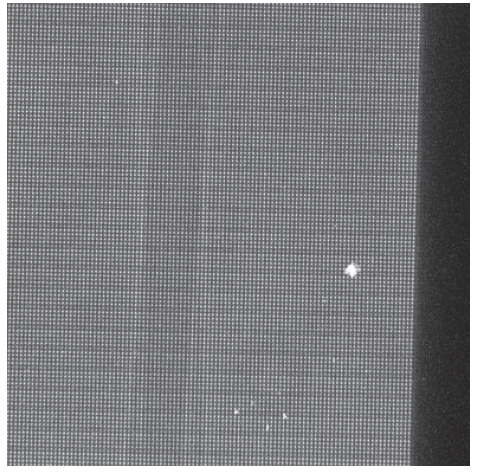

Figure 2. Image of the scene : two crossing nylon fibers (at the center of the image) and the edge of a hex key (dark zone on the right side of the image).

Two images were grabbed: one without the sample and one with the sample. For both images, we calculated the center of mass and the amplitude of each spot. The ratio of the amplitudes is proportional to the absorption of the sample and the difference of location is proportional to the deflection generated by the sample. Figure 3 illustrates the absorption and deflections generated by the samples and measured by our system, with a resolution of $10 \mu \mathrm{m}$ per pixel.

If we look at the nylon fibers, we can clearly observe deflections in the horizontal direction of $3 \mu \mathrm{rad}$ typically and an absorption of roughly $5 \%$ per nylon fiber. If we look at the hex key border, we can clearly observe a total absorption and a completely random deflection measurement.

In conclusion, we demonstrated that our Hartmann system is able to generate absorption images of objects ranging from weak absorption to full absorption level, like in standard mammography. Furthermore, it is also able to generate phase images of low absorption objects, with ultra-high sensitivity $(1 \mu \mathrm{rad})$ and resolution $(20 \mu \mathrm{m})$.

To validate our system for mammography applications, we will reproduce this experiment on more advanced samples, like a breast phantom, and compare the phase measurement to simulations. We will consider the CIRS breast phantom (model 012A), as it contains small speckles of different chemical compositions (calcium carbonate and alumina), and is therefore well suited to validate our system on breast microcalcifications 


\section{Acknowledgements}
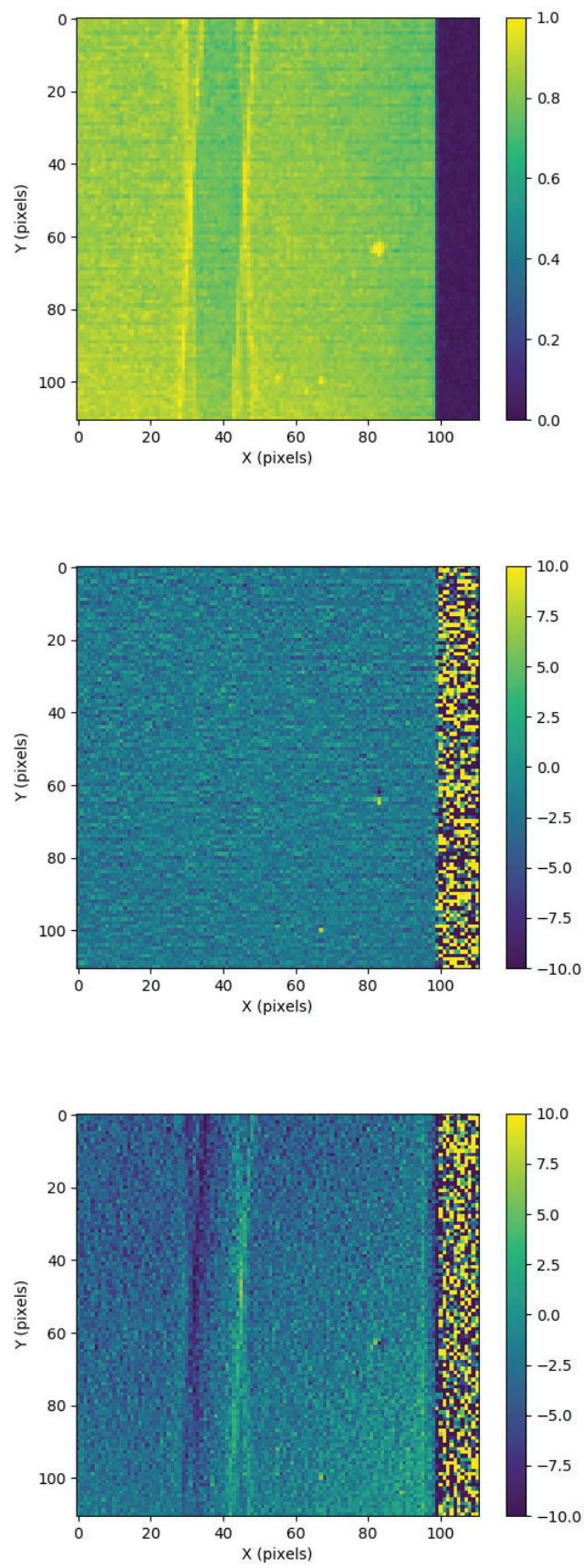

Figure 3. (Top) Measured absorption (unitless). (Middle) Measured deflection along Y ( $\mu \mathrm{rad})$. (Bottom) Measured deflection along X ( $\mu \mathrm{rad})$.

\section{References}

[1] Ghammraoui B. et al. "Investigating the feasibility of classifying breast microcalcifications using photon-counting spectral mammography: a simulation study”, Med. Phys. 44(6), (2017)

[2] Bravin, A., Coan P. and Suortti, P. "X-ray phase-contrast imaging: from pre-clinical applications towards clinics”, Phys. Med. Biol. 58, R1R35, (2013)

[3] Olivo A. et al. "Low-dose phase-contrast mammography with conventional x-ray sources”, Med. Phys. 40 (9), (2013) 


\title{
Multi-focal holographic SAX microscopy
}

\author{
Haw Hsiao ${ }^{1 *}$, Tso-Hua Wu ${ }^{5}$, Kuang-Yuh Huang ${ }^{1}$, Yuan Luo ${ }^{2,3,4}$ \\ ${ }^{1}$ Institute of Mechanical Engineering, National Taiwan University, Taipei 10051, Taiwan, R.O.C \\ ${ }^{2}$ Institute of Medical Device and Imaging, National Taiwan University, Taipei 10051, Taiwan, \\ R.O.C \\ ${ }^{3}$ YongLin Institute of Health, National Taiwan University, Taipei, 10087, Taiwan, R. O. C. \\ ${ }^{4}$ Molecular Imaging Center, National Taiwan University, Taipei, 10672, Taiwan, R. O. C. \\ ${ }^{5}$ Institute of NanoEngineering and MicroSystems, National Tsing Hua University, Hsinchu 30013, \\ Taiwan, R.O.C
}

\begin{abstract}
Multiplexed volume holographic grating (MVHGs) are diffractive elements with high wavelength and angle sensitivity. In our previous work, a non-axial-scanning confocal microscopy which combines MVHGs in illumination and corresponding confocal pinhole is proposed, in which two images at different depths can be taken simultaneously. Saturation excitation (SAX) microscopy is a super-resolution technique, which is able to breakthrough the diffraction limit of light and penetrate deeply into the sample. Here, a multi-focal super-resolution microscopy system which combines MVHGs and implementation of SAX microscopy to obtain high resolution images beyond diffraction limit at two different depths simultaneously is proposed.

Keywords: Multiplex holography, Volume gratings, Confocal microscopy, Saturation excitation, Super resolution.

\section{Introduction}

Optical microscopy is a prominent tool widely used in life sciences, since researchers can exploit it to observe biological specimens non-invasively. However, there exists a limitation of spatial resolution resulting from wave nature of light, which makes it difficult to clearly observe cells smaller than approximately half the illumination wavelength.

Super resolution microscopy, or nano-scopy, is an emerging field which is studied to breakthrough the

can be implemented by a simple modification of a conventional confocal microscope.

In our previous study [6], by combining confocal laser scanning microscopy with multiplexed volumetric holographic gratings (MVHGs) in illumination, images at two distinct depths were simultaneously obtained. Here, we proposed a multi focal holographic SAX microscopy fundamentally based on the setup in our previous work, which is able to obtain high resolution images at different depths using MVHGs.
\end{abstract} diffraction limitation of optical microscopy. Recent researches in super-resolution microscopy, including stimulated emission depletion (STED) [1] microscopy, saturated structured illumination microscopy (SSIM) [2], photoactivated localization microscopy (PALM) [3], saturation excitation (SAX) [4] microscopy and stochastic optical reconstruction microscopy (STORM) [5], have improved the spatial resolution to achieve submicron level imaging beyond the reach of conventional light microscopy.

SAX microscopy is known for its simple setup. It takes a single excitation beam to obtain super-resolution and

*107522605@ntu.edu.tw; phone+886978273649

\section{System setup}

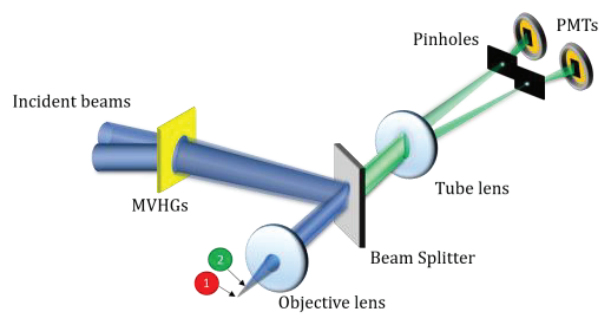

Figure 1. Multi-focal holographic confocal microscopy (MFHCM) system. 
Figure 1 shows a schematic diagram of our previous work multi-focal holographic confocal microscopic system (MFHCM). At the illumination arm (blue), an incident collimated continuous-wave $(\mathrm{CW})$ laser beam $(\lambda=488 \mathrm{~nm})$ was used to reconstruct two diffracted beams from MVHGs. Reflected by a dichroic mirror, these two diffraction beams are focused at different depth by an objective lens. At imaging arm (green), two fluorescence light beams are respectively separated by a beam splitter and pass through corresponding pinholes to remove outof-focus light. Fluorescent light from different depths is simultaneously projected onto the detector.

Fig. 2(a) [7] shows recording process of MVHGs. To record multiple gratings, by relocation of lens L0 and rotation of the signal beam with a small angle, interference pattern was sequentially recorded onto the photopolymer substrate. Recording material is a phenanthrenquinone (PQ-) doped poly methyl methacrylate (PMMA) polymer.

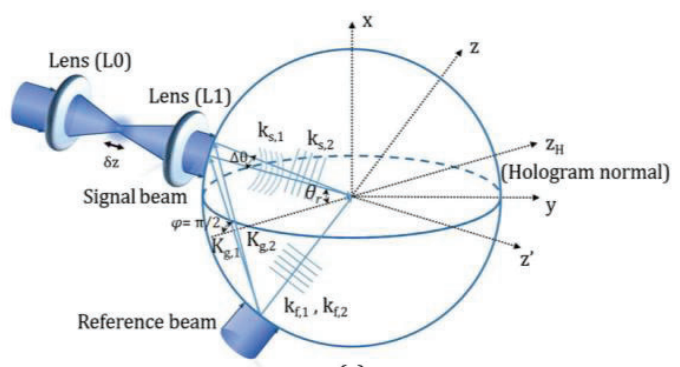

(a)

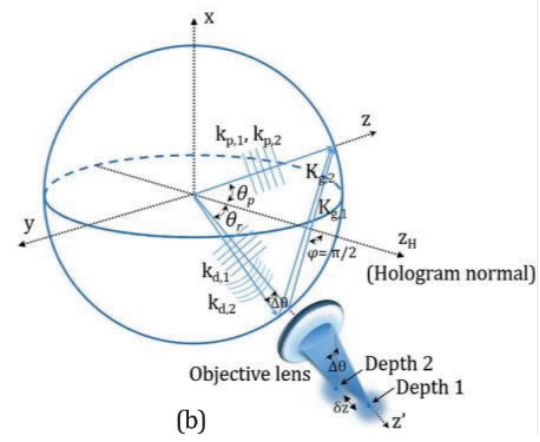

Figure 2. K-sphere diagram of (a) recording process and, (b) reconstruction condition. $\mathrm{k}_{\mathrm{p}, 1}$ and $\mathrm{k}_{\mathrm{p}, 2}$ are two probe beams satisfying the Bragg condition to simultaneously produce two diffracted beams, $\mathrm{k}_{\mathrm{d}, 1}$ and $\mathrm{k}_{\mathrm{d}, 2}$, which produce two foci at different depths in the sample space through an objective lens [6].
In this study, two acousto-optical modulators (AOM) and a lock-in amplifier are added into the system mentioned above. The schematic diagram of the proposed system is shown in Figure 3. The laser intensity was modulated temporally by exploiting two AOMs. Laser beam is divided into two by a beam splitter and each beam passes through an AOM which imposes Doppler effect in optical frequency of the diffracted light beams. The two AOMs were driven in different frequencies, and then two diffracted beams were superimposed, which is able to achieve high signal-to-noise ratio (SNR) temporal sinusoidal modulation of the excitation intensity. Modulated laser was then entered MFHCM system. Fluorescence signals from samples were detected by PMT, while harmonic demodulation of the signals was performed using a lock-in amplifier.

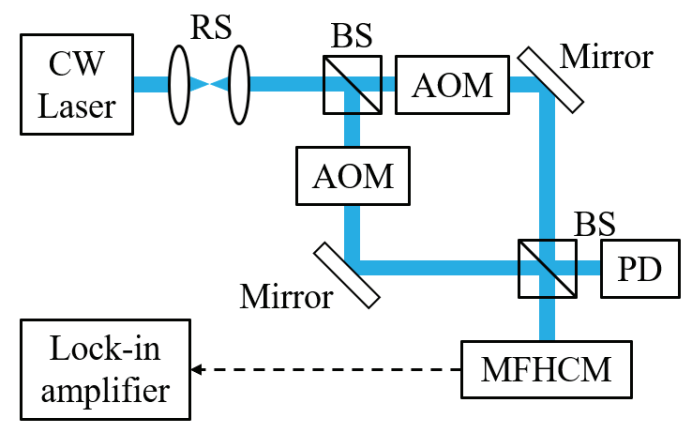

Figure 3. Multi-focal SAX microscopy system. RS: relay system; BS: beam splitter; AOM: acousto-optical modulator; PD: photodetector; MFHCM: multi-focal holographic confocal microscopy.

\section{Results}

The lateral resolution of the previous system (MFHCM) was measured by using Air Force Resolution Chart (AFRC). An image taken is shown in Figure 4(a), in which the smallest resolvable element is group 10 element 1, which corresponds to 488 nanometers in lateral resolution, while contrast of the dashed line is $76.9 \%$. 


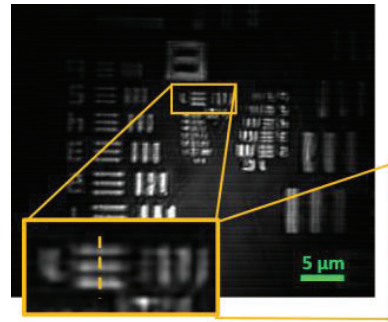

$0.488 \mu \mathrm{m}$

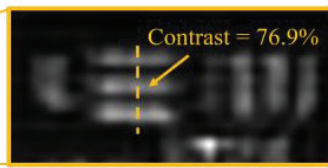

(b)
Figure 4. Lateral resolution and contrast of MFHCM system shown using AFRC standard. (a) The lateral resolution of the system can achieve $488 \mathrm{~nm}$. (b) The contrast along the dashed line is $76.9 \%$, calculated from (b).

\section{Conclusion}

Here, super-resolution microscopy which shows capability of simultaneously obtaining improved resolution images at distinct depths by combination of MVHGs and SAX microscopy is proposed. Based on confocal optical setup, two acousto-optical modulators are added for excitation modulation and a lock-in amplifier was added for harmonic demodulation. The proposed system has advantage of multidepth imaging because of multiplexed grating and saturation excitation will allow obtain the super resolution images.

In the future work, the current objectieve lens will be replaced by a high NA objective lens to obtain images which bypass the diffraction limit of light.

\section{References}

[1] Stefan W. H. and J. Wichmann, " Breaking the Diffraction Resolution Limit by Stimulated Emission: Stimulated-Emission-Depletion Fluorescence Microscopy ", Opt. Lett. 19, 780 (1994).

[2] M. G. L. Gustafsson, " Nonlinear Structuredillumination Microscopy: Wide-field Fluorescence Imaging with Theoretically Unlimited Resolution ", Proc. Natl. Acad. Sci. U. S. A. 102, 13081-6 (2005).

[3] Eric B., George H. P., Rachid S., O.W. Lindwasser, Scott O., Juan S. B., Michael W. D., Jeniffer L.S., and Harald F.H., " Imaging Intracellular Fluorescent Proteins at Nanometer Resolution ", Science 313, 1642-5 (2006).

[4] Katsumasa F., Minoru K., Shogo K., Masahito Y., and Satoshi K., " High-Resolution Confocal Microscopy by Saturated Excitation of Fluorescence ", Phys. Rev. Lett. 99, 228105
[5] Michael J. R., Mark B., and Xiao-wei Z., " Stochastic Optical Reconstruction Microscopy (STORM) Provides Sub-diffraction-limit Image Resolution ", Nat Methods. 2006 Oct; 3(10): 793-795.

[6] Po-Hao W., Vijay. R. S., Jau-Min W., King-Bin S., and Yuan L., " Non-axial-scanning Multifocal Confocal Microscopy with Multiplexed Volume Holographic Gratings ", Optics Lett. 42, 2: 346-349 (2017)

[7] Yuan L., Paul J. G., Jonathan M. W., Jennifer K. B., George B., and Raymond. K. K. " Laser-induced Fluorescence Imaging of Subsurface Tissue Structures with a Volume Holographic Spatial-spectral Imaging System ", Opt. Lett. 33, 566 (2008).

[8] George B. and Demetri. P., " Volume Holographic Multiplexing Methods Holographic Data Storage " (Springer, 2000), Vol. 21. 


\title{
Real-time 3D interactive microscopy
}

\author{
Silvio Bianchi ${ }^{\mathrm{a},{ }^{*},}$ Roberto Di Leonardo ${ }^{\mathrm{a}, \mathrm{b}}$ \\ ${ }^{a}$ NANOTEC, Institute of Nanotechnology,CNR, National Research Council, Italy \\ bepartment of Physics, "Sapienza" University of Rome, Italy \\ *silvio.bianchi.phys@gmail.com
}

\begin{abstract}
Holographic Microscopy allows for 3D visualization of colloidal/biological samples with a high framerate. We developed a 3-axis version of HM which significantly improves the axial resolution. Using GPUs we are able to analyze the holograms in real time and track objects over a large field of view. Combining this technique with optical traps we can achieve a full 3D interaction with the sample.
\end{abstract}

Keywords: holography, microscopy, micromanipulation

\section{Introduction}

Digital Holographic Microscopy allows for 3D visualization of colloidal and biological samples. Contrary to most scanning 3D microscopies, such as confocal fluorescence microscopy, digital holographic microscopy relies on a single snap to numerically reconstruct the field on the entire volume so that the framerate is limited only by the camera itself. We developed a three-axes-three-colors modification of inline holographic microscopy that improves significantly the axial resolution of standard in-line digital holographic microscopy.

\section{Description}

The working principle of our technique is shown in Fig.1. Three tilted colored beams (red, green and blue) illuminate the sample. The fringe pattern produced by the sample are recorded by an RGB camera. The field corresponding to each color channel, which is numerically reconstructed over the entire volume, refocuses in a blob elongated along the propagation axis. The overlap (i.e. the product of the field amplitudes for each color channel) produces a volumetric image whose axial resolution is greatly improved [1]
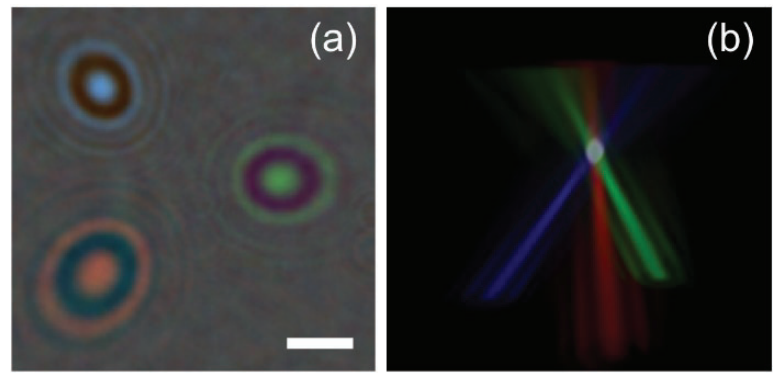

Figure 1. (a) An RGB image of a the fringe pattern (the hologram) produced by a 2 micron silica bead. (b) $3 \mathrm{D}$ reconstruction of each color channel.

We can estimate the resolution improvement with the following simple argument. Assuming that for each color the reconstructed scattered field has a Gaussian transverse profile, the overlap will be also a 3D Gaussian having a ratio between the axial $\left(\mathrm{w}_{\mathrm{z}}\right)$ and transverse $\left(\mathrm{w}_{\mathrm{x}}\right)$ standard deviations 


$$
\frac{w_{z}}{w_{x}} \approx\left(\frac{1}{\sin (\alpha)^{2}}-\frac{1}{2}\right)^{1 / 2}
$$

Where $\alpha$ is the tilt angle of the incident beams.

\section{Application to a biological system}

Our technique seems to be particularly suited for observing the dynamics of swimming cells [1,2]: for instance, E. coli bacteria have a body length of approximately $2 \mu \mathrm{m}$ and propelled themselves at an average speed of about $20 \mu \mathrm{m} / \mathrm{s}$ by spinning a bundle of helical flagella. A reasonable time resolution for tracking would be such that the cell body is displaced by $1 / 10$ of its size between frames, giving a required frame rate of about $100 \mathrm{fps}$. This requirement has to be combined with a submicron spatial resolution in both transverse and axial directions to allow precise tracking of both position and orientation of the swimming cell. Also, if the trajectory has be observed for at least a few seconds, a threedimensional field of view of about $50 \times 50 \times 50 \mu^{3}$ is required. The combination of all these requirements rules out the possibility of using microscopy techniques relying on a mechanical scan of the focal plane, i.e., confocal microscopy, since they hardly reach video-rate frame rates for a single plane.

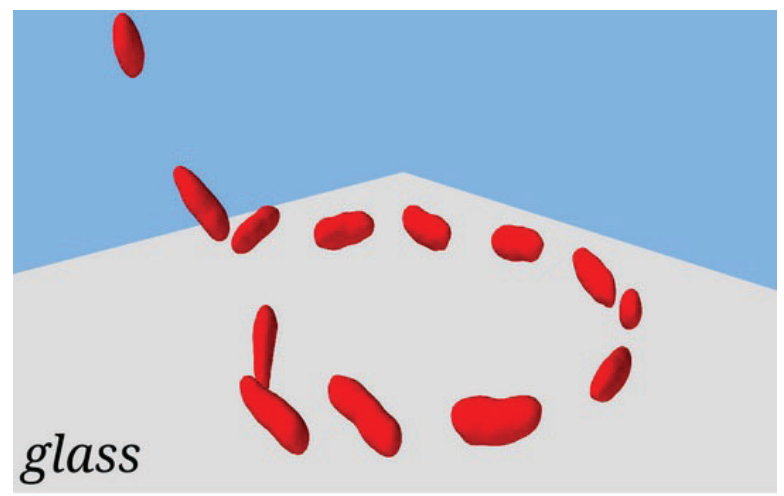

Figure 2. Sequence of reconstructions of an E. coli cell (red) approaching a solid wall and swimming close it.

\section{Real-time 3D imaging and micromanipulation}

As other microscopy techniques, our method can be easily combined with holographic optical trapping. Numerical reconstruction of the sample, as well as phase mask generation for holographic optical traps are particularly suited for parallel computing. We exploit the computational power of Graphic Processing units (GPU) to speed-up the computation/analysis of holograms and visualize and interact in real-time the sample.

\section{References}

[1] F. Saglimbeni, S. Bianchi, A. Lepore, and R. Di Leonardo, "Three-Axis Digital Holographic Microscopy for High Speed Volumetric Imaging”, Opt. Express 22 (2014).

[2] S. Bianchi, F. Saglimbeni, A. Lepore, and R. Di Leonardo, "Polar Features in the Flagellar Propulsion of E. Coli Bacteria", Phys. Rev. E 91 (2015).

[3] S. Bianchi, F. Saglimbeni, and R. Di Leonardo, "Holographic imaging reveals the mechanism of wall entrapment in swimming bacteria”, Phys Rev. X 7 (2017). 


\title{
Label-free imaging of live cell morphology by path-length stabilized quantitative phase microscope
}

\author{
Toyohiko Yamauchi ${ }^{\mathrm{a}}$,, Osamu Yasuhiko ${ }^{\mathrm{a}}$, Hidenao Yamada ${ }^{\mathrm{a}}$ \\ ${ }^{a}$ Hamamatsu Photonics K.K., 5000 Hirakuchi Hamakita-ku, Shizuoka, 434-8601, Japan \\ ${ }^{b}$ Beckman Laser Institute, University of California Irvine, 1002 Health Sciences Rd, Irvine CA, 92612, \\ USA
}

\begin{abstract}
We have constructed a compact quantitative phase microscope (QPM) with a stabilization system of the optical path disturbance due to environmental noise. We have successfully obtained optical phase images of live cells without optical tables. QPM is in principle a label-free but the users can also put the fluorescence imaging to correlate with the QPM images.

Keywords: quantitative phase microscope, label-free imaging, live imaging
\end{abstract}

\section{Introduction}

Cell morphology and its dynamics are widely studied intrinsic properties of live cells. For example, the motility of cancer cells correlates with their metastatic properties, and the membrane fluctuations of red blood cells correlate with their membrane stiffness. In order to quantitatively visualize the dynamic morphology of live cells, we have developed a compact microscope named "Low-Coherent Quantitative Phase Microscope" (LC-QPM), which has the active stabilization of the path-length disturbance and works without optical tables [1].

Quantitative phase microscopes, also known as digital holography microscopes, have been applied to cell biology because optical thickness of live cells can be obtained non-invasively[2-3]. Among the QPMs, our compact setup with low-coherent illumination is able to obtain full-field distribution of optical thickness without speckle noise or halo. Our microscope resolves the phase of the light-field and is able to visualize the membrane morphology in a sub-wavelength resolution.

In this presentation, we show noiseless quantitative phase images and time-lapse movies obtained with live cell culture. The cells were kept intact during the image acquisition and showed their membrane fluctuations in a sub-wavelength resolution. Moreover, because our setup has an option to add the fluorescence imaging capability, some of our results come with an additional labeled fluorescence information.

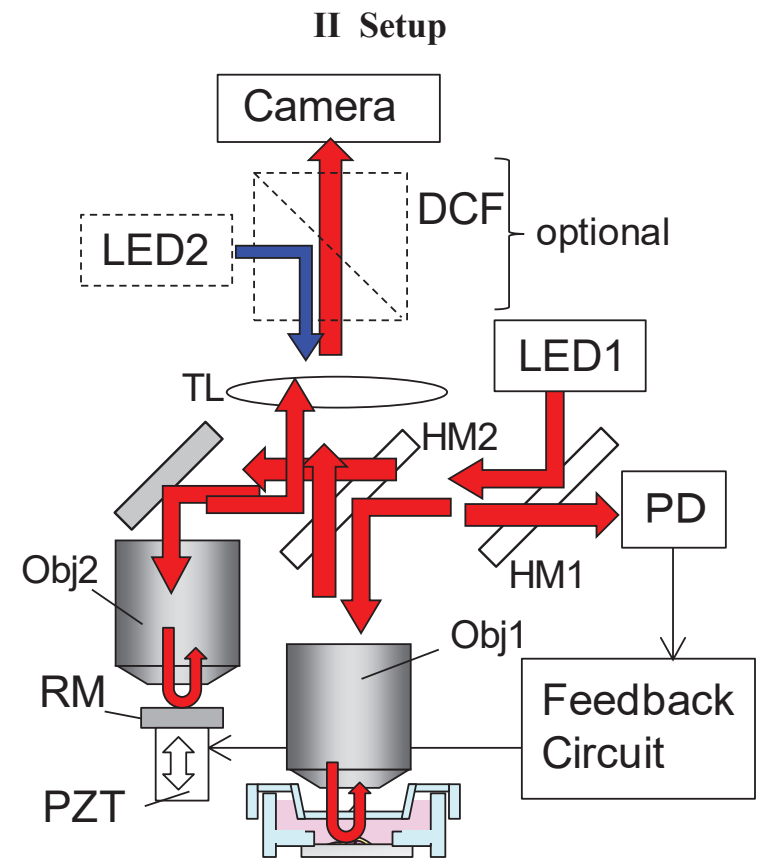

Fig.1: Schematic illustration of our QPM. Objs: Objective Lenses, RM: Reference Mirror, PZT:

Piezoelectric Transducer, PD: Photo detector, DCF:

Dichroic Filter. HMs: Half Mirrors

Figure 1 illustrates our LC-QPM based on a Linnik interferometer. The light from a light-emitting diode (LED) is incident on the Linnik interferometer, with objective lenses in both the sample and reference arms. The reflected light from the reference mirror and the sample are projected onto the camera. When the path- 
length of the sample and the reference arm are balanced, full-field interference fringes appear on the camera.

The photo detector (PD) in the setup provides the signal for the feedback control. The mechanical vibration in the interferometer is compensated and the circuit precisely controls the quarter wavelength phase-shifting [1]. The piezoelectric transducer in the reference arm is modulated at $20 \mathrm{kHz}$ with a small amplitude of 10 20 nanometers, and the fundamental and higher harmonic frequency components of the modulation are detected by the PD. The circuit processes the signal from the PD by digitally implemented lock-in amplifiers and the ratio of the fundamental and the second harmonic components tells the offset phase in the interferometer. The circuit is capable of calculating the offset phase at a sampling rate of $20 \mathrm{kHz}$, and it controls the piezo to cancel the deviation, and enables well-calibrated stepwise quarter wavelength phase shifting.

Our software made by Python is able to process the stream of the interferogram from the camera and display the unwrapped and background-corrected phase images in real time (6 unwrapped and background-corrected phase images per second for 1280 x 960 pixels). Because the OPL is stabilized during the camera exposure, users are able to choose the camera for their application from a wide variety of option, including the ones with a rolling shutter.

In order to observe biomedical samples by means of our reflection-type setup, we prepared a specially fabricated half mirror coated glass-bottom culture dish. During cell imaging, the temperature of the dish was kept at 37 degree Celsius.

As an option, the users can put a commercially available dichroic filter cube (DCF) with an additional fluorescent excitation LED in order to correlate the label-free modality by QPM and the conventional labeled modality by fluorescence imaging.

\section{Results}

Fig. 2 (a) shows a quantitative phase image of a live $\mathrm{CHO}$ cells. The objective lens was a 20x one (Olympus, LUCPLFLN 20X, NA = 0.45) and a CCD camera (Basler, acA1300-30um, 1/3 inch, 1280 x 960 pixels, 30 fps) was used for the image acquisition. We adopted a near- infrared LED (CREE, XPEFAR-L1, center wavelength = $730 \mathrm{~nm}$ ) as a light source in order to minimize cell damage by light irradiation. The uniformity visualized in Fig. 2 (b) shows 1.8 nanometers of the standard deviation in the background and this flatness without any halo enables the visualization of the cell boundaries by a simple thresholding. Moreover, we have processed the segmentation of individual cell areas by means of watershed algorithm (Fig. 2 (c)).
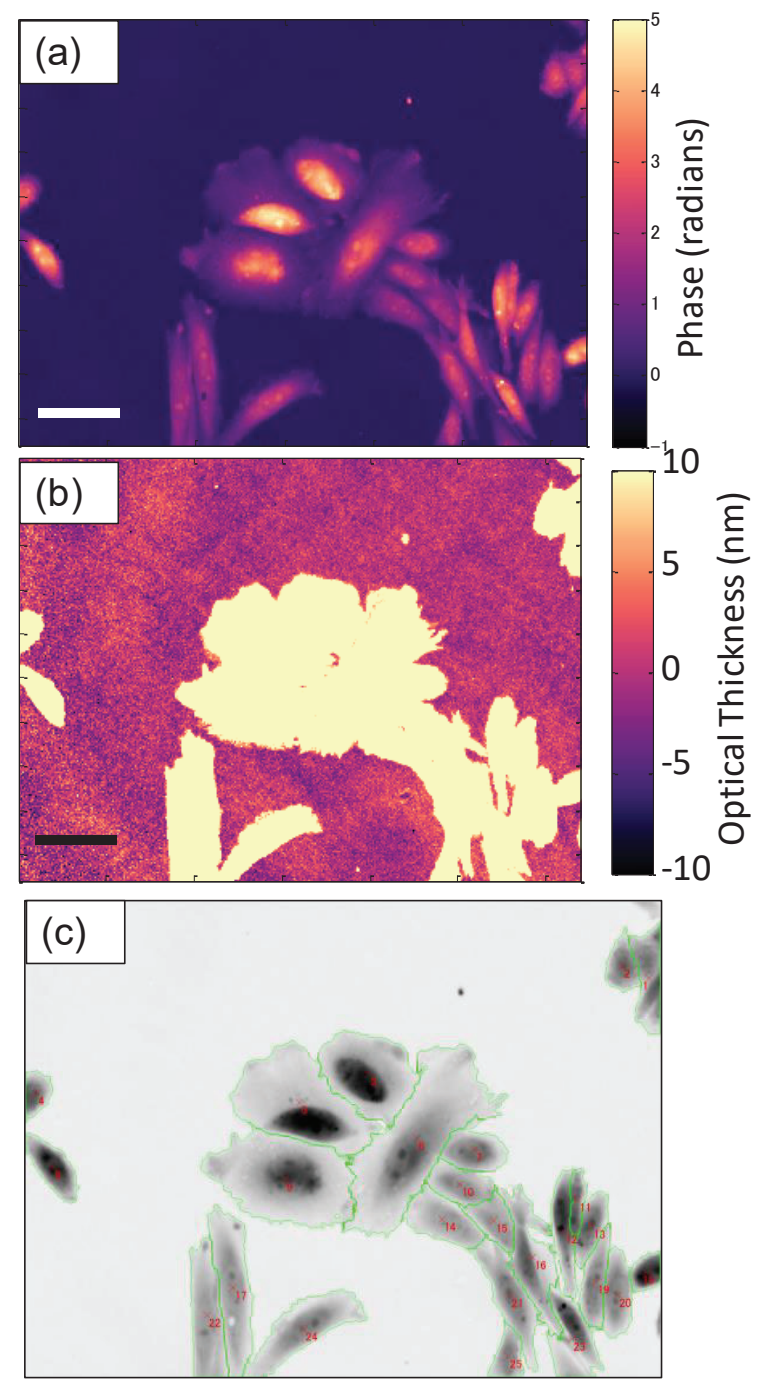

Fig. 2: (a) Full-field phase distribution of the live CHO cells. (b) Optical thickness (contrast enhanced).

(c) Individual cell segmentation from the optical thickness. 
An example of simultaneous QPM and fluorescence imaging is shown in Fig.3 where the optical thickness, segmented cell boundaries from optical thickness, and the fluorescence stained cell nuclei are superimposed. The imaging condition for the QPM imaging is the same as the Fig. 2 and the nuclear staining fluorescent dye was DRAQ5 (ex: $625 \mathrm{~nm}$, em: 700 800 nm).

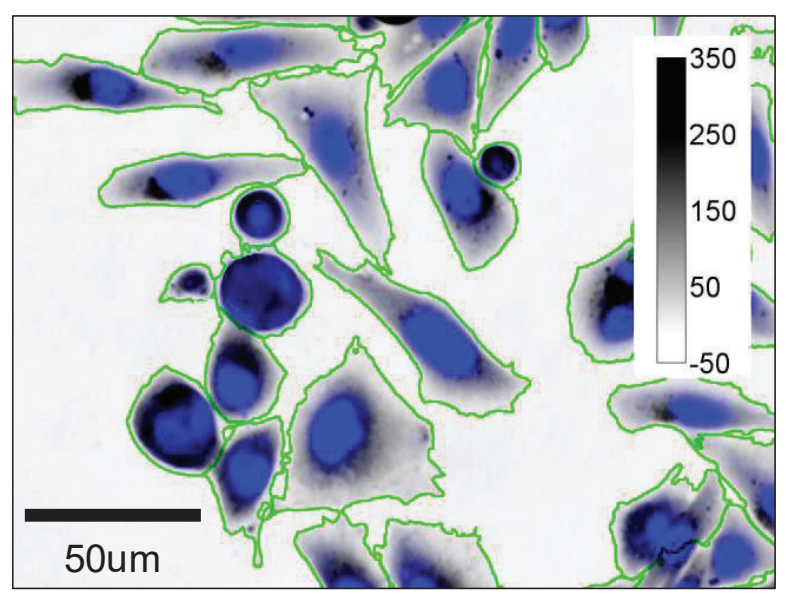

Fig. 3: Superimposed image of optical thickness (gray scale, colorscale is shown in nanometer), cell boundary estimated from optical thickness (green lines), and nuclear staining (blue).

\section{Conclusion}

We have successfully obtained optical phase images of live cells by our low-coherent quantitative phase microscope. The optical thickness image was free of background speckle noise and the halo. From the optical thickness images, we have segmented individual cell area by means of watershed algorithm. Moreover, simultaneous imaging of the optical thickness and the fluorescence was successfully implemented.

Our ongoing projects for the future improvement include the more downsizing of the setup, the implementation of the Mach-Zehnder type microscope in a same concept, and the biological study of filopodial connection by cell membrane reflection [4-6].

\section{References}

[1] Hidenao Iwai, et. al, "Quantitative phase imaging using actively stabilized phase-shifting low-coherence interferometry", Optics Letters 29, 2399-2401 (2004).

[2] M. K. Kim, "Principles and techniques of digital holographic microscopy," SPIE Reviews 1, 018005 (2010).

[3] G. Popescu, et. al, "Erythrocyte structure and dynamics quantified by Hilbert phase microscopy", J. Biomed. Opt. 10, 060503 (2005).

[4] T. Yamauchi, et. al, "Transportable and vibration-free full-field low-coherent quantitative phase microscope", Proc. SPIE, 105031U (2018).

[5] T. Yamauchi, et. al, "Speckle-free and halo-free low coherent Mach-Zehnder quantitative-phase-imaging module as a replacement of objective lens in conventional inverted microscopes", Proc. SPIE, 105031V (2018).

[6] T. Yamauchi, et. al, "Label-free imaging of the dynamics of cell-to-cell string-like structure bridging in the free-space by low-coherent quantitative phase microscopy", Proc. SPIE, 85711G (2013). 


\title{
Holographic Light Sheet Microscopy for Live Caenorhabditis elegans
}

\author{
Ting-Yu Hsieh ${ }^{* 1}$, Ju-Hsuan Chien ${ }^{1}$, Jui-ching $\mathrm{Wu}^{4}$, Yuan Luo ${ }^{1,2,3}$ \\ 1. Institute of Medical Device and Imaging, National Taiwan University, 10051, Taiwan, R. O. C. \\ 2.Molecular Imaging Center, National Taiwan University, Taipei, 10672, Taiwan, R. O. C \\ 3. YongLin Institute of Health, National Taiwan University, Taipei, 10087, Taiwan, R. O. C. \\ 4. National Taiwan University Department of Clinical Laboratory Sciences and Medical \\ Biotechnology, National Taiwan University,10048, Taiwan, R. O. C. \\ Author e-mail address: r07458002@ntu.edu.tw
}

\begin{abstract}
:
To image a live Caenorhabditis elegans (C. elegans) requires fine optical sectioning capacity. In general, confocal microscopy is a gold standard system for three-dimensional (3D) imaging; however, confocal needs long acquisition time and serious photobleaching and phototoxicity. Therefore, to obtain high-speed optical sectioning, we report holographic light sheet microscopy(HLSM) to observe 3D images of a live C. elegans with high resolution. Then, we compare brightfield microscopy and LSM using same C.elegans. In our approach, a volume hologram is utilized to record combined wavefront from a standard light sheet microscopic illumination setup to replace an objective lens as well as a cylindrical lens to provide a further compact system.
\end{abstract}

Key Word-- Optical-sectioning microscopy ; light sheet microscopy.

\section{Introduction}

To achieve high contrast images in biology samples by using optical sectioning is the thing that many microscopies have done it. For subcellular observation, confocal microscopy is commonly used. However, to improve resolution and contrast, confocal microscopy uses a pinhole to remove the out-of-focus light but sacrificing the scanning time and photo-bleching. Recently, light sheet microscopy (LSM) [1-7] selectively illuminates the interest plane of the sample that decreases the photobleaching and phototoxicity.

LSM combines the benefits of the standard wide-field microscopy and the confocal microscopy. The lateral resolution of LSM is the same as wide-field microscopy; in contrast, the axial resolution of LSM is comparable with confocal microscopy. In addition, LSM acquires optical sectioning images at a specific layer with a wide field of view in real time, and results in low photobleaching.
Hence, we experimentally demonstrate 3D images of C. elegans by using our LSM. In our experiment, we can get a 3D optical sectioning image with oocysts and the embryos in the C. elegans. By illuminating the sample in a form of thin sheet of light along the z-axis. To stack all the images at different layers together, a 3D high-contrast image can be easily acquired.

In this paper, our LSM includes a volume hologram $(\mathrm{VH})$ which is utilized to record combined wavefronts to replace a standard LSM illumination module consisting of an objective lens and a cylindrical lens which lead to a further compact system. By using this compact holographic light sheet microscopy(HLSM), 3D images of a C. elegans can be obtained.

\section{LSM setup}

To generate a light sheet, a cylindrical lens was used to reshape a Gaussian beam. In Figure.1, a 532nm laser (Cobolt AB, Cobolt Samba, 05-01 series, Sweden) beam passes through a spatial filter. The laser beam is then expanded using a 4-f relay system. Through a cylindrical lens, a light sheet is formed and illuminates the sample 
directly by passing an objective lens. For microscopic detection path, we utilized a commercial microscope (BX51, Olympus, Japan) which is equipped with a water immersion objective lens (UMPLAN FLN 20×, Olympus) and a sCMOS CCD. In our experimental measurements, our optical sectioning thickness of our LSM is $3.25 \mu \mathrm{m}$ and the lateral resolution is $0.62 \mu \mathrm{m}$.

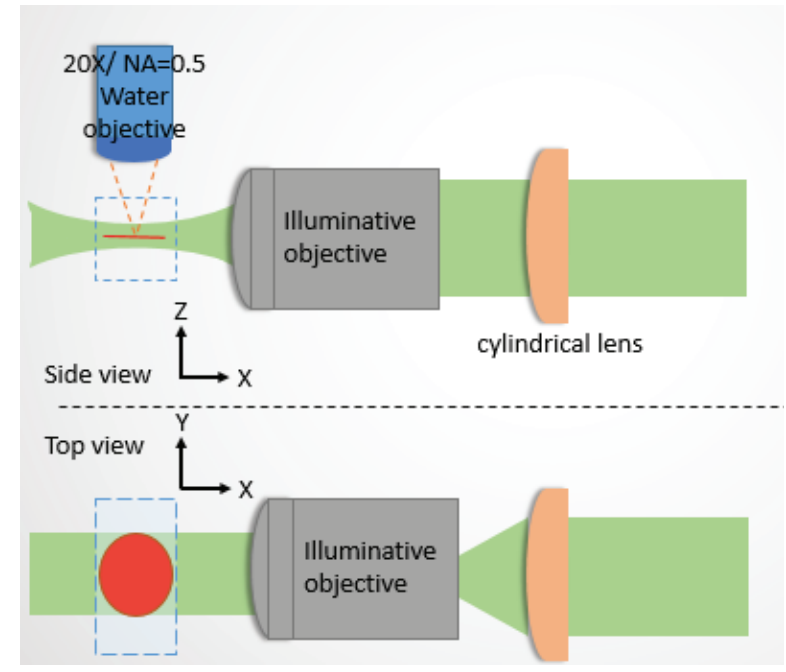

Figure 1. Schematic drawing of the illumination light sheet illumination optical path

To simplify the illumination module of the LSM, we utilized a $\mathrm{VH}$ to subsitute the cylindrical lens and objective lens. In Figure.2(a), we recorded the interference pattern generated from the light sheet beam profile and a reference beam on a $\mathrm{VH}$ with a $488 \mathrm{~nm}$ laser at 30 degree angle. And then the reconstruction setup shown in Figure.2(b), we reconstructed the the light sheet beam pattern by illuminating the VH by a $532 \mathrm{~nm}$ laser at 33.03 degree. Therefore, the HLSM setup is more compact.
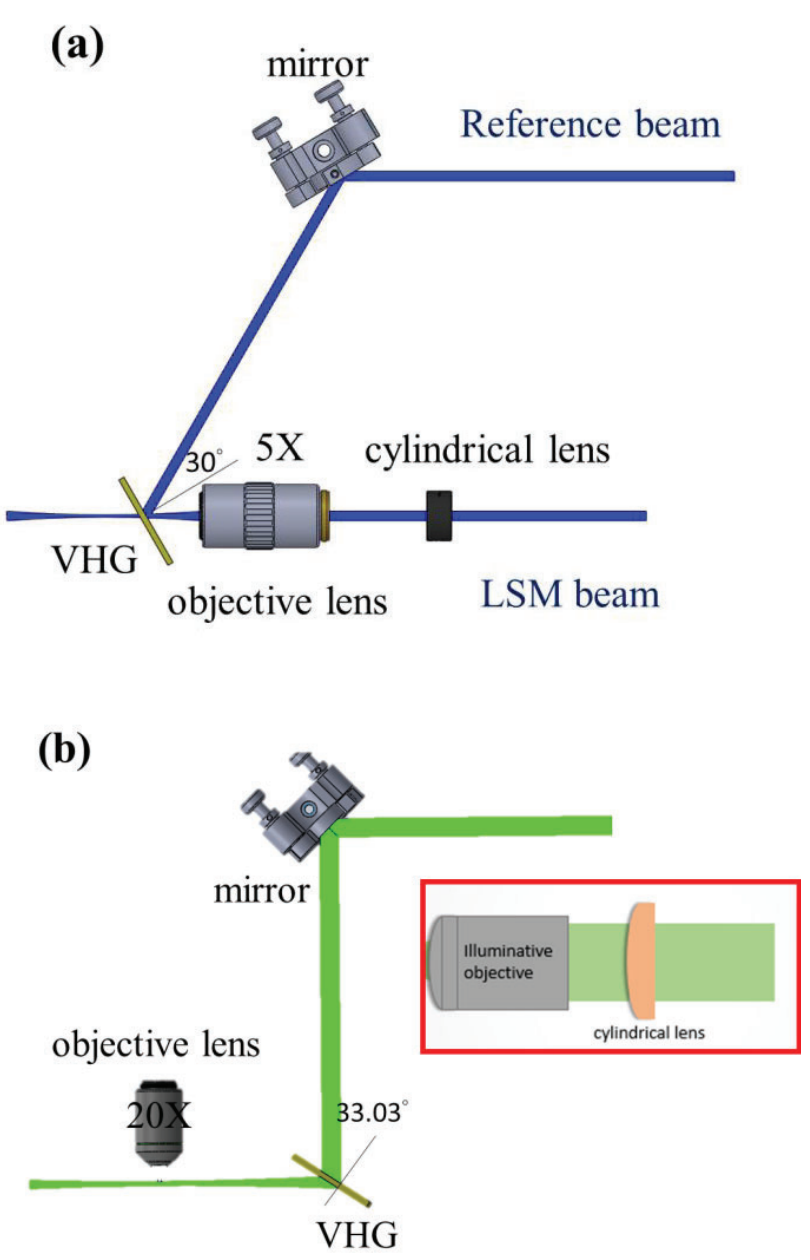

Figure 2 (a) Recording setup of light sheet illumination by using $488 \mathrm{~nm}$ laser. (b) Reconstruction setup by illuminating the recorded $\mathrm{VH}$ by a $532 \mathrm{~nm}$ laser to replace a cyclindrical lens and an objective lens.

\section{Experimaenal results}

To image a live $C$. elegans, we use a sample holder in the horizontal position and loaded the worms together with low melting agarose and with could see the oocyctes and embryo in a live C. elegans like Figure. 3 . 


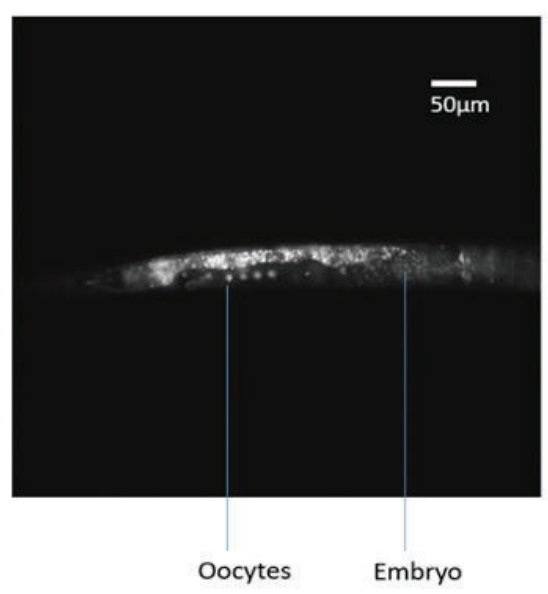

Figure. 3 LSM image data of a live C. elegans.

In addition, we reconstruct all the image and get a vedio of a moving C. elegans in real time as shown in the video link.

(https://www.youtube.com/watch?v=ICsN4PyxNKY)

Further, we compared our light sheet microscopy with a conventional bright-field microscope by using the same $C$. elegans' sample. In the conventional brightfield microscopy, fine features in the worm are barely observed clearly (Figure. 4). However, using our light sheet microscopic system, both fine features of oocytes and embryos in $C$. elegans( Figure. 5) can be observed with high image contrast and quality.

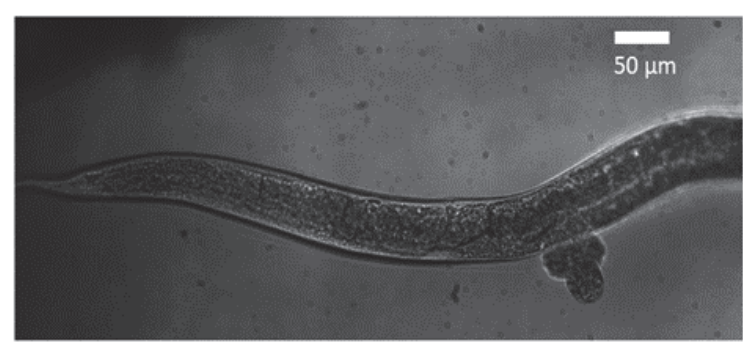

Figure. 4 C. elegans image using conventional bright-field microscopy

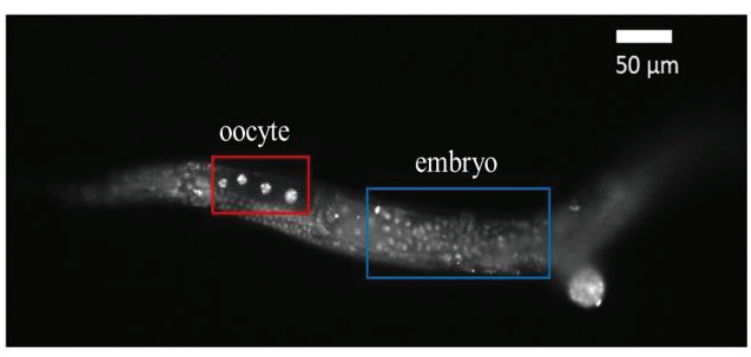

Figure. 5 C. elegans image using light sheet microscopy.

In HLSM setup, a reconstruction beam in a form of a thin sheet of light as shown in the video link below is generated by illuminating the $\mathrm{VH}$ by a $488 \mathrm{~nm}$ laser as shown in the buttom video. The probe beam is a zero order term of the $\mathrm{VH}$. The reconstruction beam is light sheet(LS) beam of the VH.

(https://www.youtube.com/watch?v=77XI S19CE)

During the above reconstruction process, the reconstruction beam illuminated $\mathrm{VH}$ at the 30 degree angle in $488 \mathrm{~nm}$ laser, and a transmission beam(zero order term) is shown in the solid line in Figure.6.

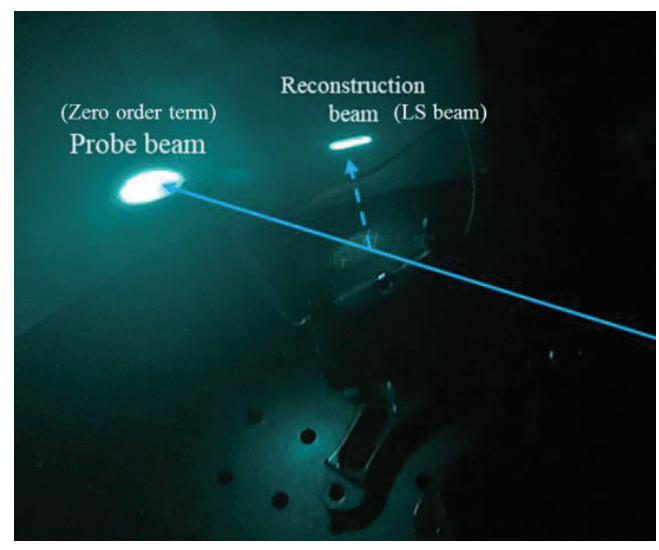

Figure. 6 Illuminated with $488 \mathrm{~nm}$ laser and got a LS beam.

When VH is illuminated at the 33.03 degree angle with a $532 \mathrm{~nm}$ laser, only two beams are generated by the $\mathrm{VH}$ which, are a probe beam (zero order term) and reconstruction beam (LS beam indicated by a dash line) in Figure 7. 


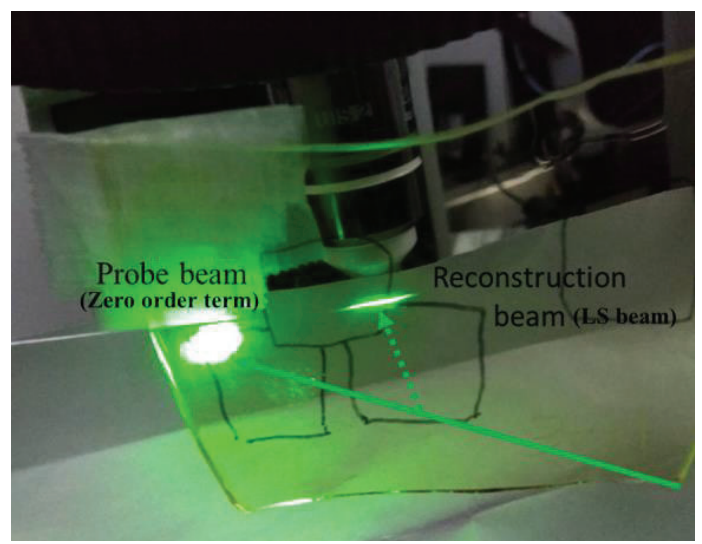

Figure. 7 Illuminated with $532 \mathrm{~nm}$ laser and got a LS beam

\section{Conclusion}

For a real-time $3 \mathrm{D}$ volumetric imaging, we have demonstrated a compact and fast HLSM system. By implementing holographic light sheet illumination module and the special design sample holder, fine features of oocytes and embryos of an anesthetized $C$. elegans would be easily observed in vivo. Compared with conventional bright-field microscopy, the imaging technique presented here open the doors to high imagimg speed of in vivo $C$. elegans with a high contrast as well as real-time operation. In addition, we utilized one $\mathrm{VH}$ to simplify the illumination module of the LSM to replace two optical components, an objective lens and a cylindrical lens. Therefore, a compact HLSM to image $C$. elegans is realized.

\section{References}

[1] Engelbrecht, C.J. and E.H. Stelzer, "Resolution enhancement in a light-sheet-based microscope (SPIM)". Optics letters, (2006). 31(10): p. 1477-1479..

[2] Huisken, J., et al., "Optical sectioning deep inside live embryos by selective plane illumination microscopy". Science, (2004). 305(5686): p. 1007-1009.

[3] Chardès, C., et al., "Setting up a simple light sheet microscope for in toto imaging of $\mathrm{C}$. elegans development". Journal of visualized experiments: JoVE, (2014)(87)
[4]Wu, Y., et al., "Inverted selective plane illumination microscopy (iSPIM) enables coupled cell identity lineaging and neurodevelopmental imaging in Caenorhabditis elegans". Proceedings of the National Academy of Sciences,

[5] Wu, Y., et al., "Spatially isotropic four-dimensional imaging with dual-view plane illumination microscopy." Nature biotechnology, (2013). 31(11): p. 1032.

[6] Ju-Hsuan Chien, "Design and Develop Light Sheet Microscopy for Live Canenorhabditis elegans" .National Taiwan University Master Thesis (2018)

[7] Ju-Hsuan Chien, Ting-Yu Hsieh, Jui-ching Wu , Yuan Luo," Light Sheet Microscopy for Live Caenorhabditis elegans",Optics \& Photonics Taiwan, the International Conference (OPTIC 2018) 


\title{
Simple intensity equalization methods in SLM generated multispots
}

\author{
Xiangyu Quan*a, Manoj Kumar, Osamu Matoba ${ }^{a}$, Yasuhiro Awatsuji ${ }^{b}$ and Hiroaki Wake ${ }^{\mathrm{c}}$ \\ ${ }^{\mathrm{a}}$ Graduate School of System Informatics, Kobe University, Rokkodai 1-1, Nada, Kobe 657-8501, \\ Japan \\ ${ }^{b}$ Faculty of Electrical Engineering and Electronics, Kyoto Institute of Technology, Matsugasaki, \\ Sakyo-ku, Kyoto 606-8585, Japan \\ 'Division of System Neuroscience, Kobe University of Graduate School of Medicine, Kusunoki-cho \\ 7-5-1, Chuo-ku, Kobe, 650-0017, Japan
}

\begin{abstract}
Multispots in three-dimensions generated by phase mode spatial light modulators (SLMs) are very useful in laser processing or light induced biological treatments, such as optogenetics. So far, the intensity of the focus spots varies depending to the distance from optical axis. In order to equalize intensities in all generated spots, simple feedback method was applied. Further, look up table was created by dividing imaging area in sectors. Experiment results show improvement in the random spots at some degree, however, further analyzation is required.
\end{abstract}

Keywords: computer generated hologram, optogenetics, laser processing

\section{Introduction}

Wavefront modulation using various kinds of spatial light modulators (SLMs) are studied, and used in many fields. In laser processing, spot by spot processing was replaced by line to line or plane to plane. Thus, high speed processing is possible. In recent years, light induced biological researches are very active in worldwide. Such as in optogenetics, light with certain wavelengths can open or close ion gates on the cell membrane so the stimulated neural signal can be generated manually. Using phase mode SLM can create high resolution, cell specific 3D multispots with few tens of milliseconds fps. However, the light intensity on every locations are not always the same. Whether it is because the diffraction efficiency drops by increased diffraction angle, or the limited aperture size works as a low pass angular spectrum filter, it always seems that spots created close to optical axis brighter than further ones. In order to solve this problem, some optimizations are made to SLM phase patterns. GS method is mostly used in such case. Some other methods are also applied to improve speed and accuracy. However, time consuming iterative algorithms are not always suitable to stimulate nerve cells in living animals, as the activity is quick and unpredictable. In order to speed up the optimization, we propose feedback system to quickly monitor generated spots and make appropriate changes.
And further, if we have this previous knowledge on the imaging system, look up table (LUT) can be applied instead of feedback process.

In this manuscript, two cases which using feedback and LUT are demonstrated. Enhanced intensity distribution in different locations are confirmed in both cases.

\section{Method}

Figure 1 shows the principle of spot illumination on the desired location using an SLM. In order to put image of SLM on the back focal plane of the objective lens, $4 \mathrm{f}$ system has been applied to illumination light.

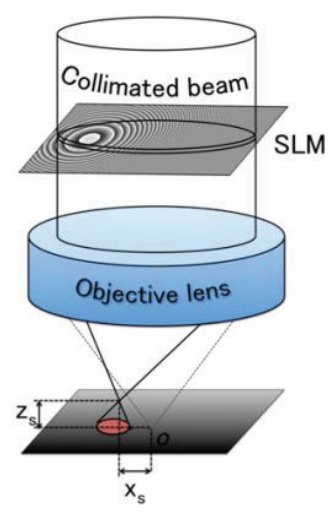

Figure 1. Principle of generating focused spot in 3D space. 
if the incoming wavefront $u_{\text {in }}$ has a specific phase distribution, described as

$$
u_{i n}(x)=\sum_{k} w_{k} \exp \left(-\frac{i \pi x^{2}}{\lambda h_{k}}\right) \exp \left(i 2 \pi x \frac{1}{g_{x k}}\right),
$$

after the objective lens, the wavefront at the front focal plane (FFP) becomes

$$
u_{F F P}(X)=\sum_{k} w_{k} \exp \left\{\frac{i \pi}{\lambda H_{k}}\left[\left(X-G_{x k}\right)^{2}\right]\right\} .
$$

Here, $H_{k}=\frac{f_{O L}^{2}}{h_{k}}, G_{x k}=\frac{f_{O L} \lambda}{g_{x k}}$, and the $k$ th focused spot falls on the position $\left(G_{x k}, G_{y k}, H_{k}\right)$ in the sample space.

For feedback method, first we set $\mathrm{w}_{\mathrm{k}}=1$ for all spot. Then from the obtained results, we analyze average intensity for all spots, and set $\mathrm{w}_{\mathrm{k}}=\frac{I_{k}}{I_{\text {average }}}$ to new pattern. This cycle usually only needs to be performed once. Without feedback, we divide an image in sectors, and give $\mathrm{w}_{\mathrm{k}}$ according to previously known spots' distributions to creating LUT. This does not require feedback, and usually LUT only depends on the optical set-up.

\section{Results and Discussion}

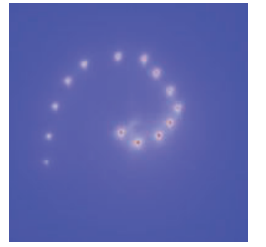

(a)Initial distribution

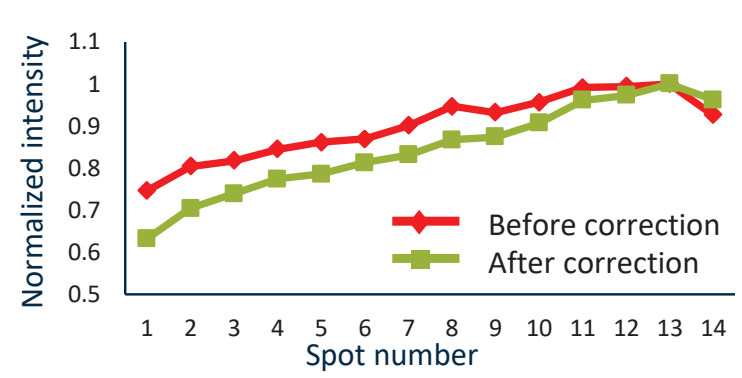

(d)

Figure 2 Feedback method to correct intensity, and the experiment result.

In order to simplify the experiments, we set $h_{k}=\infty$ in eq. (1). With optical axis on the center of the Figure 2 (ac), randomly generated 14 spots have uneven intensity distributions. As mentioned in the method section, we analyzed the average intensity among 14 spots, and decide $\mathrm{w}_{\mathrm{k}}$ according to actual intensity. For weak spots, $\mathrm{w}_{\mathrm{k}}$ becomes bigger than 1, and vice versa. As in Figure 3(d), after feedback, the plot line has flattened compared to initial distribution. $10 \%$ of improvement happened to the first spot which was furthest from the center. However, the graph could not make perfect flat line due to limitations of phase mode SLM.

The LUT was made by scanning the whole imaging area with a single spot as in Figure 3(a). And to each sectioned area, $w_{k}$ is decided according to spot intensity placing within the area. The array of $w_{k}$ is then made. After applying the $w_{k}$ to new pattern, disproportional spot intensity on the right side is improved at some degree. However, the effect is further to be analyzed.

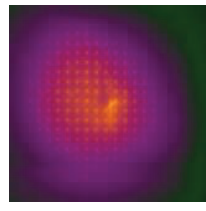

(a)Initial distribution

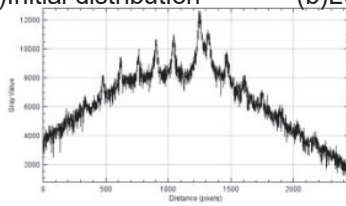

(d) diagonal pot of (a)

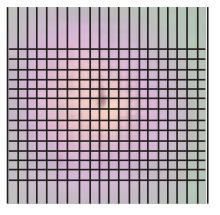

(b) LUT

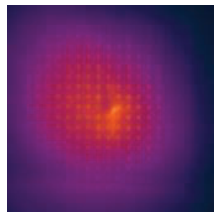

(c)After correction

(e) diagonal pot of (c)

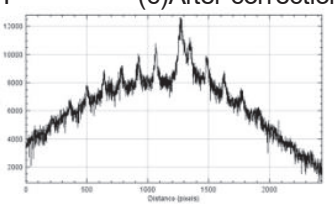

Figure 3 LUT method to correct intensity, and the experiment result.

\section{References}

Hayasaki, Y., Sugimoto, T., Takita, A. and Nishida, N., 2005. Variable holographic femtosecond laser processing by use of a spatial light modulator. Applied Physics Letters, 87(3), 031101.

Quan, X., Kumar, M., Matoba, O., Awatsuji, Y., Hayasaki, Y., Hasegawa, S. and Wake, H., 2017. 3D stimulation and imaging-based functional optical microscopy (SIFOM) of biological cells. Opt. Lett. 43, 5447-5450

Takahashi, H., Hasegawa, S. and Hayasaki, Y., 2007. Holographic femtosecond laser processing using optimal-rotation-angle method with compensation of spatial frequency response of liquid crystal spatial light modulator. Applied optics, 46(23), 5917-5923. 


\title{
A new signal processing in laser Doppler flowmetry
}

\author{
Elena Zharkikh*, a,b, Evgeny Zherebtsov ${ }^{\mathrm{a}, \mathrm{b}}$, Igor Kozlov ${ }^{\mathrm{b}}$, Angelina Zherebtsova ${ }^{\mathrm{b}}$, Viktor Dremin ${ }^{\mathrm{b}}$, \\ Andrey Dunaev ${ }^{\mathrm{b}}$, Igor Meglinski ${ }^{\mathrm{a}}$ \\ ${ }^{a}$ Opto-Electronics and Measurement Techniques Research Unit, University of Oulu, Oulu, Finland \\ ${ }^{\mathrm{b}}$ Orel State University named after I.S. Turgenev, 95 Komsomolskaya St., Orel, 302026, Russia
}

\begin{abstract}
Due to simplicity and ease of use, the laser Doppler flowmetry (LDF) has found applications in various areas of research related to non-invasive diagnosis of blood microcirculation. In fact, LDF still has a number of unsolved problems that limit its day-to-day use in clinical practice. Current report discusses the new approach for the LDF signal processing, that allows to receive a new diagnostic information. The proposed algorithm provides information on the quantitative distribution of the red blood cells velocities in the probing volume of biological tissue when applying different provocative effects.
\end{abstract}

Keywords: laser Doppler flowmetry, Doppler spectrum decomposition, blood microcirculation, signal processing, frequency of Doppler shift

\section{Introduction}

Laser Doppler flowmetry (LDF), for the first time used in 1972 for the analysis of blood microcirculation ${ }^{1}$, is currently one of the most common technologies for assessing the perfusion of biological tissues ${ }^{2}$. The method is based on probing tissue with laser radiation and recording a stochastic photocurrent resulting from optical mixing on photodetector a frequency-shifted (scattered from moving components) and non-shifted (scattered from static components) light ${ }^{3}$. LDF has found its application in a wide variety of studies ${ }^{2,4,5}$, which is facilitated by the non-invasiveness of the method, ease of use, and the ability to record the signal in real time.

However, despite all the advantages of this technology, there are unresolved issues preventing its wider introduction into clinical practice. The main challenge of the technology that limits its use is the overcoming the large inter- and intra-individual variability of the LDF signal ${ }^{6,7}$ due to differences in the optical properties of tissues, the structure of the vascular bed and physiological factors, which makes it impossible to measure perfusion in absolute units. The resulting signal (index of microcirculation) is recorded in the so-called perfusion

*Correspondence: e.zharkikh@oreluniver.ru (arbitrary) units. This results in poor reproducibility of the signal dimension, which significantly complicates the calibration of LDF devices during their manufacture, as well as their further metrological control ${ }^{8}$.

In the traditional processing of the LDF signal, the power spectrum is weighted by frequency, and as a result, the output signal is proportional to the concentration of red blood cells in the probing volume and their mean velocity 9 . In this case, integration over the wide frequency range is usually applied $(20-24000 \mathrm{~Hz})$. Previous studies have shown that the frequency range of Doppler spectrum integration has a significant effect on the recorded signal ${ }^{10}$, and in some cases, a narrower integration range is preferable ${ }^{11}$.

In this paper, a new approach to the processing of the LDF signal, which allows for the evaluation of the contribution of various frequency components to the total resulting perfusion signal, is considered and tested.

\section{Materials and Methods}

An in-house laser Doppler flowmetry setup was developed. The system includes one-mode laser of 1064 $\mathrm{nm}$ wavelength, two photodiodes, an analog amplifier with current-to-voltage converter, data acquisition card and $\mathrm{PC}$ with installed visual programming environment NI LabVIEW. Delivery of radiation to the skin and 
collecting of the backscattered light were performed by optical fibers. The scattered radiation was transformed in photocurrent on a photoconverter and then received signal was amplified by use of custom electronic board and digitized on data acquisition card NI USB $6211^{12,13}$.

Signal processing was carried out in PC with NI LabVIEW environment, and the result was displayed on the screen and recorded in a data file. The power spectrum of the signals was evaluated by a classic algorithm based on Fast Fourier Transform ${ }^{14}$.

Two different functional tests were chosen as a provocative effect: an arterial occlusion test and an experiment with local tissue pressure loading. Volunteers were pre-adapted to the room temperature of $24-25^{\circ} \mathrm{C}$ and were in a state of physical and mental rest. All measurements were performed daily from 11:00 until 13:00 to avoid any influence of circadian rhythms on the blood circulation.

The first part of the study included the occlusion of the brachial artery, which was carried out by clamping the forearm with a tonometer cuff with a pressure of $220 \mathrm{~mm}$ $\mathrm{Hg}$. Fourteen healthy young volunteers (mean age is 21 years) participated in the study. LDF signals were obtained and integrated by following sub-bands: $60-400$ Hz; 400-800 Hz; 800-1600 Hz; 1600-3200 Hz; 3200-6400 Hz. The signal was recorded during three consecutive stages: baseline measurements of skin perfusion for 3 min, arterial occlusion $-3 \mathrm{~min}$ and postocclusive period recording for $5 \mathrm{~min}$.

The second part of the experiment included the study of the effect of locally applied pressure ${ }^{15,16}$ on the LDF signal and its distribution over the frequency sub-bands. Special tooling developed and manufactured using a 3Dprinter was used in the study for loading tissue with weights ${ }^{17}$. The tooling was placed coaxially to optic fiber. At the beginning of the study, the signal was recorded without tissue loading with weights. The weights were then added evenly to the tooling until the pressure was 40 $\mathrm{kPa}$. The next stage of the experiment was the sequential unloading of the tissue in the same order in which the loading occurred. The study was conducted on 7 young healthy volunteers and lasted about $50 \mathrm{~min}$.

\section{Results and Discussion}

During the occlusion test, an increase in the LDF signal was observed in the frequency ranges of $800-1600 \mathrm{~Hz}$ and $1600-3200 \mathrm{~Hz}$.

The research results are presented on a time-resolved graph with corresponding calculated frequencies of the Doppler shift (Figure 1).

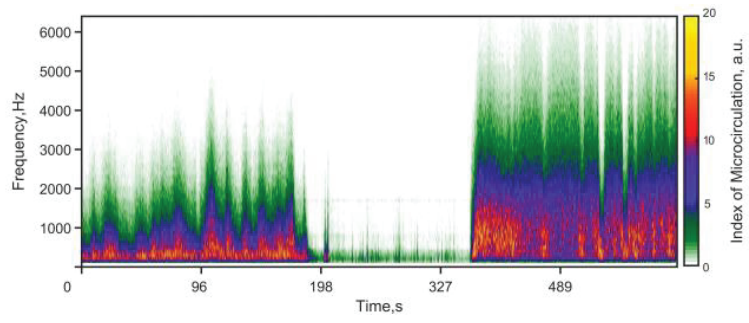

Figure 1. The distribution of the index of microcirculation in frequency and amplitude in time during the occlusion test.

The obtained measurement results after removal of the occlusion can be explained by post-occlusive reactive hyperemia, i.e. an increase in the number of fast moving red blood cells.

In the second part of the study with the local loading test, it was shown that the index of microcirculation starts to decrease with the pressure of $5 \mathrm{kPa}$. At the same time, in the low-frequency range (up to $1000 \mathrm{~Hz}$ ), an increase in the signal amplitude is observed at small values of the applied pressure. The results of the experiment are presented in Figure 2.

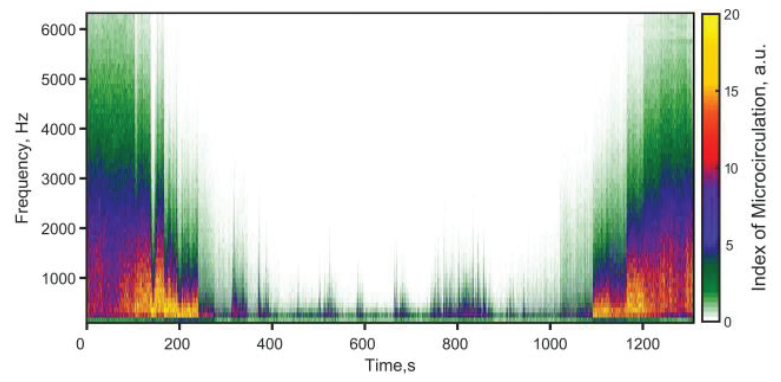

Figure 2. The distribution of the index of microcirculation in frequency and amplitude in time during the local pressure loading test.

The obtained result may be related to the fact that an increase in pressure applied to the skin slows down the 
blood flow. Thus, more slowly moving red blood cells appear in the probed volume of biotissue.

\section{Conclusions}

Using the developed algorithm, the distribution of the red blood cells velocities during functional tests was evaluated. The proposed method allows for evaluation of the contribution of each frequency sub-band to the total LDF signal.

The performed experiments demonstrated the difference in the response of different frequency subbands of the Doppler shift to provocative effects. It was shown that the largest contribution to the increase in the LDF signal in the period of post-occlusive reactive hyperemia is made by the frequency ranges from 400 to $3200 \mathrm{~Hz}$. During the test with local loading of the skin, an increase in the signal integrated over low-frequency range (up to $1000 \mathrm{~Hz}$ ) was demonstrated, while the highfrequency components of the signal decreased.

The proposed approach to analyzing the LDF signal allows obtaining new data on microcirculatory blood flow. Investigation of the microcirculation index distribution over the frequency sub-bands could potentially have diagnostic value in conditions associated with microvascular disorders.

\section{Acknowledgments}

This study was funded by the Russian Science Foundation (the research project 18-79-00237). Evg. Zh. (design of the experiment and data processing) acknowledges support from the Academy of Finland, research project No 318281 .

\section{References}

[1] Riva C., Ross B., Benedek G.B., "Laser Doppler measurements of blood flow in capillary tubes and retinal arteries," Investigative ophthalmology, 11(11), 936-944 (1972)

[2] Leahy M.J., Nilsson G.E., "Laser Doppler flowmetry for assessment of tissue microcirculation: 30 years to clinical acceptance," Proc. SPIE 7563, 75630E (2010)

[3] Leahy M.J., De Mul F.F.M., Nilsson G.E., Maniewski R., "Principles and practice of the laser-Doppler perfusion technique," Technology and Health Care, 7(2-3), 143-162 (1999)

[4] Mizeva I., Makovik I., Dunaev A., Krupatkin A., Meglinski I., "Analysis of skin blood microflow oscillations in patients with rheumatic diseases," Journal of Biomedical Optics, 22(7), (2017)

[5] Zharkikh E.V., Mizeva I.A., Makovik I.I., Dremin V.V., Zherebtsov E.A., Potapova E.V., Dunaev A.V., "Blood flow oscillations as a signature of microvascular abnormalities," Proc. SPIE 10685, 106854C (2018)

[6] Fredriksson I., Fors C., Johansson J., "Laser Doppler Flowmetry - a Theoretical Framework," (2007)

[7] Dunaev A.V., Sidorov V.V., Krupatkin A.I., Rafailov I.E., Palmer S.G., Sokolovski S.G., Stewart N.A., Rafailov E.U., "The study of synchronization of rhythms of microvascular blood flow and oxygen saturation during adaptive changes," Proc. SPIE 8935, 89350A (2014)

[8] Dunaev A.V., Zherebtsov E.A., Rogatkin D.A., Stewart N.A., Sokolovski S.G., Rafailov E.U., "Novel measure for the calibration of laser Doppler flowmetry devices," Proc. SPIE 8936, 89360D (2014)

[9] Bonner R., Nossal R., "Model for laser doppler measurements of blood flow in tissue," Applied Optics, 20(12), 2097-2107 (1981)

[10] Obeid A.N., "In vitro comparison of different signal processing algorithms used in laser Doppler flowmetry," Medical \& Biological Engineering \& Computing, 31(1), 43-52 (1993)

[11] Qu X., Ikawa M., Shimauchi H., "Improvement of the detection of human pulpal blood flow using a laser Doppler flowmeter modified for low flow velocity," Archives of Oral Biology, 59(2), 199-206 (2014)

[12] Kozlov I.O., Zherebtsov E.A., Zherebtsova A.I., Dremin V.V., Dunaev A.V., "Investigation of Doppler spectra of laser radiation scattered inside hand skin during occlusion test," Journal of Physics: Conference Series, 929(1), 012063 (2017)

[13] Volkov M.V., Kostrova D.A., Margaryants N.B., Gurov I.P., Erofeev N.P., Dremin V.V., Zharkikh E.V., Zherebtsov E.A., Kozlov I.O., Dunaev A.V., "Evaluation of blood microcirculation parameters by combined use of laser Doppler flowmetry and videocapillaroscopy methods," Proc. SPIE 10336, 1033607 (2017) 
[14] Michelson G., "Principle, validity, and reliability of scanning laser Doppler flowmetry," Journal of Glaucoma, 5(2), 99-105 (1996)

[15] Cracowski J.L., Roustit M., "Current Methods to Assess Human Cutaneous Blood Flow: An Updated Focus on Laser-Based-Techniques," Microcirculation, 23(5), 337-344 (2016)

[16] Jan Y.K., Shen S., Foreman R.D., Ennis W.J., "Skin blood flow response to locally applied mechanical and thermal stresses in the diabetic foot," Microvascular Research, 89, 40-46 (2013)

[17] Zherebtsov E.A., Kandurova K.Y., Seryogina E.S., Kozlov I.O., Dremin V.V., Zherebtsova A.I., Dunaev A.V., Meglinski I., "The influence of local pressure on evaluation parameters of skin blood perfusion and fluorescence," Proc. SPIE 10336, 1033608 (2017) 


\title{
Visualization of transcutaneous bilirubin, hemoglobin, and melanin based on hyperspectral diffuse reflectance imaging
}

\author{
Izumi Nishidate*a ${ }^{\mathrm{a}}$, Masashi Minakawa ${ }^{\mathrm{a}}$, MD. Abdul Wares ${ }^{\mathrm{a}}$, Kazuya Nakano ${ }^{\mathrm{b}}$, and Hideaki \\ Haneishi ${ }^{b}$ \\ ${ }^{\mathrm{a}}$ Graduate School of Bio-Applications \& Systems Engineering, Tokyo University of Agriculture and \\ Technology. \\ ${ }^{\mathrm{b}}$ Center for Frontier Medical Engineering, Chiba University
}

\begin{abstract}
We investigated a method to visualize transcutaneous bilirubin, hemoglobin, and melanin based on hyperspectral imaging. In the method, the Monte Carlo simulation-based multiple regression analysis for an absorbance spectrum in the visible wavelength region is used to specify the concentrations of bilirubin, oxygenated hemoglobin, deoxygenated hemoglobin, and melanin. Bile duct ligation in rats demonstrated the remarkable change in bilirubin concentration whereas rats under varying fraction of inspired oxygen showed well-known hemodynamic changes in skin tissue. The results in this study indicate potential of the method for simultaneous imaging of multiple chromophores in skin tissue.
\end{abstract}

Key words: bilirubin, melanin, hemoglobin, hyperspectral imaging, Monte Carlo simulation

\section{Introduction}

Quantitative evaluation of biological chromophores is useful for detecting various skin diseases including cancers, monitoring health status and tissue metabolism, and clinical and physiological assessments of vascular functions. Major chromophores in the superficial skin layer are melanin, oxygenated hemoglobin, and deoxygenated hemoglobin, which show distinctive optical absorption properties in the visible wavelength range. When the concentration of each chromophore varies, the corresponding change may be made on diffusely reflected light from the skin tissue in this wavelength range. Steady-state diffuse reflectance spectrum with a continuous-wave light can be easily acquired using a white light source, inexpensive optical components, and a spectrometer. Therefore, diffuse reflectance spectroscopy (DRS) has been widely used for the evaluation of human skin chromophores [1-6].

In addition to melanin and hemoglobin, bilirubin is also an important chromophore for evaluating health conditions such as neonatal jaundice, liver cirrhosis, and hepatitis. Bilirubin is a hemoglobin breakdown product and has a broad absorption spectrum with a maximum absorption in the range of $400 \mathrm{~nm}$ to $500 \mathrm{~nm}$. A condition in which the serum bilirubin concentration *inishi@cc.tuat.ac.jp; phone 81423887065 exceeds the holding capacity of the serum albumin is known as hyperbilirubinemia or jaundice. It is responsible for the yellowish skin color in jaundice. In most infants, hyperbilirubinemia reflects a normal transitional phenomenon called physiological jaundice. In some infants, however, significant hyperbilirubinemia may cause bilirubin to accumulate in the brain tissue, potentially causing irreversible brain damage called kernicterus [7]. It is therefore recommended to monitor carefully the serum bilirubin or transcutaneous bilirubin [8,9] levels on neonate jaundice, especially in the first 24 hours.

In the present study, we newly propose a method to estimate simultaneously transcutaneous bilirubin concentration $\left(C_{\mathrm{bil}}\right), \quad$ oxygenated hemoglobin concentration $\left(C_{\mathrm{HbO}}\right)$, deoxygenated hemoglobin concentration $\left(C_{\mathrm{HbR}}\right)$, and melanin concentration $\left(C_{\mathrm{m}}\right)$ based on the diffuse reflectance spectroscopy. The proposed method utilizes multiple regression analysis aided by Monte Carlo simulation [10] for diffuse reflectance spectra of skin tissue. Using the absorbance spectrum as a dependent variable and the extinction coefficients of bilirubin, melanin, oxygenated hemoglobin, and deoxygenated hemoglobin as independent variables, multiple regression analysis 
provides regression coefficients. Concentrations of bilirubin, melanin, oxygenated hemoglobin, and deoxygenated hemoglobin are then determined from the regression coefficients using empirical formulae that are deduced numerically in advance. To confirm the feasibility of a method by which to evaluate hyperbilirubinemia and hemodynamics in skin tissues, we performed in vivo experiments using rat dorsal skin after bile duct ligation under the normoxia and while changing the fraction of inspired oxygen $\left(\mathrm{FiO}_{2}\right)$ to include conditions of normoxia, hypoxia, and anoxia.

\section{Principle}

In the proposed method, the Monte Carlo simulationbased multiple regression analysis for an absorbance spectrum in the visible wavelength region (460-590 nm) is used to specify the concentrations of bilirubin $\left(C_{\mathrm{bil}}\right)$, oxygenated hemoglobin $\left(C_{\mathrm{HbO}}\right)$, deoxygenated hemoglobin $\left(C_{\mathrm{HbR}}\right)$, and melanin $\left(C_{\mathrm{m}}\right)$. Using the absorbance spectrum calculated from the measured diffuse reflectance spectrum as a response variable and the extinction coefficients of bilirubin, oxygenated hemoglobin, deoxygenated hemoglobin, and melanin, as predictor variables, multiple regression analysis provides regression coefficients. Concentrations of bilirubin, oxygenated hemoglobin, deoxygenated hemoglobin, and melanin, are then determined from the regression coefficients using conversion vectors that are numerically deduced in advance by the Monte Carlo simulations for light transport in skin. Total hemoglobin concentration $\left(C_{\mathrm{HbT}}\right)$ and tissue oxygen saturation $\left(\mathrm{StO}_{2}\right)$ are simply calculated from the oxygenated hemoglobin and deoxygenated hemoglobin.

\section{Materials and Methods}

Male Wister rats were used for the animal experiments. Anesthesia of rats was performed with isoflurane and maintained at a depth such that the rat had no response to toe pinch. After induction of anesthesia, the dorsal regions were shaved and a depilatory agent was applied on the rat dorsal skin. As the first experiment, we performed the measurements of diffuse reflectance spectra of rat dorsal skin with obstructive jaundice. Obstructive jaundice was induced in rats after common bile duct ligation. Simultaneously with hyperspectral imaging, transcutaneous bilirubin level was measured by the transcutaneous jaundice meter (JM-105Konica Minolta, Inc, Tokyo, Japan).

\section{Results and Discussion}

Figure 1 shows the typical images of transcutaneous bilirubin concentration $C_{\text {bil }}$ obtained from the proposed method during bile duct ligation. The estimated value of $C_{\text {bil }}$ increases monotonically and reaches around 18 $\mathrm{mg} / \mathrm{dl}$ at 66 hours after the onset of bile duct ligation, which showed the same tendency as the transcutaneous bilirubin concentration measured by the bilirubinometer.

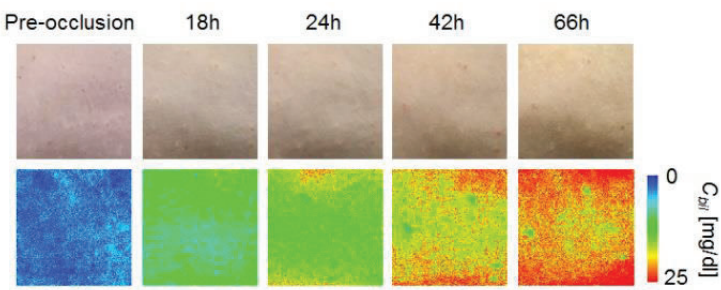

Fig.1 Typical time courses of color image and bilirubin concentration image $C_{\mathrm{bil}}$ obtained from a rat dorsal skin after common bile duct ligation.

\section{Conclusion}

In summary, a method for visualizing skin chromophores of in vivo skin tissues based on hyperspectral diffuse reflectance imaging was demonstrated. In vivo experiments using rat dorsal skin after bile duct ligation showed good correlation in transcutaneous bilirubin concentration between the proposed method and a commercially available transcutaneous bilirubinometer, which indicates the ability of the proposed method for evaluate transcutaneous bilirubin concentration.

\section{References}

[1] G. Zonios, et. al., J. Invest. Dermatol. 117, 1452 (2001).

[2] G. Stamatas, et. al., J. Biomed. Opt. 9, 315 (2004).

[3] P. R. Bargo, et. al., J. Biomed. Opt. 10, 034018 (2005).

[4] I. Nishidate, et. al., J. Biomed. Opt. 9, 700 (2004).

[5] I. Nishidate, et. al., Opt. Lett. 36, 3239 (2011).

[6] K. Yoshida, et. al., Biomed. Opt. Exp. 5, 3901 (2014).

[7] F. Groenendaal, et. al., Pediatrics. 114, 297 (2004).

[8] F. F. Rubaltelli, et. al., Pediatrics. 107, 1264 (2001).

[9] M. J. Maisels, et. al., Pediatrics. 113, 1638 (2004).

[10] L. Wang, et. al., Comput. Methods Programs

Biomed. 47(2), 131 (1995). 


\title{
High-Efficient Holographic Photopolymer Based on Fluorinated Epoxy
}

\author{
Resin
}

\author{
Diqin Zhang, ${ }^{\mathrm{a}, \mathrm{c}}$, Yu Zhao ${ }^{* b}$, Yan Ye ${ }^{\mathrm{b}}$, Yuxia Zhao ${ }^{* a}$ \\ ${ }^{a}$ Technical Institute of Physics and Chemistry, Chinese Academy of Sciences, Beijing 100190, China \\ ${ }^{b}$ Institute of Fluid Physics, China Academy of Engineering Physics, Mianyang, Sichuan 621900, \\ China \\ ${ }^{\mathrm{c}}$ University of Chinese Academy of Sciences, Beijing 100049, China
}

\begin{abstract}
A series of fluorinated epoxy resin (FTGE) with a low refractive index $(\sim 1.45)$ were synthesized and applied into the fabrication of holographic recording media based on photopolymer. The results indicated that the introduction of FTGE significantly enhanced the refractive index modulation and sensitivity of the holographic photopolymer. And one sample (No.38) showed the most excellent holographic optical characteristics among all samples. Using sample $\mathbf{3 8}$ as recording media, a holographic grating with high diffraction efficiency (over 90\%) was fabricated within 90s under the illumination of a $532 \mathrm{~nm}$ laser with the exposure intensity of $0.53 \mathrm{~mW} / \mathrm{cm}^{2}$. Meanwhile, 3.7\% diffraction efficiency was obtained under the exposure of a single pulse (532 nm, $150 \mathrm{ps}$ pulse width) with the exposure dosage of $\sim 26 \mathrm{~mJ} / \mathrm{cm}^{2}$. After a coin was recorded in $30 \mu \mathrm{m}$ thick film through a simple reflecting optical exposure by a 532 $\mathrm{nm}$ laser, its hologram was clearly visible to the naked eyes. This study indicated that fluorinated epoxy resin was benefit to enhance the holographic performances and high-quality holographic recording media can be achieved for practical application.
\end{abstract}

Keywords: fluorinated epoxy resin, holography recording, photopolymer, high diffraction efficiency, refractive index modulation

\section{Introduction}

Holography is widely used in many fields, including data storage, microscopy, lithography, head-up display, threedimensional imaging, interference metrics and anticounterfeiting. For these applications, holographic recording media with excellent optical characteristics are prerequisite. Recently photopolymers are well-recognized as the most practical holographic recording materials, since they have the advantages of high sensitivity, high diffraction efficiency, large dynamic range and simple fabrication process, etc.

Many researches had made efforts to manufacture highefficient holographic recording photopolymers. One of the most common methods is to improve the difference between refractive indexes of the writing polymer and the matrix $\left(\mathrm{n}_{\text {polymer }}-\mathrm{n}_{\text {matrix }}\right)$ to obtain a high refractive index modulation $(\Delta \mathrm{n})$. Tomita et al. doped hyperbranched organic nanoparticles (HBPs) with high refractive index $(\mathrm{n}=1.82)$ into photopolymer material, and gained a volume holographic grating with $\sim 100 \%$

*yuxia.zhao@mail.ipc.ac.cn; phone 8610 82543569;

yu_zhao@caep.cn; phone; phone 868162495703 diffraction efficiency, $\Delta \mathrm{n}$ about 0.022 in $10 \mu \mathrm{m}$ thickness of recording media under $532 \mathrm{~nm}$. Marvin et al. synthesized a monomer BPTPA with high refractive index $(\mathrm{n}=1.6)$, and obtained a transmission holographic grating with high refractive index modulation $(\Delta \mathrm{n}=0.029)$ with 60 wt $\%$ BPTPA in formula under $405 \mathrm{~nm}$. Satoh et al. improved the compatibility of fluorine matrix with monomer through chemical modifying to enhance refractive index modulation, and successful recorded and reconstructed 750 multiplexed holograms on a $0.4 \times 50 \times 50 \mathrm{~mm}$ fluorine-based photopolymer with less than $10^{-2}$ bit error rates under $532 \mathrm{~nm}$.

This study indicated that fluorinated epoxy resin with a low refractive index using as component of matrix was benefit to enhance the performances of holographic photopolymer. Combining with formula optimization, high-quality holographic recording media can be achieved for practical application.

\section{Method}

A series of fluorinated epoxy resin (FTGE) were synthesized and applied into the fabrication of holographic 
recording media based on photopolymer. All samples contained similar proportion of components (monomer, photoinitiator and photosensitizer) except one component (FTGE) of filmforming resins, as listed in Table $\mathbf{1}$ particularly.

Table 1 The type and ratio of FTGE in a series of samples

\begin{tabular}{|c|c|c|}
\hline Sample & Type of FTGE & $\begin{array}{c}\text { Ratio of FTGE } \\
(\mathrm{wt} \%)\end{array}$ \\
\hline 29 & Peta-FTGE & 8.25 \\
\hline 30 & Peta-FTGE & 4.53 \\
\hline 33 & Peta-FTGE & 24.26 \\
\hline 34 & Peta-FTGE, TTA21P & 8.39 \\
\hline 35 & Buta-FTGE & 8.13 \\
\hline 36 & Buta-FTGE & 22.60 \\
\hline 37 & Prop-FTGE & 8.13 \\
\hline 38 & Prop-FTGE & 22.74 \\
\hline 39 & Buta-FTGE, TTA21P & 7.57 \\
\hline 40 & Prop-FTGE, TTA21P & 8.17 \\
\hline 43 & Prop-FTGE, TTA21P & 11.36 \\
\hline
\end{tabular}

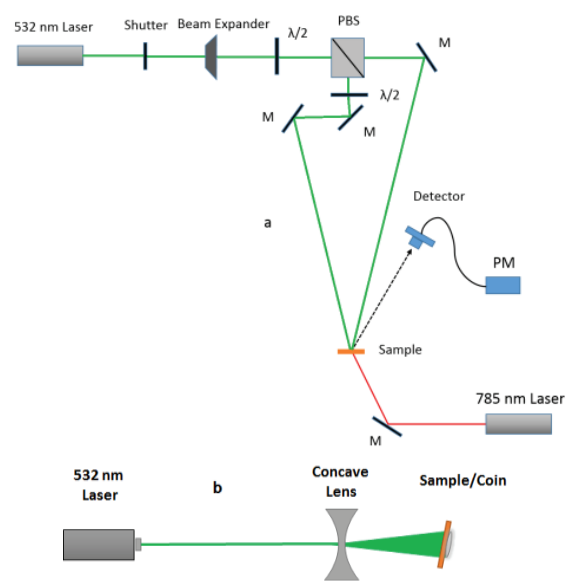

Figure 1 Holographic experimental setups (a) diffraction effciency test; (b) holographic recording of coin.

The performances of these formula were investigated, including diffraction efficiency, sensitivity, and image recording and reconstruction. Continuous holographic exposure experiments were carried out using a diode laser $(\lambda=532 \mathrm{~nm})$ as the coherent recording beam and a He-Ne laser $(\lambda=785 \mathrm{~nm})$ as the probe beam (Figure $\mathbf{1}$ a). In single-pulse holographic exposure experiments, a $532 \mathrm{~nm}$ pulsed laser (Nd:YAG, pulse width of $150 \mathrm{ps}$ ) was used as the light source. In addition, the image of a coin was recorded in $30 \mu \mathrm{m}$ thick films through a simple reflecting holographic exposure using another $532 \mathrm{~nm}$ pulsed laser (Nd:YAG, pulse width of $10 \mathrm{~ns}$ ) (Figure $1 \mathbf{b}$ ).

\section{Results and Discussion}

\section{Refractive index of FTGE}

The refractive indexes of three FTGE are measured by Abbe refractometer and listed in Table 2. Due to the different content of fluorine in them, the refractive index fluctuated in a range of 1.444-1.457. Compared with the index of a common epoxy resin TGE $(\sim 1.480)$, it is indicated that the introduction of fluorine significantly decreases the refractive index of FTGE ( 1.450), which is lowered about 0.03. Obviously, using FTGE as matrix to match common monomers will be benefit to obtain an enhanced refractive index modulation on holography performance.

Table 2 Refractive index of FTGE $\left(\mathrm{T}_{\text {room }}=21{ }^{\circ} \mathrm{C}\right.$ )

\begin{tabular}{|c|c|c|c|c|}
\hline & TGE & Peta-FTGE & Buta-FTGE & Prop-FTGE \\
\hline $\mathrm{n}$ & 1.480 & 1.444 & 1.453 & 1.457 \\
\hline
\end{tabular}

\section{Continuous holographic exposure}

The samples are under the illumination of a $532 \mathrm{~nm}$ diode laser with the exposure intensity of $0.5-4 \mathrm{~mW} / \mathrm{cm}^{2}$, ever 8 seconds a count. The curves of diffraction efficiency and exposure dosage are showed in Figure 2.

The amount of fluorinated epoxy resin Peta-FTGE in samples was $33>34 \approx 29>30$, while the order of sensitivity was $33>34>29>30$. Meanwhile the samples with Buta-FTGE or Prop-FTGE showed the same trend in the relationship between FTGE wt $\%$ and sensitivity (Figure 2a-2c). The results indicated that the sensitivity of samples was improved with the increased proportion of FTGE. Three samples with the highest ratio ( $>22 \%$ ) of FTGE all presented the highest sensitivity in three types of fluorinated epoxy resin. Besides, No.38 showed the most excellent holographic sensitivity among all samples and gained high diffraction efficiency (over 90\%) within $90 \mathrm{~s}$ exposure under $532 \mathrm{~nm}$ laser with the intensity of 0.53 $\mathrm{mW} / \mathrm{cm}^{2}$. However, phase separation occurred when the content of fluorine resin was further increased in the photopolymer materials because of the high surface energy of fluorine.

\section{Single-pulse holographic exposure}

The samples No. 33, 36, 38 with FTGE $(>22 \%)$ were examined under transient exposure of a single pulse $(532 \mathrm{~nm}$, 
$150 \mathrm{ps}$ pulse width) with the exposure dosage of (I) 38.9-39.9 $\mathrm{mJ} / \mathrm{cm}^{2}$, (II) $25.5-26.9 \mathrm{~mJ} / \mathrm{cm}^{2}$ and (III) $12.0-13.1 \mathrm{~mJ} / \mathrm{cm}^{2}$. As shown in Figure 3, No. 38 exhibits a diffraction efficiency of more than $1 \%$, which is two orders of magnitude larger than those of No. 33 and $\mathbf{3 6}$ under the triple exposure dosage. In addition, No. 38 presents $3.7 \%$ diffraction efficiency under the exposure dosage of $25.5-26.9 \mathrm{~mJ} / \mathrm{cm}^{2}$. Such a sensitivity is enough to record transient events and fast objects.
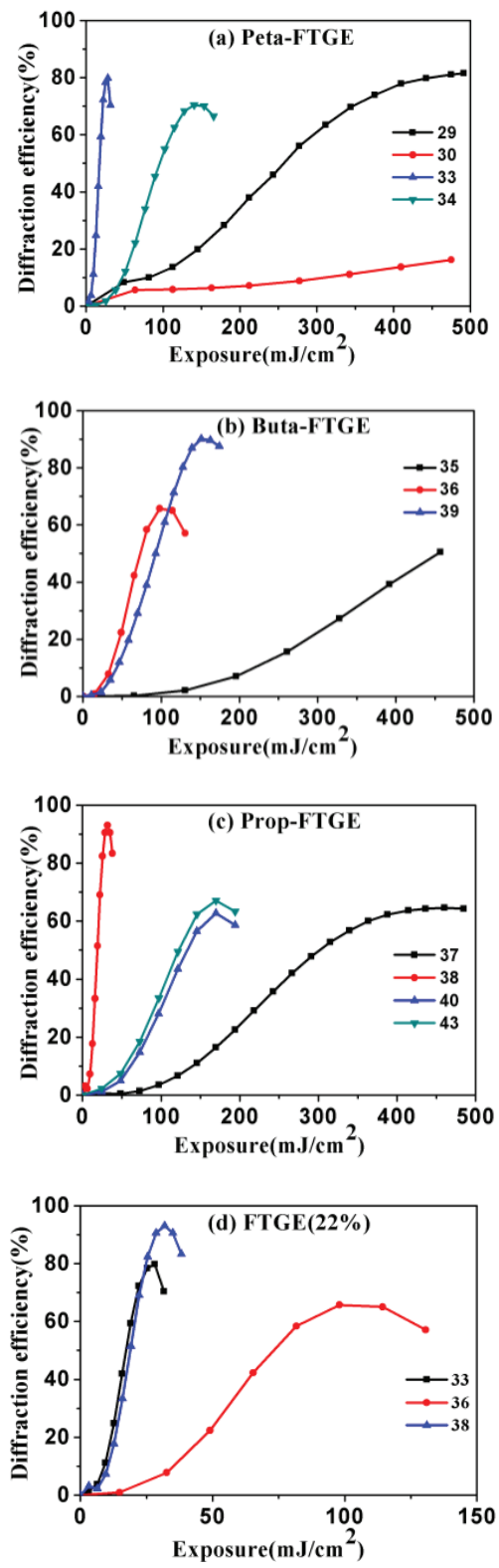

Figure 2 Exposure characteristic curves of samples with variable ratio of (a) Peta-FTGE, (b)
Buta-FTGE, (c) Prop-FTGE, and different (d) FTGE under the illumination of a $532 \mathrm{~nm}$ diode laser

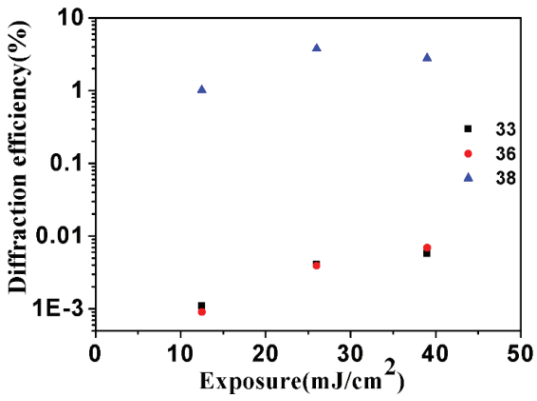

Figure 3 Diffraction efficiency of samples 33, 36, 38 with different FTGE under the exposure of a single pulse laser (532 nm, $150 \mathrm{ps}$ pulse width) with different exposure dosages.

\section{Hologram recording}

Most of holograms of coin recorded in $30 \mu \mathrm{m}$ thick films were blurry, while hologram in sample No. $\mathbf{3 8}$ was clearly visible to naked eyes as shown in Figure 4. This result was consistent with previous holographic experiments, and indicated No. $\mathbf{3 8}$ also has good performance in image recording and reconstruction.

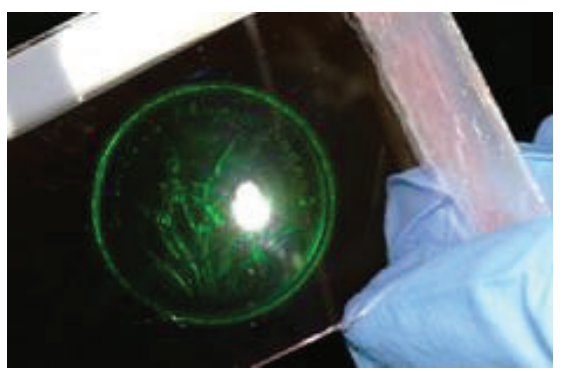

Figure 4 The visible hologram recorded in sample 38 exposure by a $532 \mathrm{~nm} \mathrm{Nd:YAG} \mathrm{laser} \mathrm{with}$ pulse width of $10 \mathrm{~ns}$

\section{Conclusion}

The introduction of fluorinated epoxy resin (FTGE) with a low refractive index $(\sim 1.45)$ significantly enhanced the refractive index modulation and sensitivity of the holographic photopolymer samples. In addition, the sensitivity of samples was improved with the increased proportion of FTGE before phase separation of materials. Through the research of a series 
of formulations, one sample (No.38) showed the most excellent holographic optical characteristics including diffraction efficiency, sensitivity, and image recording and reconstruction among all samples, which would be potential to fabricate highquality holographic recording media for practical application.

\section{References}

Chen G. N., Ni M. L., Peng H. Y., Huang F H, Liao Y G, Wang $\mathrm{M} \mathrm{K}$, and Xie X L., "Photoinitiation and Inhibition under Monochromatic Green Light for Storage of Colored 3D Images in Holographic Polymer-Dispersed Liquid Crystals," ACS Applied Materials \& Interfaces, 9(2), 18101819 (2017).

Cody D., Gribbin S., Mihaylova E., and Naydenova I., "LowToxicity Photopolymer for Reflection Holography," ACS Applied Materials \& Interfaces, 8 (28): 18481-18487 (2016). Friedrich K. B., Rainer H., Thomas R., Marc S. W., and Thomas F., "From the Surface to Volume: Concepts for the Next Generation of Optical-Holographic Data-Storage Materials,” Angew. Chem. Int. Ed., 50, 4552 - 4573 (2011).

Kowalski B. A, McLeod R. R., "Design concepts for diffusive holographic photopolymers," Journal of Polymer Science Part B: Polymer Physics, 54 (11): 1021-1035 (2016).

Li X., Chen L. W., Li Y., Zhang X. H., Pu M. B., Zhao Z Y, Ma X. L., Wang Y Q, and Luo X G., "Multicolor 3D metaholography by broadband plasmonic modulation," Science Advances, 2(11), e1601102 (2016).

Satoh, K., Aoki, K., Hanazawa, M., Kanemura, T., Lim, P. B. and Inoue, M., "Properties of New Fluorinated Holographic Recording Material for Collinear Holography,” Japanese Journal of Applied Physics, 48, 03 A030 (2009).

Marvin D. A., David J. G., Sudheendran M., Chen W., and Philip D. N., "High Dynamic Range $(\Delta \mathrm{n})$ Two-Stage Photopolymers via Enhanced Solubility of a High Refractive Index Acrylate Writing Monomer," ACS Appl. Mater. Interfaces, 10, 1217-1224 (2018).

Tomita, Y., Urano, H.,Taka A., and Yasuhiro K., "NanoparticlePolymer Composite Volume Holographic Gratings Dispersed with Ultrahigh-Refractive-Index Hyperbranched Polymer as Organic Nanoparticles," Opt. Lett., 41, 1281-1284(2016).

Yu Z., Jie Z., Yan Y., Luo Z.X., Jun L., Zeren L., and Jianhua Z., "Sensitive polyvinyl alcohol/acrylamide based photopolymer for single pulse holographic recording," Materials Letters, 138, 284-286 (2015). 


\title{
Fast-physical optics modeling of two-photon microscopy with 3D- structured illumination
}

\author{
Rui Shi *ab, Site Zhang ${ }^{\mathrm{b}}$, Christian Hellmann ${ }^{\mathrm{c}}$, and Frank Wyrowski ${ }^{\mathrm{a}}$ \\ ${ }^{a}$ Applied Computational Optics Group, Friedrich Schiller University Jena, Jena, Germany, \\ bightTrans International UG, Jena, Germany, \\ ${ }^{\mathrm{c} W y r o w s k i}$ Photonics UG, Jena, Germany,
}

\begin{abstract}
We perform a fast-physical optics modeling of two-photon microscopy with 3D-structured illumination in the context of field tracing. The Local Plane Interface Approximation (LPIA) algorithm, a free space propagation algorithm and the Fourier Modal Method (FMM) are all combined. We analyze the contrast, inhomogeneity and the temporal focusing of the 3D-structured illumination pattern in the focal region, which should be accounted for in image processing. We find that various effects influence the contrast, inhomogeneity and the temporal focusing of the pattern, such as the aberration of the real lens system, the pulsed laser and the Gaussian illumination profile. We also investigate the tolerance of the alignment of the microscopy system.
\end{abstract}

Keywords: field tracing, microscopy system, structured illumination, physical optics modeling, pulsed laser

\section{Introduction}

Recent advancements in microscopy techniques such as super-resolution imaging[1-4], multiphoton excitation[5], correlative microscopy, adaptive optics, image processing etc. opens up new possibilities towards imaging single live cells in deep tissue with high resolution. Structured illumination microscopy (SIM)[3,4] is one of the fluorescence imaging techniques that can tackle this challenge. However, in the case of thick samples the SIM technique suffers from out-offocus fluorescence background signal, which significantly reduces the signal-to-noise ratio (SNR). To overcome this obstacle, it has been suggested to use twophoton excitation[5]. For the analysis and optimization of this kind of high-NA microscopy system, a fully vectorial physical optics modelling is required that includes polarization, coherence, aberration and interference effects.

In this work, we perform a fast-physical optics modeling of the real microscopy system[6] in the context of field tracing[7]. The Local Plane Interface Approximation (LPIA) algorithm[8], a free space propagation algorithm[9] and the Fourier Modal Method (FMM)[10] are all combined. We analyze the contrast, inhomogeneity and the temporal focusing of the 3Dstructured illumination pattern in the focal region, based on the aberration of the real lens system, pulsed laser, the Gaussian illumination profile and the alignment of the microscopy system. We also investigate the tolerance of the alignment of the microscopy system.

\section{Theoretical Background}

\section{Evaluation criteria}

Following the definition of electric energy density in [11], we define the contrast as one criterion to evaluate the quality of the illumination pattern as:

$$
\mathrm{c}=\frac{w_{\text {Ave }}^{\mathrm{Max}}-w_{\text {Ave }}^{\mathrm{Min}}}{w_{\text {Ave }}^{\mathrm{Max}}+w_{\text {Ave }}^{\mathrm{Min}}}
$$

where $w_{\text {Ave }}^{\text {Max }}$ and $w_{\text {Ave }}^{\text {Min }}$ are the average values of the maxima and minima of electric energy density respectively. The best contrast leads to $\mathrm{c}=1$.

We also define the inhomogeneity, which is another important criterion for the quality of the illumination pattern, as:

$$
\sigma=\frac{w_{\mathrm{Max}}^{\mathrm{Max}}-w_{\mathrm{Min}}^{\mathrm{Max}}}{w_{\mathrm{Max}}^{\mathrm{Max}}+w_{\mathrm{Min}}^{\mathrm{Max}}}
$$


where $w_{\text {Max }}^{\text {Max }}$ and $w_{\text {Min }}^{\text {Max }}$ are the maximum and minimum values of the maxima of electric energy density respectively. The smallest inhomogeneity leads $\sigma=0$.

\section{Field tracing}

The schematic of illumination part of SIM system is shown in Fig.1. The structured illumination is achieved via interfering three coherent beams in the focal region. The three beams are generated by a grating illuminated by a continuous or pulsed laser beam. The period of the grating is chosen so that demanded period of the illumination pattern at sample plane can be obtained. Then the diffracted light goes through the first and second lens and interfere with each other at the back focal plane of the second lens to form an intermediate image. Then the intermediate image is demagnified by the 4-f setup which consists of the tube lens and high-NA objective lens to form the illumination pattern at the sample plane. The fully vectorial modeling is performed in the context of field tracing as shown in Fig.2[12]. We trace the field through different optical elements in different regions by different operators by switching between different domains with use of the bi-directional operator denoted

$B$ in space and spectral domain, $B$ in spatial-frequency and spectral domain, in order to maximize the accuracy and minimize the numerical effort.

In the case of structured illumination microscopy, we generate the electric field in spatial-frequency and spectral domain, and then propagate the field by free space operator $P_{1}$ in spatial-frequency and spectral domain which can be obtained rigorously by Spectrum of Plane Wave(SPW)[9]. When the field is diffracted from

the grating, we trace it by $B_{1}$ operator which is calculated rigorously by FMM in spatial-frequency and spectral domain[10]. Then it is propagated again by free space operator. We make an assumption for the specific application, SIM, that field which enters into the lens is in the geometric zone. Therefore, the geometric inverse Fourier transform[13] can be applied to accelerate to whole modeling procedure. Then, the LPIA is applied to propagate the field through the curved interfaces of the lens in space domain. A geometric Fourier transform is followed to obtain the field in spatial-frequency and spectral domain. We repeat the free space propagation, inverse geometric Fourier transform, LPIA and geometric Fourier transform to obtain the field at the sample plane in spatial-frequency and spectral domain. And then the regular inverse Fourier transform is performed to obtain the field in spatial and spectral domain including the diffraction and then another Fourier transform is performed to obtain the field in space-time domain. The whole modeling procedure as shown in Fig. 2 can be write briefly in Tab.1.

Table 1. Field tracing procedure.

\begin{tabular}{|l|l|}
\hline \multicolumn{1}{|c|}{ Steps } & \multicolumn{1}{c|}{ P/B-Operators } \\
\hline $1 ; 3 ; 7 ; 11 ; 15 ; 19$ & Free space propagation \\
\hline $5 ; 9 ; 13 ; 17$ & LPIA \\
\hline 2 & FMM \\
\hline $6 ; 10 ; 14 ; 18$ & Fourier transform \\
\hline $4 ; 8 ; 12 ; 16 ; 20$ & Inverse Fourier transform \\
\hline
\end{tabular}

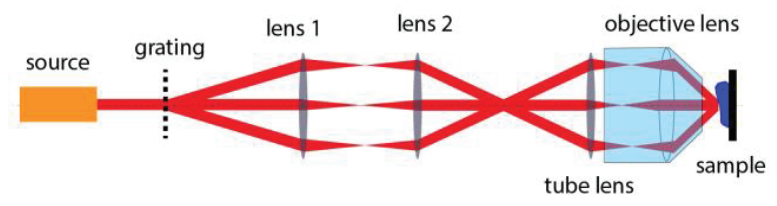

Figure 1. Schematic of the configuration of microscopy system with structured illumination. Collimated laser beam illuminates the grating. Then the diffracted beam goes through the first and second lens and interfere with each other at the back focal plane of the second lens to form an intermediate image. Then the intermediate image is demagnified by the 4-f setup which consists of the tube lens and high-NA objective lens to form the illumination pattern at the sample plane. 


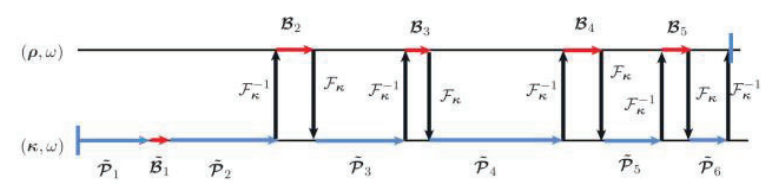

Figure 2. Field tracing diagram. $(\rho, \omega)$ denotes

the operator is in spatial and spectral domain

$(\kappa, \omega)$ denotes the operator is in spatial-frequency

and spectral domain.

\section{Initial Results}

Results are obtained via the software VirtualLab Fusion.[14]. The illumination wavelength is $800 \mathrm{~nm}$ for continuous laser and $800 \mathrm{~nm}$ for center wavelength of 100 fs pulsed laser. The illumination profile is plane wave. We show the illumination pattern near the focal plane by $0 \mathrm{~nm}, 200 \mathrm{~nm}, 400 \mathrm{~nm}$ axial shift in Fig.3 for the continuous laser illumination. In the initial results, an ideal lens is used which does not include any aberration. The contrast and inhomogeneity are changing at different axial position as expected. The illumination pattern of the pulsed laser case in shown in Fig.4. We can see the inhomogeneity increases because of the wide spectrum of the pulsed laser for all the axial position near the focus. It should be taken account during the image processing. Further results will be added into the presentation in the conference.

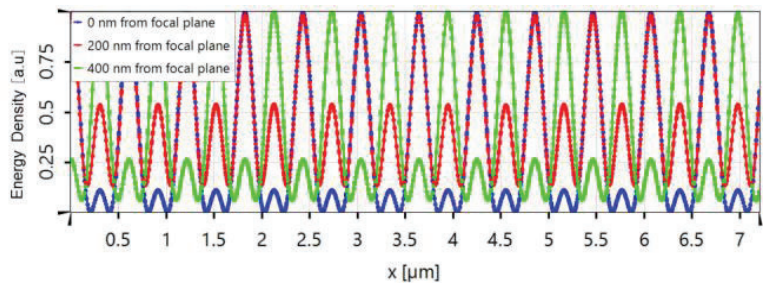

Figure 3. The interference pattern at different axial position.

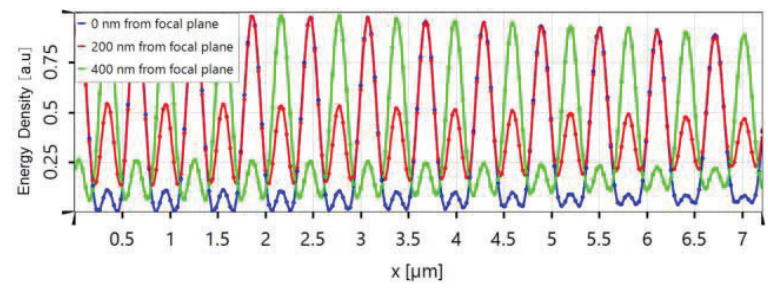

Figure 4. The interference pattern at different axial position. The inhomogeneity increases compared with Fig.3 when the light source is pulsed laser of $100 \mathrm{fs}$ with a center wavelength $800 \mathrm{~nm}$.

\section{Summary and Conclusion}

A fully vectorial physical optics is performed to modeling the real microscopy system using commercial lens and gratings.

The influence of the contrast and inhomogeneity and temporal focusing of the 3D-structued illumination pattern from the aberration of the microscopy system, the pulsed laser, and the spatial Gaussian wave profile are investigated and analyzed.

It is found that all the three factors have the impact on the quality of the illumination pattern which should be taken account for the image processing. The influence from the alignment of the microscopy system is also investigated.

\section{References}

[1] Hell, S. W. and Wichmann, J., "Breaking the diffraction resolution limit by stimulated emission: stimulated emission-depletion fluorescence microscopy," Opt. Lett. 19, 780-782 (1994).

[2] Betzig, E., Patterson, G. H., Sougrat, R., Lindwasser, O. W., Olenych, S., Bonifacino, J. S., Davidson, M. W., Lippincott-Schwartz, J., and Hess, H. F., "Imaging intracellular fluorescent proteins at nanometer resolution," Science 313(5793), 1642-1645 (2006).

[3] Rainer Heintzmann, C. G. C., "Laterally modulated excitation microscopy: improvement of resolution by Using a diffraction grating," (1999). 
[4] Gustafsson, M. G. L., "Surpassing the lateral resolution limit by a factor of two using structured illumination microscopy," Journal of Microscopy (2000).

[5] Q. Song, K. Isobe, F. Kannari, H. Kawano, A. Kumagai, A. Miyawaki, and K. Midorikawa, "Temporal Focusing Microscopy with 3D Structured Illumination for Enhanced Axial Resolution in Deep Imaging," in 2015 European Conference on Lasers and Electro-Optics - European Quantum Electronics Conference, (2015)

[6] Kurvits, J. A., Jiang, M., and Zia, R., "Comparative analysis of imaging congurations and objectives for fourier microscopy," J. Opt. Soc. Am. A 32, 2082 2092 (2015).

[7] Wyrowski, F. and Kuhn, M., "Introduction to field tracing," Journal of Modern Optics 58(5-6), 449466(2011).

[8] v. Pfeil, A., Wyrowski, F., Drauschke, A., and agedal, H., "Analysis of optical elements with the local planeinterface approximation," Appl. Opt. 39, 3304-3313 (2000).

[9] Zhang, S., Asoubar, D., Hellmann, C., and Wyrowski, F., "Propagation of electromagnetic felds between non-parallel planes: a fully vectorial formulation and an efficient implementation," Appl. Opt. 55, 529
(2016).

[10] Moharam, M. G., Gaylord, T. K., Grann, E. B., and Pommet, D. A., "Formulation for stable and efficient implementation of the rigorous coupled-wave analysis of binary gratings," J. Opt. Soc. Am. A 12, 10681076(1995).

[11] B. Richards and E. Wolf. "Electromagnetic diffraction in optical systems. ii. structure of the image field in an aplanatic system," Proceedings of the Royal Society of London A: Mathematical, Physical and Engineering Sciences, 253(1274):358379 (1959)

[12] Rui Shi, Norik Janunts R. H. C. H. F.W., "Fastphysical optics modeling of microscopy system with structured illumination," (2018)

[13] Wyrowski, F. and Hellmann, C., "The geometric fourier transform," Proc. DGaO (2017).

[14] Fast physical optics software "Wyrowski VirtualLab Fusion", LightTrans GmbH, Jena, Germany. 


\title{
Development of a Long-Wavelength Swept-Source Optical Coherence
}

\section{Tomography System for High-Content Ex Vivo Tissue Imaging}

\author{
Chuan-Bor Chueh ${ }^{\mathrm{a}}$, Ting-Yen Tsai ${ }^{\mathrm{a}}$, Yin-Peng Huang ${ }^{\mathrm{b}}$, Ting-Hao Chen ${ }^{\mathrm{a}}$, You-Nan Tsai ${ }^{\mathrm{a}}$, \\ Ching-Yu Wang, ${ }^{\mathrm{a}}$, Teng-Chieh Chang, ${ }^{\mathrm{a}}$, Yi-Ping Hung ${ }^{\mathrm{b}}$, Hsiang-Chieh Lee ${ }^{\mathrm{a}, \mathrm{c},{ }^{*}}$ \\ ${ }^{a}$ Graduate Institute of Photonics and Optoelectronics, National Taiwan University, Taipei 10617, \\ Taiwan \\ ${ }^{\mathrm{b}}$ Graduate Institute of Networking and Multimedia, National Taiwan University, Taipei, Taiwan \\ ${ }^{\mathrm{c}}$ Molecular Imaging Center, National Taiwan University, Taipei 10617, Taiwan \\ Email: hclee2@,ntu.edu.tw
}

\begin{abstract}
Optical coherence tomography (OCT) is becoming one of the most important noninvasive imaging technique, enabling rapid volumetric imaging of tissue architectures. Laser wavelength in third near infrared (NIR) optical window regime promises the potential for deep penetration depth due to lower scattering coefficient in tissue. In this paper, we have developed a high speed and long-wavelength swept-source optical coherence tomography (SS-OCT) imaging system with a $1.7 \mu \mathrm{m}$ wavelength swept laser. With a specifically designed fiber optic scanning microscope where two objectives with different magnification ( $5 \mathrm{X}$ and $10 \mathrm{X}$ ), it allows multiscale imaging of the imaging tissue specimens. In addition, the imaging field of the $1.7 \mu \mathrm{m} \mathrm{SS-OCT} \mathrm{system} \mathrm{is} \mathrm{extended} \mathrm{using} \mathrm{the} \mathrm{mosaic} \mathrm{scanning} \mathrm{protocol} \mathrm{leveraging} \mathrm{the} \mathrm{high} \mathrm{precision}$ stage in the sample arm. The $1.7 \mu \mathrm{m}$ SS-OCT imaging technology exhibits great potential for deep tissue imaging where the light attenuation due to tissue scattering is reduced.
\end{abstract}

Keywords: optical coherence tomography, tunable laser, biological and medical imaging, multiple scattering, light

\section{Introduction}

Optical coherence tomography (OCT) provides highresolution cross-section images of biological tissues without requiring any exogenous contrast agents. The 1.7 $\mu \mathrm{m}$ wavelength light has a smaller scattering coefficient compared with the $0.8 \mu \mathrm{m}$ to $1.3 \mu \mathrm{m}$ wavelength regions. With this characteristic, we are able to retrieve a deeper tissue signal owing to that longer wavelength light can travel further. In addition, it will collect less unwanted scattered light from adjacent, and thus the signal-to-noise (SNR) ratio in deeper tissue region might get higher [1]. It was testified that the $1.7 \mu \mathrm{m}$ wavelength laser has the possibility to improve the penetration depth in the observation of turbid scattering tissue owing to its lower signal attenuation in the tissue [2-5]. Despite higher water absorption with a longer wavelength, a local minimum of water absorption occurs at 1.5 to $1.85 \mu \mathrm{m}$ which are also called third near-infrared spectral window [6]. Therefore, since the scattering coefficient becomes smaller and absorption coefficient becomes larger as the wavelength becomes longer, the total signal attenuation at $1.7 \mu \mathrm{m}$ in turbid scattering tissue is still smaller than that at 1.3 $\mu \mathrm{m}[7-9]$. In this paper, a prototype $1.7 \mu \mathrm{m}$ swept-source OCT (SS-OCT) system was built enabling coregistered OCT imaging with different imaging resolutions. In the sample arm, two objectives with different numerical apertures (NA) were mounted to a power turret allowing seamless change of the imaging resolution and depth-offield (DOF). Preliminary results of the OCT images of the human finger and tape (Scotch, 3M) were demonstrated below.

\section{Method}

Figure 1 shows the schematic diagram of the $1.7 \mu \mathrm{m}$ SSOCT system. The sweep source laser (Santec) used in this system has a central wavelength of $\sim 1.7 \mu \mathrm{m}$ and supports OCT imaging with an axial resolution of $\sim 13.7 \mu \mathrm{m}$ in air (full-width at half-maximum, FWHM). The SS laser light output was divided by a fiber coupler (FC1) into the reference and sample arms through a Michelson interferometer. The light exiting the fiber terminal in each arm subsequently passed through the customized collimator. In the sample arm, other than using the galvanometer scanning mirror, a gimbal-less dual-axis 
micro-electro-mechanical system (MEMS) mirror (Mirrorcle) was used to provide high-speed beam scanning over the specimen surface. After the telescope, two turret-mounted $5 \mathrm{X}$ and $10 \mathrm{X}$ objectives to provide multiscale OCT imaging of the tissue specimens. The backscattered light from the sample will pass through FC2 and interfere with the light reflected from the FC3 of the reference arm. The interference light will go through FC4 to the dual balanced photodetector. A 12-bit digitizer (ATS9350, AlazarTech) was used to convert the analog electric signal from the detector into a digitized electric signal for the following signal processing and image reconstruction. An in-house developed user interference based on $\mathrm{C}++$ programming language was used for the instrument control and data acquisition.

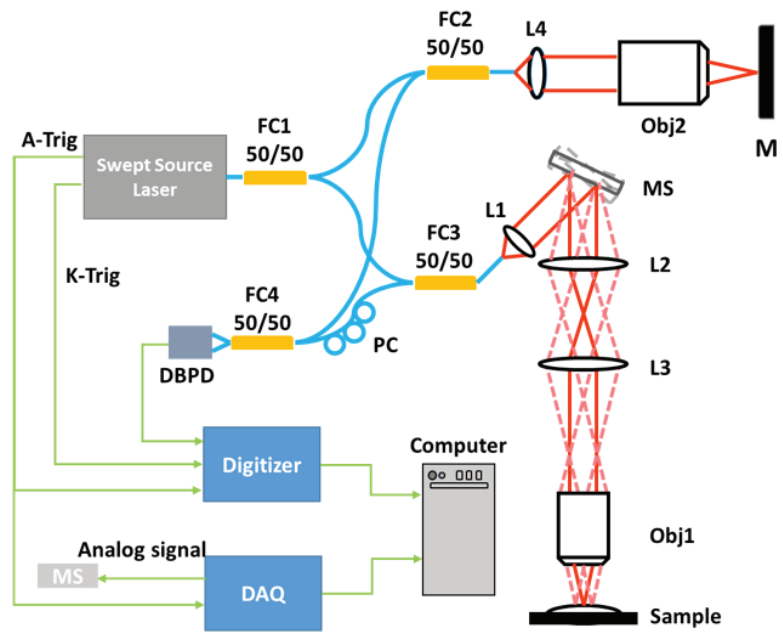

Figure 1. Schematic diagram of the $1.7 \mu \mathrm{m}$ OCT system. FC: fiberoptic coupler; L1 and L4: collimating lens; L2 and L3: achromatic lens; Obj1, and Obj2: objective; M: mirror; MS: micro-electro-mechanical system (MEMS) mirror; DBPD: Dual Balanced Photodetector; PC: polarization controller.

\section{Result}

The prototype $1.7 \mu \mathrm{m}$ SS-OCT system was used to acquire volumetric OCT images of a variety of imaging samples for the preliminary validation of the system performance, including a roll of scotch tapes and human finger as shown in Figure 2. The selected OCT images show that the developed $1.7 \mu \mathrm{m}$ SS-OCT is capable of providing OCT imaging with sufficient signal intensity even at deeper imaging depth.
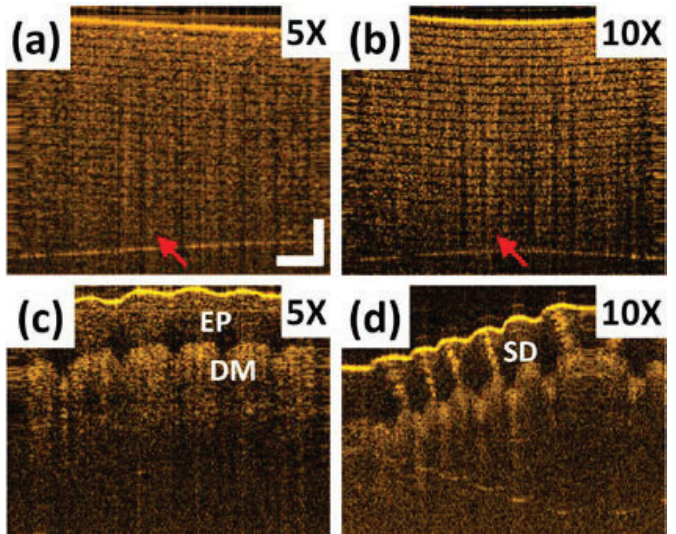

Figure 2. Example cross-sectional OCT images acquired with the $1.7 \mu \mathrm{m}$ SS-OCT system with the near-infrared objectives implemented in the sample arm. (a) 5X, (b) 10X scotch tape, (c) $5 \mathrm{X}$, (d) $10 \mathrm{X}$ ventral surface of the finger. Scale bars: $0.5 \mathrm{~mm}$.

\section{Acknowledgment}

The authors acknowledge the support from the Young Scholar Fellowship Program by the Ministry of Science and Technology (MOST) of the Republic of China (ROC), Taiwan (MOST-107-2636-E-002-003.

\section{Reference}

[1] M. Yamanaka, T. Teranishi, H. Kawagoe, and N. Nishizawa, "Optical coherence microscopy in $1700 \mathrm{~nm}$ spectral band for high-resolution labelfree deep-tissue imaging," Scientific Reports, Article vol. 6, p. 31715, 08/22/online 2016.

[2] L. Carrion, M. Lestrade, Z. Q. Xu, G. Touma, R. Maciejko, and M. Bertrand, "Comparative study of optical sources in the near infrared for optical coherence tomography applications," (in English), Journal of Biomedical Optics, vol. 12, no. 1, Jan-Feb 2007.

[3] U. Sharma, E. W. Chang, and S. H. Yun, "Longwavelength optical coherence tomography at 1.7 $\mu \mathrm{m}$ for enhanced imaging depth," Optics Express, vol. 16, no. 24, pp. 19712-19723, 2008/11/24 2008.

[4] V. M. Kodach, J. Kalkman, D. J. Faber, and T. G. van Leeuwen, "Quantitative comparison of the OCT imaging depth at $1300 \mathrm{~nm}$ and 1600 
nm," (in English), Biomedical Optics Express, vol. 1, no. 1, pp. 176-185, Aug 22010.

[5] K. Hiroyuki, Y. Masahito, M. Shuichi, Y. Yoshiaki, and N. Norihiko, "Full-range ultrahigh-resolution spectral-domain optical coherence tomography in $1.7 \mu \mathrm{m}$ wavelength region for deep-penetration and high-resolution imaging of turbid tissues," Applied Physics Express, vol. 9, no. 12, p. 127002, 2016.

[6] L. A. Sordillo, Y. Pu, S. Pratavieira, Y. Budansky, and R. R. Alfano, "Deep optical imaging of tissue using the second and third near-infrared spectral windows," (in English), Journal of Biomedical Optics, vol. 19, no. 5, May 2014.

[7] N. G. Horton et al., "In vivo three-photon microscopy of subcortical structures within an intact mouse brain," Nature Photonics, vol. 7, p. 205, 01/20/online 2013.

[8] S. Ishida and N. Nishizawa, "Quantitative comparison of contrast and imaging depth of ultrahigh-resolution optical coherence tomography images in 800-1700 nm wavelength region," Biomedical Optics Express, vol. 3, no. 2, pp. 282-294, 2012/02/01 2012.

[9] H. Kawagoe, M. Yamanaka, and N. Nishizawa, "Axial resolution and signal-to-noise ratio in deep-tissue imaging with $1.7-\mu \mathrm{m}$ high-resolution optical coherence tomography with an ultrabroadband laser source," 2017, vol. 22, p. 11: SPIE. 


\title{
Label-fee dynamic imaging of mitochondria and related organelles inside live cells with simultaneous dual-wavelength photothermal microscopy
}

\author{
Jun Miyazaki* and Yasunobu Toumon \\ ${ }^{\mathrm{f}}$ Faculty of Systems Engineering, Wakayama University, 930 Sakaedani, Wakayama, 640-8510, Japan
}

\begin{abstract}
Various organelles inside live cells was observed without use of any label by means of a highly sensitive photothermal microscopy. Simultaneous dual-wavelength photothermal imaging allows us to identify mitochondria and related organelles which are presumably attributed to autophagosomes or autolysosomes. Real-time and time-lapse imaging revealed morphological dynamics of mitochondria and related organelles under physiological treatments. Morphological change of mitochondria from filamentous to round structure was clearly observed after membrane potential depolarization induced by chemical inhibitor. In contrast to fluorescence imaging, this method can observe the dynamics of various organelles, especially mitochondria without labeling, Therefore, it would be useful to understand their essential function in biomedical sciences and has a potential for an efficient diagnostic tool of mitochondria related deceases.
\end{abstract}

Keywords: photothermal microscopy, label-free imaging, mitochondria, live cell imaging

\section{Introduction}

Optical microscopy is indispensable for visualizing intracellular structure and biological tissues in biomedical research and medical diagnosis. Since optical methods are non-invasive, they are especially useful to reveal the structure and the dynamics of organelles in live cell and organs. Fluorescence microscopy is currently one of the most popular methods for culler imaging owing to its unprecedented sensitivity of background-free detection. Fluorescence molecules such as fluorescent proteins, organic dyes, and semiconductor nanocrystals are used to label specific targets. However, there is a possibility that the label-target complex may have different behavior from the native target. In addition, photobleaching of fluorescence signal during measurement is a major problem in fluorescence microscopy, especially in timelapse imaging.

In this study, we performed photothermal (PT) imaging of live cells to visualize distribution and dynamics of organelles inside cells without use of any label. PT microscopy can visualize non-fluorescent endogenous chromophores in cells with high sensitivity and high spatial resolution. Non-fluorescent molecules are usually less affected by photobleaching compared to fluorescence molecules. We demonstrated that simultaneous dual-wavelength PT images allowed us to identify mitochondria and related organelles. Furthermore, real time and time-lapse imaging were conducted to observe their dynamics and morphological changes.

\section{Experimental setup}

\section{Experiment}

In PT microscopy, two laser beams with different wavelengths are usually used for pumping and probing [1]. The pump beam increases the temperature, $\Delta T$, around the focal point of the optical absorbing sample, which results in variations in the local refractive index (typically $\Delta n \sim$ $10^{-4}$ with $\Delta T=1 \mathrm{~K}$ for water). Variation in the refractive index is detected using the probe beam by means of the pump-and-probe technique.

Figure 1 shows the experimental setup of our PT microscopy. A 780-nm laser diode (LD) was used for probing. For two color imaging, 520 and 640-nm LDs were used for pumping with their intensity modulated at different frequencies (300 and $400 \mathrm{kHz})$ for the simultaneous lock-in detection. The combined pump and probe beams were directed to a Galvano scanner and focused on a sample through an objective lens with the numerical aperture of 1.25. Focused laser beams was scanned sequentially point-by-point and the pixel information is assembled into an image. Spatially divided balanced detection scheme was implemented using a 


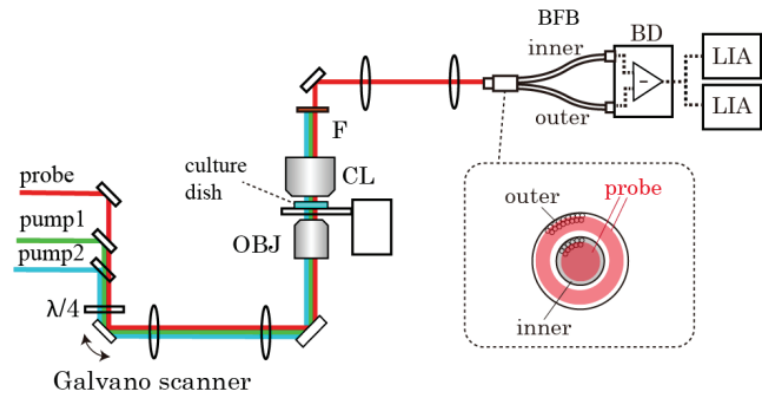

Figure 1. Photothermal microscope setup with spatially segmented balanced detection. using a. OBL: objective lens, CL: condenser lens, F: color filter, BFB: bifurcated fiber bundle, BD: balanced detector, LIA: lock-in amplifier.

bifurcated fiber bundle to improve the signal to noise ratio[2, 3].

\section{Results and discussion}

Figure 1 shows (a) bright field and (b,c) PT images of live HeLa cells. The bright field image was acquired using a CMOS camera with a LED-backlight. PT images from two channels are merged, where green and red color represent the signal intensity at the wavelength of 520 and $640 \mathrm{~nm}$, respectively. The image (c) is a magnified view of the image (b). Image acquisition time was 6 and $2 \mathrm{~s}$ in (b) and (c), respectively. It is obvious that PT microscopy provides higher contrast images inside the cell than the bright field image. Filamentous and punctuate structures are observed around cell nucleus which appeared as a circular dark shadow. The filament structures are attributed to mitochondria [4]. The contrast agent of PT signal is presumed to be cytochrome $\mathrm{c}$ in the mitochondria membrane. Because cytochrome c has an absorption peak around $520 \mathrm{~nm}$, signal intensity at the wavelength of $520 \mathrm{~nm}$ is larger than that at $640 \mathrm{~nm}$. Mitochondria appear as green in the merged images. In contrast, PT signal from punctate structures at the wavelength of $640 \mathrm{~nm}$ is larger than that at $520 \mathrm{~nm}$ and thus they appear orange in the merged images. The punctate structures are localized around mitochondria. We acquired moving images with $0.5 \mathrm{fps}$ to observe the dynamics of mitochondria and punctate structures, and found that the movement of the punctate structures are correlated with the mitochondria dynamics. We also
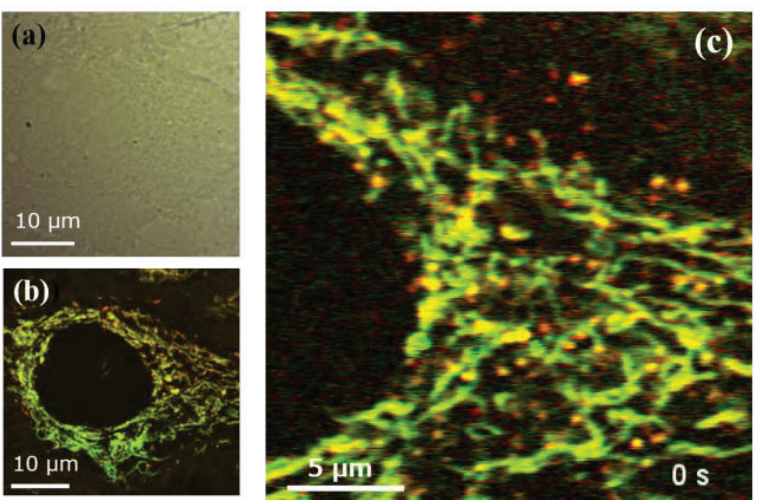

Figure 2. (a) bright field and $(b, c)$ two color photothermal images of a HeLa cell.

confirmed that cells are hardly affected by the temperature variation during measurement.

To ascertain the origin of the punctate structures, we observed morphological change of mitochondria under physiological treatments. Mitochondrial membrane depolarization is induced by adding $20 \mu \mathrm{M}$ carbonyl cyanide m-chlorophenylphydrazone (CCCP), a ionophore and decoupler of the respiratory chain. Figure 3 shows morphological translation of mitochondria from filamentous to round structure after depolarization as shown in Fig. 3 [5]. However, in contrast to mitochondria, punctate structures do not exhibit morphological change. These findings suggest that the punctate structure are different from mitochondria, but functionally related because they are colocalized. Although further studies will be needed to fully understand the origin, we consider that one of the possible candidates of the punctate structure is autophagosomes or autolysosome. There organelles result from mitochondria though autophagy process and assumed to contains cytochrome c. Thus, they would serve as PT contrast agents. Since $\mathrm{pH}$ environment inside these organelles is acidic, absorption spectrum of cytochrome c would differ from that in mitochondria. Therefore, intensity ratio of PT signal ratios at the two wavelengths are considered to be different between these organelles and mitochondria.

Dynamics observation of mitochondrial is of great importance in biomedical applications because it can be markers for identifying some myopathies or cancer [6]. This label-free method has a potential for an efficient diagnostic tool of mitochondria related deceases and 

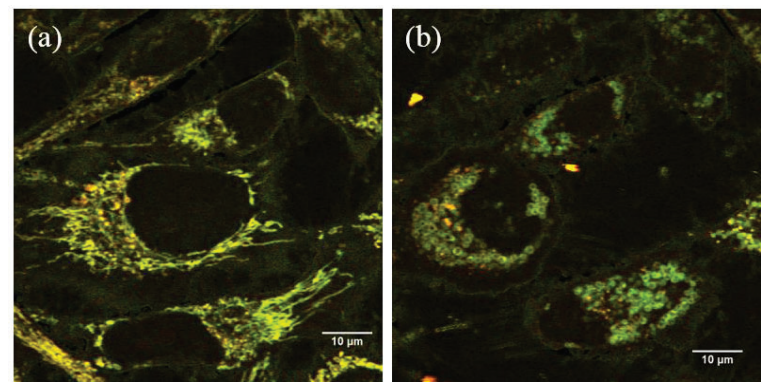

Figure 3. Photothermal images of HeLa cells (a)

before and (b) after inducing membrane depolarization with $20 \mu \mathrm{M}$ CCCP loading.

would promote understanding their essential function in biomedical sciences.

\section{References}

[1] P. Vermeulen, L. Cognet, and B. Lounis, "Photothermal microscopy: optical detection of small absorbers in scattering environments," J Microsc, 254(3), 115-121 (2014).

[2] J. Miyazaki, T. Iida, S. Tanaka et al., "Fast 3D visualization of endogenous brain signals with high-sensitivity laser scanning photothermal microscopy," Biomed Opt Express, 7(5), 170210 (2016).

[3] J. Miyazaki, H. Tsurui, K. Kawasumi et al., "Sensitivity enhancement of photothermal microscopy with radially segmented balanced detection," Optics Letters, 40, (2015).

[4] D. Lasne, G. A. Blab, F. De Giorgi et al., "Label-free optical imaging of mitochondria in live cells," Opt Express, 15(21), 14184-93 (2007).

[5] W. H. Gao, Y. M. Pu, K. Q. Luo et al., "Temporal relationship between cytochrome c release and mitochondrial swelling during UVinduced apoptosis in living HeLa cells," Journal of Cell Science, 114(15), 2855-2862 (2001).

[6] R. J. Giedt, P. Fumene Feruglio, D. Pathania et al., "Computational imaging reveals mitochondrial morphology as a biomarker of cancer phenotype and drug response," Scientific Reports, 6, (2016). 


\title{
Lensless digital holographic imaging through diffusive glass with different diffusion angles
}

\author{
Fumito Araki ${ }^{\mathrm{a}}$, Hidenobu Arimoto ${ }^{\mathrm{b}}$, Wataru Watanabe*a \\ a Department of Electrical \& Electronic Engineering, College of Science and Engineering, \\ Ritsumeikan University, 1-1-1 Nojihigashi, Kusatsu, Shiga, 525-8577 Japan \\ ${ }^{\mathrm{b}}$ Electronics and Photonics Research Institute, National Institute of Advanced Industrial Science and \\ Technology, AIST Tsukuba Central 5, 1-1-1 Higashi, Tsukuba, Ibaraki 305-8565 Japan
}

\begin{abstract}
Optical imaging through diffusive or scattering media has attracted much attention. Lensless digital holography is used to reconstruct the intensity and phase of an object located behind a diffuser. We investigate the influences of different diffuser angles of a diffusive glass plate on reconstruction of phase and intensity information of an object through a diffusive glass.
\end{abstract}

Keywords: back-propagation, complex amplitude, computational imaging, diffuser, digital holography, lensless imaging, scattering imaging, speckle

\section{Introduction}

Optical imaging through diffusive or scattering media has attracted much attention. Some groups have reportedly reconstructed intensity information of objects from intensity information of transmitted light of objects and ground glass [1]. Lensless digital holography enables reconstruction of intensity and phase images of objects through a diffusive glass plate $[2,3]$. This report describes reconstruction of phase and intensity information of an object behind diffusive glass by changing the diffusive angle of diffusive glass plates.

\section{Reconstruction of complex amplitude of an object by lensless digital holography}

Lensless digital holography is based on phase-shifting Mach-Zehnder interferometry. The experimental setup of such apparatus was described in an earlier report [3]. A He-Ne laser light source produces a $632.8 \mathrm{~nm}$ wavelength beam that is separated by a beam splitter into two beams: a reference beam and a beam passing through the sample, i.e., the sample beam. Then, the two beams are spatially overlapped to produce a hologram. Four-step phase shifting was performed using a piezo-transducer. From four digital holograms produced using four-step phase- shifting method, we calculated the quantitative phase [3]. Then, lensless digital holography enables the imaging sensor to record amplitude and phase information of the object. The object wavefront is then retrieved through numerical reconstruction. Consequently, the object intensity and phase are obtainable at an arbitrary distance.

\section{Experimental results}

We used a positive USAF test target as an object to be reconstructed. A holographic diffuser (HD) was inserted between the object and the beam splitter to visualize the object behind the diffusive glass plate. First, we attempted to reconstruct the object intensity and phase merely by calculating the backward propagation from the imaging sensor to the test chart without considering the HD effect with a diffusion angle of $1 \mathrm{deg}$ ( $1 \mathrm{~mm}$ thickness). We set the distance between the image sensor and the HD to 11.2 $\mathrm{cm}$, and the HD and the test chart to $6.5 \mathrm{~cm}$. According to the experimentally obtained result depicted in Fig. 1, it was not possible to reconstruct the test chart amplitude and phase merely by performing backward propagation calculations from the imaging sensor to the test chart.

*wata-w@fc.ritsumei.ac.jp; phone 81775994343 


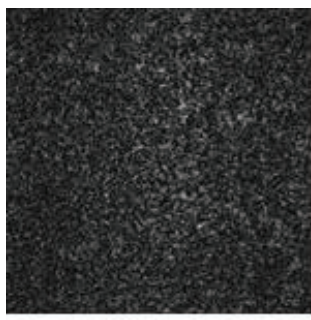

(a)

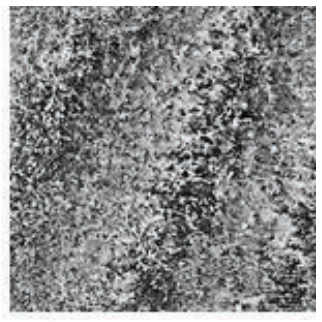

(b)
Figure 1. Reconstructed (a) intensity and (b) phase of the object at the sample plane merely by calculating backward propagation from the imaging sensor to the test chart.

To obtain information related to the test chart, we used a procedure described earlier in the literature [3]. Images of the phase distribution and the intensity distribution on the test chart are presented respectively in Figs. 2(a) and 2(b). Experiment results revealed the test chart intensity and phase through the HD.

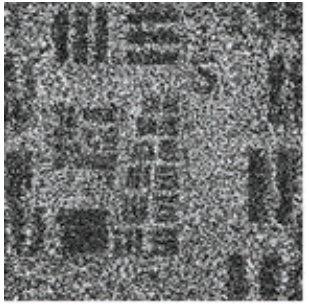

(a)

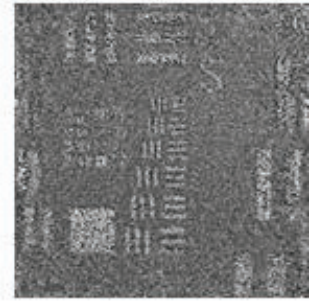

(b)
Figure 2. Reconstructed (a) intensity and (b) phase of the object through HD using a method described in the literature [3].

We investigated the quality of reconstructed intensity information of the object behind the HD by changing the diffusive angle of diffusive glass plates. The diffusion angles of the HDs were 1, 10, and 20 deg with $1 \mathrm{~mm}$ thickness. We also changed the distance between the HD and the test chart and reconstructed the object intensity information. The distance between the HD and the test target was $3.2 \mathrm{~cm}$ and $6.5 \mathrm{~cm}$. At a greater HD diffusion angle, the reconstructed image quality was degraded.

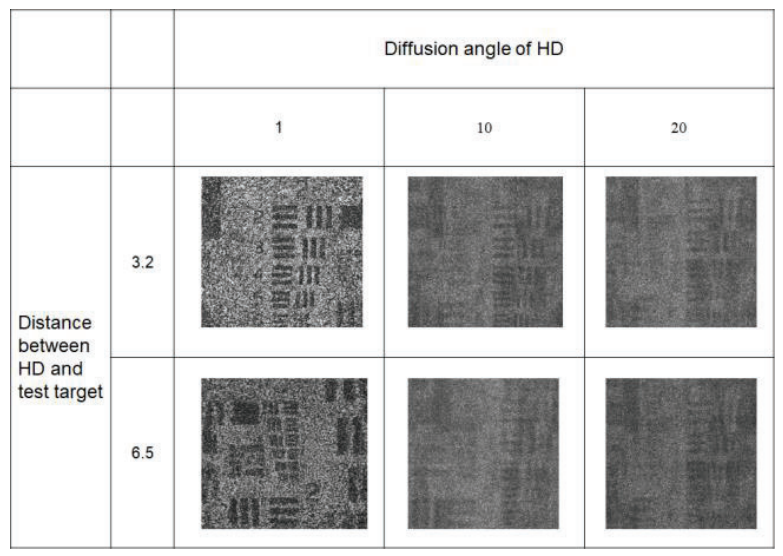

Figure 3. Reconstructed intensity of the object through different diffusion angles of $\mathrm{HD}$.

\section{Conclusion}

Lensless digital holography was used to reconstruct the phase and intensity of an object located behind the holographic diffuser. We investigated reconstruction of phase and intensity information of the object behind a diffusive glass by changing the diffusive angle of diffusive glass plates.

\section{Funding}

This work was supported by JSPS KAKENHI Grant-inAid for Scientific Research (C) Grant Number 17K05083.

\section{References}

[1] Singh, A., D. Naik, G. Pedrini, M. Takeda and W. Osten. 2014. Looking through a diffuser and around an opaque surface: A holographic approach. Opt. Express 22(7):7694-7701.

[2] Harm, W., C. Roider, A. Jesacher, S. Bernet and M. RitschMarte. 2014. Lensless imaging through thin diffusive media. Opt. Express 22(18):22146.

[3] Igarashi, A., H. Arimoto and W. Watanabe. 2018. Reconstruction of complex amplitude by lensless phaseshift digital holography through an opaque glass plate. Proceedings of SPIE 10711, in Biomedical Imaging and Sensing Conference, 1071118. 


\title{
Lensless Phase Difference Microscopy with Designed Imaging Device*
}

\author{
Xu Cao ${ }^{\mathrm{a}}$, Cheng Yang ${ }^{\mathrm{a}, \mathrm{b}}$, Zhijian Huang ${ }^{\mathrm{a}}$, Haowen Ma ${ }^{\mathrm{a}, \mathrm{b}}$, Tao Yue ${ }^{\mathrm{a}, \mathrm{b}}$, Xuemei Hu ${ }^{\mathrm{a}^{*}}$, Feng Yan ${ }^{\mathrm{a}, \mathrm{b}}$ \\ ${ }^{a}$ Department of Electronics and Engineering, Nanjing Univeristy, Nanjing, 210023, China \\ ${ }^{b}$ Nanjing University Institute of Sensing and Imaging Engineering, Nanjing, 210023, China
}

\begin{abstract}
In this paper, we propose a lensless phase difference microscopy (LPDM) using an imaging sensor with grating structure on the top of light-sensitive areas. Designing the period of grating appropriately, the proposed sensors can capture the intensity and phase difference information simultaneously using the contact imaging scheme. Benefitting from the scheme, the LPDM can achieve large space-bandwidth product with very low complexity and cost.
\end{abstract}

Keywords: microscopy, lensless imaging, phase difference imaging, image sensor, gratings

\section{Introduction}

Optical microscopy plays an important role in many research areas, especially for the biological cell and tissue imaging. Different from the conventional optical microscopies, which usually require complex and expensive devices, the lensless microscopy can take images without using the optical lens and any other professional optical elements, except the light sources and image sensors. Generally, there are two kinds of lensless microscopies, the diffraction imaging [1] and contact imaging [2] (a.k.a. shadow imaging), which records the light intensity transmitted through the specimen with very short distance between the samples and the image sensors (usually several wavelengths). While the computational complexity and reconstruction errors of diffraction imaging limit its widely application in practice, the lensless contact imaging microscopy can easily achieve extremely large space-bandwidth product (SPB) with very low system complexity and budget without complex computations.

The phase information can provide plenty of structure and refractive index information of samples and enhance the contrast of the images, and thus be widely used in the biological research and medical diagnosis. Existing phase resolved microscopies like phase contrast microscopy [3], DIC microscopy [4], and phase shifting microscopy [5] require complex light paths to derive the phase information. Recently, series of quantitative phase imaging microscopies are proposed to derive the accurate phase values with a set of specific devices. Besides, the light field microscopy also provides a way to capture the phase information using either the camera array [6] or microlens array [7]. Although these methods can capture the phase information, the SBP of them are limited by the traditional optical lenses, while the contact imaging scheme can achieve large SBP, but cannot derive the phase information direction, for there is no phase sensitive elements in the system.

In this paper, we propose a phase difference imaging sensor, which contains a diffractive structure on the top of the light-sensitive pixels. By setting the period of the gratings being twice the pixel intervals, the response of the adjacent two rows of the image sensor to the parallel light of different angles is different. In other words, the phase difference information can be extracted from the difference of adjacent two rows of the images captured by proposed LPDM sensors. The proposed sensor can capture both the intensity and phase difference information simultaneously using a very simple contact imaging scheme without complex computations.

*xuemeihu@nju.edu.cn 86-15210588703 
The proposed method is of three main advantages: (1) it can realize the phase resolved large SBP microscopy; (2) the system complexity and cost are markedly lower than the conventional microscope with phase information; (3) the phase different information can be derived easily using a very simple algorithm.

\section{Body}

We propose to realize simultaneously phase difference imaging and intensity imaging through designing the layout of the metal electronic on the image sensor.

As in Fig. 1, the period of the diffractive structure is twice the period of the pixels. The layout of the metal wire that we designed is shown in Fig. 1. The blue lines denote the designed diffractive grating and the yellow block denotes the pixel layout. As is shown, the period of the blue metal wire is twice the period of the pixel. (a)

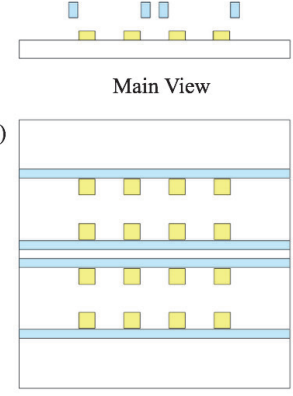

Top View (b)

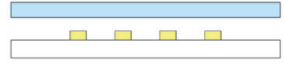

Side View

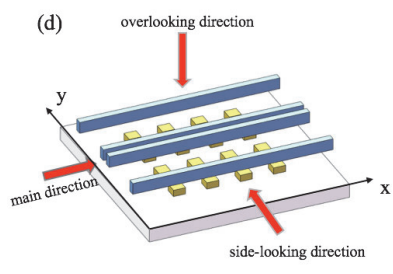

3D View
Fig. 1 The layout of the designed diffractive metal grating.

The metal diffractive grating would generate periodic light patterns on the sensor. The period of the diffractive light patterns equals the diffractive grating and the pixel difference in each period could encode the phase information of the incident light. To simulate and validate the effect of the diffractive metal pattern on the image sensor, we simulate the light intensity distribution on the sensor with the finite-difference time-domain simulation software (FDTD). As shown in Fig. 2, when the direction of the light on the sensor is perpendicular to the blue metal, along the Y direction of the sensor. As shown, the intensity distribution of the light on the image sensor is periodic, changing along the $\mathrm{Y}$ direction, and the period equals the blue metal. In each period, there is two rows of pixels and the odd row pixels are uniformly different from the even row pixels periodically. The simulation results experimentally demonstrate our basic intuition on designing the layout of the electronics related metal. As parallel light incidents in different direction, the periodic pattern diffracted by the metal grating is translated along the $\mathrm{Y}$ dimension and the angle information could be encoded in the difference between the even row and odd row. Through employing the designed novel imaging sensor under the lensless imaging scheme, we could realize simultaneously phase difference and intensity imaging.
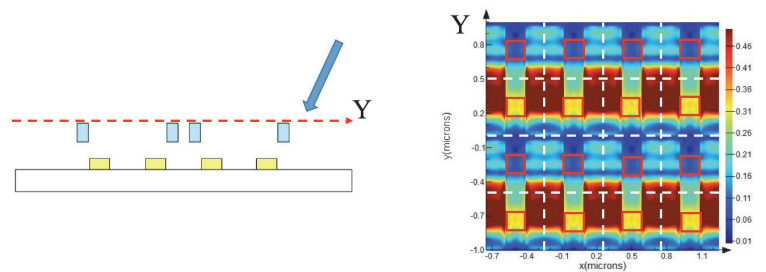

Fig.2 The FDTD simulation result: (a) the light incidence direction scheme, (b) the light intensity distribution on the image sensor. The red box denotes the pixel of the area.

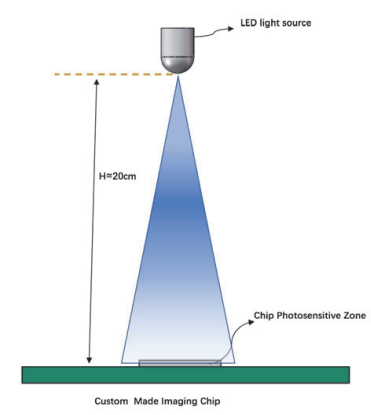

Fig. 3. Lensless imaging setup of our experiments.

The experimental setup for lensless imaging is shown in Fig. 3, we carried out physical sensor and captured real data with that, as shown in Fig. 3. Light incident perpendicularly on the sensor, the phase difference of the sample would cause the light to incident on the sensor with different angle and the difference of the even and odd rows can encode the phase difference information of the sample. As shown in Fig. 4(a), the sample on the sensor is the breast cancer cell MCF-7, immersed in the nutrient solution. As in Fig. 4(b-c), through comparing the image in the solution and out of the solution we could find out that periodic streak patterns appear where there is 
phase difference. Both the phase difference caused by the nutrient solution and the phase of the cancer cell could introduce the difference among the odd rows and the even rows. We calculate the ratio of the row and even column along the dashed red line in Fig. 4(a). As shown in Fig. 4(d). The slowly varying ratio reveals the phase difference of the nutrient solution and the rapid change along it reveals the phase difference caused by the sample. We locate the position of the rapid change along the dashed red line and plot out the corresponding area as shown in Fig. 4(f-m). Further demonstrating the phase information encoding ability of the proposed sensor design.
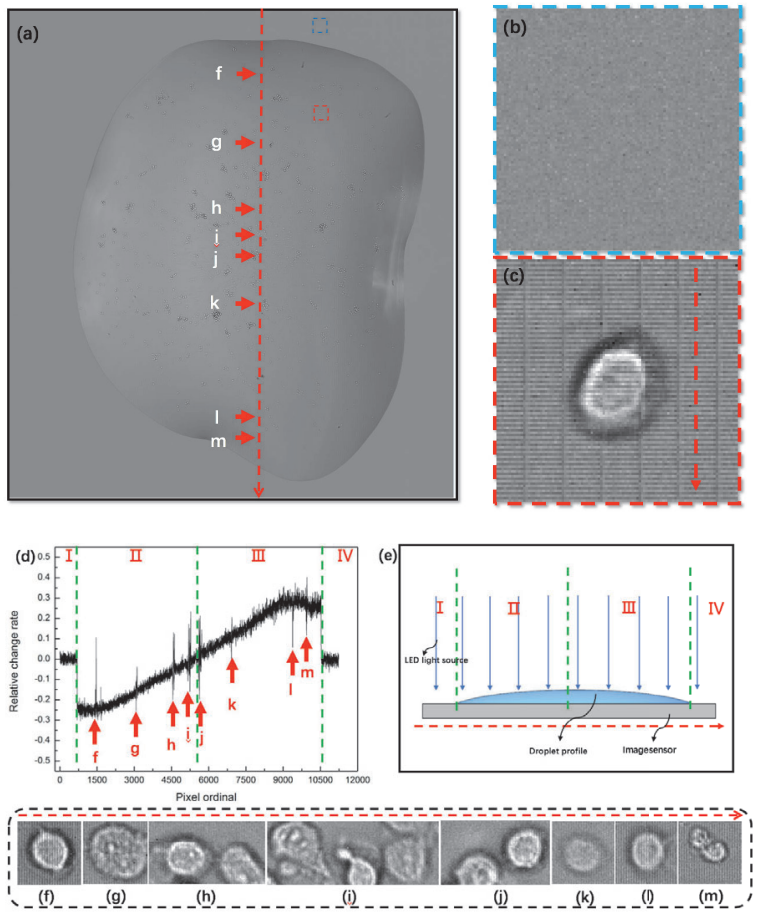

Fig. 4. Experimental captured data and phase difference analysis.

To directly visualize the phase difference encoding ability of our sensor, we rendered the phase difference result as shown in Fig. 5. We first interpolate the odd and even rows of the image to the resolution of the image, calculate the average of them. Generate the RGB image with $\mathrm{R}$ color channel the bilinear-interpolated odd-row image, G color channel the bilinear-interpolated even-row image and $\mathrm{B}$ channel the average image of the above interpolated image. The color along the $\mathrm{Y}$ dimension shown the phase difference cause by the sample on the sensor. Further demonstrating the phase resolving ability of our sensor.

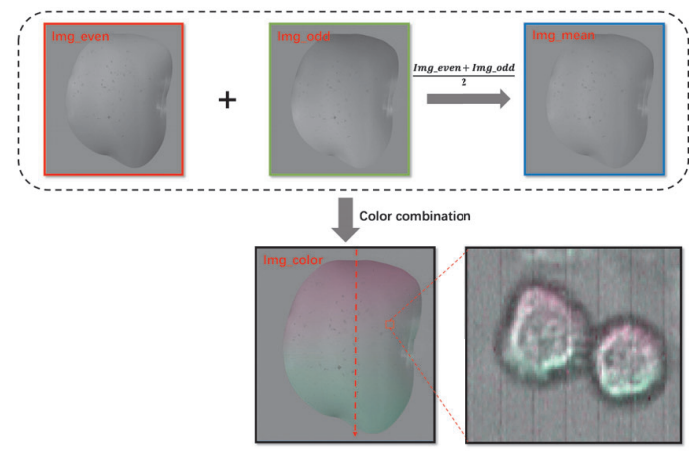

Fig. 5. Phase difference rendered imaging.

In conclusion, we designed the layout the electronic metal on the sensor and realized simultaneously intensity and phase difference imaging. Phase information would be further recovered with computation imaging method, which we leave as future work. We believe our method would inspire new generation compact of lensless phase imaging method.

\section{References}

[1] S. Isikman, S. S. S. Seo, I. Sencan, A. Erlinger, and A. Ozcan. Lensfree cell holography on a chip: From holographic cell signatures to microscopic reconstruction. 404-405 (2009).

[2] D. Lange, C. W. Storment, C. A. Conley, and G. T. A. Kovacs. A microfluidic shadow imaging system for the study of the nematode caenorhabditis elegans in space. Sensors and Actuators B: Chemical, 107(2):904-914 (2005).

[3] F. Zernike. Phase contrast, a new method for the microscopic observation of transparent objects part I. Physica. 9 (7): 686-698 (1942).

[4] W. Fang. Nomarski differential interference-contrast microscopy. ZEISS Information. 70: 114-120 (1968).

[5] K. Creath, V phase-measurement interferometry techniques, in: Progress in Optics, Elsevier, 349-393 (1988).

[6] X. Lin, J. Wu, G. Zheng, and Q. Dai, Camera array based light field microscopy. Biomedical optics express, 6(9): 3179-3189 (2015).

[7] M. Levoy, R. Ng, A. Adams, M. Footer, M. Horowitz. Light Field Microscopy. ACM Transactions on Graphics 25(3) (2006). 


\title{
Suppression of speckle noise in digital holography using speckle correlation properties of out-of-plane direction
}

\author{
Hideki Funamizu*a, Yoshiki Sakazume ${ }^{\text {a }}$, Jun Uozumi ${ }^{b}$ and Yoshihisa Aizu ${ }^{\text {a }}$ \\ ${ }^{a}$ Muroran Institute of Technology, 27-1 Mizumoto, Muroran, Hokkaido 050-8585, Japan \\ ${ }^{\mathrm{b}}$ Faculty of Engineering, Hokkai-Gakuen University, South 26, West 11, \\ Chuo-ku, Sapporo, Hokkaido 064-0926, Japan
}

\begin{abstract}
In this study, we present the suppression of speckle noise in digital holography using speckle correlation properties of the out-of-plane direction. In this method, it is assumed that digital holograms of diffuse objects are recorded on an image sensor. Using multiple holograms recorded by moving an image sensor to the out-of-plane direction, the speckle noise of a reconstructed image is suppressed and therefore the image quality is improved by the proposed method.
\end{abstract}

Keywords: Digital holography, Noise reduction, Speckle, Correlation function

\section{Introduction}

Digital holography is a useful technique for the recording and reconstruction of complex amplitudes of an optical field [1]. In this technique, an interference pattern of two coherent wavefronts, which we call a hologram, is detected and digitized by an image sensor such as CCD or CMOS camera and digital holograms are acquired in computer. The wavefront is reconstructed from the digital holograms numerically. One of the issues in digital holography is a speckle noise in the reconstructed images because of the use of coherent optical source such as laser light. To suppress this noise, several methods have been proposed such as a spatial filtering method, the use of optical sources with low coherence, multiple holograms using wavelength, polarization, illumination angle and a rotation of a diffuser.

In this study, we present a noise reduction of digital holography using speckle correlation properties in the out-of-plane direction. In this method, it is assumed that digital holograms of diffuse objects are recorded on an image sensor. Using multiple holograms recorded by moving an image sensor to the out-of-plane direction, the speckle noise of a reconstructed image is suppressed and therefore the image quality is improved.

\section{Experiment}

Now, we consider the optical system of an in-line Mach-Zehnder interferometer, which is shown in Figure 1.
A laser light is expanded by an objective lens, collimated by a lens and incident on a beam splitter $\mathrm{BS}_{1}$. The reflected light is expanded and collimated by an objective lens and lens again and is used as a reference wave. The other wave is incident on a test object. A speckle pattern generated from the test object is used as the object wave. The two optical waves are coupled by a beam splitter $\mathrm{BS}_{2}$ and interfere each other, and then a digital hologram is detected on an image sensor.

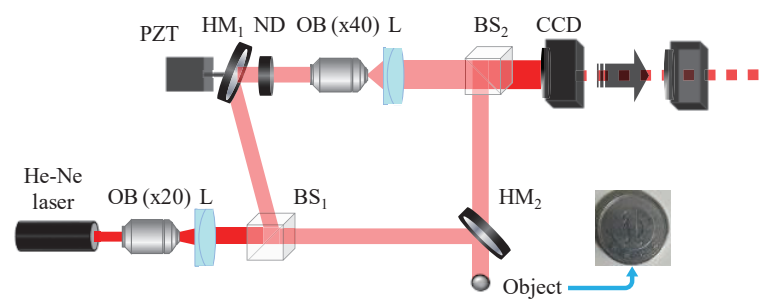

Fig.1 Experimental setup. OB: objective lens; L: lens; BS: beam splitter; HM: half mirror; ND: neutral density filter; PZT: piezoelectric transducer.

In this experiment, a He-Ne laser is used as an optical source and the test object is a one yen coin. Digital hologram is detected on a CCD camera with $1392 \times 1040$ pixels and pixel pitch $4.65 \times 4.65 \mu \mathrm{m}^{2}$. The distance between the CCD camera and the test object is $320.6 \mathrm{~mm}$. In the reconstruction process, the single FFT method is applied to the digital hologram. To eliminate the DC terms and the twin image of the reconstructed image, we use the four-step phase-shifting method, in which the phase of the reference wave is shifted by the piezoelectric 
transducer devices and the phase shift value is the integral multiple of $\pi / 2$. The multiple holograms are recorded by moving the CCD camera to the out-of-plane direction. In the intervals of the movement of the CCD camera, we use the three values $\Delta z=1.48[\mathrm{~mm}], 0.87[\mathrm{~mm}]$ and $0.52[\mathrm{~mm}]$, which are determined by the cross-correlation coefficient of speckle intensities in the out-of-plane direction [2] obtained from the experiment shown in Figure 2 and correspond to $0.25,0.50$ and 0.75 in the cross-correlation coefficient of speckle intensities. For each crosscorrelation coefficient, 36 digital holograms without the DC terms and the twin image are obtained after the fourstep phase-shifting method.

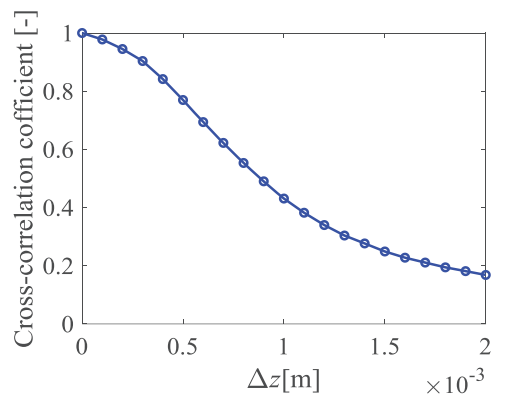

Fig. 2 Speckle correlation in the out-of-plane direction.

In the single FFT method, the pixel size of the reconstruction plane is changed by the distance between the test object and the CCD camera, which causes the mismatch of the pixels of the object images reconstructed from digital holograms with different positions. To adjust the pixel size of the reconstruction plane, we used the zero-padding method. Applying the averaging process on the basis of intensity to the object images reconstructed from multiple holograms, we demonstrated the reduction of speckle noises of the reconstructed image.

\section{Result}

Figure 3 shows the experimental results of the intensity distributions of reconstructed images. Figures 3 (a) - (d) are the intensity distributions of the object waves reconstructed from one, five, eleven and thirty-six holograms for the cross-correlation value 0.25 , respectively. It is visually seen from these figures that the speckle noises on the reconstructed image are suppressed with an increase in the number of holograms. Figures 3 (e) and (f) are the results of the averaging process using the thirty-six holograms for the correlation value 0.50 and
0.75. It is found in these figures that the suppression of speckle noise is not seen visually. To show the dependence of the noise reduction on the cross-correlation coefficient clearly, we use the speckle contrast on the reconstructed image as the evaluation function, which are shown in Figure 4. From this figure, we confirmed that the speckle contrast is lower for the low correlation value. These results mean that the image quality of the reconstructed image is better in the lower speckle correlation.

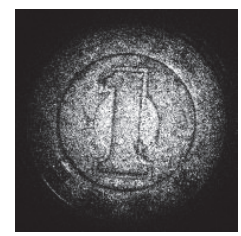

(a)

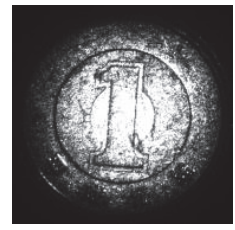

(d)

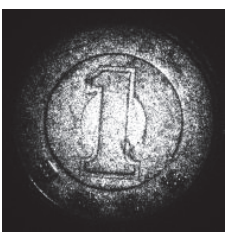

(b)

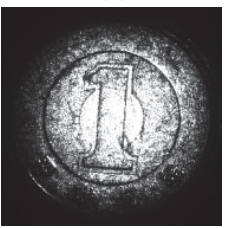

(e)

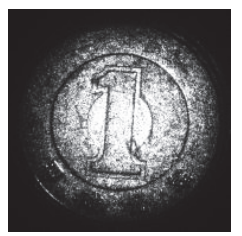

(c)

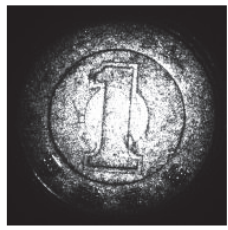

(f)
Fig.3 Experimental results. The intensity distribution reconstructed from (a)1 hologram, (b) 5 holograms, (c) 11 holograms, and (d) - (f) 36 holograms, respectively. The values of the cross-correlation coefficient of speckle intensities of (a) - (d), (e) and (f) are set to $0.25,0.50$ and 0.75 , respectively.

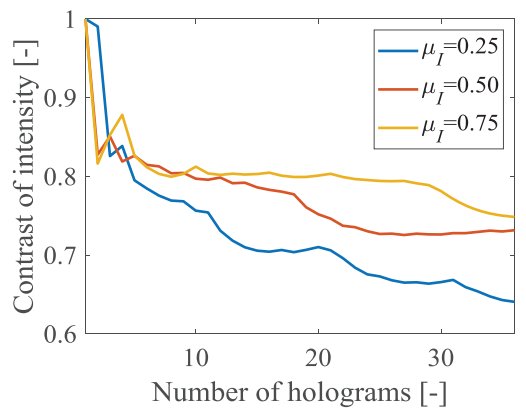

Fig.4 Effect of averaging process on speckle noise in Fig. 3 (d) - (f).

\section{References}

[1] U. Schnars and W. Jueptner: Digital Holography (Springer, 2005).

[2] J. W. Goodman: Speckle phenomena in optics, (Roberts \& Company, 2007). 


\title{
Scan-less Full-field Fluorescence Microscopy by using 2D Spectral Disperser and Dual-comb Optical Beats
}

\author{
Takahiko Mizuno ${ }^{\mathrm{a}, \mathrm{b}}$, Eiji Hase ${ }^{\mathrm{a}, \mathrm{b}}$, Takeo Minamikawa ${ }^{\mathrm{a}, \mathrm{b}}$, \\ Hirotsugu Yamamoto ${ }^{\mathrm{b}, \mathrm{c}}$, Takeshi Yasui*a,b \\ ${ }^{a}$ Tokushima University, 2-1 Minami-Josanjima, Tokushima-city, Tokushima 770-8506, Japan \\ bJST, ERATO MINOSHIMA Intelligent Optical Synthesizer (IOS), 2-1 Minami-Josanjima, \\ Tokushima-city, Tokushima 770-8506, Japan \\ 'Utsunomiya University, 7-1-2, Yoto, Utsunomiya, Tochigi, Japan 321-858
}

\begin{abstract}
We propose scan-less fluorescence imaging performed with a combination of dual-comb optical beating and 2D spectral disperser (2D-SD). Dual-optical combs with slightly different frequency spacing generate dense optical beats in the RF region (dual-comb beating). The frequency of dual-comb beating is dependent on wavelength, therefore the dualcomb beating gets one to one correspondent relation of a unique beat frequency and a position on the sample by 2D mapping using 2D-SD. The fluorescence excited by 2D-mapped beats is modulated by the same frequency of each beat. Accordingly, 2D fluorescence images can be reconstructed from RF spectrum of frequency multiplexed fluorescence signals.
\end{abstract}

Keywords: fluorescence microscopy, spectroscopy, optical frequency comb

\section{Introduction}

A biological sample usually has micro-meter order thickness, there includes structures for depth direction. In confocal fluorescence laser-scanning microscopy (CFLM), confocal pinhole is put at a conjugate position of the object plane, then it enables the image acquisition witch depth limited by a size of Airy-disc. However typical CFLM is based on a laser scanning imaging, thus it is necessary a mechanical scanning of confocal spot. It limits a maximum frame rate of imaging.

Recently, fluorescence imaging using radiofrequency-tagged emission (FIRE) was reported [1]. In this method uses a group of line focus spots that has varied optical beat frequencies (1D-FMI: 1D frequency modulated illumination). It has a potential to largely reduce the image acquisition time by mapping the image into the radio-frequency spectrum using the beating of digitally synthesized optical fields. However, due to the limited frequency-multiplexing of the beat signals, only the line image could be mapped into the radio-frequency spectrum; the beam scanning across the line image is still

*yasui.takeshi@tokushima-u.ac.jp phone+81-88-656-7377 required for $2 \mathrm{D}$ fluorescence imaging. If the frequencymultiplexing density of the beat signals is increased further, the resulting beat signals can be used for mapping of the 2D image sufficiently. However, it is difficult to largely increase the degree of frequency-multiplexing in the digitally synthesized optical beats.

For 2D scan-less fluorescence imaging using FMI, we focus on the optical beat signals between dual optical combs with slightly different frequency spacing (DCOB; dual-comb optical beating) for densely frequencymultiplexed beat signals in the RF region. The number of the signal channel useable as discrete in such dual-comb optical beats reaches more than 10,000, which are sufficient for mapping all pixels of a $2 \mathrm{D}$ image. We mapped these dual-comb optical beats into 2D space by using a 2D spectral disperser (2D-SD), which was composed of a virtually imaged phased array and a diffraction grating. The $2 \mathrm{D}$ fluorescence image was reconstructed from the radiofrequency-multiplexed fluorescence signal based on one-to-one correspondence between the 2D images pixels and the beat frequencies.

In this paper, 2D scan-less fluorescence imaging using DCOB was described. We constructed the imaging 
set-up, and it was demonstrated by getting a fluorescence image.

\section{Method}

Figure 1 shows a conceptual of the scan-less full-field fluorescence microscopy using DCOB and 2D-SD. Combs are dispersed to 2D spatial by 2D-SD. Each comb modes formed discrete spots dependent on optical frequency respectively (2D spectrograph). Thus the optical frequency of a one of the spot has 1 by 1 correspondence with the position of the spot. When two optical combs are superimposed and through the 2D-SD, each spots are overlapped on the object plane. By setting the repetition frequency of optical combs as slightly different, overlapped spots makes beating in RF region. Each beat frequencies have 1 by 1 correspondence with the position of the spot. Typical fluorophore can response for the RF modulated excitation, therefore fluorescence is modulated same frequency dependent on the position respectively. For imaging, temporal waveform of fluorescence is received by single photo detector, the amplitude spectrum of the waveform is reconstructed to the fluorescence image.

The number of pixels are dependent on the number of comb modes, typically the number is huge. Each mode has extremely high stability and narrow band width. Thus the degree of multiplexing can be improved until the limit of over-lapping of $\mathrm{CW}$ modes by each band width. Typically line-width is less than $1 \mathrm{MHz}$ and the comb optical band width is few $\mathrm{THz}$ then their can stuff over 10,000 modes, it is enough number for image pixels.

\section{Setup}

Figure 2 shows an optical setup of the scan-less fluorescence microscope using DCOB and 2D-SD. We used Er-fiber OFC lasers $\left(\lambda_{\mathrm{c} 1}=\lambda_{\mathrm{c} 2}=1570 \mathrm{~nm}, \Delta \lambda_{1}=\Delta \lambda_{2}=\right.$ $15 \mathrm{~nm}, P_{1}=120 \mathrm{~mW}, P_{2}=164 \mathrm{~mW}, f_{\text {rep1 }}=100.386960$ $\mathrm{MHz}, f_{\text {rep2 }}=100.385982 \mathrm{MHz}, \Delta f_{\text {rep }}=978 \mathrm{~Hz}, f_{\text {ceo }}=21.4$ $\mathrm{MHz}$ ). Wavelength of each OFCs were converted by PPLN devices to $\lambda_{\mathrm{c}}=523 \mathrm{~nm}, \Delta \lambda=5.2 \mathrm{~nm}, P_{\mathrm{SH} 1}=6.0$ $\mathrm{mW}, P_{\mathrm{SH} 2}=4.5 \mathrm{~mW}$. Each combs were superimposed by beam splitter, it was fed into a 2D-SD composed by a virtually imaged phased array (VIPA, FSR $=14.9 \mathrm{GHz}$, finess $=100$ ) and a grating (groove density $=1200$ $1 \mathrm{p} / \mathrm{mm}$ ). Excitation wavelength range was limited after the grating to $4.5 \mathrm{~nm}$. The 2D spectrograph from 2D-SD was input to fluorescence microscope setup. The integration power of the $2 \mathrm{D}$ spectrograph was get $600 \mu \mathrm{W}$ on the sample plane. A fluorescence from the sample was separated by a dichroic mirror $\left(\lambda_{\text {cut }}=562 \mathrm{~nm}\right)$ and fluorescence filter $\left(\lambda_{\text {pass }}=593 \mathrm{~nm}, B W_{\mathrm{FWHM}}=40 \mathrm{~nm}\right)$, and detected by photomultiplier tube. A fluorescence image was reconstructed from the amplitude spectrum that was Fourier transformed from an acquired temporal fluorescence waveform. When a confocal pinhole array puts on the conjugate plane in the fluorescence detection arm, it can be enabled to give a scan-less confocal imaging ability.

\section{Results}

For proof of imaging principle, fluorescence imaging of the fluorophore masked with a negative type test chart was performed. This experiment was focused on demonstration of wide-field imaging without a confocal pinhole array. Figure 3 shows the sample arrangement. A $300 \mu \mathrm{mol} / 1$ Rhodamine $6 \mathrm{G}$ aqueous solution was used as a fluorophore, and the $2 \mathrm{D}$ spectrograph was masked by a negative test chart. Fig. 4(a) shows a temporal

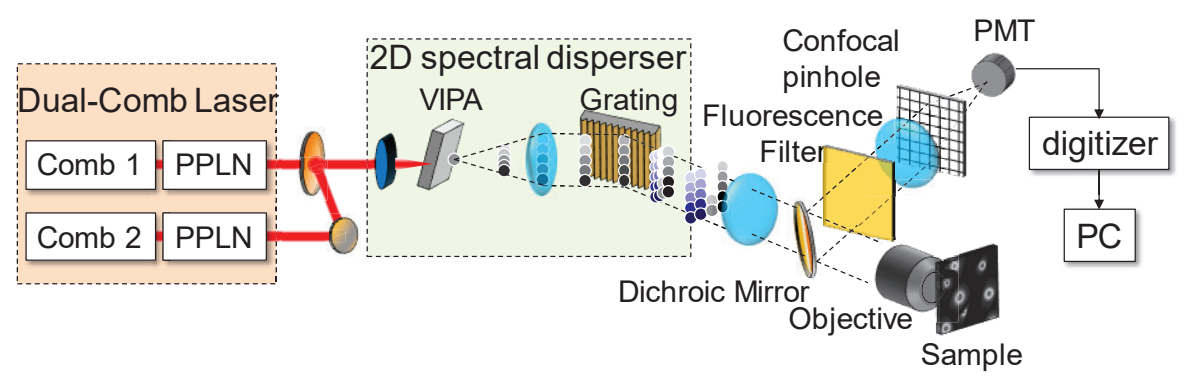

Fig. 2. Experimental setup. 
fluorescence waveform, Fig. 4(b) is a RF spectrum, and Fig 4(c) is a reconstructed fluorescence image, respectively. The number of integration for interference waveforms was 100,000 (acquiring time is $98 \mathrm{~s}$ ). The structure of a test chart can be cleary observed. In this experiment, we used an excitation power that is $600 \mu \mathrm{W}$. Excitation power per pixel is only $20 \mathrm{nW}$. By using more high excitation power, a time for image acquiring is became to short, it can achieve high-speed wide-field fluorescence imaging.

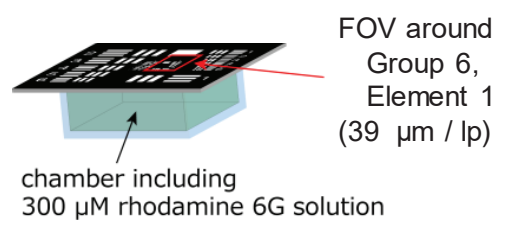

Fig. 3. Sample alignment.

(a) temporal interferences

(b) RF spectrum
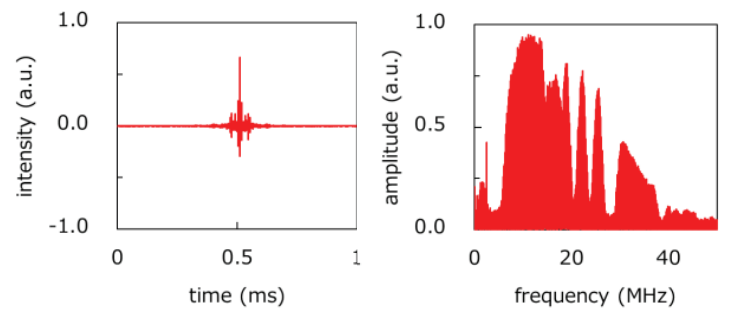

(c) reconstructed image

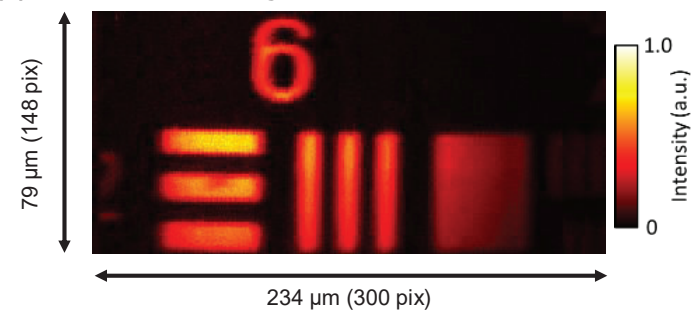

Fig. 4. Experimental results. (a) temporal fluorescence waveform, (b) RF spectrum, and (c) reconstructed image.

\section{Conclusion}

Scan-less full-field fluorescence microscopy using DCOB and 2D-SD was proposed. In this paper, we showed a performance test of fluorescence imaging for proof of principle. In future, it can be expected to apply to high-speed imaging and fluorescence lifetime imaging.

\section{Acknowledgements}

This work was supported by The Mazda Foundation, JSPS KAKENHI Grant Number JP18K13768, Exploratory Research for Advanced Technology (ERATO), Japanese Science and Technology Agency (MINOSHIMA Intelligent Optical Synthesizer Project, JPMJER1304),

\section{References}

[1] Diebold, E. D., Buckley, B. W., Gossett, D. R. and Jalali, B. 2013. Digitally synthesized beat frequency multiplexing for sub-millisecond fluorescence microscopy. Nature Photonics 7:806-810. 


\title{
Femtosecond laser microsurgery of cells using confocal surface detection
}

\section{system}

\author{
Makoto Miura $^{\mathrm{a}}$, Satoshi Hasegawa ${ }^{\mathrm{a}}$, Masashi Iwanaga ${ }^{\mathrm{b}}$, Yoshio Hayasaki ${ }^{\mathrm{a}}$ \\ ${ }^{a}$ Center for Optical Research and Education (CORE), \\ Utsunomiya University, 7-1-2 Utsunomiya, 321-8585, Japan \\ ${ }^{b}$ Department of Agrobiology and Bioresources, Faculty of Agriculture, \\ Utsunomiya University, 350 Utsunomiya, 321-8505, Japan
}

\begin{abstract}
Laser ablation has been used to subcellular surgery in a living cell, such as the dissection or in-activation of cellular organelles in order to understand cell dynamics. In the surgery, it is necessary to control carefully the focus position according to a shape of individual cells and organelles. In this paper, we demonstrate femtosecond laser processing with a confocal surface detection system for the subcellular surgery. As a basic experiment, femtosecond laser processing of quartz glass was performed to evaluate the performance of confocal surface detection system.
\end{abstract}

Keywords: femtosecond laser processing, laser microsurgery of cell, confocal microscope

\section{Introduction}

The human body is composed of about 60 trillion cells. In order to maintain normal life activities, cells need to maintain their function. Abnormal cell function causes abnormal proliferation of cells and causes serious diseases. Therefore, analysis of the cellular function with the molecular level is an important for prevention of diseases, development of therapeutic drugs, and regenerative medicine.

In order to find the cell abnormality, it is necessary to separate specific cells from tissues. After the cell dissection, DNA, RNA, protein, and metabolite contained in cells were analyzed by polymerase chain reaction (PCR) and proteome analysis. In the cell dissection, femtosecond laser has been used. When femtosecond laser pulses are tightly focused inside a cell, the intensity in the focal volume becomes high enough to cause nonlinear absorption, through multiphoton, tunneling and avalanche ionization processes, which exponentially increases the free electron density. The free electron leads to a laser ablation of the cell due to the generation of plasma [1]. Currently, the laser ablation has been used to subcellular surgery in a living cell [2-3], such as the dissection or in-activation of cellular organelles, cytoskeletal filaments, and chromosomes and the photostimulation of $\mathrm{Ca} 2+$ waves in neuronal cells.
Studying the function of organelles is important to understand cell dynamics. In the surgery, it is necessary to control carefully the focus position according to a shape of individual cells and organelles for obtaining desired results.

In this paper, we demonstrate femtosecond laser processing with a confocal surface detection system for the subcellular surgery.

\section{Experimental setup}

Figure 1 shows an experimental setup. The setup was mainly composed of a femtosecond laser processing system, a transmitted optical microscope, and a confocal microscope. The laser processing system (Coherent, Micra and Legend Elite) was used to perform the subcellular surgery. The femtosecond pulse with a center wavelength of $800 \mathrm{~nm}$, a duration of $50 \mathrm{fs}$, and a repetition frequency of $1 \mathrm{kHz}$ was illuminated to a cell through a pulse energy controller composed of a half wave plate (HWP) and a polarizing beam splitter (PBS), and a beam expanding optics. The pulse was focused by a water-immersion objective lens (OL, 60×, NA 1.0). A living cell (cultured insect cells, Sf9) was fixed to a motorized stage. A white light LED and CMOS image sensor were used to obtain the transmitted optical image. The confocal microscope was used to detect the cell surface. A laser diode with a wavelength of $1060 \mathrm{~nm}$ was 


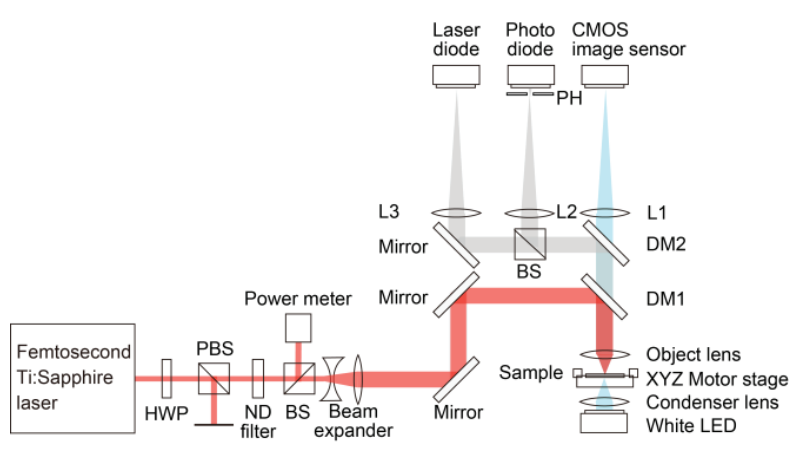

Figure 1. Experimental setup.

illuminated to the cell. An intensity of the laser reflected from the cell surface was measured by an avalanche photo diode through a pinhole $(\mathrm{PH})$ with a size of $50 \mu \mathrm{m}$.

\section{Experimental result}

Femtosecond laser processing of quartz glass was performed to evaluate the performance of confocal surface detection system as a basic experiment. Figure 2 shows the intensity of the signal obtained from the confocal surface detection system with respect to the distance between the focus position and the sample surface. The distance of $0 \mu \mathrm{m}$ was defined as the position where maximum intensity was obtained. The positive and negative value of the distance means the focus position was set to inside and outside of the sample on the basis of the surface, respectively. From the result, a resolution of the depth direction was estimated as $5 \mu \mathrm{m}$ at full width at half maximum (FWHM) of the profile. Inset shows the scanning electron microscope (SEM) image of the structure fabricated by the femtosecond laser processing with the variety of the distance. The structure was only observed at the distance of $0 \mu \mathrm{m}$ because the pulse energy was set to near the threshold $(60 \mathrm{~nJ})$ to perform the laser ablation. Consequently, a precise control of the focus position was realized using the signal obtained from the confocal detection system.

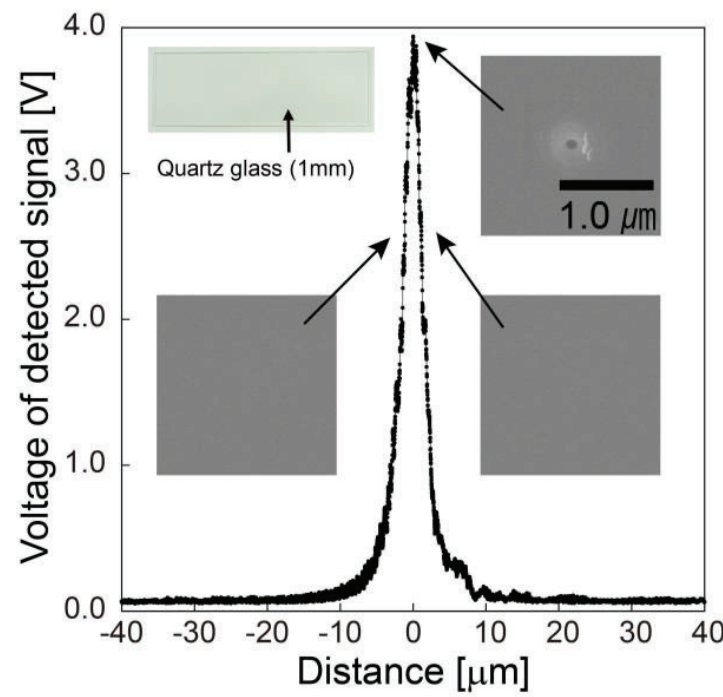

Figure 2. Intensity of the signal obtained from the confocal surface detection system versus the distance between the focus position and the sample surface.

\section{Summary}

Femtosecond laser processing with a confocal surface detection system was demonstrated for the subcellular surgery. The resolution of the depth direction was estimated as $5 \mu \mathrm{m}$ from FWHM of the intensity profile obtained by the confocal surface detection system. The laser ablation was only performed at the distance where the intensity obtained from the confocal detection system was maximum.

\section{References}

[1] A. Vogel, J. Noack, G. Hüttman, and G. Paltauf, "Mechanisms of femtosecond laser nanosurgery of cells and tissues," Appl. Phys. B 81(8), 1015-1047 (2005).

[2] Y. Zhu, and $\mathrm{H} \mathrm{He}$, "Molecular response of mitochondria to a short-duration femtosecond-laser stimulation," Biomed. Opt. Express, 8(11), 4967-4973 (2017).

[3] D. Stevenson, B. Agate, X. Tsampoula. P. Fischer, C. T. A. Brown, W. Sibbett, A. Riches, F. Gunn-Moore, and K. Dholakia, "Femtosecond optical transfection of cells: viability and efficiency," Opt, Express, 14(8), 7125-7133 (2006). 


\title{
Enhanced sensitivity of non-enzymatic glucose sensor using different concentrations of Al-doped $\mathrm{ZnO}$ nanorod
}

\author{
Zi-Hao Wang ${ }^{\mathrm{a}}$, Chih-Chiang Yang*b, Hsin-Chieh Yuc ${ }^{\mathrm{c}}$, Shi-Wei Luo ${ }^{\mathrm{d}}$ and Yan-Kuin Su ${ }^{\text {bd }}$ \\ aAdvanced Optoelectronic Technology Center, National Cheng Kung University, Tainan, Taiwan \\ ${ }^{\mathrm{b}}$ Department of Electrical Engineering, Kun Shan University, Tainan, Taiwan \\ 'Institute of Lighting and Energy Photonics, College of Photonics, National Chiao Tung University, Tainan, Taiwan \\ ${ }^{\mathrm{d}}$ Institute of Microelectronics Engineering, National Cheng Kung University, Tainan, Taiwan
}

\begin{abstract}
In this study, zinc oxide $(\mathrm{ZnO})$ nanaords doped with different concentrations $(0 \%, 1 \%, 2 \%)$ of aluminum (Al) were prepared by hydrothermal method. The effects of different concentrations of Al-doped $\mathrm{ZnO}$ nanorods on electrical properties and responses to different concentrations $(0 \mathrm{mM}$ to $12 \mathrm{mM})$ of glucose were investigated. The results show that Al-doped $\mathrm{ZnO}$ changes the energy gap of pure $\mathrm{ZnO}$ and enhances the carrier concentration. Nanorods also changes the surface area of the element due to the influence of doping. The preparation method is simple, safe and non-toxic. After the measurement results, the Al doping improves the sensitivity, stability and reliability of glucose. In the future, this research method can be used to enhance the non-enzymatic electrochemical sensor of glucose.
\end{abstract}

Keywords: aluminum, $\mathrm{ZnO}$ nanaords, non-enzymatic, glucose sensor

\section{Introduction}

In recent years, electrochemical biosensors for semiconductor and metal oxide nanostructures have attracted great attention as new instruments in healthcare, which can greatly enhance early diagnostic capabilities and thus achieve positive health management. Among the many materials studied, nanostructured $\mathrm{ZnO}$ is relatively easy to prepare and has a high aspect ratio because of its low cost. However, nanomaterials can be divided into zero-dimensional, one-dimensional and two-dimensional according to dimensions.

$\mathrm{ZnO}$ has high biocompatibility and a rich source of natural resources, so zinc oxide has received considerable attention in biosensors [1]. Zinc oxide has an Isoelectric point (IEP) of approximately ( 9.572). Nanostructures have a high catalytic effect, however, zinc oxide nanostructures show for electrochemical applications due to their unique properties, including non-toxicity, thermal stability and chemical stability, extensive surface area and fast electron transport. There is great hope.

Doping $\mathrm{ZnO}$ with elements such as aluminum (Al) [2], gallium (Ga) [3] and indium (In) [4] can exhibit n-type characteristics and is helpful in optics and electricity. The resistance of undoped pure zinc oxide is quite high. Doping can increase the carrier concentration and increase the electron mobility. In addition, doping can increase chemisorption and chemical desorption, and doping is cheaper than other methods [5]. In metal doping, the ionic radius of $\mathrm{Al}(0.54 \mathrm{~nm})$ is similar to the ionic radius of $\mathrm{Zn}(0.74 \mathrm{~nm})$. $\mathrm{Al}^{3+}$ can replace $\mathrm{Zn}^{2+}$ to avoid deformation and prevent $\mathrm{ZnO}$ lattice distortion [6]. $\mathrm{ZnO}$ nanostructures have been used in many types of enzyme sensors [7]. Because zinc oxide has good biocompatibility, it can effectively adsorb glucose oxidase, but the enzyme will fall off due to repeated longterm measurement [8]. In the enzyme-type glucose sensor, the $\mathrm{ZnO}$ nanostructure mainly plays the role of electron transfer rather than catalyzing glucose. Although the $\mathrm{ZnO}$ nanostructure enhances the ability of glucose enzyme to adsorb on the electrode, glucose oxidase is still susceptible. Environmental temperature, $\mathrm{pH}$, humidity, etc., due to the shortcomings caused by glucose enzymes, the application of $\mathrm{ZnO}$ to non-enzymatic glucose sensors began to receive attention.

\section{Experimental}

The experimental procedure is as follows. The first step is to clean the Corning glass substrate, the second and third steps are respectively evaporating chromium and gold metal layers using an electron beam evaporation machine (E-beam), and the fourth step is to use magnetron sputtering (RF-sputter). A zinc oxide seed 
layer is deposited on the chromium gold substrate, and the fifth step is to grow a one-dimensional zinc oxide nanopillar structure by hydrothermal method.

The experiment adopts low temperature hydrothermal method to grow Al-doped $\mathrm{ZnO}$ nanorods. Firstly, the required reaction solvent is prepared, and the reactant aluminum nitrate, zinc nitrate and hexamethylenetetramine are dissolved in deionized water, and evenly distributed by ultrasonic oscillator. The two reactants were mixed and poured into Teflon. The autoclave sealed in the Teflon cup was placed in an oven for six hours. After the reaction was completed, the sample after the growth was completed was taken out from the solvent to deionized water. The residual salt is removed and dried by a hot plate.

\section{Results and Discussion}

Cyclic voltammetry (CV) was used to measure the electrocatalytic activity of different concentrations of Aldoped $\mathrm{ZnO}$ nano-pillars for glucose oxidation. Figure 1 shows the $\mathrm{CV}$ response of electrodes of different concentrations of $\mathrm{Al}$-doped $\mathrm{ZnO}$ nanopillars and $\mathrm{Pt}$ electrodes observed by adding $0 \mathrm{mM}$ to $12 \mathrm{mM}$ glucose to the $\mathrm{NaOH}$ solution at a scan rate of $10 \mathrm{mV} / \mathrm{s}$. A clear oxidation peak can be seen from the figure, and the oxidation peak potential also changes with the glucose concentration. The sensitivity of pure, Al 1\% and Al 2\% calculated from Fig. 2 are 34.5, 38.9 and $48.5 \mu \mathrm{A} / \mathrm{cm}^{2}$ $\mathrm{mM}^{-1}$, respectively.

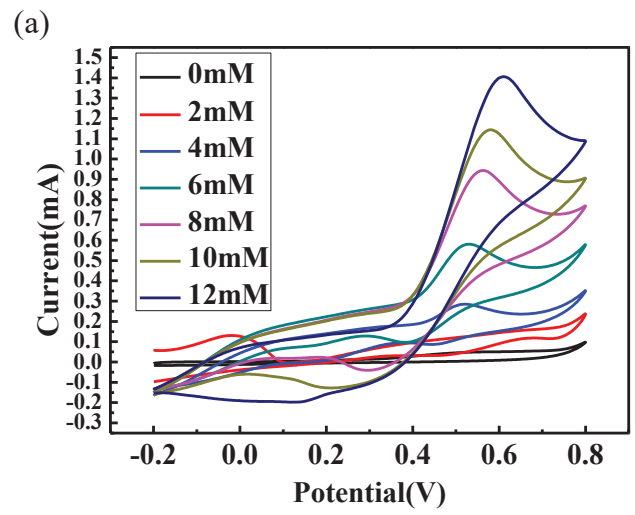

(b)

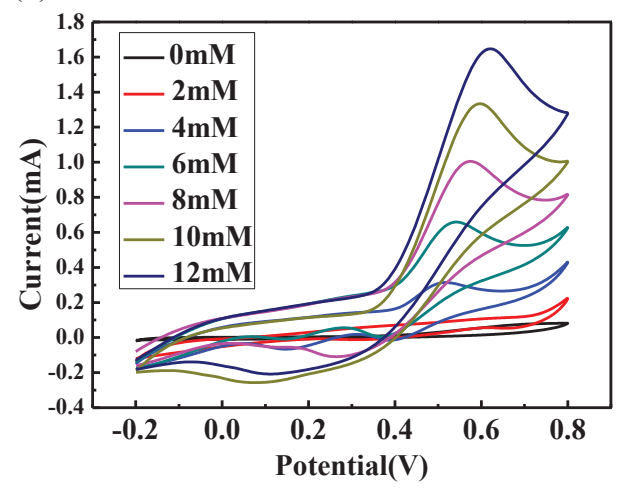

(c)

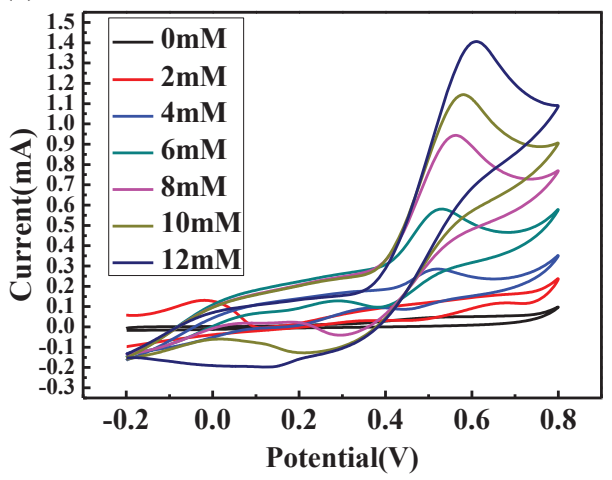

Figure 1. CVs of Al-depod $\mathrm{ZnO}$ nanorods electrode in $0.1 \mathrm{M} \mathrm{NaOH}$ containing $0 \mathrm{mM} \sim 12 \mathrm{mM}$ glucose at $10 \mathrm{mV} / \mathrm{s}$. (a) pure $\mathrm{ZnO}$ nanorods, (b) $\mathrm{Al} 1 \%$ doped $\mathrm{ZnO}$ nanorods, (c) $\mathrm{Al} 2 \%$ doped $\mathrm{ZnO}$ nanorods.

\section{Conclusions}

In this study, the work functions of pure $\mathrm{ZnO}$ and $\mathrm{Al}$ doped $\mathrm{ZnO}$ were $5.3 \mathrm{eV}$ and $4.75 \mathrm{eV}$, respectively. The reduction of the work function makes it easier to pass electrons, and $\mathrm{Al}^{3+}$ provides more carriers than $\mathrm{Zn}^{2+}$, which increases the carrier concentration and improves the detection sensitivity. Al doping enhances the sensitivity of non-enzymatic glucose sensors, indicating its high potential to develop non-enzymatic sensors. 


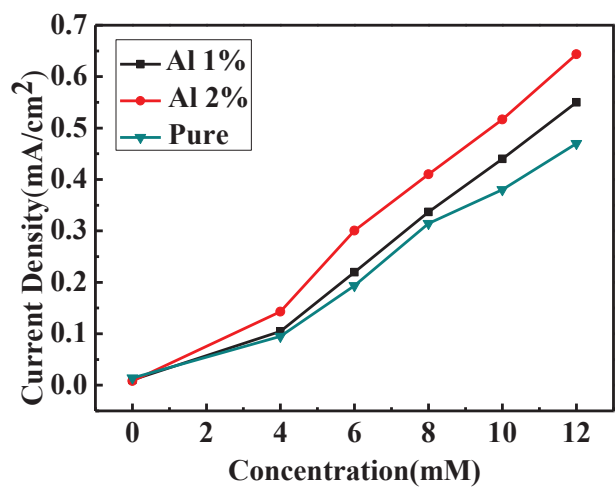

Figure 2. Peak current of CVs in different concentrations of Al-doped $\mathrm{ZnO}$ nanorods.

\section{Acknowledgments}

This research was supported by the Ministry of Science and Technology of Taiwan, ROC under Contract No. MOST 104-2221-E-168-011-MY3 and 107-2221-E$006-185$-MY3 and the Green Energy Technology Research Center, Department of Electrical Engineering, Kun Shan University, Tainan, Taiwan. The Featured Area Research Center Program is within the framework of the Higher Education Sprout Project by the Ministry of Education (MOE) in Taiwan, under Contract MOE 107N-270-EDU-T-142.

\section{References}

[1] Wang, J. X., Sun, X. W., Wei, A., Lei, Y., Cai, X. P., Li, C. M., and Dong, Z. L. "Zinc oxide nanocomb biosensor for glucose detection," Applied Physics Letters, 88(23), 233106 (2006).

[2] Chen, M., Wang, X., Yu, Y. H., Pei, Z. L., Bai, X. D., Sun, C., Huang, R. F., and Wen, L. S. "X-ray photoelectron spectroscopy and auger electron spectroscopy studies of Al-doped $\mathrm{ZnO}$ films," Applied Surface Science, 158(1-2), 134-140 (2000).

[3] Bundesmann, C., Ashkenov, N., Schubert, M., Spemann, D., Butz, T., Kaidashev, E. M., Lorenz, M., and Grundmann, M. "Raman scattering in $\mathrm{ZnO}$ thin films doped with $\mathrm{Fe}, \mathrm{Sb}, \mathrm{Al}, \mathrm{Ga}$, and Li," Applied Physics Letters, 83(10), 1974-1976 (2003).
[4] Chiang, C. K., Fincher Jr, C. R., Park, Y. W., Heeger, A. J., Shirakawa, H., Louis, E. J., Gau, S. C., and MacDiarmid, A. G. "Electrical conductivity in doped polyacetylene," Physical review letters, 39(17), 1098 (1977).

[5] Gizeli, E. "Design considerations for the acoustic waveguide biosensor," Smart Materials and Structures, 6(6), 700 (1997).

[6] Ansari, A. A., Alhoshan, M., Alsalhi, M. S., and Aldwayyan, A. S. "Prospects of nanotechnology in clinical immunodiagnostics," Sensors, 10(7), 65356581 (2010).

[7] Bae, S. Y., Na, C. W., Kang, J. H., and Park, J. "Comparative structure and optical properties of Ga-, In-, and Sn-doped $\mathrm{ZnO}$ nanowires synthesized via thermal evaporation," The Journal of Physical Chemistry B, 109(7), 2526-2531 (2005).

[8] Lin, S. Y., Chang, S. J., and Hsueh, T. J. "ZnO nanowires modified with $\mathrm{Au}$ nanoparticles for nonenzymatic amperometric sensing of glucose," Applied Physics Letters, 104(19), 193704 (2014). 


\title{
Development of photoplethysmogram sensor with an LED and sampling
}

\section{rate assessment}

\author{
Mitsutoshi Fukumoto ${ }^{\mathrm{a}}$, Hidenobu Arimoto*b ${ }^{*}$, Wataru Watanabe**a \\ a Department of Electrical \& Electronic Engineering, College of Science and Engineering, \\ Ritsumeikan University, 1-1-1 Nojihigashi, Kusatsu, Shiga, 525-8577 Japan \\ ${ }^{\mathrm{b}}$ Electronics and Photonics Research Institute, National Institute of Advanced Industrial Science \\ and Technology, AIST Tsukuba Central 5, 1-1-1 Higashi, Tsukuba, Ibaraki 305-8565 Japan
}

\begin{abstract}
Because the heart rate is affected by the autonomic nervous system, various psychological conditions are reflected by measuring heart rate variation. A photoplethysmogram (PPG) is used to estimate the skin blood flow using light. We developed a PPG sensor prototype. We used it to study the R-R interval from pulse rate variation with our PPG signals and to extract LF/HF using FFT and autoregressive (AR) methods. We compared the results with those obtained using an ECG to assess the accuracy and sampling rate.
\end{abstract}

\section{Introduction}

The heart rate (HR) of a human is not constant: it varies constantly. The $\mathrm{R}-\mathrm{R}$ interval (RRI) fluctuation measured using an electrocardiograph (ECG), called heart rate variability (HRV), is a well-known phenomenon that reflects autonomic nervous function. Actually, HRV is the physiological phenomenon of different time intervals between heartbeats. Conventionally, HRV is found through digital processing of electrocardiographic (ECG) signals.

Photoplethymysography (PPG), a non-invasive method for detection of cardiovascular pulse waves propagated around the human body [1-12], is based on the determination of optical properties of vascular tissue using a light source and photodetector. A PPG sensor uses a light emitting diode (LED) and a light receiving element using an LED. When light from the LED passes through a living body, it is partly absorbed by hemoglobin in the blood. The volume change of the blood from the increase / decrease of the measured light enables an observer to ascertain the pulse wave waveform. The PPG signal has two components: a DC component, which is more or less constant voltage, but with magnitude that changes depending on the characteristics of tissues through which the light passes (skin, cartilage, venous blood, etc.) and an AC or pulsatile component that is synchronous with $\mathrm{HR}$ and which is related to arterial blood volume. The AC pulse shapes reflect vessel compliance and cardiac performance. With PPG, pulse rate variability (PRV) correlates with HRV for longer periods of measurement. Typically, the power spectral density can be found from using fast Fourier transform (FFT) or autoregressive (AR) method from RRI. According to HRV spectral analysis, most of the HRV signal power is concentrated in two frequency ranges: HF and LF. The HF band $(0.15-0.4 \mathrm{~Hz})$ is associated with parasympathetic activity. The LF band $(0.04-0.15 \mathrm{~Hz})$ is modulated by baroreflex-induced cardiac autonomic outflow. Furthermore, by obtaining the LF / HF ratio, one can ascertain the state of the autonomic nerve.

A PPG sensor prototype is used in this study to compare measurements with those obtained with an ECG. With the PPG, the peak signal variation is PRV. To assess the PPG prototype capabilities, we compared PPG (PRV) to the ECG (HRV). We extract LF/HF ratios, which are stress indices, and verify that our PPG sensor yields the same results as those from the ECG.

\section{Experimental protocol}

We fabricated a PPG sensor using a $528 \mathrm{~nm}$ wavelength LED. The PPG measurement unit works in reflection mode; the LED illuminates the skin of a finger. Then a photodetector placed near the LED on the same side of the finger monitors the variation of light

\footnotetext{
*arimoto-h@aist.go.jp; phone 81298617880

**wata-w@fc.ritsumei.ac.jp; phone 81775994343
} 
absorption by the skin microvascular bed. The prototype circuit is presented in Fig. 1. An Arduino detects the PPG signal. We used this self-made PPG device to measure HRV using ECG and PRV simultaneously. Signal processing was performed using MATLAB.

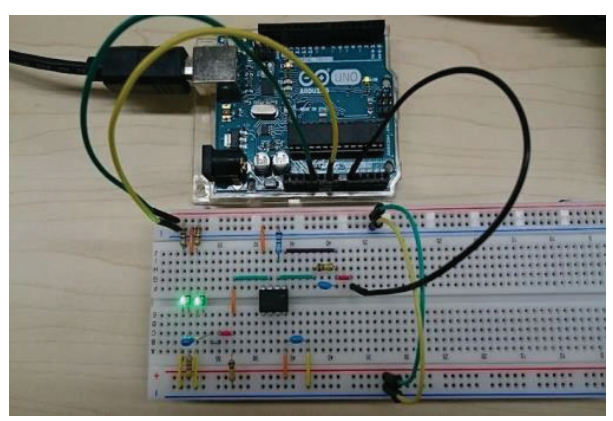

Figure 1. Photograph of the PPG circuit prototype operated by Arduino.

\section{Experiment results}

Figure 2 shows a typical PPG signal detected by the PPG prototype. Because R-peak intervals can be extracted, HRV can be derived from ECG data reliably. In fact, HRV data are obtainable with short-duration measurements. With PPG sensors, however, the peak interval accuracy is limited by the sampling rate. With PPG, PRV correlates with HRV for longer measurement periods, but not for short-duration measurements. Therefore, we measured PPG signals for $5 \mathrm{~min}$.

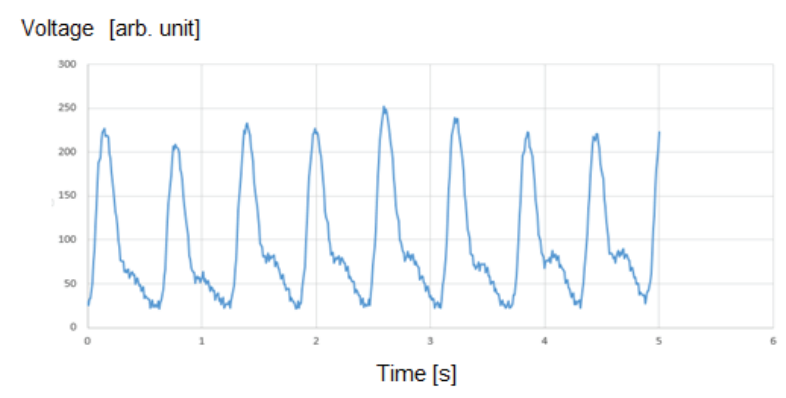

Figure 2. Typical PPG signal detected by the PPG device prototype.
Figures 3(a) and 3(b) respectively show RRI obtained using EEG and PPG. Clearly, PRV obtained with PPG correlates with HRV calculated from ECG signals.

Then, LF and HF results were compared with ECG and PPG. The LF/HF found using PPG correlated significantly with that calculated from ECG signals: the difference of LF/HF between the two devices was 0.03 . Figures 4(a) and 4(b) show a power spectrum calculated using (a) FFT and (b) AR extracted using our PPG.

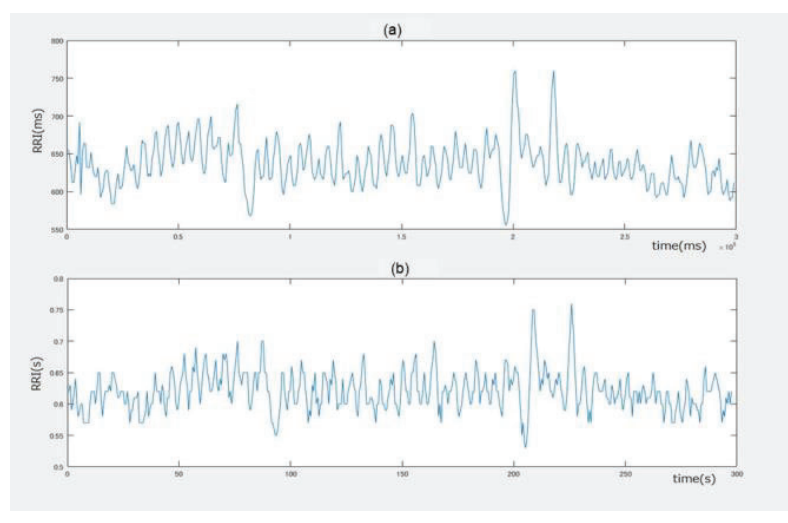

Figure 3. Comparison of R-R intervals (RRI) found using the PPG and the electrocardiograph: (a) HRV derived from the ECG; (b) pulse-to-pulse intervals (PPI) found using our PPG sensor. (a)

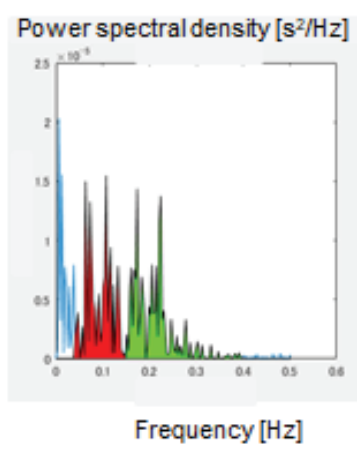

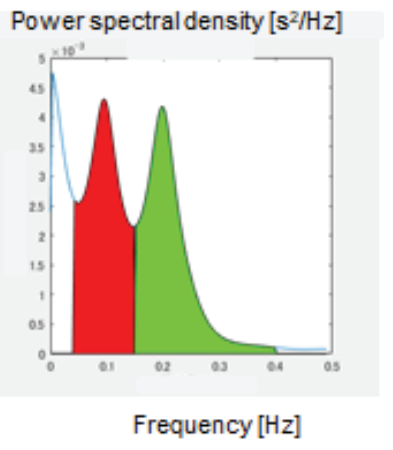

(b)
Figure 4. Power spectrum calculated using (a) FFT and (b) AR using our PPG sensor prototype. 
To assess our PPG prototype capabilities, we compared PPG (PRV) with ECG (HRV). We extracted LF/HF ratios and verified that our PPG sensor yields the same results as those obtained using the ECG.

Table 1. Results obtained using our PPG sensor and ECG

\begin{tabular}{cccc}
\hline & $\begin{array}{c}\text { PPG sensor } \\
\text { prototype }\end{array}$ & $\begin{array}{c}\text { Commercially } \\
\text { available ECG }\end{array}$ & Error \\
\hline Average RRI [ms] & 619.1 & 636 & 16.9 \\
\hline $\begin{array}{c}\text { LF/HF ratio } \\
\text { estimated by FFT }\end{array}$ & 0.93 & 1.04 & 0.11 \\
\hline $\begin{array}{c}\text { LF/HF ratio } \\
\text { estimated by AR }\end{array}$ & 0.99 & 1.02 & 0.03 \\
\hline
\end{tabular}

Because the RRI changes according to the sampling time interval, some influence appears on the LF/HF. We investigated the relation between the sampling time interval and LF/HF accuracy. The measured sampling time intervals were $4 \mathrm{~ms}, 30 \mathrm{~ms}, 60 \mathrm{~ms}, 75 \mathrm{~ms}$, and 100 ms. Measurements were conducted simultaneously with those using the EEG. The LF/HF measurements were obtained accurately with $60 \mathrm{~ms}$ and $75 \mathrm{~ms}$ sampling times.

\section{Conclusion}

For this study, after a PPG circuit was prototyped, its measurement results were compared with those obtained using an ECG. The LF/HF ratio, a stress index, showed equivalent results when obtained with the PPG prototype and the ECG. Our investigation of the relation between sampling time interval and LF/HF accuracy revealed that LF/HF measurements were obtained accurately at $60 \mathrm{~ms}$ and $75 \mathrm{~ms}$ sampling times.

\section{References}

[1] Mendelson, Y. 1992. Pulse oximetry: theory and applications for noninvasive monitoring. Clin. Chem. 38:1601.

[2] Sun, Y. and N. Thakor. 2016. Photoplethysmography revisited: From contact to noncontact, from point to imaging. IEEE Trans. Biomed. Eng. 63:463.
[3] Allen, J. 2007. Photoplethysmography and its application in clinical physiological measurement. Physiol. Meas. 28:R1R39.

[4] Jonathan, E. and M. Leahy. 2010. Investigating a smartphone imaging unit for photoplethysmography. Physiol. Meas. 31(11):N79-83.

[5] Jonathan, E. and M. J. Leahy. 2011. Cellular phone-based photoplethysmographic imaging. J. Biophotonics 4(5):293-296.

[6] Scully, C. G., J. Lee, J. Meyer, A. M. Gorbach, D. Granquist-Fraser, Y. Mendelson and K. H. Chon. 2012. Physiological parameter monitoring from optical recordings with a mobile phone. IEEE Trans Biomed Eng. 59(2):303-306.

[7] Charlot, K., J. Cornolo, J. V. Brugniaux, J. P. Richalet and A. Pichon. 2009. Interchangeability between heart rate and photoplethysmography variabilities during sympathetic stimulations. Physiological Measurement 30(12):13571369.

[8] Gil, E., M. Orini, R. Bailón, J. M. Vergara, L. Mainardi and P. Laguna. 2010. Photoplethysmography pulse rate variability as a surrogate measurement of heart rate variability during non-stationary condition. Physiological Measurement 31(9):1271-1290.

[9] Lu, S., H. Zhao, K. Ju et al. 2008. Can photoplethysmography variability serve as an alternative approach to obtain heart rate variability information? Journal of Clinical Monitoring and Computing 22(1):2329.

[10] Healey, J. A. and R. W. Picard. 2005. Detecting stress during real-world driving tasks using physiological sensors. IEEE Transactions on intelligent transportation systems 6(2):156-166.

[11] Hjortskov, N., D. Rissén, A. K. Blangsted, N. Fallentin, U. Lundberg and K. Søgaard. 2004. The effect of mental stress on heart rate variability and blood pressure during computer work. European journal of applied physiology 92(1-2):84-89.

[12] Logier, R., J. De Jonckheere, A. Dassonneville and M. Jeanne. 2016. Comparison of pulse rate variability and heart rate variability for high frequency content estimation. Conf. Proc. IEEE Eng. Med. Biol. Soc. 936-939. 


\title{
Quantitative imaging of efflux pumps in planktonic and biofilm-associated bacteria through single-molecule localization microscopy
}

\author{
Tiziano Vignolini ${ }^{\mathrm{a}}$, Lucia Gardini ${ }^{\mathrm{acc}}$, Marco Capitanio*b and Francesco S. Pavone ${ }^{\mathrm{a}, \mathrm{b}, \mathrm{c}}$ \\ ${ }^{a} L E N S$, European Laboratory for Non Linear Spectroscopy, Via N. Carrara 1, 50019 Sesto \\ Fiorentino, Italy \\ ${ }^{b}$ Physics Department, University of Florence, Via G.Sansone 1, 50019 Sesto Fiorentino, Italy \\ 'INO- National Institute of Optics, National Research Council, Largo Fermi 6, 50125 Florence, Italy
}

\begin{abstract}
When bacteria switch from a planktonic to a biofilm-associated lifestyle, a wide remodeling of gene expression takes place. In particular, efflux pump genes such as the acr $\mathrm{AB}$ operon are known to be upregulated under biofilm growth when compared to planktonic bacteria, and this phenomenon plays an important role in determining an increased resistance of biofilms to antimicrobial treatments. Although efflux pump expression in biofilms has been previously investigated through indirect methods, direct observation and quantification of efflux pumps at the single-cell level has never been reported to our knowledge. Here, we implement a method involving CRISPR/Cas9-assisted genome editing coupled to single-molecule localization microscopy to obtain quantitative data on the expression and distribution of the AcrB efflux pump in both planktonic and biofilm-associated Escherichia coli cells.
\end{abstract}

Keywords: biofilms, efflux pumps, single-molecule imaging, PALM, inclined illumination, genome editing

\section{Introduction}

The innate resistance of Gram-negative bacteria to antibiotics and other antimicrobials is a consequence of combinatorial effects of two permeability barriers (the outer and inner membranes), their ability to efflux antibiotics out of the cell and their capacity to form antibiotic tolerant biofilms that are up to 100 times more resistant than planktonic (suspended) cells ${ }^{1}$. In particular, multidrug efflux pumps such as the AcrAB-TolC complex found in many gram-negative species play an important role in resistance determination because of their wide range of substrates, which encompass both antimicrobials and other xenobiotics as well as endogenous metabolites ${ }^{2,3}$. Moreover, the genes encoding for efflux pump systems appear to be overexpressed under exposure to several antibiotics and during biofilm growth ${ }^{4}$, even though regulation of these genes is a complex phenomenon, and previous analysis of efflux pump expression via RNA microarrays and transcriptional fusions to reporter genes has yielded contradictory results ${ }^{4-6}$.
Another important phenomenon which occurs in biofilms and which contributes to antimicrobial resistance is the differentiation of isogenic bacteria in different subpopulations with varying gene expression patterns ${ }^{7}$. One instance of this phenomenon is the spontaneous manifestation of so-called "persisters" within the biofilms of several different species, which are a subpopulation of bacteria displaying extremely high levels of antimicrobial resistance which manage to survive and eventually rebuild a biofilm even after a near-total elimination ${ }^{8-11}$. Direct observation of cell-to-cell variability in gene expression within a biofilm has never been reported to the best of our knowledge.

The last decade has seen a great proliferation and popularization of single molecule and super-resolution microscopy techniques, which have allowed researchers to probe cell structures and functions in previously unattainable detail ${ }^{12}$. Such techniques are continuously evolving and are giving access to information about the

*capitanio@lens.unifi.it; phone: +39055 4572054 
organization of proteins in cells with nanometer-level resolution, providing an invaluable tool for the investigation of subcellular structures in small biological entities such as bacteria. For instance, single-molecule tracking techniques (SMT) have demonstrated the possibility to follow the movements and dynamics of single proteins $s^{13,14}$, such as transcription factors ${ }^{15}$ and cytoskeletal components $^{16}$ within living bacterial cells. On the other hand, super-resolution microscopy based on the localization of single molecules (SMLM) allowed imaging of cellular structures, such as the FtsZ protein ring involved in bacterial cell division ${ }^{17}$, which were previously unresolvable. However, the usefulness of SMT and SMLM techniques extends beyond imaging and qualitative analysis, and has allowed researchers to perform quantitative biological measurements by counting molecules and calculating diffusion coefficients. These features make super-resolution microscopy techniques especially suitable for the study of protein distribution within bacteria, both in isolated cells and in organized communities.

\section{Body}

1. Development of an E. coli strain encoding a fluorescently-labelled efflux pump suitable for singlemolecule localization microscopy

We engineered an E. coli BW25113 strain with a chromosomally encoded fusion between the native acrB gene and the coding sequence for PAmCherry, a photoactivatable fluorescent protein ${ }^{18}$. We achieved this by employing $\lambda$ Red-mediated homologous recombination coupled with CRISPR/Cas9 selection ${ }^{19}$. This recently popularized method of chromosomal editing in bacteria has several advantages when compared to traditional recombination approaches that employ the co-insertion of an antibiotic resistance cassette to select for recombinant clones $^{20}$. Most notably, the traditional method can either leave behind an extra gene (the antibiotic selection marker) or, when followed by Flp-mediated excision of the cassette, a so-called "scar" sequence that can affect the expression rate of neighboring genes in unpredictable ways ${ }^{21}$. Instead,
CRISPR/Cas9 selection allows for the seamless integration of arbitrary sequences in the host chromosome, avoiding the introduction of unnecessary mutations.

We replaced the STOP codon of the native acrB gene in E. coli BW24113 with a codon-optimized PAmCherry sequence, fused to the acrB sequence by a linker coding for Gly-Gly-Gly. Recombination was performed according to the no-SCAR protocol ${ }^{19}$. After recombination, the plasmids were cured via replica plating and the correct insertion was verified by sequencing.

Functionality of the fluorescently labelled AcrB protein was verified by measuring the MIC of chloramphenicol, an antibiotic which is a specific substrate of AcrB, via the microdilution method in LB medium ${ }^{22}$. Growth of the acrBPAmCherry strain was tested at different chloramphenicol concentrations, alongside the wild-type BW25113 strain and two deletion mutants ( $\triangle \mathrm{acrB}$ and $\Delta$ tolC). The MIC for both the acrB-PAmCherry strain and the WT strain was measured at $8 \mu \mathrm{g} / \mathrm{ml}$ while the MIC for both deletion mutants was $2 \mu \mathrm{g} / \mathrm{ml}$.

The pCas9-CR4 plasmid (Addgene plasmid \# 62655 ; http://n2t.net/addgene:62655 ; RRID:Addgene_62655) and the pKDsgRNA-ack plasmid (Addgene plasmid \# 62654 ; http://n2t.net/addgene:62654 ; RRID:Addgene_62654) were gifts from Kristala Prather, Massachusetts Institute of Technology, Department of Chemical Engineering. The recombination template, a codon-optimized PAmCherry sequence flanked by $\sim 200$ bp-long homology arms, was custom designed and ordered as a gStrand from Eurofins Genomics (https://www.eurofinsgenomics.eu/). All of the E. coli strains used in this study were gifts from Laura Piddock, University of Birmingham, Institute of Microbiology and Infection

\section{Single-molecule localization microscopy of efflux pumps on planktonic and biofilm-associated bacteria}

We used the PALM (PhotoActivated Localization Microscopy) super-resolution microscopy technique ${ }^{23}$ to directly visualize and count individual instances of AcrB on 
planktonic bacteria before and after exposure to an antibiotic, as well as within fully-grown biofilms.

PALM relies on the FIONA (Fluorescence Imaging with One Nanometer Accuracy) principle by Selvin et al. ${ }^{24}$, and employs chemical control over fluorophore photophysics to resolve the position of individual fluorescent molecules in a crowded environment. By using photo-activatable fluorescent proteins, it is possible to stochastically excite only a small fraction of the fluorophores in a sample and resolve them individually. By performing this operation for several thousands of frames and localizing the center of each point spread function with the FIONA method, it is possible to obtain a detailed and complete map of the fluorescently labeled proteins with an accuracy of a few nanometers.

As with all single-molecule localization methods, PALM is a widefield microscopy technique that needs high acquisition speeds and high signal-to-background ratios in order to provide the best results; as such, limiting the background fluorescence originating from out-of-focus planes is of crucial importance. Optimized illumination techniques such as Highly Inclined and Laminated Optical sheet (HILO) microscopy ${ }^{25}$ provide the means to perform widefield image acquisition while keeping the out-of-focus fluorescence low ${ }^{26,27}$.

We developed a combination of PALM and HILO to count and localize with high precision the AcrB-PAmCherry fusion proteins expressed by E. coli grown to mid-log phase in the absence of antibiotics, and after different times of exposure to sub-inhibitory concentrations of chloramphenicol .

PALM microscopy was performed on fixed bacteria on a custom-made microscopy setup by using a $405 \mathrm{~nm}$ laser to switch the activation of individual PAmCherry molecules and a $532 \mathrm{~nm}$ laser to excite fluorescence. Images were taken until complete photobleaching of PAmCherry was observed. Raw image stacks were analyzed with the ThunderSTORM ImageJ plugin $^{28}$ in order to reconstruct super-resolved molecular maps such as the one in Figure 1.
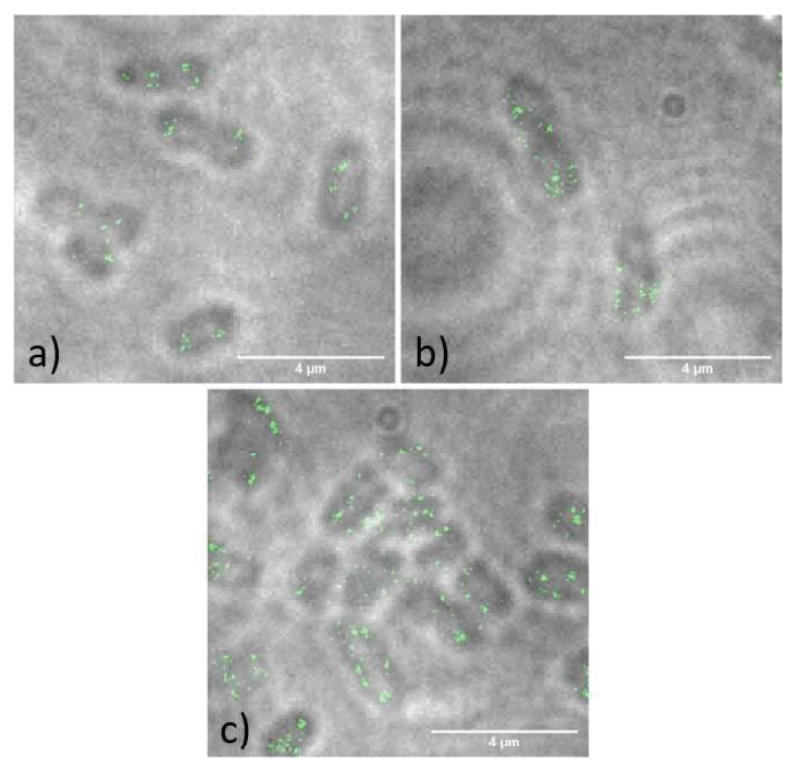

Figure 1. Super-resolution maps of AcrB-PAmCherry after a) no exposure, b) 40 minutes of exposure and c) 80 minutes of exposure to chloramphenicol.

We used the same technique to perform super-resolution imaging of AcrB in live E. coli biofilms. Bacteria were inoculated in glass-bottomed chambers containing sterile M9 minimal medium with added glucose, let grow for $>24 \mathrm{~h}$ at $37^{\circ} \mathrm{C}$ with no agitation, and then directly imaged in the same medium without mechanically perturbing the biofilm. Figure 2 is an example of a super-resolved image of AcrB obtained in a biofilm. 


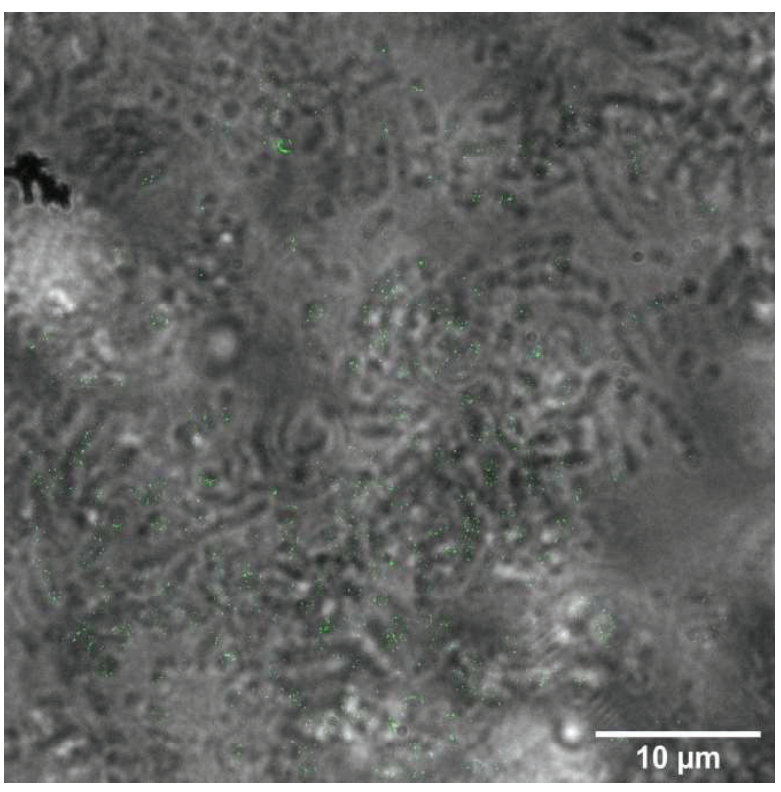

Figure 2. Super-resolution map of AcrB-PAmCherry in a biofilm.

\section{Results and Discussion}

Here, we presented a method that allows us to directly localize and quantify expressed proteins on both isolated bacteria and complex biofilm environments. By using stateof-the-art genome editing techniques which minimize unwanted transcriptional and translational side effects on labelled proteins, and by taking advantage of the quantitative nature of single-molecule localization techniques, we are able to observe protein expression and distribution in single bacterial cells with unprecedented accuracy. The preliminary results presented here will allow us to extract valuable data on the regulation of antibiotic resistance systems in bacteria, and to investigate the still largely unexplored differentiation of isogenic bacteria in the biofilm environment.

\section{Acknowledgements}

This work was supported by the European Union's Horizon 2020 research and innovation program under grant agreement no 654148 Laserlab-Europe; and EMPIR-
Horizon 2020 under grant agreement 15HLT01 MetVBadBugs.

\section{References}

[1] Høiby, N., Bjarnsholt, T., Givskov, M., Molin, S. and Ciofu, O., "Antibiotic resistance of bacterial biofilms,” Int. J. Antimicrob. Agents (2010).

[2] Ruiz, C. and Levy, S. B., "Regulation of acrAB expression by cellular metabolites in Escherichia coli," J. Antimicrob. Chemother. 69(2), 390-399 (2014).

[3] Piddock, L. J. V., "Multidrug-resistance efflux pumps - Not just for resistance," Nat. Rev. Microbiol. (2006).

[4] Soto, S. M., "Role of efflux pumps in the antibiotic resistance of bacteria embedded in a biofilm," Virulence (2013).

[5] Bailey, A. M., Webber, M. A. and Piddock, L. J. V., "Medium plays a role in determining expression of acrB, marA, and soxS in Escherichia coli," Antimicrob. Agents Chemother. (2006).

[6] Ma, D., Alberti, M., Lynch, C., Nikaido, H. and Hearst, J. E., "The local repressor AcrR plays a modulating role in the regulation of acr $\mathrm{AB}$ genes of Escherichia coli by global stress signals," Mol. Microbiol. (1996).

[7] Webb, J. S., Givskov, M. and Kjelleberg, S., "Bacterial biofilms: Prokaryotic adventures in multicellularity," Curr. Opin. Microbiol. (2003).

[8] Harms, A., Maisonneuve, E. and Gerdes, K., "Mechanisms of bacterial persistence during stress and antibiotic exposure," Science (80-. ). 354(6318) (2016).

[9] Pu, Y., Zhao, Z., Li, Y., Zou, J., Ma, Q., Zhao, Y., Ke, Y., Zhu, Y., Chen, H., Baker, M. A. B., Ge, H., Sun, Y., Xie, X. S. and Bai, F., "Enhanced Efflux Activity Facilitates Drug Tolerance in Dormant Bacterial Cells," Mol. Cell (2016).

[10] Shah, D., Zhang, Z., Khodursky, A., Kaldalu, N., Kurg, K. and Lewis, K., "Persisters: A distinct physiological state of E. coli," BMC Microbiol. 6 (2006). 
[11] Kim, J. S. and Wood, T. K., "Persistent persister misperceptions," Front. Microbiol. 7(DEC) (2016).

[12] Huang, B., Bates, M. and Zhuang, X., "SuperResolution Fluorescence Microscopy," Annu. Rev. Biochem. 78(1), 993-1016 (2009).

[13] Gardini, L., Capitanio, M. and Pavone, F. S., "3D tracking of single nanoparticles and quantum dots in living cells by out-of-focus imaging with diffraction pattern recognition," Sci. Rep. 5 (2015).

[14] Gardini, L., Calamai, M., Hatakeyama, H., Kanzaki, M., Capitanio, M. and Pavone, F. S., "ThreeDimensional Tracking of Quantum Dot-Conjugated Molecules in Living Cells," [Nanoscale Imaging], Humana Press, New York, NY, 425-448 (2018).

[15] Elf, J., Li, G. W. and Xie, X. S., "Probing transcription factor dynamics at the single-molecule level in a living cell," Science (80-. ). 316(5828), 1191-1194 (2007).

[16] Kim, S. Y., Gitai, Z., Kinkhabwala, A., Shapiro, L. and Moerner, W. E., "Single molecules of the bacterial actin MreB undergo directed treadmilling motion in Caulobacter crescentus," Proc. Natl. Acad. Sci. 103(29), 10929-10934 (2006).

[17] Holden, S. J., Pengo, T., Meibom, K. L., Fernandez Fernandez, C., Collier, J. and Manley, S., "High throughput 3D super-resolution microscopy reveals Caulobacter crescentus in vivo Z-ring organization," Proc. Natl. Acad. Sci. 111(12), 4566-4571 (2014).

[18] Subach, F. V., Patterson, G. H., Manley, S., Gillette, J. M., Lippincott-Schwartz, J. and Verkhusha, V. V., "Photoactivatable mCherry for high-resolution two-color fluorescence microscopy," Nat. Methods (2009).

[19] Reisch, C. R. and Prather, K. L. J., "The no-SCAR (Scarless Cas9 Assisted Recombineering) system for genome editing in Escherichia coli," Sci. Rep. (2015).

[20] Sharan, S. K., Thomason, L. C., Kuznetsov, S. G. and Court, D. L., "Recombineering: A homologous recombination-based method of genetic engineering," Nat. Protoc. (2009).
[21] Knöppel, A., Näsvall, J. and Andersson, D. I., "Compensating the Fitness Costs of Synonymous Mutations," Mol. Biol. Evol. (2016).

[22] Clinical and Laboratory Standards Institute (CLSI)., "Methods for Dilution Antimicrobial Susceptibility Tests for Bacteria That Grow Aerobically: Approved Standard-Ninth Edition M07-A9" (2012).

[23] Hess, S. T., Girirajan, T. P. K. and Mason, M. D., "Ultra-high resolution imaging by fluorescence photoactivation localization microscopy," Biophys. J. 91(11), 4258-4272 (2006).

[24] Selvin, P. R., Lougheed, T., Tonks Hoffman, M., Park, H., Balci, H., Blehm, B. H. and Toprak, E., "Fluorescence Imaging with One-Nanometer Accuracy (FIONA)," Cold Spring Harb. Protoc. 2007(10), pdb.top27 (2007).

[25] Tokunaga, M., Imamoto, N. and Sakata-Sogawa, $\mathrm{K}$., "Highly inclined thin illumination enables clear single-molecule imaging in cells," Nat. Methods 5(2), 159-161 (2008).

[26] Vignolini, T., Curcio, V., Gardini, L., Capitanio, M. and Pavone, F. S., "Characterization and improvement of highly inclined optical sheet microscopy," Prog. Biomed. Opt. Imaging - Proc. SPIE 10500 (2018).

[27] Vignolini, T., Gardini, L., Curcio, V., Capitanio, M. and Pavone, F. S., "Optimization of Highly Inclined Optical Sheet Illumination for Super-Resolution Microscopy," Biophys. J., 114 (3), 14a (2018).

[28] Ovesný, M., Křížek, P., Borkovec, J., Švindrych, Z. and Hagen, G. M., "ThunderSTORM: A comprehensive ImageJ plug-in for PALM and STORM data analysis and super-resolution imaging," Bioinformatics (2014). 


\title{
Implementation of Imaging Post Amplifier on Scan-less Confocal Dual-
}

\section{Comb Microscope}

\author{
Takuya Tsuda ${ }^{\mathrm{a}, \mathrm{b}}$, Takahiko Mizuno ${ }^{\mathrm{a}, \mathrm{b}}$, Takeo Minamikawa ${ }^{\mathrm{a}, \mathrm{b}}$, \\ Hirotsugu Yamamoto ${ }^{\text {b,c }}$, Takeshi Yasui*a,b \\ ${ }^{a}$ Tokushima University, 2-1 Minami-Josanjima, Tokushima-city, Tokushima 770-8506, Japan \\ bJST, ERATO MINOSHIMA Intelligent Optical Synthesizer (IOS), 2-1 Minami-Josanjima, \\ Tokushima-city, Tokushima 770-8506, Japan \\ 'Utsunomiya University, 7-1-2, Yoto, Utsunomiya, Tochigi, Japan 321-858
}

\begin{abstract}
In the biomedical field, a confocal microscopy is widely used as a powerful tool. Recently, dual-comb microscopy was proposed for scan-less confocal phase imaging, which was achieved by a combination of a dual-comb spectroscopy (DCS) and a 2D spectral disperser. For improving of acquisition speed of confocal amplitude and phase imageing, we combine dual-comb microscopy and post-amplification technique. The proposed method significantly improves signal-tonoise ratio in the rapid scan-less imaging.
\end{abstract}

Keywords: Confocal microscopy, Interference microscopy, Spectroscopy.

\section{Introduction}

Optical microscopy has been widely used as a powerful tool in life science research and industrial inspection. A confocal laser scanning microscopy (CLM) has attracted attention due to its three-dimensional imaging capability with confocality and spray light elimination $^{1,2}$. Scan-less CLM was achieved by a combination of one-dimensional (1D) spectral encoding CLM with line-field $\mathrm{CLM}^{3}$ or two-dimensional (2D) spectral encoding ${ }^{4,5}$, enabling rapid image acquisition and robustness to external disturbances. If the depth resolution in these scan-less CLM images can be further decreased beyond the limit of the confocality, namely wavelength order, CLM will become further powerful tool in a variety of application fields.

Recently, we proposed dual-comb microscopy (DCM) for scan-less confocal amplitude and phase imaging by introducing dual-comb spectroscopy (DCS) to scan-less $\mathrm{CLM}^{6}$. In this method, after the image pixels of the sample is encoded on an optical frequency comb (OFC) by $2 \mathrm{D}$ spectral encoding (2D-SE), DCS of the imageencoded OFC passing through the confocal pinhole gives the mode-resolved amplitude and phase spectra with confocality. Based on one-to-one correspondence *yasui.takeshi@tokushima-u.ac.jp phone+81-88-656-7377 between the image pixels and OFC modes, the confocal amplitude and phase images are decoded from the moderesolved amplitude and phase spectra, respectively. However, the optical intensity of the image-encoded OFC is relatively low due to $2 \mathrm{D}$ multiple focal points, confocal pinhole, and/or signal loss in 2D-SE. This hampers high signal-to-noise ratio in the rapid image acquisition.

\section{Method and Setup}

Figure 1(a) shows a principle of $\mathrm{DCM}^{6}$ based on 2D spectral encoding ${ }^{4,5}$. Here, OFC is used as an optical carrier of amplitude and phase with discrete ultramultichannel for one-to-one correspondence with image pixels. Each OFC mode is individually diffracted at different solid angles by a 2D spectral disperser for a wavelength-to-space conversion. The spatially dispersed OFC modes form a 2D spectrograph of the OFC and are then focused at different positions of a sample as a $2 \mathrm{D}$ array of focal spots. After being reflected by the sample, the $2 \mathrm{D}$ spectrograph is spatially overlapped again for the space-to-wavelength conversion. The bidirectional conversion between the wavelength and $2 \mathrm{D}$ space modulates the spectral shape of the OFC depending on the $2 \mathrm{D}$ information of the sample. In this way, a one-toone correspondence is established between the $2 \mathrm{D}$ image 
pixels and the OFC modes. The image-encoded OFC was interfered with the local OFC, and then was coupled to a single-mode fiber, giving the confocality. By passing through an EDFA, the image-encoded and the local OFCs were amplified. The mode-resolved amplitude and phase spectra of the 2D-image-encoded OFC are acquired by DCS. Finally, the confocal 2D image of the sample is decoded from the mode-resolved OFC spectra.

Figure 1(b) shows a schematic diagram of the experimental setup. A dual femtosecond Er-fiber OFC (OFC1 or signal comb, $f_{\text {ceol }}=21.4 \mathrm{MHz}, f_{\text {rep } 1}=100.389180 \mathrm{MHz}$; OFC2 or local comb, $f_{\text {ceo } 2}=21.4 \mathrm{MHz}, f_{\text {rep } 2}=100.388201 \mathrm{MHz} ; \Delta f_{\text {rep }}=f_{\text {rep } 1}-f_{\text {rep2 }}=$ $979 \mathrm{~Hz}$ ) is used for DCM light source. A 2D spectral disperser composed of a virtually imaged phased array (VIPA, FSR $=15$ $\mathrm{GHz}$, finesse $=100$ ) and a diffraction grating (groove density $=$ $1200 \mathrm{lp} / \mathrm{mm}$ ), was used for generation of the 2D spectrograph on a sample by help of relay lenses and an objective lens. The reflected light inversely propagates the same optics for the spatial overlapping of each OFC mode. After coupling into a SMF with the OFC2, the OFC1 and OFC lights were amplified by EDFA, and interferes with each other to generate an interferogram. Confocality is given by the SMF coupling. Finally, the mode-resolved amplitude and phase spectra for the OFC1 light are obtained by Fourier transform of the acquired interferogram.

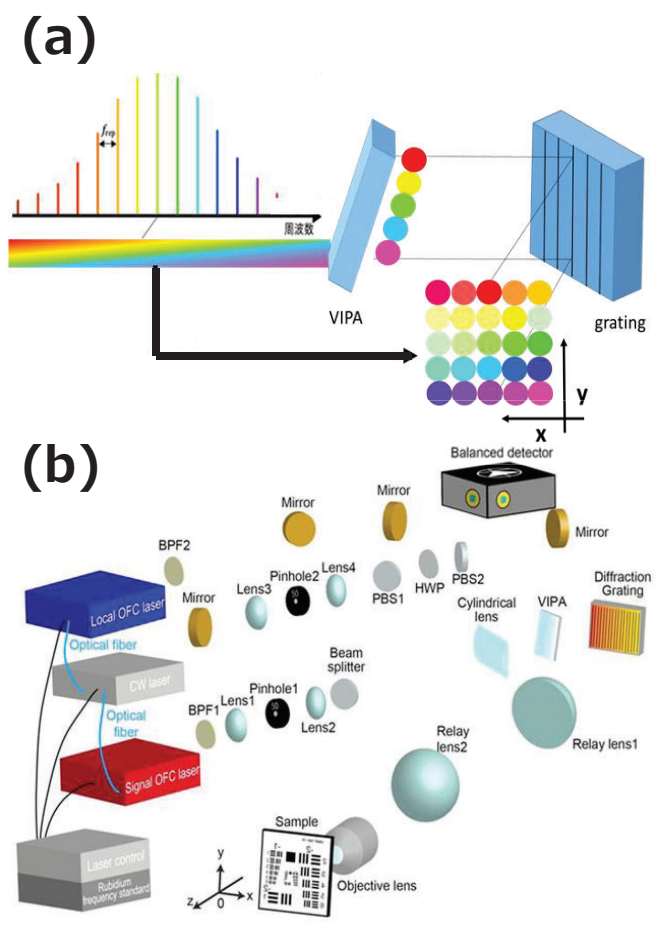

Fig. 1. (a) conceptual diagram, (b)Experimental setup.

\section{Imaging Results}

For proof of concept, we performed imaging of test chart. Figure 2 shows experimental results. Figures 2(a)(d) show the experimental results without and with the post-amplification, respectively. From the comparison of the amplitude and phase spectra between them, the amplitude signal was increased while the phase noise was reduced. Such effect was clearly confirmed in the confocal amplitude and phase image: No images of the sample appeared without the post-amplification while the sample was significantly visualized with the postamplification. The post-amplification increases the SNR up to 13-times higher value.

(a)

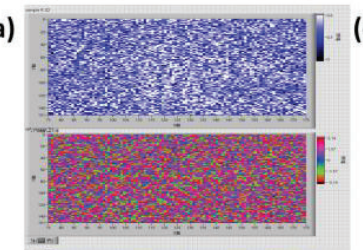

(b)

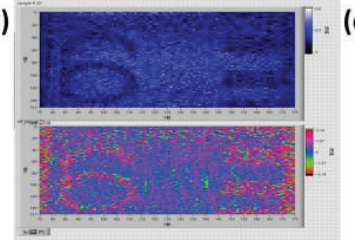

Fig.2. simple test chart imaging (a) single shot image

(b)10 average image and test chart imaging with

EDFA (c) single shot image (d)10 average image.

\section{Conclusion}

We have proposed combination of DCM with postamplification technique for improving the imaging SNR. By using post-amplification, the SNR increases up to 13times higher SNR, and scan-less confocal imaging of scattering object was successfully demonstrated. The proposed method will greatly enhance the applicability of DCM for practical applications.

This work was supported by Exploratory Research for Advanced Technology (ERATO), Japanese Science and Technology Agency (MINOSHIMA Intelligent Optical Synthesizer Project, JPMJER1304).

\section{References}

[1] P. Davidovits and M. D. Egger, "Photomicrography of 
corneal endothelial cells in vivo," Nature 244, 366367 (1973).

[2] G. J. Brakenhoff, P. Blom, and P. Barends, "Confocal scanning light microscopy with high aperture immersion lenses," J. Microsc. 117, 219-232 (1979).

[3] J. Kim, D. Kang, and D. Gweon, "Spectrally encoded slit confocal microscopy," Opt. Lett. 31, 1687-1689 (2006).

[4] K. K. Tsia, K. Goda, D. Capewell, and B. Jalali, "Simultaneous mechanical-scan-free confocal microscopy and laser microsurgery," Opt. Lett. 34, 2099-2101 (2009).

[5] K. K. Tsia, K. Goda, D. Capewell, and B. Jalali, "Performance of serial time-encoded amplified microscope," Opt. Express 18, 10016-10028 (2010).

[6] E. Hase, T. Minamikawa, T. Mizuno, S. Miyamoto, R. Ichikawa, Y.-D. Hsieh, K. Shibuya, K. Sato, Y. Nakajima, A. Asahara, K. Minoshima, Y. Mizutani, T. Iwata, H. Yamamoto, and T. Yasui, "Scan-less confocal phase imaging based on dual-comb microscopy," Optica 5, 634-643 (2018). 


\title{
Dynamic phase imaging of Haematococcus pluvialis cells by transport of intensity equation
}

\author{
Lu Zhang ${ }^{\mathrm{a}}$, Qijian Tang ${ }^{\mathrm{a}}$, Dingnan Deng ${ }^{\mathrm{c}}$, Ming Tao ${ }^{\mathrm{b}}$, Xiaoli Liu*, ${ }^{*}$, Xiang Peng ${ }^{\mathrm{a}}$ \\ ${ }^{a}$ College of Physics and Optoelectronic Engineering, Shenzhen University, Guangdong, 518060, \\ China \\ ${ }^{b}$ Key laboratory of Marine Bioresource and Eco-environmental Science, Shenzhen University, \\ Guangdong, 518060, China \\ 'Jiaying University, Guangdong, 514000, China
}

\begin{abstract}
Dual-camera combined with transport of intensity equation is a compact and effective system for dynamic quantitative phase imaging. The living cells called Haematococcus pluvialis cells has gained increasing attention because of its metabolite high-value astaxanthin, a super anti-oxidant. In this paper, a rectify method based on checkboard calibration for correcting the alignment error of dual-camera images is introduced to achieve accurate TIE phase imaging with sub-pixel aligning accuracy. The experiments of observing dynamic Haematococcus pluvialis cells and measuring quantitative phase of micro-lens array are performed to verify the efficiency of this method for high-speed and high-accuracy phase imaging.
\end{abstract}

Keyword: Transport of intensity equation, Phase imaging, FoV alignment, Dynamic imaging, Haematococcus pluvialis

\section{Introduction}

Quantitative phase recovery using transport of intensity equation (TIE) firstly proposed by Teague in 1983 belongs to non-interferometric phase imaging [1], which is deduced by Helmholtz equation under paraxial approximation, and expressed the quantitative relationship between the variation of optical intensity along the optical axis. Haematococcus pluvialis cells are one of the best organisms to produce natural antioxidant astaxanthin. How to make better use of Haematococcus pluvialis cells to produce more astaxanthin is a research hotspot in related fields [2-3]. The life of Haematococcus pluvialis cells can be divided into a green swimming phase and a red stationary phase that produces astaxanthin. The TIE phase imaging method can be used to quantitatively observe and analysis the Haematococcus pluvialis cells. But the traditional TIE does not satisfy the conditions for observing living cells, so a system for obtaining dynamic by using a dual-camera combined with TIE is a flexible

* $\underline{1 x 1 @ \text { szu.edu.cn }}$ and effective method [4]. However, the field of views (FoVs) of two cameras should be accurately aligned and it is hard to achieve complete alignment only by optical system adjustment in this system. Then phase correlation-based digital FoV correction is proposed to recognize and compensate the scale, rotation, and translation between the under-focus and over-focus images [5].

In this paper, we improve a rectify method for correcting FoVs of dual-camera system, a checkerboard target is used to extract the corresponding target landmarks in the calibration process, and the scale, rotation, translation parameters are facile to obtain by minimizing the misalignment error between the corresponding target landmarks in under-focus and over-focus images, and the accuracy of correction is improved to the sub-pixel level. Meanwhile, dynamic quantitative phase imaging for living Haematococcus pluvialis cells with TIE is performed. 


\section{Basic Principle}

TIE is derived from the Helmholtz equation, this equation expresses quantitative relationship between the change of intensity in the direction of optical axis and phase on the plane perpendicular to optical axis [6],

$$
-k \frac{\partial I(x, y)}{\partial z}=\nabla \cdot[I(x, y) \nabla \varphi(x, y)]
$$

where $\nabla$ is the two-dimensional gradient operator, $k$ is the wave number, $I(x, y)$ is the focus intensity perpendicular to optical axis, $\varphi(x, y)$ is the phase of object.

If the differential of intensity along axial direction is known, phase can be obtained by solving the Poisson equation with Fast Fourier Transform (FFT) [6]. Usually, collecting two intensity images along optical axis direction for numerical difference approximation instead of intensity's differential along axial direction. The relationship between phase and height $h(x, y)$ of object is expressed by following equation

$$
h(x, y)=\frac{\lambda}{n_{o}-n} \frac{\varphi(x, y)}{2 \pi}
$$

where $n_{0}$ is the refractive index of object, $n$ is the refractive index of surrounding medium.

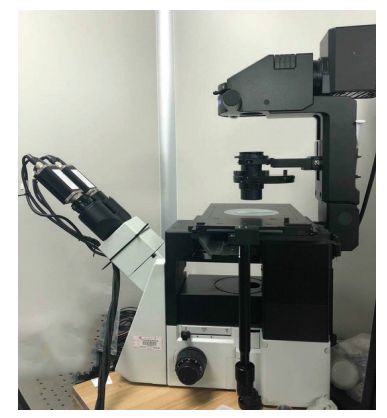

Figure 1. The system schematic.

The dual-camera TIE imaging system is shown in Figure 1. Two cameras are mounted on the microscopic eyepiece for image acquisition, and a c-mount spacer is mounted on one camera in order to obtain under-focus image and over-focus image respectively with two cameras. Due to the error of microscopy system fabrication and installation, there exist the misalignment of FoVs between two cameras. The phenomenon will affect the accuracy of recovering phase by TIE, the FoVs hence needs to be corrected. Therefore, we presented the misalignment error with a transformation consisting of scale, rotation and translation.

A checkerboard target is used in the calibration process, shown in Figure 2, the red crosses in the figure are the corresponding target landmarks that extracted by Harris algorithm, $X_{i}^{(1)}=\left[\begin{array}{l}x_{i}^{(1)} \\ y_{i}^{(1)}\end{array}\right](i=1,2, \ldots, N)$ is taken as the coordinate of target landmark in the under-focus image, $N$ is the number of target landmarks, and $X_{i}^{(2)}=\left[\begin{array}{c}x_{i}^{(2)} \\ y_{i}^{(2)}\end{array}\right]$ is the coordinate of target landmarks in the over-focus image. Then the least-square fitting method is used to minimize the misalignment error function $E(s, R, T)$

$$
\min E(s, R, T)=\sum_{i=1}^{N}\left\|X_{i}^{(1)}-\left(s * R * X_{i}^{(2)}+T\right)\right\|^{2}
$$

where $s$ is a scale factor, $R$ is rotation matrix, $T$ is translation matrix. These unknown parameters can be solved out through equation (3).
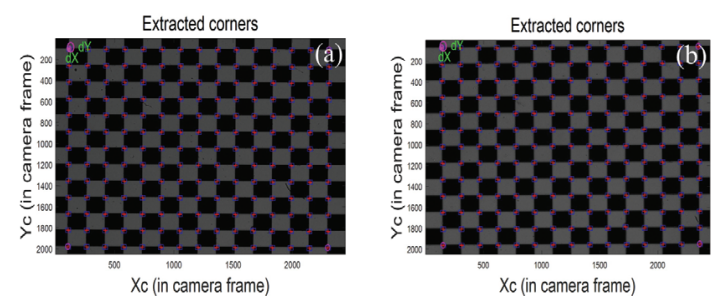

Figure 2. (a)Under-focus image and (b) over-focus image of the checkerboard

\section{Results and Discussion}

In our experiments with the system in Figure 1, the relative standard deviation of the two FoVs after correction is 0.8681 , which indicates that the accuracy of correction is at the level of sub-pixel.

The accuracy of this dual-camera microscopy system combined with TIE is verified by micro-lens array of known size firstly. In the experiment, cameras collect under and over defocus image respectively, the defocus distance is $\pm 10 \mu \mathrm{m}$, and the focus image is the average of 
under and over defocus images. According to the equation (1), the phase of micro-lens array can be calculated by TIE, the phase distribution is shown in Figure 3.

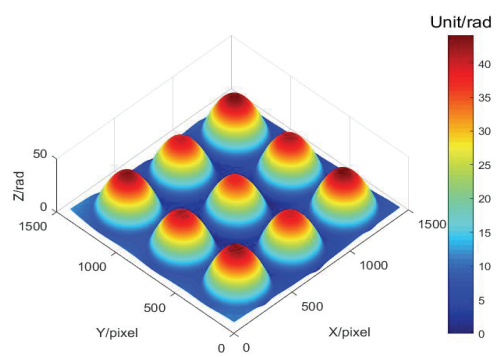

Figure 3. The phase of micro-lens array.

The refractive index of micro-lens array is $n_{o}=1.458$, the medium is air, so $n=1$. Substituting $n_{o}$ into equation (2) and obtaining the height distribution of micro lens. Then, according to the equation (4), the radius of curvature of micro lens can be calculated.

$$
R O C=\frac{h}{2}+\frac{D^{2}}{8 h}
$$

where $h$ is the height of highest point of a single lens, $D$ is the diameter with the value $123 \mu \mathrm{m}$ in the experiments.

We choose one micro lens and extract its curvature radius is $306 \mu \mathrm{m}$ by experiment. The reference value given by the manufacturer is $315 \mu m \pm 5 \%$. It can be seen that the system of obtaining phase is in the range of error tolerance.
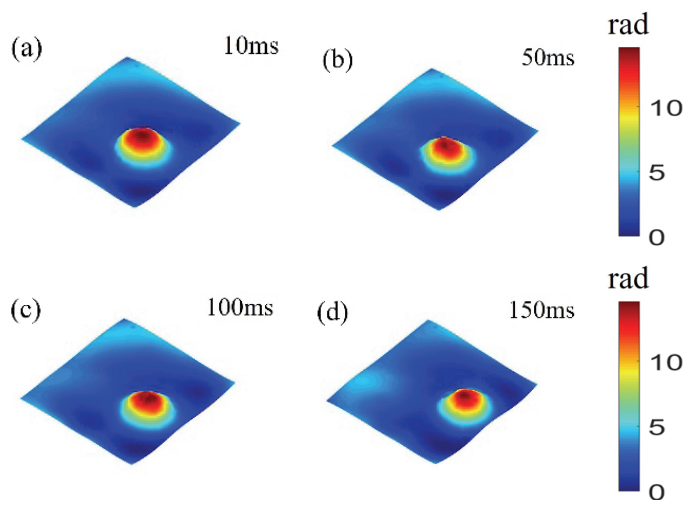
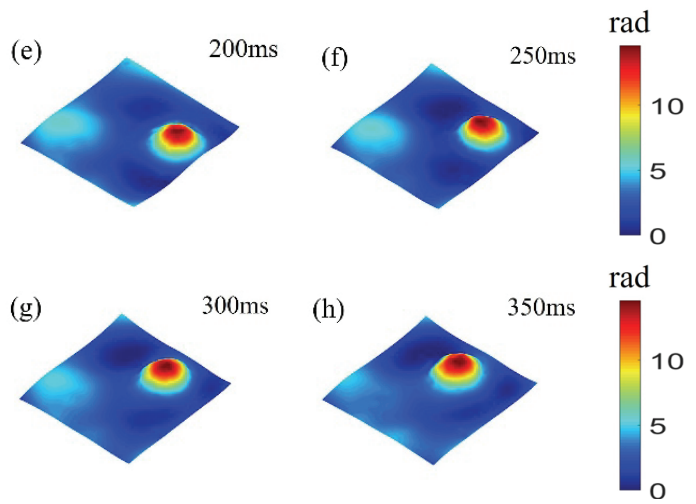

Figure 4. The phase of Haematococcus pluvialis cell at different time.

Figure 4 shows the movement track of a Haematococcus pluvialis cell within $350 \mathrm{~ms}$. Test results demonstrate that the cell with flagella is constantly swimming, and the phase is quantitatively observed.

\section{References}

[1] Teague M R. "Deterministic phase retrieval: a Green's function solution," J.opt.soc.am.a, Papers 73(11), 1434-1441 (1983).

[2] Weiwei W, Kang X, Ming T, et al., "A Novel Organic Electrochemical Transistor-Based Platform for Monitoring the Senescent Green Vegetative Phase of Haematococcus pluvialis Cells," Sensors, Papers 17(9), 1997- (2017).

[3] Boussiba S, Vonshak., "Astaxanthin accumulation in the green alga Haematococcus pluvialis. Plant Cell Physiol," Plant \& Cell Physiology, Papers 32(7), 1077-1082 (1991).

[4] Tian, X, et al., "Real-time quantitative phase imaging based on transport of intensity equation with dual simultaneously recorded field of view," Optics Letters, Papers41(7), 1427-1430 (2016).

[5] Qingtao G, Qi W, Shouyu W, et al., "Digital field of view correction combined dual-view transport of intensity equation method for realtime quantitative imaging," Optical Engineering, Papers 57(6), 063102 (2018).

[6] Ichikawa K, Lohmann A W, Takeda M., "Phase retrieval based on the irradiance transport equation and the Fourier transform method: Experiments," Applied Optics, Papers 27(16), 3433-3436, (1988). 


\title{
In vivo multimodal optical biopsy of skin cancer
}

\author{
Yulia Khristoforova*a, Ivan Bratchenko ${ }^{a}$, Oleg Myakinin ${ }^{\mathrm{a}}$, Dmitry Artemyev ${ }^{\mathrm{a}}$, Anastasiya Lykina ${ }^{\mathrm{a}}$, \\ Semen Konovalov ${ }^{\mathrm{a}}$, Dmitry Raupov ${ }^{\mathrm{a}}$, Lyudmila Shamina ${ }^{\mathrm{a}}$, Alexander Moryatov ${ }^{\mathrm{b}, \mathrm{c}}$, Dmitry \\ Kassirov $^{\text {b,c }}$, Anastasiya Andreeva ${ }^{\mathrm{b}, \mathrm{c}}$, Andrey Orlov ${ }^{\mathrm{c}}$, Sergey Kozlov, ${ }^{\mathrm{b}, \mathrm{c}}$, Valery Zakharov ${ }^{\mathrm{a}}$ \\ ${ }^{a}$ Department of Laser and Biotechnical Systems, Samara National Research University, 34 \\ Moskovskoe shosse, Samara, 443086, Russia \\ ${ }^{b}$ Department of Oncology, Samara State Medical University, 89 Chapaevskaya str., Samara, 443099, \\ Russia \\ bamara Regional Clinical Oncology Dispensary, 50 Solnechanya str., Samara, 443031, Russia \\ Abstract
}

A multimodal method for skin cancer diagnosis based on Dermatoscopy image analysis, Hyperspectral Imaging, Raman/Autofluorescense analysis is presented. More than 300 patients of Samara Regional Clinical Oncology Dispensary were involved in this clinical study. The diagnostic accuracy more than $96 \%$ (depended from tissue type) has been demonstrated for the joint optical analysis. The achieved accuracy of the multimodal optical biopsy has been compared with the preliminary diagnostics accuracy by oncologist.

Keywords: optical biopsy, multimodality, Raman spectroscopy, hyperspectral visualization, dermatoscope, skin cancer

\section{Introduction}

Skin cancer is one of the most common cancer form with the annual upward trend of the disease incidence worldwide. Success of the recovery from skin cancer is increased (higher) at the early detection of a tumor. However, the preliminary accuracy of the skin cancer diagnosis don't exceed $60 \%$ by traditionally methods because of similarity with benign skin lesions ${ }^{1}$. In order to perform noninvasive analysis of skin tumors we propose to apply multimodal optical approach for screening suspicious skin areas in the clinical practice to identify skin malignancy. This approach bases on the application of dermatoscopy (DS) image analysis ${ }^{2}$, Raman Spectroscopy (RS) in combination with Autofluorescence analysis $(\mathrm{AF})^{3}$, hyperspectral Imaging (HSI $)^{4}$. DS image analysis allows one to estimate lesion's asymmetry, border, color and size due to the magnified RGB image. $\mathrm{RS}$ and AF spectroscopy application makes it possible to detect changes in tissue chemical composition as compared to healthy skin. The HSI tissue image allows one to study as neoplasm morphology as skin chromophores distribution that allows for finding the stage of tumor growth. Therefore, that approach helped us to analyze both morphological and biochemical

*khristoforovajulia@gmail.com; phone+7 8462674550 features of neoplasms based on the advantages of all modalities. The efficiency of the proposed approaches were compare with preliminary diagnostics by the specialized oncologists.

\section{Clinical study}

The in vivo study of skin tumors was performed at Samara Regional Clinical Oncology Dispensary. There were more than 300 patients with skin tumors (Caucasian, I and II skin phenotype).

At the first stage, a specialized oncologist explored each patient. The $\mathrm{PhD}$ in Medical sciences with 16-year of practical experience participated in this study. The oncologist determined a provisional diagnosis of the studied skin neoplasm on the basis of visual examination. Then, the optical multimodal approach has been tested for skin cancer diagnosis. It should be noted, that the provisional and instrumental examinations were established independently of each other.

We perform in vivo clinical study of the skin using optical methods RS/AF, HIS and DS image analysis.

\section{DS image analysis}

To register DS images of the neoplasms multichannel dermatoscope was used. DS image is an epidermis imaging with optical magnification allowed to analyze of the neoplasms based on the ABCD rules. Developed 
dermatoscope based on the Basler acA1920-25uc camera (RGB, 12 bit/px, 1920*1080) has a resolution of $\sim 13$ $\mu \mathrm{m} / \mathrm{px}$ on the surface of the skin. The device body has been designed and printed on a 3D printer. Tissue lighting is carried out by white LED: 4 x LEDs FM-5630WDS460W-R80 (39 lm, $4000 \mathrm{~K}$ ) (two polarized LEDs and two unpolarized ones).

\section{RS/AF analysis}

The spectroscopic system that combined principles of $\mathrm{RS}$ and $\mathrm{AF}$ in the near infrared region includes thermally stabilized LML-785.0RB-04 diode laser module (785 \pm 0.1 nm central wavelength), InPhotonics optical Raman probe, QE65 Pro portable spectrometer, excitation and collection optical fibers and PC. Spectra registration using QE65 Pro setup was carried out in the 780-1000 nm region with the spectral resolution of $0.2 \mathrm{~nm}$, cooling up to $-15^{\circ} \mathrm{C}$.

\section{HSI}

To register hyperspectral images of skin tissues an acousto-optical camera was used. The HSI camera has been designed in Scientific and Technological Center of Unique Instrumentation of the Russian Academy of Sciences (Moscow, Russia). A detailed description of the camera based on an acousto-optical filter can be found elsewhere ${ }^{5}$. Using HSI camera it is possible to obtain image at an arbitrary predetermined wavelength $\lambda$ in the range $440-750 \mathrm{~nm}$ with sufficiently high spectral resolution $(\delta \lambda=2.5 \mathrm{~nm}$ at $\lambda=633 \mathrm{~nm})$ and spatial resolution of $0.14 \mathrm{~mm}$.

*Tyamada@narxlab.com; phone 1222 555-1234

\section{Results}

Figure 1 shows multimodal study of melanoma by DS analysis, HIS and RS/AF. Dermatoscope registers polarized (fig. 1a) and unpolarized (fig. 1b) images of skin neoplasm. Linear polarization is used to filter out light directly reflected from skin surface, as it is necessary for deeper epidermis structure visualization and for light absorption estimation at different wavelengths in visible range. Unpolarized image is used to estimate texture features of skin surface.

On the basis of the HS image the melanin (M, fig. 1c) and hemoglobin (Hb, fig. 1d) indexes are calculated for each neoplasm. According to the images, the regions with the highest concentration of melanin and hemoglobin may be observed. The combination with the index of blood filling, for instance, gives us a visual representation of the degree of malignancy of the neoplasm.

The registered Raman (fig. 1e) and AF (fig. 1f) spectra were processing on the basis of regression analysis using the Partial Least Square Discriminant Analysis (PLSDA). PLS-DA method allows for constructing the regression model to classify neoplasms spectra based on the significant spectral differences ${ }^{6}$.

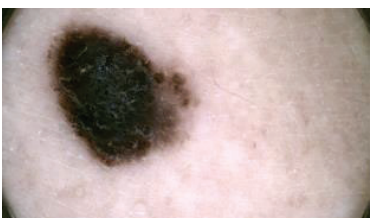

a

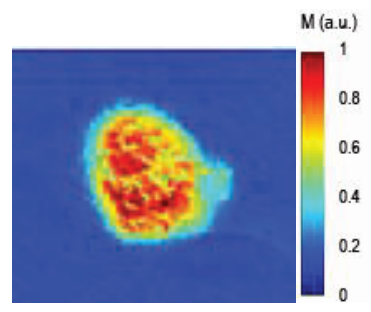

c

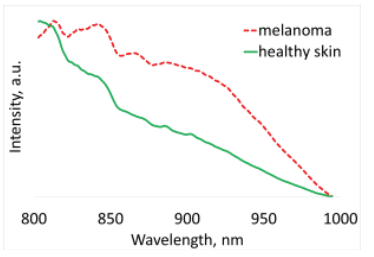

e

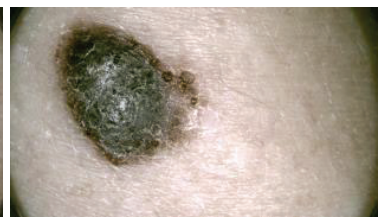

b

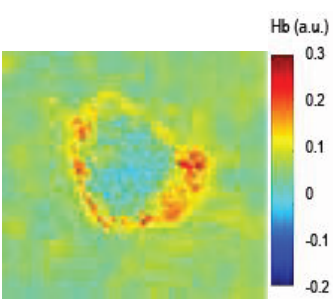

d

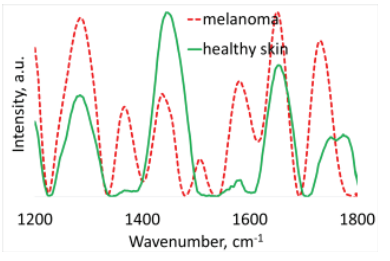

f
Figure 1. Multimodal analysis of melanoma: (a) polarized and (b) unpolarized dermatoscopic image (29 mm*16 mm image size), (c) melanin and (d) hemoglobin index, calculated by in vivo HS image, (e) $\mathrm{AF} / \mathrm{RS}$ spectra and (f) Raman spectra.

\section{Conclusions}

Application of the multimodal optical approach may allow for noninvasive studying of skin neoplasms features in more details. Dermatoscope is useful instrument for studying of the neoplasms at the preliminary inspection vision stage. Using of the dermatoscope may help doctors to improve results of screening analysis by $10-15 \%$. It is a very fast and low-cost process but only a well-trained 
oncologist may obtain high diagnostics accuracy. HSI allows for obtaining neoplasm comprehensive view with skin chromophores distribution both inside the tumor and on the border with the surrounding healthy skin. The processing of hyperspectral data is carried out in real time and does not require operator participation. In general, the simplicity of the equipment and the speed of processing results may allow for adapting the proposed method for tasks of mass screening of skin tumors. In contrast, The $\mathrm{RS} / \mathrm{AF}$ collects information from a local points of a tissue area. So, HSI shows tumor area with a suspicious properties of malignancy that may be examined in more details by RS/AF. Moreover, RS/AF method shows the higher accuracy (more than 90\%) among other methods presented in this study. The proposed joint $\mathrm{DS}+\mathrm{RS} / \mathrm{AF}+\mathrm{HIS}$ analysis leads to a high (more than 98\%) accuracy that approves its high clinical potential to study skin tumors. The application of different optical methods can significantly improve tissue malignancy detection.

Table 1. Results of the skin neoplasms differentiating

\begin{tabular}{|c|c|c|c|c|c|c|}
\hline \multirow{2}{*}{ Classes } & \multicolumn{5}{|c|}{ Accuracy, \% } \\
\cline { 2 - 6 } & $\begin{array}{c}\text { DS } \\
\text { analysis }\end{array}$ & $\begin{array}{c}\text { RS/AF } \\
\text { spectroscopy }\end{array}$ & HSI & Physician & $\begin{array}{c}\text { Oncologist's } \\
\text { diagnostics }\end{array}$ & DS+RS/AF+HSI \\
\hline $\begin{array}{c}\text { Malignant vs } \\
\text { benign }\end{array}$ & $65 \%$ & $90 \%$ & $80 \%$ & $50 \%$ & $60 \%$ & $>98 \%$ \\
\hline $\begin{array}{c}\text { Melanoma vs } \\
\text { other neoplasms }\end{array}$ & $75 \%$ & $90 \%$ & $80 \%$ & $50 \%$ & $80 \%$ & $>96 \%$ \\
\hline
\end{tabular}

\section{References}

[1] Argenziano, G., Soyer, H.P., "Dermoscopy of pigmented skin lesions - a valuable tool for early diagnosis of melanoma," Lancet Oncol. 2(7), 443-449 (2001).

[2] Myakinin, O.O., Zakharov, V.P., Bratchenko, I.A., Artemyev, D.N., Neretin, E.Y., Kozlov, S.V., "Dermoscopy analysis of RGB-images based on comparative features," Proc. SPIE 9599, 95992B (2015).

[3] Cicchi, R., Cosci, A., Rossari, S., Kapsokalyvas, D., Baria, E., Maio, V., Massi, D., De Giorgi, V., Pimpinelli, N., Pavone, F. S., "Combined fluorescence-Raman spectroscopic setup for the diagnosis of melanocytic lesions," Journal of Biophotonics 7(1-2), 86-95 (2013).
[4] Calin, M.A., Sorin, V., Savastru, D., Dragos, M., "Hyperspectral Imaging in the Medical Field: Present and Future,” Applied Spectroscopy Reviews 49, 435-447 (2014).

[5] Machihin, A., Pozhar, V., “A. Double-AOTF-based aberration-free spectral imaging endoscopic system for biomedical applications," Journal of Innovative Optical Health Sciences 8(3), 1541009 (2015).

[6] Haaland, D.M., Thomas, E. V., "Partial least-squares methods for spectral analyses. 1. Relation to other quantitative calibration methods and the extraction of qualitative information," Analytical Chemistry 60(11), 1193-1202 (1988). 


\title{
Differentiation of the microvasculature with different blood flow speed based on variable interscan time analysis in OCT angiography skin imaging
}

\author{
Ting-Yen Tsai ${ }^{\mathrm{a}}$, Yin-Peng Huang ${ }^{\mathrm{b}}$, Ting-Hao Chen ${ }^{\mathrm{a}}$, Chuan-Bor Chueh ${ }^{\mathrm{a}}$, Hsuan-Yuan Peng ${ }^{\mathrm{a}}$, \\ Meng-Shan $\mathrm{Wu}^{\mathrm{a}}$, Yi-Chun Wu ${ }^{\mathrm{a}}$, Yi-Ping Hung ${ }^{\mathrm{b}}$, Meng-Tsan Tsaic ${ }^{\mathrm{c}}$, Hsiang-Chieh Lee, ${ }^{\mathrm{a}, \mathrm{d}, \text {, }}$ \\ ${ }^{\mathrm{a}}$ Graduate Institute of Photonics and Optoelectronics, National Taiwan University, Taipei 10617, \\ Taiwan \\ ${ }^{\mathrm{b}}$ Graduate Institute of Networking and Multimedia, National Taiwan University, Taipei 10617, \\ Taiwan \\ ${ }^{c}$ Department of Electrical Engineering, Chang Gung University, Taoyuan 33302, Taiwan \\ ${ }^{\mathrm{d}}$ Molecular Imaging Center, National Taiwan University, Taipei 10617, Taiwan \\ *Email: hclee2@,ntu.edu.tw
}

\begin{abstract}
OCT-Angiography (OCTA) images are based on the nature that light backscattered from the vascular and neighboring tissue exhibiting different temporal scattering features. Therefore, by analyzing the variation of the OCT signal intensity in the OCT images acquired at different time points, regions exhibiting blood flow can be differentiated from neighboring static tissue. When compared to conventional approach such as Doppler OCT, the quantification of blood flow with OCTA is challenging. In this paper, we built a the swept-source OCT system with a $1060 \mathrm{~nm}$ wavelength-swept laser providing a high imaging speed (200,000 Ascans/sec) over the biological samples. Most importantly, repetitive OCT images can be acquired at the same locations, enabling OCTA imaging with different effective interscan time with so called variable interscan time analysis (VISTA) algorithm to highlight the microvasculature with different blood flow speed. Preliminary results of the OCTA imaging of the mouse ear skin are demonstrated in this paper.
\end{abstract}

Keywords: OCT, angiography, blood vessel, vascular image, correlation equation.

\section{Introduction}

Optical coherence tomography (OCT) provides micrometer-scale, cross-sectional imaging of the biological specimens in real time [1]. For biomedical applications, the microstructure information of the tissue can be obtained with resolutions approaching that of excisional biopsy and histopathology without the need to remove the tissue specimens or apply additional contrast agents on the tissue.

Recently, the functional extension of OCT has enabled micro-angiography of biological specimens in vivo with different approaches for example optical microangiography (OMAG) [2, 3] or OCT angiography (OCTA) $[4,5]$. The OCT based angiography technique measures the changes in amplitude (or intensity), phase, or complex value of the OCT signals resulting from the dynamic scattering of the red blood cells in the vessels or microvascular network relative to the static scattering of surrounding tissues, which serves as an endogenous contrast to decouple the moving blood from the static tissues. This approach is able to provide depth-resolved micro-angiograms with a capillary-level resolution $(\sim 10$ $\mu \mathrm{m})$, delineating the 3-D micro-vessel network within the tissue. In this paper, leveraging the high imaging speed, we performed variable interscan time analysis (VISTA) on the OCT images acquired to generate multiple OCTA images with different effective interscan time to identify the microvasculature with different blood flow speed.

\section{Method}

\section{OCT system}

In this study, we build up the swept-source OCT system (SS-OCT) with a central wavelength $1060 \mathrm{~nm}$ swept source laser (Axsun Technologies), sweep rate of $200 \mathrm{kHz}$ and average output power of $18 \mathrm{~mW}$. The imaging resolution is $6 \mu \mathrm{m}$ (lateral) and $5.2 \mu \mathrm{m}$ (axial) respectively. The sample arm was assembled with a collimator, a pair of close-spaced galvanometer scanner and an achromatic doublet lens. An eagle eye optics design with matched optics as the sample arm was 
implemented for the reference arm. The schematic diagram of the developed high speed SS-OCT instrument is shown in Fig.1.

\section{Experimental animal and method}

In the preliminary feasibility study, a mouse ear skin model was used (ICR, age 12 13 weeks). The experimental procedure was approved by the Institutional Animal Care and Use Committee (IACUC) at Chang Gung University. The mouse was anesthetized with the isoflurane during the imaging session. The hair on the ear skin was removed with the hair removal cream before the imaging session. An OCTA imaging protocol where 5 repetitive OCT frames were acquired at the same location was implemented allowing subsequent variable interscan time analysis of the OCT images. A 3D OCT volume composed of $1000 \times 5 \times 500$ Ascans was acquired over a surface imaging area of $5.1 \times 5.1 \mathrm{~mm}^{2}$..

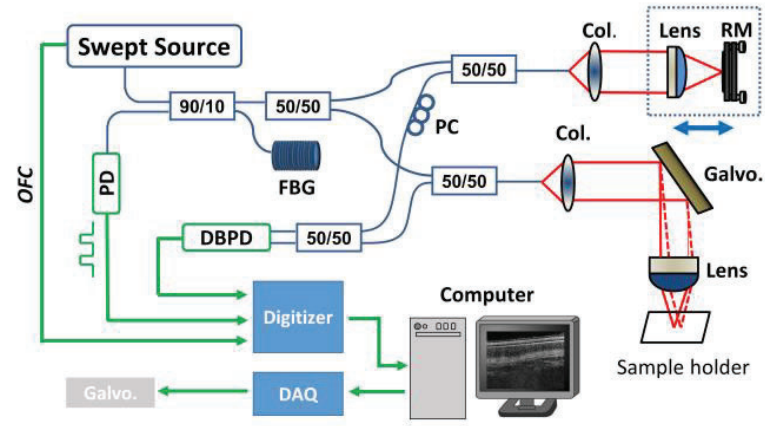

Figure 1. (a) SS-OCT setup. FC: fiber coupler, Lens: achromatic doublet, FBG: fiber bragg grating, Col: collimator, RM: reference mirror, PC: polarization controller, DBPB: dualbalanced photo diode, PD: photodetector.

\section{Results}

Figure 2 shows the example coregistered, crosssectional OCT and OCTA image of the mouse ear skin where layer tissue architecture can be observed. In Fig. 2(b), the OCTA image was generated with an effective interscan time of 10 milliseconds (ms). Figure 3 shows the depth projected OCTA images of the mouse ear skin with an effective interscan time of (a) $10 \mathrm{~ms}$ and (b) 20 ms. Here, the OCTA images with different effective interscan time contain information relative to the blood flow speed. With a longer interscan time, microvasculature with a lower blood flow speed can be identified as highlighted by the red stars annotated in Fig. 3(b). The preliminary results demonstrated in this study showed the promise to extend the OCTA imaging with blood flow information with the VISTA techniques.

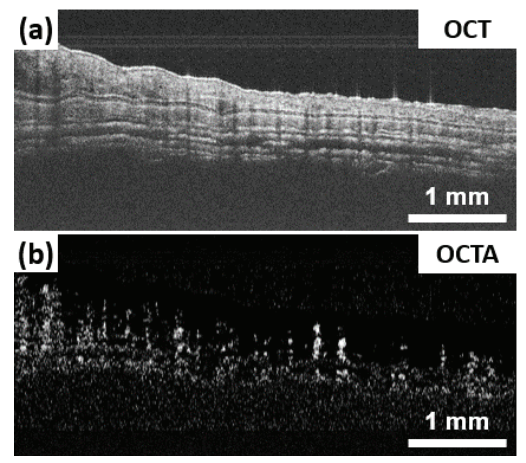

Figure 2. Example coregistered, cross-sectional (a) OCT and (b) OCTA images of the mouse ear skin.

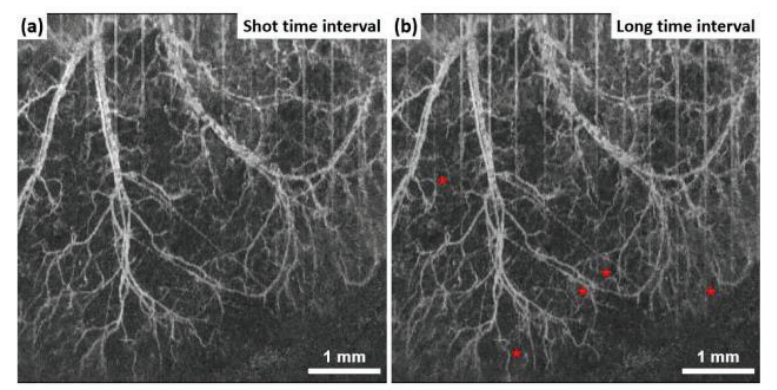

Figure 3. Depth projected OCTA image of the mouse ear skin with (a) shorter and (b) long effective interscan time. Red stars:

\section{Acknowledgment}

The authors acknowledge the support from the Young Scholar Fellowship Program by the Ministry of Science and Technology (MOST) of the Republic of China (ROC), Taiwan (MOST-107-2636-E-002-003)

\section{References}

D. Huang et al., "Optical coherence tomography," Science, vol. 254, no. 5035, pp. 1178-1181, 1991.

[2] W. J. Choi, R. Reif, S. Yousefi, and R. K. Wang, "Improved microcirculation imaging of human skin in vivo using optical microangiography with a correlation mapping mask," Journal of biomedical optics, vol. 19, no. 3, p. 036010, 2014.

[3] R. Poddar, D. Y. Kim, J. S. Werner, and R. J. Zawadzki, "In vivo imaging of human 
vasculature in the chorioretinal complex using phase-variance contrast method with phasestabilized 1- $\mu \mathrm{m}$ swept-source optical coherence tomography," Journal of biomedical optics, vol. 19, no. 12, p. 126010, 2014.

[4] A. H. Kashani et al., "Optical coherence tomography angiography: A comprehensive review of current methods and clinical applications," Prog Retin Eye Res, vol. 60, pp. 66-100, Sep 2017.

[5] M.-T. Tsai, J.-W. Zhang, Y.-H. Liu, C.-K. Yeh, K.-C. Wei, and H.-L. Liu, "Acoustic-actuated optical coherence angiography," Optics letters, vol. 41, no. 24, pp. 5813-5816, 2016.

Proc. of SPIE Vol. 11140 1114001-185 


\title{
Non-invasive measurement of blood glucose by attenuated total reflection spectroscopy with fixed-wavelength quantum cascade lasers
}

\author{
Takuya Koyama*, Saiko Kino, Yuji Matsuura \\ Graduate School of Biomedical Engineering, Tohoku University, 6-6-05 Aoba Sendai, Miyagi, \\ 980-8579, Japan \\ Email: takuya.koyama.r2@dc.tohoku.ac.jp
}

\begin{abstract}
For non-invasive blood glucose measurement, a measurement system based on mid-infrared ATR spectroscopy equipped with a combination of quantum cascade lasers (QCLs) as light sources and hollow-optical fibers as a beam delivery media is developed. Firstly the optical absorption of human lips in the mid-infrared wavelength region is measured using FT-IR. The spectrum shows the wavenumber of $1152 \mathrm{~cm}^{-1}$ exhibits strong absorption of glucose. Then we also confirmed that there is no absorption at the wavenumber of $1186 \mathrm{~cm}^{-1}$ from the spectrum of glucose solution. Finally we measured absorption of human lips using the QCL with these wavelengths. As a result, the measured differential absorption follows the change of blood glucose well and the correlation factor between the absorbance and the blood glucose level is 0.55 .
\end{abstract}

Keywords: ATR spectroscopy, blood glucose, quantum cascade laser, hollow optical fiber

\section{Introduction}

A various non-invasive technique to measure blood glucose have been proposed because conventional blood sampling is painful and has a risk of infection [1]. We proposed a measuring system based on infrared attenuated-total-reflection (ATR) spectroscopy [2]. However, it is a large-scale system and expensive in the cost and therefore, it is difficult to use the system as a simple health-care product.

Recently development of quantum cascade lasers (QCLs) that enable downsizing of the mid-infrared spectroscopy systems has been rapidly advanced. Although some groups have proposed to use QCLs for measurement of blood glucose level [3-5], those groups use wavelength-tunable QCLs that are high in the cost because a movable grating is equipped. The grating device also makes the system large and the system needs careful handling because the mechanical stability is limited low. In this paper, we build a system using single-wavelength QCLs to develop a small and low-cost system and report the results of in-vivo measurement of blood glucose levels based on mid-infrared ATR spectroscopy.

\section{Experimental setup}

Figure 1 schematically shows the experimental setup used in this study for infrared ATR measurement. We used a couple of distributed feedback (DFB)-QCLs (Hamamatsu Photonics, L12009) emitting CW power of $20 \mathrm{~mW}$. The laser beam is collimated with an aspheric lens with a focal length of $6 \mathrm{~mm}$ and it is delivered to an ATR prism by a $26 \mathrm{~cm}$-long hollow-optical fiber with an inner diameter of $2 \mathrm{~mm}$. The hollow optical fiber is composed of silver and dielectric thin films on the inside of a flexible plastic tubing and the thickness of the dielectric layer is designed to make the transmission loss at wavelength of 9-10 $\mu \mathrm{m}$.

The trapezoid ATR prism is made of non-toxic ZnS crystal with a thickness of $1.6 \mathrm{~mm}$ and a length of $24 \mathrm{~mm}$ and the incident light reflects 15 times at the top and bottom surfaces. The output beam from the prism is lead to an InAsSb semiconductor detector. In contrast to FT-IR based systems equipped with a liquid-N2 cooled $\mathrm{HgCdTe}$ detector, the system with high powered QCLs needs only a small semiconductor detector working at room temperature and this makes the system compact and low cost.

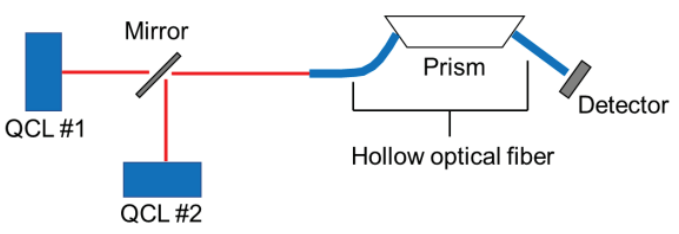

Figure 1. Experimental System 
Each of the QCLs in Fig. 1 emits only a single wavelength and therefore, it is very important to choose a combination of wavelengths that strongly reflect absorption of glucose. We firstly investigated the absorption spectrum of human lip mucosa using an FT-IR-based ATR system. Figure 2 shows an absorption spectrum of lip mucosa in an absorption band in midinfrared region 1000-1200 $\mathrm{cm}^{-1}$. There are some absorption peaks that coincide with these of glucose and the strongest one is seen at around $1080 \mathrm{~cm}^{-1}$. However, this peak comes from a $\mathrm{C}-\mathrm{O}$ bond in glucose molecules and it is strongly affected by the other components such as phosphoric acids, nucleic acids, and proteins that also involve $\mathrm{C}-\mathrm{O}$ bonds $[6,7]$. On the other hand, we found that the intensity of the absorption peak at $1155 \mathrm{~cm}^{-1}$ that came from the pyranose ring structure of glucose showed a strong correlation with the blood glucose level [2]. Therefore, one of the QCL wavelengths was set to a wavelength near 1155 $\mathrm{cm}^{-1}$.

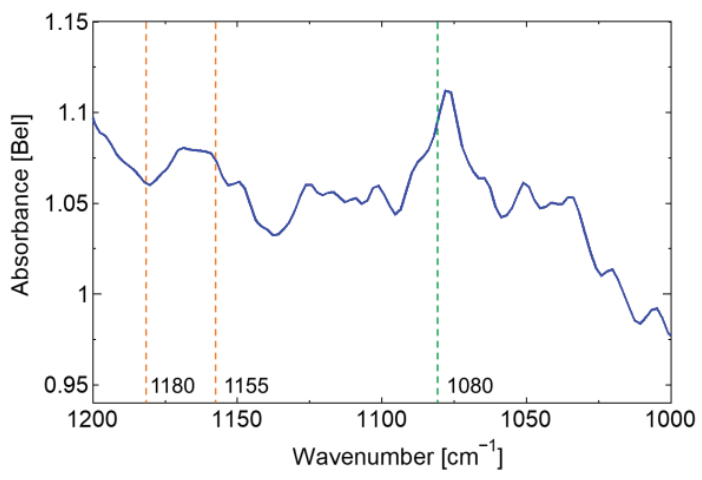

Figure 2. Absorption spectrum of human lip measured by FT-IR

According to previous study, it is known that the baseline greatly fluctuates because of changes in the surface condition of lip mucosa. To remove this influence, an absorption at another wavelength where glucose show no absorption should be measured as a background. Figure 3 shows an absorption spectrum of $10 \%$-glucose solution measured by an FT-IR-based ATR system. One can see that between two absorption bands of glucose, there is a point at $1180 \mathrm{~cm}^{-1}$ where the absorption becomes minimum.

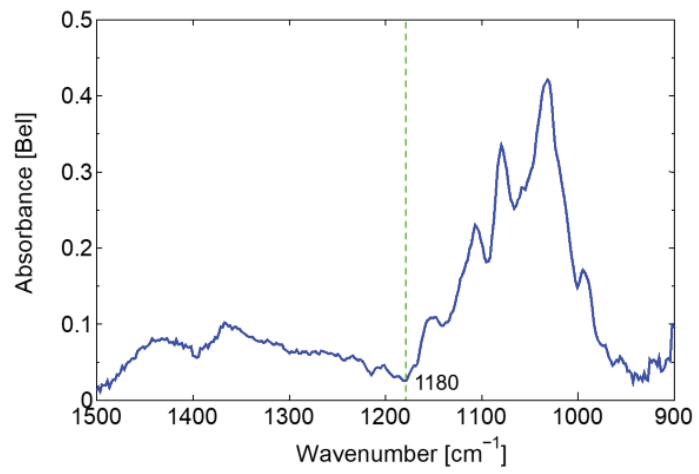

Figure 3. Absorption spectrum of 10\%-glucose solution measured by FT-IR

From the above investigation, we chose two wavelengths, 1152 $\mathrm{cm}^{-1}$ for absorption and $1186 \mathrm{~cm}^{-1}$ for background to estimate glucose absorption. We tested applicability of this method by numerical simulations based on absorption spectra of lip mucosa measured by using an FT-IR-based ATR system. In the simulation, differential absorption between $1152 \mathrm{~cm}^{-1}$ and 1186 $\mathrm{cm}^{-1}$ were calculated and the correlation to blood glucose levels measured by blood sampling was evaluated. Figure 4 shows 128 data points which were measured for one subject for 14 days, and the correlation coefficient between the absorption and the blood glucose level is 0.51 . From this result, we found the feasibility of the blood glucose measurement system using two single wavelengths.

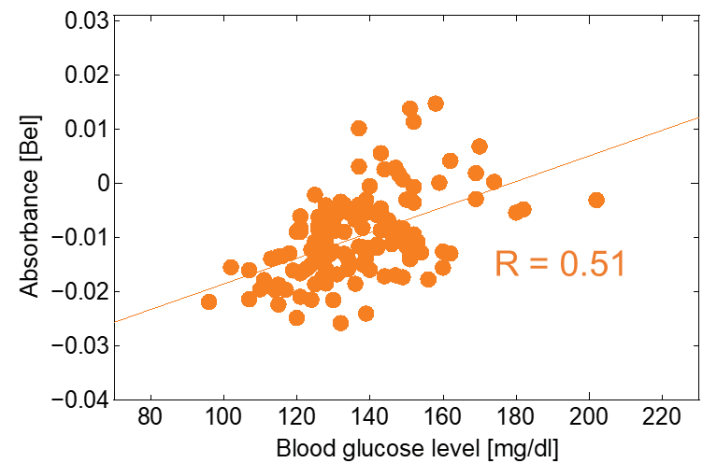

Figure 4. Correlation between the differential absorption and the blood glucose level

\section{In-vivo measurement of blood glucose}

We performed in-vivo measurement of the system shown in Fig. 1. In the measurement, two QCLs with the wavelengths of 
$1152 \mathrm{~cm}^{-1}$ and $1186 \mathrm{~cm}^{-1}$ were switched by using a flipper mirror. For each wavelength, the detected power was averaged for 5 second. Along with the optical measurement, the blood glucose levels were measured by blood sampling using a selfmonitoring-of-blood-glucose (SMBG) system (J\&J, One Touch Verio Vue).

Figure 5 shows changes of differential absorptions of a healthy adult for 120 minutes after taking a meal. One can see that both of the optical absorption and the blood glucose level decrease from the high values right after taking meal and the change in the optical absorption follows that of the blood sampling well. From calculation of correlation between those two results, we obtained the correlation coefficient $\mathrm{R}$ of 0.55 and we confirmed the feasibility of the system using a couple of single-wavelength QCLs.

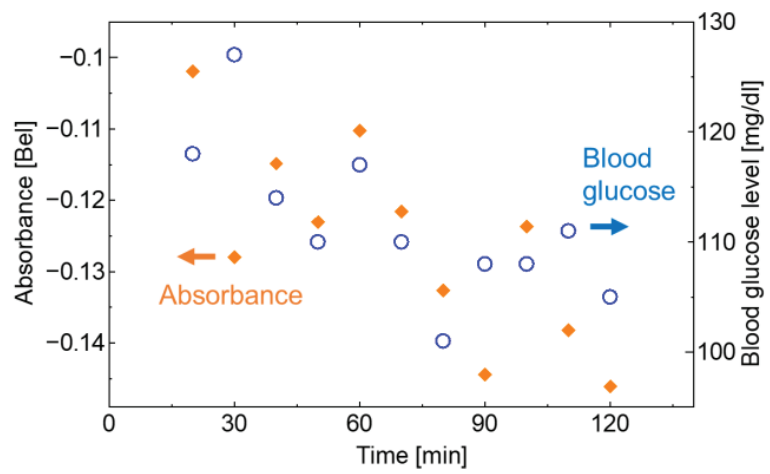

Figure 5. changes in the differential absorption and the blood glucose level.

\section{Conclusion}

For non-invasive measurement of blood glucose, we proposed an ATR measurement system using fixed-wavelength QCLs emitting different wavelengths. To choose a wavelength combination, we firstly investigated absorption spectra of human lip mucosa measured by using an FT-IR-based ATR system. As a result, we chose two wavelengths, $1152 \mathrm{~cm}^{-1}$ that coincides with an absorption peak of glucose, and $1186 \mathrm{~cm}^{-1}$ where the glucose absorption becomes minimum. From numerical simulation using spectral absorptions measured by the FT-IR system, we found that the differential absorption between the above wavelengths showed a good correlation to blood glucose levels. Then we performed in-vivo measurement using the ATR system equipped with two single-wavelength QCLs and found that the measured optical absorption follows the blood glucose well. For further improvement, we are working on suppression of measurement errors by optimizing the shapes of the laser beam and prisms.

\section{References}

[1] Vashist, S. K., "Non-invasive glucose monitoring technology in diabetes management: A review," Anal. Chim. Acta 750, 16-27 (2012).

[2] Kino, S., Omori, S., Katagiri, T., and Matsuura, Y., "Hollow optical-fiber based infrared spectroscopy for measurement of blood glucose level by using multi-reflection prism," Biomed. Opt. Express 7(2), 701-708 (2016).

[3] Liakat, S., Bors, K. A., Xu, L., Woods, C. M., Doyle, J., and Gmachl, C. F., "Noninvasive in vivo glucose sensing on human subjects using mid-infrared light," Biomed. Opt Express 5(7), 2397-2404 (2014).

[4] Brandstetter, M., Volgger, L., Genner, A., Jungbauer, C., and Lendl, B., "Direct determination of glucose, lactate and triglycerides in blood serum by a tunable cascade laser-based mid-IR sensor," Appl. Phys. B-Lasers O. 110, 233-239 (2013).

[5] Pleitez, M. A., Lieblein, T., Bauer, A., Hertzberg, O., Von Lilienfeld-Toal, H., and Mäntele, W., "In Vivo Noninvasive Monitoring of Glucose Concentration in Human Epidermis by Mid-Infrared Pulsed Photoacoustic Spectroscopy," Anal. Chem. 85, 1013-1020 (2013).

[6] Wood, B. R., "The importance of hydration and DNA conformation in interpreting infrared spectra of cells and tissues," Chem. Soc. Rev. 45, 1980-1998 (2016).

[7] Garidel, P., "Mid-FTIR-Microspectroscopy of stratum corneum single cells and stratum corneum tissue," Phys. Chem. Chem. Phys. 4, 5671-5677 (2002). 


\title{
Phase-shifting digital holography with burst-imaging method*
}

\author{
Takumi Ujiie*, Yoshio Hayasaki \\ Center for Optical Research and Education (CORE), Utsunomiya University, 7-1-2 Utsunomiya-shi, \\ Tochigi, 321-0904, Japan
}

\begin{abstract}
Phase-shifting digital holography has a feature that a hologram can be acquired with a spatial resolution of an image sensor. The interference images should be acquired at multiple times while the phase-shifting is given to the reference light. Therefore, some idea for imaging a moving object have been discussed. The phase-shifting digital holography with burst-imaging method is proposed for imaging a moving object. The phase-shifting digital holography at 885 fps is demonstrated by using a high-speed camera and a high-speed phase modulation based on a piezoelectric element.
\end{abstract}

Keywords: digital holography, phase-shifting method, burst-imaging method, high-speed camera, imaging

\section{Introduction}

Digital holography (DH) is a technique of requiring a hologram in a computer from interference fringes formed by object light and reference light to obtain a reconstructed image. The phase-shifting (PS) method is used to obtain the holography. The PS method has a feature that the hologram can be acquired with the same resolution of the image sensor without any unnecessary diffracted lights. It requires to acquire multiple images while giving the PSs to the reference light. Therefore, the parallel PS method ${ }^{1}$ and multiple camera method ${ }^{2}$ were demonstrated for imaging a moving object. However, the former required a special device for the parallel PS. And the later required a calibration (a lateral and axial alignment) between cameras.

In this research, we propose to apply a burst-imaging method to the PS in the DH. It was performed by the synchronization between a camera with the frame rate of $8 \mathrm{k}$ fps and a piezoelectric transducer. The performance was evaluated by a measurement of a moving object.

\section{Experimental setup}

The experimental setup is shown in Fig.1. This is a Michelson interferometer in which the light source is Ne-He laser (Melles Griot, 05-LHR-011). A piezoelectric transducer (PZT) (PI, P753.31C) was used for the phase modulation of the reference light. The function generator (NF CORPORATION, WF1974) simultaneously set an arbitrary voltage to the PZT and a trigger signal to the high-speed camera (IDT, MotionPro Y4) when a trigger seed signal was given by the personal computer (PC) (UNITCOM, Lesance DT). The interference fringes were required by the high-speed camera with the maximum frame rate of 8800 fps when requiring $512 \times 512$ pixels. Then the phase modulation was given by continuous driving of the PZT. The driving speed was determined in advance to have an adequate relation with the frame rate of the high-speed camera. Four interference fringes were captured for the PS and the hologram was calculated from the images. Finally the reconstruction was obtained from the hologram using the angular spectrum method.

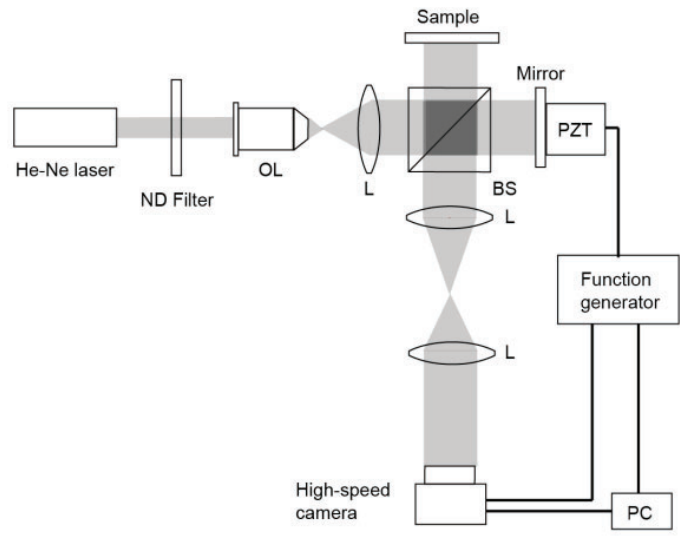

Figure 1. Experimental system. 


\section{Experimental results}

\section{Calibration of PZT}

Figure 2 shows the temporal trace of the phase modulation of the reference light after a step voltage of $3 \mathrm{~V}$ is applied to the PZT. The step voltage started at $6.25 \mathrm{~ms}$ after the trigger signal was applied to the high-speed camera. The high-speed camera captured 100 interference fringes at $8800 \mathrm{fps}$. It was confirmed that a slew rate of when a step voltage was applied to the PZT depended on the applied voltage and the weight of optical components put on the PZT in addition with the original performance of the PZT and the slope of this slew rate can be regarded as almost linear. The slope of the approximate straight line between $6.93-7.03 \mathrm{~ms}$ was $1770 \pi \mathrm{rad} / \mathrm{s}$. Then, the 4-step PSDH was performed under the camera frame rate of $3540 \mathrm{fps}$ and the trigger signal delay of $6.93 \mathrm{~ms}$.

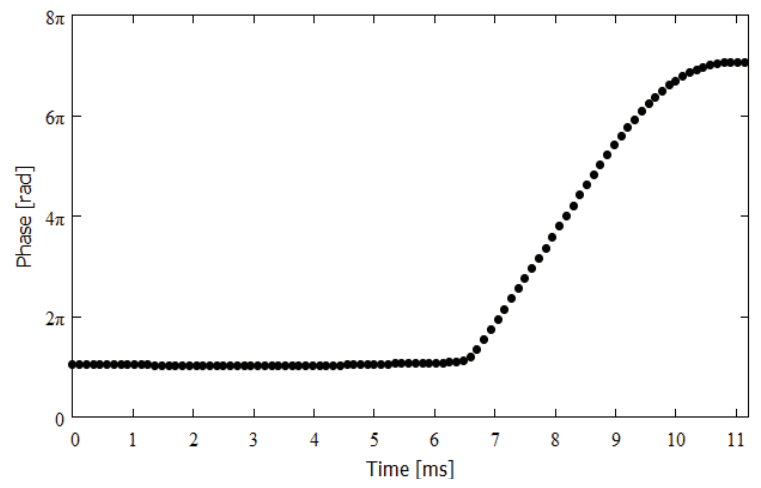

Figure 2. Calibration of PZT

\section{Hologram capturing and reconstruction}

Figure 3 shows the amplitude and phase distributions of the hologram image.
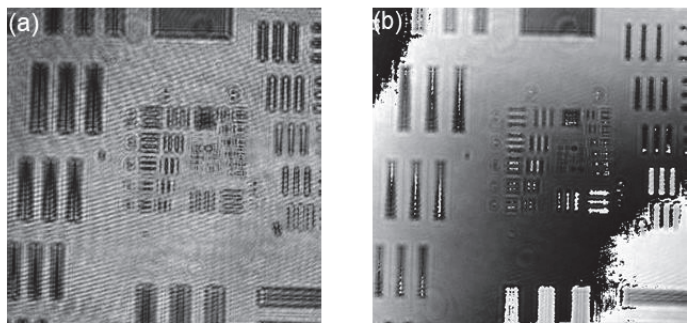

Figure 3. (a) Amplitude and (b) phase distribution of the hologram image.
Figure 4 shows the amplitude and phase distributions of the reconstruction image.
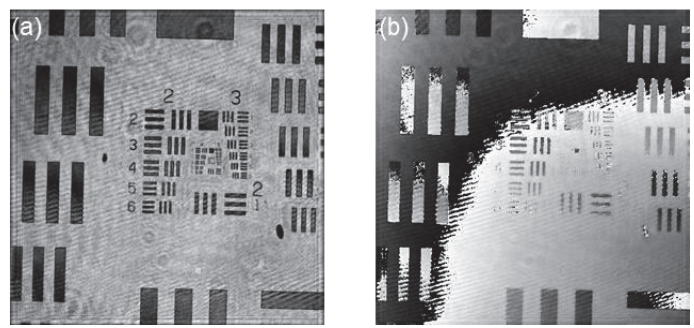

Figure 4. (a) Amplitude and (b) phase distribution of the reconstruction image.

\section{IIII Conclusion}

We performed the phase-shifting method in the digital holography with the burst-imaging method. The 4-step phaseshifting digital holography was performed on a test target at 885 fps. There is a possibility that the proposed method can image a moving object.

\section{References}

[1] Y. Awatsuji, M. Sasada and T. Kubota, "Parallel quasi-phaseshifting digital holography," Appl. Phys. Lett. 85, 1069 (2004).

[2] J. Zhang, Y. Xie, G. Li, Y. Ye, and B. E. Saleh, "Single-shot phase-shifting digital holography," Opt. Eng. 53, 1123316 (2014). 


\title{
Total protein measurement features in venous and capillary blood using Raman spectroscopy method
}

\author{
A.A. Lykina* ${ }^{a *}$ D.N. Artemyevª I.A. Bratchenko ${ }^{a}$, T.P. Kuzmina ${ }^{\text {b }, ~ I . L . ~ D a v y d k i n ~}{ }^{b}$, V.P. Zakharov ${ }^{a}$ \\ a Samara University, 34 Moskovskoye shosse, Samara, Russian Federation 443086; ' Samara State Medical University, 89 \\ St. Chapayevskaya, Samara, Russian Federation, 443099
}

\begin{abstract}
This work is devoted to the study of human blood protein fractions by Raman spectroscopy. Whole venous and capillary blood samples were used as the tested samples. For the pure Raman spectra analysis, the autofluorescence background was subtracted using baseline correction with asymmetric least squares. The spectral features were used for total protein concentration measurement of venous and capillary blood. PLS regression method was utilized for spectral data analysis with different protein concentrations. The study allowed for revealing differences between spectral features of venous and capillary blood, which are changes in the relative Raman intensities of venous and capillary blood and Raman bands appearance on $1377 \mathrm{~cm}^{-1}, 1350 \mathrm{~cm}^{-1}, 1587 \mathrm{~cm}^{-1}, 1550 \mathrm{~cm}^{-1}$ and $1650 \mathrm{~cm}^{-1}$ corresponding to oxyhemoglobin and carboxyhemoglobin bonds.
\end{abstract}

Keywords: Raman spectroscopy, whole blood, total protein, capillary blood, Partial Least Squares

\section{Introduction}

Proteins are high molecular weight polypeptides consisting of amino acids and are part of all human body fluids. Changes in the blood protein amount, as well as the certain fractions quantity, allows for drawing a conclusion about the human body state and pathology presence, also it helps to define the treatment efficiency ${ }^{1}$.

Various spectral methods allow for obtaining an individual spectral "fingerprint" of the tested sample chemical compounds and these techniques are used for qualitative evaluation of blood protein. Raman spectroscopy (RS) is one of the most sensitive optical method $^{2-4}$, therefore this approach has been used for various blood proteins analysis. The aim of this study was to compare spectral characteristics of whole venous and capillary blood (a mixture of plasma and formed elements, such as erythrocytes). Regression model of the Projection on Latent Structures (PLS) method was constructed for the determination of total protein concentration by the analysis of whole venous and capillary blood Raman spectra.

\footnotetext{
*aalykina163@gmail.com; phone+7(927) 686 1773
}

\section{Materials and methods}

1. Experimental setup and test samples

Our experimental setup for Raman spectra registration includes thermally stabilized semiconductor laser $785 \mathrm{~nm}$ (200 mW), an optical Raman probe (PBL 785), a spectrograph (Shamrock SR-500i-D1-R) with an integrated digital camera (ANDOR DU416A-LDC-DD), and computer. The diameter of the laser probing beam was $1 \mathrm{~mm}$. RS analysis was performed with an acquisition time of 60 secs.

12 samples of human venous and capillary blood were tested in this study. All samples were obtained from a biochemical laboratory. The tested samples were stored in a laboratory refrigerator at $+4{ }^{\circ} \mathrm{C}$. During experiments, the blood was placed in an aluminum cylindrical cuvette (volume $0.15 \mathrm{ml}$ and $0.55 \mathrm{ml}$ ). A cuvette with a volume of $0.15 \mathrm{ml}$ was used for capillary blood samples due to the small volume of the sample; A $0.55 \mathrm{ml}$ cuvette was used for venous blood samples.

\section{Data processing methods}

Pure Raman spectrum was obtained by autofluorescence removal from the raw data signal using baseline correction with asymmetric least squares smoothing method. Initially, Raman spectral data were preprocessed 
using normalization to Standard Normal Variate, smoothing by Savitzky-Golay filter and mean centering of the data. For the data processing, Partial Least Squares (PLS) regression methods ${ }^{5}$ were utilized. The root mean squared error $(\mathrm{RMSE})^{6}$ and the squared correlation coefficient $\left(\mathrm{R}^{2}\right)^{6}$ were calculated for blood compounds concentrations study. Data processing was carried out using $\mathrm{TP}^{\mathrm{T}}$ cloud ${ }^{\text {beta }}$ (http://tptcloud.com) cloud software module for multivariate analysis.

\section{Results and Discussion}

In this study the Raman spectra registration of human blood was carried out. The RS consists of the basic bands of blood protein fractions: $570 \mathrm{~cm}^{-1}$ (deformation vibrations of $\mathrm{FeO}_{2}$ - group), $670 \mathrm{~cm}^{-1}$ and $750 \mathrm{~cm}^{-1}$ (vibrations of pyrrole), $850 \mathrm{~cm}^{-1}$ (tyrosine vibrations), $950 \mathrm{~cm}^{-1}$ (deformation vibrations of $\mathrm{CH}$ ), $1002 \mathrm{~cm}^{-1}$ (phenylalanine vibration), $1120 \mathrm{~cm}^{-1}$ (deformation vibrations of $\mathrm{C}-\mathrm{N}$ group), $1130 \mathrm{~cm}^{-1}$ (deformation vibrations of $\mathrm{CH}$ ), $1210 \mathrm{~cm}^{-1}$ (Amide III, $\beta$-helix), $1227 \mathrm{~cm}^{-1}$ (deformation vibrations of $\mathrm{CH}$ group), $1260 \mathrm{~cm}^{-1}$ (Amide III, $\alpha$-helix), $1350 \mathrm{~cm}^{-1}$ (vibrations of tryptophan vibrations), $1377 \mathrm{~cm}^{-1}$ (vibrations of pyrrole), $1450 \mathrm{~cm}^{-1}$ (deformation vibrations of $\mathrm{CH}$ ), $1550 \mathrm{~cm}^{-1}$ (vibrations of phenylalanine), $1587 \mathrm{~cm}^{-1}$ (deformation vibrations of CC), $1650 \mathrm{~cm}^{-1}$ (Amide I, $\beta$-helix) ${ }^{3,4}$. Registered signals of blood samples revealed a coincidence of the positions of Raman peaks of biomolecules over the entire spectral range, presented in Fig. 1.

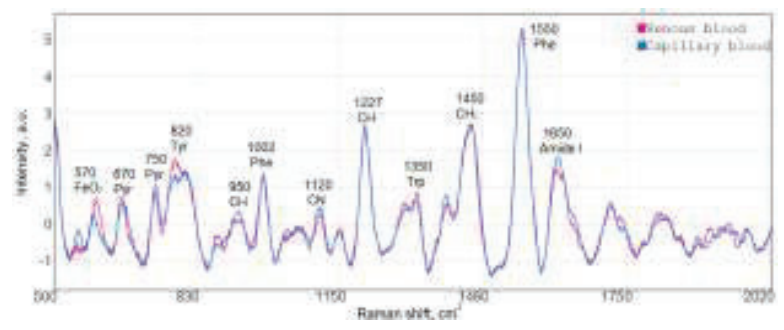

Fig. 1 Registered spectral signals of blood venous and capillary blood, processed by AF background removal (Pyr- pyrrole, Phephenylalanine, Trp- tryptophan, Tyr-tyrosin)

A model was constructed to determine the total protein from the spectral characteristics of venous and capillary blood samples, using regression analysis. Prediction accuracy of total blood protein was obtained from venous blood samples: $\quad\left\{\mathrm{LV}=4 \quad \mathrm{RMSE}_{\mathrm{c}}=4.5 \quad \mathrm{~g} / \mathrm{L}\right.$, $\left.\mathrm{RMSE}_{\mathrm{cv}}=13 \mathrm{~g} / \mathrm{L}\right\}$, and from capillary blood samples $\left\{L V=3 \quad \mathrm{RMSE}_{\mathrm{c}}=3.5 \mathrm{~g} / \mathrm{L}, \quad \mathrm{RMSE}_{\mathrm{cv}}=12 \mathrm{~g} / \mathrm{L}\right\}, \quad$ using regression analysis.

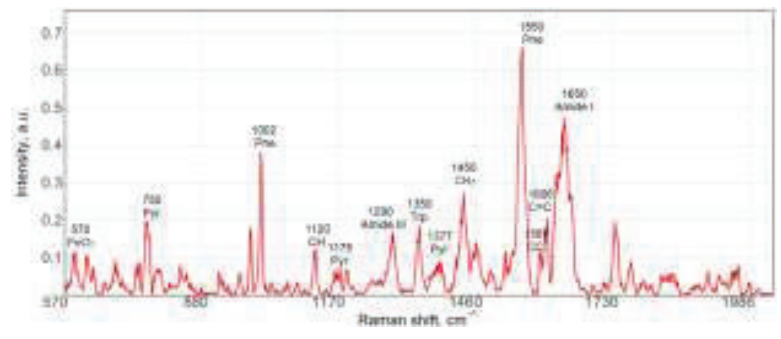

Fig. 2 VIP-variables PLS model for separation of capillary blood samples from venous blood samples

(Pyr- pyrrole, Phe- phenylalanine, Trp- tryptophan, Tyr-tyrosin) (LV 2 Spec. 97 \% Sens. $97 \%$ )

A model for separating capillary blood samples from venous blood was constructed by a PLS analysis using VIP scores (Fig.2). The VIP variables of the PLS model identified Raman peaks at $1377 \mathrm{~cm}^{-1}$ and $1587 \mathrm{~cm}^{-1}$, which belong to pyrrole oscillations and deformation vibrations of the $\mathrm{CC}$ group corresponding to oxyhemoglobin molecules ${ }^{7}$. Raman peak on $1606 \mathrm{~cm}^{-1}$ belongs to the vibrations of the CC group, which is part of the carboxyhemoglobin ${ }^{7}$. The constructed regression model makes it possible to distinguish capillary blood among venous blood samples according to spectral characteristics with a posteriori probability of $97.4 \%$, where the sensitivity is $97.5 \%$, and the specificity is $97 \%$.

\section{Conclusion}

Basing on the study, the Raman spectroscopy method is advantageous in the determination of the total protein concentration using small volumes $(0.15-0.55 \mathrm{ml})$ of blood samples. It was established that the prediction accuracy of protein concentration for samples of venous and capillary blood varied by 1-2 g/L. Moreover, according to results for calibration samples of venous and capillary samples, the method has a high potential and effectiveness in the analysis of various concentrations of blood protein solutions in clinical studies. 


\section{Acknowledgements}

The Ministry of Education and Science of the Russian Federation supported this research.

\section{References}

[1] Hanlon, E.B., Koo, R.T.W., Motz, J.T., Fitzmaurice, M., Kramer, J.R., Itzkan., I., Dasar, R.R., Feld, M.S., "Prospects for in vivo Raman spectroscopy," Phys. Med. Biol. Papers 45(2), R1-R59 (2000)

[2] Artemyev, D.N., Bratchenko, I.A., Khristoforova, J.A., Lykina, A.A., Myakinin, O.O., Kuzmina T.P., Zakharov, V.P., "Blood proteins analysis by Raman spectroscopy method," Proc. SPIE 98871Y, 1-8 (2016).

[3] Annika, M.K., Tae-Woong, J.Oh. and Hunter, M., "Blood analysis by Raman spectroscopy," Optics letters. Papers 27(22), 2004-2006 (2002).

[4] Gelder, J. D., Gussem, K.De., Vandenabeele, P., Moens L., "Reference database of Raman spectra of biological molecules," J. Raman Spectroscopy Papers 38(9), 1133-1147 (2007)

[5] Esbensen, K., [Infrared Detectors], Multivariate Data Analysis, In Practice 4-th Ed., (2000).

[6] Wold, S., "PLS-regression: a basic tool of chemometrics." Chemometrics and Intelligent Laboratory Systems Papers 58(2), 109-30 (2001).

[7] Gao, X., Zeng, Ch., Liu, H., Lu Y., "Micro-Raman spectroscopy study on the allosteric regulation of inositol hexakisphosphate on hemoglobin" Anal. Methods Papers 7, 2977-2984 (2015). 


\title{
Moisture measurement of oral mucosa based on near-infrared diffuse reflection spectroscopy by using optical fiber probe
}

\author{
Shintaro Sugawara* and Yuji Matsuura \\ Graduate School of Biomedical Engineering, Tohoku University \\ 6-6-05 Aoba, Sendai 980-8579, Japan
}

\begin{abstract}
An optical measurement method of oral mucosa moisture based on near-infrared absorption spectroscopy is proposed. A measurement setup composed of a white light source, an optical fiber probe, and a fiberoptic spectrometer is fabricated and firstly, biological samples simulating oral mucosa are measured to obtain calibration plot. Then moisture of lip mucosa surface is successfully measured by using the proposed system.
\end{abstract}

Keywords: near-infrared spectroscopy, absorption spectroscopy, oral mucosa

\section{Introduction}

Moisture measurement of oral mucosa is useful for healthcare monitoring because excessive dried mucosa brings decrease of immune functions that causes invasion of viruses and bacteria. A common method that has been used for measurement of oral mucosa is based on electrical impedance of mucosa. Some systems using this method have already in the market and mainly used in dental clinics for measurement of tongue surface. In this type of system, however, an electrical probe should be touch on the measured surface and it is not desirable in a view point of personal hygiene. Furthermore, the method cannot be applied to the pharynx and larynx where vomiting reflex occurs. Therefore, in this report, we proposed an optical measurement method of oral mucosa moisture without touching it. The proposed system is based on near-infrared absorption spectroscopy detecting the absorption of water.

\section{Experiment}

Figure 1 shows absorption spectra of hemoglobin and water that are main components of living tissue in visible to near infrared region [1]. One can see some absorption peaks of water in this region and we chose the wavelength of $1450 \mathrm{~nm}$ for measurement of oral mucosa moisture because of the clear and large absorption and of the small absorption of hemoglobin that makes incident light deeply penetrate into human tissue.

Figure 2 schematically shows experimental setup used for measurement of mucosa moisture. The fiber probe used for the experiment is composed of 7 optical fibers. Each fiber is a step-index multimode fiber with the core diameter of $0.2 \mathrm{~mm}$ and numerical aperture of 0.22 . The central fiber is used for radiation of light and surrounding 6 fibers are used for detection of diffusively reflected light from the sample. A halogen lamp is used as a light source and the spectra of reflected light is measured by a fiber-optic spectrometer. In our preliminary experiment, we found that only diffuse reflected light is detected with this probe by tilting the fiber to the sample 30 degree and therefore, this arrangement is adopted in the system in Fig. 2.

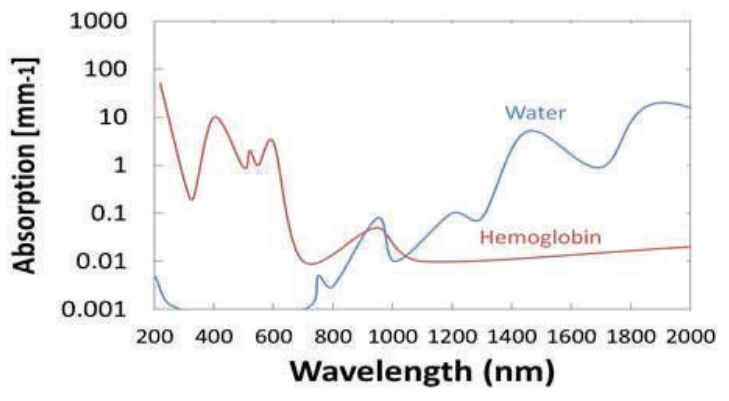

Figure 1. Absorption spectra of hemoglobin and water 


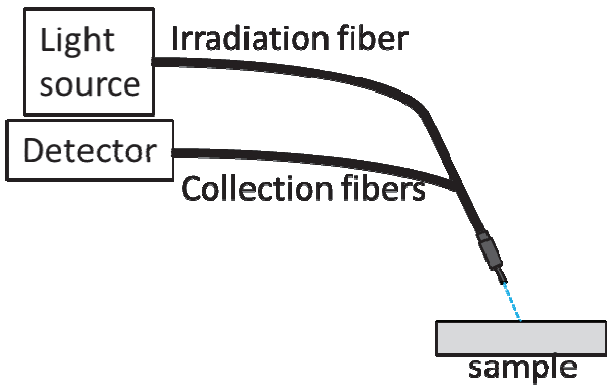

Figure 2. Measurement system

We firstly measured biological phantoms representing oral mucosa that are made of mixture of water and gelatin. We prepared water gels with a thickness of $3 \mathrm{~cm}$ with 6 different moisture contents and absorption spectra of the gels measured by the system are shown in Fig. 3. We confirmed that the absorption peak at $1450 \mathrm{~nm}$ becomes large with increasing the water content.

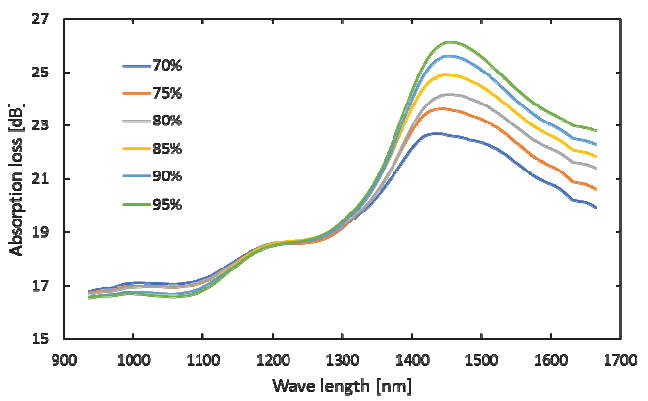

Figure 3. Absorption spectra of phantom gels

Figure 4 shows a calibration plot of the peak height to the water content. We calculated differential absorptions between $1450 \mathrm{~nm}$ and $1270 \mathrm{~nm}$ where the water absorption becomes very small. We confirmed highly linear correlation between the measured absorptions of the phantoms and water contents and this shows the feasibility of the system for measurement of surface moisture of the samples.

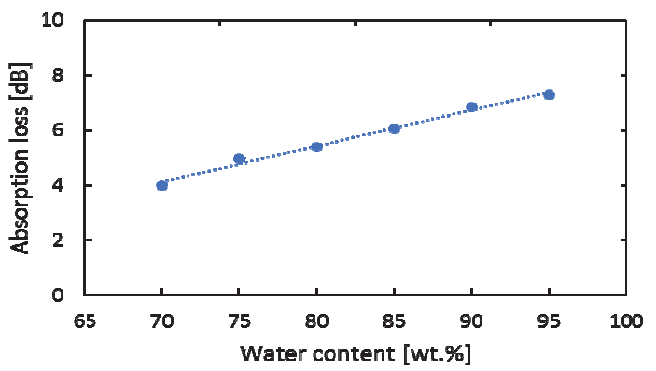

Figure 4. Calibration plot

Secondly, we performed in-vivo measurement using human lip mucosa as a sample. In this experiment, the distance between the end of fiber probe and lip surface is kept to be $2 \mathrm{~mm}$ by attaching a spacer on the end of fiber probe. Figure 5 shows absorption spectra of human lip mucosa measured consecutively for 3 times. In the measured spectra that are similar to the one measured for biological phantoms shown in Fig. 3, clear absorption peak of water at $1450 \mathrm{~nm}$ is observed. It is also seen that the peak intensity slightly decreases with measurement times because the mucosa surface is kept exposed to air during the 3 measurements that makes the mucosa surface dry. From the differential absorption between $1450 \mathrm{~nm}$ and $1270 \mathrm{~nm}$, we estimate the moisture of the lip mucosa as $95 \%$ by using the calibration plot shown in Fig. 4.

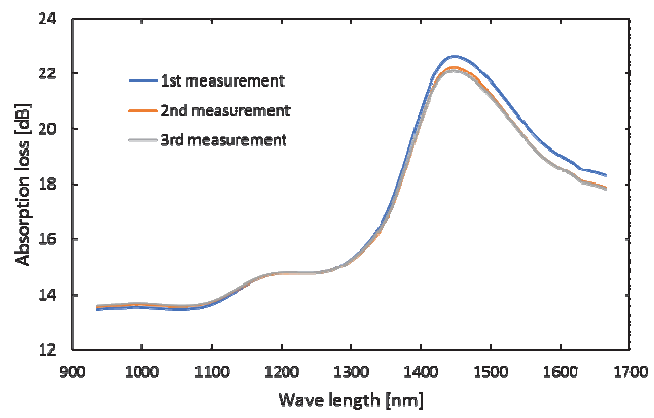

Figure 5. Absorption spectra of human lip mucosa

\section{Conclusion}

We proposed an optical measurement method of oral mucosa moisture based on near-infrared absorption spectroscopy detecting the absorption of water. We 
fabricated a measurement setup composed of a white light source, an optical fiber probe, and a fiberoptic spectrometer and firstly measured biological sample simulating oral mucosa to obtain calibration plot. Then we successfully measured moisture of lip mucosa surface by using the proposed system.

\section{References}

[1] M. S. Patterson, B. C. Wilson, and D. R. Wyman, "The propagation of optical radiation in tissue. 2 . Optical properties of tissues and resulting fluence distributions," Lasers Med. Sci., vol. 6, pp. 379-390 (1991). 


\title{
Optical microscopy using annular full-color LED for quantitative phase and spectroscopic imaging of biological tissues
}

\author{
Taro Masunaria ${ }^{a}$ Masaki Hisaka*b \\ ${ }^{a}$ Graduate School of Biomedical Engineering, Osaka Electro-Communication University, 1130-70 \\ Kiyotaki, Shijonawate, Osaka, 575-0063, Japan \\ ${ }^{b}$ Department of Biomedical Engineering, Osaka Electro-Communication University, 1130-70 \\ Kiyotaki, Shijonawate, Osaka, 575-0063, Japan
}

\begin{abstract}
We developed an optical microscope using oblique illumination and active image processing, which has the advantages of separate phase and amplitude contrast imaging, reduction of multi-scattering effects in biological tissues, and spherical aberration-free imaging. This microscope imaged tissue cells by processing oblique illumination images in a Fourier space taken from all directions azimuthally. In this research, a ring-shaped annular full-color RGB LED light source was developed to attain high-speed imaging and to obtain spectroscopic and quantitative phase structures of biological samples. Reconstructive imaging of a pine pollen structure captured with the annular full-color LED shortened the measurement time and obtained absorption and phase-contrast images for each RGB color.
\end{abstract}

Keywords: phase-contrast optical microscopy, oblique illumination, full-color LED, active processing

\section{Introduction}

Phase-contrast imaging of living tissues is one of the most important techniques to visualize low-contrast and transparent phase structures, such as induced pluripotent stem cells (iPSCs). Recently, optical microscopy using an oblique illumination and active image processing has been investigated for imaging biological phase structures ${ }^{[1,2]}$. This microscopy has several features such as separate phase and amplitude contrast imaging, spherical aberration-free imaging, reduction of multi-scattering effects. In this research, we observed the amplitude and phase images of biological specimens by fabricating an annular LED light source in which RGB full-color LEDs were arranged in a ring shape to obtain high-speed spectroscopic and quantitative phase structures of biological samples.

\section{Annular full-color RGB LED}

Figure 1 shows a ring-shaped annular full-color RGB LED light source. The RGB LED is surface-mount type, and the size is $3.5 \mathrm{~mm}$ width $\times 2.8 \mathrm{~mm}$ depth. Three elements of red light (R: $630 \mathrm{~nm})$, green light (G: $523 \mathrm{~nm}$ ), and blue light (B: $466 \mathrm{~nm}$ ) were packaged in a circular region, which were made by white scattering material, with a diameter of $2.2 \mathrm{~mm}$ in the center. The 40 full-color LEDs were placed at an interval of $9.0^{\circ}$ along the outer side of a circular board with a diameter of $10.0 \mathrm{~cm}$ [Fig. 1 (a)]. The sequential blinking of these LEDs was controlled by three 8-bit shift registers via a microcomputer.

Figure 1 (b) shows a side view of the annular LED light source. To make an effective oblique illumination on the sample, each LED was fixed at an inclination angle $\theta=$ $37^{\circ}$ to the circular circuit board, corresponding to a numerical aperture (N.A.) of 0.6 .
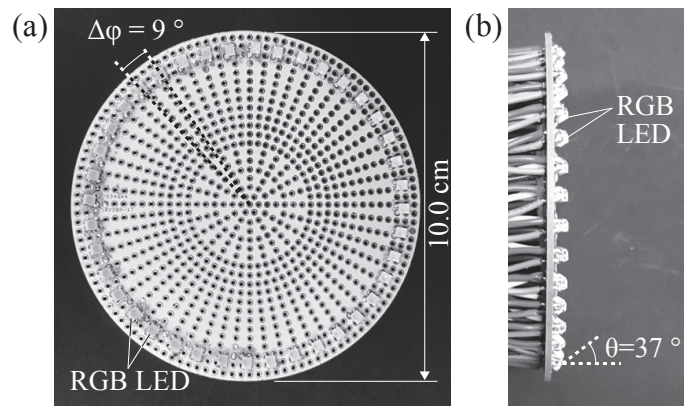

Figure 1. Prototype of the ring-shaped annular RGB full-color LED light source. (a) top view, (b) side view.

*hisaka@osakac.ac.jp; phone 8172 876-5501 


\section{Experimental setup}

An optical microscope using the annular full-color RGB LED light source and an objective lens with an annular aperture was constructed, as shown in Fig. 2. Each LED light irradiated a pine pollen biological sample at an oblique angle of $\theta=37^{\circ}$. The illumination light passed through the sample, and the transmitted light formed the image by an objective lens of N.A. $=0.6$ with $40 \times$ magnification, of which the front lens was limited to an annular aperture. The azimuthally oblique image was captured using a charge-coupled device camera at $256 \times$ 256 pixels. A series of 120 images $(3$ colors $\times 40$ azimuthal angles) was captured, while each element of 40 LEDs was flashed sequentially by angular steps of $\Delta \varphi=$ $9^{\circ}$.

The RGB LED has three elements enclosed in a plastic package, but their arrangement positions are spatially different. The irradiation intensity by the RGB LED was uniform due to the diffusion plate positioned at the center of the package. Strict configuration of the angular disposition to the circuit board is unnecessary.

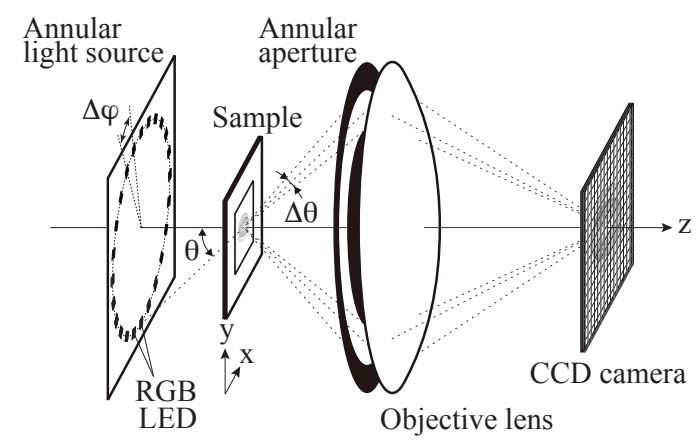

Figure 2. Optical setup for the optical microscope using a circular full-color RGB LED light source.

\section{Experimental results and investigation}

Amplitude and phase-contrast images were reconstructed by applying a figure-8-shaped spatial frequency filter to 40 oblique illumination images of each color and by superposing the 40 different azimuthal spectra in the Fourier plane. Figure. 3 shows the amplitude and phase-contrast images that depicted the air sac and the pollen body in the pine pollen. However, the phase image of the air sac differed, depending on the light source wavelength. In addition, the measurement time was decreased to $1 / 5.6$ times compared to the mechanical scanning of a light source ${ }^{[2]}$.

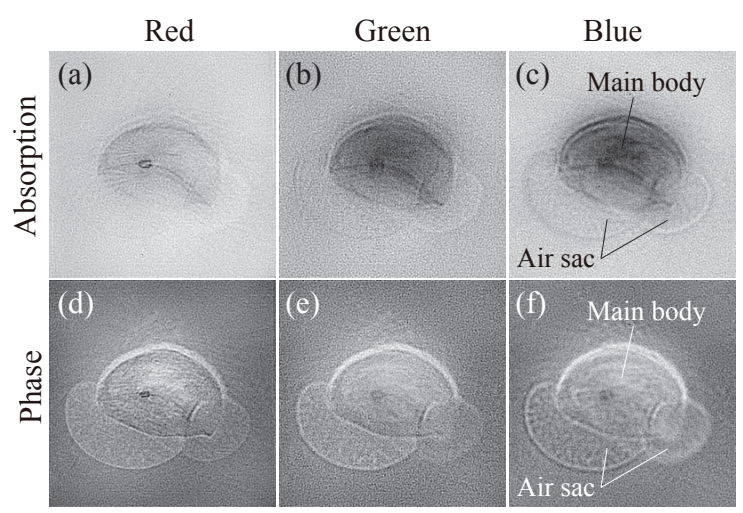

Figure 3. Reconstructed images of a pine pollen. (a)(c) Absorption images and (d)-(f) phase images for RGB color.

\section{Summary}

An optical microscope using annular RGB LED light source was constructed, and phase and absorption contrast cross-sectional images of a pine pollen sample were obtained using an active filter in the Fourier plane. We succeeded in shortening the measurement time by electrically lighting the spatially arranged LED light source. In addition, there is a possibility of acquiring spectroscopic information and quantitative phase information for biological tissues by introducing a multicolor light source.

\section{Acknowledgments}

We are very grateful to Y. Nakahigashi for his valuable cooperation in our experiments.

\section{References}

[1] Ikuta, T., "An aberration-free imaging technique based on focal depth extension," Journal of Electron Microscopy, 47(5), 427-432 (1998)

[2] Miyake, Y., Hisaka, M. and Ikuta, T., "Phase-contrast optical microscopy for biological tissues using oblique laser illumination," The 5th Asian and Pacific-Rim Symposium on Biophotonics (APBP'15), APBPp2-1 (2015). 


\title{
Non-invasive blood glucose measurement
}

\author{
by using fast swept-wavelength quantum cascade laser
}

\author{
Naoto Shibata*a, Saiko Kino ${ }^{\mathrm{a}}$, Naota Akikusa $^{\mathrm{b}}$, Atsushi Sugiyama ${ }^{\mathrm{b}}$ and Yuji Matsuura ${ }^{\mathrm{a}}$ \\ ${ }^{\mathrm{a} G r a d u a t e}$ School of Biomedical Engineering, Tohoku University, 6-6-05 Aoba, Sendai 980-8579, \\ Japan

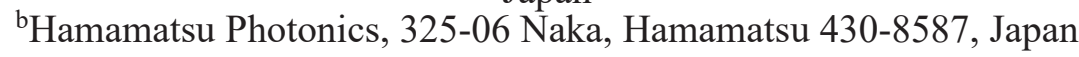

\begin{abstract}
For non-invasive measurement of blood glucose, we proposed an ATR absorption spectroscopy system using a QCL with a MEMS-based external cavity that enables a rapid sweep of emitting wavelength. By using this system, we measured absorption of human lip mucosa and as a result, we observed that the change of the peak intensity follows that of blood glucose well and we confirmed the correlation between the peak intensity and blood glucose level.
\end{abstract}

Keywords: blood glucose measurement, quantum cascade laser, hollow optical fiber

\section{Introduction}

Development of a non-invasive measurement system of blood glucose levels has been strongly desired. We proposed a mid-infrared attenuated-total-reflection (ATR) spectroscopy system composed of a Fourier-transform infrared spectrometer (FT-IR) and hollow optical fibers. By using this system, we showed that finger print spectra of glucose were found in absorption spectra of human lips and that good correlation was obtained between the absorption intensity and blood glucose levels [1].

We also proposed an ATR system using fixedwavelength quantum cascade lasers (QCL) to make the system compact and low cost [2]. On the other hand, recently a QCL with a MEMS-based external cavity that enables a rapid sweep of the emitting wavelength has been developed and by using this, precise measurement of blood glucose based on ATR spectra of glucose's absorption band is expected. Here we show the system configuration and measured results for glucose solution and human lips.

\section{Experiment}

Figure 1 schematically shows our measurement system using a swept-wavelength QCL. The pulsed QCL has a MEMS grating that rapidly sweeps the emitting wavelength from the wavenumber of $1000 \mathrm{~cm}^{-1}$ to 1200 $\mathrm{cm}^{-1}$ and the pulse peak power is around $200 \mathrm{~mW}$. The light from the QCL is introduced into a trapezoid ATR prism (24 mm in length and $2.4 \mathrm{~mm}$ in height) made of $\mathrm{ZnS}$ by using a hollow optical fiber with an inner diameter of $1 \mathrm{~mm}$. The light from ATR prism was led to a $\mathrm{HgCdTe}$ detector by another hollow optical fiber.

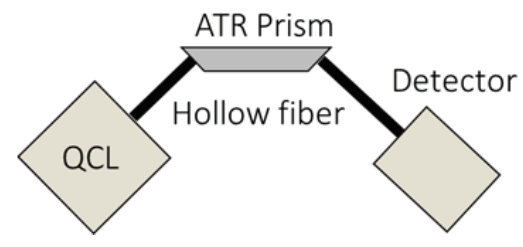

Figure 1 Measurement system

Prior to blood glucose measurement, we tested the accuracy of the system by measurement of glucose solution. In this test, a solution is put on the prism surface and the absorption spectrum was calculated using the one measured without a solution as background. Figure 2 shows the absorption spectra of glucose solutions with different concentrations compared to the one measured using an FT-IR-based system.

Although one sees some noises on the spectra measured by the QCL system, some absorption peaks of glucose are clearly observed. From this result, we 
confirmed possibility of blood glucose measurement with the swept-wavelength QCL system.

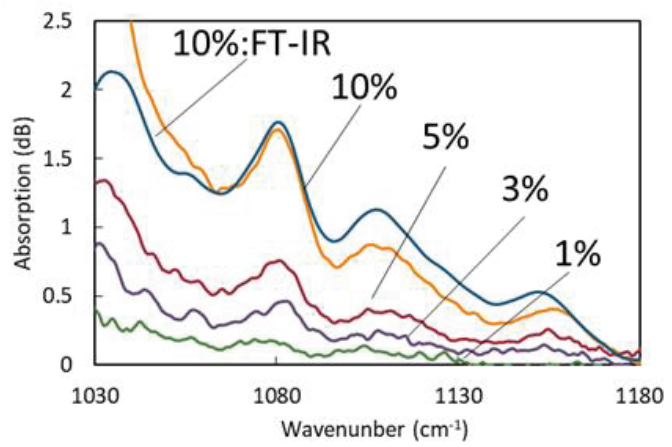

Figure 2 Absorption spectra of glucose solution

Then we measured absorption spectra of human lips using the QCL system. In the measurement spectrum, a test subject holds the prism with upper and lower lips for $10-15 \mathrm{sec}$ that is much shorter than what is necessary for measurement with the FT-IR system (30$60 \mathrm{sec}$ ) and this makes the measurement error due to movement of human lips smaller. Figure 3 shows an absorption spectrum of human lips measured by the QCL system compared to the one obtained by the FTIR system. The spectrum obtained by the QCL system shows a good coincidence with the one taken by the FT-IR spectrum. From this result, we confirmed that absorption spectra of human lips are correctly measured by using the QCL system.

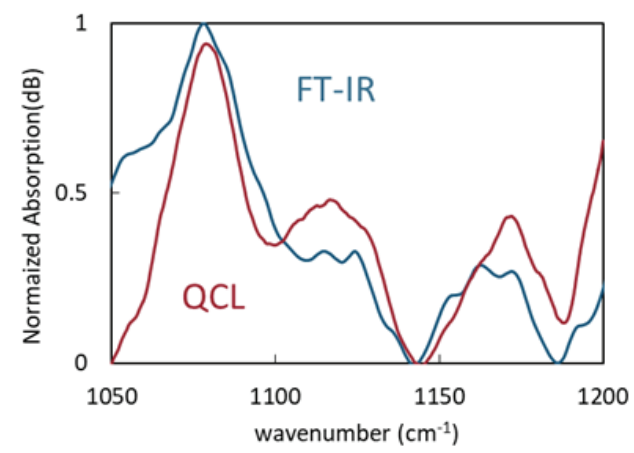

Figure 3 Absorption spectra of human lips

Figure 4 shows change of the peak area of absorption at $1080 \mathrm{~cm}^{-1}$ and $1160 \mathrm{~cm}^{-1}$ after meal compared to the blood glucose levels measured by blood sampling. In the optical measurement, absorption obtained with 3 consecutive measurements were averaged to reduce effect of measurement errors. It is seen that a better coincidence is observed between the absorption of $1160 \mathrm{~cm}^{-1}$ and blood glucose level. This is because the absorption peak at $1080 \mathrm{~cm}^{-1}$ comes from $\mathrm{C}-\mathrm{OH}$ bonds and it is strongly affected by proteins and lipids that also contains $\mathrm{C}-\mathrm{OH}$ bonds [3].

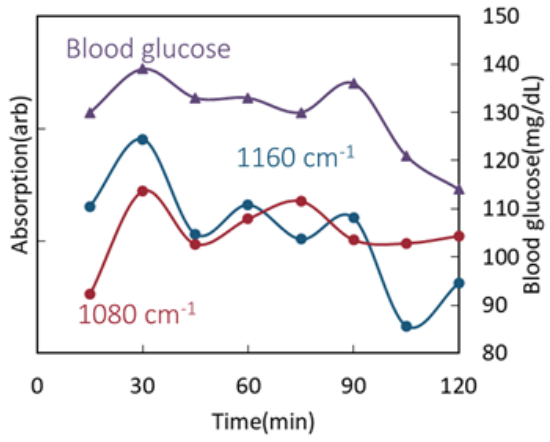

Figure 4 Changes in the absorbance after a meal measured by the QCL system compared to blood glucose level.

Figure 5 shows a correlation plot between the blood glucose estimated from the measured absorption and the one taken by blood sampling. The measurement was performed for a single subject for 3 days. Blood glucose levels are estimated by linear regression using the peak area of the $1160 \mathrm{~cm}^{-1}$ peak. A good correlation with the correlation factor $\mathrm{R}^{2}=0.93$ was obtained and we confirmed the feasibility of the swept-wavelength QCL system for blood glucose measurement.

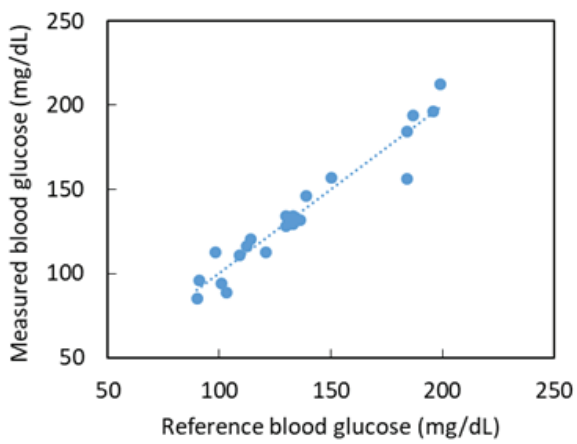

Figure 5 Correlation between blood glucose levels estimated from optical measurement and that from blood sampling. 


\section{Conclusion}

For non-invasive measurement of blood glucose, we proposed an ATR absorption spectroscopy system using a QCL with a MEMS-based external cavity that enables a rapid sweep of emitting wavelength. The system is composed of the QCL, hollow optical fibers, and a trapezoid ATR prism and by using this system, we measured absorption of human lip mucosa. As a result, we observed some absorption peaks that comes from glucose molecular on the measured spectra. The change of the peak intensity follows that of blood glucose well and we confirmed the correlation between the peak intensity and blood glucose level. We are working on improvement of measurement accuracy by analyzing the light distribution in the prism.

\section{References}

1, S. Kino et al., "Hollow optical-fiber based infrared spectroscopy for measurement of blood glucose level by using multi-reflection prism," Biomed. Opt. Express, vol. 7, no. 2, pp. 701-708 (2016)

2, K. Yoshioka et al., "Blood glucose measurement with multiple quantum cascade lasers using hollow-optical fiber-based ATR spectroscopy," Proc. SPIE 10251, 102511U (2017).

3, I. Poljanšek, et al., "Characterization of phenolformaldehyde prepolymer resins by in line FT-IR spectroscopy," Acta Chim. Slov., vol. 52, pp. 238-244 (2005). 


\title{
New library of spectral reflectance and point spread function developed by Monte Carlo simulation with nine-layered skin model
}

\author{
Kaustav Das*, Yuta Kobori, Tomoki Hashisaka, Takehiro Ohya, \\ Tomonori Yuasa, Hideki Funamizu, Yoshihisa Aizu \\ Muroran Institute of Technology, Muroran, Hokkaido 050-8585, Japan
}

\begin{abstract}
We developed new library of spectral reflectance curves and point spread functions of intensity distribution on skin surface by using Monte Carlo simulation with nine-layered skin tissue model. From this library, absorption and scattering coefficients in dermal layer are found to be the most contributing factors in the pattern of spectral curves and point spread functions. This library is a useful tool for investigating the contribution of optical properties on the skin reflectance phenomena.
\end{abstract}

Keywords: skin tissue, spectra, point spread function, Monte Carlo simulation, library file.

\section{Introduction}

Light rays incident on the human skin surface results in occurrence of two processes, the first one is absorption and the second one is scattering that include reflection and transmission. For investigating spectral reflectance curves and point spread functions (PSF) of reflected intensity on skin surface, we developed new library of them in the programming surroundings of Monte Carlo simulation (MCS) [1] in the nine-layered skin tissue model [2]. It has been suspected that predominantly the conditions of absorption coefficient in dermal and subcutaneous layers, and scattering coefficients in the upper dermal layer are assumed to be the controlling factors for any such pattern of spectra and PSFs. The investigation of such patterns is demonstrated in the simulated results.

\section{Nine-layered model and MCS}

The intricate structure of human skin tissue can be classified into three layers such as epidermis, dermis and subcutaneous layer in a general way. But, in a detailed and definite way, skin tissue is classified into nine layers which was used for simulations, as can be seen in Fig. 1. These layers with particular thickness are; layer one $\left(\mathrm{L}_{1}\right)$ : stratum corneum $(0.015 \mathrm{~mm})$, layer two $\left(\mathrm{L}_{2}\right)$ : stratum granulosum and stratum spinosum $(0.060 \mathrm{~mm})$, layer three $\left(\mathrm{L}_{3}\right)$ : stratum basale $(0.015 \mathrm{~mm})$, layer four $\left(\mathrm{L}_{4}\right)$ : papillary dermis $(0.045 \mathrm{~mm})$, layer five $\left(\mathrm{L}_{5}\right)$ : subpapillary dermis $(0.175 \mathrm{~mm})$, layer six $\left(\mathrm{L}_{6}\right)$ : upper blood net dermis $(0.080 \mathrm{~mm})$, layer seven (L7): reticular dermis $(0.600 \mathrm{~mm})$, layer eight (L8): deep blood net dermis $(0.100 \mathrm{~mm})$ and layer nine $\left(\mathrm{L}_{9}\right)$ : subcutaneous tissue $(6.000 \mathrm{~mm})$. In MCS with the help of quite accurate and organized coding, the phenomenon of light propagation in human skin tissue has been mimicked as genuinely as

*18096009@mmm.muroran-it.ac.jp; phone 81 143-46-5348; possible. To carry out MCS, five parameters were required for each layer: scattering coefficient $\mu_{s}$, absorption coefficient $\mu_{a}$, anisotropy parameter $g$, refractive index $n$ and thickness $t$. The five parameters for each of nine layers means totally 45 values to be specified for MCS execution. This is quite complicated and impractical for development of the library. We propose a new approach in which the two main parameters $\mu_{a}$ and $\mu_{s}$ are varied by magnification while values of $g, n$, and $t$ are fixed. For $\mu_{a}$ and $\mu_{s}$, their magnification $\mathrm{M}_{a}$ and $\mathrm{M}_{s}$ are changed, respectively, as given in Table 1.

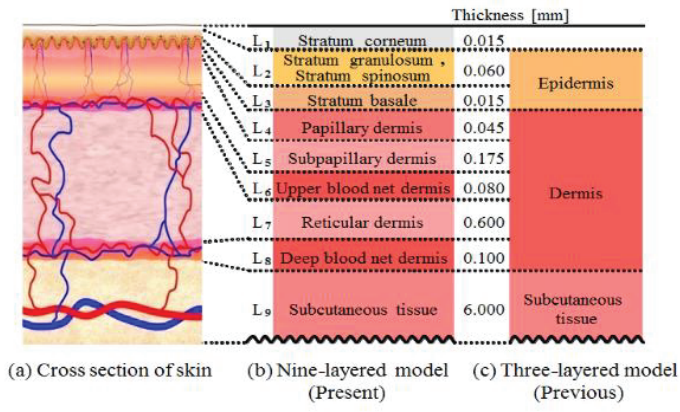

Fig. 1 Schematic structure of skin tissue, nine-layered model, and three-layered model.

Table 1 Different magnification of $\mathrm{M}_{a}$ and $\mathrm{M}_{s}$.

\begin{tabular}{|c|c|c|}
\hline Layers & $\mathrm{M}_{a}$ & $\mathrm{M}_{s}$ \\
\hline $1 \sim 3$ & $\begin{array}{l}\mu_{a} L_{1-3} \\
0.6 \sim 1.6\end{array}$ & $\begin{array}{l}\mu_{S} L_{1-3} \\
0.6 \sim 1.4\end{array}$ \\
\hline $4 \sim 6$ & \multirow{2}{*}{$\begin{array}{l}\mu_{a} L_{4-9} \\
0.2 \sim 3.6\end{array}$} & $\begin{array}{l}\mu_{S} L_{4-6} \\
0.3 \sim 9.3\end{array}$ \\
\hline $7 \sim 9$ & & $\begin{array}{c}\mu_{s} L_{7-9} \\
0.1 \sim 1.0\end{array}$ \\
\hline
\end{tabular}




\section{Results and discussion}

Figs 3 and 4 shows spectra and PSFs in 700nm with different magnification of $\mathrm{M}_{a}$ which indicates different conditions of $\mu_{a}$ in dermal and subcutaneous layers ( $L_{4}$ $L_{9}$ ), keeping all other parameters fixed to be the initial

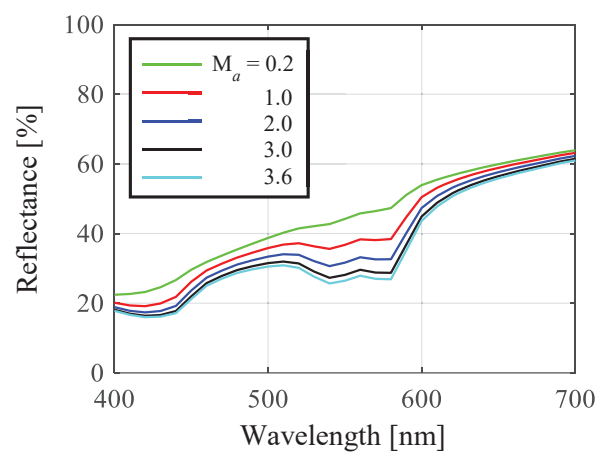

Fig. 3 Spectra simulated with different magnification of $\mathrm{M}_{a}$ in dermal and subcutaneous layers $\left(L_{4} L_{9}\right)$.

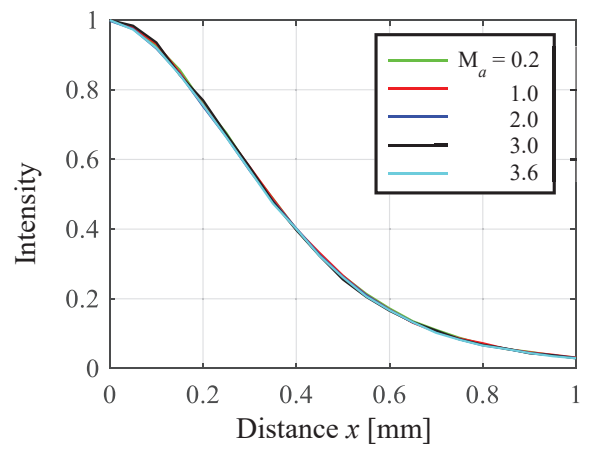

Fig. 4 PSFs simulated with different magnification of $\mathrm{M}_{a}$ in dermal and subcutaneous layers $\left(L_{4}-L_{9}\right)$.

Distance $x=0$ corresponds to the illumination point.

values [2]. With the change in $M_{a}$ value from the small to large, the spectral reflectance reduces from high to low. But in PSFs, there was no change in the pattern. Figs 5 and 6 demonstrate spectra and PSFs with different magnification of $\mathrm{M}_{s}$ which refers to different condition of $\mu_{s}$ in the upper dermal layer $\left(L_{4} L_{6}\right)$, keeping all other parameters fixed to be the initial values [2]. With the increase in $\mathrm{M}_{s}$ value, the spectral reflectance becomes higher, and for PSFs, low $\mathrm{M}_{s}$ values produce spread out of point spread functions and higher $\mathrm{M}_{s}$ values produce stiffer point spread functions. Hence, the created library files are useful for understanding the tendency of spectra and PSFs with changing condition of $\mu_{a}$ in dermal and subcutaneous layers and $\mu_{s}$ in the upper dermal layer. These library files can be referred in future to characterise the nine-layered model in a less complicated way.

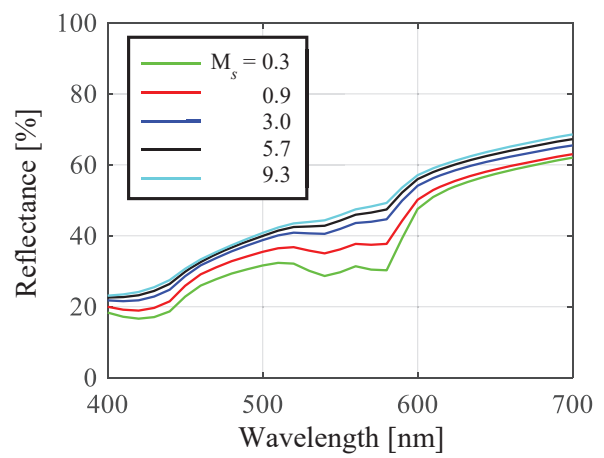

Fig. 5 Spectra simulated with different magnification of $\mathrm{M}_{s}$ in the upper dermal layer $\left(L_{4-} L_{6}\right)$.

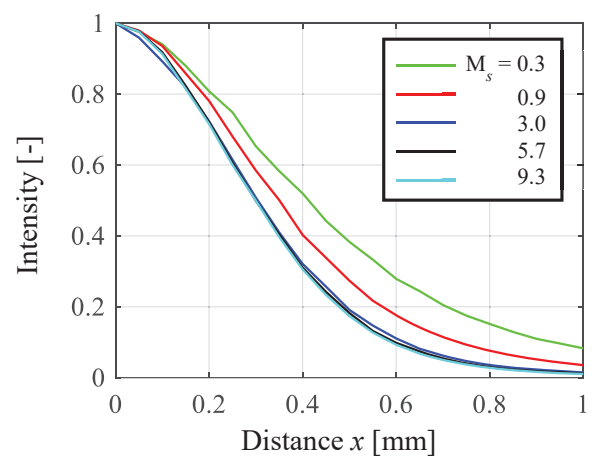

Fig. 6 PSFs simulated with different magnification of $\mathrm{M}_{s}$ in the upper dermal layer $\left(L_{4}-L_{6}\right)$. Distance $x=0$ corresponds to the illumination point.

\section{Conclusion}

We have developed new library of spectra and PSFs for various values of $\mu_{a}$ and $\mu_{s}$. The change in condition of absorption coefficient in dermal and subcutaneous layers had some contribution to the change in pattern of spectra but had no contribution to the pattern of PSFs of reflected intensity on skin surface. The change in condition of scattering coefficient in the upper dermal layer had some contribution to the change in pattern of both spectra and PSFs of reflected intensity on skin surface to some extent. All these features can be seen in the library files and furthermore investigations are needed to enrich these library files.

\section{References}

Wang, L., Jacques, S. L., and Zheng, L., "MCML-Monte Carlo modeling of photon transport in multilayered tissues," Comput. Methods Programs Biomed. 47, 131-146 (1995).

Maeda, T., Arakawa, N., Takahashi, M., and Aizu, Y., "Monte Carlo simulation of spectral reflectance using a multilayered skin tissue model," Opt. Rev. 17, 223-229 (2010). 


\title{
Early diagnosis of teeth caries with non-invasive optical coherence
}

\section{tomography}

\author{
Yen-Li Wang ${ }^{\mathrm{a}}$, Chia-Yen Ke ${ }^{\mathrm{b}}$, Hsiang-Chieh Lee ${ }^{\mathrm{c}, \mathrm{d}}$, Ya-Ju Lee ${ }^{\mathrm{b}}$, and Meng-Tsan Tsai ${ }^{\mathrm{e} \text {, } *}$ \\ a Department of Periodontics, Chang Gung Memorial Hospital, Taoyuan, Taiwan \\ ${ }^{\mathrm{b}}$ Institute of Electro-Optical Science and Technology, National Taiwan Normal University, Taiwan \\ ${ }^{\mathrm{c}}$ Graduate Institute of Photonics and Optoelectronics, National Taiwan University, Taiwan \\ ${ }^{\mathrm{d}}$ Department of Electrical Engineering, National Taiwan University, Taiwan \\ ${ }^{e}$ Department of Electrical Engineering, Chang Gung University, Taoyuan, Taiwan \\ ${ }^{\mathrm{f}}$ Department of Dermatology, Chang Gung Memorial Hospital, Linkou, Taiwan \\ *Email: mttsai@mail.cgu.edu.tw
}

\begin{abstract}
In this study, optical coherence tomography (OCT) was implemented for characterizing the early demineralization on enamel topology. $37 \%$ phosphoric acid was used to simulate the acid environment in the oral cavity and accelerates the acid effect. After temporally applying the phosphoric acid on the teeth surface, the changes in morphology and optical property of teeth can be identified with OCT. The OCT results illustrate the scattering intensity significantly increased during teeth demineralization. Therefore, the results obtained before and after acid application are quantitatively compared, based on the estimation of scattering coefficient and surface roughness. The estimated scattering coefficient increased with time during acid application and the same trend also can be found from the results of surface roughness. The results indicate that the demineralization on the teeth surface can be detected, enabling to further detect the earlystage cavities.
\end{abstract}

Keywords: optical coherence tomography, tooth, caries, scattering

\section{Introduction}

A tooth is a hard structure, enabling to cut and crush food before swallowing and digesting. For human teeth, there are four types including incisors, canines, premolars and molars, and each type provides different functions for food chewing. The tooth can be divided into three parts such as crown, neck, and root. The crown is the outmost part of tooth above the cementoenamel junction without surrounding the soft tissue. The neck is below the crown and forms the line between the crown and root which acts as a support for the tooth and fasten in the jawbone. In regard to the tooth anatomy, it is composed of enamel, dentin pulp, and cementum. The enamel is the hardest and outmost part of teeth and the primary composition of enamel is hydroxyapatite that is a crystalline calcium phosphate. When sucrose in food reacts with bacteria on the crown surface with the production of acid, the acid causes calcium loss in the enamel layer of crown. In contrast, saliva acts as a reservoir of minerals to remineralize the enamel layer against the demineralization process. Normally, the speeds of demineralization and remineralization in teeth are equal to keep the teeth healthy. However, if the teeth are under the acid condition for the long-term exposure, it results in unbalance between demineralization and remineralization. Therefore, in the beginning of dental caries, demineralization starts at the teeth surface and gradually spread into the dentin layer. Dental caries cause teeth breakdown as a results of acids induced by bacteria in the oral cavity that dissolves the teeth structure. In the early-stage sign of caries, it is appearance of a white or yellow spot on the enamel surface, representing existence of a microcavity.

\section{Methods}

\section{System setup}

To identify the morphological change of teeth during demineralization, a high-resolution spectral-domain OCT system was implemented for teeth scanning as shown in Figure 1. The used broadband light source is a 
superluminescence diode (M-D-890-UBB-HP, Superlum, Ireland), covering a spectral range of $150 \mathrm{~nm}$ with a center wavelength of $900 \mathrm{~nm}$. An isolator is connected to the output end of broadband light source to prevent from the back reflection and then, a fiber-based Michelson interferometer is connected to the output end of the isolator. A two-axis galvanometer (GVS002, Thorlabs, New Jersey, United States) is utilized to provide lateral scanning and a scanning lens is used to focus the optical beam in the sample. Dispersion induced by the scanning lens (LSM02, Thorlabs, New Jersey, United States) is compensated by a dispersion compensator. Finally, the interference signal is detected by a spectrometer, consisting of a beam expander, diffraction grating, achromatic lens and linescan camera (spL4096-140km, Basler, Ahrensburg, Germany). To synchronize the lateral scanning and frame acquisition, a DAQ board with multichannel analog output is used. The line rate of linescan camera is set to $100 \mathrm{kHz}$, corresponding to a system frame rate of $100 \mathrm{~Hz}$. Additionally, the measured axial and transverse resolutions are 3 and $4 \mu \mathrm{m}$, respectively. For 3D OCT imaging, a volume data is consisting of 500 B-scans in which each B-scan is composed of 1000 A-scans.

\section{Results and Discussion}

To understand the difference between healthy and demineralized teeth, the tooth was scanned before the acid application for $120 \mathrm{~s}$. Before the acid application, the tooth was scanned with OCT and a volume dataset with a size of $1.2 \times 1.2 \times 1 \mathrm{~mm}^{3}$ was recorded. Subsequently, $37 \%$ phosphoric acid gel was applied for $120 \mathrm{~s}$ to induce tooth demineralization and then, the gel was removed from the tooth surface with tissue. Additionally, the demineralized tooth was cleaned with the brush and the ultrasound cleaner. Again, the same tooth area as imaged before the acid application was scanned with OCT. Figures 1(a) and 1(b) show the 2D OCT images at the same location of tooth obtained before and after the acid application for $120 \mathrm{~s}$, respectively. Moreover, the corresponding en-face OCT images extracted from 3D images of Figs. 2(a) and 2(b) are shown in Figs. 1(c) and 1(d), respectively. From Fig. 1, it can be noted that the backscattered intensity around the tooth surface became stronger after the acid application. The previous reports

have indicated that the acid-induced demineralization causes an appearance of cobblestone on the etched enamel surface. On the other hand, the enhanced backscattered intensity on the tooth surface as shown in Figs. 1(b) and 1(d) can be identified, implying the enhanced backscattered intensity was as a result of the appearance of cobblestone. The results are in coincidence with the previous study in which the attenuation coefficient of the demineralized teeth is larger than that of normal teeth. Here, our results illustrate higher attenuation coefficients for demineralized teeth were caused by the stronger scattering on the demineralized enamel surface, limiting the optical penetration in the deeper depth.
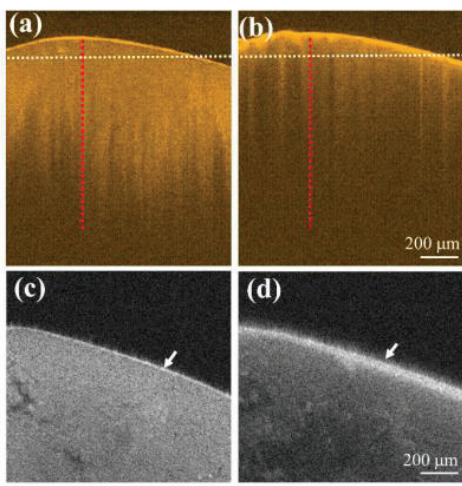

Fig. 1. 2D OCT images of the tooth obtained (a) before the acid application and (b) after acid application for $120 \mathrm{~s}$. En-face images of the tooth extracted from 3D OCT images obtained (a) before the acid application and (b) after acid application for $120 \mathrm{~s}$. The white lines indicate the depth of en-face images. (e) and (f) the OCT depth profiles indicated by the red lines in (a) and (b) respectively.

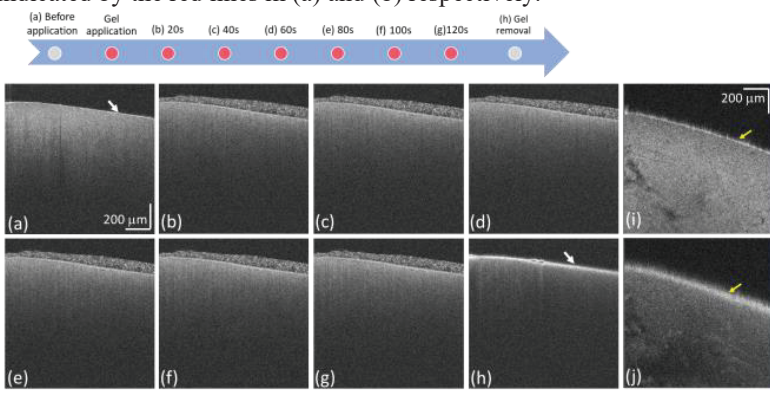

Fig 2. Time-series OCT images of one healthy tooth obtained (a) before the application of $37 \%$ phosphoric acid gel, after the application of $37 \%$ phosphoric acid gel at the various time points of (b) $20 \mathrm{~s}$, (c) $40 \mathrm{~s}$, (d) 60 $\mathrm{s}$, (e) $80 \mathrm{~s}$, (f) $100 \mathrm{~s}$, (g) $120 \mathrm{~s}$ and (h) after removal of the acid gel. (i),(j) the corresponding en-face images extracted from the 3D images of (a) and (h).

Figure 2 shows time-series OCT images of one healthy tooth obtained before the application of $37 \%$ phosphoric acid gel, after the application of $37 \%$ 
phosphoric acid gel at the various time points of $20 \mathrm{~s}, 40 \mathrm{~s}$, $60 \mathrm{~s}, 80 \mathrm{~s}, 100 \mathrm{~s}, 120 \mathrm{~s}$ and $(\mathrm{h})$ after removal of the acid gel. (i),(j) the corresponding en-face images extracted from the 3D images of (a) and (h).

\section{Conclusion}

The estimated scattering coefficient increased with time during acid application and the same trend also can be found from the results of surface roughness. The results indicate that the demineralization on the teeth surface can be detected, enabling to further detect the early-stage cavities.

Proc. of SPIE Vol. $111401114001-206$ 


\title{
Development of silicone-based skin tissue phantom and its spectral reflectance properties
}

\author{
Tomonori Yuasa ${ }^{1}$, Teru Kuwahara ${ }^{1}$, Takaaki Maeda ${ }^{2}$, Hideki Funamizu ${ }^{1}$, and Yoshihisa Aizu ${ }^{1}$ \\ ${ }^{1}$ Muroran Institute of Technology, Mizumoto, Muroran, Hokkaido 050-8585, Japan \\ ${ }^{2}$ National Institute of Technology, Kushiro College, Kushiro, Hokkaido 084-0916, Japan
}

\begin{abstract}
We developed a silicone-based three-layered skin tissue phantom that has no temporal deterioration of spectral characteristics. In this paper, we prepared some different samples having different concentrations of scatterer and absorber for each of three layers. By using these samples, we set various phantoms by different combinations of three layers and, then, measured spectral reflectance and LAB values. To confirm the usefulness, we discussed its optical properties and the range of concentrations.
\end{abstract}

Kevwords: human skin. skin tissue phantom. spectral reflectance.

\section{Introduction}

Optical measurement of the human skin is actively used for medical treatments in dermatology and developments of cosmetics. When light is entered into skin tissue, it propagates with receiving effects of absorption and scattering. Thus, skin tissue conditions are reflected on the spectral reflectance. It is known that the skin tissue phantom is useful tool for validation experiments on spectral reflectance measurements of skin. We first fabricated agar-based phantom [1]. Then, we further developed silicone-based [2] phantom and confirmed that its spectral reflectance was similar to that with human skin. In this study, we prepared multiple sample layers with varying concentrations of absorber and scatterer, constructed various three-layer phantoms combining them, and measured their spectral reflectance and $\mathrm{LAB}$ values. By constructing data as a library of the measured spectral reflectance and component concentration in various combination patterns of three layers, it can be used as guideline for future improvement in manufacturing of skin phantom.

\section{Silicone-based skin tissue phantom}

Human skin has a complicated structure, then it is often treated as a simplified three-layered model of epidermis, dermis and subcutaneous tissue when considering optical properties. Figure 1 shows a cross section of skin and the three-layered skin tissue model. Figure 2 shows conditions of the three-layered phantom. The base material of each layer is the lucent silicon, which is made by adding scatterer and absorber. As substitutions of absorber ( melanin and hemoglobin ) and scatterer in skin tissue, color pigments and $\mathrm{TiO}_{2} / \mathrm{BaSO}_{4}$ are used in this model, due to the similarity of their spectral properties. These materials are added to the lucent silicone according to each layer's condition. The concentration of absorber and scatterer is diluted, because the thickness of each layer of a phantom model is different from that of real human skin tissue.

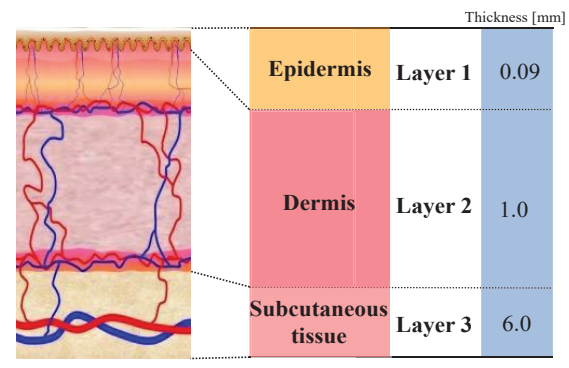

$\begin{array}{ll}\text { (a) Cross section of skin } & \text { (b) Three-layered model }\end{array}$

Figure 1. Three-layered skin tissue model.

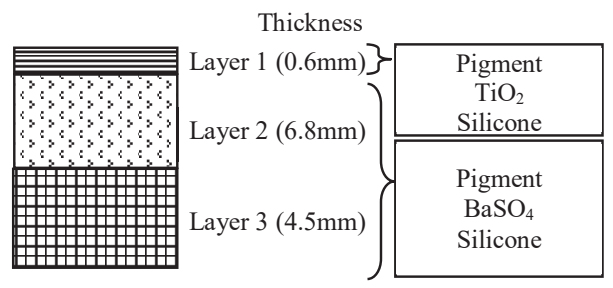

Figure 2. Structure of a silicone-based skin tissue phantom model. 


\section{Results and Discussion}

We measured spectral reflectance curves as the concentrations of scatterer and absorber change for each layer. And we evaluated their characteristics as a skin model by comparing results of silicone-based skin tissue phantom and light propagation by Monte Carlo simulation (MCS). Figure 3 (a) shows spectral reflectance curves with concentration change of scatterer in the 1st layer. Figure 3 (b) shows spectral reflectance curves obtained by MCS with change of scattering coefficient in the 1st layer. Figure 4 (a) shows spectral reflectance curves with concentration change of absorber in the first layer. Figure 4 (b) shows spectral reflectance curves obtained by MCS with change of absorption coefficient in the 1st layer. In both cases, the second and third layers are kept in the standard state of optical properties as our phantom. It can be seen that the skin phantom shows the same changing trend as MCS in the results.

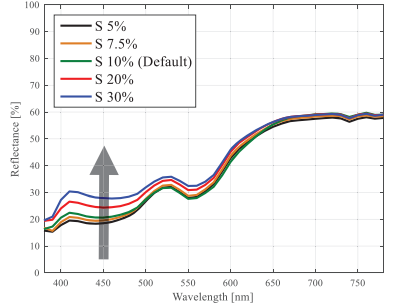

(a) Results of phantom

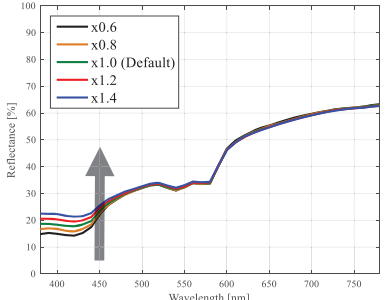

(b) Results of MCS
Figure 3. Spectral reflectance curves with concentration change of scatterer.

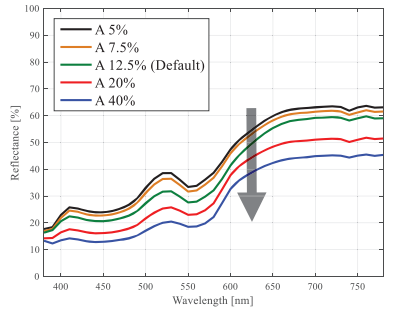

(a) Results of phantom

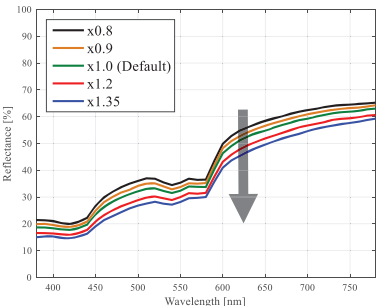

(b) Results of MCS
Figure 4. Spectral reflectance curves with concentration change of absorber.

We prepared five kinds of single layer samples with different concentrations of absorber and scatterer for each layer. Table 1 shows dye component concentrations of the five different sets prepared for each layer. The combination of each layer was changed, and the LAB values were measured totally for 125 combinations. We investigated whether it is possible to satisfy the distribution range of $\mathrm{LAB}$ values that are typically found in the real human skin, by comparing them with data of 459 people of SOCS [3].
Figure 5 shows $a^{*} b^{*}$ values of phantom and SOCS, respectively. The $\mathrm{a}^{*}$-value of phantom ranges from 7 to 18 which is well corresponding to the range for SOCS. However, the $\mathrm{b}^{*}$-value of phantom demonstrates a range from -5 to 24 which is different from that for SOCS. The phantom needs to be improved to have larger $b^{*}$-value. To do this, we consider preparation of further color pigments having different spectral properties.

Table 1. Five different concentrations of absorber and scatterer in each of three layers.

\begin{tabular}{|c|c|c|c|c|c|c|}
\hline \multirow{2}{*}{} & \multicolumn{2}{|c|}{ Layer 1 } & \multicolumn{2}{c|}{ Layer 2 } & \multicolumn{2}{c|}{ Layer 3 } \\
\cline { 2 - 7 } & $\mathrm{A}[\%]$ & $\mathrm{S}[\%]$ & $\mathrm{A}[\%]$ & $\mathrm{S}[\%]$ & $\mathrm{A}[\%]$ & $\mathrm{S}[\%]$ \\
\hline $\mathrm{A}$ & 5 & 10 & 0.25 & 1.25 & 0.25 & 1.75 \\
\hline $\mathrm{B}$ & 40 & 10 & 0.25 & 1.25 & 2.25 & 1.75 \\
\hline $\mathrm{C}$ & 12.5 & 10 & 0.75 & 1.25 & 0.70 & 1.75 \\
\hline $\mathrm{D}$ & 12.5 & 5 & 0.75 & 0.25 & 0.70 & 0.75 \\
\hline $\mathrm{E}$ & 12.5 & 30 & 0.75 & 2.25 & 0.70 & 2.75 \\
\hline
\end{tabular}

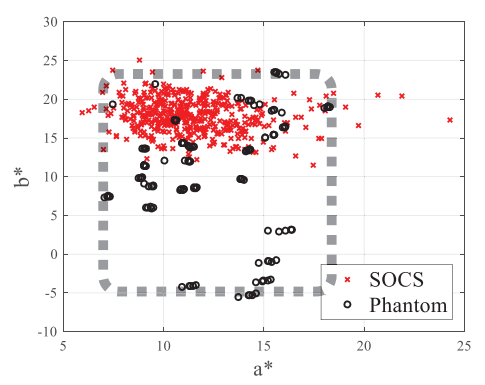

Figure 5. $a * b *$ values of phantoms and SOCS data.

\section{Conclusion}

We made several sample layers with different concentrations of absorber and scatterer. And, we constructed various three-layer phantoms combining them and measured their spectral reflectance and LAB values. Then, it is convenient to recombine the first layer with different concentrations of absorber and scatterer. We made 125 combined samples, and investigated their color properties. We confirmed that our phantom realize human skin color partly. More realistic color may be developed near future.

\section{References}

[1] I. Nishidate, Y. Aizu, H. Mishina, Journal of Biomedical Optics, 9 (4), 700-710 (2004).

[2] S. Iva, A. Tanabe, T. Maeda, H. Funamizu, Y. Aizu, Opt. Rev., 21 (3), 353-358 (2014).

[3] ISO/TR 16066:2003, Graphic technology - Standard object colour spectra database for colour reproduction evaluation. 


\title{
Study on the option of the optimal time length of measurement in resting-state fNIRS brain imaging
}

\author{
Chia-Cheng Wang ${ }^{\text {a }}$, Guan-Cheng Dong ${ }^{\text {a }}$ Jung-Chih Chen ${ }^{\text {a }}$, Chia-Yen Lee ${ }^{\text {b }}$, Ching-Cheng Chuang *a \\ * Corresponding author \\ a Institute of Biomedical Engineering, National Chiao Tung University, Hsinchu 30010, Taiwan \\ b Department of Electrical Engineering, National United University, Miaoli 36063, Taiwan
}

\begin{abstract}
Resting-state functional near-infrared spectroscopy (rs-fNIRS) has become a powerful tool for evaluating the functional connectivity (FC) and networks of the brain. However, how short period of the rs-fNIRS scanning time can adequately present the stable resting-state functional network features of the brain is still unclear, especially in the prefrontal cortex (PFC) which play an important role in complex brain functions. In this study, the 10-min duration of the rs-fNIRS signal of PFC was measured from 38 participants and then the time series was divided into 10 parts from 1 to 10 min with a 60 -sec increment. The optimal time length in rs-fNIRS measurement was investigated by analyzing the correlation, significant difference and connectome between different durations of rs-fNIRS scanning time. The results show that FC maps exhibited high similarity achieved after $2.0 \mathrm{~min}$ fNIRS data acquisition duration. Our findings suggest that the rs-fNIRS provide high potential to apply to clinical neuroscience.
\end{abstract}

Keyword: Resting-state functional near-infrared spectroscopy, functional connectivity, prefrontal cortex

\section{Introduction}

Resting-state functional near-infrared spectroscopy (fNIRS) has become a powerful tool for evaluating the functional connectivity (FC) and networks of the brain. By measuring the brain's hemodynamic spontaneous fluctuations can reflect the characteristics of low frequency $(<0.1 \mathrm{~Hz})$ spontaneous neural activity [1]. The fNIRS technique displays several unique advantages compared to functional magnetic resonance imaging (fMRI), such as quietness, less expensive, non-ionizing radiation imaging, higher temporal resolution and better portability for use [2].

However, to facilitate the use of fNIRS-based imaging technique in the study of brain networks in adults, one critical step is to determine the minimum time length that is capable of providing functional brain connectivity and statistics result. Regrettably, the current resting-state fNIRS field is still unclear. Previous fMRI-derived brain imaging studies have suggested that $5 \sim 7 \mathrm{~min}$, or $\geq 9 \mathrm{~min}$ BOLD data can yield stable correlation strengths [3]. The empirical conclusions will provide critical information for the brain functional connectivity in adults with normal brain development and the clinical implementation of the fNIRS technique [4].
In the present study, functional brain network connectome and statistics analysis were applied to a series of incrementally time length of resting-state fNIRS imaging data. These include collecting resting-state fNIRS time series data, prepoccessing, estimating the correlation coefficient matrix, and analyzing optimal time length using correlation, significant difference [5]

\section{Method}

\subsection{Participants}

In this study, 38 healthy young adults (16 males and 22 females), age between 22 to 25 years (the mean age was $23 \pm 2$ years old) with no history of neurological diseases, were recruited from National Chiao Tung University, Taiwan. All participants provided written informed consent. The study was in accordance with the latest version of the Declaration of Helsinki, and approved by the Institutional Review Board (IRB) in National Chiao Tung University, Taiwan.

\subsection{Data acquisition}

A continuous wave near-infrared system (ETG-4000, Hitachi Medical Co., Tokyo, Japan) was used to measure 
the time course of $\mathrm{HbO}_{2}$ and $\mathrm{Hb}$ concentrations. The system included 17 laser sources and 16 detectors, with each source including two wavelengths (690 and $830 \mathrm{~nm}$ ) of near-infrared light and the sampling rate was set to 50 Hz. The sources and detectors were systematically placed on the left and right hemispheres of the participant's head, with spatial separation between adjacent sources and detectors at $3 \mathrm{~cm}$. The configuration resulted in 52 measurement channels that covered the frontal, temporal lobes of the cerebral cortex. The positions of the probes were consistent with the international 10-20 system of electrode layout.

\subsection{Data analysis}

In our study, the optical signal of each channels was filtered through a bandpass filter within 0.01 to $0.08 \mathrm{~Hz}$ to reduce the effects of systematic noise and obtain hemodynamic fluctuations after raw data of $\Delta \mathrm{HbO}_{2}$ and $\Delta \mathrm{Hb}$ were obtained from the system. Then the average data was obtained for group-level analysis to reduce the effects of individual differences. The distribution of the average filtered signals of the time series transformed into correlation matrix, which shows the correlation coefficient of individuals in different channels. The two sample $t$-test and correlation analysis is used to recognize the completion of brain functional connectivity (FC).

\subsubsection{Functional connectivity}

The low-frequency change of hemoglobin concentration was analyzed by calculating the Pearson's correlation coefficient $r X Y$ :

$$
r_{X Y}=\frac{\sum_{i=1}^{n}\left(X_{i}-\bar{X}\right)\left(Y_{i}-\bar{Y}\right)}{\sqrt{\sum_{i=1}^{n}\left(X_{i}-\bar{X}\right)^{2}\left(Y_{i}-\bar{Y}\right)^{2}}}
$$

In Eq. (1), $X_{i}$ and $Y_{i}$ are the temporal response of grouplevel oxygenation dynamics $\left(\Delta \mathrm{HbO}_{2}, \Delta \mathrm{Hb}\right.$ and $\left.\Delta \mathrm{tHb}\right)$ of each detection channels; and then calculated the grouplevel FC maps at different fNIRS signal acquisition durations in resting-state.

\subsubsection{Significant differences}

The spectrum of the low-frequency $(0.01$ to $0.08 \mathrm{~Hz})$ of $\Delta \mathrm{HbO}_{2}, \Delta \mathrm{Hb}$, and $\Delta \mathrm{tHb}$ were obtained from time series signal by using FFT method. Then the spectrum analysis of $\Delta \mathrm{tHb}$ of each channel in group-level was structured from spectrum curve to determine the significantly different between relatively short and longer 10.0-min signal durations by using two-sample $t$-test with $p$ value $<$ 0.001 .

\section{Results and Discussion}

\subsection{Functional connectivity}

For each participant, we measured hemodynamic signal changes from multiple regions of the cerebral cortex and then calculated the group-level FC maps at different fNIRS signal acquisition durations (Fig. 1(a)), were calculated with Pearson's correlation coefficient that the formula was described in Eq. (1). The results of correlation matrices represent the strength of resting-state functional connectivity for $\Delta \mathrm{HbO}_{2}$ in group-level analysis. Visually, the spatial patterns of these FC maps exhibited high similarity across variable data collection lengths. Quantitatively, when compared to the relatively longer 10.0-min data acquisition duration, these results showed that FC was accurate trend achieved after 2.0-min fNIRS imaging duration (Fig. 1(b)).

a

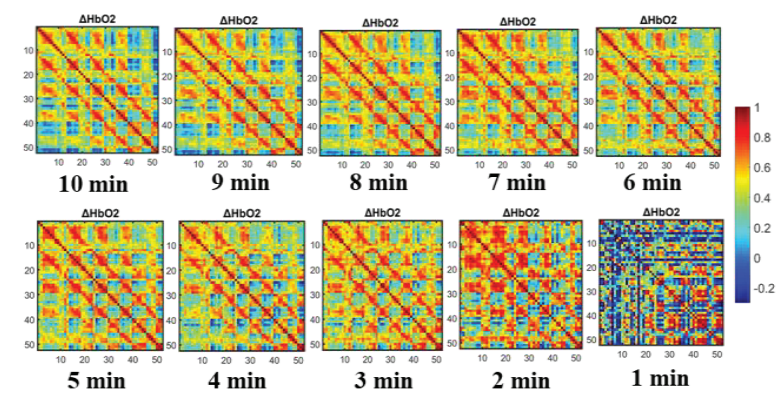

b

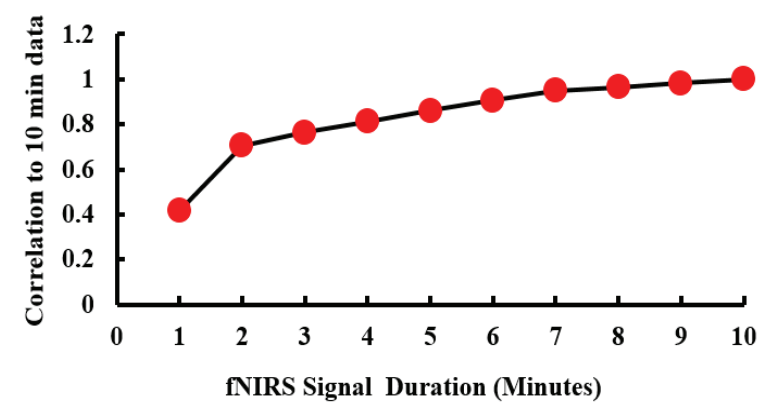


Fig. 1. The correlation matrices derived from different durations of rs-fNIRS scanning time. (a) The FC maps of $\Delta \mathrm{HbO}_{2}$ calculated by Pearson correlation for fNIRS signal acquisition durations ranging from 1.0 to $10 \mathrm{~min}$ with 60 sec bins. Visually, the spatial patterns of these FC maps exhibited high similarity across variable data collection lengths. (b) The plots of the correlation strength calaulated for FC maps of $\Delta \mathrm{HbO}_{2}$ between short and long (10.0 min) signal durations. The red-filled circles indicate significant strong correlation of the spatial FC maps associated with a given fNIRS signal acquisition duration with the FC map when computed using 10.0 min of fNIRS data.

\subsection{Significant differences}

Then the spectrum analysis of $\Delta \mathrm{HbO}_{2}$ of each channel in group-level across variable data collection lengths. Table 1 shows the significantly different channels based on two-sample $t$-test calculated from spectrum curve data between relatively shorter and longer 10.0-min fNIRS signal durations. The results of this analysis indicate that there is no significant difference $(P>0.05)$ after 7 minutes fNIRS signal acquisition duration.

Table 1. Two-sample $t$-test data for spectrum curve analysis associated with fNIRS signal acquisition duration. Data are $P$ values.

\begin{tabular}{|c|c|c|c|c|c|c|c|c|c|}
\hline $\begin{array}{l}\text { Compared with } \\
10 \text { minutes }\end{array}$ & $\begin{array}{c}9.0 \mathrm{~min} \\
\text { fNIRS } \\
\text { Signal } \\
\text { Acquisition }\end{array}$ & $\begin{array}{c}8.0 \mathrm{~min} \\
\text { fNIRS } \\
\text { Signal } \\
\text { Acquisition }\end{array}$ & $\begin{array}{c}7.0 \mathrm{~min} \\
\text { fNIRS } \\
\text { Signal } \\
\text { Acquisition }\end{array}$ & $\begin{array}{c}6.0 \mathrm{~min} \\
\text { fNIRS } \\
\text { Signal } \\
\text { Acquisition }\end{array}$ & $\begin{array}{c}5.0 \mathrm{~min} \\
\text { fNIRS } \\
\text { Signal } \\
\text { Acquisition }\end{array}$ & \begin{tabular}{|c|}
$4.0 \mathrm{~min}$ \\
fNIRS \\
Signal \\
Acquisition
\end{tabular} & \begin{tabular}{|c|}
$3.0 \mathrm{~min}$ \\
fNIRS \\
Signal \\
Acquisition
\end{tabular} & $\begin{array}{c}2.0 \mathrm{~min} \\
\text { fNIRS } \\
\text { Signal } \\
\text { Acquisition }\end{array}$ & $\begin{array}{c}1.0 \mathrm{~min} \\
\mathrm{fNIRS} \\
\text { Signal } \\
\text { Acquisition }\end{array}$ \\
\hline P-value & 0.935653 & 0.253915 & 0.071413 & $1.57 \mathrm{E}-87$ & 7.87E-78 & $1.6 \mathrm{E}-71$ & $5.6 \mathrm{E}-219$ & $1.6 \mathrm{E}-183$ & \begin{tabular}{|l|l|}
$1.9 \mathrm{E}-295$ \\
\end{tabular} \\
\hline
\end{tabular}

\section{Conclusions}

Our result demonstrated that functional connectivity during resting-state condition of the brain could be successfully measure by fNIRS scanning with high temporal resolution. The results of this analysis indicate FC was accurate trend achieved after 2.0-min fNIRS imaging duration and then the spectrum analysis is no significant difference $(P>0.05)$ after 7 minutes fNIRS signal acquisition duration. Although there are still some limitations and unresolved issues [6], our finding provides direct evidence for healthy adult studies involving to the choice of the optimal resting-state fNIRS scanning duration in functional brain connectivity.

\section{References}

[1] L. Cai, Q. Dong, and H. J. Niu, "The development of functional network organization in early childhood and early adolescence: A resting-state fNIRS study," Developmental Cognitive Neuroscience, vol. 30, pp. 223235, Apr 2018.

[2] S. L. Novi, R. B. M. L. Rodrigues, and R. C. Mesquita, "Resting state connectivity patterns with near-infrared spectroscopy data of the whole head," Biomedical Optics Express, vol. 7, no. 7, pp. 2524-2537, Jul 12016.

[3] S. Sasai et al., "A NIRS-fMRI study of resting state network," Neuroimage, vol. 63, no. 1, pp. 179-193, Oct 152012.

[4] J. Y. Wang, Q. Dong, and H. J. Niu, "The minimum resting-state fNIRS imaging duration for accurate and stable mapping of brain connectivity network in children," Scientific Reports, vol. 7, Jul 252017.

[5] Z. Li et al., "Dynamic functional connectivity revealed by resting-state functional near-infrared spectroscopy," Biomedical Optics Express, vol. 6, no. 7, pp. 2337-2352, Jul 12015.

[6] Y. Hoshi, "Functional near-infrared spectroscopy: current status and future prospects," J. Biomed. Opt. 12(6), 062106 (2007).

[7] Tomasi, D. G., Shokri-Kojori, E. \& Volkow, N. D. Temporal Evolution of Brain Functional Connectivity Metrics: Could 7 Min of Rest be Enough? Cerebral Cortex (2016).

[8] E. Sakakibara et al., "Detection of resting state functional connectivity using partial correlation analysis: A study using multi-distance and whole-head probe nearinfrared spectroscopy," Neuroimage, vol. 142, pp. 590601, Nov 152016. 


\title{
Complex Measurements of Fluorescence and Speckle Contrast in
}

\section{Laboratory Mice during Pancreas Ischemia Modeling}

\author{
Valery Shupletsov ${ }^{\mathrm{a}}$,Ksenia Kandurova*a , Evgeniya Seryogina ${ }^{\mathrm{a}}$, Gennadii Piavchenko,c, \\ Viktor Dremin ${ }^{\mathrm{a}, \mathrm{d}}$, Andrian Mamoshin ${ }^{\mathrm{a}, \mathrm{e}}$, Andrey Dunaev ${ }^{\mathrm{a}}$ \\ ${ }^{a}$ Research and Development Center of Biomedical Photonics, Orel State University named after I.S. \\ Turgenev, Orel, Russia \\ ${ }^{b}$ Center for Preclinical Research, J.-s.c. "Retinoids", Moscow, Russia \\ ${ }^{c}$ Histology, Cytology and Embriology Department, I.M. Sechenov First Moscow State Medical \\ University (Sechenov University), Moscow, Russia \\ ${ }^{\mathrm{d}}$ Optoelectronics and Measurement Techniques Unit, University of Oulu, Oulu, Finland \\ ${ }^{\mathrm{e} O r e l}$ Regional Clinical Hospital, Orel, Russia
}

\begin{abstract}
The paper describes the experiment at pancreas ischemia model carried out by combining the methods of fluorescence spectroscopy and laser speckle contrast imaging in laboratory mice. The measurement was performed before and after ischemia to evaluate the relationship between the changes of blood flow and metabolic activity. The results showed high positive correlation between fluorescence intensity at excitation wavelength of $365 \mathrm{~nm}$ and speckle contrast. At the same time, $450 \mathrm{~nm}$ fluorescence intensity and speckle contrast showed high negative correlation as well as two kinds of fluorescence maxima. The proposed approach seems promising for further studies and future clinical testing.
\end{abstract}

Keywords: fluorescence spectroscopy, speckle contrast, mice, pancreas, ischemia

\section{Introduction}

Currently, acute destructive pancreatitis is one of the urgent problems in abdominal surgery due to its high complications probability and mortality. Average mortality rate of pancreatitis is $20-45 \%$; however, it can reach up to $85 \%$ in infected pancreatic necrosis and $100 \%$ in fulminant form ${ }^{1}$.

The main factors of acute pancreatitis progression include microcirculation disorders, ischemic reperfusion injury and the transition from apical apoptosis to necrosis. One of the most important factors among them is ischemic-reperfusion injury of the pancreas. Violations of microhemodynamics is leading mechanism, which triggers progression of pathological processes in pancreas tissue $^{2,3}$. This causes tissue anoxia, which leads to cell metabolism disorders.

One of the main problems surgeons face in this pathology is lack and low quality of diagnostic information in obtained clinical condition during the surgical operations. This problem is even more relevant to minimally invasive surgical operations. In last decade, this approach became *kandkseniya@gmail.com; phone+79102682946 more widespread for diagnosing and surgical treatment of the patients with the pathologies of abdominal organs. Compared with open surgery, minimally invasive surgery allows one to decrease the operative morbidity and mortality, as well as to lower the recovery time of the patient and rehabilitation cost ${ }^{4}$, because the challenges of the intraoperative collection and processing of the diagnostic information in real time are not solved well yet. Informative rapid diagnostic methods will allow the surgeon to timely assess the patient's condition during surgical operations and choose the right tactics. Therefore, development and verification of novel diagnostic techniques and criteria for visualization and analysis of inflamed and necrotic tissues in minimally invasive surgery is rapidly growing field of research.

At present, a gold standard for tissue recognition is a histological examination of samples obtained by biopsy. This method is used as a reference for other techniques and for accurate diagnosing. However, obtaining results takes much time and the method itself may cause several types of complications. Existing widespread diagnostic methods allows either obtain the information about the 
pathological state of tissue later or obtain intraoperative information about anatomical and morphological features rather than morphological changes in tissue. The development and implementation of the method combining the advantages of both approaches is necessary for improving the quality of diagnostics ${ }^{5}$.

Possible solution is optical non-invasive methods, which are rapidly applied in more and more areas, including practical medicine ${ }^{6}$. This approach includes various spectroscopic and imaging techniques for studying biological tissues in vivo in real time without invasive acquiring the sample of this tissue. These methods allow to evaluate metabolic processes, chromophores content, blood perfusion and oxygenation ${ }^{7}$. Optical methods can increase the prevalence and effectiveness of minimally invasive abdominal interventions in clinical practice by providing additional diagnostic information for surgeon in real time.

One of the most widespread optical spectroscopic methods for in vivo diagnostics is fluorescence spectroscopy (FS). The method is based on the excitation of fluorescence of endogenous and exogenous tissue fluorophores with UV or visible optical radiation to record the fluorescence emission spectra. The rate of metabolic processes in biotissue manifests in changes of concentration of certain endogenous fluorophores involved in tricarbonic acid cycle. In particular, the reduced form of NADH coenzyme has a fluorescence intensity maximum at a wavelength of $490 \mathrm{~nm}$ when excited with $365 \mathrm{~nm}$ radiation. Oxidized FAD has a maximum of fluorescence intensity in the range of 520$540 \mathrm{~nm}$ when excited with $450 \mathrm{~nm}$ radiation ${ }^{8}$.

The metabolic processes depend on proper tissue oxygen supply delivered by blood. The information about blood microcirculation in organs tissue is important to determine normal tissue and inflammatory or necrosis processes during the surgery. Therefore, many optical techniques are developed for this purpose as well as for studying of biochemical reactions closely related to blood supply disorders, at the moment, the most effective methods are diffuse reflectance spectroscopy ${ }^{9}$ and imaging $^{10}$, laser Doppler imaging ${ }^{11}$ and laser specklecontrast imaging (LSCI) ${ }^{12}$.

LSCI method is based on the properties of laser radiation. When laser radiation falls on the inhomogeneous surface random interference of coherent light occurs, which creates a randomly changing picture of the intensity, known as speckle. Temporal and spatial statistics of speckle patterns provide information on the motion of scattering particles.

As the metabolic activity and blood microcirculation are closely interrelated, it makes sense to study both of these parameters together. Multimodal approach seems more promising for complex diagnostics of different aspects of pathological processes in tissues ${ }^{13,14}$.

The aim of the work is to study the possibility of combined application of optical spectroscopic and imaging techniques for multimodal diagnosing of the pancreas tissue state.

\section{Materials and Methods}

The experiment was performed on model mice. The experiment was approved by Ethics committee of Orel State University (record of the meeting №10 of 16.10.2017).

The equipment included two setups implementing FS and LSCI methods each (Fig. 1, 2).

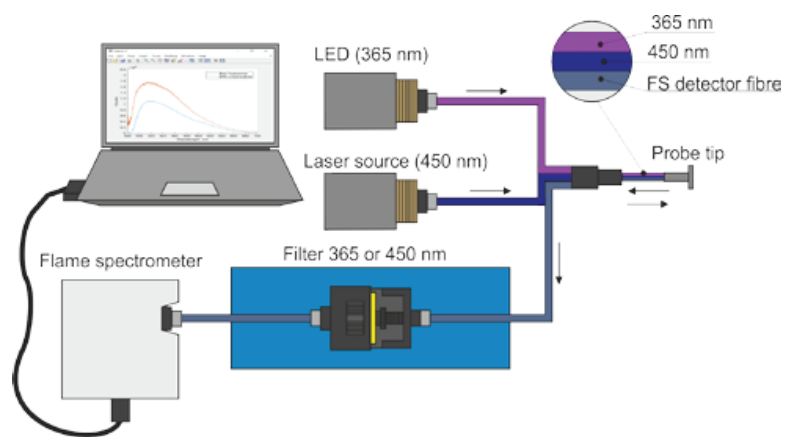

Figure 1. The schematic illustration of FS setup

The first setup implements FS method. A 365 nm UV light source (LED) and $450 \mathrm{~nm}$ blue light source (laser diode) were used to excite the emission of NADH and FAD coenzymes showing the metabolic activity. FS channel has been composed of two emitting and one collecting fibers. The fibers were combined in a single fiber optical probe. The optical filters were used to exclude backscattered source radiation and to obtain only fluorescence spectra.

Collected light was recorded by a CCD spectrometer in the range of 350-820 $\mathrm{nm}$ (FLAME, Ocean Optics, USA). A personal computer was used to control the system and 
automate the data acquisition process. The data processing has been performed using custom-developed software in MATLAB software.

Figure 2 shows the scheme of LSCI setup.

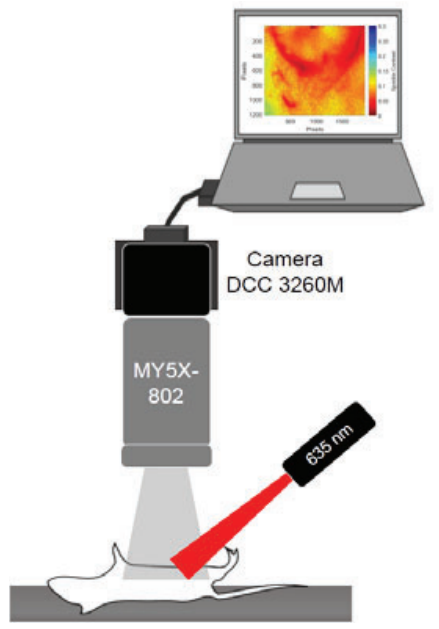

Figure 2. The schematic illustration of LSCI setup

$10 \mathrm{~mW}$ laser source working at $635 \mathrm{~nm}$ wavelength (Edmund Optics Inc., USA) was used to illuminate the object. CMOS-camera DCC 3260M (Thorlabs, Inc., USA) with $1936 \times 1216$ pixels and $5.86 \mu \mathrm{m}$ pixel size combined with $34 \mathrm{~mm}$ Mitutoyo Plan Apochromat Objective MY5X-802 (Thorlabs, Inc., USA) recorded raw speckle images. The obtained images were processed with a custom-developed algorithm in the offline mode. The standard spatio-temporal algorithm has been used for speckle contrast images obtaining. The calculation of the average speckle contrast of the image was performed using the Eq. 1:

$$
K=\left\langle\frac{\sigma_{N}}{\langle I\rangle_{N}}\right\rangle_{k},
$$

where \langle\rangle - the symbol of averaging; $N$ - the window of averaging $N \times N(N=7) ; k$ - the number of consecutive frames $(k=20) ;\langle I\rangle_{N}$ - average intensity in the window $N \mathrm{x} N ; \sigma_{N}-$ standard deviation in the window $N \mathrm{x} N$.

Experimental studies were performed on clinically healthy male Balb/c mice. Mice were obtained from the "Andreevka" vivarium FSBI SCBT FMBA of Russian
Federation. Before the transfer to the clear zone of the laboratory and the experiment, the animals were kept in quarantine for 14 days. When placed in quarantine, the veterinarian conducted a primary assessment of the animals condition with the introduction of the examination results in the relevant statement and then examined animals daily. The basic rules of maintenance and care corresponded to the standards of the sanitary rules for the arrangement, equipment and maintenance of experimental biological clinics and in the guide "Laboratory animals"15 and GLP principles.

During the study, mice were anesthetized with Zoletil 100 (Vibrac, France) at standard dose. Each animal was fixed on a special platform in the position on the back. A transverse laparotomy procedure was performed; the operative access was made to the upper part on the back wall of the abdominal cavity in the retroperitoneal space. After that, the complex of organs containing the pancreas was carried out. The body of the pancreas was isolated and ligature from polyester thread was placed. In addition, a cotton swab soaked in a $0.9 \%$ solution of sodium chloride was placed in the operating field. Then, the animal was placed under the optical system to visualize the area and record the frame sequences. After that, the fiber probe was placed in the middle of visualized area and three pairs of fluorescence spectra were registered. The registration of images and spectra was made before ligating and after 1, 6, 11 .. $66 \mathrm{~min}$ after ligating of pancreas vessels.

\section{Results and Discussion}

The setup showed in Figure 2 was used to record the sequence of frames in mice. For each stage, a sequence of 20 frames was recorded. After image processing with the space-time algorithm spectral contrast images were obtained (Fig. 3).

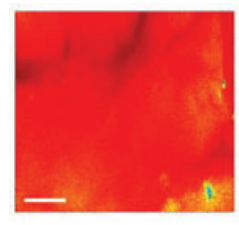

a)

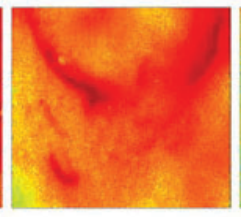

b)

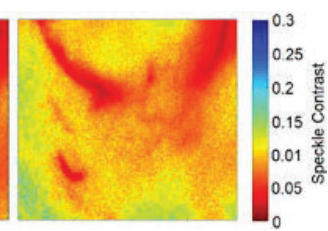

c)
Figure 3. Speckle images from mice before (a), $26 \mathrm{~min}$ (b) and 56 min (c) after ligating pancreas. Scale bar equals $2 \mathrm{~mm}$. 
The average contrast ratio of the speckle image in the area of 100x100 pixels was calculated using the Eq. 1 to acquire average speckle contrast values (Fig. 4).

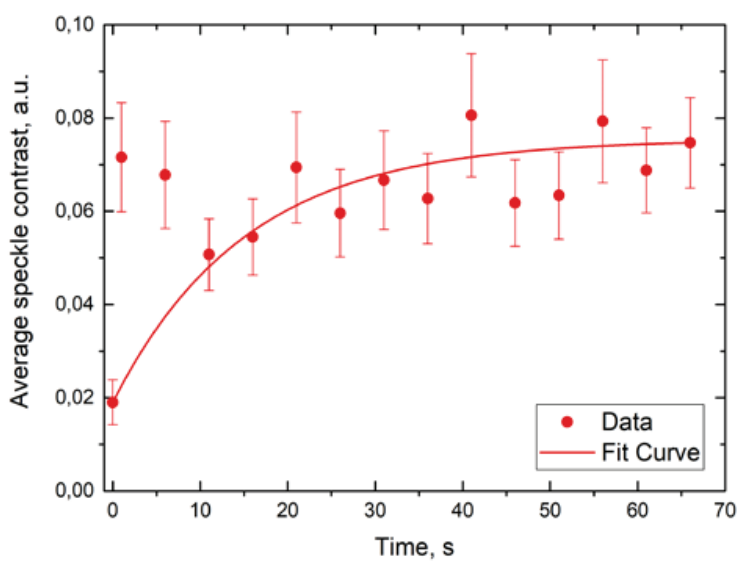

Figure 4. Average speckle contrast value of 100x100 pixels area during ligation process.

Figure 4 shows gradual increasing of speckle contrast value after ligating the pancreas, which indicates the slowing of blood flow and development of tissue ischemia. Slow change of speckle contrast and its oscillations indicate that the venous blood flow still occurred as the blood left the vessels remained without arterial supply.

The results of measurements by FS method are shown in Figure 5.

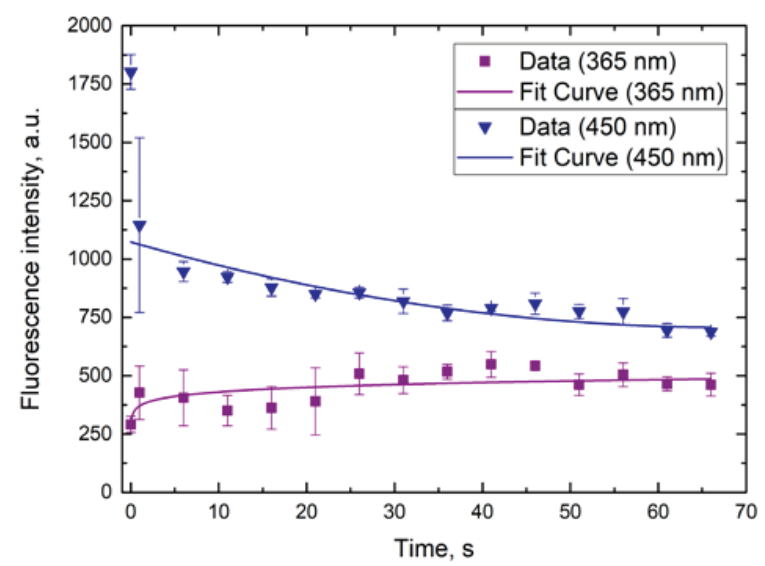

Figure 5. Average fluorescence intensity maxima measured during the experiment.

Figure 5 shows that intensity of fluorescence excited by $365 \mathrm{~nm}$ radiation increases as well as average speckle contrast. At the same time, the fluorescence excited by $450 \mathrm{~nm}$ radiation greatly decreased after ligation and continued to drop slowly. Such pattern indicate that progressing tissue ischemia has led to accumulation of $\mathrm{NADH}$, which is associated with a lack of oxygen. Meanwhile, the consumption of FAD increases which manifests in its fluorescence decrease. These effects are a sign of hypoxia and poor blood supply of pancreas tissue.

The Pearson correlation coefficient was calculated to prove the interconnection of data obtained. The results of analysis are presented in Table 1.

Table 1. Pearson correlation between fluorescence intensity maxima and average speckle contrast.

\begin{tabular}{|c|c|c|}
\hline $\begin{array}{c}\text { Data } \\
\text { Sample 1 }\end{array}$ & $\begin{array}{c}\text { Data } \\
\text { Sample 2 }\end{array}$ & $\begin{array}{c}\text { Pearson Correlation } \\
\text { Coefficient for } \mathbf{p} \leq \mathbf{0 , 0 1}\end{array}$ \\
\hline $\begin{array}{c}\text { Average } \\
\text { speckle } \\
\text { contrast }\end{array}$ & $\begin{array}{c}365 \mathrm{~nm} \\
\text { fluorescence }\end{array}$ & 0.6908 \\
\hline $\begin{array}{c}365 \mathrm{~nm} \\
\text { fluorescence }\end{array}$ & $\begin{array}{c}450 \mathrm{~nm} \\
\text { fluorescence }\end{array}$ & -0.67575 \\
\hline $\begin{array}{c}\text { Average } \\
\text { fluorescence }\end{array}$ & $\begin{array}{c}\text { speckle } \\
\text { contrast }\end{array}$ & -0.80386 \\
\hline
\end{tabular}

One can see almost high positive correlation between average speckle contrast and fluorescence emission (excited at $365 \mathrm{~nm}$, as well as negative high correlation between average speckle contrast and fluorescence emission (excited at $450 \mathrm{~nm}$ ) and between both of fluorescence intensities.

\section{Conclusion}

Obtained results prove the possibility of using multimodal approach to evaluate metabolic activity and microcirculation rate by both spectroscopic and imaging methods.

Combined measurements of fluorescence intensity and speckle contrast demonstrate close dependence of cell metabolism on oxygen supply. This result shows the necessity of further development of methodology and criteria for optical diagnostic for future prospects of clinical implementation. More research are necessary to reveal the correlation more clearly and find out the coefficients, which determines it. 
The experimental results of this study will be used for adjusting the techniques of FS and LSCI for application in standard minimally invasive surgery tools. Especially it is planned to integrate this techniques in a laparoscope. The addition of more spectroscopy (diffuse reflectance spectroscopy) and imaging (fluorescence imaging, hyperspectral imaging) methods for further studies seems promising as well.

\section{Acknowledgements}

This study was supported by the Russian Science Foundation under project № 18-15-00201.

\section{References}

[1] Al Mofleh, I. A., "Severe acute pancreatitis: Pathogenetic aspects and prognostic factors," World J. Gastroenterol. (2008).

[2] Broe, P. J., Zuidema, G. D. and Cameron, J. L., "The role of ischemia in acute pancreatitis: studies with an isolated perfused canine pancreas.," Surgery (1982).

[3] Prinz, R. A., "Mechanisms of acute pancreatitis Vascular etiology," Int. J. Pancreatol. (1991).

[4] Chahal, P. and Baron, T. H., "Endoscopic palliation of cholangiocarcinoma," Curr. Opin. Gastroenterol. 22(5), 551-560 (2006).

[5] Kandurova, K., Dremin, V. V., Zherebtsov, E. A., Dunaev, A. V., Mamoshin, A. V., Alyanov, A. L., Muradyan, V. F. and Potapova, E. V., "Application of the fluorescence spectroscopy for the analysis of the state of abdominal cavity organs tissues in mini-invasive surgery," Biophotonics Photonic Solut. Better Heal. Care VI (2018).

[6] Kennedy, G. T., Okusanya, O. T., Keating, J. J., Heitjan, D. F., Deshpande, C., Litzky, L. A., Albelda, S. M., Drebin, J. A., Nie, S., Low, P. S. and Singhal, S., "The optical biopsy: A novel technique for rapid intraoperative diagnosis of primary pulmonary adenocarcinomas," Ann. Surg. 262(4), 602-609 (2015).

[7] Lakowicz, J. R., [Principles of fluorescence spectroscopy] (2006).

[8] Bartolomé, F. and Abramov, A. Y., "Measurement of mitochondrial nadh and fad auto fluorescence in live cells," Methods Mol. Biol. 1264, 263-270 (2015).

[9] Potapova, E. V, Dremin, V. V, Zherebtsov, E. A.,
Makovik, I. N., Zherebtsova, A. I., Dunaev, A. V, Podmasteryev, K. V, Sidorov, V. V, Krupatkin, A. I., Khakhicheva, L. S. and Muradyan, V. F., "Evaluation of microcirculatory disturbances in patients with rheumatic diseases by the method of diffuse reflectance spectroscopy," Hum. Physiol. 43(2), 222-228 (2017).

[10] Nishidate, I., Ishizuka, T., Mustari, A., Yoshida, K., Kawauchi, S., Sato, S. and Sato, M., "Evaluation of Cerebral Hemodynamics and Tissue Morphology of in Vivo Rat Brain Using Spectral Diffuse Reflectance Imaging," Appl. Spectrosc. (2017).

[11] Essex, T. J. H. and Byrne, P. O., "A laser Doppler scanner for imaging blood flow in skin," J. Biomed. Eng. (1991).

[12] Sdobnov, A., Bykov, A., Popov, A., Meglinski, I. A. and Zherebtsov, E., "Investigation of speckle pattern dynamics by laser speckle contrast imaging," Biophotonics Photonic Solut. Better Heal. Care VI (2018).

[13] Dremin, V. V, Zherebtsov, E. A., Sidorov, V. V, Krupatkin, A. I., Makovik, I. N., Zherebtsova, A. I., Zharkikh, E. V, Potapova, E. V, Dunaev, A. V, Doronin, A. A., Bykov, A. V, Rafailov, I. E., Litvinova, K. S., Sokolovski, S. G. and Rafailov, E. U., "Multimodal optical measurement for study of lower limb tissue viability in patients with diabetes mellitus," J. Biomed. Opt. 22(8) (2017).

[14] Mayevsky, A., Walden, R., Pewzner, E., Deutsch, A., Heldenberg, E., Lavee, J., Tager, S., Kachel, E., Raanani, E., Preisman, S., Glauber, V. and Segal, E., "Mitochondrial function and tissue vitality: bench-tobedside real-time optical monitoring system," J. Biomed. Opt. (2011).

[15] Council of the Organisation for Economic cooperation and Development., "OECD Series on Principles of Good Laboratory Practice and Compliance Monitoring" (1998). 


\title{
Study on properties of near-infrared excitation upconversion nanoparticles for optogenetic applications
}

\author{
Tai-Ling Huang ${ }^{a}$, Wei-Che Li ${ }^{\text {a }}$, Jen-Hsuan Wang ${ }^{\text {a }}$, Chia-Yen Lee ${ }^{\mathrm{b}}$, Ching-Cheng Chuang \#, a Jung- \\ Chih Chen *, a \\ * Corresponding author; \# Co-corresponding author \\ ${ }^{\text {a }}$ Institute of Biomedical Engineering, National Chiao Tung University, Hsinchu 30010, Taiwan \\ ${ }^{\mathrm{b}}$ Department of Electrical Engineering, National United University, Miaoli 36063, Taiwan
}

\begin{abstract}
Lanthanide-doped upconversion nanoparticles (UCNPs) have attracted great attention for biomedical applications due to good biocompatibility and unique anti-Stokes optical property. Channelrhodopsin-2 (ChR2) was used to change membrane potential that can triggered by blue light. However, blue light can't penetrate human tissue directly. For purpose of controlling the cardiomyocytes to produce depolarization after ChR2 gene transfer. In this study, UCNP were used co-precipitation method to synthesis which convert near-infrared (NIR) into visible emission. In order to confirm the effect between the UCNP and ChR2 transgene in cardiomyocyte, we recorded preliminary membrane potential results by high speed camera. These results demonstrated that UCNP extended elongation of membrane potential under NIR excitation indeed. It showed that UCNP we prepared can be applied to cardiac optogenetics.
\end{abstract}

Keywords: Upconversion nanoparticles, Optogenetics, Near-infrared (NIR)

\section{Introduction}

Lanthanide-doped upconversion nanoparticles (UCNPs) can covert low-energy near-infrared (NIR) excitation to high energy visible emission that can active specific lightresponsive proteins like Channelrhodopsin-2 (ChR2) is one of an ion channel can enhance $\mathrm{Ca}^{2+}$ permeability [1]. Because penetration depth of NIR is deeper than visible [2], which can be a potential way in cardiac optogenetics. Commonly, most of UCNPs doped with $\mathrm{Yb}^{3+}$ are excited at $980 \mathrm{~nm}$ wavelength, but continuous laser irradiation only at $980 \mathrm{~nm}$ would lead a risk of substantial overheating because of water absorbs, low penetration depth and therefore cause tissue damaged [3] is a major obstacle of UCNPs. Considering the reason that excitation at $980 \mathrm{~nm}$ water absorbs around 20 times more than at $808 \mathrm{~nm}$ [4]. Therefore, it is necessary to develop UCNPs with high upconversion luminescence intensity at $808 \mathrm{~nm}$ can avoid overheating and enhanced upconversion luminescence. An excitation wavelength around $808 \mathrm{~nm}$ were developed by utilizing $\mathrm{Nd}^{3+}$ ions as sensitizers[5]. Furthermore, upconversion luminescence intensity of UCNPs is essential, so many researchers devoted to breakthrough the upconversion intensity and efficiency, symbolic way is to form a core-shell structure of UCNP.

In this study, we synthesized $\mathrm{NaYF}_{4}: \mathrm{Yb} / \mathrm{Tm} @$ $\mathrm{NaYF}_{4}: \mathrm{Yb} / \mathrm{Nd}$ core-shell structure with co-precipitation methode can excitation by $808 \mathrm{~nm}$, which can produce strong blue luminescence. We measured optical mapping of membrane potentials to test $\mathrm{ChR} 2$ transgene in cardiomyocyte triggered by UCNP which excited at 808 $\mathrm{nm}$ and $980 \mathrm{~nm}$ wavelength, also discussed characteristics of UCNP.

The results reveal UCNPs can assisting cardiac optogenetics. On the basis of these distinctive advantages, UCNPs have attracted biomedical interest that can control $\mathrm{ChR} 2$ transgene in cardiomyocyte in non-invasive way.

\section{Experimental}

\section{Materials}

Yttrium (III) acetate hydrate (99.9\%, ALFA), Ytterbium (III) acetate hydrate (99.9\%, ALFA), Thulium (III) acetate hydrate (99.9\%, ALFA), Neodymium (III) acetate hydrate (99.9\%, ALFA), Oleic acid (OA; 90\%, Aldrich), 1-Octadecene (ODE; 90\%, ACROS), Ammonium fluoride ( $98 \%$, Merck), Sodium hydroxide (98\%, Merck), Cyclohexane (99\%, Fisher), Methanol (99.9\%, MA), Hydrochloric acid (99\%, Sigma-Aldrich).

\section{Synthesis of NaYF4:Yb/Tm as a core structure}

core/shell structure upconversion noanoparticles were synthesized by co-precipitation method. As-synthesized 
core nanoparticles solution contained $\mathrm{OA}(6 \mathrm{~mL})$ and ODE $(15 \mathrm{~mL})$ first, then Yttrium acetate hydrate, Ytterbium acetate hydrate and Thulium acetate hydrate was added and stirred at $45{ }^{\circ} \mathrm{C}$ for 60 mins. The mixture was heated to $150{ }^{\circ} \mathrm{C}$ for $1 \mathrm{hr}$ to form yellow solution. After cooled down to $45^{\circ} \mathrm{C}, 7.9 \mathrm{~mL}$ of $\mathrm{NaOH}$ and $2 \mathrm{~mL}$ of $\mathrm{NH}_{4} \mathrm{~F}$ was added to the solution stirred for $1 \mathrm{hr}$. Then solution was heated at $110{ }^{\circ} \mathrm{C}$ to remove methanol for 20 mins. Next the solution was heated to $280^{\circ} \mathrm{C}$ with $1.5 \mathrm{hrs}$ under the argon atmosphere. Finally, the solution were collected by centrifugation for 10 mins and stock in cyclohexane.

\section{Synthesis of NaYF4:Yb/Tm@NaYF4:Yb/Nd core/sell structure}

To prepare the shell solution, a flask contained OA (5.1 $\mathrm{mL})$ and ODE $(11.9 \mathrm{~mL})$, then Yttrium acetate hydrate, Ytterbium acetate hydrate and Neodymium acetate hydrate was added and stirred at $45{ }^{\circ} \mathrm{C}$ for 30 mins. Subsequently heated to $150{ }^{\circ} \mathrm{C}$ for $1 \mathrm{hr}$. After cooled down to $45^{\circ} \mathrm{C}$, the as-synthesized $\mathrm{NaYF}_{4}: \mathrm{Yb} / \mathrm{Tm}$ core nanoparticles solution $(6 \mathrm{~mL}), \mathrm{NaOH}(1.7 \mathrm{~mL})$ and $\mathrm{NH}_{4} \mathrm{~F}(6.715 \mathrm{~mL})$ was added to the flask stirred at $45{ }^{\circ} \mathrm{C}$ for $1 \mathrm{hr}$. Then the solution was heated at $110{ }^{\circ} \mathrm{C}$ to remove methanol for 20 mins. Finally heated to $305{ }^{\circ} \mathrm{C}$ continuously stirred for $1.5 \mathrm{hrs}$ under the argon atmosphere. At the end, the solution were collected by centrifugation for 10 mins and stock in cyclohexane.

\section{Results and Discussion}

\section{Characteristics of UCNP}

$\mathrm{NaYF}_{4}: \mathrm{Yb} / \mathrm{Tm} @ \mathrm{NaYF}_{4}: \mathrm{Yb} / \mathrm{Nd}$ were analyzed by FE-SEM. We could see the morphology of the UCNPs were hexagonal, and the size distribution were highly uniform (see Figure 1), the diameter of core/shell nanoparticles were around $80 \mathrm{~nm}$.

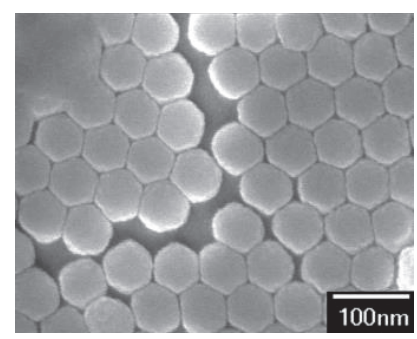

Fig. 1. SEM images of $\mathrm{NaYF}_{4}: \mathrm{Yb} / \mathrm{Tm} @ \mathrm{NaYF}_{4}: \mathrm{Yb} / \mathrm{Nd}$

In order to analysis elements of noanoparticles, $\mathrm{NaYF}_{4}: \mathrm{Yb} / \mathrm{Tm} @ \mathrm{NaYF}_{4}: \mathrm{Yb} / \mathrm{Nd}$ were further analyzed by energy dispersive X-ray spectrometer (EDS) to determine the elements of nanoparticles. As shown in Fig. 2, the UCNPs did really be synthesized and the elements actually include $\mathrm{Yb}, \mathrm{Tm}$ and $\mathrm{Nd}$.

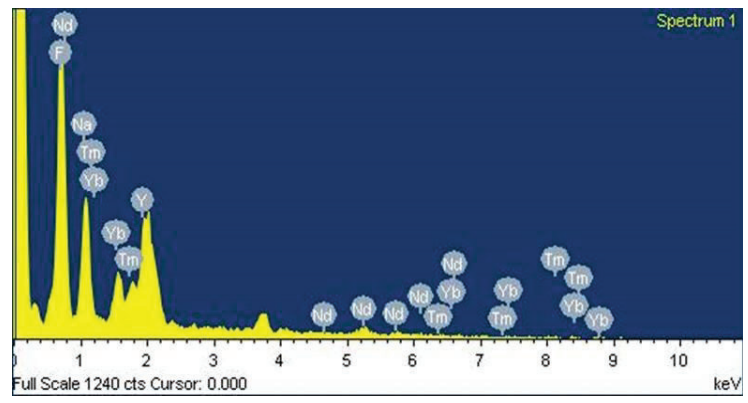

Fig. 2. Elements analysis of

$\mathrm{NaYF}_{4}: \mathrm{Yb} / \mathrm{Tm} @ \mathrm{NaYF}_{4}: \mathrm{Yb} / \mathrm{Nd}$ by EDS

measurement

UV-VIS spectroscopy was used to detect the absorption peaks of $\mathrm{NaYF}_{4}: \mathrm{Yb} / \mathrm{Tm} @ \mathrm{NaYF}_{4}: \mathrm{Yb} / \mathrm{Nd}$ nanoparticles in Figurs 3. We could see that core/shell nanoparticles had absorption peaks near $794 \mathrm{~nm}$ and $804 \mathrm{~nm}$, because the absorption peaks of $\mathrm{Nd}^{3+}$ ions is around $800 \mathrm{~nm}$, and absorption cross-section of $\mathrm{Nd}^{3+}$ ions at $800 \mathrm{~nm}$ is 25 -fold larger than that of $\mathrm{Yb}^{3+}$ [4]. So that we could use nearinfrared laser with an excitation wavelength of $808 \mathrm{~nm}$ in this experiment.

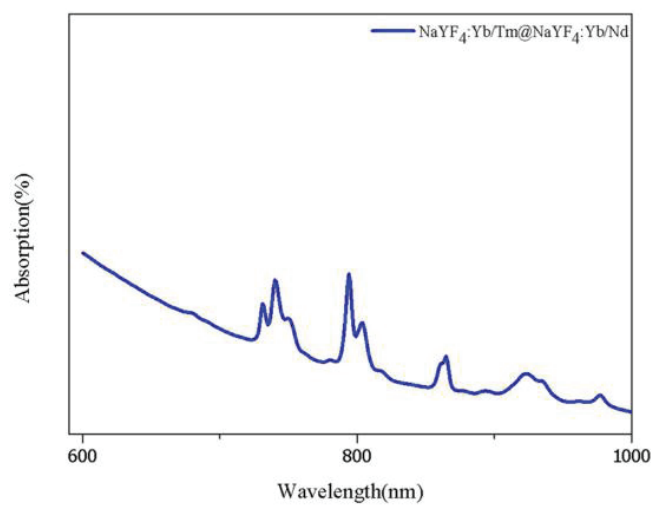

Fig. 3. UV-VIS spectra of $\mathrm{NaYF}_{4}: \mathrm{Yb} / \mathrm{Tm} @ \mathrm{NaYF}_{4}: \mathrm{Yb} / \mathrm{Nd}$

We used Photoluminescence to see the spectra of emission peaks. As shown in Fig. 4, core/shell nanoparticles were excited by $808 \mathrm{~nm}$ laser $(1 \mathrm{~W})$, which could be converted to $470 \mathrm{~nm}$ blue light emission. Because $\mathrm{Nd}^{3+}$ ions can absorb $808 \mathrm{~nm}$ near-infrared laser energy $\left({ }^{4} \mathrm{I}_{9 / 2} \rightarrow{ }^{4} \mathrm{~F}_{5 / 2}\right)$ to inner sensitizer $\mathrm{Yb}^{3+}$ 
$\left({ }^{2} \mathrm{~F}_{7 / 2} \rightarrow{ }^{2} \mathrm{~F}_{5 / 2}\right)$, finally transfer energy to activator $\mathrm{Tm}^{3+}$ $\left({ }^{1} \mathrm{G}_{4} \rightarrow{ }^{3} \mathrm{H}_{6}\right)$ then release energy light.

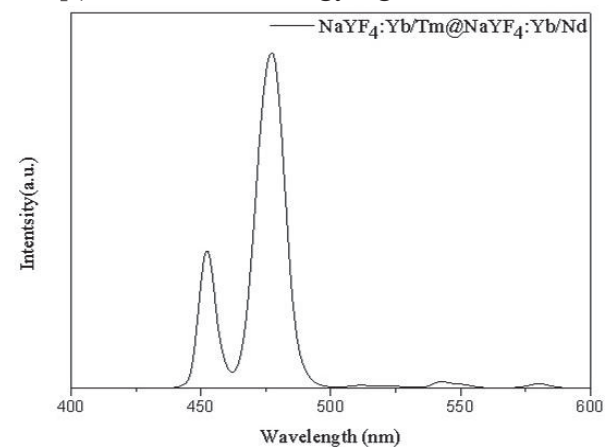

Fig. 4. Spectra of $\mathrm{NaYF}_{4}: \mathrm{Yb} / \mathrm{Tm} @ \mathrm{NaYF}_{4}: \mathrm{Yb} / \mathrm{Nd}$ by Photoluminescence spectroscopy

\section{Optical mapping for the change of cell membrane potential}

After virus transfection, the change of membrane potential of cardiomyocytes were detected by optical mapping (see Figure 5), cells were incubated with $\mathrm{NaYF}_{4}: \mathrm{Yb} / \mathrm{Tm} @ \mathrm{NaYF}_{4}: \mathrm{Yb} / \mathrm{Nd}$ for immediately and 6 hrs, and then stained with the potential-sensitive dye. Core/shell UCNPs were excited by $808 \mathrm{~nm}$ and $980 \mathrm{~nm}$. We could see the action potential duration (APD) of the cell membrane potential was significantly longer than control. This demonstrated that the opening of the ion channel ChR2 after activation require a period of time. From the results, the prolongation of APD might cause the pacing frequency of cardiomyocytes to slow down.

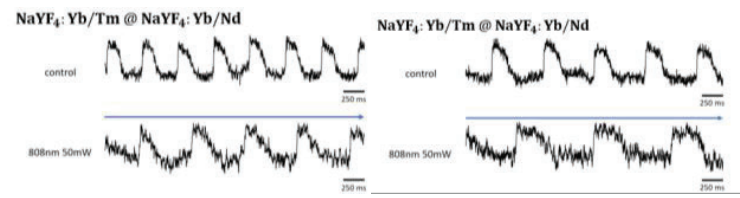

Fig. 5. Optical mapping images of $\mathrm{NaYF}_{4}: \mathrm{Yb} / \mathrm{Tm} @ \mathrm{NaYF}_{4}: \mathrm{Yb} / \mathrm{Nd}$ in cardiomyocytes (left: immediately, right: 6 hours)

\section{Conclusions}

In this study, we successfully synthesize a core/shell $\mathrm{NaYF}_{4}: \mathrm{Yb} / \mathrm{Tm} @ \mathrm{NaYF}_{4}: \mathrm{Yb} / \mathrm{Nd}$ nanoparticles for optogenetics applications through thermal coprecipitation process. We can see that the size of core/shell UCNPs are around $80 \mathrm{~nm}$; and core/shell UCNPs can covert $808 \mathrm{~nm}$ and $980 \mathrm{~nm}$ excitation to $470 \mathrm{~nm}$ emission. For optical mapping, $808 \mathrm{~nm}$ and 980 $\mathrm{nm}$ NIR are able to excite UCNPs controlling cardiomyocytes. Preliminary results expressed that excitation of upconversion nanoparticles provide a good potential for cardiac optogenetics pacing.

\section{References}

[1] J. S. Meyer and L. F. Quenzer., [Psychopharmacology: Drugs, the brain, and behavior], Sinauer Associates, Incorporated, 2013.

[2] A. Bansal, H. Liu, M. K. Jayakumar, S. AnderssonEngels, and Y. Zhang, "Quasi-Continuous Wave NearInfrared Excitation of Upconversion Nanoparticles for Optogenetic Manipulation of C. elegans," Small, vol. 12,no. 13, pp. 1732-1743, Apr 062016.

[3] Ye-Fu Wang, Gao-Yuan Liu, Ling-Dong Sun, JiaWen Xiao, Jia-Cai Zhou, and Chun-Hua Yan, " $\mathrm{Nd}^{3+}{ }_{-}$ Sensitized Upconversion Nanophosphors: Efficient In Vivo Bioimaging Probes with Minimized Heating Effect [4] Wen, Shihui, et al. "Advances in highly doped upconversion nanoparticles." Nature communications 9.1 (2018): 2415.

[5] M. K. Jayakumar, N. M. Idris, K. Huang, and Y. Zhang, "A paradigm shift in the excitation wavelength of upconversion nanoparticles," Nanoscale, vol. 6, no. 15, pp. 8441-8443, Aug 072014.

[6] J. G. Bernstein and E. S. Boyden, "Optogenetic tools for analyzing the neural circuits of behavior," Trends in cognitive sciences, vol. 15, no. 12, pp. 592-600, 2011

[7] T. Bruegmann et al., "Optogenetic control of heart muscle in vitro and in vivo," Nat Methods, vol. 7, no. 11, pp. 897-900, Nov 2010.

[8] S. A. Park, S.-R. Lee, L. Tung, and D. T. Yue, "Optical mapping of optogenetically shaped cardiac action potentials," Scientific reports, vol. 4, 2014.

[9] J. G. Bernstein and E. S. Boyden, "Optogenetic tools for analyzing the neural circuits of behavior," Trends in cognitive sciences, vol. 15 , no. 12, pp. 592-600, 2011 


\title{
Tapered optical fiber temperature sensor coated with DNA based biopolymer
}

\author{
Sanggwon Song, Seongjin Hong, Aeri Jung and Kyunghwan Oh* \\ Photonics Device Physics Laboratory, Institute of Physics and Applied Physics, Yonsei University, \\ 50 Yonsei-ro Seodaemun-gu, Seoul, 120-749, Republic of Korea
}

\begin{abstract}
We demonstrated a biocompatible temperature sensor using single mode tapered fiber and DNA-CTMA(DNA-Cetyl tri-methyl ammonium) solid film. DNA-CTMA was coated on the tapered region, which is an interferometer. The spectral change with temperature was measured due to the refractive index changes of DNA-CTMA according to temperature. The range of the temperature change was from 35 to $65^{\circ} \mathrm{C}$, and the sensitivity was $-0.91 \mathrm{~nm} /{ }^{\circ} \mathrm{C}$. Furthermore, confirmed that the stain sensitivity of this sensor was $-19 \mathrm{pm} / \mathrm{u} \varepsilon$.
\end{abstract}

Keywords: Fiber Sensor, Fiber optics, Interferometer, Biomaterials, DNA

\section{Introduction}

Fiber optic sensors are promising alternative as a biosensor because of its non-toxic bio-compatibility inherent electromagnetic wave immunity, mechanical flexibility, and small form factor ${ }^{1-3}$. Especially, tapered optical fiber sensors have been researched due to its small form factor and high temperature sensitivity of -3.88 $\mathrm{nm} / \mathrm{C}$ compared to other types of optical fiber sensor such as FBG(Fiber bragg grating), and EFPI(extrinsic FabryPerot interferometer).

Recently the authors have introduced an optical fiber sensor based on DNA-CTMA thin solid film ${ }^{4,5}$. It shows a high spectral shift $-0.22 \mathrm{~nm} / \mathrm{C}$, and small form factor $14.5 \mathrm{~mm}$. We adopted the tapered type of optical fiber sensor, which enables highly localized micro sensing and higher spectral sensitivity depending on temperature.

In this paper, we report a smaller size, and higher spectral sensitivity of temperature optical fiber sensor based on tapered optical fiber, and DNA-CTMA film.

\section{Experimental set up and Results}

The tapered fiber we used is shown as in Figure 1. a DNA-CTMA film was coated on the tapered region. The film has a thermo-optic coefficient that changes with temperature. ${ }^{4,5}$
The fabrication process of the sensor was made by tapering the SMF using a Vytran(GPX-3000) and dropcasting the DNA-CTMA: BuOH solution on the tapered section. It is then completed by drying and baking.

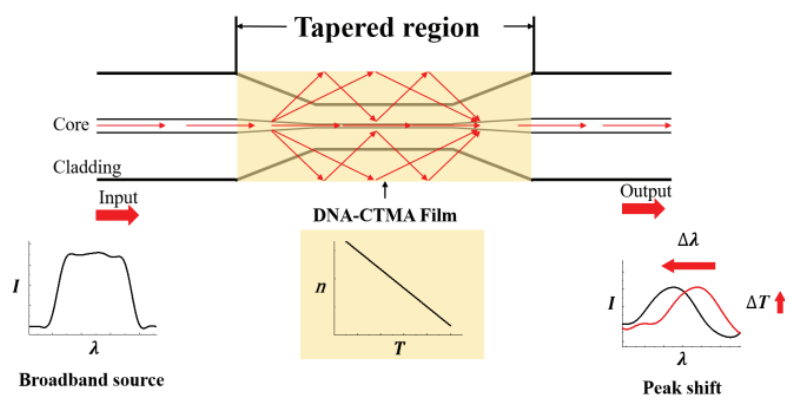

Figure 1. Schematic diagram of tapered fiber

Temperature sensor with DNA-CTMA film.

When the broadband source is injected into this sensor, the higher order mode and the fundamental mode are combined to form an interference pattern after passing through the tapered region, and the spectrum shift according to the surrounding environment can be observed through the optical spectrum analyzer(OSA). We show a schematic diagram of this experiment in Figure 2. the sensor was immersed in a water bath to simulate the biocompatible. 


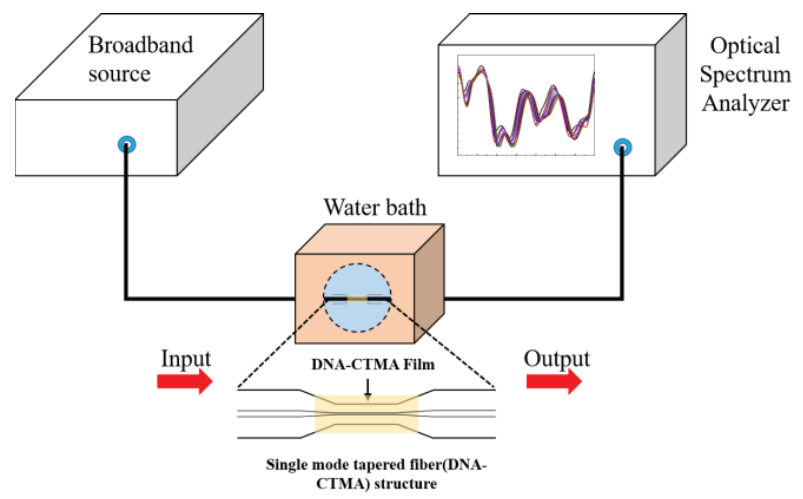

Figure 2. Schematic diagram of the experimental measurement setup.
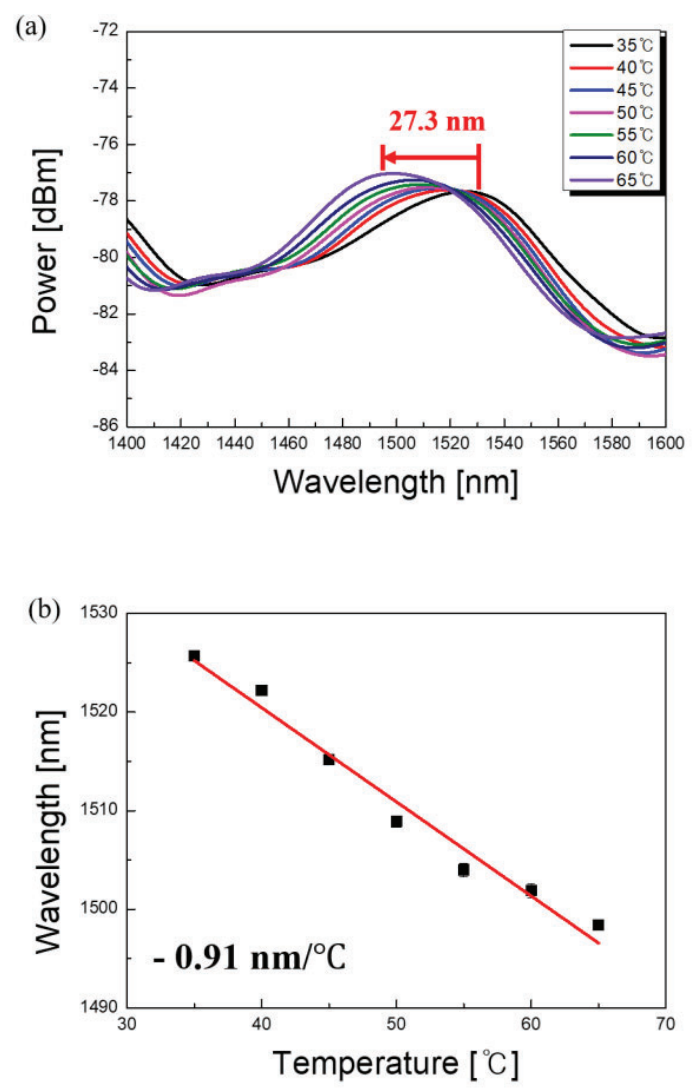

Figure 3. (a) Spectral shifts between $35^{\circ} \mathrm{C}$ and $65^{\circ} \mathrm{C}$, (b) temperature dependence of wavelength.
The temperature was controlled from 35 to $65^{\circ} \mathrm{C}$. The DNA-CTMA film is not dissolved in water and Figure 3 shows the shift of the spectra measured according to the temperature changes. The spectrum was blue shifted by $27.3 \mathrm{~nm}$ and the temperature sensitivity of the sensor was $-0.91 \mathrm{~nm} /{ }^{\circ} \mathrm{C}$. Figure 4 shows the strain sensitivity of the fabricated sensor and a linear slope of $-19 \mathrm{pm} / \mathrm{u} \varepsilon$ was obtained.

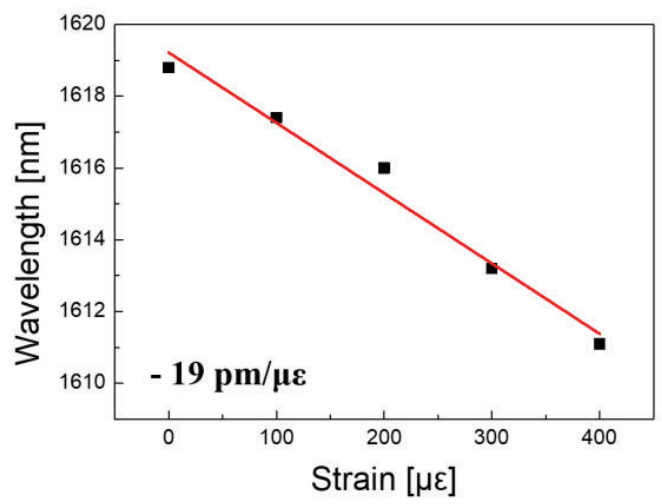

Figure 4. Strain dependence of wavelength.

\section{Conclusion}

In conclusion, we demonstrated a temperature sensor range from 35 to $65^{\circ} \mathrm{C}$ using DNA-CTMA with tapered fiber and thermo-optic coefficient. The total length of the sensor is $2.5 \mathrm{~mm}$ and the length of the tapered region is $350 \mathrm{um}$. The temperature sensitivity of the sensor was $0.91 \mathrm{~nm} / \mathrm{C}$, and the strain sensitivity was $-19 \mathrm{pm} / \mathrm{u} \varepsilon$, which is very small and has a high sensitivity biocompatible sensor.

\section{References}

[1] Lee, B. "Review of the present status of optical fiber sensors." Optical fiber technology 9(2), 57-79 (2003).

[2] Tsai, W. H., and Chun, J. L. "A novel structure for the intrinsic Fabry-Perot fiber-optic temperature sensor." Journal of Lightwave Technology 19(5), 682-686 (2001). [3] Xue, Y., Yu, Y. S., Yang, R., Wang, C., Chen, C., Guo, J. C., Zhang, X. Y., and Sun, H. B. "Ultrasensitive temperature sensor based on an isopropanol-sealed optical microfiber taper." Optics letters 38(8), 1209-1211 (2013). 
[4] Hong, S., Jung, W., Nazari, T., Song, S., Kim, T., Quan, C., and Oh, K. "Thermo-optic characteristic of DNA thin solid film and its application as a biocompatible optical fiber temperature sensor." Optics letters 42(10), 1943-1945 (2017).

[5] Hong, S., Jung, W., Kim, T., and Oh, K. "Compact Biocompatible Fiber Optic Temperature Microprobe Using DNA-Based Biopolymer." Journal of Lightwave Technology 36(4), 974-978 (2018).

Proc. of SPIE Vol. $111401114001-222$ 


\title{
High spatial resolution ion imaging with potentiometric sensor using an electron beam*
}

\author{
Wataru Inami*a,b, Kiyohisa Nii ${ }^{b}$, Satoru Shibano ${ }^{\mathrm{c}}$, Hikaru Tomita ${ }^{\mathrm{b}}$, Yohimasa Kawata ${ }^{\mathrm{a}, \mathrm{b}}$ \\ ${ }^{a}$ Research Institute of Electronics, Shizuoka University, 3-5-1 Johoku Hamamatsu 432-8011, Japan \\ ${ }^{\mathrm{b}}$ Graduate School of Integrated Science and Technology, \\ Shizuoka University, 3-5-1 Johoku, Hamamatsu 432-8561, Japan \\ ${ }^{c}$ Graduate School of Science and Technology, Shizuoka University, 3-5-1 Johoku, Hamamatsu 432- \\ 8561, Japan
}

\begin{abstract}
We have developed high spatial resolution ion imaging system using a focused electron beam. The system consists of inverted electron microscope and electrolyte/insulator/semiconductor (EIS) structure. The ion sensor is formed by depositing an ion sensitive membrane on a n-type (or p-type) silicon substrate. The flat band voltage shift of the ion sensor substrate is caused when ions attach to the sensor surface. The flat band voltage shift can be measured by irradiating the focused electron beam. The spatial resolution of the system depends on the spot size of the irradiated beam. Since the spot size of the focused electron beam is very small, high spatial resolution can be obtained.
\end{abstract}

Keywords: ion imaging, high resolution, potentiometric sensor, chemical sensor, biosensor

\section{Introduction}

The LAPS is an ion imaging sensor using light ${ }^{1}$. The spatial resolution of the LAPS is determined by the spot size of focused light and the lateral diffusion of photocarriers generated by the light irradiation in the semiconductor substrate. By using an electron beam with a spot size smaller than the diffraction limit of the light, the spatial resolution is much improved.

\section{Ion imaging by potentiometric sensor using focused electron beam}

\section{Measurement system}

Figure 1 shows the schematic diagram of an ion imaging system using a potentiometric sensor with focused electron beam. To increase the spatial resolution, a focused electron beam is used to measure the ion concentration. In the case of using an electron beam, since the electron beam can be focused to a spot of nm size, the spatial resolution is greatly improved. The principle of ion concentration measurement of this system is the same as LAPS. The ion imaging system using electron beam also measures flat band voltage shift of an ion sensor substrate like LAPS. The flat band voltage shift occurs when ions adhere to the ion sensitive layer surface. This amount of the shift depends on the interface potential between the ion sensitive membrane and the electrolyte.

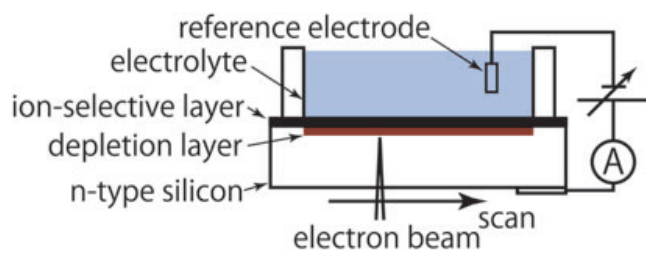

Figure 1. The schematic diagram of the high spatial resolution ion imaging system using focused electron beam.

A bias voltage is applied to an ion sensor by the power supply to form depletion state. The ion sensor is composed of semiconductor silicon and an ion sensitive membrane. Aluminum was deposited on the backside of the ion sensor as an electrode using a vacuum thermal evaporator. As the hydrogen ion sensitive membrane, materials such as $\mathrm{SiO}_{2}, \mathrm{Si}_{3} \mathrm{~N}_{4}, \mathrm{Al}_{2} \mathrm{O}_{3}, \mathrm{Ta}_{2} \mathrm{O}_{5}$, etc. are used. Other ions can also be measured by changing these materials of the ion selective layer. When irradiated with focused electron beam, the electron-hole pair is excited in the ion sensor, charge separation occurs, and current flows in the external circuit. The current flowing through the external circuit is amplified by the I-V amplifier and the voltage is acquired in a computer using $\mathrm{A} / \mathrm{D}$ converter. 
A 2D image of ion concentration distribution is constructed by raster scanning the focused electron beam.

\section{Principle of ion concentration measurement}

Figure 2 shows principle of ion concentration measurement by electron beam irradiation. A bias voltage is applied to the ion sensor substrate, and a depletion layer is formed below the ion selective layer. When ions adhere to the ion sensitive layer ( $\mathrm{SiN}$ ) surface of the ion sensor having the depletion layer, the interface potential between the ion sensitive layer and the electrolyte is shifted. This shift changes the bias voltage. Due to this shift, the bias voltage applied to the ion sensor substrate changes, and the thickness of the depletion layer changes. The ion adsorption - desorption on the surface of the ion sensitive membrane is explained by the binding model $^{2}$. The amount of current flowing through the external circuit depends on the thickness of the depletion layer. Therefore, by measuring the current amount, the ion concentration depending on the thickness of the depletion layer can be obtained. (a)

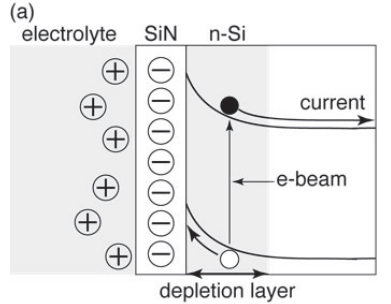

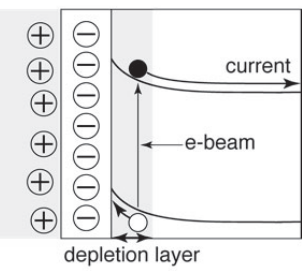

Figure 2. Change in depletion layer thickness due to $\mathrm{Ph}$ difference. (a) high $\mathrm{pH}$, (b) low $\mathrm{pH}$.

\section{Experiment result and discussion}

\section{Evaluation the spatial resolution}

We evaluated the spatial resolution of the ion imaging system using knife-edge spatial resolution test. To fabricate the knife-edge, half of the sensor surface was covered with photoresist. The sensor part not covered with photoresist can measure ion concentration. The sensor part covered with photoresist is not responsive to ions. A pH standard solution was dropped onto the ion sensor. Figure 3 shows the experimental result of the knife-edge spatial resolution test. In Fig. 3(a), the top left part of the image is dark, and the bottom right part is bright. The dark part is covered with photoresist. From this result, it was found that the ion imaging system using focused electron beam can map the ion distribution. Figure 3(b) shows the line profile along the yellow rectangle in the edge image of Fig. 3(a). Here, the spatial resolution was given by calculating the distance between the points of $20 \%$ and $80 \%$ of the maximum intensity value of the line profile curve. From the result, the spatial resolution of the ion imaging system we developed was estimated to be $60 \mu \mathrm{m}$. This resolution is much lower than the spot size. The cause is scattering of the electron beam, because we confirmed that the electron beam is greatly scattered in the ion sensor by Monte-Carlo simulation ${ }^{3}$.

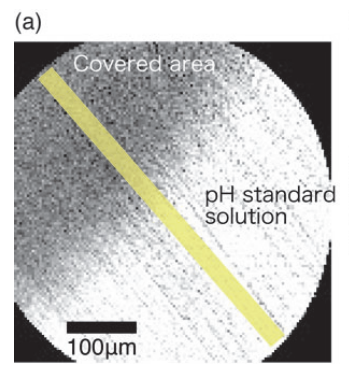
(b)

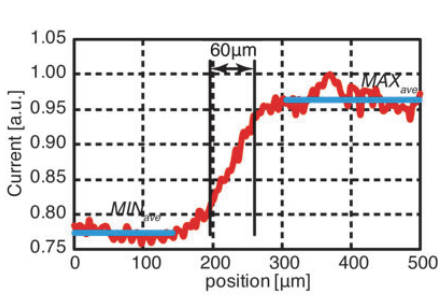

Figure 3. The experimental result of the knife-edge spatial resolution test of the ion imaging system using electron beam.

\section{Conclusions}

We have developed the high spatial resolution ion imaging system and evaluated the spatial resolution. We succeeded in obtaining the ion concentration distribution image with the system using an electron beam. From the result of knife-edge spatial resolution test, the spatial resolution of the system was $60 \mu \mathrm{m}$. Degradation in the spatial resolution occurs due to electron beam scattering and diffusion of carriers inside the ion sensor substrate. The spatial resolution of the current system is limited by the scattering of the electron beam, not the spot size. Therefore, it is possible to improve the spatial resolution by thinning the ion sensor substrate.

\section{Acknowledgement}

Funding from the A-STEP is gratefully acknowledged.

\section{References}

[1] Hafeman, D. G., Parce, J. W. and McConnell, H. M., "Light-addressable potentiometric sensor for biochemical systems," Science 240, 1182-1185 (1988).

[2] Harame, D. L., Bousse, L. J., Shott, J. D. and Meindl, J. D., "Ion-sensing devices with silicon nitride and 
borosilicate glass insulators," IEEE Trans. Electron Dev. ED-34, 1700 - 1707 (1987).

[3] Joy, D. C., [Monte Carlo Modeling for Electron Microscopy and Microanalysis], Oxford Univ. Press, New York, Oxford, (1995). 


\title{
Single-shot recording of both front and rear surfaces of object by digital holography using a polarization-imaging camera *
}

\author{
Kohei Arao*a ${ }^{\mathrm{a}}$, Tatsuya Hirakawa ${ }^{\mathrm{a}}$, Takahito Fukuda ${ }^{\mathrm{a}}$, Shogo Mochida ${ }^{\mathrm{a}}$, Yasuhiro Awatsuji ${ }^{\mathrm{a}}$, \\ Kenzo Nishio ${ }^{\mathrm{a}}$, Osamu Matoba ${ }^{\mathrm{b}}$ \\ ${ }^{a}$ Kyoto Institute of Technology, Matsugasaki Goshokaido-cho, Sakyo-ku, Kyoto 606-8585, Japan \\ ${ }^{\mathrm{b}}$ Kobe University, Rokkodai 1-1, Nada, Kobe 657-8501, Japan
}

\begin{abstract}
We propose a technique based on digital holography that can record three-dimensional images of both front and rear surfaces of an object with a single-shot exposure using a polarization-imaging camera. Two object waves from both the surfaces and the reference wave are introduced to the camera simultaneously. The polarization of the two object waves are adjusted to orthogonal each other. We can record two interference fringe images containing the information of both the surfaces as a single hologram using the polarization-imaging camera by a space division multiplexing of the holograms. We have succeeded in experimentally demonstrating the proposed method for the case of the recording both surfaces of a die.
\end{abstract}

Keywords: holography, digital holography, three-dimensional image, polarization, omini-directional image

\section{Introduction}

Three-dimensional (3D) imaging is a topic of significant interest. In recent years, digital holography [1] has been researched as a hopeful technique of $3 \mathrm{D}$ imaging. In general, only one surface of an object can be recorded by using this technique, because only the surface is illuminated. In order to record both front and rear surfaces of the object, use of multiple cameras or rotating the object on stage is needed. However, recording the $3 \mathrm{D}$ images of both the surfaces with a single-shot exposure using a single camera is difficult by applying these methods.

In this paper, we propose a method to record the $3 \mathrm{D}$ images of both the surfaces of the object with a singleshot exposure by digital holography using a single camera. Also we experimentally demonstrate the proposed method.

\section{Proposed method}

Figure 1 shows a schematic of a principle of the proposed method. Two object waves from both front and rear surfaces of an object are introduced to a single camera. The polarization of the waves is adjusted to orthogonal to each other. Two interference fringe images are generated on the plane of the camera. One is resulted from the superposition of the object wave from the front surface and the reference wave. The other is resulted from the superposition of the object wave from the rear surface and the reference wave. We record the two fringe images using a polarization-imaging camera. Because a micro-polarizer array is attached on this camera as shown in Fig.1, we can simultaneously record the two interference fringe images generated by the two linearlypolarized light wave on pixel-by-pixel using this camera, independently. Consequently, we can record two holograms containing the information of both the surfaces with a single-shot exposure by space-division multiplexing of the holograms.

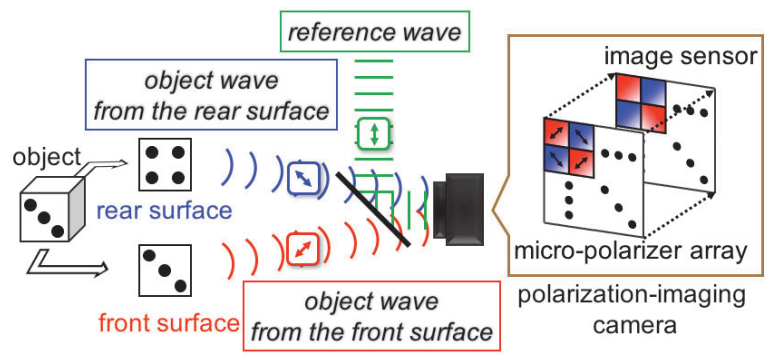

Figure 1. Schematic of a principle of a proposed method.

Figure 2 shows a schematic flow of the reconstruction of the two images of both front and rear surfaces of the object. First, the pixels detecting each linearly-polarized 
light waves are extracted from the recorded hologram and the extracted pixels are rearranged in each image corresponding to each linearly polarized light. Second, we get two holograms containing the information of both the surfaces by interpolating the blanked pixels. Finally, by applying the image reconstruction algorithm of digital holography to the holograms [2], we obtain two reconstructed images.

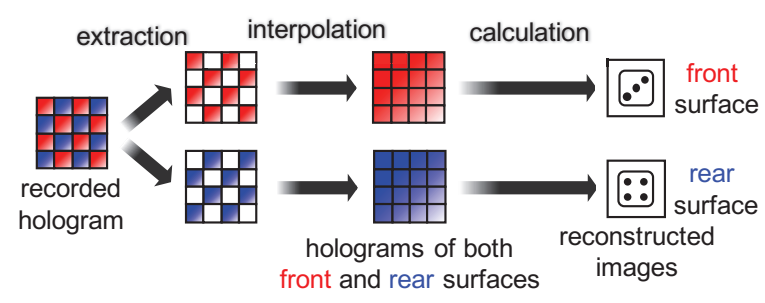

Figure 2. Schematic flow of the reconstruction of the two images of both front and rear surfaces of an object.

\section{Experimental setup}

Figure 3 shows an example of the proposed method. We can make the two object waves from the front and rear surfaces the orthogonal polarization each other by using a polarizing beam splitter PBS2 and two quarter wave plates QWP1 and QWP2. After that, we record two holograms generated by the object wave from the front surface and the reference wave and by the object wave from the rear surface and the reference wave using the polarization-imaging camera. Every $2 \times 1$ pixels of the camera have a $2 \times 1$ micro-polarizer, which was developed for parallel two-step phase-shifting digital holography [3]. We can reconstruct two $3 \mathrm{D}$ images of the both surfaces from the single hologram recorded by the camera. In addition, we set a slight angle between two object waves and reference wave by tilting the beam splitter in this setup in order to separate the desired image from the unwanted images, which are the 0th order diffraction wave and the conjugate image.

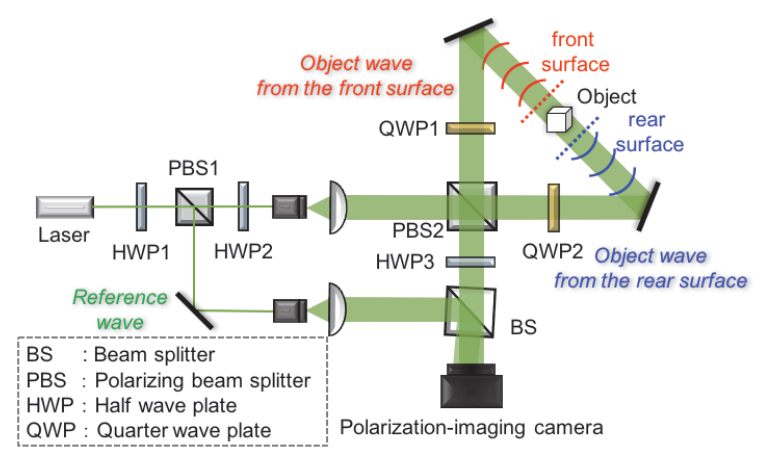

Figure 3. An example of the optical system of the single-shot recording of both front and rear surfaces of an object using a polarization-imaging camera.

\section{Experimental result}

We used a Nd: $\mathrm{YVO}_{4}$ laser operated at $532 \mathrm{~nm}$ for a light source. The angle between the object waves and the reference wave was set at $2^{\circ}$.

Figure 4 shows the reconstructed images of the front and rear surfaces of the object. We recorded a die whose the front surface and the rear surface are defined as 3 and 4, respectively. As shown in Figs.4 (a) and (b), we can recognize 3 and 4 on the reconstructed image of the front surface and that of the rear surface, respectively. Thus, we succeeded in recording both the surfaces of the object with a single-shot exposure using the proposed method.

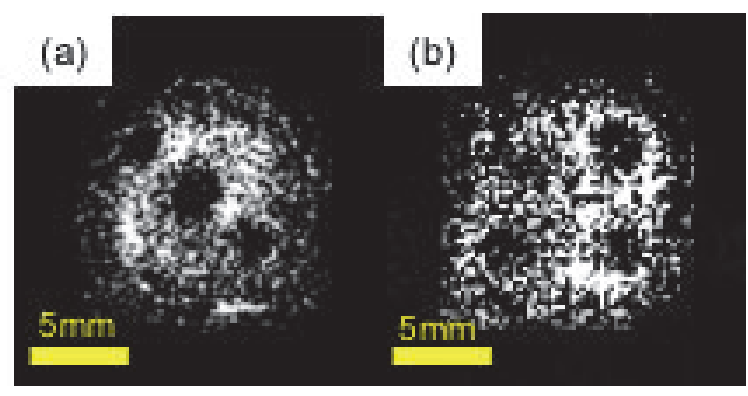

Figure 4. Reconstructed images of both front and rear surfaces of the object. (a) Front surface (b) rear surface

\section{Conclusion}

We have proposed the method for recording both the surfaces of the object with a single-shot exposure by digital holography using a polarization-imaging camera. 
Also we have succeeded in experimentally demonstrating the proposed method. This technique will contribute 3D imaging of dynamic objects in a wide variety of applications including biomedical application such as living cells, particles in flows.

This study was partially supported by Japan Society for the Promotion of Science (JSPS) KAKENHI Grant-in-Aid for Challenging Exploratory Research.

\section{References}

[1] J. W. Goodman, and R. W. Lawrence, Appl. Phys. Lett. 11, 77 (1967).

[2] M. Takeda, et al., J. Opt. Soc. Am. 72, 156 (1982).

[3] T. Tahara, et al., Opt. Express 18, 18975 (2010). 


\title{
Imaging of sound by a digital holographic microscope
}

\author{
Kazuki Shimizu ${ }^{\mathrm{a}}$, Yuki Takase ${ }^{\mathrm{a}}$, Peng Xia ${ }^{\mathrm{b}}$ Yasuhiro Awatsuji*a, Kenzo Nishio ${ }^{\mathrm{a}}$, \\ Sudheesh K. Rajput ${ }^{\mathrm{c}}$, Osamu Matoba ${ }^{\mathrm{c}}$ \\ ${ }^{a}$ Kyoto Institute of Technology, Matsugasaki Goshokaido-cho, Sakyo-ku, Kyoto, 606-8585, Japan \\ ${ }^{\mathrm{b}}$ National Institute of Advanced Industrial Science and Technology, Tsukuba, 305-8568, Japan \\ ${ }^{c}$ Kobe University,1-1 Rokkodai, Nada, Kobe, 657-8501, Japan
}

\begin{abstract}
We proposed a technique for recording sound wave in microscopic field using digital holography and experimentally demonstrated motion-picture phase imaging of a sound wave. Frequency of the sound wave was obtained by applying Fourier transformation to the temporal change of pixel value extracted from the reconstructed phase motion picture. By using a parallel phase-shifting digital holography, we succeeded in recording a sound wave, emitted from a speaker vibrating at a frequency of $1,000 \mathrm{~Hz}$, propagating in a microscopic field.
\end{abstract}

Keywords: digital holography, three-dimensional imaging, microscopy, sound field

\section{Introduction}

Observation of sound is useful for various acoustic designs. In general, a sound field is measured by microphone array [1], Schlieren photography [2], and so on. Although a microphone array can measure near field sound, high resolution measurement is difficult. Schlieren photography is a simple way to visualize a sound field. However, it is difficult to quantitatively visualize a sound field. Digital holography [3] can overcome the difficulties. It has been reported visualizing sound waves from a tuning fork [4], a human voice [4] and the sound emitted from a whistle [5]. However, visualizing a sound field in a microscopic field has not been reported yet. In this paper, we propose a technique for recording sound wave in microscopic field using digital holography. Also, we recorded phase motion picture of a sound field emitted from a speaker in a microscopic field. Frequency of the sound is obtained from the phase motion picture. We experimentally demonstrated a sound field imaging by a digital holographic microscope.

\section{Parallel phase-shifting digital holography}

In digital holography, an interference image generated by an object wave and a reference wave is recorded with an image sensor. The complex amplitude distribution, that consists of the intensity and the phase, of the object wave at arbitrary depth is reconstructed by a computer. A digital holographic microscopy is an application of digital holography to a microscopy. A digital holographic microscopy is a technique that achieves motion-picture quantitative phase imaging in a microscopic field. In particular, a parallel phase-shifting digital holographic microscopy [6] is a technique that can obtain a motion picture of the high-quality complex amplitude images of specimens moving at high speed. Then, we employ parallel phase-shifting digital holographic microscopy to visualize a sound field. Figure 1 shows a schematic of the principle of parallel phase-shifting digital holography. The technique records the information of the multiple phase-shifted holograms required for phase-shifting digital holography [7] in a single hologram. The information recorded every $2 \times 2$ pixels of an image sensor by space-division multiplexing of the holograms. The complex amplitude distribution of the object wave on the image sensor is obtained by applying the calculation used in the phase-shifting interferometry to the multiple holograms. 
Multiple phase-shifted holograms

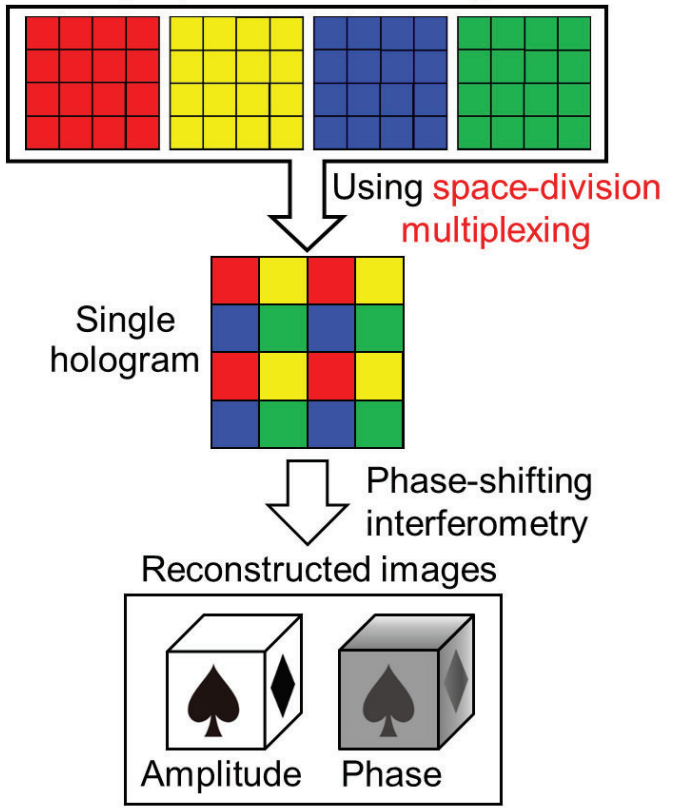

Fig.1. Schematic of the principle of parallel phaseshifting digital holography.

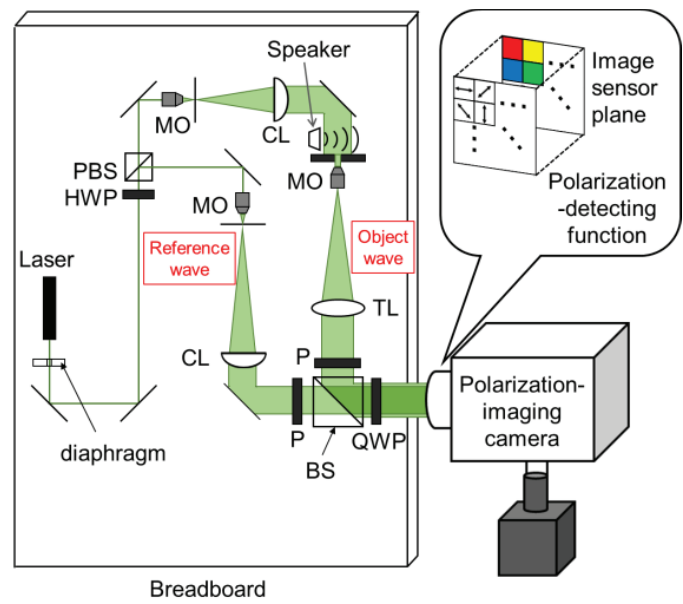

Fig. 1. Schematic of the parallel phase-shifting digital holographic microscope. PBS, polarizing beam splitter; HWP, half wave plate; QWP, quarter wave plate; P, polarizer; $\mathrm{MO}$, microscope objective; TL, tube lens.

\section{Experiment}

We experimentally demonstrated motion-picture phase imaging of a sound field in a microscopic field by a parallel phase-shifting digital holographic microscope. The schematic of the microscope is shown in Figure 2. We recorded a sound having a single frequency of 1,000 $\mathrm{Hz}$ and emitted from a speaker. We used JBL GO as the speaker and put the speaker before the microscope objective as shown in Fig. 2. A Nd:YVO4 laser operated at $532 \mathrm{~nm}$ is used as a light source. We used Photron FASTCAM SA2-P as a polarization imaging camera. The lateral magnification of the microscope was 10 . The recording frame rate, the shutter speed, and the total recording time were $3,000 \mathrm{fps}, 0.167 \mathrm{~ms}$, and $0.1 \mathrm{~s}$, respectively. Figure 3 shows the reconstructed phase image. Figure 4(a) shows temporal change of pixel value of a single point of the reconstructed phase image. As seen from this figure, it can be confirmed that the phase changes at a constant frequency. By subtracting the local average phase, we can eliminate low-frequency components then the signal component is enhanced as shown in Figure 4(b). To confirm that the phase changing at a constant frequency is due to sound, Fourier transformation was employed to the temporal change of phase. The spectrum distribution of Fig. 4(b) was shown in Figure 5. A peak was obtained at $1,000 \mathrm{~Hz}$. Thus, it was confirmed that the phase changing at a constant frequency was due to sound. Figure 6 shows the spectrogram of Fig. 4(b). The spectrogram shows temporal change of the spatial frequency. It was confirmed that the frequency is constant. Thus, we experimentally demonstrated the sound field imaging by the parallel phase-shifting digital holographic microscope.

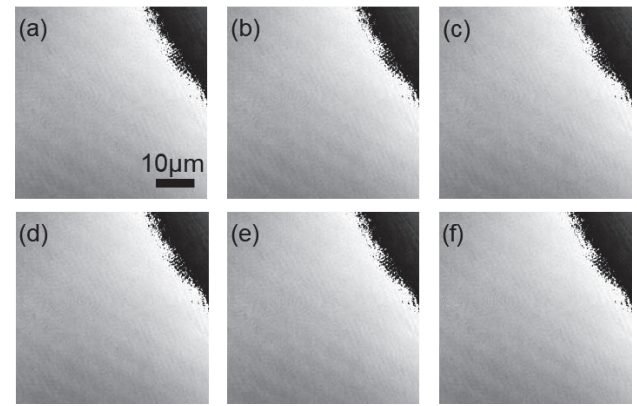

Fig. 3. Reconstructed phase images of the sound emitted from the speaker. (a) $0.0 \mathrm{~ms}$. (b) $0.33 \mathrm{~ms}$. (c) $0.67 \mathrm{~ms}$. (d) $1.0 \mathrm{~ms}$. (e) $1.33 \mathrm{~ms}$. (f) $1.67 \mathrm{~ms}$. 

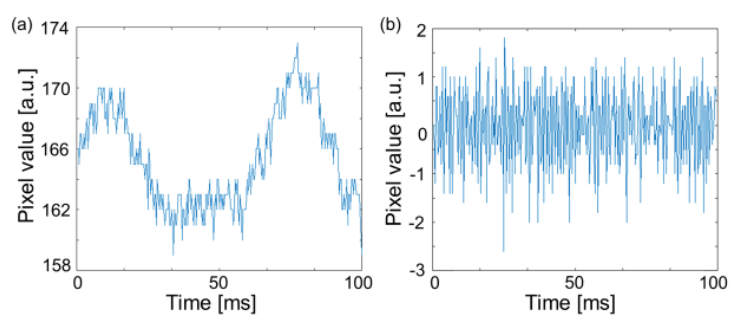

Fig. 4. Temporal change of the pixel value of the phase image. (a) Temporal change of pixel value of the reconstructed phase image. (b) Temporal change of the pixel value after subtracting the local average phase.

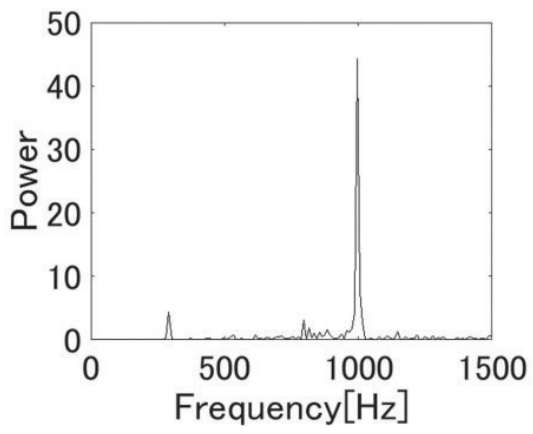

Fig. 5. Spectrum of Fig.4(b).

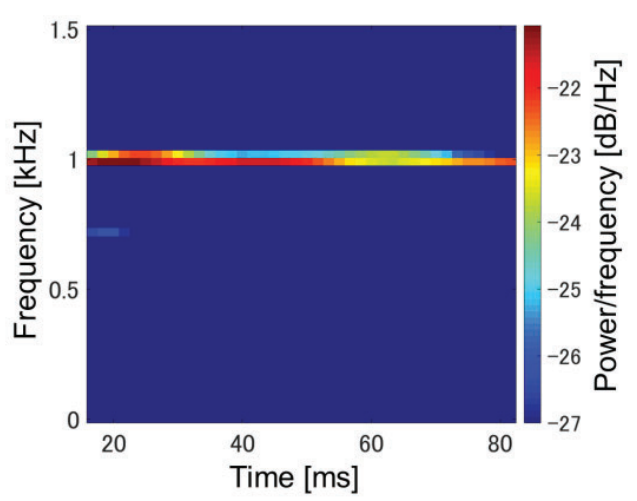

Fig. 6. Spectrogram of Fig.4(b).

\section{Conclusion}

We have proposed a technique for recording sound wave in microscopic field using digital holography. Also, we have succeeded in experimentally demonstrating phase imaging of sound field in a microscopic field by the parallel phase-shifting digital holographic microscope.
This study is expected in various applications such as measurement of shock wave, the effect of sound waves on microorganisms, analysis of sound waves in acoustics and so on.

This study was partially supported by Japan Society for the Promotion of Science (JSPS) KAKENHI Grant-in-Aid for Challenging Exploratory Research.

\section{References}

[1] A. Henning et al., Exp. Fluids 45, 1073 (2008).

[2] M. Raffel, Exp. Fluids 56, 60 (2015).

[3] J. W. Goodman and R. W. Lawrence, Appl. Phys. Lett. 11, 77 (1967).

[4] O. Matoba et al., Opt. Lett. 39, 6549 (2014).

[5] K. Ishikawa et al., Opt. Lett. 41, 991 (2018).

[6] Y. Awatsuji et al., Appl. Phys. Lett. 85, 1069 (2004).

[7] I. Yamaguchi and T. Zhang, Opt. Lett. 22, 1268 (1997).

[8] K. Shimizu et al., Proc. SPIE 10711, 1071115, (2018). 


\title{
Glucose concentration measurement with enzymatic modified cuvette by heterodyne interferometer
}

\author{
You-Xuan Li ${ }^{\mathrm{a}}$, Pei-Chen Lai ${ }^{\mathrm{a}}$, Yu-Han Chen ${ }^{\mathrm{a}}$, Cheng-chih Hsu ${ }^{\mathrm{a}, \mathrm{d}}$, Chyan-Chyi Wu ${ }^{\mathrm{b}}$, Ching-Liang \\ Dai $^{\mathrm{c}}$ \\ ${ }^{a}$ Department of Photonics Engineering, Yuan Ze University, 135, Yuan-Tung Road, Chung-Li, \\ 32003 Taiwan \\ ${ }^{\mathrm{b}}$ Department of Mechanical and Electromechanical Engineering, Tamkang University, New Taipei \\ 25137, Taiwan \\ ${ }^{\mathrm{c}}$ Department of Mechanical Engineering, National Chung Hsing University, Taichung 402, Taiwan \\ d Department of Electro-Optical Engineering, National United University, Miaoli City 36063, Taiwan
}

\section{Abstract}

In this study, an enzymatic modified cuvette was fabricated and adopt to measure the glucose concentration with heterodyne interferometer. Based on the optical configuration, the calibration of the modified cuvette was no longer required. The results indicated that the resolution of the proposed method was approximated of $3 \mathrm{mg} / \mathrm{dl}$. The calibration curve showed high linearity within the dynamic range of $0 \sim 500 \mathrm{mg} / \mathrm{dl}$.

Keywords: Glucose concentration, Heterodyne interferometer, Glucose oxidase, Enzyme, Glucose sensor.

\section{Introduction}

Diabetes has been described as a silent epidemic disease resulting from its discovery and need to more efforts to achieve efficient diagnostic and treatment [1]. Excess glucose in the blood, which causes multiple complications such as cardiovascular disorders [2] and kidney failure [3]. Therefore, there is a critical need for personal monitoring of the blood glucose level.

Optical and electrochemical analyses have been widely investigated for glucose concentration measurement. Most of the optical method [4-5] depended on the colorchanged in an indicator that reflects the glucose concentration. To quantify the glucose level of those methods [4-5], the spectroscopic detection with high wavelength resolution is essential requirement and not to suitable for personal use. Otherwise, electrochemical analyses applied amperometric method directly converted the electrochemical signal into corresponding glucose concentration [6-7]. Unfortunately, the calibration of the resistance of the electrode in the personal glucometer is difficult.

In this study, we proposed an alternative glucose concentration measurement method which integrated

*1hsu920624@gmail.com; phone 88634638800 enzymatic modified cuvette (EMC) into a heterodyne interferometer. Based on the high sensitivity and wide dynamic response of the heterodyne interferometer, proposed method can provide the sensitivity of $0.01^{\circ}$ for $1 \mathrm{mg} / \mathrm{dl}$ glucose concentration variation and resolution of $3 \mathrm{mg} / \mathrm{dl}$.

\section{Principles}

The optical configuration of the proposed method was showed in figure 1 . The heterodyne light source was divided into two paths, one was reference path and another was test path. For the reference path, the light beam will propagate: $\mathrm{BS} \rightarrow \mathrm{P} \rightarrow \mathrm{EMC}_{(\text {air })} \rightarrow \mathrm{AN}_{\mathrm{r}} \rightarrow \mathrm{D}_{\mathrm{r}}$. Meanwhile, the testing path will be $\mathrm{BS} \rightarrow \mathrm{EMC}_{(\text {solution) }} \rightarrow \mathrm{AN}_{\mathrm{t}} \rightarrow \mathrm{D}_{\mathrm{t}}$. Therefore, the reference and testing signals detected by $\mathrm{D}_{\mathrm{r}}$ and $\mathrm{D}_{\mathrm{t}}$ can be written as:

$$
\begin{aligned}
& I_{r}=\frac{1}{2}\left[1+\cos \left(\omega t+\phi_{B S}+\phi_{P}+\phi_{G O_{x}}+\frac{2 \pi}{\lambda} d\right)\right], \\
& I_{t}=\frac{1}{2}\left[1+\cos \left(\omega t+\phi_{B S}+\phi_{G O_{x}}+\frac{2 \pi}{\lambda} n d\right)\right],
\end{aligned}
$$

where $\phi_{B S}, \phi_{P}$, and $\phi_{G O x}$ are the phase difference coming from $\mathrm{BS}, \mathrm{P}$, and $\mathrm{GO}_{\mathrm{x}}$ respectively; $n$ is the refractive 
index of sample and $d$ is the path length. Before and after the sample injection into the EMC, the phase differences between reference path and testing path can be written as:

$$
\begin{aligned}
& \phi_{\text {before }}=-\phi_{P}, \\
& \phi_{\text {after }}=\frac{2 \pi}{\lambda}(n-1) d-\phi_{P} .
\end{aligned}
$$

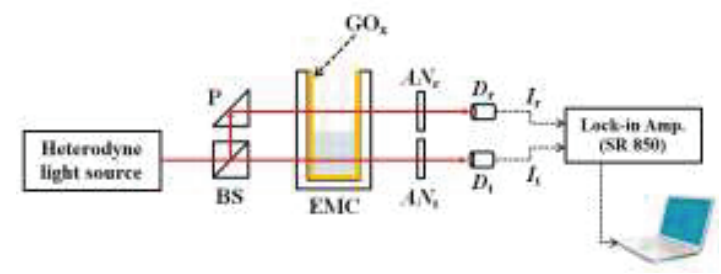

Figure 1. Measurement configuration of the proposed method. P: prism; $\mathrm{BS}$ : beam splitter; $\mathrm{GO}_{\mathrm{x}}$ : glucose oxidase; EMC: enzymatic modified cuvette; AN: analyzer.

To compare $\phi_{\text {after }}$ and $\phi_{\text {before }}$, the phase difference coming from the chemical reaction between $\mathrm{GO}_{\mathrm{x}}$ and glucose can be written as:

$$
\phi=\phi_{\text {after }}-\phi_{\text {before }}=\frac{2 \pi}{\lambda}(n-1) d .
$$

It obvious that the phase difference $\phi$ is related to the refractive index of sample as the path length is obtained in advance. Theoretically, the phase difference $\phi$ is proportional to the refractive index changed of the sample and also to the concentration variation of testing sample [8]. In practice, the phase difference $\phi$ can be obtained immediately upon sending the testing and reference signals into the lock-in amplifier.

\section{Results and Discussion}

The inner surface of a commercial cuvette was modified by GOx followed previous work [9]. The $\mathrm{pH}$ value and concentration of GOx were controlled at 7.5 and $10 \mu \mathrm{g} / \mathrm{ml}$. The glucose solutions of various concentrations were prepared by dissolving glucose anhydrous in a distilled water $(0,10,50,300,400$, and $500 \mathrm{mg} / \mathrm{dl}$ ). The glucose-free solution (distilled water only) was used to blank control.

Figure 2 indicated the dynamic response of the proposed method. It is obviously that the phase variation strongly related to the glucose concentration. The response time were within $5 \mathrm{~s}$ for all samples.

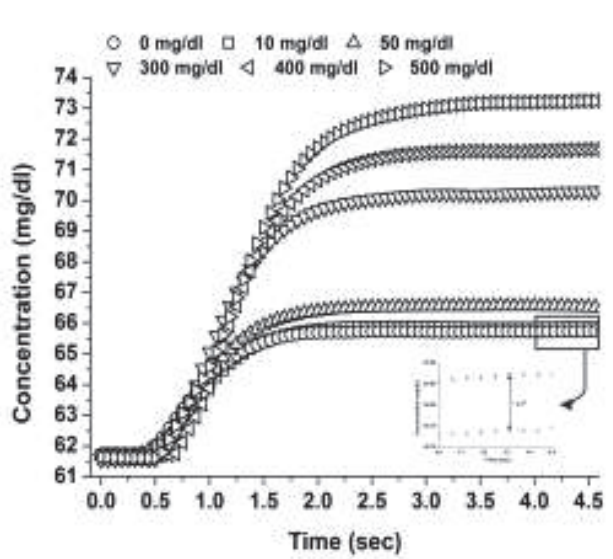

Figure 2. Dynamic response curves of the proposed method.

Figure 3 showed the calibration curve of the proposed method. The slope of calibration curve indicated the sensitivity of the proposed method and can be approximated of $0.01^{\circ}$ for $1 \mathrm{mg} / \mathrm{dl}$ glucose concentration variation.

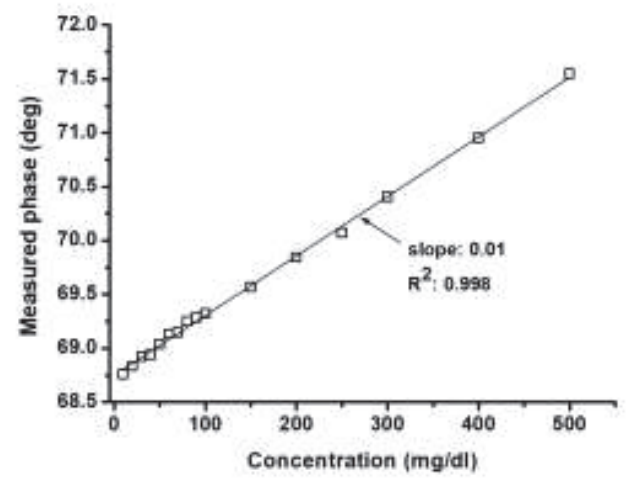

Figure 3. Calibration curve of the proposed method.

The resolution $|\Delta c|$ of the proposed method can be obtained by calculating the ratio of the phase error $|\Delta \phi|$ of the proposed method to the slope $s$ of the calibration curve, which is represented by

$$
|\Delta c|=\frac{|\Delta \phi|}{s} .
$$

According to the optical configuration and error analysis [10], the phase error $|\Delta \phi|$ of the proposed method can be approximated of $0.03^{\circ}$. Therefore, the 
resolution of the proposed method can be approximated of $3 \mathrm{mg} / \mathrm{dl}$.

\section{Conclusion}

This study proposed an enzymatic modified cuvette and integrated into a heterodyne interferometer. Based on the optical configuration of the measurement system, the calibration procedure is easy and rapid. The results showed that the high sensitivity, high resolution, wide measurement range, and rapid measurement.

\section{References}

[1] Nwaneri C., "Diabetes mellitus: a complete ancient and modern historical perspective," WebmedCentral Diabetes 2015 , http://www.webmedcentral.com/article_view/4831 17 (February 2015).

[2] Appleton S. L., Seaborn C. J., Visvanathan R., Hill C. L., Gill T. K., Taylor A. W., Adams R. J., "Diabetes and cardiovascular disease outcomes in the metabolically healthy obese phenotype," Diabetes Care 36(8), 2388-2394 (2013).

[3] Wanner C., inzucchi S. E., Lachin J. M., Fitchett D., von Eynatten M., Mattheus M., Johansen O. E., Woerle H. J., Broedl U. C., Zinman B., "Empagliflozin and progression of kidney diease in type 2 diabetes," The New England Journal of Medicine 375, 323-334 (2016).

[4] Choi J., Kang D., Han S., Kim S. B., Rogers J. A., "Microfluidic networks: thin, soft, skin-mounted microfluidic networks with capillary bursting valves for chrono-sampling of sweat," Advance Healthcare Materials 6(5), 1601355 (2017).

[5] Connelly J. T., Rolland J. P., Whitesides G. M., "Paper machine for molecular diagnostic," Analytical Chemistry 87(15), 7595-7601 (2015).

[6] Mishra R. K., Vinu Mohan A. M., Soto F., Chrostowski R., Wang J., "A microneedle biosensor for minimally-invasive transdermal detection of nerve agents," Analyst 142(6), 918-924 (2017).

[7] Kim J., Jeerapan I., Imani S., Cho T. N., Bandodkar A., Cinti S., Mercier P. P., Wang J., "Noninvasive alcohol monitoring using a wearable tattoo-based iontophoretic-biosensing system," ACS Sensors 1(8), 1011-1019 (2016).
[8] Lin T. C., Lu Y. L., and Hsu C. C., "Fabrication of glucose fiber sensor based on immobilized GOD technique for rapid measurement," Optics Express 18, 27560-27566 (2010).

[9] Hsu C. C., Lan W. L., Chen Y. C., Sung Y. Y., "Precision measurement of the refractive index and thickness of GOx thin film at different pH levels," Optics and Lasers in Engineering 51, 388-393 (2013).

[10] Su D. C., Hsu C. C., "Method for determining the optical axis and (ne, no) of a birefringent crystal," Applied Optics 41(9), 3936-3940 (2002). 